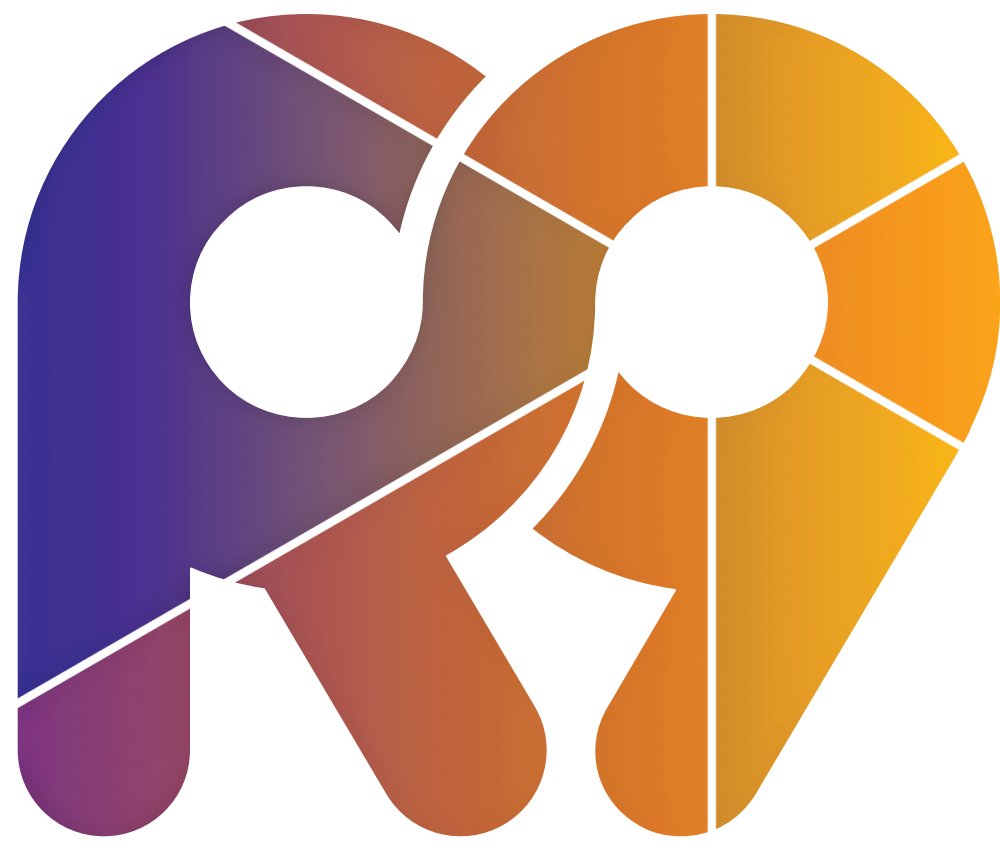

NINTH INTERNATIONAL CONFERENCE ON RADIATION IN VARIOUS FIELDS OF RESEARCH

June 14 - 18, 2021 | Hunguest Hotel Sun Resort | Herceg Novi | Montenegro

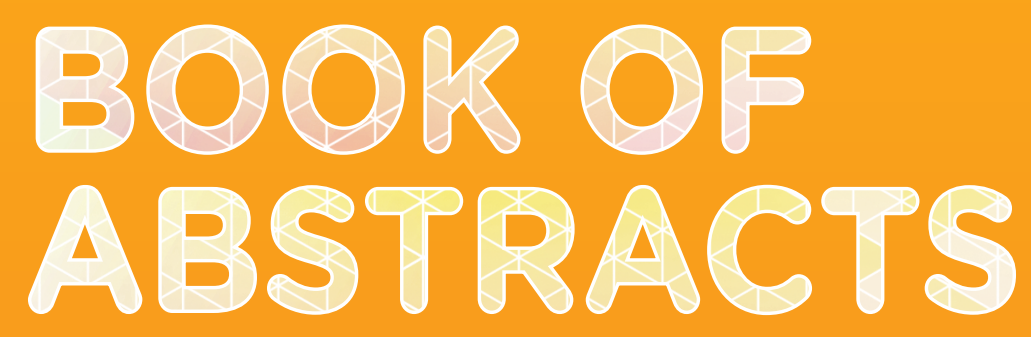




\section{TABLE OF CONTENTS}

Click on the title of the abstract to access it

\section{BIOCHEMISTRY}

Šaćira Mandal, Adlija Čaušević, Sabina Semiz, Plasma leptin concentrations and lipid profile in Bosnian healthy and Type 2 diabetic individuals

Iliana Koleva, Veselin Ivanov, Yanka Karamalakova, Galina Nikolova, C-reactive protein and ROS promote chronic ochratoxin-induced $\mathrm{H}$ nephrotoxicity in mice 2

Yanka Karamalakova, Ekaterina Georgieva, Tzvetelin Georgiev, Bilyana Tacheva, Muhammad Akram, Veselin Ivanov, Galina Nikolova, Lemna minor L. extract ameliorates the intracellular C-reactive protein and ROS levels in progressive bleomycin-induced pulmonary fibrosis

Anna A. Oleshkevich, Specific features of change in enzymate activity in the acoustic field 4

Aušra Nemeikaitė-Čènienė, Violeta Jonušienė, Lina Misevičienè, Audronė Marozienė, Narimantas Cenas Čènas, The use of rate constants of flavoenzyme-catalyzed singleelectron reduction of nitroaromatics in the analysis of their cytotoxicity mechanisms ........ 5

Aušra Nemeikaitè-Čènienė, Jonas Šarlauskas, Violeta Jonušienè, Lina Misevičienė, Audronè Marozienè, Mindaugas Lesanavičius, Narimantas Čènas, QSARs in prooxidant aerobic cytotoxicity of heteroaromatic $N$-oxides: Role of flavoenzyme-catalyzed singleelectron reduction

Safija Herenda, Edhem Hasković, Denis Hasković, Ena Hasković, Inhibitory effect of terpenes on acetylcholinesterase activity

\section{BIOENGINEERING}

Svetozar Stoichev, Avgustina Danailova, Ivan Iliev, Inna Sulikovska, Velichka Strijkova, Kirilka Mladenova, Tonya Andreeva, Effect of graphene oxide incorporation into polymeric matrix of polysaccharide multilayered microcapsules 8

\section{BIOINFORMATICS}

Ivan P. Scheglakov, Anna A. Oleshkevich, Yulia L. Gordeeva, Comparative analysis of indicators of homeostasis in domestic animals (dogs)

\section{BiOMATERIALS}

Milica Jeremic Knezevic, Aleksandar Knezevic, Daniela Djurovic Koprivica, Aleksandra Maletin, Bojana Milekic, Tatjana Puskar, Analysis of elastic properties of dental impression materials. 
Oksana Kadyseva, Vladimir Bykov, Olga Strelova, Alexander Grebenyuk, Determination of physical and chemical parameters that can be used to predict the hemostatic activity of chitosan without conducting in vivo experiments

Monica Vidotto, Timor Grego, Ina Erceg, Božana Čolović, Maja Dutour Sikirić, Nadica Maltar-Strmečki, Effects of saline solution and simulated body fluid on ion substituted hydroxyapatites EPR spectra.

Tatiana Tozar, Mihai Boni, Simona Nistorescu, Mihail Lucian Pascu, Angela Staicu, Hydrogels photo-crosslinking by $266 \mathrm{~nm}$ pulsed laser radiation.

Roxana Cristina Popescu, Mihaela Tudor, Diana Savu, Nanoparticle radiosensitization experiments at IFIN-HH, Romania

\section{BIOMEDICAL ENGINEERING}

Tihomir Georgiev, Zhivko Bliznakov, Nikolay Dukov, Kristina Bliznakova, Use of $\mathrm{X}$-ray contrast materials with inkjet printing for creation of breast anthropomorphic phantoms

Mirjana R. Cvijović, Milorad M. Murić, Vladica V. Čudić, Ana P. Čudić, Mirjana M. Petrović, Marina M. Kremić, Multi-stage filters improved solution for air pollutants accelerating cardiopulmonary diseases in Zlatibor District 16

\section{BIOMEDICINE}

Gordana Šošić, Mirjana Varjačić, Nikola Jović, Tanja Novaković, Predictors of the basal frequency of micronucleus, nucleoplasmic bridges and nuclear buds at the beginning of pregnancy in a population of healthy pregnant women and pregnant women with thrombophilia

Ingrida Domarkiene, Gabriele Zukauskaite, Ausra Matuleviciene, Justas Arasimavicius, Vaidutis Kucinskas, Laima Ambrozaityte, ADAPT - genomic initiative to analyse adaptation in the group of Lithuanian Chernobyl liquidators.

Gabrielė Žukauskaitė, Ingrida Domarkienė, Aušra Matulevičienè, Evelina Marija Vaitėnienè, Justas Arasimavičius, Vaidutis Kučinskas, Laima Ambrozaitytė, Genome association study of the Lithuanian Chernobyl catastrophe liquidators indicates potentially protective alleles for multifactorial diseases.

Darko Grujičić, Ljiljana Mirkov, Kristina Virijević, Nikola Pivljaković, Dragoslav Marinković, Olivera Milošević-Djordjević, Homozygous-recessive characteristics (HRCs) and biochemical parameters as biomarkers of cardiovascular disease risk .20

Elena I. Yarygina, Vita Laga, Anna A. Oleshkevich, Antibody isolation technique from chicken egg yolks

Aleksandra Maletin, Milica Jeremic-Knezevic, Daniela Djurovic-Koprivica, Tanja Veljovic, Bojana Ramic, Bojana Milekic, Jovana Laban-Terzija, Forming of peri-implant soft tissue in dental implant prosthetic

Iliana Koleva, Yanka Karamalakova, Maria Angelova, Galina Nikolova, C-reactive protein levels and oxidative stress markers as predictors of spontaneous preterm birth in women from South Bulgaria 
Iliana Koleva, Maria Angelova, Galina Nikolova, Yanka Karamalakova, Relationship between maternal inflammatory and oxidative disturbances in spontaneously declared preterm births.

Daniela Djurovic Koprivica, Tatjana Puskar, Milica Jeremic Knezevic, Bojana Milekic, Aleksandra Maletin, Jovana Laban Terzija, Materials for taking impressions in implant prosthetic dentistry....

Dejana Milojevic, Aleksandar Milojevic, The use of bioidentical hormone therapy and the quality of women's health after the reproductive period

Donika Ivanova, Zvezdelina Yaneva, Investigation of the dependence between ROS production and apoptosis induction in leukemia lymphocytes after the application of redox-modulators in low concentrations

Igor Belyaev, Biological and health effects of non-ionizing radiation that is used by $5 \mathrm{G} . . .28$

\section{BIOPHYSICS}

Yuriy Volkov, Boris Roshchin, Aleksandr Nuzhdin, Viktor Asadchikov, Yuriy Ermakov, Alexey Tikhonov, Veijo Honkimäki, Structural effects in phospholipids monolayers in presence of poly-D-lysines

Nia Petrova, Svetla Todinova, Stefka Taneva, Dimitrina Koleva, Petar Petrov, Violeta Velikova, Sashka Krumova, Functional and structural consequences of foliar application of single-walled carbon nanotubes on photosynthetic membranes 30

Avgustina Danailova, Sashka Krumova, Ariana Langari, Regina Komsa-Penkova, Georgi Golemanov, Ina Gyosheva, Emil Gartchev, Kameliya Kercheva, Alexey Savov, Stefka Taneva, Svetla Todinova, Thermal stability of red blood cell and plasma proteins derived from women with miscarriages: Microcalorimetric approach

Anna A. Oleshkevich, Svetlana Komarova, Photo-redox effect of alkaline hydrolysates of wool from small cattle

Ariana Langari, Svetla Todinova, Velichka Strijkova, Tonya Andreeva, Sashka Krumova, Stefka Taneva, Regina Komsa-Penkova, Georgi Golemanov, Galya Georgieva, Ultrastructural alteration of platelets and erythrocytes derived from women with spontaneous abortions: Atomic force microscopy study

Arvi Freiberg, Kõu Timpmann, Margus Rätsep, Liina Kangur, Color-tuning in $\mathrm{Ca}^{2+-b i n d i n g ~}$ photosynthetic bacteria

Charilaos Xenodochidis, Milena Draganova, Milena Georgieva, George Miloshev, Plamen Zagorchev, Development of a system for in vitro irradiation of biological objects with electromagnetic radiation

Mina Raileanu, Mihaela Bacalum, Liviu Craciun, Tiberiu Esanu, Ioana Porosnicu, 3D tumour spheroids for the prediction of the effects of radiation and treatment with antimicrobial peptides 36

\section{CANCER RESEARCH}

Irma Ivette Santana Martinez, Kristof Zarschler, Stephan Holger, Michael Bachmann, Bispidines as versatile bifunctional chelators for ${ }^{64} \mathrm{Cu}$-labeling of biomolecules. 
Wojciech Szymanowski, Kamila Buzun, Anna Szymanowska, Agnieszka Gornowicz, Roman Lesyk, Krzysztof Bielawski, Anna Bielawska, The cytotoxic potential of a novel 2-thioxo-4thiazolidinone derivative used with anti-HER2 antibodies in AGS gastric cancer cells .......38

Anna Szymanowska, Kamila Buzun, Wojciech Szymanowski, Robert Czarnomysy, Agnieszka Gornowicz, Mariusz Mojzych, Anna Bielawska, Krzysztof Bielawski, The proapoptotic effect of novel pyrazolo[4,3-e]tetrazolo[4,5-b][1,2,4]triazine derivatives in HT-29 colon cancer cells

Kamila Buzun, Agnieszka Gornowicz, Robert Czarnomysy, Roman Lesyk, Krzysztof Bielawski, Anna Bielawska, The pro-apoptotic effect of new 2-thioxo-4-thiazolidinone derivative Les-3331 on MCF-7 and MDA-MB-231 cell lines

Dominika Radomska, Dominik Radomski, Robert Czarnomysy, Krzysztof Bielawski, Anticancer activity of novel selenoesters in MCF-7 and MDA-MB-231 human breast cancer cells.

Dominik Radomski, Dominika Radomska, Anna Szymanowska, Robert Czarnomysy, Krzysztof Bielawski, Anna Bielawska, Evaluation of pro-apoptotic activity of novel derivatives of 1,2,4-triazine sulfonamides in DLD-1 colon cancer cells .

Donika Ivanova, Zvezdelina Yaneva, Empirical modelling of the antiproliferative activity of palbociclib on leukemia lymphocytes.

Milena Georgieva, Zlatina Gospodinova, Milena Keremidarska-Markova, Trayana Kamenska, Galina Gencheva, Natalia Krasteva, PEGylated nanographene oxide-based nanoparticles as smart nanocarriers for colon cancer photothermal therapy 44

Milena Georgieva, Zlatina Gospodinova, Trayana Kamenska, Galina Gencheva, Natalia Krasteva, PEGylated graphene oxide nanoparticles as a tool for the regulation of cancer cell invasion and growth....

Margarita Pustovalova, Lina Alhaddad, Taisia Blokhina, Andreyan Osipov, Sergey Leonov, Irradiation-surviving NSCLC cells exhibit partial EMT-program activation and altered DNA damage response depending on their p53 status .... 46

Katia R.B. Melo, Fabio D. Nascimento, Mariana S. Araujo, Marcela M.P. Alvarez, Renan P. Cavalheiro, Carlos M.V. Palomino, Maria Aparecida S. Pinhal, Helena B. Nader, Ivarne L.S. Tersariol, Guacyara Motta, Interaction of plasma kallikrein-kinin system proteins with breast cancer cells

Simona-Cristina Anghelina, Mihaela Temelie, Liviu Crăciun, Diana Savu, Investigating proton irradiation effects on glioblastoma cell lines 48

\section{COVID 19}

Nikolay Kokodii, Ihor Krasovskyi, Stanislav Pogorelov, Vladimir Timaniuk, Dmytro Kuzmin, Katerina Ivanova, About the mathematical models of the Covid-19 epidemic...... 49

Roisin Brennan, Michaela Davis, An investigation into occupational burnout among radiographers in Ireland due to the COVID-19 pandemic and the strategies used to cope with it

Mitko Mitev, Multiple thromboses as late complications in a patient with pneumonia caused by the COVID-19 infection. 
Jozef Sabol, Otakar Mika, Use of previous experience and knowledge from protection against CBRN to protection against COVID-19 pandemic

\section{ENVIRONMENTAL CHEMISTRY}

Inga Zinicovscaia, Alexey Safonov, Evaluation of biosorption and bioaccumulation capacity of cyanobacteria Arthrospira (Spirulina) Platensis for radionuclides

Dmitrii Grozdov, Inga Zinicovscaia, Nikita Yushin, Metal removal from zinc-containing effluents using Shewanella xiamenensis biofilm formed on zeolite

Gheorghe Sarbu, Constantin Popa, On numerical solution of nonlinear parabolic multicomponent convection-diffusion problems 55

Ivana Rasic Misic, Snezana Tosic, Emilija Pecev-Marinkovic, Jelena Mrmosanin, Stefan Petrovic, Testing the effectiveness of removing toxic elements by clinoptilolite .56

Grzegorz Boczkaj, Kirill Fedorov, Elvana Cako, Zhila Honarmandrad, Advanced treatment of water and wastewater by hybrid technologies utilizing cavitation phenomenon..... 57

Jędrzej Proch, Przemysław Niedzielski, Multi-mode Sample Introduction System (MSIS) as an interface between high performance liquid chromatography (HPLC) and inductively coupled plasma optical emission spectrometry (ICP OES) in arsenic speciation analysis .58

\section{EnVironmental Physics}

Jan Kisiel, Kinga Polaczek-Grelik, Agata Walencik-Łata, Katarzyna Szkliniarz, Underground laboratories in the Baltic Sea Region - EUL Project

Dragana Baturan, Selena Samardžić, Robert Lakatoš, Uranija Kozmidis Luburić, Measurements of the effective illumination in the conference hall and the questionnaire survey about the lighting perceptions of participants..... .60

Konstantin Lovchinov, Gergana Alexieva, Miroslav Petrov, Hristo Nitchev, Nikolay Tyutyundzhiev, Sensitivity of electrochemically deposited $\mathrm{ZrO}_{2}$ layers on a quartz resonator 61

Yuriy Kylivnik, Svyatoslav Vuchkan, Ihor Syika, Hanna Vasylyeva, Olexander Sych, Purification of aqueous solutions from strontium ions by natural and synthetic sorbents under increased radiation background 62

Margarita Kuqali, Dhurata Kuqi, Ramadan Firanj, A preliminary study of the electromagnetic field exposure of the population living near high voltage power lines of 110 $\mathrm{kV}$ and $220 \mathrm{kV}$ in Tirana City, Albania.

Mirjana Radenkovic, Dusan Topalovic, Tatjana Trtic Petrovic, Visa Tasic, Srboljub Stankovic, Vojislav Stanic, Evaluation of black carbon in fine atmospheric particulate matter on various filter types by multi-wavelength light absorption technique

A.S. Doroshkevich, V.A. Alexandrov, A.I. Lyubchyk, B.L. Oksengendler, A.K. Kirillov, T.A. Vasilenko, T.E. Konstantinova, A.V. Shilo, N.N. Nikiforova, A.A. Nabiyev, M.A. Balasoui, D. Mardare, C. Mita, V.I. Bodnarchuk, D. Chicea, S.I. Lyubchyk, Advanced adsorption energy generating systems based on polarized substrates 65 
Konstantin Vergel, Inga Zinicovscaia, Nikita Yushin, Assessment of atmospheric deposition in Central Russia, Vladimir and Yaroslavl regions, using moss biomonitors, neutron activation analysis and GIS technologies

Emina Ramic, Jasna Huremovic, Sabina Zero, Estimating the impact of acid pH solution on the metal desorption from lichen Evernia prunastri (L.) Ach.

Dušan Topalović, Mirjana Radenković, Viša Tasić, Vojislav Stanić, Predrag Božović, Optimization of the source apportionment solution using the rotational tools in US EPA PMF 5.0 software 68

\section{FOOD SAFETY AND HEALTH}

Yanka Karamalakova, Ekaterina Georgieva, Veselin Ivanov, Galina Nikolova, EPR characterization of the ROS-associated toxicity in gamma irradiated Lemna minor L....... 69

Emilija Pecev-Marinković, Snežana Tošić, Aleksandra Pavlović, Ivana Rašić Mišić, Jelena Mrmošanin, Stefan Petrović, ICP-OES method for determination of selected elements in infant formulas and cereals based baby food 70

Ulyana Bliznyuk, Polina Borshchegovskay, Timofey Bolotnik, Victoria Ipatova, Alexander Konstantinov, Michael Yemelyanov, Igor Rodin, Felix Studenikin, Oleg Khmelevsky, Alexander Chernyaev, Oleg Shinkarev, Dmitry Yurov, Research of changes in the chemical properties of food products as a result of exposure to ionizing radiation at different doses 71

Ivana D. Rašić Mišić, Snezana B. Tošić, Aleksandra N. Pavlović, Emilija T. PecevMarinković, Jelena M. Mrmošanin, Health risk estimation of potentially toxic elements in complementary fruit-based food 72

Daria Petrukhina, Ibragim Medzhidov, Vladimir Kharlamov, Mariya Pomyasova, Oxana Tkhorik, Sergei Gorbatov, Valentin Shishko, Aleksandr Shesterikov, Victor Tikhonov, Alexander Tikhonov, Igor Ivanov, Experience in the modernization of a non-thermal plasma source for pre-sowing seed treatment.....

Ralitsa Mladenova, Katerina Aleksieva, Nikolay Solakov, Kamelia Loginovska, Study of gamma radiation effects on free radicals generation and antioxidant potential of beebread.

Egon Andoni, Enkeleda Ozuni, Majlind Sulce, Dorjana Beqiraj, Albana Munga, Pellumb Zalla, Evaluation of heavy metals in different tissues of red mullet

\section{HEALTH AND ENVIRONMENT}

Tatiana Plakhova, Maria Vyshegorodtseva, Anastasia Konyukhova, Irina Seregina, Roman Svetogorov, Anna Romanchuk, Vladimir Ivanov, Stepan Kalmykov, Nanoceria solubility in simulated biological systems: The key mechanisms and analogy to actinide dioxides........ 76

Agna Nadine Maia de Sena Carvalho, João Pedro, Gabriel Luís Miguel, Physical-chemical and isotopic characterization $(\delta 18 \mathrm{O}, \delta 2 \mathrm{H}$ and $3 \mathrm{H})$ of surface and groundwater in the Luanda Region for the integrated management of water resources

Irena Stoilova, Radostina Madzharova, Maya Krastanova, Physical factors in the treatment of occupational spine injuries .78

Vedada Čeljo, The role of pharmacists in the health care team in hospital practice 79 
Galya Toncheva, Magdalena Boradjieva, Antoaneta Saravanska, Vidka Divarova, Kiril Gavazov, An extraction-chromogenic system for iron(III) based on 4-nirocatechol ...........80

Galina Nikolova, Yanka Karamalakova, Veselina Gadjeva, Nasko Vasilev, Ivan Fasulkov, Pencho Penchev, Yordanka Ilieva, Oxidative stress parameters affecting fertility of Bulgarian Murrah buffalos depending on age and season

Yanka Karamalakova, Galina Nikolova, Veselina Gadjeva, Nasko Vasilev, Ivan Fasulkov, Pencho Penchev, Yordanka Ilieva, Real-time biomarkers affecting fertility of Bulgarian Murrah buffalos depending on age and season

Agna Nadine Maia de Sena Carvalho, Maria Lúcia Pereira Antunes, Jose Antonio Silva Souza, Tran Hoang Mai, Do Quoc Hung, Evaluation of the presence of radionuclides of natural origin (NORM) and rare earths in the Brazilian Red Mud .83

Vera Vukanić, Horizontal distribution and abundance of Copepoda in the shore in the immediate vicinity of shellfish farms in the inner bays of the Boka Kotorska Bay - Southern Adriatic 84

\section{MATERIALS SCIENCE}

Assemgul Kissabekova, Alma Dauletbekova, Irina Kudryavtseva, Aleksandr Lushchik, Sergey Omelkov, Magdalena Baran, Yaroslav Zhydachevskyy, Cathodoluminescence of $\mathrm{Bi}^{3+}$-doped lanthanide niobates.

Ekaterina Filippova, Plasma modification of thin polycaprolactone films .86

Ivana Lončarević, Ljuba Budinski Petković, Zorica Jakšić, Slobodan Vrhovac, Percolation in irreversible deposition of mixtures on a triangular lattice

Andrei Racu, Marius Stef, Irina Nicoara, Daniel Vizman, Gabriel Buse, Photoluminescence and Judd-Ofelt analysis of $\mathrm{ErF}_{3}-$ doped $\mathrm{BaF}_{3}$ crystals

Melise Karatay Kutman, Ozge Kozgus Guldu, Coskun Harmansah, F. Zumrut Biber Muftuler, Synthesis of $\mathrm{Fe}_{3} \mathrm{O}_{4}$ magnetic nanoparticles by using green chemistry

Alexey Buzmakov, Artem Grigorev, Yuri Volkov, Victor Asadchikov, Optimization of the Xray microtomography reconstruction quality and acquisition time in the study of biocompatible scaffolds

Aleksei Krasnikov, Magdalena Baran, Assemgul Kissabekova, Aleksandr Lushchik, Volodymyr Tsiumra, Leonid Vasylechko, Svetlana Zazubovich, Yaroslav Zhydachevskyy, Origin of luminescence centers in $\mathrm{Bi}^{3+}$-doped niobates. 91

Denis Zolotov, Victor Asadchikov, Alexey Buzmakov, Marina Chukalina, Irina Dyachkova, Anastasia Ingacheva, Dmitry Nikolaev, Igor Schelokov, The development of spectral tomography using the crystal-analyzer scheme. . .92

Nataliya Krutyak, Dmitry Spassky, Dina Deyneko, Vitali Nagirnyi, Energy transfer and luminescence properties of novel $\mathrm{Na}_{3.6} \mathrm{Y}_{1.8-\mathrm{x}}\left(\mathrm{PO}_{4}\right)_{3}$ :Dy ${ }^{+}$phosphor.

Dmitry Spassky, Nataliya Krutyak, Alisa Ukhanova, Fedor Fedyunin, Vladimir Morozov, Nina Kozlova, Evgeniia Zabelina, Valentina Kasimova, Oleg Buzanov, Alexei Belik, Influence of temperature on the optical and luminescent properties of scintillation crystals based on GAGG:Ce 
Jelena Bijelic, Akos Kukovecz, Zvonko Jaglicic, Sugato Hajra, Manisha Sahu, Igor Djerdj, The electric and magnetic properties study of nanocrystalline triple perovskite $\mathrm{Sr}_{3} \mathrm{Co}_{2} \mathrm{WO}_{9}$ prepared by the modified sol-gel route

Jelena Tamuliene, Narimantas Čènas, Modified approach for evaluation of standard singleelectron reduction potential of nitroaromatic compounds in aqueous medium 96

Evgeni Shablonin, Anatoli Popov, Gatis Prieditis, Evgeni Vasil'chenko, Aleksandr Lushchik, Thermal annealing of structural defects in fast-neutron-irradiated $\mathrm{Al}_{2} \mathrm{O}_{3}$ single crystals.

Ivana Mladenović, Nebojša Nikolić, Jelena Lamovec, Dana Vasiljević Radović, Vesna Radojević, Influence of parameters of the pulsating current (PC) regime on morphological, structural and hardness characteristics of copper coatings electrodeposited on $\mathrm{Si}(111)$ 98

Snežana Papović, Milan Vraneš, Jovana Panić, Aleksandar Tot, Nikolet Baganj, Sanja Mutić, Jasmina Anojčić, Physicochemical characterisation of commonly used organic UV filters from cosmetic products and development of their detection by liquid chromatography UV-diode array .99

Nicoleta Florea, Mihai Badea, Cristina Burducea, Liviu-Stefan Craciun, Tiberiu-Relu Esanu, Raluca-Maria Marginean, Paul Mereuta, Constantin Mihai, Calin Mircea Rusu, Andreea-Elena Serban, Preparation and characterization of molybdenum solid targets for high current cyclotron production of medical ${ }^{99 m}$ Tc radionuclides. 100

Svetlana Gramatiuk, Anna Kurbatova, Yulia Ivanova, The clinical case of stem cell therapies for the treatment of radiation-induced ulcer 101

Andriy Kovalskiy, Roman Holovchak, Yaroslav Shpotyuk, Jozef Cebulski, Influence of irradiation conditions on optical photoresponse of chalcogenide glass thin films 102

Vojislav Jovanović, Suzana Samaržija-Jovanović, Branka Petković, Tijana Jovanović, Gordana Marković, Milena Marinović-Cincović, Vesna Teofilović, The effect of UVirradiation on the thermal stability of modified urea-formaldehyde resins with thermally activated montmorillonite .103

\section{Medical Devices}

Yuri Kovalenko, Sergii Miroshnichenko, Andrii Nevhasymyy, Compact universal digital $\mathrm{X}$-ray diagnostic system 104

Sergey Taskaev, Neutron source VITA for Boron Neutron Capture Therapy and other applications..... 105

T.Yu Zelenyak, V.A. Kinev, P.P. Gladyshev, T.N. Vershinina, A.C. Doroshkevich, P.S. Dzhumaev, R.Sh. Isayev, M. Balasoiu, A. Stanculescu, Development and RBS study of ultra-sensitive high-speed perovskite film X-ray detectors for radiation technologies ..... 106

\section{Medical Imaging}

Vera Artiko, Therapy of neuroendocrine tumors with 90Y DOTA-TATE

Sergii Balashov, Yury Kovalenko, Organizational and technical ways of providing the population with accessible and high-quality radiodiagnosis in conditions of scarce resources .108 
Josip Ivacic, Aleksandar Knezevic, Daniela Djurovic Koprivica, Aleksandra Maletin, Milica Jeremic Knezevic, Visibility of the lateral pterygoid muscle in temporomandibular disorders by magnetic resonance imaging 109

Ana Lazarova, The role of contrast-enchased computer tomography in the management of intestinal obstruction

Tatiana Kiseleva, Yulia Stepanova, Natalya Guseva, Ksenya Lugovkina, High-frequency ultrasound scanning in eyelids assessment 111

Daria Kiseleva, Yulia Stepanova, Naida Sultanova, Possibilities of sonoelastography in the evaluation of the lower third of face soft tissue changes determining the most effective method of correction 112

Yulia Stepanova, Vlada Raguzina, Tatiana Baitman, Aleksandr Gritskevich, Radiology diagnostics at the stages of extracorporal resection of a single kidney in the treatment of renal cell cancer 113

Stevan Vrbaski, Renata Longo, Adriano Contillo, Quantitative analysis of the breast tissue chemical composition based on the spectral decomposition of X-ray tomographic breast images

D. Mihaylov, V. Stoyanov, D. Thomas, M. Mitev, Various examination methods applied for the detection of pathological changes in the nasal mucosa and patency of the nasal passages in patients with the reduced sense of smell

Yulia Stepanova, Nora Arutiunian, Naida Sultanova, Alexey Kopyltsov, Radiological examination at the stages of surgical treatment of the consequences of the soft tissue augmentation with vaseline oil for aesthetic purposes 116

\section{Medical Physics}

Aslı Bozkurt, Şule Kaya Keleş, Gaye Özgür Çakal, Ayşe Kaşkaş, Dose measurement in radiotherapy using various thermoluminescence dosimeters ....

Matteo Franchini, The FOOT experiment - Light nuclei fragmentation measurements ...118

Marija Jeremic, Milovan Matovic, Dragoslav Nikezic, Dragana Krstic, Tatjana Miladinovic, Suzana Pantovic, Measurement of urinary excretion and blood clearance after peptide receptor radionuclide therapy

Carlo Mancini-Terracciano, Francesco Collamati, Riccardo Faccini, Giuseppe Iaccarino, Riccardo Mirabelli, Francesca Nicolanti, Massimiliano Pacilio, Elena Solfaroli Camillocci, Antonella Soriani, Silvio Morganti, WIDMApp (Wearable Individual Dose Monitoring Apparatus): An innovative approach for individual dose monitoring in Molecular Radiotherapy 120

Natasa Brasik, Effective dose as a reporting tool in radiology: Myth or reality . 121

Ivan Tsanev, Borislav Pavlov, Peicho Petkov, Leandar Litov, Investigation of the applicability to study the FLASH effect with protons on LINAC-4 accelerators at CERN and with carbon ions on NUCLOTRON at JINR (Dubna) 122

Maria Piziorska, Marcin Szymański, Oskar Madetko, Wioletta Ślusarczyk-Kacprzyk, Wojciech Bulski, Cyber Knife - A proposal of dosimetric audit with shane phantom....... 123 
Maria Poncyljusz, Wojciech Bulski, The differences in dose distribution caused by uncorrected rotations

Kristina Bliznakova, Yanka Baneva, Georgi Todorov, Computational breast models dedicated to contrast enhanced spectral mammography

\section{Medicine - CASE Reports}

Miona Vuletic, Milca Veličković, Aleksandar Acović, Vladimir Ristić, Tamara Ristić, Tatjana Kanjevac, A rare type of mesiodens - case report: Diagnosis and treatment....... 126

Ana Lazarova, A rare case of orbital mucocele

Rossen Hadjiev, Margarita Tankova, Margarita Taushanova, Lyubomir Damyanov, Clinical case of ovarian cancer after in-vitro fertilization: Forensic medical malpractice - clinical case and data review 128

\section{MiCROWAVE, LASER, RF, UV AND SOLAR RAdiATIONS}

Volodymyr Timaniuk, Ella Romodanova, Stanislav Pogorelov, Tatiana Dubko, Mykola Kokodii, Physical processes of the action of laser radiation on biological systems............ 129

Michaela Lastovickova, Testing femtosecond delay line for diffractive imaging and atomic, molecular and optical sciences as ELI Beamlines 130

Zsófia Szilágyi, Györgyi Kubinyi, Erika Szabó, Bertalan Pintér, Brahim Selmaoui, György Thuróczy, Genotoxicity assessment of combined exposure to WiFi and UV radiation on human keratinocytes in vitro 131

Bertalan Pintér, Zsófia Szilágyi, Erika Szabó, Györgyi Kubinyi, Yves Le Drean, György Thuróczy, The effect of intermediate frequency electromagnetic field at $22 \mathrm{kHz}$ on human fibroblast cells in vitro 132

Nina Rubtsova, Sergey Perov, Olga Belaya, 2-5G electromagnetic field chronic exposure biological effects assessment

Andra Dinache, Tatiana Tozar, Adriana Smarandache, Ionut Andrei, Mihai Boni, Mihail Lucian Pascu, Angela Staicu, The effect of laser radiation on drug emulsions 134

\section{NEUROSCIENCE}

Mihaela Temelie, Tiberiu Esanu, Liviu Craciun, Nicoleta Moisoi, Diana Savu, Analysis of DNA damage response following proton radiation exposure in an in vitro neuronal model .135

\section{NEUTRON AND HEAVY ION RADIATIONS}

Menekşe Şenyiğit, Determination of neutron flux distribution in a ${ }^{252} \mathrm{Cf}$ irradiator using GATE 136

Elizabeth Musacchio Gonzalez, Time to energy conversion method for neutron Time of Flight measurements.

Valeria Monti, Roberto Bedogni, Marco Costa, Andrea Pola, Davide Bortot, Alessandro Lega, Saverio Altieri, Single moderator active neutron spectrometer for therapeutic beam characterization in Boron Neutron Capture Therapy facilities 138 
I.A. Chepurchenko, A.S. Doroshkevich, M. Kulik, T.YuZelenyak, A.I. Madadzada, A.N. Lihachev, M.A. Balasoui, E.V. Lychagin, P. Badica, M. Stef, B. Jasinska, T.V. Phuc, L.H. Khiem, P.L. Tuan, The concept of the development and practical testing of the "Fast neutron activation analysis (FNAA)" method at the EG-5 accelerator, FLNP JINR 139

\section{NUCLEAR MEDICINE}

Milovan Matovic, Miroslav Ravlic, Vladimir Zdravkovic, Aleksandar Vukadinović, Marija Jeremić, Sanja Vraneš-Đurić, Our solution for automatic capsules filling with high activities of ${ }^{131} \mathrm{I}$ 140

Gohar Hovhannisyan, Tiruhi Bakhshiyan, Photonuclear production of 47Sc isotope 141

Gleb Aleshin, Bayirta Egorova, Lyubov Zamurueva, Sofia Khabirova, Anastasia Zubenko, Olga Fedorova, Stepan Kalmykov, Copper, zinc and bismuth complexes with new macrocyclic chelators for radiopharmaceuticals: In vitro and in vivo stability ..... 142

Milena Dimcheva, Quality control of non-imaging intraoperative gamma probes

Sofia Khabirova, Gleb Aleshin, Evgeny Anokhin, Anna Shchukina, Anastasia Zubenko, Radiolabelled strontium hexaferrite nanoparticles for radiopharmaceutical applications 144

Alexander Madumarov, Nikolay Aksenov, Gospodin Bozhikov, Consideration of reactor and photonuclear pathways to produce $195 \mathrm{mPt}$ for theranostics applications ....

Vojislav Antic, Milos Mladenovic, Mirjana Petrovic, Zivorad Savic, The concept of developing a security plan for nuclear medicine facilities in the Republic of Serbia ......... 146

Aleksandr Vasiliev, Stanislav Ermolaev, Aino Skasyrskaya, Elena Lapshina, Boris Zhuikov, Express production of medical Bi-213 using various Ac/Bi generators.

Ilya Chuprakov, Alexander Madumarov, Vladislav Rozhkov, Alexei Zhemchugov, Alexei Sabelnikov, Gospodin Bozhikov, Alexander Bodrov, Nikolay Aksenov, Production of 99mTc via ${ }^{100} \mathrm{Mo}(\gamma, \mathrm{n}){ }^{99} \mathrm{Mo}$ reaction using ${ }^{100} \mathrm{MoO}_{3}$ target at microtron $\mathrm{MT}-25$. 148

\section{Pharmaceutical Sciences}

Marina Krysko, Olga Strelova, Iuliia Slustovskaia, Hair samples' retrospective analysis for the determination of drug consumption by applying enzymatic hydrolysis methods 149

Iryna Timanyuk, Iryna Bondarieva, Volodymyr Malyi, Feasibility of introducing telepharmacy elements in low-income countries 150

Viktoria Tihonova, Anna Saushkina, Estimation of the reproducibility for the identificational method of paracetamol-based drugs 151

Anna Zhigalina, Ol'ga Strelova, Alexsandr Grebenuk, Elizaveta Cekhanskaya, The development of a certified reference material for Pharmaceutical Quality Assurance based on genistein. 152

\section{RADIATION CHEMISTRY}

Krzysztof Piechocki, Marcin Kozanecki, Kaloian Koynov, Justyna Piechocka, Diffusion in POEGMA hydrogels prepared by Radiation Induced Crosslinking Polymerization ..... 153 
Irina G. Antropova, Natalia V. Panferova, Eldar P. Magomedbekov, Reduction of silver ions using chitosan and investigation of their reactivity

Aleksandar Lazarević, Sanja Petrović, Jelena Stanojević, Dragan Cvetković, Jelena Zvezdanović, TBA-MDA test for lipid peroxidation detection induced by UV-A and UV-B irradiation of Protoporphyrin IX in SUV liposomes

155

\section{RADIATION DETECTORS}

Toshiyuki Onodera, Keitaro Hitomi, Press molded TlBr semiconductor crystals for flat panel gamma-ray imaging detectors.... 156

Alexander Macris, Kevin McKay, Cheryl Brabec, William Charlton, Sheldon Landsberger, Development of a shielding design for a boron-10 zinc sulfide silver-activated $\left[{ }^{10} \mathrm{~B} / \mathrm{ZnS}(\mathrm{Ag})\right]$ detector using radiation counting and MCNP modeling ..... .157

Conny Egozi, Francis Martinez, Brandon De Luna, Sheldon Landsberger, A preliminary investigation for the use of gamma-gamma coincidence spectrometry to determine ${ }^{239} \mathrm{Pu}$ 158

Marco Miliucci, Silicon Drift Detectors system for high precision nuclear physics spectroscopy measurements: The SIDDHARTA-2 experiment at the DAФNE Collider ... 159

Jovana Knezevic, Dusan Mrdja, Kristina Bikit, Istvan Bikit, Jaroslav Slivka, Sofija Forkapic, Predrag Kuzmanovic, Corrections of HPGe detector efficiency curve due to true coincidence summing by program EFFTRAN and Monte-Carlo simulations 160

Abdulrahman Albarodi, M. Bilge Demirköz, Aziz Ulvi Çalışkan, Uğur Kılıç, Deniz Orhun Boztemur, Ahmet Baran Can, Egecan Karadöller, Mehmet Güntekin Kabuli, Levent Tavacioğlu, Design and characterization of a compact radiation monitor for space rockets 161

Daniel Nolasco Altamirano, Teodoro Rivera Montalvo, Olivia Amanda García Garduño, Alejandro Alonso Sotolongo, Juan Zárate Medina, Characterization of $\mathrm{GdAlO}_{3}$ as beta radiation detector. 162

Juan Román-Raya, Catalina A. Rodríguez-Cano, Isidoro Ruiz-García, Alberto J. Palma, Miguel A. Carvajal, Damián Guirado, Angular dependence of Light-Dependent Resistors irradiated by $6 \mathrm{MV}$ photon beams 163

Isidoro Ruiz-García, Pablo Escobedo, Juan Román-Raya, Stefan Illić, Damian Guirado, Alberto José Palma, Miguel Ángel Carvajal, Temperature compensation in dose-rate measurements based on commercial photodiodes using a modified reader unit 164

Roberto Bedogni, Alessandro Lega, Alessandro Calamida, Alessia Cemmi, Ilaria Di Sarcina, Semiconductor detectors for monitoring radiation processing. 165

Abisai Alejandro Barrera Angeles, Jesús Gerardo Gutiérrez Márquez, Teodoro Rivera Montalvo, Daniel Nolasco Altamirano, Marco Antonio Ugalde Valdés, Alfredo Morales Hernández, Daniel Barrera Hernández, $\mathrm{LaAlO}_{3}: \operatorname{Pr}^{3+}$ powder TL response for electron beam. 166

\section{RADIATION EFFECTS}

Tamara Fuciarelli, David Rollo, Radiation exposure causes developmental alterations in size and shape of wings and structures associated with song production in male crickets (Acheta domesticus). 
Svetlana Sosnina, Pavel Okatenko, Mikhail Sokolnikov, Malignant neoplasms in the offsprings of female Mayak workers

Marko Gerić, Jelena Popić, Goran Gajski, Vera Garaj-Vrhovac, Interventional radiology unit teams occupationally exposed to low-dose ionizing radiation: A cytogenetic view.... 169

Riccardo Pagliarello, Ilaria Di Sarcina, Elisabetta Bennici, Giuseppe Ferrara, Luca Nardi, Eugenio Benvenuto, Alessia Cemmi, Silvia Massa, Use of gamma radiation for the characterization of biofortified tomato plants intended for space cultivation 170

Tsveta Angelova, Christo Angelov, Svetla Gateva, Gabriele Jovtchev, Does altitude have an effect on pigment content of wild growing plants in Rila Mountain? 171

Giulio Metelli, Ilaria Di Sarcina, Marco Garegnani, Giuseppe Ferrara, Alessia Cemmi, Luca Nardi, Eugenio Benvenuto, Ground-based preliminary tests to simulate space microgravity and radiation effects on plants in the seed to seedling transition 172

Yevgenya Stepanova, Igor Kolpakov, Vitaliy Vdovenko, Valentina Kondrashova, Victor Zygalo, Olena Leonovych, Role of glutathione S-transferase M1 (GSTM1), P1 (GSTP1), and T1 (GSTT1) gene polymorphisms in the development of the bronchial hyperreactivity in children residing at radiologically contaminated territories of Ukraine . .173

Ingars Reinholds, Elina Pajuste, Liga Avotina, Guntars Vaivars, Einars Sprugis, Rossi Mikko, Kettunen Heikki, Effect of electron beam radiation on the mechanical and thermomechanical properties of proton exchange membranes .174

Rudolfs Janis Zabolockis, Elina Pajuste, Liga Avotina, Gunta Kizane, Investigation of neutron-irradiated beryllium pebble oxidation in dry and humid air at elevated temperatures for LOVA/LOCA estimation and corresponding safety protocol development 175

Beata Ziaja-Motyka, Electronic, structural and magnetic transitions in X-ray irradiated solid materials . .176

Veronika Deffner, Sabine Hoffmann, Peter Scholz-Kreisel, Nora Fenske, Uncertainties in radiation exposure estimates of the German uranium miners' cohort 177

Thiwanka Weerakkody, Champa Dissanayake, A.G. Chandrapala, Maheshika Kalpage, Thilaka Attanayaka, Indrani Dissanayake, Kasun Binduhewa, Terrestrial background radiation study in Bathalegoda, North-Western province of Sri Lanka.... 178

\section{RADIATION MEASUREMENTS}

Cassidy Reis, Sheldon Landsberger, Optimization of neutron activation analysis of rareearth elements

Davide Bortot, Andrea Pola, Stefano Pasquato, Davide Mazzucconi, Anna Brusa, Carlo Chiesa, Fabio Zanellati, Leonardo Baldassarre, Radiometric characterization for the decommissioning of the Scanditronix MC17 medical cyclotron of the Italian National Cancer Institute of Milano 180

Davide Mazzucconi, Davide Bortot, Andrea Pola, Stefano Agosteo, Valeria Conte, Experimental characterization of a wall-less TEPC for nanodosimetric measurements ....181

Edyta Dąbrowska-Szewczyk, Pawel Kukolowicz, Anna Zawadzka, Piotr Kowalczyk, Rafal Podgorski, Michal Wojasiński, Tomasz Ciach, Tomasz Zawistowski, Influence of beam spoiler and air gap on dose distribution in build-up region for X6 MV static field 182 
Nuray Yavuzkanat, Modeling Geant4 based GATE simulation for total gamma efficiency of phoswich type detector system

Dragana Jordanov, Laslo Nadjdjerdj, The new matrix method for deriving counting rate equations describing coincidence summing of gamma and $\mathrm{X}$-rays for germanium spectrometers .184

Katarzyna Szkliniarz, Kinga Polaczek-Grelik, Agata Walencik-Łata, Jan Kisiel, Measurements of natural background radiation in the underground laboratories of the BSUIN and EUL projects 185

Mirjana Đurašević, Aleksandar Kandić, Igor Čeliković, Zorica Obradović, Tamara Milanović, Ivana Vukanac, Comparison of coincidence summing correction factors calculated by EFFTRAN and GESPECOR software 186

Monia Vadrucci, Fabio Borgognoni, Alessia Cemmi, Ilaria Di Sarcina, Beatrice D’orsi, Luigi Picardi, Characterization and applications of electron and X-ray beams of the REX facility 187

Dorottya Jakab, Zsuzsa Endrődi, Tamás Pázmándi, László Tósaki, Péter Zagyvai, Evaluation of the precision of radioactive deposition measurements 188

Ciprian Cosar, Aurelian Luca, Catalin Matei, Maria Sahagia, Monte Carlo simulation methods for gamma-ray metrology 189

Peter Necz, Balázs Gyulai, József Krausz, György Thuroczy, Broadband and band-selective measurements of radiofrequency EM field with drone system around $5 \mathrm{G}$ base station.... 190

Anna Pantya, Tamás Pázmándi, Laura Horváth, Péter Zagyvai, Optimization of thyroid measurements with Monte Carlo simulation in in-vivo monitoring 191

Ulyana Bliznyuk, Polina Borchegovskaya, Felix Studenikin, Alexander Chernyaev, Vadim Khankin, Verification method to increase uniformity of foodstuff irradiation 192

Lidiya Karl, Tatiana Lashchenova, Albert Marenny, Monitoring studies to assess the condition of drinking water supply sources in potentially radon-hazardous areas by specific gross alpha activity.... 193

Yordanka Karakirova, Velislava Yordanova, Optimizing the size of cylindrical sucrose solid state/EPR dosimeters for high energy ionizing radiation

Agnieszka Szymak, Piotr Moska, Konrad Tudyka, Grzegorz Poręba, Grzegorz Adamiec, The internal dose rate in quartz grains: Implications for luminescence dating 195

Robert Csaba Begy, Codrin Fabian Savin, David-Karoly Sule, The effect of water gases on radon measurements in electrostatic cell radon monitor 196

Tomas Nemeš, Dušan Mrđa, Ištvan Bikit, Random coincidence and angular correlation corrections in the sum-peak method using Monte Carlo simulations - A new approach ...197

Katarina Karadzic, Radiation monitoring system 198

Predrag Kuzmanović, Igor Čeliković, Sofija Forkapić, Nataša Todorović, Leposava Filipović Petrović, Dušan Mrđa, Jovana Knežević, Determination of radon emanation for phosphogypsum produced in Serbia.... 199 


\section{RADIATION ONCOLOGY}

Florina-Gianina Elfarra, Radu Mutihac, Ileana Radulescu, Thyroid scintigraphy using Technetium-99m 200

Mimoza Ristova, Cancer in the countries of the SEE (Balkans) region and the prospective of the Particle Therapy Center - SEEIIST 201

\section{RAdiation Physics}

Lisa Centofante, Antonietta Donzella, Aldo Zenoni, Matteo Ferrari, Michele Ballan, Stefano Corradetti, Fabio D'Agostini, Giordano Lilli, Mattia Manzolaro, Alberto Monetti, Luca Morselli, Daniele Scarpa, Alberto Andrighetto, Study of the ion source complex radioactive contamination in the SPES facility .202

Tomasz Wasowicz, Antti Kivimäki, Robert Richter, A competition between two- and three-body dissociation channels in photo-double-ionization of tetrahydro-2H-pyran and 3,4-dihydro-2H-pyran molecules .203

Michal Jurkowski, Tomasz J. Wasowicz, Ionization and fragmentation of the six-membered heterocycles containing oxygen - Comparative studies of electron and photon impact on the 3,4-dihydro-2H-pyran molecules 204

Roman Vernydub, Olena Kyrylenko, Oksana Konoreva, Oleksandr Radkevych, Dmytro Stratilat, Volodymyr Tartachnyk, Radiation defects in GaP, GaAsP, InGaN LEDs ...........205

Dragana Krstic, Dragoslav Nikezic, Ana Krstic, Milena Zivkovic, Marija Jeremic, The application of voxel and ORNL phantoms to the internal and external dosimetry..... 206

Ioana Lalau, Mihail-Razvan Ioan, Modelling the radioluminescence of alpha particles in the air by the Monte Carlo method 207

\section{RAdiation Protection}

Mikhail Osipov, Evgeniy Fomin, Mikhail Sokolnikov, Computed tomography in population living close to the nuclear production complex: Cohort profile. .208

Ileana Radulescu, Mihail-Razvan Ioan, Aurelian Luca, Evaluation of various atmospheric radon monitors

Ioanna Kyriakou, Ioanna Tremi, Alexandros Georgakilas, Dimitrios Emfietzoglou, Microdosimetric investigation of the radiation quality of low-medium energy electrons using Geant4-DNA 210

Teodora Todorova, Iviyana Ivanova, Mariya Yovkova, Maria Dimitrova, Petya Parvanova, Martin Dimitrov, Stephka Chankova, DNA repair capacity of Saccharomyces cerevisiae after single and combined treatment with zeocin and different concentrations of Clinopodium vulgare L. extract 211

Teodoro Rivera, Eduardo Uruchurtu, Rocio Toledo, Ricardo Garcia, Isidro Gomez, Radiation dose optimization in interventional cardiology using artificial intelligence ..... 212

Amaya Sinha, Jose Parga, Sheldon Landsberger, Calculating safe handling radiation dosage limits from neutron activation analysis 213

Ganna Grygorova, Vladimir Klochkov, Svetlana Yefimova, Redox-active oxide nanoparticles improving short- and long-term survival of X-ray irradiated rats 214 
Behnam Khanbabaee, Annette Röttger, Rolf Behrens, Stefan Röttger, Sebastian Feige, Oliver Hupe, Hayo Zutz, Paula Toroi, Paul Leonard, Liset de la Fuente Rosales, Pete Burgess, Vincent Gressier, Jose Luis Gutierrez Villanueva, Rodolfo Cruz Suárez, Dirk Arnold, Support for a European Metrology Network on reliable radiation protection: Gaps in radiation protection metrology

Na Hye Kwon, Dong Oh Shin, Jin Sung Kim, Dong Wook Kim, Sang Hyoun Choi, A survey of the current status in the management of radioactive wastes from linear accelerators in Korea 216

Csilla Rudas, Tamás Pázmándi, Case study with CARC software for verifying compliance with atmospheric release criteria of nuclear installations

Graeme Turkington, Kelum A. A. Gamage, James Graham, Modelling the sensitivity of semiconductor detectors for in-situ monitoring of strontium-90 contamination at nuclear decommissioning sites 218

Jelena Stanković Petrović, Marko Krajinović, Nikola Kržanović, Miloš Živanović, Andrea Kojić, Predrag Božović, TLD-10o post-irradiation fading characteristics according to IEC 62387:2020 standard. 219

Monica Vidotto, Emanuela Bortolin, Nadica Maltar-Strmečki, An Optically Stimulated Luminescence study of costal sand as a potential fortuitous dosimeter 220

Nataliya Uzlenkova, Maryna Kornet, Nataliya Skorobogatova, Olexandr Brazhko, Alevtyna Kryvko, Olena Maslennikova, Mykhailo Zavhorodnii, S-heteryl modified cysteamine protects total body irradiation-induced hematopoietic system injury in rats 221

Jozef Sabol, Ten years of the Fukushima nuclear accident: Global impact of a lesson learnt. 222

\section{RADIOBIOLOGY}

Nely Metlyaeva, Andrey Bushmanov, Irina Galstyan, Michail Konchalovsky, Anahit Davtyan, Olga Shcherbatykh, Features of individual psychological adaptation and periods of staged hematological syndrome in two patients with severe acute radiation sickness and moderate acute radiation sickness and acute leukemia who suffered from the Chernobyl accident (22 years and 30 years of observation) 223

Viktoria Panfilova, Olga Kolganova, Olga Chibisova, The effect of proton and gamma irradiation on the behavior, learning ability and pregnancy in rats .224

Svetlana V. Belkina, Marianna D. Pronkevich, Inhibition of cell recovery from densely ionizing radiation by copper sulfate.... 225

Polina Kuptsova, Galina Zhurakovskaya, Olga Pereklad, Marianna Pronkevich, Participation of molecule excitation in the biological effects of ionizing radiation............226

Anna Rzianina, Gennady Mytsin, Karine Voskanyan, Victor Gaevskiy, Enhancement of Yirradiation genotoxicity in human lung carcinoma A 549 cells in the presence of gold nanoparticles

Anzhelika Melnikova, Luidmila Komarova, Research on the combined effects of radiation and chemotherapy on tumor cells .228

Albena Staynova, Dimka Georgieva, Ljubomira Popova, Rositsa Hristova, Cytogenetic follow-up study for over 4 years of three individuals accidentally exposed to ${ }^{60} \mathrm{Co}$ in Bulgaria 
Nevena Aneva, Nora Kostova, Ilonka Ivanova, Ljubomira Popova-Hadjiiska, Rositsa Hristova, Effect of curcumin on proteasome activity in irradiated or non-irradiated lymphocytes. .230

Ilonka Ivanova, Hristo Hristov, Hristo Tsonev, Rositsa Hristova, Evaluating change in genomic frequency of translocations in 20 patients exposed to low-dose X-ray irradiation during routine vertebroplasty. 231

Nora Kostova, Dimka Georgieva, Albena Staynova, Ljubomira Popova, Ilonka Ivanova, Rositsa Hristova, Does curcumin protect cellular DNA against $\gamma$-ray induced damage? 232

Ekaterina Koryakina, Marina Troshina, Raisa Baykuzina, Vladimir Potetnya, Alexey Solovev, Sergey Koryakin, Cytogenetic effects of protons at low doses 233

Marina Troshina, Ekaterina Koryakina, Vladimir Potetnya, Aleksey Solovev, Vladimir Pikalov, Sergey Koryakin, Cytogenetic effects in mammalian cells irradiated with carbon SOBP beam.

Andreyan Osipov, Nelly Babayan, Natalia Vorobyeva, Bagrat Grigoryan, Anna ChigasovaGrekhova, Margarita Pustovalova, Sofya Rodneva, Yuriy Fedotov, Gohar Tsakanova, Rouben Aroutiounian, DNA double-strand break repair efficiency in cancer cells exposed to laser-driven ultrashort electron beams 235

Goran Gajski, Mirta Milić, Marko Gerić, Marijana Nodilo, Željka Knežević, Natko Beck, Vera Garaj-Vrhovac, Saveta Miljanić, Mariá Ranogajec-Komor, Đurđica Milković, Blood and buccal micronuclei as biomarkers in monitoring of children exposed to diagnostic radiation in clinical settings. 236

Mirta Milić, Nada Oršolić, Nikola Borojević, Anica Horvat Knežević, Vesna Benković, Protective role of isoflurane after combined exposure with 1 or 2 Gy of ionizing radiation assessed with alkaline comet assay in vivo 237

Vesna Benkovic, Nikola Borojević, Nada Oršolić, Gordana Brozović, Anica Horvat Knežević, Mirta Milić, Combined exposure to halothane and 1 or 2 Gy ionizing radiation causes synergistic effect in DNA damage in both blood and liver of Swiss albino mice ....238

Roberta Stoica, Mihai Radu, Beatrice Mihaela Radu, Functional changes in brain microvascular endothelial cells upon low-energy accelerated proton-irradiation .239

Dmytro Ganzha, Dmytro Ganzha, Comparison of methods for assessing the effect of chronic radiation exposure on the veining of leaves of Phragmites australis (Cav.) Trin. ex Steud .240

Gina Manda, Dana Niculae, Ionela-Victoria Neagoe, Radu Serban, Dragos-Andrei Niculae, Ramona Dusman, Elena Milanesi, Maria Dobre, Hypoxia and antioxidant signaling in human colon carcinoma HCT116 cells exposed in vitro to $64-\mathrm{Cu}$...... 241

Evgeniya Blinova, Vladislav Nikiforov, Mariya Yanishevskau, Alexandr Akleyev, Genetic markers associated with the development of stochastic effects .242

Mihaela Tudor, Roxana Popescu, Mihaela Temelie, François Chevalier, Diana Savu, Direct and bystander effects of human chondrosarcoma cell line irradiated with protons .243 
Dávid Kis, Eszter Persa, Tünde Szatmári, Rita Hargitai, Ilona Barbara Csordás, Géza Sáfrány, Katalin Lumniczky, Acute and chronic health effects of extracellular vesicles after irradiation ....

Nataliya Maznyk, Tetiana Sypko, Viktor Starenkiy, Inna Gukova, Nataliia Bohatyrenko, Serhii Artiukh, Liudmyla Cherkasko, Irina Krugova, Larysa Zabobonina, Approaches for the estimation of the cytogenetic effects in lymphocytes of cancer patients due to combined radiation and chemotherapy.... 246

\section{RADIOCHEMISTRY}

Emilie Baudat, Céline Gautier, Isabelle Billard, Pascal Fichet, Optimization of Sr-90 precipitation in nitric acid using experiment design and speciation modeling for radioactive waste characterization 247

Igor Smirnov, Maria Karavan, Dynamic test of alkaline HLW processing with hydroxycalix[6]arenes based solvent .248

Nikolai Andreadi, Artem Mitrofanov, Petr Matveev, Nataliya Borisova, The role of ligand conformational mobility in the process of $\mathrm{An}(\mathrm{III}) / \mathrm{Ln}(\mathrm{III})$ extraction....

Igor Smirnov, Ahmed Hamdy Aly Harb, Igor Balantsev, Maria Karavan, Yttrium-90 separation in carbonate media by solvent extraction.... .250

Vladislav Timoshenko, Alexandr Brechalov, Yuri Ermolenko, Igor Smirnov, Fast method for studying the extraction of the main HLW components with crown ethers in new fluorine-containing diluents 251

Artem Mitrofanov, Andreadi Nikolay, Vadim Korolev, Petr Matveev, Stepan Kalmykov, Development of a DFT functional for quantum radiochemistry 252

Alexander Boyarintsev, Sergey Stepanov, Sergey Frankiv, Nikita Chervyakov, Purification of uranium(VI) from impurities of fission product surrogates by solvent extraction in the CARBEX process 253

Alexander Boyarintsev, Sergey Stepanov, Sergey Frankiv, Nikita Chervyakov, Oxidative dissolution of $\mathrm{U}_{3} \mathrm{O} 8$ in carbonate-alkaline solutions 254

Radu A. Leonte, Diana S. Cocioaba, Ramona D. Dusman, Bogdan G. Burghelea, Simona I. Baruta, Roxana M. Cornoiu, Liviu S. Craciun, Dana Niculae, Production of Cu-64 on an automated irradiation and processing system - Process validation 255

Ramona-Daniela Dusman, Diana Cocioaba, Alina Raicu, Mirela Mihon, Livia Chilug, Radu Leonte, Liviu Craciun, Dana Niculae, Production of Zr-89 radioisotope using a variable energy cyclotron (TR-19) 256

Nikolay Aksenov, Prospects and challenges of chemistry of superheavy element research at FLNR 257

Aleksandr Bodrov, Gospodin Bozhikov, Nikolay Aksenov, Yurii Albin, Radiochemical separation of heavy actinides produced in multi-nucleon transfer reactions .258

Emre Uygur, Kadriye Büşra Karatay, Emine Derviş, Cansu Kayaş, Fazilet Zümrüt Biber Müftüler, The synthesis of encapsulated radiolabeled compounds to be used for diagnosis of Parkinson's disease 


\section{RADIOECOLOGY}

Vladimir Kukarskih, Nadezhda Devi, Makar Modorov, Nadezhda Shimalina, Ludmila Mikhailovskaya, Vera Pozolotina, Effects of ionizing radiation and weather-climatic factors on radial growth of Scots pine in the East Ural Radioactive Trace .260

Olena Volkova, Vladimir Belyaev, Serge Pryshlyak, Vladimir Skyba, Parameters of ${ }^{137 C s}$ specific activity changes in the fish from the Kiev water reservoir. 261

Dmitrii Kremenchutskii, Influence of rainfall on the temporal variability of atmospheric beryllium-7 ( $7 \mathrm{Be})$ activity concentration 262

Gennady Batrakov, Dmitrii Kremenchutskii, Yuliya Kurinnaya, Influence of meteorological parameters on the temporal variability of bismuth-214 (214Bi) on atmospheric aerosols. 263

Anna Semenkova, Irina Tonyan, Victoria Krupskaya, Anna Romanchuk, Stepan Kalmykov, Fine effects governing Cs sorption onto aluminosilicates. .264

Ekaterina Tyupina, Artem Pryadko, Daria Marchenko, Polina Parshina, Pavel Kozlov, Features of application and durability of metallic silver on bentonite and silica aerogel for obtaining radioiodine getters. .265

Damir Borković, Ines Krajcar Bronić, Andreja Sironić, Jadranka Barešić, Comparison of sampling and measurement methods for atmospheric ${ }^{14} \mathrm{C}$ activity 266

Natallia Kuzmina, Mikhail Zhemzhurov, Development of the decommissioning concept of the specialized enterprise for radioactive waste management UE "Ekores" in the Republic of Belarus .267

Ludmila Mikhailovskaya, Vera Pozolotina, Makar Modorov, Vladimir Kukarskih, Valentina Guseva, Zinaida Mikhailovskaya, Nadezhda Shimalina, Oleg Tarasov, Accumulation of ${ }^{90} \mathrm{Sr}$ by Pinus sylvestris and Betula pendula in the zone of radioactive contamination (East Ural Radioactive Trace, Russia). .268

Nemeer Padiyath, Prasoon Raj, Diana Francis, Soil-to-plant transfer factors in arid regions using stable isotopes of caesium and strontium in local vegetables .269

Mihajlo Vićentijević, Dubravka Vuković, Vujadin Vuković, Marija Pavlović, Jasna Kureljušić, Dragan Živanov, Branislava Slavata, RH analysis of natural radionuclides in animal feed and animal feed additives 270

Robert Csaba Begy, Codrin Fabian Savin, David-Karoly Sule, Radiological survey of spring water from post-volcanic areas in Romania

Robert Csaba Begy, Codrin Fabian Savin, Szabolcs Kelemen, Daniel Veres, Octavian Liviu Muntean, Cristian Valeriu Malos, Tibor Kovacs, Investigation of the effect of anthropogenic land use on the Pănăzii Lake (Romania) catchment area using Cs-137 and Pb-210 radionuclides 272

Prasoon Raj, Nemeer Padiyath, Yacine Addad, Diana Francis, Baseline soil-to-plant transfer of NORM radionuclides in Abu Dhabi date-palms 273

Olga Komissarova, Tatiana Paramonova, Natalia Kuzmenkova, Leonid Turykin, Transfer from radioactively contaminated soil and accumulation of ${ }^{137} \mathrm{Cs}$ in agricultural crops with edible belowground part 


\section{RADIOLOGY}

Aleksandr Khalikov, Natalya Mamedova, Fedor Pak, Dose planning system for the proton beam therapy complex

Natalya Kuzora, Aleksandr Khalikov, Natalya Mamedova, Djan Karlin, Proton beam therapy complex at $1000 \mathrm{MeV}$ proton beam 276

Viktor Myakynkov, Results of the radiology monitoring of breasts in women with gynecological malignant tumors

Călin Mircea Rusu, Mihai Straticiuc, Mihaela Bacalum, Mihai Radu, Monte Carlo simulations of biological samples irradiation using a new setup at the 3 MV Tandetron ${ }^{\mathrm{TM}}$ of IFIN-HH 278

Živorad Savić, Katarina Savić, Sofija Savić, Mirjana Petrović, Vojislav Antić, Diagnosis of non-traumatic hemorrhages in the brain parenchyma. 279

\section{RADIOTHERAPY}

Ella Moreau, Eugen Andreiadis, Vincent Blet, Development of functionalized resins for the purification of innovative radiopharmaceuticals 280

Svitlana Yefimova, Pavel Maksimchuk, Kateryna Hubenko, Alexander Sorokin, Antioxidant behavior of $\mathrm{GdYVO}_{4}: \mathrm{Eu}^{+}$nanoparticles under X-ray and UV irradiation: Proposed mechanism 281

Iryna Bespalova, Pavel Maksimchuk, Kateryna Hubenko, Alexander Sorokin, Svetlana Yefimova, Creation of complexes of "scintillation nanoparticles $\mathrm{LaF}_{3}: \mathrm{Tb}^{3+}$-Rose Bengal" for X-ray activated PDT .282

Mikołaj Tarchalski, Paweł Kukołowicz, Optical system for quick and precise assessment of mechanical isocenter of accelerators .283

Grigorii V. Merzlikin, Sergey V. Akulinichev, Ivan A. Yakovlev, Comparison of the results of calculations using GEANT4 and SRNA .284

Julya Zuenkova, Lev Izurov, Multimodal approach in treatment of skin cancer ..... .285

Zoran Stefanovski, Petar Chakalaroski, Violeta Klisarovska, Determination of tissue volume in $100 \%$ isodose for application with brachytherapy cylinder .286

Kinga Polaczek-Grelik, Aneta Kawa-Iwanicka, Comparison of dose distribution verification methods for high-precision radiotherapy .287

Gaia Franciosini, Patrizia De Maria, Micol De Simoni, Marta Fischetti, Michela Marafini, Massimiliano Pacilio, Damiana Rubeca, Alessio Sarti, Angelo Schiavi, Adalberto Sciubba, Vincenzo Tombolini, Marco Toppi, Giacomo Traini, Antonio Trigilio, Vincenzo Patera, Prostate cancer FLASH therapy treatments with electrons of high energy: A feasibility study .288

Luiza Garaeva, Vladimir Burdakov, Eva Kuus, Andrey Volnitskiy, Alina Garina, Hau Nhan Tran, Georgy Andreev, Andrey Lubinskiy, Konstantin Shabalin, Victor Ezhov, Dmitry Lebedev, Nicolay Verlov, Andrey Konevega, Tatiana Shtam, Boron compound as a sensitizer to proton radiation for cancer cells in vitro .289 
Petar Chakalaroski, Violeta Klisarovska, Zoran Stefanovski, Case report of a renal cell carcinoma skin metastasis treated locally with contact brachytherapy .

\section{RADON AND THORON}

Agata Kowalska, Piotr Maciejewski, Hydrogeochemical background of ${ }^{222} \mathrm{Rn}$ in groundwaters from fore-sudetic monocline area in Poland

Robert Lakatoš, Sofija Forkapić, Selena Samardžić, Kristina Bikit-Schroeder, Aleksandra Mihailović, Jordana Ninkov, Assessment of geogenic radon potential based on granulometric analysis of soil in Vojvodina region .292

Ivana Vukanac, Igor Čeliković, Gordana Pantelić, Miloš Živanović, Jelena Krneta Nikolić, Comparison of indoor radon measurement methods ..... .293

Dusica Spasic, Ljiljana Gulan, High indoor radon concentration in residential houses....294

Ana Sofia Silva, Maria de Lurdes Dinis, Characterization of radioactivity in thermal springs and the implementation of the European Directive 2013/59/Euratom .295

\section{OTHER TOPICS}

Eni Bushi, Klejda Hudhra, Andreas Kakouris, Delina Xhafaj, Using experts' consensus to evaluate the Readiness Factors of Lean Implementation in Healthcare 296

Vladyslav Seminko, Pavel Maksimchuk, Ganna Grygorova, Yuri Malyukin, $\mathrm{Re}^{3+-}$ doping and $\mathrm{pH}$ effect on the redox activity of nanoceria $\left(\mathrm{CeO}_{2-\mathrm{x}}\right)$.

Ibrahim Alrammah, Preliminary criticality safety assessment for a nuclear fuel production facility. 298

Mirjana Đurašević, Zorica Obradović, Katarina Rajković, Achieved results in radiation protection education of the Center for Permanent Education INN "Vinča”. .299

Kateryna Hubenko, Pavel Maksimchuk, Svetlana Yefimova, Controlled redox-activity of small orthovanadate nanocrystals .300

Pavlo Maksymchuk, Kateryna Hubenko, Vladyslav Seminko, Svetlana Yefimova, Pro-oxidant action of preliminarily UV-activated orthovanadate nanocrystals................ 301

Aida Šapčanin, Emina Ramić, Selma Korać, Mirsada Salihović, Spices as acid-base indicators in volumetric analysis 302

Mikhail Zhemzhurov, Natallia Kuzmina, Environmentally appropriate technologies for the final disposal of radioactive waste of the Belarusian NPP 303

Evgeny Domarov, Nikoly Kuksanov, Denis Vorobiev, Michail Golkovskii, Yurii Golubenko, Sergy Fadeev, Alexy Korchagin, Rustam Salimov, Alexy Semenov, ELV series accelerators and their applications in radiation technological processes 304

Paweł Czerski, Izabela Chmielewska, Jarosław Rachubik, ILC for ${ }^{90} \mathrm{Sr}$ determination in feedstuffs

Gordana Markovic, Milena Marinović-Cincović, Vojislav Jovanović, Suzana SamaržijaJovanović, Vesna Teofilović, Marija Marković, Jelena Pavličević, Jaroslava BudinskiSimendić, Irradiation resistance of elastomeric composites based on NR/CSM blend and waste rubber powder. 


\title{
Plasma leptin concentrations and lipid profile in Bosnian healthy and Type 2 diabetic individuals
}

\author{
Šaćira Mandal¹, Adlija Čaušević², Sabina Semiz3,4 \\ 1 University of Sarajevo, Faculty of Pharmacy, Department of Natural Sciences in Pharmacy, Sarajevo, Bosnia \\ and Herzegovina \\ 2 University of Sarajevo, Faculty of Pharmacy, Department of Biochemistry and Clinical Analysis, Sarajevo, \\ Bosnia and Herzegovina \\ 3 College of Medicine and Health Sciences, Khalifa University, Abu Dhabi, United Arab Emirates \\ 4 Faculty of Engineering and Natural Sciences, International University of Sarajevo, Sarajevo, Bosnia and \\ Herzegovina
}

\section{https://doi.org/10.21175/rad.abstr.book.2021.1.1}

Leptin is a hormone secreted from adipose tissue (AT) that plays important role in metabolism of carbohydrate, proteins, and lipids. Also, leptin has been implicated in energy homeostasis and regulation of food intake. Previous studies, demonstrated that plasma leptin improved glucose and lipid metabolisms independently of the food intake reduction. In humans, leptin concentrations are correlated with insulin resistance (IR) independently of body weight or adiposity, both in normoglycemic and in diabetes patients. Objective of this study was to evaluate the relationship between plasma leptin concentration and lipid profile in healthy and diabetic individuals. Twenty-six participants were including in this study, 13 of them were newly diagnosed and non-treated Type 2 diabetes (T2D) patients and 13 matched healthy controls. Metabolite parameters (glucose, glycated haemoglobin, lipid profile: total cholesterol, triacylglycerol, high density lipoprotein, low density lipoprotein and very low density lipoprotein) and hormone concentrations (leptin and insulin) were measured by using standard IFCC protocols and ELISA kit, respectively. Plasma leptin concentration increased significantly $(\mathrm{p}<0.001)$ in diabetic patients compared to controls. Values of other analysed biochemical parameters were highly significantly different between cases and controls $(\mathrm{p}<0.001)$, except for VLDL-cholesterol concentrations in controls ( $p>0.05$ ). Our data suggest that plasma leptin concentrations were affected by the increased levels of glucose, insulin and lipid profile in Bosnian study population. Therefore, leptin can be used as a marker of glucose and lipid control in newly diagnosed patients. 


\title{
C-reactive protein and ROS promote chronic ochratoxin-induced $\mathrm{H}$ nephrotoxicity in mice
}

\author{
Iliana Koleva, Veselin Ivanov, Yanka Karamalakova, Galina Nikolova
}

Medical Faculty, Trakia University, Stara Zagora, Bulgaria

https://doi.org/10.21175/rad.abstr.book.2021.1.2

Nephrotoxicity induced by Ochratoxin A (OTA) and ameliorating effects of Azadirachta Indica oil were investigated in mice chronically exposed for 28 days to OTA. Experimental groups were as follows: a) control; b) OTA -treated; c) Azadirachta Indica oil-treated, and d) OTA plus Azadirachta Indica oil-treated (OTA-A. indica oil). The mice in the control group were administered with only a daily oral administration of $0.9 \%$ sodium chloride solution. OTA was administered $1.25 \mathrm{mg} / \mathrm{kg}$ b.w., i.p., given in every two days. Azadirachta Indica oil was administered $120 \mathrm{mg} / \mathrm{kg}$ b. w. i.p., in every two days. A. indica (120 mg/kg b.w., i.p.) administered $2 \mathrm{~h}$ before OTA-administration. By the end of the chronic OTA-nephrotoxicity experiment, no mortality was observed in the study groups. Levels of C-reactive protein (CRP) and reactive oxygen species (ROS) were examined in renal tissue by ELISA assay and electron paramagnetic resonance spectroscopic method. OTA-exposure resulted in significant increases in C-RP $(\mathrm{p}<0.05)$ and ROS levels $(\mathrm{p}<0.04)$ compare to controls, which is associated with an increased risk of chronic kidney disease.

The results obtained in the groups $A$. indica and the combination of $A$. indica + OTA with the oxidative-protective action inhibited OTA-induced nephrotoxicity by statistically significantly reduced values of reactive protein $(\mathrm{p}<0.000)$, ROS production $(\mathrm{p}<0.005)$, and endogenous antioxidant activation. Renal damage was evaluated using CRP assays and ROS products analysis. We showed that $A$. indica preserved renal function and decreased renal oxidative damage. Therefore, $A$. indica oil prophylaxis may be an interesting strategy for the prevention of nephrotoxicity.

Keywords: Nephrotoxicity, Ochratoxin A, Azadirachta Indica oil, CRP, ROS 


\title{
Lemna minor L. extract ameliorates the intracellular C-reactive protein and ROS levels in progressive bleomycin-induced pulmonary fibrosis
}

\author{
Yanka Karamalakova', Ekaterina Georgieva', Tzvetelin Georgiev², \\ Bilyana Tacheva ${ }^{3}$, Muhammad Akram ${ }^{4}$, Veselin Ivanov5, Galina Nikolova ${ }^{1}$ \\ 1 Chemistry and Biochemistry Department, MF, Trakia University, Stara Zagora, Bulgaria \\ 2 Physiology, Pathophysiology and Pharmacology Department, MF, Trakia University, Stara Zagora, Bulgaria \\ 3 Medical Physics, Biophysics and Radiology Department, MF, Trakia University, Stara Zagora, Bulgaria \\ 4 Eastern Medicine Department, Government College University, Faisalabad, Pakistan \\ 5 Neurology, Psychiatry and Disaster Medicine Department, MF, Trakia University, Stara Zagora, Bulgaria
}

\section{https://doi.org/10.21175/rad.abstr.book.2021.1.3}

The protective effect of Lemna minor L. (L. minor) roots extract to Bleomycin-Induced progressive pulmonary fibrosis (BLM-PF) in mice was investigated. The LME (at $200 \mathrm{mg} / \mathrm{mL}$ concentration) antioxidant capacity was quantified by catalase-like activity (CAT), superoxide dismutase-like activity (SOD), total antioxidant capacity (TAC), DPPH absorption (98\%), and DPPH radical-scavenging activity (97.3\%), by using spectrophotometrical and EPR analysis. The progressive model (29 days) was used to investigate the BLM-PF; after 16 days of BLM-administration, the PF was registered. Pulmonary toxicity was induced by intraperitoneal injection of animals once daily with BLM (0.069 U/mL; $0.29 \mathrm{U} / \mathrm{kg} \mathrm{BW} ; \mathrm{n}=12 \mathrm{IRC} / \mathrm{b}$ mice) for 4 weeks. The L. minor was administered once a day, 4 weeks, $2 \mathrm{~h}$ prior $(200 \mathrm{mg} / \mathrm{mL}$; $0.341 \mathrm{mg} / \mathrm{kg} /$ day; in $\mathrm{n}=6 \mathrm{IRC} / \mathrm{b}$ mice $)$. The 4 groups were as follows: 1) control group- tap to water and normal food; 2) BLM-administration; 3) L. minor - administration; 4) L. minor protection + BLM-administration.

The physiological status and behavior of animals were monitored daily and on the 3oth day the mice were sacrificed (Nembutal $50 \mathrm{mg} / \mathrm{kg}$ i.p.). The lung samples were removed $\left(\mathrm{pH}=7.4,4^{\circ} \mathrm{C}\right.$ ) and analyzed for biochemical parameters (SOD, CAT, GSH, GPX1, malondialdehyde (MDA)). C-reactive protein (CRP) was investigated by using Canine C-ELISA Kit- 557826-D. The ROS levels were measured by spin-adduct-reduction between phenyl N-tertiary-butyl nitrone (PBN in DMSO) and generated radicals, by EPR-EMXmicro, X-band spectrometer after double integration of the corresponding spectra. The results present BLM-oxidative toxicity and a statistically significant decrease in SOD ( $p<0.03)$, CAT ( $<<0.05)$ GPX1 $(p<0.05)$ enzyme activity, and a two-fold increase in MDA ( $<<0.05)$, CRP ( $<<0.05)$ and ROS- levels $(\mathrm{p}=0.004)$, compared to group 1 . In opposite in groups 3 and 4 , the highly-toxic BLM-effects were significantly decreased for all parameters in pulmonary cells ( $\mathrm{p}<0.05$, $\mathrm{t}$-test), relative to the controls. In conclusion, were indicated that $L$. minor treatment stimulates endogenous activity, and effectively reduced CRP- inflammation and scavenging ROS products. A positive correlation was registered between CRP and ROS ( $r=0.49$, $t-$ test). The L. minor ameliorates ROS-formations and neutralized the BLM-induced oxidative toxicity probably by suppressing the body/pulmonary cell residual inflammation processes.

Keywords: L. minor, BLM, CRP, ROS

Acknowledgments: The study was supported by scientific projects 2/2020 of Medical Faculty, Trakia University, Bulgaria. 


\title{
Specific features of change in enzymate activity in the acoustic field
}

\author{
Anna A. Oleshkevich \\ Moscow Skryabin State Academy of Veterinary Medicine and Biotechnology, Moscow, Russia
}

\section{https://doi.org/10.21175/rad.abstr.book.2021.1.4}

Ultrasonic (US) methods are an integral part of a comprehensive diagnostic examination of animals and humans that determines the relevance of studying their physiological effects in biological objects and revealing the active frequencies' spectra for different tissues of domestic animals for modulated ultrasound. In addition, it is necessary to determine the biochemical and physiological mechanisms of changes in the body, tissues and individual cells when using US-exposure. Therefore, healthy domestic animals of different sexes, ages and breeds were formed into groups according to the principle of physiological analogues. The blood of animals was used for biochemical studies. The samples were sonicated with ultrasound in the ISATA intensity ranged from 0.05 to $2.0 \mathrm{~W} / \mathrm{cm}^{2}$ at frequencies of $0.88 \mathrm{MHz}$ and $2.64 \mathrm{MHz}$. The signal for modulation was applied in the frequency of $0.01-1000 \mathrm{~Hz}$, with a step of $5-10 \mathrm{~Hz}$. The temporary exposure was $3 \mathrm{sec}-15 \mathrm{~min}$. Before determining the enzyme activity in blood plasma, whole blood samples were exposed to ultrasound, and after cell separation, plasma biochemical analysis was performed. The activity of serum enzymes was measured after irradiation of the blood serum of the same animals.

Analysis of the US effect on the activity of homeostasis enzymes showed that exposure to continuous ultrasound with an intensity of $0.4-0.7 \mathrm{~W} / \mathrm{cm}^{2}(\mathrm{p}<0.05)$ for $60 \mathrm{sec}$ and longer caused an increase in the activity of alkaline phosphatase (ALP) and lactate dehydrogenase (LDH) in cat's blood plasma; an increase in the activity of aspartate aminotransferase (AST), creatine kinase (CK), alkaline phosphatase and LDH - in blood plasma from dogs; and the activity of AST, CK and ALP in horse blood plasma also increased. A decrease in activity was revealed after treatment of blood plasma of dogs (alanine aminotransferase, ALT) and horses (AST, CK and LDH). A unidirectional combined change in the activity of blood plasma enzymes in cats was found $(\mathrm{p}<0.05)$ at an intensity of $0.7 \mathrm{~W} / \mathrm{cm}^{2}$ : the activity of alkaline phosphatase and $\mathrm{LDH}$ was doubled after exposure to continuous ultrasound, as well as with the imposition of $800 \mathrm{~Hz}$ modulation. A simultaneous decrease in the activity of several enzymes in the blood serum of cats does not occur under all applied modes.

A combined change in the activity of dogs' blood plasma enzymes was established after exposure to continuous ultrasound with an intensity of $0.4 \mathrm{~W} / \mathrm{cm}^{2}$ : an increase in the activity of AST, CK, ALP and LDH from 1.6 (AST) to 2.7 times (LDH).

A multidirectional change in the activity of horse blood plasma enzymes was established after treatment with continuous ultrasound. The intensity range $0.4-0.7 \mathrm{~W} / \mathrm{cm}^{2}$ initiates a decrease in the activity of ALT (by 1.5 times) and an increase in the activity of other enzymes - AST, CK, ALP, LDH - from 1.6-1.75 to 2.6 times (LDH). Inhibition by 1.3 times of the activity of both CK and LDH can be caused by US-irradiation of $1.0 \mathrm{~W} / \mathrm{cm}^{2}$. In this case, the alkaline phosphatase activity increases 2.2 times.

A more active effect was revealed in blood serum under the influence of modulated ultrasound. The ability to simultaneously increase/decrease the activity of several enzymes has been established. 


\title{
The use of rate constants of flavoenzyme-catalyzed single-electron reduction of nitroaromatics in the analysis of their cytotoxicity mechanisms
}

\author{
Aušra Nemeikaitè-Čènienè1, Violeta Jonušiené ${ }^{2}$, \\ Lina Misevičienè3, Audronè Marozienè3, Narimantas Cenas Čènas3
}

\author{
1 State Research Institute Center for Innovative Medicine, Vilnius, Lithuania \\ 2 Institute of Biosciences of Vilnius University, Vilnius, Lithuania \\ 3 Institute of Biochemistry of Vilnius University, Vilnius, Lithuania
}

\section{https://doi.org/10.21175/rad.abstr.book.2021.1.5}

Enzymatic formation and redox cycling of free radicals is an intrinsic property of nitroaromatic compounds $\left(\mathrm{ArNO}_{2}\right)$. If the main mode of their action is redox cycling and subsequent oxidative stress, then the cytotoxicity can be described by regression log (cytotoxic concentration) $=a-b E^{1_{7}}-$ $c \log D$, where $E^{1}$ is the single-electron reduction potential of compound, and $\log D$ is its octanol/water distribution coefficient at $\mathrm{pH}$ 7.0 ([1-3], and references therein). The deviation from the limits predicted by the redox cycling activity may point to additional mechanisms of cytotoxicity or therapeutic action of $\mathrm{ArNO}_{2}$. However, the direct determination of $E^{1}{ }_{7}$ values of $\mathrm{ArNO}_{2}$ by pulseradiolysis is complicated. On the other hand, $E^{1}{ }_{7}$ may be calculated from the geometric average of their bimolecular reduction rate constants by single-electron transferring flavoenzymes such as NADPH:cytochrome P-450 reductase, ferredoxin:NADP+ oxidoreductase, or NADPH:adrenodoxin reductase/adrenodoxin complex [1-3]. Using this approach, we obtained the previously unavailable $E^{1}{ }_{7}$ values of nitroaromatic antiandrogens nilutamide $(-0.408 \mathrm{~V})$, flutamide $(-0.399 \mathrm{~V}), 2-$ hydroxyflutamide (-0.394 V) and (3-amino-2-hydroxy-2-methyl- $N$-(4-nitro-3-trifluoromethyl) phenyl) propanamide (-0.377 V), and antiviral agents nitazoxanide (-0.380 V) and niclosamide ($0.339 \mathrm{~V}$ ). With an exception of more cytotoxic niclosamide, the cytotoxicity of these compounds in mouse hepatoma MH22a cells closely followed the regression previously obtained for the model nitroaromatic compounds $(n=15)$ with available $E^{1}{ }_{7}$ values [3]. Taken together with the protection by antioxidants, it points to the prevailing oxidative stress-type cytotoxicity mechanism. On the other hand, the cytotoxicity of examined compounds in human colon carcinoma HCT-116 cells was almost by one order of magnitude higher than expected. This points to the prevalent other modes of action of above nitroaromatic antiandrogens and antiviral agents, whose mechanisms warrant further investigation, and to a definite potential of repurposing of these drugs.

\section{References}

[1] N. Čėnas, A. Nemeikaitė-Čènienė, E. Sergedienė, H. Nivinskas, Ž. Anusevičius, J. Šarlauskas, Biochim. Biophys. Acta 1528 (2001) 31-38.

[2] N. Čènas, A. Nemeikaitè-Čènienè, J. Šarlauskas, Ž. Anusevičius, H. Nivinskas, L. Misevičienè, A. Marozienè, In: Ecotoxicology of Explosives (G.I. Sunahara, G. Lotufo, R.G. Kuperman, J. Hawari, Eds.), CRC Press, Boca Raton, London, New York, 2009, pp. 211-226.

[3] A. Nemeikaitè-Čénienè, J. Šarlauskas,V. Jonušienė, L. Misevičienė, A. Marozienè, A.V. Yantsevich, N. Čènas, Chemija 31 (2020) 170-177.

Acknowledgments: This work was supported by the European Social Fund (Measure No. 09.33-LMT-K-712, Grant No. DOTSUT-34/09.3.3.-LMT-K712-01-0058/LSS-600000-58). 


\title{
QSARs in prooxidant aerobic cytotoxicity of heteroaromatic $\mathrm{N}$-oxides: Role of flavoenzyme-catalyzed single-electron reduction
}

\author{
Aušra Nemeikaitè-Čènienè1, Jonas Šarlauskas², \\ Violeta Jonušienè 3 , Lina Misevičienè², Audronè Maroziené², \\ Mindaugas Lesanavičius ${ }^{2}$, Narimantas Čènas ${ }^{2}$
}

\author{
1 State Research Institute Center for Innovative Medicine, Vilnius, Lithuania \\ 2 Institute of Biochemistry of Vilnius University, Vilnius, Lithuania \\ 3 Institute of Biosciences of Vilnius University, Vilnius, Lithuania
}

\section{https://doi.org/10.21175/rad.abstr.book.2021.1.6}

Derivatives of tirapazamine and other heteroaromatic $N$-oxides $(\operatorname{ArN} \rightarrow \mathrm{O})$ exhibit antitumour, antibacterial, and antiprotozoal activities, which are attributed to reductive activation and free radical generation ([1], and references therein). Although $\mathrm{ArN} \rightarrow \mathrm{O}$ are typically considered as potential hypoxia-selective antitumour agents, in some cases they possess high anticancer activity under oxic conditions. Besides, their oxidative stress-type mammalian cell cytotoxicity may be important side effect in antimicrobial and antiparasitic action.

Extending our previous studies [2], we aimed to clarify the role of flavoenzyme-catalyzed singleelectron reduction of $\operatorname{ArN} \rightarrow \mathrm{O}$ in their aerobic cytotoxicity. We synthesized a series of $\mathrm{ArN} \rightarrow \mathrm{O}$ with available and unavailable values of single-electron reduction potential $\left(E^{1_{7}}\right)(n=18)$. Their reactivity $\left(k_{\mathrm{cat}} / K_{\mathrm{m}}\right)$ towards single-electron transferring flavoenzymes NADPH:cytochrome P-450 reductase, ferredoxin:NADP ${ }^{+}$oxidoreductase, and NO synthase (nNOS) increased with their $E_{7}{ }_{7}\left(\Delta \log k_{\text {cat }} / K_{\mathrm{m}}\right.$ $\left./ \Delta E^{1}{ }_{7} \approx 10 \mathrm{~V}^{-1}\right)$. The reactivity of $\mathrm{ArN} \rightarrow \mathrm{O}$ with unavailable $E^{1}{ }_{7}$ values correlated with the $\sigma$ values of their substituents. The reactivity of $\mathrm{ArN} \rightarrow \mathrm{O}$ was similar to that of quinones with the same $E^{1}{ }_{7}$ values. Their toxicity in murine hepatoma MH22a and human carcinoma HCT-116 cells increased with their $E^{1}{ }_{7}$ and/or the average of their $\log k_{\text {cat }} / K_{\mathrm{m}}$ in single-electron enzymatic reduction. However, it exceeded the cytotoxicity of quinones with the same redox activity by one order of magnitude. The protective effects of antioxidants demonstrated the prooxidant character of cytotoxicity. The enhanced cytotoxicity of $\operatorname{ArN} \rightarrow \mathrm{O}$ was partly attributed to the action of cytochromes P-450, because their inhibitors protected against the toxicity, but potentiated the cytotoxicity of quinones. Another factor is the action of $\mathrm{NAD}(\mathrm{P}) \mathrm{H}$ :quinone oxidoreductase (NQO1), which reduces quinones in twoelectron way and contributes to their detoxification. Its inhibitor, dicoumarol, enhanced the cytotoxicity of quinones and protected against the cytotoxicity of $\mathrm{ArN} \rightarrow \mathrm{O}$. We found that NQO1 atypically reduces $\mathrm{ArN} \rightarrow \mathrm{O}$ in mixed single- and two-electron way, and possibly contributes to their redox cycling. The multiparameter regression analysis shows that there exists a statistically significant relationship between $k_{\text {cat }} / K_{\mathrm{m}} \mathrm{ArN} \rightarrow \mathrm{O}$ towards NQO1 and their cytotoxicity. These data show that apart from the typical representatives of single-electron transferring flavoenzymes, NQO1 is another important target of $\mathrm{ArN} \rightarrow \mathrm{O}$, and provide certain guidelines for their rational synthesis.

\section{References}

[1] X. Shen, K.S. Gates, Chem. Res. Toxicol. 32 (2019) 348-361.

[2] A. Nemeikaitè-Čèniené, J. Šarlauskas, V. Jonušienè, A. Marozienè, L. Misevičienè, A.V. Yantsevich, N. Čènas, Int. J. Molec. Sci. 20 (2019) 4602.

Acknowledgments: This work was supported by the European Social Fund (Measure No. 09.33-LMT-K712, Grant No. DOTSUT-34/09.3.3.-LMT-K712-01-0058/LSS-600000-58). 


\title{
Inhibitory effect of terpenes on acetylcholinesterase activity
}

\author{
Safija Herenda ${ }^{1}$, Edhem Haskoviće ${ }^{2}$, Denis Hasković ${ }^{3}$, Ena Hasković 4
}

\begin{abstract}
1Department of Chemistry, Faculty of Science, University of Sarajevo, Sarajevo, Bosnia and Herzegovina 2Department of Biology, Faculty of Science, University of Sarajevo, Sarajevo, Bosnia and Herzegovina 3 Clinical Center of the University of Sarajevo, Organizational Unit - Clinical Pathology, Cytology and Human Genetics, Sarajevo, Bosnia and Herzegovina

4Clinical Center of the University of Sarajevo, Organizational Unit - Clinical Chemistry and Biochemistry, Sarajevo, Bosnia and Herzegovina
\end{abstract}

\section{https://doi.org/10.21175/rad.abstr.book.2021.1.7}

Cholinesterases are a group of enzymes that hydrolyze choline esters faster than other carboxylic acid esters. Acetylcholinesterase is found in the tissue of the central nervous system, synaptic ganglia, motor plates, in the stroma of erythrocytes. The physiological role of acetylcholinesterase is the hydrolysis of its natural substrate - acetylcholine (ACh). Numerous sesquiterpenes act as phytoalexins, antibiotic compounds produced by plants in response to attacks by microorganisms. They are formed from a common precursor, farnesyl pyrophosphate, using various cyclization methods, in many cases followed by carbon skeletal rearrangement. Thujopsene is a natural chemical compound, classified as sesquiterpene. It is found in essential oil, and its antioxidant, antifungal, anticancer and antimicrobial properties are known. In this paper, the electrochemical processes of acetylcholinesterase on a glassy carbon electrode (GC) were investigated using a cyclic voltammetry method. The effect of different substrate concentrations was monitored by cyclic voltammetry, and the effect of different scan rates on the appearance of voltammograms was examined. Cyclic voltammograms can provide information on the rate of charge transfer, the processes of charge transport, and the interactions that occur between enzyme segments at specific sites. The kinetic parameters were examined by chronoamperometry, and the results showed the inhibitory properties of Thujopsene on enzymatic activity. 


\title{
Effect of graphene oxide incorporation into polymeric matrix of polysaccharide multilayered microcapsules
}

\author{
Svetozar Stoichev, Avgustina Danailova', Ivan Iliev², \\ Inna Sulikovska², Velichka Strijkova3, Kirilka Mladenova4, Tonya Andreeva1
}

\author{
1 Institute of Biophysics and Biomedical Engineering, Bulgarian Academy of Sciences, Sofia, Bulgaria \\ 2 Institute of Experimental Morphology, Pathology and Anthropology with Museum, Bulgarian Academy of \\ Sciences, Sofia, Bulgaria \\ 3 Institute of Optical Materials and Technologies, Bulgarian Academy of Sciences, Sofia, Bulgaria \\ 4 Faculty of Biology, Sofia University, Sofia, Bulgaria
}

\section{https://doi.org/10.21175/rad.abstr.book.2021.2.1}

Polyelectrolyte multilayered (PEM) microcapsules have a variety of applications, the most significant being in systems for delivery of therapeutic and bioactive substances. PEMs microcapsules have many advantages including site-specific drug delivery, controlled sustained drug release for prolonged period of time, effective drug protection from external factors, reduction of adverse side effects and reduced toxicity. The interest in PEM microcapsules is mainly due to the possibility of fine design and modification of their properties (size, composition, morphology, permeability, hardness, etc.).

The present study is focused on characterization of the morphology, mechanical properties and biocompatibility of PEM microcapsules composed of natural polyelectrolytes (chitosan/alginate/hyaluronic acid), and of the effect of graphene oxide (GO) incorporation in the polymer matrix. GO is known as the thinnest and most robust material with unique electrical, thermal and mechanical features. We have fabricated a number of hybrid GO-polysaccharide multilayered capsules by layer-by-layer assembling technique onto $\mathrm{CaCO}_{3}$ core, followed by core decomposition in mild conditions. Hybrid microcapsules with different composition were thus constructed by varying the number of the deposited layers, as well as the number or localization of the incorporated GO-layers into the polysaccharide matrix. The shape, morphology and surface roughness were evaluated by atomic force microscopy (AFM) and the PEM microcapsules elasticity by nanoindentation. AFM data demonstrated that the incorporation of GO nanosheets in the polymer matrix affects the thickness, surface roughness, and elasticity of the hybrid GO-PEM microcapsules. Analyses of the viability and proliferation of fibroblast cells after treatment with hybrid PEM microcapsules revealed pronounced concentration-depending citotoxic and antiproliferative effect. The constructed multilayered hybrid microcapsules made of natural polyelectrolytes and carbon nanomaterial could have an application in drug delivery.

Acknowledgments: This work was supported by grant КП-06-M21/4, Competition for financial support for basic research projects of young scientists and postdoctoral fellows - 2018, Bulgarian National Science Fund. Research equipment of Distributed Research Infrastructure INFRAMAT, part of Bulgarian National Roadmap for Research Infrastructures, supported by Bulgarian Ministry of Education and Science was used in this investigation. 


\title{
Comparative analysis of indicators of homeostasis in domestic animals (dogs)
}

\author{
Ivan P. Scheglakov, Anna A. Oleshkevich, Yulia L. Gordeeva
}

Moscow Skryabin State Academy of Veterinary Medicine and Biotechnology, Moscow, Russia

https://doi.org/10.21175/rad.abstr.book.2021.3.1

Statistical analysis of the results of biochemical studies of blood serum of animals helps to determine the quantitative and qualitative characteristics, to establish the frequency of occurrence (repeatability) of the given parameters. Also, the analysis of indicators can reveal the presence of links between parameters; reliably evaluate them, ensuring in the future the reliability and timeliness of forecasting.

For the study, groups of animals with metabolic and cardiovascular diseases were formed. The total number of domestic dogs of various breeds and ages selected for research was 123 individuals.

A biochemical study of blood tests in dogs included the determination of the main 30 markers of the body's metabolism (enzyme activity). The deviation of the values of the selected indicators from the norm is a symptom of the development/presence of various diseases or disorders of the regulation of metabolism, the work of systems and organs.

Statistical analysis was performed using the method of correlation analysis in the "STATISTICA 6.0 " system. The correlation coefficients of the main biochemical parameters were determined both among themselves and with the values of the concentrations of trace elements. The coefficient characterizes the strength of the relationship between indicator indicators and its direction.

Examples of indicator values found when determining the correlation dependence (results are given in parentheses; $\mathrm{p}<0.05$ ):

- $\quad$ triglycerides - cholesterol (o.8);

- gamma glutamyltransferase - direct bilirubin (0.932);

- gamma glutamyltransferase - total bilirubin (0.939);

- $\quad$ calcium - lipase (0.64);

- $\quad$ phosphorus - urea (0.98);

- $\quad$ potassium - lipase (0.51);

- $\quad$ sodium - uric acid (0.51);

- $\quad$ sodium - lipase (0.58);

- $\quad$ alanine aminotransferase - direct bilirubin (0.689).

The data obtained as a result of the study indicate that there is a close relationship between the values of the activity of homeostasis enzymes and the indicators of mineral metabolism (the concentration of a number of trace elements in the blood serum). 


\title{
Analysis of elastic properties of dental impression materials
}

\section{Milica Jeremic Knezevic, Aleksandar Knezevic ${ }^{1,2}$, Daniela Djurovic Koprivica1, Aleksandra Maletin', Bojana Milekic3, Tatjana Puskar3}

\author{
1 University of Novi Sad, Faculty of Medicine Novi Sad, Novi Sad, Serbia \\ 2 Clinical Centre of Vojvodina, Medical Rehabilitation Clinic, Novi Sad, Serbia \\ 3 University of Novi Sad, Faculty of Medicine, Dentistry Clinic of Vojvodina, Novi Sad, Serbia
}

\section{https://doi.org/10.21175/rad.abstr.book.2021.4.1}

Background. In dentistry, it is important that the impression material remains attached to the impression tray during the recording of the impression. Partial detachment may cause gross distortions of the impression which may remain undetected and will almost certainly lead to illfitting appliances or restorations.

Impression materials must have adequate elastic properties and adequate tear resistance, coupled with a rigidity which is low enough to enable the impression to be removed.

If a material is rigid after setting it may not be possible to remove it from undercut areas. This obviously has a negative effect on the ability to achieve an adequate impression, but more seriously may undermine the viability of the remaining teeth as they may be subjected to a considerable stress if an attempt is made to remove the impression.

Methods. The properties which are most important are rigidity and elasticity, since they determine whether an impression material can be used to record undercuts. When standing teeth are to be recorded, or when the patient has deep soft-tissue undercuts, the set impression material must be flexible enough to be withdrawn past the undercuts and elastic enough to give recovery and an accurate impression. Hence, impression materials are classified as being elastic or non-elastic.

Results. The impression recorded with the elastic material accurately records the true shape of the tooth with the correct degree of undercut. The impression recorded with the plastic material has been grossly distorted during removal and has not recorded any undercut. The impression recorded with the viscoelastic material gives a distorted shape.

Conclusion. The degree of distortion depends on the severity of the undercut, the thickness of the impression material and the time for which the impression is maintained in a compressed state. 


\title{
Determination of physical and chemical parameters that can be used to predict the hemostatic activity of chitosan without conducting in vivo experiments
}

\author{
Oksana Kadyseva, Vladimir Bykov², Olga Strelova', Alexander Grebenyuk
}

1 St. Petersburg State Chemical and Pharmaceutical University, St. Petersburg, Russia

2 N. Petrov NMRC of Oncology Health Department Russian Federation, St. Petersburg, Russia

\section{https://doi.org/10.21175/rad.abstr.book.2021.4.2}

Bleeding is the main cause of death as a result of injuries and wounds, so the development of effective hemostatics remains an urgent task of emergency medicine. Currently, local hemostatic agents (LGA) based on the chitosan biopolymer are widely used as means of providing emergency care for bleeding. At the same time, there is no single standard for evaluating the physicochemical and biological properties of chitosan. None of its traditionally controlled parameters, such as molecular weight and the degree of deacetylation, is crucial in determining the ability to initiate hemostasis. In this regard, the most reliable test for evaluating the effectiveness is still conducting in vivo experiments on large animals.

In our work, using NMR spectroscopy, spectrophotometry, viscometry, inductively coupled plasma mass spectrometry, as well as methods for assessing hemostasis by in vitro and in vivo experiments, we determined the physicochemical parameters of chitosan, which are related with its hemostatic properties. These indicators were tested when evaluating the quality of chitosan raw materials, as well as LGA in the form of bandages and granules obtained on its basis.

It was found that the ability to initiate hemostasis was shown by those samples of chitosan in which, at $\mathrm{pH}$ values of the solution close to physiological, a significant part of the molecules passed from the conformational state «rigid rod» to the state "globule», which was accompanied by an abrupt change in the light transmission of the solution. For effective samples, the value of light transmission determined by spectrophotometry at $\mathrm{pH} 7.36$ was $42.5-66.1 \%$, and for inefficient samples $>85.0 \%$. In this case, the viscosity average molecular weight and the degree of deacetylation affect the dynamics of changes in the conformation of the chitosan molecule, but the relation is not linear and depends on the viscosity of solutions and their temperature. The parameters of the molecular mass distribution determined by the DOSY NMR method did not demonstrate a connection with the manifestation of hemostatic properties of chitosan. The results obtained are consistent with the results of in vitro and in vivo tests. 


\title{
Effects of saline solution and simulated body fluid on ion substituted hydroxyapatites EPR spectra
}

\author{
Monica Vidotto', Timor Grego',2, Ina Erceg1, \\ Božana Čolović3, Maja Dutour Sikirić1 ${ }^{\text {, Nadica Maltar-Strmečki1 }}$
}

\author{
1 Ruđer Bošković Institute, Zagreb, Croatia \\ 2 University Hospital Centre Zagreb, Zagreb, Croatia \\ 3 Vinča Institute, Belgrade, Serbia
}

\section{https://doi.org/10.21175/rad.abstr.book.2021.4.3}

Hydroxyapatite ( $\left.\mathrm{HA}, \mathrm{Ca}_{10}\left(\mathrm{PO}_{4}\right)_{6}(\mathrm{OH})_{2}\right)$, a calcium orthophosphate, due to its similarity to the inorganic part of human's hard tissue, is among the most frequently used hard tissue regeneration biomaterial [1]. As such, the effects of HA ageing in media similar to blood plasma need to be studied, specifically after sterilization with $\gamma$-radiation, to better understand the possible structural changes it would undergo in the human body. Moreover, such changes in ion substituted HAs are particularly interesting since so called biological HA is in fact non-stoichiometric poorly crystalline, calcium deficient, Na-, Mg- and carbonate substituted HA (Dorozhkin, 2012) [2].

In this study, HA and 3 different substituted HAs were irradiated to $25 \mathrm{kGy}$ with Co-6o $\gamma$-rays and their electron paramagnetic (EPR) spectra were recorded 1, 14 and 28 days after treatment with saline solution and simulated body fluid (SBF) [3]. The ion substituted HAs were doped with $\mathrm{Mg}$ and Si: $2 \% \mathrm{Mg} ; 2 \% \mathrm{Mg}+0.4 \% \mathrm{Si}$ and $2 \% \mathrm{Mg}+1.25 \% \mathrm{Si}$. EPR spectra were analysed, and the most pronounced peaks assigned. The effects of ageing in the two media are presented and discussed.

\section{References}

[1] Conrado A, Ginebra M-P, and J. An. Biomineralization and Biomaterials: Fundamentals and Applications. Amsterdam, Netherlands: Woodhead Publishing, 2016.

[2] Dorozhkin, S.V., 2012., in: Calcium Orthophosphates: Applications in Nature, Biology, and Medicine. Pan Stanford, Singapore.

[3] Kokubo, T., Takadama, H., 2006. How useful is SBF in predicting in vivo bone bioactivity? Biomaterials 27, 2907-2915. https://doi.org/10.1016/j.biomaterials.2006.01.017

Acknowledgments: This study was supported by Croatian Science Foundation, Grant HRZZ- IP-2018-011493 and Croatian-Serbian bilateral project "Ion substituted hydroxyapatites for bone tissue engineering". 


\title{
Hydrogels photo-crosslinking by $266 \mathrm{~nm}$ pulsed laser radiation
}

\author{
Tatiana Tozar, Mihai Boni, \\ Simona Nistorescu, Mihail Lucian Pascu, Angela Staicu
}

National Institute for Laser, Plasma, and Radiation Physics, Magurele, Ifov, Romania

https://doi.org/10.21175/rad.abstr.book.2021.4.4

In the last years, efforts have been made for the development of smart wound dressings with enhanced efficiency in the treatment of major skin wounds. The requirements of an ideal wound care system are: capability of the long-term release of medicine, to maintain high humidity at the wound site, non-toxicity, to be removed without affecting the wound, to allow the exchange of gases and water vapor, to be impermeable to bacteria and cost-effective. A solution for an ideal wound dressing are hydrogels These are formed from polymers, natural or artificial, and can offer optimized therapeutic action and controlled release of the drugs. They can be formed via photopolymerization, solution polymerization/cross-linking, bulk polymerization, suspension polymerization, or inversesuspension polymerization. The hydrogels obtained by polymers exposure to UV radiation offer the advantages of temporal and spatial control, free of chemical additives, and simultaneously sterilization is obtained. This method produces free radicals in polymer solution due to UV laser interaction with a photoinitiator.

This study is addressing the hydrogel formation by photocrosslinking gelatin chains functionalized with methacrylate groups when Irgacure 2959 was used as a photoinitiator. The solution was exposed to the fourth harmonic of an Nd:YAG laser at energies between 0.25 and $1 \mathrm{~mJ}$. The crosslinking process was monitored in real-time by laser-induced fluorescence and offline by UV-Vis and FTIR absorption spectroscopy. The dimerization reaction was evaluated at intermediate times; the changes from dilute to the semi-dilute polymer solution and to fully crosslinked hydrogel were monitored.

Additionally, the photodegradation of Irgacure 2959 was investigated in the same condition used to form the hydrogels. Its photodegradation was evaluated at $1 \mathrm{~min}, 5 \mathrm{~min}, 10 \mathrm{~min}, 20 \mathrm{~min}$, and 30 min. The photodegradation of Irgacure 2959 was evidenced by the fluorescence spectra changes in peak intensity and peak wavelength, *the UV-Vis absorption spectra, where the absorbance band of Irgacure 2959 vanished and a new band at shorter wavelengths appeared. Also, the FTIR spectra analysis showed the degradation of the photosensitizer and facilitated photoproducts identification.

Acknowledgments: This work was supported by a grant of the Romanian Ministry of Education and Research, CNCS-UEFISCDI, project PN-III-P1-1.1-PD-2019-1117 and by the Ministry of Research, Innovation and Digitization, project NUCLEU Program 16N/o8.02.2019. 


\title{
Nanoparticle radiosensitization experiments at IFIN-HH, Romania
}

\section{Roxana Cristina Popescuํ, Mihaela Tudor'1,2, Diana Savu1}

\author{
1 Horia Hulubei National Institute for Research and Development in Physics and Nuclear Engineering, \\ Magurele, Romania \\ 2 Faculty of Biology, University of Bucharest, Bucharest, Romania
}

\section{https://doi.org/10.21175/rad.abstr.book.2021.4.5}

Translational cancer radiotherapy aims to deliver a high dose at the tumor site, to inhibit the development of cancer cells, while, in the meantime, trying to protect the healthy cells. In order to reach this goal and to minimize the adverse systemic effects, targeted nanoparticle therapies have been proposed as a solution.

We have employed different types of radiation therapy in combination with iron oxide nanoparticles (IONP) to be used as radiosensitizers.

Due to the secondary reactive species production following the interaction with radiation, intermediate Z IONP determined the alteration of the tumor cells metabolic function, DNA damage, protein expression, division or even induction of cell death.

We have proved that the sensitizing response is influenced by the nanoparticles targeting ability and intracellular localization, as well as radiation type and properties, like energy, dose and flow.

Acknowledgments: This work was supported by Romanian Ministry of Research National grants no. PN 19060203, 543 PED/2019. 


\title{
Use of X-ray contrast materials with inkjet printing for creation of breast anthropomorphic phantoms
}

\author{
Tihomir Georgiev, Zhivko Bliznakov, Nikolay Dukov, Kristina Bliznakova
}

Medical University of Varna, Varna, Bulgaria

https://doi.org/10.21175/rad.abstr.book.2021.5.1

Introduction. In recent years the anthropomorphic phantoms have become an essential part in the fields of biomedical technology and radiology, allowing studies without patients' involvement.

Aim. We evaluate the potential use of two radiopaque materials for manufacturing anthropomorphic phantoms with inkjet technology.

Materials and methods. Two radiopaque materials, potassium iodide (KI) and sodium tungstate dihydrate (STD), were used to print anthropomorphic breast phantoms, based on real patient data. HP Officejet 5510 inkjet printer was used for the study, with plain office and flat filter paper. Four experiments with different KI solutions were performed. The first model was printed with mixture of KI and water, $0.6 \mathrm{~g}$ per $1 \mathrm{ml}$ of water, mixed with cartridge ink in 1:1 ratio. The produced four identical physical breast phantoms each with $4.2 \mathrm{~mm}$ thickness were imaged on Canon CXDI X-ray. The second experiment involved $2 \mathrm{ml} \mathrm{KI}$ solution injected directly into $19 \mathrm{ml}$ genuine ink cartridge and printing of calibration phantom on flat filter paper. Images were taken with Siemens Somatom Definition CT. The third experiment involved KI, $0.45 \mathrm{~g}$ per $1 \mathrm{ml}$ of water injected directly into an empty cartridge. Images from patient data, segmentations from patient data, and a calibration phantom were printed and imaged at Siemens Somatom Definition CT. The fourth experiment involved the use of alternative ink prepared with a mixture of $0.894 \mathrm{~g}$ of STD dissolved in $1 \mathrm{ml}$ of water and was used for comparison with KI. Finally, the printed patterns representing glandular and adipose tissues in the physical phantoms were compared in terms of Hounsfield Units (HUs) to literature results.

Results and discussion. Phantoms identical in pattern were evaluated at several regions of interest and the corresponding attenuations were found to have good match. Increasing KI concentration provides better contrast but Hus are higher than theoretical. Furthermore, the results for the STD corresponded to the theoretical calculations that STD attenuation would be lower than the equivalent KI phantom, however, STD is more stable to light and humidity over the time.

Conclusions. Both contrast agents are easily soluble in water. In-house developed ink mixtures can be used to replicate radiology characteristics of the breast tissues. The inkjet printing technology is suitable for manufacturing of anthropomorphic phantoms.

Keywords: Anthropomorphic breast phantoms, inkjet printing, X-ray contrast materials mixtures, X-ray imaging

Acknowledgments: This work is supported by the Bulgarian National Science Fund under grant agreement DN 17/2. 


\title{
Multi-stage filters improved solution for air pollutants accelerating cardiopulmonary diseases in Zlatibor District
}

\author{
Mirjana R. Cvijović1, Milorad M. Murićé, Vladica V. Čudić3, \\ Ana P. Čudić4, Mirjana M. Petrović5, Marina M. Kremić6
}

\author{
1 University of Belgrade, Faculty of Medicine, Institute for Microbiology and Immunology, Belgrade, Serbia \\ 2 Academy of Vocational Studies of Western Serbia, Užice, Serbia \\ 3 PWW LLC, Office in Belgrade, Belgrade, Serbia \\ 4 Vrije Universität, Amsterdam, Amstelveen, North Holland, Netherlands \\ 5 Clinical Center of Serbia, Clinic for Endocrinology, Diabetes and Metabolic Diseases, Belgrade, Serbia \\ 6 Health Center for Adults Užice, Užice, Serbia
}

\section{https://doi.org/10.21175/rad.abstr.book.2021.5.2}

Exposure to air pollutants: sulfur dioxide, nitrogen dioxide, suspended particles (PM10, PM2.5, ultrafine particles), soot, nickel, arsenic, lead, cadmium, total sediment have negative consequences to human health, particularly pulmonary and cardiovascular diseases[1]. Premature deaths accelerated by air pollution in Serbia are around 6,00o people per year. The air quality in Užice and Kosjerić is classified as the $3^{\text {rd }}$ category. Cardiovascular diseases have the largest contribution, $57.8 \%$ in total deaths in Zlatibor district during 2019.

Air quality in Zlatibor district was analyzed for the period between 2017 untill 2021. in Užice and Kosjerić. Extremely high values of polycyclic aromatic hydrocarbons-benzopyrene occasionally occurred, within PM10, (daily doses $28.0 \mathrm{ng} / \mathrm{m} 3$ ), in the center of Užice (winter of 2018). The concentrations of $\mathrm{Ni}$ and $\mathrm{Cd}$ were occasionally above the limit values. Maximum daily Ni values in PM 10 were $68.9 \mathrm{ng} / \mathrm{m} 3$. The highest measuring values of soot were in the center of Užice during the winter period. The soot most likely comes from incomplete combustion of fuels containing carbon, car exhaust gases. Some epidemiological studies indicate that the health of inhabitants in Užice and Kosjerić is strongly affected by air pollutants, socio-economical status, and meteorological conditions, cold winters. The values of PM10 and PM 2.5 are extremely high during the heating period in Kosjerić. The cement dust has negative impact to human health in Kosjerić, also. The aim of this paper is to try to use some technical/technological improvements to reduce air pollution in Zlatibor district and prevent cardiopulmonary diseases accelerated by air pollutants. The solution will be addressed to production of improved multi-stage filters for ventilation, deducting systems in the industry and heating plants.

Keywords: Air pollution, Health impact, suspended particles PM1O and PM 2.5, PAH, nickel, soot, multifunctional filters, environmental cardiology

\section{References}

[1] T. Bourdrel, M-A. Bind, Y. Béjot, O. Morel, J-F, Argacha, Cardiovascular effects of air pollution, Archives of Cardiovascular Disease (2017) 110, 634-642. 


\title{
Predictors of the basal frequency of micronucleus, nucleoplasmic bridges and nuclear buds at the beginning of pregnancy in a population of healthy pregnant women and pregnant women with thrombophilia
}

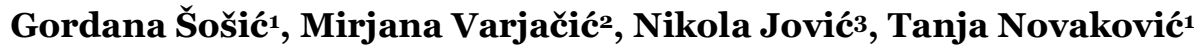

\author{
1 Department for Cytogenetic Diagnosis, Clinic of Obstetrics and Gynecology, Clinical Center "Kragujevac", \\ Kragujevac, Serbia \\ 2 Department of Pathology of Pregnancy, Clinic of Obstetrics and Gynecology, Clinical Center "Kragujevac", \\ Kragujevac, Serbia \\ 3 Clinic of Obstetrics and Gynecology, Clinical Center “Kragujevac”, Kragujevac, Serbia
}

https://doi.org/10.21175/rad.abstr.book.2021.6.1

Aim. This study aims to analyze the correlation between risk factors and the presence of cytogenetic biomarkers: micronuclei, nucleoplasmic bridges and nuclear buds in peripheral blood lymphocytes and to determine the predictors of basal frequencies of these biomarkers in pregnant women in the first trimester of gestation.

Materials and methods. In a population of 74 pregnant women (37 healthy women and 37 women with inherited thrombophilia), we determined the frequency of micronucleus, nucleoplasmic bridges and nuclear buds using the cytokinesis-block micronucleus assay. The correlation of risk factor with cytogenetic biomarkers was determined by the Spearman rank correlation $(\mathrm{p}<0.01$, $\mathrm{p}<0.05$ ). Univariate and multivariate linear regression analysis identified significant predictors of the frequency of cytogenetic biomarkers.

Results. A statistically significant positive correlation was observed between the presence of thrombophilia and the frequency of micronucleus, nucleoplasmic bridges, and nuclear buds $(\mathrm{p}<0.05)$, previous miscarriages with micronucleus and nucleoplasmic bridges frequency $(\mathrm{p}<0.01)$, the presence of relatives with thrombosis and non-O blood type with micronucleus frequency $(p<0.05)$. Significant predictors of micronucleus frequency were thrombophilia, previous miscarriage and non-O blood groups ( $\mathrm{p}=0.000 ; \mathrm{p}=0.042 ; \mathrm{p}=0.008)$. Predictors of nucleoplasmic bridges frequency were thrombophilia, previous miscarriage, number of miscarriages and pregnancy to order $(\mathrm{p}=0.000 ; \mathrm{p}=0.001 ; \mathrm{p}=0.002 ; \mathrm{p}=0.020)$. Predictors of nuclear buds frequency were thrombophilia and degree of education ( $\mathrm{p}=0.011 ; \mathrm{p}=0.015)$. Thrombophilia contributes most to individual variability in micronucleus frequency concerning nucleoplasmic bridges and nuclear buds (34.5\%vs.21.2\%vs.7.3\%).

Conclusion. In the study population, thrombophilia is the most significant predictor and contributes most to the variability of cytogenetic biomarkers relative to other factors studied.

Keywords: Micronucleus, nucleoplasmic bridges, nuclear buds, pregnant women, thrombophilia 


\title{
ADAPT - genomic initiative to analyse adaptation in the group of Lithuanian Chernobyl liquidators
}

\author{
Ingrida Domarkiene, Gabriele Zukauskaite, Ausra Matuleviciene, \\ Justas Arasimavicius, Vaidutis Kucinskas, Laima Ambrozaityte
}

\author{
Department of Human and Medical Genetics, Institute of Biomedical Sciences, Faculty of Medicine, Vilnius \\ University, Vilnius, Lithuania
}

\section{https://doi.org/10.21175/rad.abstr.book.2021.6.2}

Background. Among the remaining survivors of the Chernobyl catastrophe liquidators from Lithuania, there are not only those who are seriously ill, but also those who are aging relatively healthy. They are exclusive as an object of research, because not only they survived extreme conditions, but also adapted to the life-lasting effects of ionizing radiation. Reaction to ionizing radiation may depend on specific individual genomic variation. Thus, we postulate that adaptation responsible for survival and longevity can be determined by the unique set of effect (protective and pathogenic) variants. Research Project Adaptive genetic mechanisms - a comprehensive study of whole genome variation in the group of the Lithuanian Chernobyl catastrophe liquidators (ADAPT) aims to characterize whole genome variation of Chernobyl catastrophe liquidators in the context of adaptive genetic mechanisms.

Methods. Study recruits Chernobyl nuclear power plant catastrophe clean-up workers from Lithuania, who agrees to fill in the questionnaire and donate venous blood for DNA extraction and further analysis. DNA samples undergo microarray genotyping and part of the samples will be set for whole genome sequencing. This research study focuses on different types of genomic variation (SNV, CNV) and will take various quantitative and qualitative approaches. Analysis methods will evaluate genetic variation diversity and distribution, genetic architecture of current adaptation, protective/risk-determining variation profile, genomic associations and population genetic parameters involved in microevolutionary processes.

Results. First, we have established the DNA biobank and clinical data database of Chernobyl catastrophe liquidators from Lithuania. Second, we have initiated to define patterns of specific genomic variation in the genomes of Chernobyl catastrophe liquidators from Lithuania and will elucidate differences from the general Lithuanian and other populations.

Conclusions. We have already biobanked 115 DNA samples, consolidated questionnaire and clinical data in the database and started data generation process using microarray genotyping and whole genome sequencing.

\section{Acknowledgments:}

We are very grateful to the Association "Lithuanian movement "Chernobyl", especially Antanas Turčinas, for participation in volunteer recruitment for the project, and all Lithuanian Chernobyl catastrophe liquidators, who agreed to participate.

We thank dr. Giedrė Smailytė and prof. Juozas Rimantas Lazutka for cooperation on data consolidation of previous studies of the Lithuanian Chernobyl catastrophe liquidators.

The study was approved by the Vilnius Regional Biomedical Research Ethics Committee (permission No. 2019/4-1119-612), Lithuania.

The study meets all the ethical requirements, and the informed written consent of all study participants is mandatory.

This project has received funding from the Research Council of Lithuania (LMTLT), agreement No. S-MIP-20-35.

All authors declare no conflicting interests. 


\title{
Genome association study of the Lithuanian Chernobyl catastrophe liquidators indicates potentially protective alleles for multifactorial diseases
}

\author{
Gabrielè Žukauskaitè, Ingrida Domarkienè, \\ Aušra Matulevičienè, Evelina Marija Vaitènienè, \\ Justas Arasimavičius, Vaidutis Kučinskas, Laima Ambrozaitytė
}

Vilnius University, Vilnius, Lithuania

https://doi.org/10.21175/rad.abstr.book.2021.6.3

Background. Lithuanian Chernobyl catastrophe liquidators (LCCLs) are an exclusive object of research, because not only they survived extreme conditions, but also adapted to the life-lasting effects of ionizing radiation (IR). IR has the power to change the structure of the adapting genome making some genomic loci and variants associated with better adaptation and survival. Thus, reaction to IR and adaptation to its effects may depend on the unique genomic variation. The aim of this study is to define specific genomic variation of the LCCLs.

Methods. The study group included 93 LCCLs. All of the participants were 50-78-year-old men. DNA was extracted and genome-wide genotyping was performed. All participants had to fill out a questionnaire, which helped to collect data on LCCLs role in the liquidation of the Chernobyl power plant catastrophe and their clinical phenotype data. This data was used for the analysis of detected associations. To identify genetic variation unique to LCCLs, allele frequency comparison and association analysis for the set of potentially protective genome variants between study and the reference group was performed using Rstudio (Fishers' Exact test) and PLINK ( $\chi^{2}$ test) software. The reference group included 182 unrelated, self-reported healthy men of the Lithuanian descent (sample collection and genotyping was a part of the LITGEN project, funded by the European Social Fund under the Global Grant measure, agreement No VP1-3.1-SMM-07-K-01-013).

Results. Association study for the set of potentially protective genome variants was performed and three genome variants showed statistical significance. According to the scientific literature, a variant in LOXL1 gene ( $\mathrm{rs} 3825942, \mathrm{p}=0.019$ ) may have protection against exfoliation syndrome (with glaucoma) whereas two variants in $C X_{3} C R 1$ gene (rs3732378, $\mathrm{p}=0.008$ and $\mathrm{rs} 3732379, \mathrm{p}=0.015$ ) may protect against coronary artery disease in the study group of 65 years and older.

Conclusions. We have identified that the LCCLs genome variation differ from the general Lithuanian population group in the context of potentially protective genome variants. Detected genome variants may protect against some multifactorial diseases and that may explain why some of the LCCLs age relatively healthy despite large IR doses experienced.

In general, the identification of potentially protective genomic variants may be useful in analysing the genetic architecture of multifactorial diseases as well as supplement knowledge of their etiopathogenesis mechanisms.

\section{Acknowledgments:}

We are grateful to the Association "Lithuanian movement "Chernobyl" and all the Lithuanian Chernobyl catastrophe liquidators for their contribution in this study.

The study was approved by the Vilnius Regional Biomedical Research Ethics Committee (permission No. 2019/4-1119-612), Lithuania.

This study is a part of the ADAPT project, funded by the Research Council of Lithuania, agreement No. S-MIP-20-35.

All authors declare no conflicting interests. 


\title{
Homozygous-recessive characteristics (HRCs) and biochemical parameters as biomarkers of cardiovascular disease risk
}

\author{
Darko Grujičić1, Ljiljana Mirkov², Kristina Virijević ${ }^{1}$ Nikola Pivljakovićé, \\ Dragoslav Marinković3, Olivera Milošević-Djordjevićc ${ }^{14}$
}

\author{
1 University of Kragujevac, Faculty of Science, Department of Biology and Ecology, Kragujevac, Serbia \\ 2 Health Care Center, Kragujevac, Serbia \\ 3 Serbian Academy of Sciences and Arts, Belgrade, Serbia \\ 4 University of Kragujevac, Serbia, Faculty of Medical Sciences, Department of Genetics, Kragujevac, Serbia
}

\section{https://doi.org/10.21175/rad.abstr.book.2021.6.4}

Cardiovascular diseases (CVD) are a leading cause of death globally. The aim of our study was to analyse homozygous-recessive characteristics (HRCs) and some biochemical parameters such as concentration of glucose, cholesterol, triglyceride in blood in the group of CVD patients and in the control sample, as well as to evaluate whether there is a predisposition to the occurrence of CVD, when these biomarkers are concerned. This study analysed presence, distribution, and individual variability of 20 selected genetically controlled morphophysiological traits among 90 CVD patients (45 males and 45 females), average age $62.80 \pm 10.20$ and 90 healthy controls (45 males and 45 females), average age $50.39 \pm 9.17$. Our results showed a significant difference in the individual variation of HRCs between the patients and controls $\left(\chi^{2}=315.34 ; p<0.0001\right)$. Statistically significant differences were found in the frequency of 9 out of 20 analyzed HRCs. The average value of HRCs in group patients was significantly higher, comparing to the control sample $(6.37 \pm 1.71 \mathrm{vs} .4 .97 \pm 1.68$; $\mathrm{p}<0.001)$, while variability decreases compared to the control sample $\left(\mathrm{V}_{\mathrm{CVD}}=0.27 \%\right.$; $\left.\mathrm{V}_{\mathrm{C}}=0.34 \%\right)$. There is not a statistical difference in the HRC between males and females in analyzed samples. In the group of CVD patients the frequency of HRCs for males was $6.56 \pm 1.69$, while for females, it was $6.18 \pm 1.74$. In controls the frequency of HRCs for males was $5.02 \pm 1.74$, and for females it was $4.91 \pm 1.64$. This study showed difference in the type of distribution HRCs; in the CVD patients' the most were with 5 to $8 / 20$, while in control sample were with 3 to $6 / 20$ recessive traits. Regarding biochemical parameters, our results showed a significantly higher concentration of glucose and triglycerides in the blood the CVD patients compared to control sample $(t=3.14, p<0.001 ; t=4.20$; $\mathrm{p}<$ 0.0001). The distribution of ABO blood type frequencies between tested samples showed that in both samples the most present is A blood type, but significantly more present in the sample of CVD patients (51.1\% vs. 37.2\%). The results of multiple linear regression analysis of tested variables (gender, smoking, glucose, cholesterol, triglyceride, HRCs) showed that only HRCs and triglyceride concentration have a significant impact on the onset of the CVDs. Our conclusion is that the increased degree of recessive homozygosity and decreased variability homozygous-recessive characteristics of CVD patients versus controls, regardless of the gender, indicates a potential genetic predisposition for CVD, including biochemical parameters such as elevated concentration triglycerides can significantly contribute to the development of this predisposition.

Keywords: Biochemical parameters, cardiovascular disease, homozygous-recessive characteristics, genetic predisposition 


\title{
Antibody isolation technique from chicken egg yolks
}

\author{
Elena I. Yarygina, Vita Laga, Anna A. Oleshkevich
}

Moscow Skryabin State Academy of Veterinary Medicine and Biotechnology, Moscow, Russia

\section{https://doi.org/10.21175/rad.abstr.book.2021.6.5}

In most cases for further use in immunochemical reactions (in particular, for the diagnosis of avian diseases), immunoglobulins of high purity are obtained from blood serum. The use of other sources, such as eggs, is less common. However, this system has several advantages. These include the greater availability of the material and the increased concentration of IgG - up to $15-20 \mathrm{mg} / \mathrm{ml}$.

The aim of this work was to develop a method for the isolation of immunoglobulin G (IgG) from the yolk of chicken eggs, which could be used to obtain antisera.

The method of double reprecipitation of polyethylene glycol (PEG) 60oo D was taken as a basis, in which the author's modifications were made. The contact time of the yolk solution with PEG was increased to 1 hour, which made it possible to reduce the centrifugation speed to 10 thousand rpm instead of 12-16 thousand rpm. The second modification of the method was the use of gel chromatography at the last stage of IgG purification.

The purity and specificity of the preparations obtained were determined by polyacrylamide gel electrophoresis (PAGE) and immunoelectrophoresis on glass plates.

The globulin fraction obtained by this method was enriched with IgG and contained a small amount of transferrin proteins. In comparative experiments on the separation of transferrin using separation on Sephadex G-200 and ultragel A-4, no fundamental difference was found between the results ( $\mathrm{p}<0.001)$. Pure IgG was obtained in the second peak taken from the column. Fractions of the second peak were pooled, concentrated and examined for purity and specificity.

According to the results of analysis in PAGE and immunoelectrophoresis, the preparation was specific and homogeneous. It can be used as an antigen to obtain antisera and for various immunochemical studies. 


\section{Forming of peri-implant soft tissue in dental implant prosthetic}

\section{Aleksandra Maletin', Milica Jeremic-Knezevic ${ }^{1}$, Daniela Djurovic-Koprivica ${ }^{1}$,

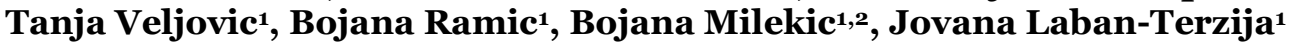

1 University of Novi Sad, Faculty of Medicine, Novi Sad, Serbia

2 Dentistry Clinic of Vojvodina, Novi Sad, Serbia

\section{https://doi.org/10.21175/rad.abstr.book.2021.6.6}

Background. Making of peri-implant soft tissue around of dental implant could be crucial of functional and esthetic integration of implant supported restoration in dental implant prosthetic.

Some of available methods for forming peri-implant soft tissue imply forming soft tissue using crown of extracted tooth, which is then remodeled and placed on abutment. Other methods are forming soft tissue with making and using temporary dedicated crown in laboratory or by using different gingiva formers. The most common method of making peri-implant soft tissue is by using different types of gingiva formers. The gingiva former is placed when the osseointegration of the implants is complete.

Soft tissue component in implant therapy is very important part for final success of an implantation. With presence of gingiva, implant and surrounding bone structures are protected from microorganisms. Process of modeling peri-implant soft tissue is critical to protect implant underlying structure during osseointegration.

Conclusion. Today, integrity, durability and shape of peri-implant soft tissue should be considered as a most important factor of final success of dental implant treatment in means of functional, physiological and esthetic aspects. 


\title{
C-reactive protein levels and oxidative stress markers as predictors of spontaneous preterm birth in women from South Bulgaria
}

\author{
Iliana Koleva', Yanka Karamalakova², Maria Angelova1, Galina Nikolova² \\ 1 Obstetrics and Gynaecology Clinic, UMHAT, Stara Zagora, Bulgaria \\ 2 Department of Chemistry and Biochemistry, Medical Faculty, Trakia University, Stara Zagora, Bulgaria
}

\section{https://doi.org/10.21175/rad.abstr.book.2021.6.7}

Premature birth (PTB) is a problem with clinical and social importance, divided into two basic types: 1) Idiopathic, spontaneous PTB ( $>80 \%$ )-genital activity is declared while preserving the integrity of the amniotic membrane or labor begins after premature amniotic sac rupture; and 2) Artificially induced (elective) PTB ( $>30 \%$ ). The ascending bacterial infection of the amniotic membranes, amniotic fluid, placenta, umbilical cord and fetus may be a basic PTB etiological cause. "Sterile" inflammation of the amniotic membranes and waters, the umbilical cord and the placenta is also possible. In clinical practice, C-reactive protein (CRP) and ROS production are the basic pathogenetic and diagnostic factors in the acute phase of inflammation.

The objective of the study was to investigate plasmatic CRP levels and ROS production/ oxidative stress as indicators for prognosis of spontaneous PTB. Patients were examined at UMBAL, Stara Zagora, Bulgaria (2017-2020) and were determined in two groups: 1) Controls- the healthy pregnant women, n1=100 (20-32y.o.) with registered singleton pregnancy, gave birth on term (BT) at gestational age 37-39+6 weeks; and 2) The preterm birth patients (PTB), n2=100 (17-38 years old) were confirmed clinically by cardiotocography for gestation $32-34^{+6}$ weeks. CRP levels were quantified in plasma by CRP-highly sensitive latex-enhanced turbidimetry in $\mathrm{mg} / \mathrm{L}$. Plasmatic ROS productions was investigated by spin- trapping EPR analyses in arbitrary units. The tested blood samples were taken by puncture of the v. cubitalis in the presence of an anticoagulant at the time of hospitalization and examined within 2 hours. Pregnant women with diabetes mellitus, antepartum hemorrhage, essential hypertension, pre-eclampsia, systemic chronic illness and infections were excluded.

Statistically significant two times elevated CRP (average $6.79 \mathrm{mg} / \mathrm{l}, \mathrm{R}=0.977, \mathrm{p}<0.05$, t-test) and ROS (average 4.33 a.u., $\mathrm{R}=0.95, \mathrm{p}<0.003$, t-test) has been noted in PTB women, compared to the NP group.

In conclusion, CRP, ROS levels and elevated oxidative stress could be used as predictors of spontaneous preterm birth in South-Bulgarian woman.

Keywords: CRP, PTB, NP

Acknowledgments: The study was supported by PhD program of Dr. Iliana Koleva-Korkelia and by scientific project 1/2020 of Medical Faculty, Trakia University, Bulgaria. 


\title{
Relationship between maternal inflammatory and oxidative disturbances in spontaneously declared preterm births
}

\author{
Iliana Koleva', Maria Angelova', Galina Nikolova², Yanka Karamalakova ${ }^{2}$ \\ 1 Obstetrics and Gynaecology Clinic, UMHAT, Stara Zagora, Bulgaria \\ 2 Department of Chemistry and Biochemistry, Medical Faculty, Trakia University, Stara Zagora, Bulgaria
}

\section{https://doi.org/10.21175/rad.abstr.book.2021.6.8}

Spontaneous preterm birth is the basic problem of perinatal mortality in Bulgaria. Pregnancy is characterized by physiological inflammatory and oxidative disturbances- determined by twice increased neutrophils levels, reactive oxygen levels, and a reduction in the levels of antioxidant enzymes. The objective of the study was to investigate and to appreciate the levels of oxidative stress and inflammatory markers in patients with spontaneous preterm birth and term birth declared cases. The case-control study was performed in UMBAL-City Hospital, Stara Zagora during 2018-2020. The study involved 200 patients, determined in two groups: Group 1- the healthy, term birth declared cases (TB), n1=100 (20-32 y.o.) at gestational age $34+0-36+6$ weeks; Group 2-The spontaneous preterm birth patients (SPTB), n2=100 (17-38 years old) confirmed clinically by cardiotocography for gestation $>32$ weeks. For this purpose we measured the levels of C-reactive protein (CRP), proinflammatory cytokines (IL-6), leukocyte activation (neutrophils count), total antioxidant status (TAC), malondyaldehyde (MDA) and ROS production.

The results showed statistically significant elevated concentrations of IL-6, CRP, Leuco

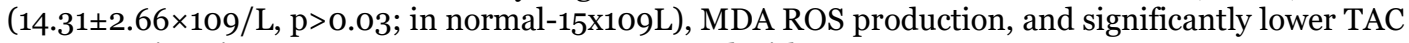
concentrations in PTB pregnant women as compared with TB women.

Key words: Leuc, SPTB, TB, leukocytosis

Acknowledgments: The study was supported by PhD program of Dr. Iliana Koleva-Korkelia and by scientific project 1/2020 of Medical Faculty, Trakia University, Bulgaria. 


\title{
Materials for taking impressions in implant prosthetic dentistry
}

\author{
Daniela Djurovic Koprivica, Tatjana Puskar, Milica Jeremic Knezevic, \\ Bojana Milekic, Aleksandra Maletin, Jovana Laban Terzija
}

University of Novi Sad, Faculty of Medicine, Novi Sad, Serbia

https://doi.org/10.21175/rad.abstr.book.2021.6.9

Background. Accuracy and trueness of dental implants definitive impressions are the main goals in therapy of partial or complete edentulism using implant supported restorations. Choosing the right impression technique as well as impression material for each individual clinical case is of great importance in a therapy process because of their direct impact on the accuracy. The impression material used for these purpose should, beside biocompatible and compatible with model cast materials, be easy to mix, rapidly setting, accurate and dimensionally stable after removal from the mouth.

Methods. Until recently the suggested materials for implant impressions included A-silicones, polyether materials, C-silicones, polysulfide materials and hydrocolloids although in modern implant dentistry only first two materials have continued to be used. Good properties which both of those two materials have in common are strain in compression, tensile strength, good accuracy and precision, very solid dimensional stability, fine elastic recovery and minimal contraction during polymerizationelastic recovery. There are also some crucial differences comparing them such as huge rigidity of poliether material in a solid bonded state and increased elastic property of additional silicone material after bonding finishing and removing impression from the oral cavity. In accordance with the stated differences, each material has its own indication considering conventional impression methods. Poliethers are usually recommended for open-tray or direct impression technique for the purpose of keeping the transfer system within the impression and, on the other hand, A-silicones are oftentimes suggested for the closed-tray or indirect impression method to facilitate the imprinting of transfers into the impression after taking it. With the desire to produce a material with the best possible characteristics, a little more than a decade ago, a new impression material was presented on the dental market. The introduced material is the combination of poliether and A-silicone and called "vinylsiloxanether", which points to its chemical structure based on these two materials (polyvinylsiloxane and polyether). The idea was to preserve all the good properties of both materials and to improve disadvantages.

Results. Vinylsiloxanter shows analogous mechanical and chemical characteristics as both component materials with the improvements of its final bonding hardness and enabled chemical bonding with polyvinylsiloxane. Because of that bond in the latest studies there are usually investigating the new combination for co called "splint" open- tray technique with splinting transfers using A-silicone and taking impression with vinylsiloxanether. There are several papers in the recent scientific literature that have examined the benefits of combined impression material and there are many controversial results. It showed as a good alternative to polyether material and more easy to handle, but investigations were primarily in in vitro conditions, and only few of them were clinical studies. Combination of new material with splinting transfers with A-silicone has proven lower accuracy comparing to the polyether and no splinting technique.

Conclusion. Although the combined impression material has proved some advantages it will have to be newly clear up through clinical studies using different impression techniques in order to take a primate as material for taking implant impressions in the future. 


\title{
The use of bioidentical hormone therapy and the quality of women's health after the reproductive period
}

\author{
Dejana Milojevic ${ }^{1}$, Aleksandar Milojevic ${ }^{2}$
}

\author{
1 MediGroup Opšta Bolnica, Belgrade, Serbia \\ 2 University Clinical Center of Serbia, Belgrade, Serbia
}

\section{https://doi.org/10.21175/rad.abstr.book.2021.6.10}

Hay to age healthy....What are bioidentical hormones? It is not a lot of things. It is certainly not a scientific term. BHTs are very similar to endogenous hormones, which are synthesized in the body, and therefore bhima therapy has clear characteristics of personalized or individual therapy, tailored to each patient. The female population is growing more and more, life expectancy has been extended, so the concept of investing in the quality of life of post-reproductive health is very important. Guidelines for the development of non-hormonal and therapy are still being developed. We suggest that clinicians and health professionals consider menopausal age relevant to a region or ethnic group to be a part of the assessment at the time of cessation or initiation of therapy. Special importance is given to monitoring women with a family history of wound. This is part of preventive health strategies - personalized menopause and personalized therapy. This position from the European Society for Menopauses and Andropause (EMAC) provides a path of care for maintaining women's health during and after menopause. It is designed for use by those involved in women's health. It includes assessment, screening for later life diseases, treatment and follow-up. Strategies must be optimized to maintain post-reproductive health due to increased longevity. These include diet and lifestyle optimization, menopausal hormone therapy and for the treatment of menopausal symptoms and skeletal preservation, tailored to individual needs with all recommended medical procedures.

Keywords: Bioidentical hormone therapy

\section{References}

1. International Diabetes Federation Diabetes Atlas, (2017) (Accessed 14 June 2018)

2. A. Menke, S. Casagrande, L. Geiss, C.C. Cowie, Prevalence of and trends in diabetes among adults in the United States, 1988-2012, JAMA 314 (2015) 1021-1029.

3. World Population Aging United Nations New York, (2015) (Accessed 14 June 2018). 


\title{
Investigation of the dependence between ROS production and apoptosis induction in leukemia lymphocytes after the application of redox-modulators in low concentrations
}

\author{
Donika Ivanova, Zvezdelina Yaneva
} Trakia University, Faculty of Veterinary Medicine, Department of Pharmacology, Animal Physiology and
Physiological Chemistry, Chemistry Unit, Stara Zagora, Bulgaria

\section{https://doi.org/10.21175/rad.abstr.book.2021.6.11}

In recent years, the purpose of many scientific studies has been to decrease the toxic side effect of chemotherapeutic drugs in cancer therapy, through combination with natural substances. There are evidences about the existence of natural compounds (redox-modulators), which could not only exhibit antioxidant properties, but have demonstrated pro-oxidant activities, depending on the environmental conditions. It is well known that the differences in the redox-homeostasis between normal and cancer cells are based on disrupted balances between the intracellular level of reactive oxygen species (ROS) and the antioxidant defined systems in cancer cells. It has been discussed that uncontrolled cellular proliferation and dysfunction of the metabolic regulation processes are linked to the specific mechanism for promotion of ROS production in cancer cells. The redox-modulators administered in cancer therapy could be used as potential target molecules, which general molecular mechanism is based on influences on the unique biochemical pathways that distinguish the cancer cells from normal ones.

The aim of the present study is to clarify the relation between cell viability, intracellular ROS production and induction of apoptosis, after application of low dose of docosahexaenoic acid, melatonin or resveratrol on leukemia lymphocytes after 24 and 48 hours incubation time. In our previous study it has been demonstrated that the investigated redox-modulator does not alter cell viability of normal lymphocytes, applied in low concentrations.

The obtained results demonstrated that $20 \%$ inhibition of cell viability of leukemia lymphocytes by docosahexaenoic acid, melatonin and resveratrol is accompanied by increased generation of ROS production and induction of apoptosis in different range. Due to the observation that non-cytotoxic effect on normal lymphocytes had been shown, the elevated levels of ROS production by the studied natural compounds is associated rather with specific activation of intracellular regulatory processes for induction of apoptosis in cancer cells, than with direct induction of harmful side effects. 


\section{Biological and health effects of non-ionizing radiation that is used by $5 \mathrm{G}$}

\section{Igor Belyaev}

Department of Radiobiology, Cancer Research Institute, Biomedical Research Center, Slovak Academy of Science, Bratislava, Slovakia

\section{https://doi.org/10.21175/rad.abstr.book.2021.6.12}

Adverse health effects of radiofrequency radiation (RF) from mobile communication including cancer risks and reproductive impacts have been reported, while some studies did not find such effects [1]. Complex dependence of the non-thermal RF effects on various physical and biological variables, such as carrier frequency, polarization, modulation, intermittence, electromagnetic stray fields, genotype, physiological traits, and cell density during exposure account for an apparent inconsistence in the published data [2]. Health effects of $5 \mathrm{G}$ mobile communication, which, in addition to $2 \mathrm{G}-4 \mathrm{G}$ signals, uses also millimeter waves (MMW), are of significant public concern. It follows from available studies that RF in general and MMW in particular, at very low intensities below the ICNIRP guidelines can induce biological effects and affect human health. We have shown that MMW inhibited repair of DNA damage induced by ionizing radiation under exposure at specific frequencies and polarizations [3]. Fundamental role of MMW in regulation of homeostasis was postulated by H. Fröhlich [4] and confirmed in many studies [2]. Available studies, together with almost complete absence of MMW in atmosphere due to effective absorption, which suggests the lack of adaptation to this type of radiation, suggest that the health effects of chronic MMW exposures may be more significant than for any other frequency range. The data showing dependence of RF/MMW effects on static and extremely low frequency magnetic fields suggested a strategy for reducing health effects from RF/MMW of mobile communication.

\section{References}

[1] Belyaev, I. et al. EUROPAEM EMF Guideline 2016 for the prevention, diagnosis and treatment of EMFrelated health problems and illnesses. Rev Environ Health 31, 363-397, doi:10.1515/reveh-2016-0011 (2016).

[2] Belyaev, I. Y. Dependence of non-thermal biological effects of microwaves on physical and biological variables: implications for reproducibility and safety standards. European Journal of Oncology - Library 5, 187-218 (2010).

[3] Belyaev, I. Y., Shcheglov, V. S., Alipov, E. D. \& Ushalov, V. D. Nonthermal effects of extremely highfrequency microwaves on chromatin conformation in cells in vitro - Dependence on physical, physiological, and genetic factors. IEEE Transactions on Microwave Theory and Techniques 48, 2172-2179 (2000).

[4] Frohlich, H. in Advances in Electronics and Electron Physics Vol. 53 (eds L. Marton \& C. Marton) 85-152 (Academic Press, 1980).

Acknowledgments: This study was supported by the Slovak Research and Development Agency (APVV-15-0250) and the Grant Agency VEGA (2/0089/18) of the Slovak Republic. 


\title{
Structural effects in phospholipids monolayers in presence of poly-D-lysines
}

\author{
Yuriy Volkov', Boris Roshchin', Aleksandr Nuzhdin', \\ Viktor Asadchikov', Yuriy Ermakov², Alexey Tikhonov'3, Veijo Honkimäki 4
}

\author{
1 FSRC Crystallography and Photonics RAS, Moscow, Russia \\ 2 Frumkin Institute of Physical Chemistry and Electrochemistry RAS, Moscow, Russia \\ 3 P.L. Kapitza Institute for Physical Problems RAS, Moscow, Russia \\ 4 European Synchrotron Research Facility, Grenoble, France
}

\section{https://doi.org/10.21175/rad.abstr.book.2021.7.1}

Langmuir monolayers of saturated phospholipids exhibit phase transition from "liquid expanded" (LE) to "liquid condensed" (LC) state with change of lateral pressure [1]. In turn, changes in lateral ordering of serine-based lipids can be initiated by adsorption of polypeptide molecules on the lipid layer [2]. We present the investigation of influence of adsorption of poly-D-lysines $\left[\mathrm{H}_{12} \mathrm{C}_{6} \mathrm{~N}_{2} \mathrm{O}\right]_{n} \times \mathrm{HBr}$ on the structure of Langmuir monolayer of dimyristoyl-phosphatidylserine (DMPS) on water with the use of synchrotron X-ray reflectometry (XRR) and Volta potential diagrams. XRR measurements have been carried out at beamline ID31, $\mathrm{ESRF}(\mathrm{E} \approx 17 \mathrm{keV}, \lambda \approx 0.18 \AA$ ). Surface pressure and Volta potential has been measured on a setup MicroTrough XS V4.o (Kibron). Detailed description of the beamline, setup and reflectometry methodics as well as some preliminary results was presented in [3].

DMPS monolayer has been formed by droplet method with calibrated amount of $0.5 \mathrm{mg} / \mathrm{ml}$ solution of $\mathrm{C}_{34} \mathrm{H}_{65} \mathrm{NO}_{10} \mathrm{PNa}$ in chloroform, corresponding to area-per-molecule $72 \AA^{2}$ (LE-phase) and $36 \AA^{2}$ (LC-phase) respectively, on a buffer solution of $10 \mathrm{mM} \mathrm{KCl}$ in triple-distilled water. Two variants of poylysine with chain length $n=12\left(\mathrm{M}_{\mathrm{r}} \sim 5000 \mathrm{Da}\right)$ and $n=200\left(\mathrm{M}_{\mathrm{r}} \sim 70000 \mathrm{Da}\right)$ in concentrations $\sim 0.27 \mathrm{uM}$ have been used for the buffer dopation, according to the expected maximal adsorption level on the interface.

According to the obtained data, surface pressure and Volta potential diagrams exhibit significant difference between states of monolayer with and without poly-D-lysines in the buffer substrate. In particular, presence of polymer leads to more frequent detection of surface clusters of lipid in LE-state. Estimations of effective dipole momentum $\mu$ yields 0.48 and 0.38 Debye respectively. Depth-graded distributions of electron concentration obtained from XRR data show significant decrease in the integral density of lipid layers, which is evident to the change of inclination angle for $\left[\mathrm{CH}_{2}\right]$ chains.

In our opinion, combined results can be explained by the increase in stiffness of monolayer in both phase states, corresponding to more intense formation of lipid clusters. Presence of polymer macromolecules changes the equilibrium at the substrate/monolayer interface, which fundamentally changes the pattern of surface filling within the lipid membrane.

\footnotetext{
References

[1] Loesche M., Sackmann E. and Mohwald H., Ber. Bunsenges. Phys. Chem. 87, 848 (1983).

[2] Ermakov Yu. A., Kamaraju K., Dunina-Barkovskaya A. et al., Biochemistry 56, 5457 (2017).

[3] Ermakov Yu.A., Asadchikov V.E., Volkov Yu.O. et al., JETP 109(5), 340 (2019).
}

Acknowledgments: This work was supported by the Ministry of Science and Higher Education within the State assignments for the institutes of RAS. The authors acknowledge Russian Foundation for Basic Research for funding theoretical part of the work (Project no. 19-04-00242). X-ray experiments have been performed in frames of Project SC-4845 on European Synchrotron Research Facility (Grenoble, France). 


\title{
Functional and structural consequences of foliar application of single-walled carbon nanotubes on photosynthetic membranes
}

\author{
Nia Petrova', Svetla Todinova', Stefka Taneva', \\ Dimitrina Koleva², Petar Petrov3, Violeta Velikova4, Sashka Krumova1
}

\author{
1 Institute of Biophysics and Biomedical Engineering, Bulgarian Academy of Sciences, Sofia, Bulgaria \\ 2 Faculty of Biology, Sofia University "St. K. Ohridski”, Sofia, Bulgaria \\ 3 Institute of Polymers, Bulgarian Academy of Sciences, Sofia, Bulgaria \\ 4 Institute of Plant Physiology and Genetics, Bulgarian Academy of Sciences, Sofia, Bulgaria
}

\section{https://doi.org/10.21175/rad.abstr.book.2021.7.2}

The interaction of nanomaterials with biological objects is becoming of a great interest in the last years due to the advancement of nanotechnology and its various applications in daily life. A number of publications explore the interaction of carbon nanotubes with plant tissues and organelles but only a few of them study their effect on the light-converting photosynthetic (thylakoid) membranes functionality and structural arrangement. In vitro and in silico studies have revealed the possibility for direct physical interaction (electron and exciton transfer, formation of chemical bonds) between photosynthetic reaction centers and carbon nanotubes, but so far this has not been proven in intact plants.

In this work, we characterize the effect of foliar application of polymer-grafted single-walled carbon nanotubes (SWCNT) (in concentration 10, 100 and $300 \mathrm{mg} / \mathrm{L}$ ) on pea plants by probing the functionality, structural organization and thermal stability of plants' thylakoid membranes.

Pulse amplitude-modulated fluorescence imaging on intact leaves revealed that concentrations above $100 \mathrm{mg} / \mathrm{L}$ of SWCNT resulted in lower number of photochemically active photosystem II reaction centers, as well as retarded generation of photoprotective state (non-photochemical quenching of chlorophyll $a$ fluorescence), however the maximal quantum yield of photosystem II in the dark- and light-adapted states was not reduced by any of the applied treatments. Both transmission electron microscopy of chloroplasts and biochemical fractionation of isolated thylakoid membranes demonstrated major restructuring of the stacked and unstacked membrane regions and increase in luminal space at $300 \mathrm{mg} / \mathrm{L}$ SWCNT. Thermally-induced denaturation of the membrane photosynthetic protein components showed a strong effect of SWCNT on the stability of the major light-harvesting complex of photosystem II, supposedly associated with its migration from the grana towards stroma lamellae.

In conclusion, our data reveal concentration dependent effect of foliar application of polymergrafted SWCNT. While the $10 \mathrm{mg} / \mathrm{L}$ concentration did not exhibit any harmful effects with respect to the tested characteristics, the 100 and $300 \mathrm{mg} / \mathrm{L}$ SWCNT concentrations resulted in reduced functionality of photosystem II and structural remodeling of the thylakoid membrane. However, it should be noted that those effects were not detrimental for the plant since it preserved the maximal quantum yield of photosystem II.

Acknowledgments: This work was financially supported by the National Science Fund, Bulgaria (grant number KП-06-H36/8, S.K.). 


\title{
Thermal stability of red blood cell and plasma proteins derived from women with miscarriages: Microcalorimetric approach
}

\author{
Avgustina Danailova', Sashka Krumova1, Ariana Langari', \\ Regina Komsa-Penkova², Georgi Golemanov², Ina Gyosheva3, Emil Gartchev3, \\ Kameliya Kercheva3, Alexey Savov3, Stefka Taneva', Svetla Todinova1
}

\author{
1 Institute of Biophysics and Biomedical Engineering, Bulgarian Academy of SciencesSofia, Bulgaria \\ 2 Department of Biochemistry, Medical University, Pleven, Bulgaria \\ 3 University Obstetrics and Gynecology Hospital "Maichin Dom", Sofia, Bulgaria
}

\section{https://doi.org/10.21175/rad.abstr.book.2021.7.3}

Red blood cells (RBCs) and blood plasma proteins are in contact with all tissues. Therefore, their properties are affected by the physiological/ pathological state of the organism and any change of their features could serve as a potential biomarker for a disease state. Differential scanning calorimetry (DSC) allows for the thermodynamic characterization of proteins that are part of complex biological systems in their native environment, as well as their specific structural or chemical alteration in different pathological conditions. Herein, we apply this method to study the thermal stability of the major proteins of RBCs and blood plasma derived from women with miscarriages (MS), as well as non-pregnant (CNP) and pregnant (CP) age-matched controls.

In structural terms the RBC is maintained by a system of cytoskeletal proteins that are connected to the RBC plasma membrane where two major proteins are found - glycophorin and band 3 protein. The main protein constituent of RBCs is the oxygen-transport metalloprotein hemoglobin that accounts for ca. $96 \%$ of the RBC's dry content. It was suggested that complications during pregnancy are related to the level of the free and the membrane bound hemoglobin, and the hematocrit. Herein we study the structural stability of hemoglobin and cytoskeletal proteins in this pathology.

The calorimetric profiles of RBCs exhibit a complex shape consisting of four well resolved endothermic transitions centered at $50{ }^{\circ} \mathrm{C}, 57^{\circ} \mathrm{C}, 63^{\circ} \mathrm{C}$ and $72{ }^{\circ} \mathrm{C}$, assigned to denaturation of spectrin, bands 2.1, 4.1, 4.2 proteins, the cytoplasmic domain of band 3 protein and hemoglobin, respectively. Freshly collected RBCs of the studied patients do not differ significantly from the controls, however in the course of aging a faster thermal destabilization of hemoglobin, band 3, bands 2.1, 4.1 and 4.2 proteins and spectrin is observed for the MS than for the control groups.

DSC profiles of blood plasma from CNP and CP women does not differ significantly from each other and are characterized by four transitions at about $50{ }^{\circ} \mathrm{C}, 62{ }^{\circ} \mathrm{C}, 70{ }^{\circ} \mathrm{C}, 82{ }^{\circ} \mathrm{C}$ and a shoulder at ca. $76^{\circ} \mathrm{C}$, which can be attributed to fibrinogen; albumin; immunoglobulins, complement protein $\mathrm{C}_{3}$, IgG and transferrin denaturation, respectively. Only slight change in the plasma calorimetric profiles was found related to increased fibrinogen and globulins level in plasma of healthy pregnant women as compared to non-pregnant ones. On the whole, the thermograms of plasma derived from MS women exhibit increased heat capacity of the globulin-assigned transitions and upshift of the denaturation temperature of albumin-assigned transition as well as reduced ratio of albumin/globulin heat capacity compared to those of the control ones.

Our data demonstrate modified DSC plasma thermograms and accelerated aging-related changes in RBCs' calorimetric profiles of women with miscarriages that might serve as markers for high-risk pregnancy.

Acknowledgments: This work is supported by grant KП-06-H21/4, Competition for financial support for basic research projects - 2018, National Science Fund (S.T.). 


\title{
Photo-redox effect of alkaline hydrolysates of wool from small cattle
}

\section{Anna A. Oleshkevich, Svetlana Komarova}

\begin{abstract}
Moscow Skryabin State Academy of Veterinary Medicine and Biotechnology - MVA by K.I (Moscow SAVMB), Moscow, Russia
\end{abstract}

\section{https://doi.org/10.21175/rad.abstr.book.2021.7.4}

The effect of a light flash on the change in the redox potential of alkaline hydrolysates of animal hair (photo-redox effect) was studied. The wool of small cattle (sheep, goats) taken from various parts of the animal's body was chosen as a model object.

The animals varied in breed, sex and age. Alkaline hair hydrolysates were prepared by heating for 30 minutes in $4 \mathrm{M}-\mathrm{NaOH}$ solution in a boiling water bath. After incubation, the hydrolysate was diluted to $\mathrm{pH}$ 12.5. The final concentration of keratin was $2 \mathrm{mg} / \mathrm{ml}$. The following were used as informatively significant parameters of the redox effect: the value of the potential of the measuring electrode relative to the auxiliary one before turning on the light flash; the value of the potential 20 seconds after the beginning of the action of light on the test solution; potential value in 20 seconds after switching off the light flash.

Research has revealed the following:

1. the redox potentials of alkaline hydrolysates of hair, wool and fluff from animals of the subfamily of Goats (Goat) lie in the same interval; the values do not go beyond $56-73 \mathrm{mV}$ ( $\mathrm{p}<$ 0.05);

2. under a flash of light, the redox potential of keratin-containing solutions of skin derivatives changes ( $\mathrm{p}>0.05)$;

3 . the range of changes in the redox potential under illumination is $\pm(5 \div 20) \mathrm{mV}$ from the initial value;

4. the change in the value of the redox potential with an increase in the concentration of the alkali solution is insignificant;

5. when the flash exposure does not exceed 300 seconds the redox shift is completely reversible, longer exposure can lead to irreversible redox shift. 


\title{
Ultrastructural alteration of platelets and erythrocytes derived from women with spontaneous abortions: Atomic force microscopy study
}

\author{
Ariana Langari' ${ }^{1}$, Svetla Todinova, Velichka Strijkova ${ }^{2,1}$, \\ Tonya Andreeva', Sashka Krumova', Stefka Taneva1, \\ Regina Komsa-Penkova3, Georgi Golemanov3, Galya Georgieva3
}

\author{
1 Institute of Biophysics and Biomedical Engineering, Bulgarian Academy of Sciences, Sofia, Bulgaria \\ 2 Institute of Optical Materials and Technologies “Acad. Yordan Malinovski”, Bulgarian Academy of Sciences, \\ Sofia, Bulgaria \\ 3 Department of Biochemistry, Medical University, Pleven, Bulgaria
}

\section{https://doi.org/10.21175/rad.abstr.book.2021.7.5}

Normal pregnancy is accompanied by significant changes in the hemostasis. The hypercoagulability of pregnancy is the prerequisite of the increased risk of thrombosis, especially in women with thrombophilic mutation. This state may induce formation of microthrombosis in uterine-placental blood vessels and subsequently to result in spontaneous abortion in the first trimester. The primary role of platelets in thrombosis is well known, but there is a growing evidence revealing the active roles of erythrocytes in hemostasis as well.

Herein, we apply atomic force microscopy (AFM) to evaluate the alteration of the morphometric (cells morphology and roughness) and nanomechanical (Young's modulus) characteristics of platelets and erythrocytes derived from women with spontaneous abortion (SA) compared to those from control pregnant (CP) and non-pregnant (CNP) women.

The shape of platelets obtained from CNP and CP participants is typical for weakly activated platelets. The morphometric parameters of $\mathrm{CP}$ platelets does not differ significantly from that of CNP expressed in reduced roughness and increased elasticity of cells membrane. The platelets derived from the SA group however are at a later stage of activation, with prominent cytoskeletal rearrangements. Considerably reduced membrane roughness and higher Young's modulus, compared to the controls ones, are found in the patients with SA, $75 \%$ of which are carriers of thrombophilic polymorphisms in (FVL, FII20210A, PLA1/A2, MTHFR or PAI-1) genes. Thus, it appears that the structural and mechanical variations in platelets from women with SA correlate with platelet hyperactivity and the carriage of thrombophilic polymorphisms.

No significant difference is found in the morphological profile of freshly isolated erythrocytes from CNP, CP and SA, women, i.e. the cells population is largely homogeneous and the typical biconcave structure is predominant. Substantial changes in the shape are observed during cells aging, encompassing almost completely biconcave as well as other morphological classes. Modification in the morphological characteristics of erythrocytes is found in the cells aging process that is more pronounced for SA than for the control groups. The membrane roughness of SA cells decreases drastically during the first 10 days and has lower value, while Young's modulus is significantly higher throughout the follow-up period as compared to the control ones. Our data clearly show accelerated erythrocyte aging in women with SA as compared to controls, reflected in altered morphological parameters, which might serve as markers in high-risk pregnancies.

In conclusion, the data clearly demonstrate ultrastructural alteration of platelets and erythrocytes derived from women with spontaneous abortions, which can be used for the development of morphometric algorithm to predict a risk of pathological conditions during early pregnancy.

\section{Acknowledgments:}

This work is supported by grant KП-06-H21/4, Competition for financial support for basic research projects - 2018, National Science Fund (S.T.).

Research equipment of Distributed Research Infrastructure INFRAMAT, part of Bulgarian National Roadmap for Research Infrastructures, supported by Bulgarian Ministry of Education and Science was used in this investigation. 


\section{Color-tuning in $\mathrm{Ca}^{2+}$-binding photosynthetic bacteria}

\section{Arvi Freiberg, Kõu Timpmann, Margus Rätsep, Liina Kangur}

Institute of Physics, University of Tartu, Tartu, Estonia

\section{https://doi.org/10.21175/rad.abstr.book.2021.7.6}

Flexible color adaptation to available ecological niches is vital for the common thriving of photosynthetic organisms. Hence, most purple bacteria living in the shade of green plants and algae apply bacteriochlorophyll-a pigments to harvest near infra-red light around $875 \mathrm{~nm}$. Exceptional are some $\mathrm{Ca}^{2+}$-containing species fit to utilize almost $100 \mathrm{~nm}$ redder quanta. The physical basis of this anomalous spectral red-shifted absorbance (equivalent to $\sim 5.5 \mathrm{kBT}$ at ambient temperature) remains unsettled. In this work, by applying several sophisticated spectroscopic techniques, we firmly establish the $\mathrm{Ca}^{2+}$-facilitated enhancement of the inter-pigment couplings in the LH1core lightharvesting pigment-protein complex as the main cause of the record red-shift. The changes in specific interactions such as hydrogen-bonding between the pigments and the surrounding protein, although present, turned out to be secondary in this regard. Apart from solving a decades-old conundrum, these results complement the list of physical principles applicable for efficient spectral tuning of photo-sensitive molecular nano-systems, native or synthetic alike. 


\title{
Development of a system for in vitro irradiation of biological objects with electromagnetic radiation
}

\author{
Charilaos Xenodochidis', Milena Draganova2,3, \\ Milena Georgieva4, George Miloshev4, Plamen Zagorchevi,3
}

\author{
1 Department of Biophysics, Faculty of Pharmacy, Medical University, Plovdiv, Bulgaria \\ 2 Department of Medical Biology, Medical Faculty, Medical University, Plovdiv, Bulgaria \\ 3 Research Institute at Medical University, Plovdiv, Bulgaria \\ 4 Laboratory of Molecular Genetics, Institute of Molecular Biology “Acad. Roumen Tsanev”, Bulgarian \\ Academy of Sciences, Sofia, Bulgaria
}

\section{https://doi.org/10.21175/rad.abstr.book.2021.7.7}

Introduction. Recently, light sources such as light-emitting diodes (LED) and broadband spectrum lamps have become commonly used in medicine thanks to the similar effects as of lowintensity lasers. Despite the proven positive effects of such therapies, deeper analysis of their effects with non-ionizing radiation is yet to be done on various physiological systems. The aim of this paper is to present a novel system for in vitro irradiation of biological systems that allows the modulation of the activity of major neuromodulators and potentiates a detailed study of the molecular mechanisms through which its effects are executed on cellular and organismal levels.

Materials and methods. All experiments were conducted with male Wistar rats weighting approximately $250 \mathrm{~g}$. Smooth muscle (SM) strips were dissected from the stomach corpus of euthanized rats and were divided into three groups. The first group was composed of non-irradiated tissues (the control group). The second and the third group were composed of SM preparations exposed to UV (LED $365 \mathrm{~nm}$ ) and red (LED $660 \mathrm{~nm}$ ) light, respectively. The power of the two sources was constant at $3 \mathrm{~W}$. The irradiation of the gastric tissues lasted $60 \mathrm{sec}$, and the effect was reported at the $15^{\text {th }}$ min after the act.

Results. We have developed a system for in vitro irradiation of biological tissues and organs and named it a wet organ bath (wob). The wob was used to test the reactivity of rat gastric smooth muscle (SM) tissues to exogenous application of serotonin (5-HT). The LED sources exploited in the system were emitting radiation at wavelengths $365 \mathrm{~nm}$ and $660 \mathrm{~nm}$, while their intensity, power density and direction were optimized experimentally. We have observed changes in the reactivity of the SM strips to 5 - HT at a concentration of $5 \mu \mathrm{M}$ after exposure for 60 sec to LED $365 \mathrm{~nm}$ and LED $660 \mathrm{~nm}$ radiation. Interestingly, in the case of LED UV-light the reactivity of the SM preparations to 5-HT was decreased by $2 \mathrm{mN}$, which was considered a significant change compared with the control group. However, SM strips irradiated with LED $660 \mathrm{~nm}$ demonstrated increased reactivity to 5-HT up to $1 \mathrm{mN}$ against non-irradiated $\mathrm{SM}$ tissues.

Conclusions. We have developed a specialized system for irradiation of biological tissues that allows fine modulation of the contractile abilities of smooth muscles and potentiates their biological functions.

Keywords: Water organ bath, electromagnetic radiation, LED, gastric smooth muscles, serotonin

Acknowledgments: Support for Charilaos Xenodochidis is under the project: "Development of Photodynamic Therapy for Neurodegenerative Diseases by Influencing the Enzyme Monoamine Oxidase A", provided by America for Bulgaria Foundation, Grant №15/22.12.2020. 


\title{
3D tumour spheroids for the prediction of the effects of radiation and treatment with antimicrobial peptides
}

\author{
Mina Raileanu, ${ }^{1,2}$, Mihaela Bacalum', \\ Liviu Craciuns, Tiberiu Esanu'3, Ioana Porosnicu4
}

\begin{abstract}
1 Horia Hulubei National Institute for R\&D in Physics and Nuclear Engineering (IFIN-HH), The Department of Life and Environmental Sciences (DFVM), Magurele, Romania

2 Faculty of Physics of the University of Bucharest, Biophysics Department, Magurele, Romania

3 Horia Hulubei National Institute for R\&D in Physics and Nuclear Engineering (IFIN-HH), Applied Nuclear Physics (DFNA), Magurele, Romania

4 National Institute for Laser, Plasma and Radiation Physics, Magurele, Romania
\end{abstract}

\section{https://doi.org/10.21175/rad.abstr.book.2021.7.8}

For multimodality therapies such as the combination of chemical compounds (in our case antimicrobial peptides) and radiation, the quantification of biological effects is key for dose prescription and response prediction. Tumour spheroids have a microenvironment that resembles more closely that of tumours in vivo and may thus be a superior in vitro cancer model than monolayer cultures. Here, the response of tumour spheroids formed from one established human cancer cell line (HCT116- human colon carcinoma) to single and combination treatments of radiation (X-ray and/or proton radiation) ( $\mathrm{O}-4 \mathrm{~Gy}$ ), and Cecropin A-Melittin (CA-Mel) hybrid has been evaluated. Response was analysed in terms of spheroid growth, cell viability and the cell cycle or senescence of the treated cells. It was found that sensitivity to radiation in spheroids was significantly less than that seen in monolayer cultures. Spheroids showed different patterns of shrinkage and regrowth when exposed to our compounds or radiation: treated spheroids shed dead cells within four days of applying our antimicrobial peptides and displayed faster growth post-exposure than samples that received radiation or no treatment. Irradiated spheroids maintained a dense structure and exhibited a longer growth delay than spheroids receiving chemical treatment or combination treatment. We suggest that, unlike radiation, which kills dividing cells, chemical-induced cell death affects cells independent of their proliferation status. In conclusion, our 3 study reveals differences in response to chemical treatment and/or radiation that were not apparent in $2 \mathrm{D}$ viability assays but that may significantly influence treatment efficacy. 


\title{
Bispidines as versatile bifunctional chelators for ${ }^{64} \mathrm{Cu}$-labeling of biomolecules
}

\author{
Irma Ivette Santana Martinez, Kristof Zarschler, \\ Stephan Holger, Michael Bachmann
}

\author{
Helmholtz Zentrum Dresden Rossendorf, Dresden, Germany
}

https://doi.org/10.21175/rad.abstr.book.2021.8.1

Introduction. Positron emission tomography (PET) represents a very sensitive functional imaging technique in nuclear medicine for diagnosis of various diseases. PET imaging requires the use of a positron emitting radionuclide that is incorporated into a biologically active molecule, the socalled radiotracer. Organically derived radiotracers incorporate nonmetal radionuclides by covalent bond formation, while metal-based radiopharmaceuticals rely on coordination chemistry.[1]

Bifunctional chelators as part of a radiometal-based tracer are responsible for stable complexation of the radiometal ion and for covalent linkage between the complex and the targeting vector. To avoid any loss of complex stability, the bioconjugation strategy must not interfere with the radiometal complexation by occupying coordinating groups. Since the functionalization of the C9 position of the very stable $\mathrm{Cu}^{\mathrm{II}}$ chelator 3,7-diazabicyclo[3.3.1]nonane (bispidine) does not disturb the metal binding centre, we prepare and characterize a set of novel bispidine derivatives equipped with suitable functional groups for diverse bioconjugation reactions. We demonstrate their functionality and versatility by conjugation to antibody-based biomolecules and validate the obtained conjugates in vitro and in vivo.

Methods. A range of novel BFCAs based on the hexadendate bis(amine) tetrakis(pyridine) bispidine-9-ol equipped with suitable functionalities for diverse bioconjugation reactions were synthetized,[2] characterized and radiolabelled with the positron-emitting nuclide ${ }^{64} \mathrm{Cu}$. In order to evaluate their kinetic inertness, ligand challenge experiments using EDTA and DOTA were performed. As a proof of concept, a dibenzocyclooctyne (DBCO)-modified ligand was site-specifically introduced into an azide-functionalized antibody fragment by means of enzyme-mediated bioconjugation and click chemistry.

Results. Bispidine ligands form stable complexes very quickly with ${ }^{64} \mathrm{Cu}^{\mathrm{II}}$ under mild conditions achieving a chemical purity higher than $99.9 \%$. No indications of transchelation were observed in ligand challenge experiments throughout the period of investigation, confirming their excellent $\mathrm{Cu}^{\mathrm{II}}$ complex stabilities. A homogenous, uniform, single-conjugated and highly defined bispidineantibody fragment was obtained by pursuing a two-step site-specific modification strategy. In vitro binding studies proofed high target specificity of the radiolabelled tracer and uncompromised functionality.

Main conclusions. Bispidines with their excellent metal chelation properties and promising bioconjugation strategies are outstanding bifunctional imaging agents. Due to their high ${ }^{64} \mathrm{Cu}^{\mathrm{II}}$ selectivity, ease of functionalisation and high stability, the bispidine derivatives reveal an enormous potential as PET imaging agent for cancer diagnosis. The DBCO-functionalized derivative was designed to undergo strain-promoted azide-alkyne coupling to azide-functionalized antibody fragments or other proteins of interest.

\section{References}

[1] a) T. Derlin, V. Grünwald, J. Steinbach, H. J. Wester, T. L. Ross, Dtsch. Arztebl. Int. 2018, 115, 175-181; b) T. I. Kostelnik, C. Orvig, Chem. Rev. 2019, 119, 902-956.

[2] a) P. Comba, M. Kerscher, K. Rück, M. Starke, Dalton Trans. 2018, 47, 9202-9220; b) S. Juran, M. Walther, H. Stephan, R. Bergmann, J. Steinbach, W. Kraus, F. Emmerling, P. Comba, Bioconjug. Chem. 2009, 2O, 347-359; c) P. Comba, L. Grimm, C. Orvig, K. Rück, H. Wadepohl, Inorg. Chem. 2016, 55, 12531-12543; d) H. Stephan, M. Walther, S. Fähnemann, P. Ceroni, J. K. Molloy, G. Bergamini, F. Heisig, C. E. Müller, W. Kraus, P. Comba, Chem. Eur. J. 2014, 20, 17011-17018. 


\title{
The cytotoxic potential of a novel 2-thioxo-4-thiazolidinone derivative used with anti-HER2 antibodies in AGS gastric cancer cells
}

\section{Wojciech Szymanowski', Kamila Buzun', Anna Szymanowska1, Agnieszka Gornowicz¹, Roman Lesyk², Krzysztof Bielawski33, Anna Bielawska1}

\author{
1 Department of Biotechnology, Faculty of Pharmacy, Medical University of Bialystok, Białystok, Poland \\ 2 Department of Pharmaceutical, Organic and Bioorganic Chemistry, Danylo Halytsky Lviv National Medical \\ University, Lviv, Ukraine \\ 3 Department of Synthesis and Technology of Drugs, Faculty of Pharmacy, Medical University of Bialystok, \\ Białystok, Poland
}

\section{https://doi.org/10.21175/rad.abstr.book.2021.8.2}

Human epidermal growth factor receptor 2 (HER2/neu, ErbB2) is a transmembrane protein with tyrosine kinase activity, that is often overexpressed in many tumors, including gastric cancer. HER2 has become an important molecular target for newly designed anticancer drugs, including monoclonal antibodies such as trastuzumab and pertuzumab. However, some cancer cells display resistance to such treatment, which means that finding new ways of increasing its effectiveness is needed. So far, scientists have observed an increase in response to therapy after the use of some monoclonal antibody in combination with a chemotherapeutic agent.

The aim of the study was to investigate the effect of anti-HER2 antibodies, trastuzumab and pertuzumab, with a novel 2-thioxo-4-thiazolidinone derivative [1] on cell viability and induction of apoptosis in AGS gastric cancer cells. We also investigated the effect of the compound 1 on mitochondrial membrane potential. Cell viability was evaluated using tetrazolium salt according to Carmichael's method. The induction of apoptosis was determined using annexin $\mathrm{V}$ and propidium iodide and the disruption of the mitochondrial membrane potential was assessed using JC-1 mitoscreen kit.

The results of the present study demonstrated that combined treatment of trastuzumab and the compound 1 led to a significant decrease in the survival of AGS gastric cancer cells compared to monotherapy or combined treatment with pertuzumab and the compound 1. Similar effect was observed in the apoptotic activity, in which the investigated compounds strongly induced apoptosis in AGS cells. Trastuzumab together with the 2-thioxo-4-thiazolidinone derivative led to the decrease of mitochondrial membrane potential in the tested cell line. 


\title{
The pro-apoptotic effect of novel pyrazolo[4,3-e]tetrazolo[4,5-b][1,2,4]triazine derivatives in HT-29 colon cancer cells
}

\author{
Anna Szymanowska1, Kamila Buzun', Wojciech Szymanowski, \\ Robert Czarnomysy², Agnieszka Gornowicz' ${ }^{1}$, Mariusz Mojzych³, \\ Anna Bielawska', Krzysztof Bielawski²
}

\author{
1 Department of Biotechnology, Faculty of Pharmacy, Medical University of Bialystok, Bialystok, Poland \\ 2 Department of Synthesis and Technology of Drugs, Faculty of Pharmacy, Medical University of Bialystok, \\ Bialystok, Poland \\ 3 Department of Organic Chemistry, Faculty of Sciences, Siedlce University of Natural Sciences and \\ Humanites, Siedlce, Poland
}

\section{https://doi.org/10.21175/rad.abstr.book.2021.8.3}

Colon cancer has been is considered as the major cause of death in many countries in the world. The currently used therapy is cytotoxic and as a search for novel anti-cancer drug candidates among $[1,2,4]$ triazine derivatives is a promising field of research. The diverse biological activities of $[1,2,4]$ triazine: antiviral, antitumor, antifungal, anti-inflammatory and hypotensive has been reported. In addition numerous studies showed that $[1,2,4]$ triazine condensed with other heterocycles possesses a broad spectrum of biological activities. The structure of tricyclic pyrazolo[4,3-e]tetrazolo[4,5-b][1,2,4]triazine derivatives is similar to CDK inhibitors which play crucial role in treatment of cancer. Hence we designed a novel series of pyrazolo[4,3-e]tetrazolo[4,5$b][1,2,4]$ triazine derivatives that might have a potential anticancer effect in colon adenocarcinoma cells.

Cytotoxic activity of novel pyrazolo[4,3-e]tetrazolo[ $[4,5-b][1,2,4]$ triazine derivatives was evaluated against HT-29 - colon adenocarcinoma cell line. Cell viability was assessed according to the Carmichael's method using tetrazolium salt after 24 hours of incubation. The induction of apoptosis was determined using annexin $\mathrm{V}$ and propidium iodide. The decrease of mitochondrial potential was measured using JC-1 MitoScreen kit.

We showed that the tested compounds led to the inhibition of cell viability. Furthermore novel synthesized derivatives had a significant cytotoxic activity in HT-29 cells comparable to roscovitine and 5-flurouracil. Moreover pyrazolo[4,3-e]tetrazolo[4,5-b][1,2,4]triazine derivatives led to the decrease of mitochondrial potential in tested cell line. Further studies will be required to understand the detailed molecular mechanism of action of novel series of pyrazolo[4,3-e]tetrazolo[4,5$b][1,2,4]$ triazine.

Acknowledgments: Research is financed as part of the "Incubator of Innovation + 2.0" project. 


\title{
The pro-apoptotic effect of new 2-thioxo-4-thiazolidinone derivative Les-3331 on MCF-7 and MDA-MB-231 cell lines
}

\author{
Kamila Buzun', Agnieszka Gornowicz', Robert Czarnomysy², \\ Roman Lesyk ${ }^{3}$, Krzysztof Bielawski², Anna Bielawska ${ }^{1}$
}

\begin{abstract}
1 Medical University of Bialystok, Faculty of Pharmacy, Department of Biotechnology, Bialystok, Poland 2 Medical University of Bialystok, Faculty of Pharmacy, Department of Synthesis and Technology of Drugs, Bialystok, Poland

3 Danylo Halytsky Lviv National Medical University, Department of Pharmaceutical, Organic and Bioorganic Chemistry, Lviv, Ukraine
\end{abstract}

\section{https://doi.org/10.21175/rad.abstr.book.2021.8.4}

Apoptosis, also known as type I programmed cell death, is a process precisely regulated at the gene level. It leads to the organized and efficient removal of cells in which DNA damage or other defects have occurred during development. The mechanism of apoptosis is complex and involves multiple signaling pathways. The intrinsic signaling cascade is initiated inside the cell and involves mitochondria. The extrinsic signaling pathways begin at the cell surface when a specific death-ligand binds to an appropriate receptor. Both pathways lead to the activation of specified initiator caspases and subsequent activation of the executioner caspases [1]. Preliminary anticancer in vitro studies on novel 2-thioxo-4-thiazolidinone derivative Les-3331 have shown high cytotoxicity and strong antiproliferative activity of the compound against human breast cancer cell lines (MCF- 7 and MDA-MB-231).

The aim of this study was estimation of Les-3331 proapoptotic effect against MCF-7 and MDA-MB-231 cancer cell lines. The flow cytometry was utilized to examine the induction of apoptosis. Next, the enzyme-linked immunosorbent assay kits were used to determine the influence of Les-3331 on caspase-8 and caspase-9 concentrations.

The obtained results showed that the novel 2-thioxo-4-thiazolidinone derivative Les-3331 induced apoptosis in both human breast cancer cells. Furthermore, Les-3331 led to an increase in caspase- 8 and caspase-9 concentration, both in MCF-7 and MDA-MB-231 cell lines. The study revealed that the probable mechanism of action of Les-3331 is related to the activation of the extrinsic and intrinsic apoptotic pathways.

\section{References}

[1] A. Gornowicz et al., "The effect of novel 7-methyl-5-phenyl-pyrazolo[4,3-e]tetrazolo[4,5-b][1,2,4]triazine sulfonamide derivatives on apoptosis and autophagy in DLD-1 and HT-29 colon cancer cells", Int. J. Mol. Sci., vol. 21, no. 15, article ID 5221, 2020

Acknowledgments: Research is financed under the project № POWR.03.02.00-00-Io51/16 from European Union funds, PO WER 2014-2020, grant № 02/IMSD/G/2019. 


\title{
Anticancer activity of novel selenoesters in MCF-7 and MDA-MB-231 human breast cancer cells
}

\author{
Dominika Radomska1, Dominik Radomski',2, \\ Robert Czarnomysy', Krzysztof Bielawski
}

\begin{abstract}
1 Medical University of Bialystok, Faculty of Pharmacy with the Division of Medical Analytics, Department of Synthesis and Technology of Drugs, Białystok, Poland

2 Medical University of Bialystok, Faculty of Pharmacy with the Division of Medical Analytics, Department of Biotechnology, Bialystok, Poland
\end{abstract}

\section{https://doi.org/10.21175/rad.abstr.book.2021.8.5}

Breast cancer is one of the most commonly diagnosed cancers and its mortality rate is in the lead of cancer-related deaths. Selenium compounds seem to give new perspectives and hope in this field. It turns out that these compounds may be more effective and selective with a simultaneous reduction of their dose and toxicity in comparison to commonly used treatment, and they can also overcome multidrug resistance. Moreover, these compounds make it possible to reduce the doses of standard drugs used in combination with conventional therapy, increasing their anticancer activity [1].

Therefore, our study aimed to evaluate the cytotoxic and antiproliferative activity of novel selenoesters with ketone and nitrile end fragments (EDAG-1, EDAG-7, EDAG-8, EDAG-10, EDA-71, and E-NS-4) against MCF-7 and MDA-MB-231 breast cancer cells and MCF-10A normal breast epithelial cells. Their cytotoxic potential was analyzed by the cell viability and DNA biosynthesis in cancer cells after 24 hours of incubation with these compounds in different concentrations. For this purpose, the MTT assay was performed according to the Carmichael's method using tetrazolium salt, and the incorporation of radioactive [3H]-thymidine into the DNA of MCF-7 and MDA-MB-231 cells was measured.

The preliminary study results prove that EDAG-1 and EDAG-8 exhibit greater cytotoxic effects than the reference compound - epirubicin. Whereas the other compounds exhibit similar or less cytotoxic activity on cancer cells than epirubicin. Furthermore, in an investigation of the incorporation of $[3 \mathrm{H}]$-thymidine into the DNA of cancer cells, it was observed that the compounds had an effect on DNA biosynthesis and led to its inhibition at low concentrations in the nanomole range. Importantly, the cytotoxic and antiproliferative activity of these compounds is in a dosedependent manner. Additionally, the results show that selenoesters with ketone end fragments are more cytotoxic than those containing nitrile end fragments, and the amount of selenoester substituents in the benzene ring has an impact on the anticancer activity of these compounds.

In conclusion, the novel selenoesters exhibit potent anticancer activity even at nanomolar concentrations but further studies are required to determine the detailed molecular mechanisms of their action.

\section{References}

[1] Radomska et al. Selenium Compounds as Novel Potential Anticancer Agents. Int J Mol Sci, 2021, 22, 1009, doi:10.3390/ijms22031009. 


\title{
Evaluation of pro-apoptotic activity of novel derivatives of 1,2,4-triazine sulfonamides in DLD-1 colon cancer cells
}

\author{
Dominik Radomski',2, Dominika Radomska², Anna Szymanowska1, \\ Robert Czarnomysy², Krzysztof Bielawski², Anna Bielawska ${ }^{1}$
}

\begin{abstract}
1 Medical University of Bialystok, Faculty of Pharmacy with the Division of Medical Analytics, Department of Biotechnology, Bialystok, Poland

2 Medical University of Bialystok, Faculty of Pharmacy with the Division of Medical Analytics, Department of Synthesis and Technology of Drugs, Bialystok, Poland
\end{abstract}

\section{https://doi.org/10.21175/rad.abstr.book.2021.8.6}

Colorectal cancer (CRC) is among the most lethal and frequently diagnosed cancers worldwide. Many researchers still search for drugs that are more effective, selective, and less toxic than current CRC treatments. The use of 1,2,4-triazine sulfonamides seem to be one of the possible solutions to this problem. These compounds exhibit a diverse and broad spectrum of their activity. There is evidence of their antimicrobial, anti-inflammatory, anesthetic, and anticancer properties, as well as their use in oncological treatment dates back to 1965 when patients were treated with altretamine in clinical trials for the first time. Several studies report that 1,2,4-triazine sulfonamide derivatives exhibited anticancer effects through inhibition of Brc-Abl kinase and carbonic anhydrase IX and XII activities [1].

We aimed to analyze the anticancer effects of novel 7-methyl-5-phenyl-pyrazolo[4,3$e]$ tetrazolo[ $[1,5-b][1,2,4]$ triazine sulfonamide derivatives (MM124 and MM137) against DLD-1 human colorectal cancer cells. Roscovitine and 5 -fluorouracil were used as reference compounds. Cell viability was measured by MTT assay following the Carmichael's method using tetrazolium salt, while the antiproliferative activity of our compounds was determined by tritium-labeled thymidine incorporation into the DNA of DLD-1 cells. Furthermore, the influence of 1,2,4-triazine sulfonamide derivatives on apoptosis process and mitochondrial potential was assessed using annexin $\mathrm{V}$ and propidium iodide, and JC-1 MitoScreen kit, respectively.

As a result of our study, we found that MM124 and MM137 have higher cytotoxic activity and lead to a decreased incorporation of $[3 \mathrm{H}]$-thymidine into cell DNA in comparison to roscovitine and 5 -fluorouracil. Moreover, treatment with these compounds resulted in an enhanced apoptosis process and a significant decrease of mitochondrial potential in human colorectal cancer cells. The obtained results seem to be very promising, although further studies are required to elucidate the detailed molecular mechanism of action of the novel 1,2,4-triazine sulfonamide derivatives.

\section{References}

[1] Szymanowska et al. [1,2,4]triazines - as potential drugs in cancer chemotherapy. Postępy Higieny $i$ Medycyny Doświadczalnej, 2021, 75, 64-84, doi:10.5604/01.3001.0014.7145.

Acknowledgments: Research is financed as a part of the "Incubator of Innovation + 2.0" project. 


\title{
Empirical modelling of the antiproliferative activity of palbociclib on leukemia lymphocytes
}

\author{
Donika Ivanova', Zvezdelina Yaneva² \\ 1 Trakia University, Faculty of Veterinary Medicine, Department of Pharmacology, Animal Physiology and \\ Physiological Chemistry, Chemistry Unit, Students Campus, Stara Zagora, Bulgaria \\ 2 Chemistry Unit, Department of Pharmacology, Animal Physiology and Physiological Chemistry, Faculty of \\ Veterinary Medicine, Trakia University, Students campus, Stara Zagora, Bulgaria
}

\section{https://doi.org/10.21175/rad.abstr.book.2021.8.7}

Chemotherapy uses both target-specific and systemic-action drugs and drug combinations to treat cancer. Cyclin-dependent kinases (CDKs) are critical regulatory enzymes implicated in the control of cell cycle transitions, from G1 to $\mathrm{S}$ phase. Palbociclib is a CDK4/6 specific inhibitor, approved by Food and Drug Administration (FDA). Its molecular mechanism of action includes phosphorylation of the tumor suppressor retinoblastoma $(\mathrm{Rb})$ and blocks cell cycle progression. Palbociclib has been clinically proven to be effective in breast cancer, but its use in acute lymphoblastic leukemia has rarely been explored. Mathematical modelling of cancer cells' response to chemotherapy assists the judicious selection of a drug type and its dosage for optimized drug administration.

The aim of the present study was to assess the applicability of two empirical mathematical models - the bi-exponential and 2-nd order polynomial model, for description of the antiproliferative behaviour of Palbociclib on leukemia lymphocytes. The effect of chemotherapeutic concentration and incubation time on cancer cell viability was investigated. The values of the model parameters, regression coefficients and error functions were determined by non-linear regression analyses. The obtained results proved a significant extend of correlation between the experimental data and the bi-exponential model. The latter outlines the possibility of applying it for prediction of the antiproliferative effect of various doses of Palbociclib, the corresponding cancer cell viability and provides ease of finding out the best therapy and a quantitative benchmark in general. 


\title{
PEGylated nanographene oxide-based nanoparticles as smart nanocarriers for colon cancer photothermal therapy
}

\section{Milena Georgieva', Zlatina Gospodinova ${ }^{2,3}$, Milena Keremidarska-Markova²,4, Trayana Kamenska², Galina Gencheva5, Natalia Krasteva²}

\author{
1 Institute of Molecular Biology “Acad. R. Tsanev”, Bulgarian Academy of Sciences, Sofia, Bulgaria \\ 2 Institute of Biophysics and Biomedical Engineering, Bulgarian Academy of Sciences, Sofia, Bulgaria \\ 3 Institute of Plant Physiology and Genetics, Bulgarian Academy of Sciences, Sofia, Bulgaria \\ 4 Sofia University "St. Kliment Ohridski”, Faculty of Biology, Sofia, Bulgaria \\ 5 Sofia University “St. Kliment Ohridski”, Faculty of Chemistry and Pharmacy, Sofia, Bulgaria
}

\section{https://doi.org/10.21175/rad.abstr.book.2021.8.8}

Smart nanomaterials that can integrate chemotherapy and photothermal therapy (PTT) into a single nanocarrier are the focus of intensive research since the last decade. Graphene derivatives appear to be one of the most promising nanomaterials for such smart nanocarriers. The $2 \mathrm{D}$ carbon lattice of graphene oxide (GO) provides a large surface area which allows loading with a high number of molecules suitable for the development of multipurpose nanoplatforms. The special optical properties of GO, namely their strong visible and near-field absorbance, enable their exploitation for cancer PTT. However, GO has the propensity to form aggregates, particularly under physiological conditions which could compromise their biocompatibility. One approach for stabilizing GO nanoparticles (NPs) in aqueous dispersions is coating the particle surface with polymeric ligands thus yielding it with a physical barrier. Polyethylene glycol (PEG) is a hydrophilic polymer widely used for the functionalization of various nanomaterials to improve their solubility and stability in physiological solutions, as well as their biocompatibility. In this study, we aimed at designing smart nanoparticles based on PEGylated nanographene oxide particles (nGO-PEG) and near-infrared (NIR) irradiation and evaluated their effect on low and high invasive colorectal carcinoma cells. Physicochemical properties of pristine and PEGylated GO NPs were thoroughly estimated. PEGylation was found to increase the particle sizes from $256.7 \mathrm{~nm}$ (pristine $\mathrm{nGO}$ ) to $324.6 \mathrm{~nm}$ (nGOPEG), the zeta potential from -32.9 to $-21.6 \mathrm{mV}$ and the absorbance of GO in the NIR region. Further, nGO showed enhanced stability in an aqueous solution, improved dispensability in a complete culture medium and amended biocompatibility after PEGylation. A strong synergic effect of nGOPEG activated with NIR irradiation for $5 \mathrm{~min}\left(1.5 \mathrm{~W} / \mathrm{cm}^{-2}\right.$ laser) was observed on cell growth inhibition of low invasive colon cancer cells (HT29) and their wound closure ability while the effect of NIR on cellular morphology was relatively weak. Our results show that PEGylation of nGO combined with NIR irradiation holds the potential for biocompatible smart nanocarrier in colon cancer cells with improved physicochemical properties and enhanced biological compatibility. Further optimization of the irradiation process and detailed screening of nGO-PEG in combination with NIR and chemotherapeutics on colon cancer cell fate is a prerequisite for high efficient combined nanoand photothermal therapy for colon cancer.

Keywords: Nano-graphene oxide (nGO), nGO-PEG, near-infrared (NIR) light, phototherapy, colon cancer 


\title{
PEGylated graphene oxide nanoparticles as a tool for the regulation of cancer cell invasion and growth
}

\author{
Milena Georgieva', Zlatina Gospodinova ${ }^{2,3}$, \\ Trayana Kamenska ${ }^{2}$, Galina Gencheva ${ }^{4}$, Natalia Krasteva ${ }^{2}$
}

\author{
1 Institute of Molecular Biology “Acad. R. Tsanev”, Bulgarian Academy of Sciences, Sofia, Bulgaria \\ 2 Institute of Biophysics and Biomedical Engineering, Bulgarian Academy of Sciences, Sofia, Bulgaria \\ 3 Institute of Plant Physiology and Genetics, Bulgarian Academy of Sciences, Sofia, Bulgaria \\ 4 Sofia University “St. KlimentOhridski”, Faculty of Chemistry and Pharmacy, Sofia, Bulgaria
}

\section{https://doi.org/10.21175/rad.abstr.book.2021.8.9}

Nowadays cancer is one of the leading causes of death in the world. Current diagnostic techniques and therapeutic approaches need improvement to provide earlier diagnosis and treatment and to overcome the limitation arising from lack of specificity and systemic toxicity. In this scenario, nanomaterials could help in optimizing the efficacy of cancer treatment. In the last decade, graphene and its derivatives unambiguously have collected research interest worldwide for various potential medical applications including in vivo diagnosis, gene and drug delivery, and cancer photothermal therapy. Graphene oxide (GO) is the water-soluble form and the most common derivative of graphene. It is characterized as an exceptional nanomaterial that possesses multiple physical properties critical for biomedical applications. The large, 2D planar surface structure and enriched oxygen-containing groups provide relatively good biocompatibility and solubility, together with its ability for loading a high number of different molecules, including drugs, are properties that are appropriate for delivering drugs within the body. Functionalization with polymer ligands further improves the solubility and stability of GO in physiological solutions and alters the reactivity of nanoparticles toward cancer cells. In this work, we have functionalized GO with polyethylene glycol (PEG) and have evaluated the effect of the as-made nanoparticles on the migratory and proliferation ability of a different type of cancer cells, including A375 melanoma cells and two colon adenocarcinoma cells with different invasion potential - Colon26 and HT29. We have compared the obtained results to those for normal kidney MDCK cells. Both types of GOs, pristine and PEGylated, suppressed cancer cells proliferation and mobility in a concentration and cell type-dependent manner while in normal MDCK cells only the highest concentrations of 200 and $500 \mu \mathrm{g} / \mathrm{ml}$ inhibit cell growth at 48 hours of exposure suggesting that cancer cells were more sensitive to GO and GO-PEG treatment than normal cells. In general, PEGylation mitigated the inhibitory effect of GO on the growth and migratory ability of all studied cancer cells. Our results proved that the effects of both GOs NPs on cancer cells proliferation and mobility were dose-, NPs- and cell-type-dependent, hence providing a rationale for future design and use of graphene-based nanomaterials for cancer research. 


\title{
Irradiation-surviving NSCLC cells exhibit partial EMT-program activation and altered DNA damage response depending on their p53 status
}

\author{
Margarita Pustovalova', Lina Alhaddad, \\ Taisia Blokhina², Andreyan Osipov², Sergey Leonov
}

\begin{abstract}
1 Moscow Institute of Physics and Technology, Dolgoprudny, Russia
2 State Research Center-Burnasyan Federal Medical Biophysical Center of Federal Medical Biological Agency, Moscow, Russia
\end{abstract}

\section{https://doi.org/10.21175/rad.abstr.book.2021.8.10}

Non-small cell lung cancer (NSCLC) represents $85 \%$ of all lung cancer cases. Ionizing radiation (IR) is used for patients diagnosed with unresectable NSCLC. However, radiotherapy remains largely palliative due to the survival of specific cell subpopulations which can acquire radioresistance. In the present study, we compared two NSCLC cells sublines, A549 (p53wt) and H1299 (p53null), survived multifraction X-ray radiation exposure (MFR) at a total dose of $60 \mathrm{~Gy}$. The surviving cells demonstrated reduced radiosensitivity and morphological changes compared to parental cells. p53 status affected the pattern of expression of Epithelial-mesenchymal Transition (EMT) markers - Ncadherin, E-cadherin and Vimentin in MFR-surviving cells compared to parental NSCLC cells, witnessing the appearance of EMT-like phenotype of MFR-surviving sublines. p53 significantly affected the 1D confined migratory behavior (wound healing) of NSCLC cells before and after MFR. Our data further emphasize the impact of p53 status on the kineticks of $\gamma \mathrm{H} 2 \mathrm{AX}$ foci and the associated efficacy of the DNA DSB repair in NSCLC cells survived after MFR. We revealed that Rad51 protein might play a principal role in MFR-surviving of p53null NSCLC cells promoting DNA DSB repair by homologous recombination (HR) pathway. The proportion of Rad51 + cells elevated in CD44high/CD166high population in MFR-surviving p53wt and p53null sublines and their parental cells. For the first time, we demonstrated that MFR exposure led to the significant decrease in the expression of p63 and p73, the p53-family members, in p53null cells, which correlated with the increase of cell polyploidy. Our data indicate that accurate assessment of the p53 status-related migration, autophagy, senescence, and EMT processes in populations of MFR-surviving cells can reveal new potential targets of NSCLC cell resistance to radiotherapy, opening up an avenue for the development of new modulators radiosensitizing tumor cells while rescuing normal tissue for patients' therapeutic benefit. 


\title{
Interaction of plasma kallikrein-kinin system proteins with breast cancer cells
}

\author{
Katia R.B. Melo, Fabio D. Nascimento, Mariana S. Araujo, \\ Marcela M.P. Alvarez, Renan P. Cavalheiro, Carlos M.V. Palomino, Maria \\ Aparecida S. Pinhal, Helena B. Nader, Ivarne L.S. Tersariol, Guacyara Motta
}

Universidade Federal de São Paulo, São Paulo, Brazil

https://doi.org/10.21175/rad.abstr.book.2021.8.11

Introduction. The activation of contact system proteins (CS) of the coagulation intrinsic pathway cascade is a phenomenon that has been studied in relation to thrombosis associated with the worst prognosis of cancer and the plasma kallikrein-kinin system (KKS) is related to CS (Campello, E. et al., 2018; Schmaier, A.H., 2016). The aim of this work is to study, in human breast epithelial cells, the interaction of high molecular mass kininogen (HK), the presence of plasma kallikrein (PKa) and bradykinin (BK) release.

Methods. The cell lineages studied were MCF-10A (non-metastatic), MCF-7 (less-metastatic) and MDA-MB-231 (highly-metastatic). The techniques employed were confocal microscopy, radioimmunoassay (RIA) and RT-PCR. The proteins/reagents used were HK-Dylight-650; anti-BK (MBK3 clone) and anti-PKa (U691.10) antibodies; inhibitors of kininases (JA2, thiorphan, captopril) and serine proteases (aprotinin, benzamidine, PMSF). The confocal microscopy analysis showed HK-Dylight-650 (200.0 nM) bound on cell surface in MCF-10A (1,947.0 \pm 0.003 pixels/cell), MCF-7 (648.0 \pm 0.002 pixels/cell) and MDA-MB-231 (1,251.00 \pm 0.002 pixels/cell). In endocytic vesicles HK-Dylight-650 and LT-green colocalized in MCF-10A (974.0 \pm 0.002 pixels/cell), MCF-7 (61.0 \pm 0.001 pixels/cell) and MDA-MB-231 (385.0 \pm 0.001 pixels/cell). In comparison the HK binding and endocytosis was MCF10A > MDAMB-231 > MCF-7. By confocal microscopy the BK was detected on surface of MCF-10A (2,654.0 \pm 0.002 pixels/cell) and 19.7\% colocalized with LT-red, MCF-7 showed BK bound (264.0 \pm 0.002 pixels/cell) and $40.9 \%$ colocalized with LT-red and MDA-MB-231 showed BK bound (109.0 \pm 0.002 pixels/cell) and 53\% colocalized with LT-red. The BK in endocytic vesicles of MDA-MB-231 allows its proliferative effect in metastatic cells. The kininogenase activity was analyzed by the amount of BK released in supernatant, after incubation with $\mathrm{HK}$, and determined by RIA. In presence of kininases inhibitors the amount of BK was MCF-10A $\left(1.13 \pm 0.01 \mathrm{ng} / 10^{6} \mathrm{cell}\right), \mathrm{MCF}-7\left(0.29 \pm 0.01 \mathrm{ng} / 10^{6} \mathrm{cell}\right), \mathrm{MDA}-\mathrm{MB}-231\left(0.78 \pm 0.00 \mathrm{ng} / 10^{6} \mathrm{cell}\right)$; in presence of both serine proteases and kininases inhibitors the BK released was MCF-1OA (0.03 $\left.\pm 0.01 \mathrm{ng} / 10^{6} \mathrm{cell}\right), \mathrm{MCF}-7$ (0.19 $\left.\pm 0.00 \mathrm{ng} / 10^{6} \mathrm{cell}\right)$ and MDA-MB-231 (o.21 $\left.\pm 0.00 \mathrm{ng} / 10^{6} \mathrm{cell}\right)$. The higher BK release in MCF-10A cells is in agreement with its proangiogenic effect. In MDA-MB231 we detected plasma prekallikrein (PK) mRNA and these cells analyzed by confocal microscopy showed PK/PKa in both non-permeabilized and permeabilized cells.

Conclusion. Our data suggest a role for plasma KKS in breast cancer through interaction of HK with cell surface that may results in endocytosis and BK release through endogenous and pericellular proteolysis by PKa. 


\title{
Investigating proton irradiation effects on glioblastoma cell lines
}

\author{
Simona-Cristina Anghelina, Mihaela Temelie, Liviu Crăciun, Diana Savu
}

Horia Hulubei National Institute for Physics and Nuclear Engineering, Măgurele, Romania

\section{https://doi.org/10.21175/rad.abstr.book.2021.8.12}

The glioblastoma multiform (GBM) is a heterogeneous and highly invasive form of malignant primary brain tumor, being the most aggressive brain cancer. The survival of patients which received surgery and/or chemo-/radiotherapy remains poor, only $3-7 \%$ of patients surviving longer than 5 years following diagnosis. Non-conventional radiotherapy such as proton irradiation represents a better choice than conventional photon irradiation due to the higher ionizing density of protons in the Bragg peak, that can induce complex DNA lesions, difficult to be repaired by mutated tumor cells. Our aim was to investigate proton irradiation effect on cellular survival of glioblastoma cell lines. Preliminary in vitro studies were performed on three different cell lines of glioblastoma (U-118 MG, U-251 MG and U-87 MG). The proton irradiation was done at the Cyclotron TR-19 facility (within IFIN-HH). The cell were irradiated with doses of 2,4 and 6 Gy at a dose-rate of $1 \mathrm{~Gy} / \mathrm{min}$. MTT assay was used to determine cell viability 5 days post-irradiation. Long-term cellular survival was determined by clonogenic assay. We observed a dose-dependent decrease of cells viability and clonogenic survival for all the cell lines. In further experiments, we will investigate the mechanisms of radioresistance with the aim to develop new antitumor strategies.

Keywords: Glioblastoma, proton irradiation

Acknowledgments: The study was supported by EEA Norway Romania grants (EEA-RO-NO-2019-0510, nr. 41/2021). 


\title{
About the mathematical models of the Covid-19 epidemic
}

\author{
Nikolay Kokodii ${ }^{1,2}$, Ihor Krasovskyi², Stanislav Pogorelov², \\ Vladimir Timaniuk², Dmytro Kuzmin², Katerina Ivanova²
}

1 Karazin Kharkiv National University, Kharkiv, Ukraine

2 National University of Pharmacy, Kharkiv, Ukraine

\section{https://doi.org/10.21175/rad.abstr.book.2021.9.1}

Relevance. Analyzing and forecasting the epidemic dynamics using mathematical models is very useful for assessing the situation and choosing methods to combat the disease. Models should:

- correctly describe the past or current epidemic dynamics in order to provide for the necessary actions when it recurs in the future;

- give a forecast of the development of the current epidemic based on information about its initial site and the current period.

But, it is difficult to make a forecast based on statistical data, which are often incomplete and have errors. Therefore, there are many models using different mathematical methods. Many models use a system of differential equations, for example, SIR, SEIR, etc.

The disadvantages of these models are:

- the need for numerical solution of systems of differential equations. The impossibility of obtaining formulas describing the ongoing processes complicates the analysis of the results obtained.

- when solving, it is necessary to select the values of several parameters, which leads to the ambiguity of the results obtained.

Purpose of work. To test a mathematical model of the COVID-19 epidemic in several countries of the world for the correct description of the epidemic dynamics and make a forecast of its development further.

Results. Based on statistical data on the COVID-19 epidemic dynamics in various countries, the mathematical model of the SIR type was tested. The selected countries had different epidemic dynamics - fast and slow growth of number of cases, with the presence and absence of a maximum during the epidemic, with different stages of development of the epidemic at the time of the analysis. We used statistical data on the epidemic dynamics in a given country from its beginning to January 2021. The correctness of the description of the epidemic dynamics and the possibility of forecasting based on data on the initial stage of the epidemic were checked.

A model was also tested using the fact that the time dependence of the number of patients is well described by the Gaussian function.

Conclusions. An unexpected fact was established that the model based on the Gaussian function describes and predicts the COVID-19 epidemic dynamics well. The epidemic dynamics forecast in Ukraine and some other countries was made. 


\title{
An investigation into occupational burnout among radiographers in Ireland due to the COVID-19 pandemic and the strategies used to cope with it
}

\author{
Roisin Brennan, Michaela Davis \\ University College Dublin School of Medicine and Medical Science, Dublin, Ireland
}

https://doi.org/10.21175/rad.abstr.book.2021.9.2

Background. Occupational burnout has been shown to have a profound impact on many professionals, however it has been reported that more healthcare workers experience it than employees in any other line of work. During the COVID-19 pandemic, radiographers are working on the frontline, imaging infected and potentially infected patients. This study aims to identify the prevalence of burnout among radiographers employed in Irish hospitals, the extent in which COVID-19 contributes to this and mechanisms in which these professionals cope with burnout arising from the pandemic.

Method. An anonymous online survey which incorporated both open and closed-ended questions was distributed to radiographers employed in eight hospitals in Ireland. The responses were analysed using descriptive statistics on SPSS Version 16, and thematic analysis utilising NVIVO12.

Results. 93 radiographers completed the survey, with 45 (48\%) respondents reported suffering from moderate to very high levels of burnout in the workplace. COVID-19 was among the various reasons for burnout, with participants stating that they are afraid of contracting the disease and that they have been affected psychologically from treating infected patients. Radiographers also declared that they are apprehensive of their colleagues who do not abide by the COVID-19 regulations. Over half of radiographers feared bringing the virus home to family most or all of the time. Unavailability of annual leave during the pandemic was widely reported in the open-ended responses. Strategies commonly adapted by radiographers within the workplace to manage burnout resulting from COVID-19 include talking to and sharing workloads with colleagues, getting sufficient breaks, requesting PPE, practising regular infection control and social distancing. Outside of the hospital, radiographers cope with burnout by going for exercise, relaxing, acquiring enough sleep, talking to family and friends and carrying out hobbies.

Conclusion. It is evident from this research that radiographers in Ireland suffer from high levels of burnout and that COVID-19 is a very prominent source of stress. Many radiographers incorporate various coping strategies in their lives, both in and out of work. However, it is clear that more work needs to be undertaken to address the significant burnout among radiographers arising from the coronavirus pandemic. Advising radiographers of ways to deal with burnout may minimise any future increasing incidences of mental health problems in the profession arising from occupational burnout. 


\section{Multiple thromboses as late complications in a patient with pneumonia caused by the COVID-19 infection}

\section{Mitko Mitev}

Department of Medical Physics, Biophysics, Roentgenology and Radiology, Faculty of Medicine, Department of Diagnostic Imaging, University Hospital 'St. Kirkovich', Trakia University - Stara Zagora, Stara Zagora, Bulgaria

\section{https://doi.org/10.21175/rad.abstr.book.2021.9.3}

Introduction. Venous and arterial thromboembolisms are associated with COVID-19, but there are few studies of lower limb ischemia as a later complication of infection. The study presents identified early and late complications caused by COVID-19, with the presence of multiple thromboses in the aorta and peripheral vessels.

Material and methods. The patient is an 80-year-old man hospitalized with COVID-19 (SARS-CoV-2). The study was conducted in 2021. MDCT Siemens Definition AS was used. Computed tomography angiography was made with Omnipaque, $100 \mathrm{ml}$. The data was processed with Syngo.via workstation and VB4OB_NFO2 software version.

Results. After computed tomography angiography, changes were found in the lungs, central and peripheral vessels. Bilateral interstitial pneumonia was diagnosed. A parietal thrombus was identified in the area of the aortic arch and acute thrombosis on the left side of the common iliac artery and on the right side of the superficial femoral artery as an acute later complication of the infection. The patient was sent for emergency surgery to remove the found blood clots in the lower limbs.

Conclusion. The application of the correct diagnostic algorithm in this clinical case with the application of CT scan with contrast helped to quickly identify early complications and to avoid more severe and later changes both in the vessels and in other organs and systems.

Keywords: Aorta, Arterial thrombosis, interstitial pneumonia, complications, COVID-19

\section{References}

[1] Cheruiyot I, Kipkorir V, Ngure B, Misiani M, Munguti J, Ogeng'o J. Arterial Thrombosis in Coronavirus Disease 2019 Patients: A Rapid Systematic Review. Ann Vasc Surg. 2021; 70:273-281. https://doi.org/10.1016/j.avsg.2020.08.087

[2] Costa VO, de Almeida GBC, Nicolini EM, de Abreu Rodrigues G, da Costad BMA, Carvalhoe GH, dos Reise ALS, Colen DP. Acute arterial occlusion of the lower limb as the main clinicalmanifestation in a patient with Covid-19 - Case Report. International Journal of Surgery Case Reports. 2020; 77:454-458 https://doi.org/10.1016/j.ijscr.2020.11.046

[3] Goldman IA, Ye K, Scheinfeld MH. Lower-extremity Arterial Thrombosis Associated with COVID-19 Is Characterized by Greater Thrombus Burden and Increased Rate of Amputation and Death. Radiology. 2020; 297:E263-E269. https://doi.org/10.1148/radiol.2020202348

[4] Schweblin C, Hachulla AL, Roffi M, Glauser F. Delayed manifestation of COVID-19 presenting as lower extremity multilevel arterial thrombosis: a case report. European Heart Journal - Case Reports. 2020; 4:1-4. https://doi.org/10.1093/ehjcr/ytaa371 


\title{
Use of previous experience and knowledge from protection against CBRN to protection against COVID-19 pandemic
}

\author{
Jozef Sabol, Otakar Mika
}

Police Academy of the Czech Republic in Prague, Prague, Czech Republic

\section{https://doi.org/10.21175/rad.abstr.book.2021.9.4}

The aim of the paper is to explore the ways of applying previous experience and knowledge gained from prevention and protection against CBRN to assure adequate protection of individuals against the risk of disease and the spread of the COVID-19 pandemic, incl. personal protective equipment and detection of coronavirus in the workplace and environment. The paper will be focused on a thorough analysis and evaluation of the current situation in the Czech Republic and in the world, taking into account modern procedures and means of disinfection of affected persons, air, premises, and equipment. Special attention will be paid to a comprehensive analysis of current needs for protection against COVID-19 and verification of methods, protective equipment, and devices recently developed.

The paper is an attempt to demonstrate how information from one area and its understanding can be successfully applied to solve or mitigate the problems in another area. 


\title{
Evaluation of biosorption and bioaccumulation capacity of cyanobacteria Arthrospira (Spirulina) Platensis for radionuclides
}

\author{
Inga Zinicovscaia1,2, Alexey Safonov3 \\ 1 Joint Institute for Nuclear Research, Dubna, Russia \\ 2 Horia Hulubei National Institute for R\&D in Physics and Nuclear Engineering, Bucharest, Romania \\ 3 Frumkin Institute of Physical Chemistry, Russian Academy of Science, Moscow, Russia
}

\section{https://doi.org/10.21175/rad.abstr.book.2021.10.1}

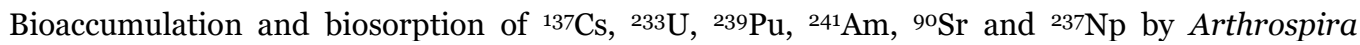
platensis CNMN-CB-O2 biomass were investigated. Bioaccumulation experiments were performed in EDTA-free Zarrouk cultivation medium and in the medium with the addition of sodium polyphosphate. Arthrospira platensis CNMN-CB-02 biomass showed high uptake capacity for ${ }^{239} \mathrm{Pu}$, ${ }^{241} \mathrm{Am}$, and ${ }^{\circ} \mathrm{Sr}$ in both media, while the uptake of ${ }^{233 \mathrm{U}}$ was significantly enhanced in the presence of polyphosphate ions. In biosorption experiments, the biomass also showed high sorption capacity for

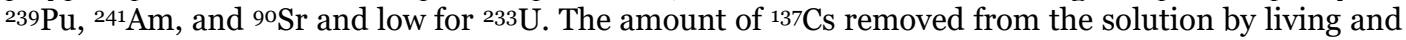
dry biomass was almost the same. The desorption studies revealed that A. platensis $\mathrm{CNMN}-\mathrm{CB}-\mathrm{O} 2$ biomass can be reused for biosorption and showed a high strength of radionuclide binding to phosphorylated biomass. A. platensis CNMN-CB-02 biomass can be considered a good candidate for radionuclide removal from salt and alkaline solutions and in in situ bioremediation strategies. 


\title{
Metal removal from zinc-containing effluents using Shewanella xiamenensis biofilm formed on zeolite
}

\author{
Dmitrii Grozdov', Inga Zinicovscaia',2, Nikita Yushin'1
}

\author{
1 Joint Institute for Nuclear Research, Dubna, Russia \\ 2 Horia Hulubei National Institute for R\&D in Physics and Nuclear Engineering, Bucharest-Magurele, \\ Romania
}

\section{https://doi.org/10.21175/rad.abstr.book.2021.10.2}

The adsorption properties of adsorbent, Shewanella xiamenensis biofilm formed on zeolite, has been investigated in order to determine its applicability for complex zinc-containing effluents treatment. The optimum conditions for metal adsorption from synthetic solutions were evaluated by changing initial $\mathrm{pH}$ of the solution, zinc concentration, temperature and time of contact. The maximum removal of metal ions present in analyzed systems was achieved occurred at $\mathrm{pH}$ range 3.0-6.0 whitin 60-150 min of sorbent-sorbate contact. The results obtained from the equilibrium studies were analyzed by three adsorption models, namely, Langmuir, Freundlich and Temkin models. Maximum adsorption capacity of adsorbent calculated from Langmuir model varied from 3.4 to $6.5 \mathrm{mg} / \mathrm{g}$. Four kinetic models: pseudo-first order, pseudo-second-order, Elovich and Weber and Morris intraparticle diffusion were applied for the description of experimentally obtained data. High coefficient of determination values calculated for pseudo-second-order and Elovich models indicate on predominant role of chemisorption in metal removal. Changes in free energy, enthalpy and entropy were also evaluated. Negative Gibbs energy values and positive $\Delta H^{\circ}$ values indicate the spontaneous and endothermic character of the sorption process. The effect of $\mathrm{pH}$ and bisorbent dosage on $\mathrm{Zn}$ (II) removal from industrial effluent with initial $\mathrm{Zn}$ (II) concentration $52.8 \mathrm{mg} / \mathrm{L}$ was tested. Maximum $\mathrm{Zn}$ (II) removal was achieved at $\mathrm{pH}$ 6.0 applying two-stage treatment system (with the addition of a new dose of biosorbent). 


\title{
On numerical solution of nonlinear parabolic multicomponent convection-diffusion problems
}

\author{
Gheorghe Sarbu' ${ }^{\text {, Constantin Popa }}{ }^{2}$
}

1 NIMRD, Constanta, Romania

2 Ovidius University, Constanta, Romania

\section{https://doi.org/10.21175/rad.abstr.book.2021.10.3}

In this work, we analyze the numerical solving of the multi-component mass transfer equations. The present test problems are two-dimensional, parabolic, non-linear, convection-diffusion equations. An implicit finite difference method was used to discretize the mathematical model equations. The algorithm used to solve the non-linear system resulted for each time step is the modified Picard iteration. The numerical performances of the preconditioned conjugate gradient algorithms (BICGSTAB and GMRES) in solving the linear systems of the modified Picard iteration were analysed in detail. For all the combinations of the parameters, we obtained a stable solution.

The values of the convergence rate obtained show that the present numerical solution is secondorder accurate in space. The values of the convergence rate and efficiency of the preconditioned GMRES and BiCGSTAB algorithms are similar those usually reported in literature for non-linear convection diffusion problems. 


\title{
Testing the effectiveness of removing toxic elements by clinoptilolite
}

\author{
Ivana Rasic Misic, Snezana Tosic, \\ Emilija Pecev-Marinkovic, Jelena Mrmosanin, Stefan Petrovic
}

University of Nis, Faculty of Sciences and Mathematics, Department of Chemistry, Nis, Serbia

\section{https://doi.org/10.21175/rad.abstr.book.2021.10.4}

The aim of this work was to examine the ion exchange characteristics of the dietary product zeolite-clinoptilolite on water samples from four locations of the River Borska. According to its chemical status, the Serbian Environmental Protection Agency marked this river in red because of its high content of $\mathrm{Ni}, \mathrm{Pb}$ and $\mathrm{Cd}$. The method of inductively coupled plasma-optical emission spectrometry (ICP-OES) was used for the simultaneous determination of 19 elements before and after the treatment of water with zeolite ( 24 hours at room temperature). The results of the analyses showed that by ionic exchange of $\mathrm{Na}^{+}, \mathrm{K}^{+}, \mathrm{Ca}^{2+}, \mathrm{Mg}^{2+}$ i $\mathrm{Ba}^{2+}$, zeolite effectively removed (100\%) $\mathrm{Cr}$, As and $\mathrm{Pb}$ from water. Minor affinity was noted for $\mathrm{Al}$ (up to 61\%) and $\mathrm{Cd}$ (up to $38 \%$ ), while the efficiency of $\mathrm{Co}$ and $\mathrm{Ni}$ removal was up to $11 \%$. Magnesium and sodium have the same exchange trend that tracks the presence of nickel, arsenic and cadmium, in tested water samples. Calcium exchange approximately tracks lead and chromium content. The barium exchange trend follows the presence of Co. Based on the efficacy of the removal of ions from the solution and the balance of ions modified from zeolite, it can be concluded that from the point of view of dietary application, clinoptilolite does not release toxic elements ( $\mathrm{As}, \mathrm{Pb}, \mathrm{Cd}, \mathrm{Al}, \mathrm{Cr}$ ), and does not capture electrolytes. 


\section{Advanced treatment of water and wastewater by hybrid technologies utilizing cavitation phenomenon}

\section{Grzegorz Boczkaj',2, Kirill Fedorov', Elvana Cako', Zhila Honarmandrad1}

1 Department of Process Engineering and Chemical Technology, Faculty of Chemistry, Gdańsk University of Technology, Gdansk, Poland

2 EkoTech Center, Gdańsk University of Technology, Gdansk, Poland

\section{https://doi.org/10.21175/rad.abstr.book.2021.10.5}

Cavitation phenomenon was utilized with spectacular effects for hybrid Advanced Oxidation Processes (AOPs). Most important developments relate to its application for treatment of water as well as industrial effluents [1-2]. Cavitation phenomenon can be generated in the liquid by intentionally formed differences in local pressure. It can be obtained by local increase of the liquid flowrate (hydrodynamic cavitation) or by ultrasounds (sonocavitation/acoustic cavitation).

The paper presents selected recent developments in the field of cavitation based AOPs with the discussion of the most important results of applicational studies on these groups of processes. During the presentation recent developments of our research group will be presented in respect to effective degradation of several pollutants present in industrial effluents, including volatile organic compounds (VOCs) and BTEXs. Special attention will be made on possibility of application of cavitation phenomenon for activation of persulfates using direct as well as catalytic route. Details on optimization of process parameters will be discussed and related to mechanisms responsible for transformation or organic pollutants during cavitational treatment [3-7].

Developed technologies will be discussed in terms of specific instrumentation used to perform the process, details of process control, economical evaluation, scale-up aspects, hybrid - cavitationcatalytic processes as well as overall advantages and limitations of cavitation based AOPs.

\section{References}

1. G. Boczkaj, A. Fernandes, 2017, Wastewater treatment by means of Advanced Oxidation Processes at basic pH conditions: A review, Chem. Eng. J. 320, 608-633.

2. M. Gągol, A. Przyjazny, G. Boczkaj, 2018, Wastewater treatment by means of advanced oxidation processes based on cavitation - A review, Chem. Eng. J. 338, 599-627.

3. K. Fedorov, M. Plata-Gryl, J. Khan, G. Boczkaj 2020, Ultrasound-assisted heterogeneous activation of persulfate and peroxymonosulfate by asphaltenes for the degradation of BTEX in water, J. Hazard. Mater. 397 (2020) 122804

4. K. Fedorov, X. Sun, G. Boczkaj, Combination of hydrodynamic cavitation and SR-AOPs for simultaneous degradation of BTEX in water, Chem. Eng. J. 2021 in press,

5. E. Cako, K. Dinesh, R. Soltani, G. Boczkaj Ultrafast degradation of brilliant cresyl blue under hydrodynamic cavitation based advanced oxidation processes (AOPs), Water Res. Ind. 24 (2020) 100134,

6. M. Gągol, R. D. C. Soltani, A. Przyjazny, G. Boczkaj 2019, Effective degradation of sulfide ions and organic sulfides in cavitation-based Advanced Oxidation Processes (AOPs), Ultrason. Sonochem. 58, 104610.

7. M. Gągol, E. Cako, K. Fedorov, R. Soltani, A. Przyjazny, G. Boczkaj, Hydrodynamic cavitation based advanced oxidation processes: Studies on specific effects of inorganic acids on the degradation effectiveness of organic pollutants, J. Mol. Liq. 307 (2020) 113002.

Acknowledgments: The authors gratefully acknowledge financial support from the National Science Centre for project UMO-2017/25/B/ST8/01364. 


\title{
Multi-mode Sample Introduction System (MSIS) as an interface between high performance liquid chromatography (HPLC) and inductively coupled plasma optical emission spectrometry (ICP OES) in arsenic speciation analysis
}

\author{
Jędrzej Proch, Przemysław Niedzielski \\ Adam Mickiewicz University in Poznań, Faculty of Chemistry, Department of Analytical Chemistry, Poznań, \\ Poland
}

\section{https://doi.org/10.21175/rad.abstr.book.2021.10.6}

The usage of a commercial in-spray chamber hydride generator, Multi-mode Sample Introduction System (MSIS) as an interface between high performance liquid chromatography and inductively coupled plasma optical emission spectrometry (HPLC-MSIS-ICP OES) is presented. The optimization of hydride generation in MSIS and chromatographic run (using anion-exchange HPLC column with phosphate buffer as a mobile phase) were carried out compositely. Within $400 \mathrm{~s}$ of a single analysis, the method allowed to determine three arsenic forms: As(III), As(V) and dimethylarsinate (DMA) with satisfying limits of detection (2.1-7.0 $\left.\mu \mathrm{g} \mathrm{L}^{-1}\right)$ and precision (2.7-5.7\%). Recovery for standard addition method was high and acceptable (95-106\%). The described method was used for environmental and food samples analyses. Besides of the mentioned hyphenated technique, perspectives of other optical systems are presented. Full results were presented in Proch \& Niedzielski, Talanta, 208 (2020) 120395 and Proch \& Niedzielski, Analytica Chimica Acta, 1147 (2021), 1-14.

Acknowledgments: The work was supported by grant no. POWR.03.02.00-00-Io26/16 co-financed by the European Union through the European Social Fund under the Operational Program Knowledge Education Development. 


\title{
Underground laboratories in the Baltic Sea Region - EUL Project
}

\author{
Jan Kisiel, Kinga Polaczek-Grelik, Agata Walencik-Łata, Katarzyna Szkliniarz
}

University of Silesia, Katowice, Poland

\section{https://doi.org/10.21175/rad.abstr.book.2021.11.1}

Recently, underground laboratories have become more and more popular. Although they were most often used to conduct scientific research, today they are, among others, a place for business development, innovation, as well as plant and mushroom breeding. In 2017, the idea of creating a network of underground laboratories operating in the Baltic Sea region was born, which became a project called BSUIN (Baltic Sea Underground Innovation Network) financed by INTERREG Baltic Sea Region. The purpose of the project was to develop a service offering of the Baltic Sea Region's (BSR) underground laboratories (UL) to increase their capability to offer technology transfer utilizing the facilities and research infrastructures of the ULs for business development. This project ended in 2020 with great success. Currently, its continuation is carried out in the EUL (Empowering Underground Laboratory network usage) project. Both projects involve 6 underground laboratories (Callio Lab in Pyhäsalmi mine (Finland), Äspö Hard Rock laboratory (Sweden), FLB-Research and Education Mine "Reiche Zeche" (Germany), KGHM CUPRUM R\&D Center in Wroclaw (Poland), the UL of Khlopin Institute in St Petersburg (Russia), and Ruskeala marble mine in Karelia (Russia)), 2 associated laboratories (Experimental Mine Barbara (Poland), Hagerbach Test Gallery (Switzerland)) and 13 partner organizations and 19 associated organizations. Projects are broken down into several work packages and these in turn into activities. One of both projects' activities is the characterization of the natural radiation background in participating underground laboratories.

During the conference, both projects emphasizing the EUL will be presented, and their main goals will be discussed. 


\title{
Measurements of the effective illumination in the conference hall and the questionnaire survey about the lighting perceptions of participants
}

\author{
Dragana Baturan, Selena Samardžić, \\ Robert Lakatoš, Uranija Kozmidis Luburić
}

Faculty of Technical Sciences, Novi Sad, Serbia

https://doi.org/10.21175/rad.abstr.book.2021.11.2

Lighting is one of the main aspects of working and living environment. Many studies show that an adequate amount of light improves mood and energy levels, while poor lighting contributes to low working capacity, depression, and other deficiencies in the body. The amount and type of lighting directly affect concentration, sleep, emotional reactions, and many other aspects of daily life. Some authors found that the detrimental impact of working in a windowless environment is a universal phenomenon. The purpose of this study is to investigate the role of natural lighting in an everyday environment. The research was conducted among employees from several companies, seminar participants, who attended expert lectures in the windowless conference room designed to accommodate gatherings of all kinds. The conference room has 350 seats. The questionnaire with 15 questions is divided into three parts: The first part is related to sociodemographic data, the second part consisted of questions about life habits regarding light perception and illumination and the third part was about the respondent's subjective experience of a space illuminated with natural and/or artificial light. For data analysis, the software for statistical data analysis IBM SPSS Statistics 20 was used. Illuminance in the conference hall is measured by a lux meter, which is a handy instrument with a sensor for light detection. The measured illuminance is directly displayed in lux (lx). Subjects had the highest mean scores for the attitude that orientation of living room due to cardinal points (east, west, north, and south) play an important role in choosing a living space. Besides, there is a strong opinion that the presence of both, natural and artificial light significantly contributing good workspace environment. However, respondents have a different attitude regarding thermal sensation under the influence of artificial light sources, indicating that they have different psychophysical perceptions of those phenomena. It also can be concluded, at the level of statistical significance, that work under artificial lighting creates a sense of lack of fresh air and eye fatigue. Illuminations measurements indicate uneven lighting at desks position in a whole conference room. Although midlevel lighting is adequate for collaborative settings such as conference rooms, where depth is more valued than speed, and provide participant to be more alert and focused, but still relaxed, in this case very low levels of lighting were recorded. Values varied from 45-123.2 lx. Having in mind that the recommended levels are 300-600 lx, it can be concluded that architectural lighting design does not meet the visual requirements. 


\title{
Sensitivity of electrochemically deposited $\mathrm{ZrO}_{2}$ layers on a quartz resonator
}

\section{Konstantin Lovchinov', Gergana Alexieva², Miroslav Petrov3, Hristo Nitchev3, Nikolay Tyutyundzhiev3}

\author{
1 Institute of Optical Materials and Technologies “Acad. J. Malinowski”, Bulgarian Academy of Sciences, \\ Sofia, Bulgaria \\ 2 Sofia University, Faculty of Physics, Sofia, Bulgaria \\ 3 Institute of Electronics, Bulgarian Academy of Sciences, Sofia, Bulgaria
}

\section{https://doi.org/10.21175/rad.abstr.book.2021.11.3}

This paper presents studies of the sensitivity of electrochemically deposited layers of $\mathrm{ZrO}_{2}$ on the gold electrodes of quartz resonators to various volatile compounds. The layers of $\mathrm{ZrO}_{2}$ is deposited by an electrochemical process which is carried out in aqueous containing $\left(\mathrm{ZrOCl}_{2}\left(5 \cdot 10^{-3} \mathrm{M}\right)\right.$ and $\mathrm{KCl}$ (o.1 M) for $\mathrm{ZrO}_{2}$ at $70^{\circ} \mathrm{C}$ and (vs SCE) using a three-electrode electrochemical cell. The morphological structure of the $\mathrm{ZrO}_{2}$ layers deposited on the polished QCM surface is studied by Scanning Electron Microscopy (SEM) and optical profilometry. The influence of the roughness on the $\mathrm{ZrO}_{2}$ and sorption properties is investigated. The resonant frequency shift $(\Delta \mathrm{F})$ of the quartz structure are using to defined sorption ability of the $\mathrm{ZrO}_{2}$ layers by measuring the presence of different concentration in the volatile compounds under study. 


\title{
Purification of aqueous solutions from strontium ions by natural and synthetic sorbents under increased radiation background
}

\author{
Yuriy Kylivnik, Svyatoslav Vuchkan², \\ Ihor Syika², Hanna Vasylyeva², Olexander Sych ${ }^{2}$
}

1 ISPE, Kyiv, Ukraine

2 Uzhgorod National University, Uzhgorod, Ukraine

\section{https://doi.org/10.21175/rad.abstr.book.2021.11.4}

The basis of the process of any adsorption purification of solutions is a sorbent or ion exchanger. Therefore, the choice of sorbent used for the extraction of heavy metal cations or radionuclides, for example strontium, from aqueous solutions is important, as well as monitoring the amount and migration of strontium in the environment. Many works are devoted to sorption materials used for this purpose. The most widely studied are natural crystalline frame aluminosilicates (zeolites), synthetic organic and inorganic ion exchangers, activated carbon and metal oxides.

The ability of natural Transkarpathian zeolite, cation exchange resin (KU 8.6), activated carbon and titanium phosphate to absorb strontium ions under conditions of increased radiation background was investigated in present work. It was shown, that upon irradiation the sorption properties of all these materials are slightly reduced. The only exception is titanium phosphate with a surface modified with $\mathrm{NH}_{4} \mathrm{OH}$.

The adsorption of strontium ions by natural Transkarpathian zeolite, cation exchange resin (KU 8.6), activated carbon and titanium phosphate from model solutions, which, along with $\mathrm{Sr}^{2+}$ ions, contain $\mathrm{Fe}^{2+}$ ions and water radiolysis products, was also studied. It was shown, that the sorption coefficients of strontium ions from the model solution are in some cases higher than from the solution of distilled water. In this case, clinoptilolite more efficiently absorbs iron ions than strontium ones. 


\title{
A preliminary study of the electromagnetic field exposure of the population living near high voltage power lines of $110 \mathrm{kV}$ and $220 \mathrm{kV}$ in Tirana City, Albania
}

\author{
Margarita Kuqali, Dhurata Kuqi, Ramadan Firanj
}

Faculty of Mathematical Engineering and Physics Engineering, Polytechnic University of Tirana, Tirana, Albania

\section{https://doi.org/10.21175/rad.abstr.book.2021.11.5}

Given the interest of the communities living near high voltage lines we performed a quantitative assessment of the electromagnetic field under the power lines of $110 \mathrm{kV}$ and $220 \mathrm{kV}$ in two residential areas nearby the city of Tirana.

Regarding to the electric field created by the $220 \mathrm{kV}$ power line, which was measured at the height of $1.6 \mathrm{~m}$ from the ground, the highest measured value was $569.72 \mathrm{~V} / \mathrm{m}$, and the lowest value was $156.81 \mathrm{~V} / \mathrm{m}$. On the other hand, regarding the magnetic field the highest recorded value was $0.6972 \mu \mathrm{T}$ and the lowest was $0.1416 \mu \mathrm{T}$.

Regarding the electric field created by the $110 \mathrm{kV}$ power line, the highest measured value which was found in the middle of the line trace was $16.49 \mathrm{~V} / \mathrm{m}$ and $16.29 \mathrm{~V} / \mathrm{m}$ for the height of $1.6 \mathrm{~m}$ and 1 $\mathrm{m}$ from the ground, respectively. Whereas, the lowest value of the electric field that was measured resulted as $0.7831 \mathrm{~V} / \mathrm{m}$ at the height of $1.6 \mathrm{~m}$ from the ground and $0.5741 \mathrm{~V} / \mathrm{m}$ for the height of $1 \mathrm{~m}$ from the ground, they both belong to the end of the line.

Regarding to the magnetic field created by the $110 \mathrm{kV}$ voltage line, the largest measured value was $0.6621 \mu \mathrm{T}$ and $0.6409 \mu \mathrm{T}$ respectively for the heights of 1.6 and $1 \mathrm{~m}$ from the ground, these values belong to the middle of the line route. Whereas the lowest values of the magnetic field measured near the points of the beginning of the conductor line the result was $0.1857 \mu \mathrm{T}$ for the height of $1.6 \mathrm{~m}$ from the ground and $0.1416 \mu \mathrm{T}$ for the height of $1 \mathrm{~m}$ from the ground.

All measured values resulted to be lower than the limits of $5 \mathrm{kV} / \mathrm{m}$ and $100 \mu \mathrm{T}$ for the general public. 


\title{
Evaluation of black carbon in fine atmospheric particulate matter on various filter types by multi-wavelength light absorption technique
}

\author{
Mirjana Radenkovic1, Dusan Topalovic, Tatjana Trtic Petrovic ${ }^{1}$, \\ Visa Tasic $^{2}$, Srboljub Stankovic', Vojislav Stanic ${ }^{1}$
}

\author{
1 Vinca Institute of Nuclear Sciences-National Institute of Republic of Serbia, University of Belgrade, \\ Belgrade, Serbia \\ 2 Mining and Metallurgy Institute, Bor, Serbia
}

\section{https://doi.org/10.21175/rad.abstr.book.2021.11.6}

Besides the evident harmful impact to human health, black carbon (BC) is considered as second important contributor to climate change due to its sunlight absorption and warming effects. It is a major component of fine atmospheric particulate matter emitted during the incomplete combustion of fossil fuels and biomass burning emissions from both natural and anthropogenic sources. Atmospheric carbon was recognized in forms of soot, black carbon, elemental carbon, inorganic carbon, organic carbon, brown carbon, etc, depending on the origin and absorption characteristics. Measurement methodologies for BC analysis in aerosol samples are mostly based on optical and thermal properties of carbon species. Here are presented results of the application of the optical analytical technique which relies on the multi-wavelenght light attenuation by black carbon component of fine particulate matter deposited on filter media. For that purpose, standard polytetrafluoroethylene (PTFE), quartz and carbon nanotube filters with different qualitative features and pore diameters were exposed to fine aerosol fraction at urban background monitoring site in heating and non heating seasons, using low-volume air samplers with $2.3 \mathrm{~m}^{3} / \mathrm{h}$ air flow. A multi wavelength absorption black carbon instrument (MABI) with $405 \mathrm{~nm}, 465 \mathrm{~nm}, 525 \mathrm{~nm}, 639 \mathrm{~nm}$, $870 \mathrm{~nm}, 940 \mathrm{~nm}$ and $1050 \mathrm{~nm}$ LEDs was used for blank and exposed filters analysis and black carbon evaluation. Differences in obtained BC values are discussed in relation to various absorption potential of different filter media, taking into account absorption coefficients dependence on the wavelength and density. Main advantage of this method is simplicity and complementarity with nondestructive nuclear analytical techniques (EDXRF, PIXE) for elemental analysis of fine aerosol fraction on specific filter media. Differentiation between black carbon coming from fossil fuels combustion and from biomass burning sources would be additional information valuable for source apportionment analysis using positive matrix factorization and reliable discussion of air pollution observed at selected receptor site. 


\section{Advanced adsorption energy generating systems based on polarized substrates}

\section{A.S. Doroshkevich1,2, V.A. Alexandrovi,3, A.I. Lyubchyk4-6, B.L. Oksengendler7, A.K. Kirillov8 ${ }^{8}$ T.A. Vasilenko ${ }^{8}$, T.E. Konstantinova ${ }^{2}$, A.V. Shilo ${ }^{2}$, N.N. Nikiforova7, A.A. Nabiyev9, M.A. Balasoui ${ }^{10}$, D. Mardare ${ }^{11}$, C. Mita ${ }^{11}$, V.I. Bodnarchuk ${ }^{1}$, D. Chicea ${ }^{12}$, S.I. Lyubchyk ${ }^{6}$}

1 Joint Institute for Nuclear Research, Dubna, Moscow Region, Russia

2 Donetsk Institute for Physics and Engineering named after O.O. Galkin, Kiev, Ukraine

3 Dubna State University, Dubna, Moscow Region, Russia

4 Research Centre in Industrial Engineering, Management and Sustainability, Lusófona University, Lisbon, Portugal

5 Nanotechcenter LLC, Kyiv, Ukraine

6 REQUIMTE, Faculdade de Ciências e Tecnologia, Universidade Nova de Lisboa, Caparica, Portugal

7 U. A. Arifov Institute of Ion-Plasma and Laser Technologies of the Academy of Sciences of the Republic of

Uzbekistan

8 Saint-Petersburg Mining University, St.-Petersburg, Russia

9 ANAS Institute of Radiation Problems, Baku, Azerbaijan

10 Horia Hulubei National Institute for R\&D in Physics and Nuclear Engineering (IFIN-HH), Bucharest,

Romania

11Alexandru Ioan Cuza University of Iasi, Faculty of Physics, Iasi, Romania

12University "LUCIAN BLAGA" of SIBIU, Sibiu, Romania

\section{https://doi.org/10.21175/rad.abstr.book.2021.11.7}

Modern chemoelectronic (hydroelectric) converters are able to convert the heat of adsorption of moisture molecules into an electrical form through specific reversible structural - phase transformations induced by adsorption effects [1-2]. Advanced converter designs have an efficiency of the order of $3.6 \mathrm{~mW} \mathrm{~h}^{-1} / \mathrm{g}$ (or $18 \mathrm{~mW} \mathrm{~h}^{-1} / \mathrm{cm}^{3}$ when calculated for $100 \%$ concentration of nanopowder). Analysis of the available data suggests that the major limiting factor in this case is the random electrical polarization of the medium providing of charge transfer process from the reaction volume of the functional layer to the electrodes. Without considering the percolation nature of ionic conductivity [3], the problem can be solved by polarization of the medium in the space between the electrodes by means of by polarized of substrates. This paper discusses the concept of an advanced device on the polarized substrates. The effectiveness of this approach was shown.

\section{References}

[1] A.S. Doroshkevich, E.B. Askerov, A.I. lyubchyk, and al. Direct transformation of the energy of adsorption of water molecules in electricity on the surface of zirconia nanoparticles // Applied Nanoscience. 9(8), 1603-1609, DOI: $10.1007 / \mathrm{s} 13204-019-00979-6$.

[2] Alekseenko VI, Volkova G.K. The adsorption mechanism of the phase transformation of stabilized zirconium dioxide. ZhTF, 2000, vol. 70, no. 9, p.57-62.

[3] E.A. Gridina, A.S. Doroshkevich, A.I. Lyubchyk, and al. The effect of percolation electrical properties in hydrated nanocomposite systems based on polymer sodium alginate with a filler in the form nanoparticles $\mathrm{ZrO}_{2}$ 3mol\% $\mathrm{Y}_{2} \mathrm{O}_{3} / /$ Advanced Physical Research. 2019, V1, №2, (pp.70-8o).

Acknowledgments: The study was performed in the scope of the H2020/MSCA/RISE/SSHARE number 871284 and the RO-JINR Projects within the framework of themes FLNP JINR: 04-4-1105-2011/2022 and $03-4-1128-2017 / 2022$. 


\title{
Assessment of atmospheric deposition in Central Russia, Vladimir and Yaroslavl regions, using moss biomonitors, neutron activation analysis and GIS technologies
}

\author{
Konstantin Vergel1, Inga Zinicovscaia ${ }^{1,2}$, Nikita Yushin'
}

1 Joint Institute for Nuclear Research, Dubna, Russia

2 Horia Hulubei National Institute for R\&D in Physics and Nuclear Engineering, Bucharest, Romania

\section{https://doi.org/10.21175/rad.abstr.book.2021.12.1}

Moss biomonitoring technique was applied to study peculiarities of the spatial distribution of atmospheric deposition of heavy metals on the territory of the Vladimir and Yaroslavl regions. During the summer 2018, samples of the terrestrial mosses Pleurosium shreberi were collected at the 126 sites evenly distributed over the territory of investigated regions. The combination of neutron activation analysis and atomic absorption spectrometry allowed to determine more than 30 elements in moss samples. To identify the main sources of air pollution multivariate data analysis technique - factor analysis was applied, while the deposition patterns of pollutants was illustrated using GIS technology. Median values of the elements were compared with the results obtained for other regions in Russia, which participated in moss survey studies. The contamination factors, Geo-accumulation Index and pollution load index were calculated for the following elements $\mathrm{As}, \mathrm{Sb}$, $\mathrm{Pb}, \mathrm{V}, \mathrm{Cd}, \mathrm{W}, \mathrm{Fe}, \mathrm{Cr}, \mathrm{Ni}$ and $\mathrm{Co}$. Ni. The dominant anthropogenic sources of air pollutants in studied regions can be considered industrial activity and transport. 


\title{
Estimating the impact of acid pH solution on the metal desorption from lichen Evernia prunastri (L.) Ach.
}

\author{
Emina Ramic1,2, Jasna Huremovic², Sabina Zero² \\ 1 University of Sarajevo, Faculty of Pharmacy, Department of Natural Sciences in Pharmacy, Sarajevo, Bosnia \\ and Herzegovina \\ 2 University of Sarajevo, Faculty of Science, Department of Chemistry, Sarajevo, Bosnia and Herzegovina
}

\section{https://doi.org/10.21175/rad.abstr.book.2021.12.2}

Evernia prunastri is lichen used as bioindicator of air pollutions for decades, moreover numerous studies showed that lichens have the possibility to accumulate metals from air in concentrations that are higher than their metabolic demand. Metals are absorbed directly through the surface of the lichen thallus. The aim of this study was to estimate the desorption of eight heavy metals $(\mathrm{Cd}, \mathrm{Cr}, \mathrm{Cu}, \mathrm{Fe}, \mathrm{Mn}, \mathrm{Ni}, \mathrm{Pb}$ and $\mathrm{Zn}$ ) from Evernia prunastri with acid $\mathrm{pH}$ solutions (4.33, and 5.12) and to determine could the lichens become secondary environment pollution source. The metal analysis was performed by flame atomic absorption spectrometry (FAAS).

Solutions used for desorption were prepared by mixing different amounts of $\mathrm{H}_{2} \mathrm{SO}_{4}, \mathrm{HNO}_{3}$, $\left(\mathrm{NH}_{4}\right)_{2} \mathrm{SO}_{4}$ and $\mathrm{NH}_{4} \mathrm{NO}_{3}$. Lichen before desorption were washed in demineralized water and air dried. Three consecutive desorption were performed with each solution, during each of the desorption lichens were in contact with the solution for 24 hours.

Results showed that using the solution with $\mathrm{pH} 4.33$, Mn was desorbed in the highest mass percentage (92.7\%) from the lichen and desorption mass percentage of $\mathrm{Mn}$ and $\mathrm{Zn}$ from the lichen by using the solution of $\mathrm{pH} 5.12$ was $88 \%$ and $67.2 \%$, respectively. The desorption mass percentage of other investigated metals were lower than $30 \%$ except for $\mathrm{Cr}$ whose mass percentage was lower than $1 \%$ with solution $\mathrm{pH} 4.33$ and below the limit of detection for the applied method by using the solution with $\mathrm{pH}$ 5.12. Results indicate that lichens can become secondary environment pollutant and that mass percentage of desorbed metals is depending on strength linked of the metal for the components of the lichen tissues. Low mass percentage of metal desorption could indicate that the metal is tightly bonded inside the lichen cell itself, either they build highly insoluble compounds or complex compounds with lower or higher stability constants. High mass percentage of metal desorption can indicate that metals are linked with an ionic bond to the corresponding chemical groups in the lichens.

Keywords: Evernia prunastri (L.) Ach., washed lichen, desorption, heavy metals, acid pH solutions, FAAS 


\title{
Optimization of the source apportionment solution using the rotational tools in US EPA PMF 5.0 software
}

\author{
Dušan Topalović1, Mirjana Radenković1, \\ Viša Tasić ${ }^{2}$, Vojislav Stanić1 ${ }^{\text {, Predrag Božović }}{ }^{1}$
}

\author{
1 Vinča Institute of Nuclear Sciences - National Institute of the Republic of Serbia, University of Belgrade, \\ Belgrade, Serbia \\ 2 Mining and Metallurgy Institute Bor, Bor, Serbia
}

\section{https://doi.org/10.21175/rad.abstr.book.2021.12.3}

Positive matrix factorization (PMF) is a dimension reduction method used to model the covariance structures of observable variables in order to impel a smaller number of latent nonnegative factors. It resolves receptor modeling problem, which is based on the chemical mass balance equation (CMB) and may discover hidden patterns in the environmental data, where each extracted factor is accompanied by an actual source of emission. In this paper, PMF source apportionment analyses of fine aerosol fraction ( $\mathrm{PM}_{2.5}$ mode) at Belgrade suburban background site, in 2016/17 year, have been performed by processing a data set of $130 \mathrm{PM}_{2.5}$ mass concentrations and twenty-one elemental concentrations and soot concentrations in each $\mathrm{PM}_{2.5}$ sample (mode). The $\mathrm{PM}_{2.5}$ mass concentrations in collected samples have been determined following SRPS EN 12341:201 procedure, elemental concentrations were obtained by PIXE alanytical technique in the frame of the regional IAEA project, in the Institute of Nuclear Research, Hungarian Academy of Sciences, and soot concentrations were analyzed by smoke stain reflectometry in accordance with ISO 9835:1993 (E). The EPA PMF program ver 5.0, was used to solve the PMF model. Since the determination of an optimal PMF solution is a strongly heuristic procedure, there is a necessity of finding a more quantitative ways to reduce the arbitrariness of this technique. In order to reduce the range of possible solutions, we have analyzed how the values of model parameters changes as a function of the number of factors. PMF modeling was performed in a robust mode. For the purpose of finding the best fit solution wich minimize the object function $\mathrm{Q}$, we varied the number of factors in the range from 4 to 8 . Additionally, influence of rotations are also analyzed in iterative steps by varying FPEAK function in the range -1 to +1 with an increment of 0.5 . Obtained results indicated significant role of $\mathrm{Q} / \mathrm{Q}$ exp ratio analysis for optimal solution choice. In optimization process, number of factors with $\mathrm{Q} / \mathrm{Q}_{\exp }$ ratio less than 1, were rejected as a possible solutions. This paper shows that optimization procedure should include examination of rotational matrix in which the rotational degree of freedom of solution is considered. Solutions with a steep change in their rotational degree of freedom were rejected. Finally, the additional improvement can be done by optimizing the parameters representing the scaled mean value (IM) and the scaled standard deviation (IS) of the each individual column in scaled residual matrix. 


\title{
EPR characterization of the ROS-associated toxicity in gamma irradiated Lemna minor $\mathrm{L}$.
}

\author{
Yanka Karamalakova ${ }^{1}$, Ekaterina Georgieva', \\ Veselin Ivanov $^{2}$, Galina Nikolova ${ }^{1}$
}

1 Department of Chemistry and Biochemistry, Medical Faculty, Trakia University, Stara Zagora, Bulgaria

2 Neurology, Psychiatry and Disaster Medicine Department, Trakia University, Stara Zagora, Bulgaria

\section{https://doi.org/10.21175/rad.abstr.book.2021.13.1}

Objectives. The EPR-X-band e-scan (Bruker ER 116 DS) spectrometer was used for selective detection/ identification of paramagnetic (superoxide anions (O2-), hydroxyl (HO•), and peroxyl (RCOO•)) substances and as radiation-dosimeter on the kinetics and antioxidant activity of irradiated Lemna minor L. (LM) species of the Bulgarian flora. Lemna Minor L. (LM), an aquatic plant that belongs to the genus Lemna/ Lemnaceae, easily used in the treatment of asthma, vitiligo, glaucoma, allergies, rheumatism, possessed strong antioxidant activity.

The chemical compound as phytol, campesterol, loliolide, dihydroactinidiolide, ascorbic acid, vanillic acid, 2,3-dihydroxybenzoic acid, caffeic acid, chlorogenic acid, and etc, exhibit LM antibacterial, anti-inflammatory, antihistamine, and anti-carcinogenic effects.

Materials and methods. The dry/ ethanol solution LM samples were single irradiated by using a $60 \mathrm{Co}$ source at doses of 1, 2, 2.5, 3, 5, 10 Gy (Gamma cell 5001, India; $1.377 \mathrm{~Gy} / \mathrm{h}$ ). The spectrophotometric antioxidant activities of LM against superoxide $(>81 \%)$, hydroxyl $(>52.7 \%)$, DPPH (89.9\%), ABTS (>55.5\%), SOD-like (>50.9\%), CAT-like (>56.2\%), LP, and NO radicals $(>56 \%)$ were maximally evaluated at a dose of $3 \mathrm{~Gy}$. The reducing power in the aqueous phase was $(>54.37 \%$, $\mathrm{R} 2=0.878)$.

The LM ability to scavenge in vitro ROS production and associated toxicity after irradiation, the spin-trap BMPO• (5-tert-Butoxycarbonyl-5-methyl-1-pyrroline-N-oxide), and free -radicals TEMPOL (4-hydroxy-2,2,6,6-tetramethylpiperidine-N-oxyl) and DPPH • (2,2-diphenyl-1picrylhydrazyl) were expressed, at different time intervals (oh, $1 \mathrm{~h}, 2 \mathrm{~h}, 24,48 \mathrm{~h}$ ). For the reference EPR-signal, the LM was replaced with $30 \mu \mathrm{L}$ deionized water.

Results. The maximal DPPH • (93\%) and TEMPOL (79.7\%) neutralization, was detected in about $1 \mathrm{~h}$, by the $3 \mathrm{~Gy}$ irradiated LM sample. The antioxidant activity of LM in water solution was similar. The maximal spin-adduct formation (DMPO-OH•, 76\%) and its neutralization to hydrogen atom were measured at 30 min for the 2.5Gy and 3Gy irradiated LM extracts. The EPR signal intensity in all analyzes sharply decrease at $1.2 \mathrm{~h}$.

In conclusion, the LM sample from Bulgarian flora showed the highest ability to scavenge in vitro ROS production and to neutralize the associated radiation-toxicity, due to its high content of nutrients, and could be useful as a cosmetic ingredient.

Key words: Lemna minor L., $\gamma$-radiation, antioxidants

Acknowledgments: The study was supported by scientific projects 2/2020 of Medical Faculty, Trakia University, Bulgaria. 


\title{
ICP-OES method for determination of selected elements in infant formulas and cereals based baby food
}

\author{
Emilija Pecev-Marinković, Snežana Tošić, Aleksandra Pavlović, \\ Ivana Rašić Mišić, Jelena Mrmošanin, Stefan Petrović
}

Faculty of Sciences and Mathematics, Department of Chemistry, Niš, Serbia

https://doi.org/10.21175/rad.abstr.book.2021.13.2

The manuscript presents results of macro elements ( $\mathrm{K}, \mathrm{Na}, \mathrm{Ca}, \mathrm{Mg}, \mathrm{P})$, the essential elements (Fe, $\mathrm{Zn}, \mathrm{Cu}, \mathrm{Cr}$ ), elements which are probably essential ( $\mathrm{Mn}, \mathrm{Si}, \mathrm{Ni}, \mathrm{Co}, \mathrm{B})$ and potentially toxic elements $(\mathrm{Pb}, \mathrm{Cd}, \mathrm{As}, \mathrm{Ba})$ determination in infant formulas and cereals baby food intended to consumption during the period from birth to second year. Infant formulas and cereals baby food have a significant role in children's nutrition because they are the major source of nutrients. The presence of contaminants in baby food may pose health risks for children. For this reason, it is important that the levels of selected elements found in consumed infant formulas and baby food be determined routinely.

Thirteen different infant formulas and twenty seven cereal baby foods from different manufacturers were collected from pharmacies and supermarkets in Niš, Serbia, in 2015/2016. Samples were prepared by microwave digestion in a laboratory microwave oven (Milestone EthosPLUS, Italy) rated at $1000 \mathrm{~W}$. Samples analyzed were carried out on an iCAP 6000 inductivelycoupled plasma optical atomic emission spectrometar (ICP OES, Thermo Scientific, UK).

Among macro elements $\mathrm{K}$ is most abundant in infant formula samples. The level of $\mathrm{K}$ were in the range of 2.19 to $3.4 \mathrm{mg} \mathrm{g}^{-1}$. After $\mathrm{K}$, the most common elements are Ca (1.49 to $4.34 \mathrm{mg} \mathrm{g}^{-1}$ ) and $\mathrm{P}$ (1.13 to 6.05 $\left.\mathrm{mg} \mathrm{g}^{-1}\right)$. The $\mathrm{Mg}$ and Na levels were among 0.157-0.299 $\mathrm{mg} \mathrm{g}^{-1}, 0.0198-0.0859 \mathrm{mg} \mathrm{g}^{-1}$, respectively. Among the essential elements, $\mathrm{Zn}\left(20.9-106.8 \mu \mathrm{g} \mathrm{g}^{-1}\right)$ and $\mathrm{Fe}\left(10.9-106.3 \mu \mathrm{g} \mathrm{g}^{-1}\right)$ are the most abundant, followed by $\mathrm{Cu}\left(1.98-12.71 \mu \mathrm{g} \quad \mathrm{g}^{-1}\right)$, $\mathrm{Mn}\left(0.343^{-2.64} \mu \mathrm{g} \quad \mathrm{g}^{-1}\right)$ and $\mathrm{Cr}\left(0.15-0.347 \mu \mathrm{g} \mathrm{g}^{-1}\right)$.

Among macro elements $\mathrm{P}$ ( 0.458 to $5.360 \mathrm{mg} \mathrm{g}^{-1}$ ) is most abundant in all samples of cereal based baby food followed by K (0.270 to $6.060 \mathrm{mg} \mathrm{g}^{-1}$ ), and $\mathrm{Ca}$ (0.279 to $2.040 \mathrm{mg} \mathrm{g}^{-1}$ ). The $\mathrm{Mg}$ and $\mathrm{Na}$ levels were among 0.072-0.597 $\mathrm{mg} \mathrm{g}^{-1}$, 0.01707-0.0699 $\mathrm{mg} \mathrm{g}^{-1}$, respectively. Among the essential elements, Fe (6.2 -70.75 mg kg-1) and $\mathrm{Zn}\left(2.74-19.7 \mathrm{mg} \mathrm{kg}^{-1}\right)$ are the most abundant, followed by $\mathrm{Mn}$ ( 0.84 to $20.52 \mathrm{mg} \mathrm{kg}^{-1}$ ), and $\mathrm{Cu}\left(0.23-4.24 \mathrm{mg} \mathrm{kg}^{-1}\right)$. Arsenic, lead, cadmium were not detected in any sample of infant baby formulas. Cd was detected only in cereals baby food. Comparing the metal ion levels and the maximum possible weekly intake, the ETWI of $\mathrm{Cd}$ was below the PTWI. Calculated values of ETWI and ETMI were lower than recommended for tolerable weekly intake (TWI) and provisional tolerable monthly intake (PTMI). 


\title{
Research of changes in the chemical properties of food products as a result of exposure to ionizing radiation at different doses
}

\author{
Ulyana Bliznyuk, Polina Borshchegovskay, Timofey Bolotnik², \\ Victoria Ipatova', Alexander Konstantinov3, Michael Yemelyanov3, \\ Igor Rodin², Felix Studenikin', Oleg Khmelevsky, \\ Alexander Chernyaevi,4, Oleg Shinkarev', Dmitry Yurov4
}

\author{
1 M.V. Lomonosov Moscow State University, Physics Department, Moscow, Russia \\ 2 M.V. Lomonosov Moscow State University, Chemistry Department, Moscow, Russia \\ 3 Federal Center for Hygiene and Epidemiology, Moscow, Russia \\ 4 Skobeltsyn Institute of Nuclear Physics of Lomonosov Moscow State University, Moscow, Russia
}

\section{https://doi.org/10.21175/rad.abstr.book.2021.13.3}

Today, one of the most important challenges around the world is to ensure the appropriate quality and safety of food products which involves extending the shelf life of foodstuffs without detriment to their nutritional qualities and taste.

Food processing with ionizing radiation is becoming an increasingly efficient and environmentally friendly method to extend the shelf life of food items/ the time of storage. However, irradiation causes/triggers the appearance of free radicals, which, in turn, affect complex organic compounds: proteins, fats, and carbohydrates. Therefore, it is important to study the chemical properties of processed food products.

Our research team is conducting research to assess the effect of ionizing radiation at different doses on the chemical properties of bulk materials and vegetable and meat products.

For example, the electron beam with an energy of $1 \mathrm{MeV}$ in the dose range from 0.25 to $6 \mathrm{kGy}$ influences the organoleptic and chemical characteristics of chilled turkey meat. The concentration of viable microorganisms decreases non-linearly with an increase in the radiation dose. It was found that at range doses of $0.25-2 \mathrm{kGy}$, there are no changes in the appearance, taste, and smell of the samples. At doses above $3 \mathrm{kGy}$, the color, consistency, and smell of the product are different compared to those of the unirradiated control samples.

Observations of oxidation as a result of irradiation at doses up to $10 \mathrm{kGy}$ of 25 saturated and unsaturated fatty acids contained in turkey meat have shown that at range doses not exceeding 3-4 $\mathrm{kGy}$, the content of fatty acids remains almost unchanged. At doses of $10 \mathrm{kGy}$, the content of most acids decreases by 1.6-1.8 times. Method of Gas Chromatography-Mass Spectrometry (GC-MS) has been used to identify volatile substances (alcohols, aldehydes, and ketones) and their concentration in irradiated turkey samples with high accuracy. It was found that the concentration of volatile substances (alcohols, ketones) decreases with an increase in the radiation dose according to an exponential law. Also, acetone was chosen as a marker, since its concentration increases linearly depending on the absorbed dose. The Fingerprint method using spectra, chromatograms, and various data from electrochemical sensors processed by image recognition methods did not reveal statistically significant differences between irradiated and non-irradiated turkey samples.

The study on the effects of ionizing radiation on the concentration of reducing sugars in potato tubers performed to determine the dose required to stop germination of potato tubers during different storage periods, suggests that the concentration of sugars depends on the time of storage of potatoes before irradiation. The earlier the treatment is carried out, the smaller the dose that inhibits germination is, and the smaller the fluctuations in the concentration of reducing sugars is relative to the initial control values during the subsequent storage period.

Acknowledgments: This research has been supported by the Interdisciplinary Scientific and Educational School of Moscow University "Photonic and Quantum technologies. Digital medicine". 


\title{
Health risk estimation of potentially toxic elements in complementary fruit-based food
}

\author{
Ivana D. Rašićc Mišić, Snezana B. Tošić, Aleksandra N. Pavlović, \\ Emilija T. Pecev-Marinković, Jelena M. Mrmošanin
}

University of Niš, Faculty of Science and Mathematics, Department of Chemistry, Niš, Serbia

\section{https://doi.org/10.21175/rad.abstr.book.2021.13.4}

The inductively coupled plasma emission spectrometric (ICP-OES) method was used for the simultaneous determination of ten elements ( $\mathrm{Zn}, \mathrm{Fe}, \mathrm{Cr}, \mathrm{Cu}, \mathrm{Mn}, \mathrm{Ni}, \mathrm{Al}, \mathrm{Ba}, \mathrm{B}$, and $\mathrm{Sr}$ ) in 23 samples of purees and juices. Potentially toxic elements can be harmful to health if present in food. Even essential and probably essential elements can be toxic if present in excessive amounts. Infants and toddlers are particularly vulnerable due to their smaller body size, developing brains, and organ systems. The determination of mineral content in baby purees and juices is very important (for both manufacturers and consumers) due to their positive as well as negative impact on children's health. The health risk assessment of potentially toxic elements on infants and toddlers was estimated by calculating the average daily dose (ADD), hazard quotient (HQ) and hazard index (HI). The values of $\mathrm{HI}<1$ for all analyzed fruit products suggest that none of the quantified potentially toxic elements may pose a health risk for selected groups of children. The health risk for the lead was not estimated since EPA has not yet established the chronic oral reference dose (RfD) for this metal. Also, the previous official provisional tolerable weekly intake (PTWI) value of $25 \mu \mathrm{g} \mathrm{Pb} / \mathrm{kg}$ body weight/day was withdrawn. New PTWI value that would be considered health protective has not been possible yet to establish. However, the lead exposure was evaluated based on the size of daily consumption of the sample with highest lead content and average toddler body weight. The value of $2.21 \mu \mathrm{g} \mathrm{Pb} / \mathrm{kg}$ body weight/day is in the lead exposure interval ( $1.1-3.10 \mu \mathrm{g} \mathrm{Pb} / \mathrm{kg}$ body weight/day) estimated by EFCA. 


\title{
Experience in the modernization of a non-thermal plasma source for pre-sowing seed treatment
}

\author{
Daria Petrukhina, Ibragim Medzhidov, Vladimir Kharlamov, \\ Mariya Pomyasova, Oxana Tkhorik, Sergei Gorbatov, Valentin Shishko, \\ Aleksandr Shesterikov, Victor Tikhonov, Alexander Tikhonov, Igor Ivanov
}

\author{
Russian Institute of Radiology and Agroecology, Obninks, Russia
}

https://doi.org/10.21175/rad.abstr.book.2021.13.5

Argonne microwave non-thermal plasma of atmospheric pressure was used in this work. In the experiments, we used the barley seeds (Hordeum vulgare L.) of the Vladimir sort, a harvest of 2018. Each investigated case of the experiment included 50 seeds in three repeats. Petri dishes with seeds were placed under the plasma flow with different impact duration on them. After the process, seeds were laid out on wet filter paper, germinated for seven days in a thermostat at a temperature of $20 \pm 0.5^{\circ} \mathrm{C}$ and then, the laboratory germination was determined. The non-thermal plasma source's core is a low-budget $2.45 \mathrm{GHz}$ magnetron microwave generator with a high-voltage power unit [1].

In the first experiment, non-thermal plasma was generated using a 6-pin resonant microwave discharger. The exposure of non-thermal plasma on seeds did not lead to a statistical change in the sprouts' length and roots of 7-day-old barley seedlings. However, after exposure for 5 and 20 minutes, the laboratory germination significantly decreased to the control value. In other cases, no changes were detected. After the first tests of the non-thermal plasma source, its deficiencies were observed that could have a negative effect on the sowing qualities of seeds after their irradiation by plasma. So, because of design specifics during prolonged work time, the discharger is overheating caused a breakdown in the coaxial line. Probably one of the reasons for this was insufficient heat rejection.

To address this problem, an octagonal spark gap was formed with improved heat removal from the discharge zone due to the formation of recirculating blowing zones. With the new geometry of the discharger, it was possible to distribute the load more uniformly, produce the stable discharge, and achieve the installation's stability, including during prolonged work operation. The exposure of seeds with Argonne microwave non-thermal plasma after device modification did not negatively affect the sowing characteristics of barley seeds. The laboratory germination of the seeds did not exceed the control value. It was also found that the plasma exposure does not affect the root and shoot length on the 7 th-day of ontogenesis.

Thus, during the work on the study of the effect of non-thermal plasma on the sowing qualities of spring barley of the Vladimir variety, it was found that the plasma treatment, in general, does not have a negative or stimulating effect on the initial growth processes of barley seeds, but reduces the infection with phytopathogenic fungi [2].

\section{References}

[1] Tikhonov V., Gorbatov S., Ivanov I., Tikhonov A. The Low-Cost Microwave Source of Non-Thermal Plasma. In: 7th International Congress on Energy Fluxes and Radiation Effects (EFRE) - 15th International Conference on Modification of Materials with Particle Beams and Plasma Flows (Tomsk 2020). IEEE, 2020. DOI: 10.1109/EFRE47760.2020.9242089

[2] Petrukhina D., Pomyasova M., Karpenko E. Research of possibility of application of non-thermal plasma for phytosanitary treat-ment of barley seeds // Machinery and Equipment for Rural Area. 2020. DOI: $10.33267 / 2072-9642-2020-9-30-33$ 


\title{
Study of gamma radiation effects on free radicals generation and antioxidant potential of beebread
}

\author{
Ralitsa Mladenova', Katerina Aleksieva1, \\ Nikolay Solakov², Kamelia Loginovska²
}

\author{
1 Institute of Catalysis, Bulgarian Academy of Sciences, Sofia, Bulgaria \\ 2 Institute of Cryobiology and Food Technologies, Agricultural Academy, Sofia, Bulgaria
}

\section{https://doi.org/10.21175/rad.abstr.book.2021.13.6}

Gamma irradiation could be used as a safe method for disinfection and shelf-life prolongation of beebread. By means of the using of ionizing radiation can be avoided proteins and sugars deterioration, no changes to flavor, taste and texture also were observed. The effect of beebread gamma treatment on antiradical activity was evaluated by Electron Paramagnetic Resonance (EPR) spectroscopy and also the composition of phenolic compounds was spectrophotometrically determined. An analysis of the main phenolic constituents before and after gamma treatment was performed by ultra-high performance liquid chromatography. Free radicals induced in beebread by ionizing rays and their influence on antioxidant properties of tested samples also were investigated.

A narrow signal which intensity increased with the dose is assign to O-centered free radicals induced by gamma rays and they are stable for 120 days after irradiation. The values of total phenolic content and total flavonoid content found in beebread increased after irradiation depending on the dose. The antiradical activity and total antioxidant capacity of extracts were expressed by the DPPH free radical scavenging activity (FRSA) and accordingly with trolox equivalent antioxidant capacity (TEAC). The EPR study shows that FRSA and TEAC increased after irradiation with $5 \mathrm{kGy}$ and 20 $\mathrm{kGy}$, while the slight decreasing was observed after irradiation at $10 \mathrm{kGy}$. The analysis of flavonoid profile in tested samples showed that the concentration of identified main groups phenolic compounds increase after gamma treatment for flavonols and phenolic acids, while quercetine glycosides increase only at $5 \mathrm{kGy}$ irradiation.

The study shows that gamma irradiation affect antioxidant activity of beebread. The effect depends on the treatment dose with the most significant increase in antioxidant potential observed in samples irradiated with $5 \mathrm{kGy}$.

Acknowledgments: The authors thank the Bulgarian National Science Fund at the Bulgarian Ministry of Education within the framework of Project "KP-o6-N 39/12" for the financial support. 


\title{
Evaluation of heavy metals in different tissues of red mullet
}

\author{
Egon Andoni, Enkeleda Ozuni, Majlind Sulce, \\ Dorjana Beqiraj, Albana Munga, Pellumb Zalla
}

Faculty of Veterinary Medicine, Agricultural University of Tirana, Tirana, Albania

\section{https://doi.org/10.21175/rad.abstr.book.2021.13.7}

The concentration levels of mercury, lead, cadmium and chrome were measured in muscle and liver tissue of Red mullet (Mullus barbatus). Fish samples were collected and purchased from the main fresh fish market of Tirana. A total of 40 samples of muscle and liver tissue were determined by using atomic absorption spectrophotometer (AAS). The results of the study showed that $\mathrm{Hg}$ levels $(p=0.001)$ varied significantly between tissues of Red mullet samples. Based on the results concentration level of $\mathrm{Hg}, \mathrm{Pb}$ and $\mathrm{Cr}$ measured in muscle samples tissue resulted to be within the permissible levels for human consumption set by EC legislation. Cadmium made an exception ( $0.09 \mathrm{mg} / \mathrm{kg} \mathrm{ww}$ ). The results of our study give important information on heavy metal contamination of Red mullet tissues. Furthermore, the data suggests that Red mullet should be the object of further monitoring with the aim to safeguard Tirana consumers' health from heavy metals contamination.

Keywords: Heavy metals, Red mullet, muscle tissue, liver tissue 


\title{
Nanoceria solubility in simulated biological systems: The key mechanisms and analogy to actinide dioxides
}

\author{
Tatiana Plakhova', Maria Vyshegorodtseva', \\ Anastasia Konyukhova', Irina Seregina', Roman Svetogorov², \\ Anna Romanchuk', Vladimir Ivanov3, Stepan Kalmykov'
}

\author{
1 Lomonosov Moscow State University, Moscow, Russia \\ 2 NRC "Kurchatov Institute", Moscow, Russia \\ 3 Kurnakov Institute of General and Inorganic Chemistry, Moscow, Russia
}

\section{https://doi.org/10.21175/rad.abstr.book.2021.14.1}

Nanocrystalline cerium dioxide (nanoceria, $\mathrm{CeO}_{2}$ ) is judged to be a very promising material for industrial and biomedical applications. Due to the extensive use, nanoceria is getting released into the environment, and exposure to humans is a major concern. Depending on particle size, shape, reaction medium, $\mathrm{CeO}_{2}$ can have both negative and positive effects on a living organism. In some case, $\mathrm{CeO}_{2} \mathrm{NPs}$ cause oxidative stress, which leads to the formation of reactive oxygen species (ROS: hydrogen peroxide, superoxide radical etc.) and so forth in living cells. Conversely, ceria NPs could also act as anti-oxidants by ROS scavenging. The antioxidant activity of $\mathrm{CeO}_{2} \mathrm{NPs}$ contributes to its promising use in the composition of neuro-, cardio-, radio-protective and anti-inflammatory drugs. It is commonly believed that the antioxidant activity of nanoceria is closely related to the changes in oxygen non-stoichiometry, e.g., the $\mathrm{Ce}(\mathrm{IV}) / \mathrm{Ce}(\mathrm{III})$ ratio. However, with the development of modern methods of solid phase analysis, the presence of Ce(III) in the structure of biologically active particles has been questioned. In this case, the key factor affecting the bioactivity of $\mathrm{CeO}_{2}$ can be solubility and associated processes (redox reactions, phase transformations, metal-ion complexation, etc.). It should be noted that $\mathrm{CeO}_{2}$ solubility study is of great significance as the main non-radioactive analog of actinide dioxides $\mathrm{AnO}_{2}(\mathrm{An}=\mathrm{Pu}, \mathrm{Th}, \mathrm{U}) . \mathrm{CeO}_{2}$ is a valence and a structural analog of $\mathrm{AnO}_{2}$ as they share the same fluorite type structure. Predicting the bioavailability of actinide-containing nanoparticles is an important task for radiological risk assessments in many nuclear industry fields.

The present study is aimed to investigate solubility and associated processes of nanosized $\mathrm{CeO}_{2}$ in solutions, simulated biological media. $\mathrm{CeO}_{2}$ samples with different nanoparticles size were prepared using a facile rapid chemical precipitation method from cerium (III) nitrate as a stock solution. The part of fresh precipitated samples was exposed to thermal treatment under $40^{\circ} \mathrm{C}, 150^{\circ} \mathrm{C}$ or freeze-dried to determine the influence of the initial solid surface state on $\mathrm{CeO}_{2}$ solubility. In a first step, $\mathrm{CeO}_{2}$ samples dissolving has been conducted in so-called pure solution, i.e., solutions with low ionic strength $(\mathrm{I}=0.01 \mathrm{M})$. Then, the influence of $\mathrm{Cl}, \mathrm{HCO}_{3^{2-}}, \mathrm{PO}_{4^{3-}}$ and $\mathrm{C}_{3} \mathrm{H}_{5} \mathrm{O}(\mathrm{COO})_{3^{3-}}$ on solubility under steady-state conditions for selected $\mathrm{CeO}_{2}$ samples was investigated. Finally, the dissolution of $\mathrm{CeO}_{2}$ in complex media such as pulmonary, phagolysosome, cell culture media, and serum were studied. For a better understanding of the solubility behavior of $\mathrm{CeO}_{2} \mathrm{NPs}$, and to enable thermodynamic modelling, during the experiment procedure both solid and aqueous phases were examined simultaneously. The solubility was determined by measuring cerium concentration in aqueous phase using inductively coupled plasma mass spectrometry or by using

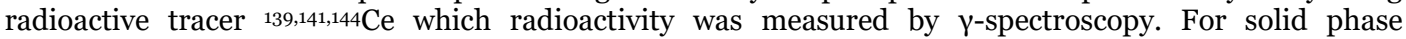
characterization a bunch of modern high-precision microscopic and spectroscopic techniques were used.

Nanoparticles of size 2, 5 and $8 \mathrm{~nm}$ were obtained in this work. No significant effect of nanoparticle size on solubility at steady state conditions in $0.01 \mathrm{NaClO}_{4}$ was observed. It was found that the solubility of $\mathrm{CeO}_{2}$ nanoparticles depends on their thermal pre-treatment: samples drying leads to a decrease in $\mathrm{CeO}_{2}$ solubility under steady state conditions. At the same time solubility kinetic virtually identical to freshly precipitated and dried samples. It was observed that solubility of $2 \mathrm{~nm} \mathrm{CeO}{ }_{2}$ nanoparticles is higher than 5 $\mathrm{nm}$ in presence of $\mathrm{PO}_{4}{ }^{3-}$ ions at the $\mathrm{pH}$ range $4-8$. At the same time under this condition the initial $\mathrm{CeO}_{2}$ phase transformation to cerium (III) phosphate is occurred. The obtained experimental data were compared with the available data on $\mathrm{PuO}_{2}$ and $\mathrm{ThO}_{2}$ solubility under similar conditions. 


\title{
Physical-chemical and isotopic characterization $\left(\delta_{1} 80, \delta_{2} \mathrm{H}\right.$ and $\left.3 \mathrm{H}\right)$ of surface and groundwater in the Luanda Region for the integrated management of water resources
}

\author{
Agna Nadine Maia de Sena Carvalho',2, João Pedro3, Gabriel Luís Miguel² \\ 1 Paulista State University, Sorocaba, Brazil \\ 2 Agostinho Neto University, Luanda, Angola \\ 3 Metropolitan Superior Polytechnic Institute of Angola, Luanda, Angola
}

\section{https://doi.org/10.21175/rad.abstr.book.2021.14.2}

Water is an essential resource for all forms of life on the planet, of the earth's water resources, only $3 \%$ is fresh water, two thirds of which is trapped in the form of polar caps and glaciers. The remaining $1 \%$ of fresh water, a fifth is found in remote and inaccessible places, and much of the rainwater resulting from flooding caused by monsoons cannot be used easily. Currently, only $0.08 \%$ of all fresh water in the world is exploited by mankind, and used for ever-increasing needs. The analysis of isotopes of chemical elements naturally present in water, have been decisive in investigating problems related to water dynamics. The isotopes of deuterium (2H), oxygen-18 (18O), both stable isotopes and tritium $(3 \mathrm{H})$, radioactive isotope, mark the parts of the hydrological cycle, making it possible to know the areas of recharging aquifer systems, the origin and age of the resources water (transit time, definition of flow lines, etc.), identify groundwater salinization mechanisms and the source of polluting sources (organic matter and / or fertilizers). Therefore, this work aims to carry out a physical-chemical and isotopic characterization of samples of groundwater and surface water in the Luanda region. For the study of the physical-chemical characterization, the parameters of $\mathrm{pH}$, dissolved oxygen, total dissolved solids, temperature and conductivity were measured. The isotopic characterization, was done by for the stable isotopes it was made by a laser isotopic water analyzer LWIA (of the English: Laser Water Isotope Analyzer), for the stable isotopes, and a liquid scintillation counter LSC (of the English: Liquid Scintillator Counting), for the radioactive isotope. The analyzes of the stable isotopes were not prepared before the measurement, they were only filtered so that the residues present did not interfere in the measurement, in the case of the analysis of the radioactive isotope, there was a distillation of the samples and subsequent enrichment of the present environmental tritium.

Keywords: Water resources, stable and radioactive isotopes, LWIA, LSC 


\title{
Physical factors in the treatment of occupational spine injuries
}

\author{
Irena Stoilova ${ }^{1}$, Radostina Madzharova², Maya Krastanova²
}

\author{
1 Department of Hygiene, Medical Ecology, Occupational Diseases and Disaster Medicine, Medical University, \\ Pleven, Bulgaria \\ 2 Department of Physical Medicine, Rehabilitation, Occupational Therapy and Sports, Medical University, \\ Pleven, Bulgaria
}

\section{https://doi.org/10.21175/rad.abstr.book.2021.14.3}

Introduction. Spinal injuries are a current health problem for people working in various sectors of the economy. Occupational injuries of the spine develop as a result of the action of harmful factors of production, the most important of which are physical stress and unfavorable production microclimate. The main clinical syndromes of the considered diseases are pain, vertebral and radicular. Modern methods of treatment of injuries include physical factors - electrotherapy, light therapy, magnetic field, massage, therapeutic gymnastics, extension therapy, peloidotherapy.

Materials and methods. The subject of the study is 105 cases of spinal cord injuries in employees in various companies in Northern Bulgaria. The persons were hospitalized in the Department of Occupational Diseases and Clinic of Physiotherapy and Rehabilitation, University Hospital - Pleven in the period 2017 - 2020. Clinical, laboratory, functional, imaging and statistical research methods were used. Treatment with physical factors was performed.

Aim of the study. To improve the treatment and prevention of occupational injuries of the spine.

Results and discussion. In $93 \%$ of patients, improvement in clinical syndromes and performance of daily activities was found. $5.5 \%$ of the subjects need surgical treatment.

Conclusion. Physiotherapy is an appropriate and successful approach in the treatment of occupational injuries of the spine.

Keywords: Occupational diseases, spine, physical factors 


\title{
The role of pharmacists in the health care team in hospital practice
}

\author{
Vedada Čeljo \\ Clinical Centre, University of Sarajevo - Clinic Pharmacy, Sarajevo, Bosnia and Herzegovina
}

https://doi.org/10.21175/rad.abstr.book.2021.14.4

Introduction. The development of the practice of the Clinic Pharmacy, from the orientation towards the drug, is in the process of being directed towards the patient. In developing countries, such as Bosnia and Herzegovina, the level of communication between doctors and pharmacists is low and needs to be improved.

Aims. The aim of this paper is to improve the present role of pharmacists in the healthcare team in hospital practice, in order to provide better quality care for the patient.

Setting. Hospital Pharmacists across Bosnia and Herzegovina were contacted by e-mails to participate in completing the questionnaire.

Method. The research is based on an anonymous questionnaire that contained nine topics.

Results. Out of the 66 hospital pharmacists who were invited to fill in the questionnaire, 40 hospital pharmacists completed the questionnaire with a response rate of $60 \%$. The highest percentage of examinees (pharmacists) who communicate with doctors are in area 2 when the dose of the drug is unsuitable for the patient or indication $(51 \%)$, and in area 7 , when the prescribed drug is not appropriate to the provided way of prescribing $(52 \%)$.

Generally, three types of barriers have been identified that play important roles: the doctorpharmacist relationship, the reduced amount of required information, the methods of communication.

Conclusion. Recommendations for improvement include an upgrade of the electronic system, an express need for procedures covering future therapeutic drug replacement, and the need for direct communication with doctors in clinics. In summation, with consideration of the individual roles of doctors and pharmacists in the health care system, acceptance of each other, effective communication, mutual respect are of utmost importance. 


\title{
An extraction-chromogenic system for iron(III) based on 4-nirocatechol
}

\section{Galya Toncheva1, Magdalena Boradjieva1, Antoaneta Saravanska², Vidka Divarova ${ }^{2}$, Kiril Gavazov ${ }^{2}$}

\author{
1 Plovdiv University Paisii Hilendarski, Plovdiv, Bulgaria \\ 2 Medical University of Plovdiv, Plovdiv, Bulgaria
}

\section{https://doi.org/10.21175/rad.abstr.book.2021.14.5}

The complex formation was studied in a water-chloroform system containing iron(III), 4-nitrocatechol (4NC) and xylometazoline hydrochloride (XMH). The optimum conditions for the extraction of iron(III) were found: concentration of $4 \mathrm{NC}$, concentration of $\mathrm{XMH}, \mathrm{pH}$, and extraction time. The stoichiometry of the extracted complex was established, and the key extractionspectrophotometric characteristics were determined: absorption maximum, molar absorptivity, distribution ratio, fraction extracted and Beer's law limits.

Keywords: Iron(III); 4-nitrocatechol; xylometazoline hydrochloride; ternary complex; solvent extraction; spectrophotometry

Acknowledgments: This work was supported by the Plovdiv University Scientific Fund (grant No SP21 HFoO4). 


\title{
Oxidative stress parameters affecting fertility of Bulgarian Murrah buffalos depending on age and season
}

\author{
Galina Nikolova', Yanka Karamalakova, Veselina Gadjeva', \\ Nasko Vasilev', Ivan Fasulkov', Pencho Penchev², Yordanka Ilieva²
}

1 Trakia University, Stara Zagora, Bulgaria

2 Agricultural Academy, Agricultural Institute, Shumen, Bulgaria

\section{https://doi.org/10.21175/rad.abstr.book.2021.14.6}

The study of oxidative damage and protection against it has become increasingly important last years. Research on the role of oxidative stress in the physiology and pathology of ruminants has also been relatively recent. Some data show that oxidative stress has a negative effect on the reproductive process in mammals. It is therefore desirable to determine and control their oxidative /antioxidant status in order to prevent pathological changes leading to problems in fertilization, embryonic development and survival of the offspring. In ruminants, the activity of oxidative stress biomarkers can also be affected by the season, which in turn can have major effects on their reproductive capacity.

In the present study we determined the degree of influence of oxidative stress on the fertility of buffaloes and buffalo heifers of the breed Bulgarian Murra during non-breeding and breeding season. For this purpose, some final products of oxidation of lipid and protein were elevated, such as malodialdehyde (MDA) and protein carbonyl content (PPC) as biomarkers of oxidative status and the levels of antioxidant enzymes -superoxide dismutase (SOD) and glutathione peroxidase (GSH-Px) were established, as well.

The study included 24 multiple-born buffaloes $>40$ days postpartum and 30 buffalo heifers of the Bulgarian Murra breed. The animals were kept on the buffalo farm of the Agricultural Institute, Shumen (Northeastern Bulgaria, latitude: 43.28N, longitude: 26.93E). The ration included concentrated fodder for lactating buffaloes, beer porridge, alfalfa hay, straw, and constant access to water. The buffaloes weighed from $460 \mathrm{~kg}$ to $650 \mathrm{~kg}$, aged from 2 to 6 years and lactating milk $1800-2100 \mathrm{~kg}$ with daily double milking. The buffalo heifers had a body weight of $450-470 \mathrm{~kg}$ and an age of $820-840$ days. The ration consisted of concentrated fodder, alfalfa hay, straw and constant access to water. Group of buffaloes $(n=24)$, non-breeding (spring and summer) season $(n=8)$ and breeding season (autumn and winter) $(n=16)$; Group of buffalo heifers $(n=30)$ non-breeding season $(\mathrm{n}=17)$ and breeding season $(\mathrm{n}=13)$.

To determine the biomarkers, blood samples were obtained from v. jugularis by means of a vacutainer on the day of starting the estrus synchronization program. Subsequently centrifuged and the serum was stored at $-20^{\circ} \mathrm{C}$ until the study.

All biomarkers characterizing the oxidative status in buffalo heifers MDA and PPC were statistically significantly higher compared to buffaloes $(\mathrm{p}<0.001)$. Increased oxidative stress was registered in buffalo heifers during the breeding season, compared to buffaloes in both seasons due to the reduced activity of SOD and GSH-Px. Lipid peroxidation (MDA) products were also statistically significantly increased in buffalo heiferss during the breeding season, followed by the non-breeding season, and in buffaloes from both seasons, followed by statistically significantly increased in SOD. Elevated levels of malondialdehyde and superoxide dismutase were reported in non-pregnant buffalo heifers, but without a statistically significant difference. However, buffalo heifers were found to have better fertility, probably due to more active antioxidant protection as an adaptive mechanism against oxidative stress. 


\title{
Real-time biomarkers affecting fertility of Bulgarian Murrah buffalos depending on age and season
}

\author{
Yanka Karamalakova1, Galina Nikolova, Veselina Gadjeva', \\ Nasko Vasilev', Ivan Fasulkov', Pencho Penchev², Yordanka Ilieva2
}

1 Trakia University, Stara Zagora, Bulgaria

2 Agricultural Academy, Agricultural Institute, Shumen, Bulgaria

\section{https://doi.org/10.21175/rad.abstr.book.2021.14.7}

Oxidative stress has a negative effect on the reproductive process in mammals. It is therefore desirable to determine and control their oxidative/antioxidant status in order to prevent pathological changes leading to problems in fertilization, embryonic development and survival of the offspring. The danger of oxidative stress stems from the fact that it is not proven by clinical symptoms. The condition is diagnosed by biomarkers of oxidative stress, such as the levels of a number of products of radical damage to cellular structures, lipids, proteins, nucleic acids and the activities of antioxidant enzymes and others. The aim of the presented study was: 1 . To measure some real time biomarkers of radical formation - oxygen species (ROS), ascorbate radicals (Asc •), nitric oxide radicals (NO •); 2. To discuss the influence of the measured real time biomarkers on fertility of buffaloes and buffalo heifers of Bulgarian Murra breed during non-breeding and breeding season.

The study included 24 multiple-born buffaloes $>40$ days postpartum and 30 buffalo heifers of the Bulgarian Murra breed. The animals were kept on the buffalo farm of the Agricultural Institute, Shumen (Northeastern Bulgaria, latitude: 43.28N, longitude: 26.93E). The ration included concentrated fodder for lactating buffaloes, beer porridge, alfalfa hay, straw, and constant access to water. The buffaloes weighed from $460 \mathrm{~kg}$ to $650 \mathrm{~kg}$, aged from 2 to 6 years and lactating milk 1800 $2100 \mathrm{~kg}$ with daily double milking. The buffalo heifers had a body weight of $450-470 \mathrm{~kg}$ and an age of $820-840$ days. The ration consisted of concentrated fodder, alfalfa hay, straw and constant access to water. Group of buffaloes $(n=24)$, non-breeding (spring and summer) season $(n=8)$ and breeding season (autumn and winter) $(\mathrm{n}=16)$; Group of buffalo heifers $(\mathrm{n}=30)$ non-breeding season $(\mathrm{n}=17)$ and breeding season $(\mathrm{n}=13)$.

To determine the real time biomarkers blood samples were obtained from v. jugularis by means of a vacutainer on the day of starting the estrus synchronization program. Subsequently centrifuged and the serum was stored at $-20^{\circ} \mathrm{C}$ until the study.

The real time biomarkers characterizing the oxidative status in buffalo heifers were statistically significantly higher compared to buffaloes ( $\mathrm{p}<\mathrm{0.001)}$. Increased oxidative stress was registered in buffalo heifers during the breeding season, compared to buffaloes in both seasons through a statistically significant increase in ROS products in blood serum. The elevated levels of ROS, ascorbate radicals, and nitric oxide were reported in nonpregnant buffalo heifers, but without a statistically significant difference. However, buffalo heifers were found to have better fertility, probably due to more active antioxidant protection as an adaptive mechanism against oxidative stress. 


\title{
Evaluation of the presence of radionuclides of natural origin (NORM) and rare earths in the Brazilian Red Mud
}

\author{
Agna Nadine Maia de Sena Carvalho', Maria Lúcia Pereira Antunes', \\ Jose Antonio Silva Souza ${ }^{2}$, Tran Hoang Mai ${ }^{3}$, Do Quoc Hung ${ }^{4}$
}

\author{
1 Sao Paulo State University (UNESP), Sorocaba, Brazil \\ 2 Federal University of Pará (UFPA), Belem, Brazil \\ 3 Institute for Technology of Radioactive and Rare Elements of Vietnam, Hanoi, Vietnam \\ 4 Center for Rare Radioactive Elements Experiment Analysis of Vietnam, Hanoi, Vietnam
}

\section{https://doi.org/10.21175/rad.abstr.book.2021.14.8}

Mining is an activity that generates revenue to leverage a country's economy but also generates waste. Waste is considered a threat to the environment, but it can be reused. Red mud is a residue generated from the processing of bauxite and is very rich in aluminum, silicon and iron. This residue has been studied as a raw material for the production of red ceramics and an input for the production of cement and can also be a secondary source of rare earths. However, there is little information about the presence of rare earths and natural radionuclides in this waste generated in Brazil. Therefore, this work aims to characterize the residue of Red Mud from the States of Pará and São Paulo / Brazil and to determine the specific activities of radionuclides of natural origin from the U-238, Th-232 and K-40 series and the presence of rare earths. The radionuclide evaluation was performed using the gamma spectrometry technique with the use of a hyper pure germanium semiconductor detector and for the evaluation of rare earths, the red mud samples were analyzed in the ICP-AES (Inductively Coupled Plasma - Atomic Emission Spectrometry ) and ICP-MS (Inductively Coupled Plasma - Mass Spectrometry). With the ICP-MS technique, the total number of rare earth elements (ETR) quantified was $911.09 \mathrm{ppm}$ and a total of $738.48 \mathrm{ppm}$ for the ICP-OES technique. For both techniques, it was observed in all samples that Cerium (Ce) is the predominant element, followed by lanthanum (La) and Neodymium (Nd). Based on the results obtained, the ICP-MS showed better efficiency in the quantification of rare earth elements (ETR) due to the factor of mass analysis per element and it was also possible to analyze the concentration of ${ }^{238} \mathrm{U}$ and ${ }^{232} \mathrm{Th}$ in the samples. In the evaluation of natural radionuclides (NORM), the specific activities of $\mathrm{U}$, Th and $\mathrm{K}$ found in the Brazilian red mud samples analyzed, present an average of $163.68 \pm 17.76 \mathrm{~Bq} / \mathrm{kg}$, $436.58 \pm 12.02 \mathrm{~Bq} / \mathrm{kg}$ and $52.15 \pm 10.59 \mathrm{~Bq} / \mathrm{kg}$, respectively for the red mud obtained in the state of Pará, and $51.15 \pm 6.28 \mathrm{~Bq} / \mathrm{kg}, 58.30 \pm 6.10 \mathrm{~Bq} / \mathrm{kg}$ and $47,98 \pm 8.40 \mathrm{~Bq} / \mathrm{kg}$, respectively for the red sludge in the state of São Paulo. The values of the activities of the radionuclides found in the mud of the state of Pará, are higher than those found in studies of Brazilian literature and similar to those of the international literature, except for the K-40. Comparing the activity results for the two samples, the red mud samples from the State of Pará show greater activity for the elements ${ }^{232} \mathrm{Th}$ and ${ }^{238} \mathrm{U}$ than the samples from the State of São Paulo.

Keywords: Red mud, rare lands, ICP-AES, ICP-MS 


\title{
Horizontal distribution and abundance of Copepoda in the shore in the immediate vicinity of shellfish farms in the inner bays of the Boka Kotorska Bay - Southern Adriatic
}

\begin{abstract}
Vera Vukanić
State University of Novi Pazar, Faculty of Biology, Novi Pazar, Serbia

https://doi.org/10.21175/rad.abstr.book.2021.14.9

Temporal and spatial variability of Copepoda was studied in 2008 at tree shallow stations (P1, P2, $\mathrm{P}_{3}$ ) in the inner bays in the Bay of Boka Kotorska (closed coastal waters of Southern Adriatic).

The research was done in the shore in the immediate vicinity of shellfish farms in the Bay of Kotor $\left(\mathrm{P}_{1}, \mathrm{P}_{2}\right)$ and Bay of Risan ( $\left.\mathrm{P}_{3}\right)$. We observed specific behavioral pattern in distribution and in an abundance of Copepoda. 45 species of Copepoda were determined during this study an we registered the species: Paracalanus parvus (Claus), Centropages kröyeri Giesbrecht, Acartia clausi Giesbrecht, Oithona nana (Giesbrecht), Microsetella norvegica (Boeck), Euterpina acutifrons (Dana), Oncaea media Giesbrecht and Oncaea subtilis Giesbrecht, participated with $39.6 \%$ in the total abundance of all Copepoda. Samples were collected seasonally at 3 stations using WP2 net of $200 \mu \mathrm{m}$ mesh by vertical and horizontal hauls. After our data were compared with the results of previous studies, we concluded that there are two characteristic maximums of abundance in total zooplankton, in spring and summer. The first one was induced by the increased abundance of Copepoda, while the second one appears in August and is caused by the dominance of Cladocera, especially the species Penilia avirostris Dana. Similar data are cited by authors for the coastal zones of other seas in Mediterranean. The present study examined the seasonal distribution and abundance of the specimens as well as the oscillations in ecological properties in the researched area. It is concluded that seasonal differences in the horizontal distribution are associated with the dynamic of the hydrographic conditions in the inner bays of the Bay of Boka Kotorska.
\end{abstract}

Keywords: Copepoda, Adriatic, Boka Kotorska Bay, horizontal distribution, coastal waters 


\title{
Cathodoluminescence of $\mathrm{Bi}^{3+}$-doped lanthanide niobates
}

\author{
Assemgul Kissabekova ${ }^{1,2}$, Alma Dauletbekova ${ }^{1}$, \\ Irina Kudryavtseva², Aleksandr Lushchik², Sergey Omelkov², \\ Magdalena Baran3, Yaroslav Zhydachevskyy ${ }^{4}$
}

\author{
1 L.N. Gumilyov Eurasian National University, Nur-Sultan, Kazakhstan \\ 2 University of Tartu, Tartu, Estonia \\ 3 Institute of Electronic Materials Technology, Warsaw, Poland \\ 4 Lviv Polytechnic National University, Lviv, Ukraine
}

\section{https://doi.org/10.21175/rad.abstr.book.2021.15.1}

Luminescence characteristics of $\mathrm{Bi}^{3+}$-doped lanthanide niobates were reported about 50 years ago [1]. The interest to these materials has been recently renewed by looking the undoped and $\mathrm{Bi}^{3+}$-doped niobates as potential candidates for numerous technological applications. Indeed, besides an intense visible luminescence, these materials have high dielectric constant, low phonon frequencies, good photoelastic and nonlinear optical properties as well as an excellent chemical, mechanical, and thermal stability. Owing to that, $\mathrm{YNbO}_{4}: \mathrm{Bi}$ and $\mathrm{GdNbO}_{4}: \mathrm{Bi}$ were proposed as potential blue phosphors for field emission displays, while $\mathrm{YNbO}_{4}$ and $\mathrm{GdNbO}_{4}$ co-doped with $\mathrm{Bi}^{3+}$ and different rare-earth ions (e.g., $\mathrm{Tm}^{3+}, \mathrm{Dy}^{3+}, \mathrm{Yb}^{3+}, \mathrm{Eu}^{3+}, \mathrm{Nd}^{3+}$ ) were found to be suitable for white light emitting diodes or solar cells. Up to now, the cathodoluminescence (CL) characteristics of lanthanide niobates were examined only at room temperature and under the excitation by low energy electrons of 1-7 keV $[2,3]$, when the penetration depth of the electron beam into phosphor does not exceed $400 \mathrm{~nm}$. In these experiments, both phosphors, $\mathrm{YNbO}_{4}: \mathrm{Bi}$ and $\mathrm{GdNbO}_{4}$ : $\mathrm{Bi}$, showed a strong and relatively broad emission bands around $\sim 2.8 \mathrm{eV}$.

The aim of the present work is to perform a detailed study of the CL characteristics of $\mathrm{Bi}^{3+}$-doped $(\mathrm{Y}, \mathrm{Lu}, \mathrm{Gd}) \mathrm{NbO}_{4}$ powders under $\sim 100 \mathrm{keV}$ pulsed electron excitation with a penetration depth of an electron beam more than $20 \mu \mathrm{m}$ that reduces the effect of surface defects on luminescence [4]. The time-resolved measurements, including CL spectra and decay kinetics were performed in a wide temperature range starting from $5 \mathrm{~K}$.

At low temperatures, all phosphors show wide emission band in the visible region with maxima around 2.7-3.0 eV. In our opinion, this emission band is the superposition of several bands with excitonic nature [5]. In addition to strong visible emission, a weak ultrafast emission $(<100 \mathrm{ps}$, limit of our measurements) covering the entire transparency region of niobates is observed as well. Similar to other materials (see, e.g., [4]), this emission could be ascribed to so-called intraband luminescence due to radiative relaxation of hot band carriers.

The obtained CL properties of niobates have been compared with those obtained earlier under photoexcitation of the same phosphors [5].

\author{
References \\ [1] G. Blasse, A. Bril, J. Chem. Phys. 48 (1968) 217-222; J. Lumin. 3 (1970) 109-131. \\ [2] S.H. Shin, D.Y. Jeon, K.S. Suh, J. Appl. Phys. 90 (2001) 5986-5990. \\ [3] X. Liu, J. Lin, J. Lumin. 122-123 (2007) 700-703. \\ [4] S. Omelkov, V. Nagirnyi, S. Gundacker et al., J. Lumin. 198 (2018) 260-271. \\ [5] M. Baran, A. Kissabekova, A. Krasnikov et al., NIMB 463 (2020) 7-15.
}




\title{
Plasma modification of thin polycaprolactone films
}

\section{Ekaterina Filippova}

\author{
Tomsk Polytechnic University, Tomsk, Russia
}

\section{https://doi.org/10.21175/rad.abstr.book.2021.15.2}

Polycaprolactone (PCL) is a biodegradable polyester and is widely used material as medical implants. Using of films based on biodegradable thin films with controlled solubility as a temporary material for stem cells may be an alternative to existing insoluble polymers. The hydrophobicity and low surface energy can limit the use of the polymer as a substrate for cell cultures and corneal implants. The purpose of this research is the study the influence of the plasma modification on PCL films properties.

The feedstock for films was obtained by dissolving PCL (Netherlands) in the chloroform $\left(\mathrm{CHCl}_{3}\right)$ 1\% solution was poured into Petri dishes (12 g) which were placed in a fume hood until complete evaporation of $\mathrm{CHCl}_{3}$ and the formation of PCL films. Modification was carried out using the atmospheric pressure low-temperature plasma. The plasma treatment time was 30 .

The surface roughness analysis showed that $R a$ of PCL films (inner) surface was $0.02 \pm 0.005 \mu \mathrm{m}$, $R q$ was $0.04 \mu \mathrm{m}, R t$ was 0.42 . PCL films (outer) surface had $R a=0.03 \pm 0.01 \mu \mathrm{m}, R q=0.5 \mu \mathrm{m}, R t=$ 0.48. The plasma modification of the material increased a roughness (1.5 times) of virgin PCL films.

The wettability of the surface analysis showed that the PCL inner side had a wetting angle of water of $72.0^{\circ} \pm 2.2$, outer side had a wetting angle of water of $73.1^{\circ} \pm 3.1, p>0.05$. The PCL film properties were close to hydrophobic. Surface modification of PCL films increased the hydrophilicity of films, reducing the contact angle of the inner side by $9^{\circ}-10^{\circ}(12 \%-14 \%)$, and increased the surface energy values mainly due to the polar component to $20.6 \pm 0.3 \mathrm{~mJ} / \mathrm{m}^{2}$.

As a result of the research, it was found that the plasma modification increases a roughness $(1.5$ times) of PCL films, the hydrophilicity of films, reducing the contact angle of the inner side by $9^{\circ}-$ $10^{\circ}(12 \%-14 \%)$, and increases the surface energy values mainly due to the polar component to $20.6 \pm 0.3 \mathrm{~mJ} / \mathrm{m}^{2}$.

Acknowledgments: The research was conducted with the financial support of the Russian Foundation for Basic Research (RFBR) as part of the project № 20-08-00648. 


\title{
Percolation in irreversible deposition of mixtures on a triangular lattice
}

\author{
Ivana Lončarević1, Ljuba Budinski Petkovićc', \\ Zorica Jakšićc ${ }^{2}$, Slobodan Vrhovac ${ }^{2}$
}

\author{
1 Faculty of Technical Sciences, Novi Sad, Serbia \\ 2 Institute of Physics, Beograd, Serbia

\section{https://doi.org/10.21175/rad.abstr.book.2021.15.3}

Percolation phenomena have been investigated for a wide variety of binary mixtures by Monte Carlo simulations. Depositing objects were made by self-avoiding lattice steps on a triangular lattice and irreversibly placed on the substrate. Percolation threshold $\theta_{\mathrm{p}}$ for mixtures of objects covering the same number of sites is always lower than $\theta_{\mathrm{p}}$ for the more compact object, and it can be even lower than $\theta_{\mathrm{p}}$ for both components. Mixtures of percolating and non-percolating objects almost always percolate, but the percolation threshold is higher than $\theta_{\mathrm{p}}$ for the percolating component. Adding a component with good percolating properties to a system of non-percolating objects makes the resulting deposit percolate. Lowest percolation thresholds are obtained for mixtures with elongated angled objects. It could be estimated that the angled objects of length between $l=6$ and $l=10$ give the largest contribution to the percolation. 


\title{
Photoluminescence and Judd-Ofelt analysis of $\mathrm{ErF}_{3}$-doped $\mathrm{BaF}_{3}$ crystals
}

\author{
Andrei Racu',2, Marius Stef', Irina Nicoara1, Daniel Vizman', Gabriel Buse1 \\ 1 Faculty of Physics, West University of Timisoara, Timisoara, Romania \\ 2 National Institute of Research \& Development for Electrochemistry and Condensed Matter, Timisoara, \\ Romania
}

\section{https://doi.org/10.21175/rad.abstr.book.2021.15.4}

In this work, we investigate the influence of erbium ions concentration on optical properties of $\mathrm{ErF}_{3}$ doped $\mathrm{BaF}_{2}$ crystals. Four concentrations of $\mathrm{ErF}_{3}$ from 0.05 to 0.5 mol\% were studied. Experimentally absorption, photoluminescence (PL) and decay measurements were done at room temperature in ultraviolet (UV) and visible (VIS) spectral range. Judd-Ofelt (J-O) analysis based on four $\mathrm{Er}^{3+}$ absorption peaks centered at $377,519,653,802 \mathrm{~nm}$ was used to calculate $\mathrm{J}-\mathrm{O}$ intensity parameters, radiative emission probabilities, the branching ratios and radiative lifetimes. For PL measurements two erbium absorption peaks at 290 and $380 \mathrm{~nm}$ were selected and pumped separately. A notable PL emission in UV was registered at $321 \mathrm{~nm}$ when pumped with $290 \mathrm{~nm}$ and can be attributed to the transition ${ }^{2} \mathrm{P}_{3 / 2} \rightarrow{ }^{4} \mathrm{I}_{15 / 2}$ of $\mathrm{Er}^{3+}{ }^{3}$ ions. The green and red emission at 539 and $668 \mathrm{~nm}$ were registered in VIS, when pumped with $380 \mathrm{~nm}$. Those are assigned to the erbium transition ${ }^{4} \mathrm{~S}_{3 / 2} \rightarrow{ }^{4} \mathrm{I}_{15 / 2}$ and ${ }^{4} \mathrm{~S}_{3 / 2} \rightarrow{ }^{4} \mathrm{I}_{15 / 2}$ respectively. A linear to exponential increase in intensity of absorption and PL spectra with concentrations was observed. The calculated and experimental radiative lifetimes were in milliseconds range for green and red emissions. Although the red and green emission of $\mathrm{Er}^{3+}$ ions doped in $\mathrm{BaF}_{2}$ crystals has been observed by other authors, the influence of $\mathrm{ErF}_{3}$ concentration on the UV emission has not been investigated so far. 


\title{
Synthesis of $\mathrm{Fe}_{3} \mathrm{O}_{4}$ magnetic nanoparticles by using green chemistry
}

\author{
Melise Karatay Kutman', Ozge Kozgus Guldu', \\ Coskun Harmansah², F. Zumrut Biber Muftuler'
}

\author{
1 Ege University, Department of Nuclear Applications, Institute of Nuclear Sciences, Izmir, Turkey \\ 2 Ege University, Ege Vocational School, Izmir, Turkey
}

\section{https://doi.org/10.21175/rad.abstr.book.2021.15.5}

Reducing and oxidizing agents used in the synthesis of nanoparticles have received great attention lately due to their applications in various fields. While physical and chemical methods are more popular for nanoparticle synthesis, green chemistry is the better option because of its eco-friendly and non-toxicity [1]. Extracts of various plants (tea, coffee, banana, etc.) have been used as reductant and capping agents during the synthesis. Tea extracts with high polyphenol contents act as both chelating/reducing and capping agents for the synthesis of nanoparticles [2].

In this study, $\mathrm{Fe}_{3} \mathrm{O}_{4}$ magnetic nanoparticles were synthesized with green tea extract which is suitable reductant for green chemistry because of its rich in active polyphenols [3, 4]. Magnetic nanoparticles were synthesized by optimizing the synthesis conditions at room temperature for $4 \mathrm{~h}[5]$.

For this purpose, different amounts of green tea extracts were used in the synthesis of $\mathrm{Fe}_{3} \mathrm{O}_{4}$ magnetic nanoparticles to increase the efficiency within the scope of optimization studies. The synthesized $\mathrm{Fe}_{3} \mathrm{O}_{4}$ magnetic nanoparticles were characterized by X-Ray Diffraction (XRD), Energy Dispersive X-Ray Spectroscopy (EDS) and Scanning Electron Microscopy (SEM) and magnetic properties of synthesized nanoparticles were analyzed via Vibrating Sample Magnetometer (VSM). Results of XRD analysis showed that patterns of $\mathrm{Fe}_{3} \mathrm{O}_{4}$ magnetic nanoparticles are consistent. EDS analysis exhibited that $\mathrm{Fe}, \mathrm{C}$ and $\mathrm{O}$ elements are present in the sample. The atomic compositions for $\mathrm{Fe}, \mathrm{C}$ and $\mathrm{O}$ were $8.89 \%, 39.39 \%$ and $51.73 \%$, respectively. The size distribution of $\mathrm{Fe}_{3} \mathrm{O}_{4}$ nanoparticles from SEM images was found range of 71-206 $\mathrm{nm}$. The magnetic properties of $\mathrm{Fe}_{3} \mathrm{O}_{4}$ nanoparticles were measured using Vibrating Sample Magnetometer (VSM) in order to better understand the magnetization. According to VSM results, the saturation magnetization (Ms) value has been measured $3.10 \mathrm{emu} / \mathrm{g}$.

It is known that novel $\mathrm{Fe}_{3} \mathrm{O}_{4}$ nanoparticles may have potential to be used in different applications in medicine and industry. These results suggest that the novel magnetic nanoparticles may find applications in $\mathrm{n}$ a wide variety of industrial sectors.

Keywords: Green synthesis, green tea, $\mathrm{Fe}_{3} \mathrm{O}_{4}$ magnetic nanoparticles, characterization

\section{References}

[1] Salam, H.A., Rajiv, P., Kamaraj, M., Jagadeeswaran, P., Gunalan, S., \& Sivaraj, R. (2012). Plants: green route for nanoparticle synthesis. Int Res J Biol Sci, 1(5), 85-90.

[2] Kharissova, O.V., Dias, H.R., Kharisov, B.I., Pérez, B.O., \& Pérez, V.M.J. (2013). The greener synthesis of nanoparticles. Trends in biotechnology, 31(4), 240-248.

[3] Guo, Q., Zhao, B., Li, M., Shen, S., \& Xin, W. (1996). Studies on protective mechanisms of four components of green tea polyphenols against lipid peroxidation in synaptosomes. Biochimica et Biophysica Acta (BBA)-Lipids and Lipid Metabolism, 1304(3), 210-222.

[4] Agarwal, R., Katiyar, S.K., Zaidi, S.I., \& Mukhtar, H. (1992). Inhibition of skin tumor promoter-caused induction of epidermal ornithine decarboxylase in SENCAR mice by polyphenolic fraction isolated from green tea and its individual epicatechin derivatives. Cancer Research, 52(13), 3582-3588.

[5] Fahmy, H.M., Mohamed, F.M., Marzouq, M.H., Mustafa, A.B.E.D., Alsoudi, A.M., Ali, O.A., \& Mahmoud, F.A. (2018). Review of green methods of iron nanoparticles synthesis and applications. BioNanoScience, 8(2), 491-503. 


\title{
Optimization of the X-ray microtomography reconstruction quality and acquisition time in the study of biocompatible scaffolds
}

\author{
Alexey Buzmakov, Artem Grigorev',2, Yuri Volkov, Victor Asadchikov \\ 1 FSRC “Crystallography and Photonics” RAS, Moscow, Moscow, Russia \\ 2 RUDN University, Moscow, Moscow, Russia
}

\section{https://doi.org/10.21175/rad.abstr.book.2021.15.6}

The biocompatible polymer materials, as well as polymer-based structures, play a pivotal role in intensive research and extensive development of advanced biomedical devices and pharmaceutical formulations. The properties of such systems depend on their spatial structure. For practical usage and modeling of biocompatible scaffolds' properties, it is important to know such parameters as cumulative porosity, pores size distributions, number of connected and isolated pores. Laboratory $\mathrm{X}$-ray microCT allows to reconstruct the spatial distribution of polymer in the sample. In this work, we provide the optimization method of microCT measurement parameters (X-ray spectrum bandwidth, number of projections, one projection exposure time, etc.) to minimize measure time, sample doze keeping good reconstruction quality.

Acknowledgments: This work was supported by Ministry of Science and Higher Education as part of work under the State Assignment FSRC "Crystallography and Photonics" Russian Academy of Science in part of the X-ray micrtoCT measurements and Russian Foundation for Basic Research (Grant No. 19-29-12045_mk) in part of the data processing. 


\title{
Origin of luminescence centers in $\mathrm{Bi}^{\mathrm{i}^{+}}$-doped niobates
}

\author{
Aleksei Krasnikov', Magdalena Baran², Assemgul Kissabekova3, \\ Aleksandr Lushchik', Volodymyr Tsiumra ${ }^{4,5}$, Leonid Vasylechko' ${ }^{6}$, \\ Svetlana Zazubovich ${ }^{1}$, Yaroslav Zhydachevskyy ${ }^{4,6}$
}

\author{
1 Institute of Physics, University of Tartu, Tartu, Estonia \\ 2 Institute of Electronic Materials Technology, Warsaw, Poland \\ 3 L.N. Gumilyov Eurasian National University, Nur-Sultan, Kazakhstan \\ 4 Institute of Physics, Polish AS, Warsaw, Poland \\ 5 Ivan Franko National University of Lviv, Lviv, Ukraine \\ 6 Lviv Polytechnic National University, Lviv, Ukraine
}

\section{https://doi.org/10.21175/rad.abstr.book.2021.15.7}

$\mathrm{Bi}^{3+}$-doped compounds, where a $\mathrm{Bi}^{3+}$ ion substitutes for a trivalent rare-earth ion, became recently the subject of special interest and extensive research due to their possible applications as scintillator and phosphor materials. The compounds co-doped with $\mathrm{Bi}^{3+}$ and trivalent rare-earth ions were found to be potentially applicable as spectral converters for solar cells and solid state light sources of new generation, so called white light-emitting diodes, owing to the presence of absorption bands in the ultraviolet spectral region and intense broad visible $\mathrm{Bi}^{3^{+}}$-related emission bands.

In $\mathrm{Bi}^{3+}$-doped compounds, two types of emission bands with strongly different characteristics can exist. The ultraviolet (UV) emission, usually ascribed to the electronic transitions from the triplet relaxed excited state (RES) of a $\mathrm{Bi}^{3+}$ ion, has a relatively small Stokes shift and FWHM. The decay kinetics of this emission indicates a large $\left(\sim 10^{2} \mathrm{meV}\right)$ energy distance $\mathrm{D}$ between the emitting and metastable levels of the triplet RES of $\mathrm{Bi}^{3+}$. The visible (VIS) emission is characterized by the large Stokes shift and FWHM, as well as by temperature dependence of the decay time indicating a small D value $(\sim 1 \mathrm{meV})$.

Despite a huge number of publications, the origin of the VIS emission is still under discussion. This emission was ascribed to the metal-to-metal charge transfer, single $\mathrm{Bi}^{3+}$ ions, $\mathrm{Bi}^{3+}$ dimers or clusters, trapped or localized excitons, etc. To understand the reasons of different origin of $\mathrm{Bi}^{3+}$-related luminescence centers in different materials, the detailed spectroscopic study and comparison of various $\mathrm{Bi}^{3+}$-doped compounds is needed.

In this work, $\mathrm{Bi}^{3+}$-doped lanthanide niobates are considered. The positions of their emission and excitation bands as well as the interpretation of experimental data reported in different papers are different. The low-temperature luminescence decay kinetics, which could allow to determine the origin, structure, and parameters of the corresponding RES, have not been studied. Therefore, we have carried out a detailed and systematic investigation of the $\mathrm{Bi}^{3+}$-related luminescence in $\mathrm{YNbO}_{4}: \mathrm{Bi}, \mathrm{LuNbO}_{4}: \mathrm{Bi}$, and $\mathrm{GdNbO}_{4}: \mathrm{Bi}$ powders with different $\mathrm{Bi}^{3+}$ contents by the methods of the steady-state and time-resolved luminescence spectroscopy at the temperatures from 4.2 to $500 \mathrm{~K}$. For comparison, the luminescence characteristics of the undoped niobates are studied as well.

In the emission spectra of $\mathrm{Bi}^{3+}$-doped niobates, two visible $\mathrm{Bi}^{3+}$-related emission bands are observed. The analysis of temperature dependences of their decay time allows us to make the conclusion on their exciton-like origin. The higher-energy VIS emission is suggested to arise from an exciton localized around a single $\mathrm{Bi}^{3+}$ ion. The intensity of the lower-energy VIS emission shows the superlinear dependence on the $\mathrm{Bi}^{3+}$ content. This allows to ascribe this emission to the radiative decay of an exciton localized around a dimer $\left\{\mathrm{Bi}^{3+}-\mathrm{Bi}^{3+}\right\}$ center. No UV emission is found which could arise from the radiative decay of the triplet RES of $\mathrm{Bi}^{3+}$. This fact as well as the exciton-like origin of the $\mathrm{Bi}^{3+}-$ related VIS emissions indicate that the triplet RES of both the $\mathrm{Bi}^{3+}$ ion and the $\left\{\mathrm{Bi}^{3+}-\mathrm{Bi}^{3+}\right\}$ dimer are located inside the conduction band of the investigated niobates. 


\title{
The development of spectral tomography using the crystal-analyzer scheme
}

\author{
Denis Zolotov', Victor Asadchikov, Alexey Buzmakov, \\ Marina Chukalina, ${ }^{1,2}$, Irina Dyachkova ${ }^{1}$, Anastasia Ingacheva ${ }^{2,3}$, \\ Dmitry Nikolaev²,3, Igor Schelokov4
}

\author{
1 FSRC "Crystallography and Photonics" RAS, Moscow, Russia \\ 2 Smart Engines Service LLC, Moscow, Russia \\ 3 Institute for Information Transmission Problems (Kharkevich Institute) RAS, Moscow, Russia \\ 4 Institute of Microelectronics Technology and High Purity Materials RAS, Chernogolovka, Russia
}

\section{https://doi.org/10.21175/rad.abstr.book.2021.15.8}

The present work demonstrated how a combination of well-known tools-a two-dimensional detector and a silicon $\mathrm{Si}(111)$ crystal analyzer- could improve the multimodality of X-ray tomographic imaging. The use of the crystal analyzer made it possible to separate two characteristic lines ( $\mathrm{K}_{\alpha}$ and $\mathrm{K}_{\beta}$ ) of the molybdenum anode from the polychromatic radiation (spectrum) of the conventional $\mathrm{X}$-ray tube. Thus, as a result of one measurement, three radiographic projections (images) can be simultaneously recorded in the transmitted and diffracted beams. The projection images of an object in a study at different wavelengths were separated in space and registered independently for further processing, which is of interest for the spectral tomography method [1].

Also, an algorithm for geometric correction of projection distortions of X-ray images obtained due to asymmetric diffraction from the chosen families of reflecting crystal planes is developed [2]. To check the correctness of the proposed algorithm, the method of three-channel image formation was used. In this approach, the final image is formed by writing to three color channels of three different images. I.e., the direct beam projection and two corrected diffraction projections are recorded in 3 channels - RGB. It allows determining directly (visually) how much the proposed procedure of compensation of geometrical distortions caused by the crystal analyzer mounted in the optical path is correct.

In addition, the use of a crystal analyzer as an energy separator of the primary beam together with polychromatic synchrotron X-ray radiation opens new opportunities for research to contrast almost any substance in the sample. In this case, the crystal can be tuned to obtain reflections before and after the absorption edge of a particular chemical element.

\section{References}

[1] Zolotov, D., Buzmakov, A., Grigoriev, M., \& Schelokov, I. (2020). Dual-energy crystal-analyzer scheme for spectral tomography. Journal of Applied Crystallography, 53(3).

[2] Buzmakov, A., Chukalina, M., Dyachkova, I., Ingacheva, A., Nikolaev, D., Zolotov, D., \& Schelokov, I. (2020). Enhanced Tomographic Sensing Multimodality with a Crystal Analyzer. Sensors, 20(23), 6970.

Acknowledgments: This research was supported by the Russian Foundation for Basic Research (Projects No.18-29-26036, No. 18-29-26020) as part of the application of crystal analyzer and the Ministry of Science and Higher Education within the State assignment FSRC "Crystallography and Photonics" RAS as part of the X-ray measurements. 


\title{
Energy transfer and luminescence properties of novel $\mathrm{Na}_{3.6} \mathrm{Y}_{1.8-\mathrm{x}}\left(\mathrm{PO}_{4}\right)_{3}: \mathrm{Dy}^{3+}$ phosphor
}

\author{
Nataliya Krutyak,2, Dmitry Spassky²,3, Dina Deyneko4, Vitali Nagirnyi² \\ 1 Physical Department, M.V. Lomonosov Moscow State University, Moscow, Russia \\ 2 Institute of Physics, University of Tartu, Tartu, Estonia \\ 3 Skobeltsyn Institute of Nuclear Physics, M.V. Lomonosov Moscow State University, Moscow, Russia \\ 4 Chemistry Department, M.V. Lomonosov Moscow State University, Moscow, Russia
}

\section{https://doi.org/10.21175/rad.abstr.book.2021.15.9}

Inorganic materials with intense luminescence are widely applied in lighting, security systems, medicine, high energy physics, etc. The requirements for their properties depend on the potential application and can differ significantly. However, for the overwhelming majority of phosphors a high light output and temperature stability of luminescence intensity are important. At present thermal quenching is still a main obstacle for phosphor applications in pcLEDs. Dy doped phosphors are attractive for such application because emission spectrum of $\mathrm{Dy}^{3^{+}}$consists of several narrow groups of lines, which covers visible spectral region. In recent years, an active search for novel materials with zero-thermal-quenching has been provided [1]. Here we present the results of the study of the luminescence properties of novel $\mathrm{Na}_{3.6} \mathrm{Y}_{1.8-\mathrm{x}}\left(\mathrm{PO}_{4}\right)_{3}: \mathrm{Dy}^{3+}$ phosphor.

A series of $\mathrm{Na}_{3.6} \mathrm{Y}_{1.8-x}\left(\mathrm{PO}_{4}\right)_{3}: x \mathrm{Dy}^{3+}(\mathrm{x}=0.01,0.05,0.1,0.3,0.4)$ phosphors were synthesized by a solid-state reaction. Characterization of crystal structure has been performed using XRD technique. Luminescence excitation and emission spectra under excitation in the UV region were measured using a laboratory setup based on a LOT-Oriel MS-257 spectrograph in the temperature range 77-500 K. Spectra in VUV region were obtained using specialized setup with Shamrock 303i (Andor Technology) monochromator. An optical vacuum cryostat allowed measurements at $\mathrm{T}=5-300 \mathrm{~K}$.

Data on unit cell parameters and space group were obtained using the XRD analysis. Under UV excitation $(350 \mathrm{~nm})$ the luminescence spectra consist of five groups of luminescence bands peaking at 487, 580, 672 and $760 \mathrm{~nm}$ corresponding to $4 \mathrm{f}-4 \mathrm{f}$ intraconfigural ${ }^{4} \mathrm{~F}_{9 / 2} \rightarrow{ }^{6} \mathrm{H}_{15 / 2},{ }^{4} \mathrm{~F}_{9 / 2} \rightarrow{ }^{6} \mathrm{H}_{13 / 2},{ }^{4} \mathrm{~F}_{9 / 2}$ $\rightarrow{ }^{6} \mathrm{H}_{11 / 2}$ and $4 \mathrm{~F}_{9 / 2} \rightarrow{ }^{6} \mathrm{H}_{9 / 2}$ transitions of $\mathrm{Dy}^{3+}$ ions, respectively. Maximal intensity has been registered for the sample with $\mathrm{x}=0.05$. Decrease of luminescence intensity has been observed for higher Dy concentrations due to concentration quenching caused by non-radiative energy transfer between nearby Dy ${ }^{3+}$ ions.

Beside emission from $\mathrm{Dy}^{3+}$ photoluminescence spectra under VUV excitation demonstrate an additional broad band at $340 \mathrm{~nm}$. The excitation spectrum for the broad band has a threshold at $\sim 7$ $\mathrm{eV}$, which presumably corresponds to the fundamental absorption edge of $\mathrm{Na}_{3.6} \mathrm{Y}_{1.8}\left(\mathrm{PO}_{4}\right)_{3}$ phosphor. Therefore, the band is attributed to intrinsic luminescence from the phosphate host. In the excitation spectrum of $\mathrm{Dy}^{3+}$ luminescence a number of narrow low-intensity bands at $3-5 \mathrm{eV}$ are observed, which represent the intracenter excitation of $\mathrm{Dy}^{3+}$. The excitation energy transfer from the host to activator ions is demonstrated.

\section{References}

[1] Y.H. Kim et al. // Nature Materials. - 2017. - Vol. 16. - 543-550.

Acknowledgments: The research was performed within the project MOBJD613. 


\title{
Influence of temperature on the optical and luminescent properties of scintillation crystals based on GAGG:Ce
}

\author{
Dmitry Spassky,2, Nataliya Krutyak3,2, Alisa Ukhanova3, \\ Fedor Fedyunin3, Vladimir Morozov4, Nina Kozlova5, \\ Evgeniia Zabelina5, Valentina Kasimova5, Oleg Buzanov6, Alexei Belik7
}

\author{
1 Skobeltsyn Institute of Nuclear Physics, M.V. Lomonosov Moscow State University, Moscow, Russia \\ 2 Institute of Physics, University of Tartu, Tartu, Estonia \\ 3 Physical Department, M.V. Lomonosov Moscow State University, Moscow, Russia \\ 4 Chemistry Department, M.V. Lomonosov Moscow State University, Moscow, Russia \\ 5 National University of Science and Technology (MISiS), Moscow, Russia \\ 6 Fomos-Materials, Moscow, Russia \\ 7 International Center for Materials Nanoarchitectonics (WPI-MANA), National Institute for Materials \\ Science, Tsukuba, Japan
}

\section{https://doi.org/10.21175/rad.abstr.book.2021.15.10}

Ce-doped $\mathrm{Gd}_{3}(\mathrm{Al}, \mathrm{Ga})_{5} \mathrm{O}_{12}$ (GAGG) garnets are well-known scintillating crystals for medical applications and high energy physics $[1,2]$. These crystals attract attention due to the combination of high density, chemical stability, very high light yield and reasonable energy resolution. Recently it was shown that GAGG:Ce crystals with partial substitution of $\mathrm{Al}$ and $\mathrm{Ga}$ cations by Sc (GASGG:Ce) demonstrate enhanced energy transfer from the host to $\mathrm{Ce}^{3+}$ emission centers [3]. The effect results in the increase of light output at low temperatures in GASGG:Ce while at ambient conditions the emission of GASGG:Ce is less intensive due to partial quenching. In the present study we show the influence of temperature on optical and luminescent properties of GAGG:Ce and GASGG:Ce.

Single crystals of GAGG:Ce and GASGG:Ce were grown by the Czochralski method at FomosMaterials (Moscow, Russia). Structural characterization of grown crystals was performed by XRD technique using a large Debye-Scherrer camera at the BL15XU beamline of SPring-8 synchrotron radiation source. Structural data allowed to specify the crystal compositions and site occupation by $\mathrm{Gd}, \mathrm{Al}, \mathrm{Ga}$ and Sc cations. Absorption spectra were measured using an Agilent Technologies Cary5000 spectrophotometer at $300 \mathrm{~K}$ and PerkinElmer Lambda 950 spectrophotometer in temperature region 80-500 K. Luminescence excitation and emission spectra under excitation in the UV region were measured using the laboratory setup based on LOT-Oriel MS257 spectrograph.

The influence of temperature on the absorption, luminescence and excitation spectra was studied. Pronounced peaks related to intracenter electron transitions between ground $4 \mathrm{f}$ and excited $5 \mathrm{~d}(1)$ and $5 \mathrm{~d}(2) \mathrm{Ce}^{3+}$ states were observed in these spectra in the crystal's transparency region. Parameters of the absorption and emission bands related to $\mathrm{Ce}^{3+}$ and their dependence on the temperature were obtained. A shift of the thermal quenching threshold of luminescence to the low-temperature region with the introduction of $\mathrm{Sc}^{3+}$ into the crystal was established. This effect is connected with the decrease of the energy gap between the lowest excited cerium level and conduction band bottom. An attempt to calculate the parameters of configurational coordinate curves related to $\mathrm{Ce}^{3+}$ emission centers in GAGG:Ce and GASGG:Ce was performed.

\author{
References \\ [1] Lecoq P. // Nucl. Instr. and Meth. in Phys. Res. A 2016. V. 809. P. 130. \\ [2] Korzhik M. et al. // Cryst. Res. Technol. 2019. V. 54. P. 1800172. \\ [3] Spassky D. et al. // CrystEngComm. 2020. V. 22. P. 2621.
}

Acknowledgments: This research was supported by Russian Foundation for Basic Research №20-02-00688. 


\title{
The electric and magnetic properties study of nanocrystalline triple perovskite $\mathrm{Sr}_{3} \mathrm{CO}_{2} \mathrm{WO}_{9}$ prepared by the modified sol-gel route
}

\author{
Jelena Bijelic'1, Akos Kukovecz², Zvonko Jaglicic3,4, \\ Sugato Hajra5, Manisha Sahu5, Igor Djerdj1
}

\author{
1 Department of Chemistry, Josip Juraj Strossmayer University of Osijek, Osijek, Croatia \\ 2 University of Szeged, Interdisciplinary Excellence Centre, Department of Applied and Environmental \\ Chemistry, Szeged, Hungary \\ 3 University of Ljubljana, Faculty of Civil and Geodetic Engineering, Ljubljana, Slovenia \\ 4 University of Ljubljana, Institute of Mathematics and Physics \& Mechanics, Ljubljana, Slovenia \\ 5 Department of Robotics Engineering, Daegu Gyeongbuk Institute of Science \& Technology (DGIST), Daegu, \\ South Korea
}

\section{https://doi.org/10.21175/rad.abstr.book.2021.15.11}

Polar perovskite materials have attracted extensive attention due to the coexistence of magnetic ground state and semiconductor band gap within the same material. For the first time triple perovskite $\mathrm{Sr}_{3} \mathrm{Co}_{2} \mathrm{WO}_{9}$ has been synthesized in the nanocrystalline form with an average crystallite size of $23 \mathrm{~nm}$ using a modified aqueous citrate sol-gel method. The crystal structure of $\mathrm{Sr}_{3} \mathrm{Co}_{2} \mathrm{WO}_{9}$ is cubic at the room temperature, space group $\mathrm{Fm}-3 \mathrm{~m}$ with with lattice parameter $a=7.9073(6) \AA$. The formation of $\mathrm{Sr}_{3} \mathrm{Co}_{2} \mathrm{WO}_{9}$ was studied by cyclic voltammetry, UV/Vis spectroscopy and in situ X-ray diffraction method subsequently analysed by the Rietveld analysis. The detected hysteresis loops reveal ferrimagnetic ordering with Curie temperature of $144 \mathrm{~K}$. The measured effective magnetic moment of $3 \mathrm{mB}$ is close to the expected value for rarely observed intermediate spin $S=1$ but can also be explained as a combination of HS (40 \%) $\mathrm{Co}^{3+}$ and LS (60 \%). The compound exhibits the semiconducting properties with the optical band gaps equal to $3.52 \mathrm{eV}$ (indirect) and $3.76 \mathrm{eV}$ (direct), respectively, calculated from UV/Vis absorption spectrum using the Tauc method. Semiconducting nature was also confirmed by $\mathrm{AC}$ conductivity measurements, which is between $10^{-5}$ and $10^{-4} \Omega^{-1} \mathrm{~cm}^{-1}$. The frequency dependent dielectric constant was explained by employing the MaxwellWagner model. The frequency dependent AC conductivity follows the universal Jonscher's power law. This material could be a good candidate for implementation as a constituent for devices where its semiconducting properties would be spin controlled. 


\title{
Modified approach for evaluation of standard single-electron reduction potential of nitroaromatic compounds in aqueous medium
}

\author{
Jelena Tamuliene', Narimantas Čènas²
}

\author{
1 Vilnius University, Institute of Theoretical Physics and Astronomy, Vilnius, Lithuania \\ 2 Vilnius University, Institute of Biochemistry, Vilnius, Lithuania
}

\section{https://doi.org/10.21175/rad.abstr.book.2021.15.12}

The cytotoxic/therapeutic activity of nitroaromatic compounds $\left(\mathrm{ArNO}_{2}\right)$ is frequently exerted through their free radical formation and subsequent oxidative stress. In this case, the activity of $\mathrm{ArNO}_{2}$ increases with their single-electron reduction potential (potential of $\mathrm{ArNO}_{2} / \mathrm{ArNO}_{2}{ }^{-} \cdot$ redox couple) in aqueous medium $\left(E_{7}^{1}\right)$ ([1], and refs. therein). Typically, the $E^{1}{ }_{7}$ values of $\mathrm{ArNO}_{2}$ are determined by pulse-radiolysis, which requires sophisticated equipment and may be complicated by insufficient compound solubility or side reactions of free radicals. Several currently developed theoretical approaches for prediction of single-electron reduction potentials, e.g., the Born-Haber cycle, are confined to an implicit solvent model or nonaqueous systems [2,3]. However, they lack sufficient accuracy, and their results may not be easily extrapolated to aqueous media. One also has to take into account the limitation of a polarizable continuum model (PCM), because the electron addition to the acceptor molecule could lead to the formation of unstable van der Walls or dipoledipole interaction-type adducts with the solvent molecules. To overcome the limitations of PCM, we performed the calculations of $E^{1}{ }_{7}$ of 12 mono- and dinitrobenzenes in aqueous solvent with the inclusion of additional water molecules by using the Born-Haber cycle protocol. We found that the calculated $E^{1_{7}}$ values depend on the number of additional water molecules. The proper selection of their number depends on the calculated value of dipole moment projections with respect to the standard orientation of molecule in Cartesian coordinates. If the number of additional water molecules is equal to the number of projections $(n=1-3)$, then the calculated $E^{1}{ }_{7}$ values are sufficiently close to the experimentally determined ones $\left(r^{2}=0.86\right)$.

\section{References}

[1] Čènas N, Nemeikaitè-Čėnienė A, Sergedienė E, Nivinskas H, Anusevičius Ž, Šarlauskas J. Biochim. Biophys. Acta 1528 (2001), pp. 31-38.

[2] Roy LE, Jakubikova E, Batista ER. Associate Directorate for Theory, Simulation, and Computation (ADTSC) LA-UR-09-01756, www.lanl.gov/orgs/adtsc/publications.php

[3] Sviatenko LM, Gorb L, Hill CF, Leszczynski J. J. Comput. Chem. 34 (2013), pp. 1094-1100, https://doi.org/10.1002/jcc.23228

Acknowledgments: This work is supported by the European Social Fund (Measure No. 09.33-LMT-K-712, grant No. DOTSUT-34/09.3.3.-LMT-K712-01-0058/LSS-600000-58). 


\title{
Thermal annealing of structural defects in fast-neutron-irradiated $\mathrm{Al}_{2} \mathrm{O}_{3}$ single crystals
}

\author{
Evgeni Shablonin'1, Anatoli Popovi,2, Gatis Prieditis', \\ Evgeni Vasil'chenko',2, Aleksandr Lushchik'
}

\author{
1 Institute of Physics, University of Tartu, Tartu, Estonia \\ 2 Institute of Solid State Physics, University of Latvia, Riga, Latvia
}

\section{https://doi.org/10.21175/rad.abstr.book.2021.15.13}

Sufficient radiation resistance against prolonged irradiation is an important requirement seriously limiting the range of materials suitable for application in the harsh environment of future fusion reactors. Due to high tolerance to neutron irradiation as well as good optical and mechanical properties, aluminium oxide in the form of single crystals or transparent polycrystalline ceramics is a promising candidate for using as optical window material. The detailed understanding of the processes of primary lattice defect formation, aggregation and thermal annealing of radiationinduced damage is necessary to improve the radiation resistance of material.

This paper presents a study of the process of annealing radiation damage in $\alpha-\mathrm{Al}_{2} \mathrm{O}_{3}$ single crystals induced by fast fission neutrons (energy $>0.1 \mathrm{MeV}$ at $\sim 60{ }^{\circ} \mathrm{C}$, fluence $\sim 6.9 \times 10^{18} \mathrm{n} / \mathrm{cm}^{2}$ ). The damage was analyzed via radiation-induced optical absorption (RIOA) in the $2-5.6 \mathrm{eV}$ region containing several absorption bands related to oxygen vacancy dimers ( $F_{2}$-type centers). The annealing of RIOA at $295-1100 \mathrm{~K}$ was registered in a stepwise regime: an irradiated sample was heated in highly pure argon atmosphere to a certain temperature $T_{\mathrm{i}}$, kept at $T_{\mathrm{i}}$ for $10 \mathrm{~min}$ and rapidly cooled down to $295 \mathrm{~K}$, at which all spectra were measured. For precise analysis of individual defectrelated bands, decomposition of the RIOA spectra into elementary Gaussians was performed. Additionally, spectrally integrated (1.9-4.1 eV range) thermostimulated luminescence with heating rate $2 \mathrm{~K} / \mathrm{s}$ was registered for temperatures up to $\sim 850 \mathrm{~K}$.

The Gaussian components of the RIOA with the maxima at 4.08, 3.45 and $2.75 \mathrm{eV}$ have been considered as a measure of the $F_{2}, F_{2}{ }^{+}$and $F_{2}{ }^{2+}$ centers, respectively. Unlike $F$ and $F^{+}$centers (an oxygen vacancy with two/one trapped electrons), the concentration of which continuously decreases at the sample heating up to $1100 \mathrm{~K}$, the concentration of dimer defects with different charge states passes the increasing stages above $500 \mathrm{~K}$ starting from the $\mathrm{F}_{2}{ }^{2+}$ centers (i.e., two spatially close $F^{+}$centers). The tentative mechanisms of such rise of the $F_{2}{ }^{2+}$ center concentration as well as the subsequent transformation/rise of dimer centers, ${F_{2}}^{2+} \rightarrow F_{2}{ }^{+} \rightarrow F_{2}$ at 650-800 K are considered. The possible sources of carriers (electrons) needed for the recharging of dimer centers are also analyzed on the basis of thermally stimulated luminescence measurements.

\section{References}

[1] E. Shablonin, A.I. Popov, G. Prieditis, E. Vasil'chenko, A. Lushchik, Thermal annealing and transformation of dimer $F$ centers in neutron-irradiated $\mathrm{Al}_{2} \mathrm{O}_{3}$ single crystals, J. Nucl. Mater. 543, 152600 (2021) 


\title{
Influence of parameters of the pulsating current (PC) regime on morphological, structural and hardness characteristics of copper coatings electrodeposited on $\mathrm{Si}(111)$
}

\author{
Ivana Mladenović ${ }^{1}$ Nebojša Nikolić1, \\ Jelena Lamovec ${ }^{2}$, Dana Vasiljević Radović ${ }^{1}$, Vesna Radojević3
}

\author{
1 University of Belgrade, Institute of Chemistry, Technology and Metallurgy, Belgrade, Serbia \\ 2 University of Criminal Investigation and Police Studies, Belgrade, Serbia \\ 3 University of Belgrade, Faculty of Technology and Metallurgy, Belgrade, Serbia
}

\section{https://doi.org/10.21175/rad.abstr.book.2021.15.14}

Electrodeposition of $\mathrm{Cu}$ was performed on $\mathrm{Si}(111)$ by the pulsating current (PC) regime in the range of the average current densities $(j$ av $)$ between 15 and $70 \mathrm{~mA} \mathrm{~cm}^{-2}$. The selected values of the average current densities were attained by varying either pause duration $\left(t_{\mathrm{p}}: 28.3,15,7.5\right.$ and $5 \mathrm{~ms}$, i.e. $j_{\mathrm{av}}$ : $15,25,40$ and $50 \mathrm{~mA} \mathrm{~cm}{ }^{-2}$ for the constant values of deposition pulse of $5 \mathrm{~ms}$ and current density amplitude $\left(j_{\mathrm{A}}\right)$ of $\left.100 \mathrm{~mA} \mathrm{~cm}^{-2}\right)$ or amplitude of the current density $\left(j_{\mathrm{A}}: 120\right.$ and $140 \mathrm{~mA} \mathrm{~cm}^{-2}$, i.e. $j_{\text {av }}$ : 60 and $70 \mathrm{~mA} \mathrm{~cm}^{-2}$ for the constant values of deposition pulse of $5 \mathrm{~ms}$ and pause duration of $5 \mathrm{~ms}$ ). Morphological and structural characteristics of the obtained coatings were examined by scanning electron microscope (SEM), atomic force microscope (AFM) and X-ray diffraction (XRD), respectively. With increasing the average current density, morphologes of the coatings changed from those with large and well defined crystal grains obtained at $j_{\mathrm{av}}$ of $15 \mathrm{~mA} \mathrm{~cm}^{-2}$ (the dominant effect of activation control) to fine-grained obtained at $j_{\text {av }}$ of $50 \mathrm{~mA} \mathrm{~cm}^{-2}$ (the mixed activation-diffusion control) and those with globules when diffusion becomes a dominant process $\left(j_{\mathrm{av}}=70 \mathrm{~mA} \mathrm{~cm}-2\right)$. The minimum roughness showed the $\mathrm{Cu}$ coating obtained at $j_{\text {av }}$ of $50 \mathrm{~mA} \mathrm{~cm}{ }^{-2}$. Simultaneously, crystal structure changed from the strong (220) to the strong (111) preferred orientation with increasing average current density. The change of surface morphology was discussed by the effect of applied parameters of the PC regime on the type of electrodeposition control, while change in crystal orientation of produced coatings was explained by various rates of growth on various crystal planes. Hardness analysis of the produced coatings was performed by application of the Chicot-Lesage (C-L) composite hardness model. By application of this model, the relative indentation depth (RID; where $\mathrm{RID}=h / d ; h$ is an indentation depth, and $d$ is a thickness of coating) of 0.14 was established as the limiting value separating the area of the absolute hardness of the Cu coatings (RID < 0.14) from the area in which application of the C-L model is necessary for a determination of the absolute hardness of coatings (RID > 0.14). For RID < 0.14, the measured composite hardness corresponded to the absolute hardness of the coating. 


\title{
Physicochemical characterisation of commonly used organic UV filters from cosmetic products and development of their detection by liquid chromatography UV-diode array
}

\author{
Snežana Papović, Milan Vraneš, Jovana Panić, \\ Aleksandar Tot, Nikolet Baganj, Sanja Mutić, Jasmina Anojčić
}

Faculty of Sciences, Department for Chemistry, Biochemistry and Environmental Protection, Novi Sad, Serbia

https://doi.org/10.21175/rad.abstr.book.2021.15.15

Chemical UV filters are widely used components of sunscreens and other cosmetic and personal care products to protect skin against UV radiation [1,2]. With regard to adverse health effects of organic UV filters, there are concerns not only about contact allergy but also systemic effects like endocrine disruption [3]. The accurate determination of an organic UV filter contaminant's physicochemical properties is essential for their releasement into the environment, and consequently their environmental impact and fate. Physicochemical properties are controlling the valid environmental models and assessments. Conversely, the predictive/interpretive value of environmental studies can be seriously compromised if the physicochemical data upon which they rely are of questionable or unknown quality. Information regarding experimentally obtained physicochemical properties of UV-filter are still very scarce and is not possible to develop adequate risk assessments. The present work focuses on frequently used UV filters (avobenzone, benzophenone-3, octocrylene and octinoxate) and evaluating the resulting thermal behaviour and structural changes. Experimental measurements of density, viscosity and electrical conductivity were performed to get insight into their transport properties in wide temperature range. Differential scanning calorimetry (DSC) and thermogravimetric (TG) analysis were used to determine their melting point and thermal behaviour. Physicochemical properties of UV-filters are important to understand which analytical methodologies are appropriate to their determination in the different environmental compartments.

\section{References}

[1] W. Uter, M. Goncalo, K. Yazar, E.M. Kratz, G. Mildau, C. Liden Contact Dermatitis, 71 (2014), 162-169.

[2] E. Manova, N. von Goetz, U. Hauri, C. Bogdal, K. Hungerbuhler Int. J. Hyg. Environ. Health, 216 (2013), 508-514.

[3] E. Manova, N. von Goetz, K. Hungerbuhler Brit. J. Dermatol., 171 (2014), 1368-1374.

Acknowledgments: This work was financed by the Provincial Secretariat for Higher Education and Scientific Research, grant number: 142-451-3167/2020-01. 


\title{
Preparation and characterization of molybdenum solid targets for high current cyclotron production of medical ${ }^{99 \mathrm{~m}} \mathrm{Tc}$ radionuclides
}

\author{
Nicoleta Florea, Mihai Badea, Cristina Burducea, \\ Liviu-Stefan Craciun, Tiberiu-Relu Esanu, Raluca-Maria Marginean, \\ Paul Mereuta, Constantin Mihai, Calin Mircea Rusu, Andreea-Elena Serban
}

Horia Hulubei - National Institute for Physics and Nuclear Engineering (IFIN-HH), Magurele, Romania

\section{https://doi.org/10.21175/rad.abstr.book.2021.15.16}

${ }^{99 \mathrm{~m}} \mathrm{Tc}$ is widely used in modern nuclear medicine and is currently obtained from the radioactive

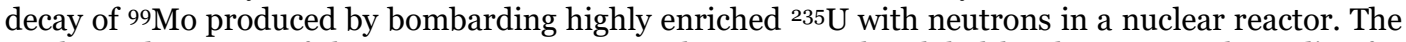
unplanned outages of the reactors cause ${ }^{99}$ Mo shortages at the global level, so research studies for alternative methods to produce ${ }^{99 \mathrm{~m} T c}$ are ongoing all over the world. The cyclotron approach via ${ }^{100} \mathrm{Mo}(\mathrm{p}, 2 \mathrm{n}){ }^{99 \mathrm{~m}} \mathrm{Tc}$ nuclear reaction was found to be the most promising one. The key challenge for the large-scale cyclotron production of $99 \mathrm{mTc}$ consists in the development of high-density ${ }^{100 \mathrm{Mo}}$ targets that are: able to support multi-hour high current cyclotron irradiation, with high density, adhesion to baseplate, and high thickness, without material losses. An "ideal" technique, to fulfill all the requirements for the target, does not exist [1-5]. The choice of target preparation technique is always a compromise between fulfilling the application's particular requirements and the cost of implementation, so extended research is needed.

In this context, the main objective of this study consists in research for the development and testing of robust molybdenum solid targets to be further used for high current cyclotron production of ${ }^{99 m} \mathrm{Tc}$ radionuclide using the ${ }^{100} \mathrm{Mo}(\mathrm{p}, 2 \mathrm{n})$ nuclear reaction. The cyclotron target should be highly bonded to a metallic baseplate and be able to sustain high cyclotron currents. For this purpose, a new approach to obtain a high-density molybdenum target using pressing-heating process was developed by making use of the scanning capability of the electron-gun part of the high vacuum deposition system, allowing the complete surface heating without inducing deformations, in high vacuum (without impurities), without the evaporation process (no material losses).

The surface morphology, roughness, hardness, impurities, adhesion performances were established by using Atomic Force Microscopy (AFM), Scanning Electron Microscopy (SEM) and Energy-Dispersive X-Ray Spectroscopy (EDX) techniques. The prepared targets were also successfully irradiated with protons at high-current cyclotron.

\section{References}

[1] H. Skliarova, S. Cisternino, G. Cicoria, M. Marengo, V. Palmieri, Innovative Target for Production of Technetium-99m by Biomedical Cyclotron, Molecules, 24, pp. 1-22, 2019.

[2] IAEA Cyclotron Based Production of Technetium-99m; IAEA: Vienna, Austria, 2017, ISBN 978-92-0-102916-4.

[3] IAEA, Accelerator-based Alternatives to Non-HEU production of 99Mo/99mTc. In Proceedings of the 3rd Research Coordination Meeting of IAEA, Vienna, Austria, June 22-26, 2015

[4] P. Gould, Medical isotope shortage reaches crisis level, Nature, 46o, pp. 312-313, 2009.

[5] P. Schaffer, F. Bénard, A. Bernstein, K. Buckley, A. Celler, N. Cockburn, J. Corsaut, M. Dodd, C. Economou, T. Eriksson, Direct Production of $99 \mathrm{~m}$ Tc via $100 \mathrm{Mo}(\mathrm{p}, 2 \mathrm{2n})$ on Small Medical Cyclotrons. Phys. Procedia., 66, pp. 383-395, 2015.

Acknowledgments: This work was supported by a grant of the Romanian Ministry of Education and Research, CNCS - UEFISCDI, project number PN-III-P1-1.1-TE-2019-0337, within PNCDI III. 


\section{The clinical case of stem cell therapies for the treatment of radiation-induced ulcer}

\section{Svetlana Gramatiuk, Anna Kurbatova, Yulia Ivanova}

Ukraine Association of Biobank, Kharkiv, Ukraine

https://doi.org/10.21175/rad.abstr.book.2021.15.17

Background and aims. While cancer therapies increasingly achieve cure and extend survival, they often result in chronic side effects in patients. Acute reactions impacting normal tissue injury early after treatment are related to oxidative stress and inflammation that alter the microenvironment which includes sensitive stem cell niches. The aim of the study is to effect of stem cell transplantation on the course of the wound process in conditions of radiation-induced ulcer.

Materials and methods. The study was based on Patient G., burned in 1960, with a 1-year history of of radiotherapy for treatment of basal cell carcinoma. The estimated tolerance doses for are which was caused by a therapeutic radiation at fractionate 50 Gy at 1 years previously. In our protocol we used autologous mesenchymal stem cells, which were isolated from the patient's peripheral blood by magnetic separation using an AutoMACS automatic system, USA.

Results. Clinical case shows that in patients with of radiation-induced ulcer, it is advisable to use step-by-step treatment: first step - restoration of the arterial blood flow in the extremity, second step - scleroobliteration of the incompetent perforator veins and the third stage - transplantation of mesenchymal stem cells into muscles along the perimeter of radiation-induced ulcer.

Conclusions. This tactic contributes to the long-lasting and sustained positive clinical effect and rapid healing of radiation-induced ulcer. 


\title{
Influence of irradiation conditions on optical photoresponse of chalcogenide glass thin films
}

\section{Andriy Kovalskiy', Roman Holovchak, Yaroslav Shpotyuk², Jozef Cebulski²}

\author{
1 Austin Peay State University, Clarksville, United States \\ 2 University of Rzeszow, Rzeszow, Poland
}

\section{https://doi.org/10.21175/rad.abstr.book.2021.15.18}

Due to their unique optical and electrical properties, chalcogenide glass thin films (ChGF) are exceptionally promising materials for IR photonics, non-linear optics, all-optical switching, micro(nano)-lithography, and phase-change memory devices. ChGF are suitable for most of these applications because of the extraordinary photo-induced effects caused by modification of electronic and/or atomic structure under irradiation. However, some of applications such as non-linear optical waveguides require low optical dispersion at high light intensities in the infrared. In this work, both metastable and transient photoinduced changes of optical transmission at the fundamental absorption edge region are compared for thermally evaporated and spin coated chalcogenide glass thin films. In-situ kinetics of metastable photobleaching and transient photodarkening as well as following light-off relaxations in As- and Ge-based thin films were studied and fitted with the stretched exponential function. Dependences of the kinetic parameters on conditions of irradiations are analyzed. It was found that for band gap light the photoresponse is much weaker for the spincoated films. However, high energy light radiation causes significant changes of near-surface structure of both types of thin films, independently on the preparation method. Mechanism of photoinduced structural relaxation is proposed based on Raman microscopy data. 


\title{
The effect of UV-irradiation on the thermal stability of modified urea-formaldehyde resins with thermally activated montmorillonite
}

\author{
Vojislav Jovanović1, Suzana Samaržija-Jovanovié1, \\ Branka Petković ${ }^{1}$, Tijana Jovanović ${ }^{2}$, Gordana Marković3, \\ Milena Marinović-Cincovićc ${ }^{4}$, Vesna Teofilović 5
}

\author{
1 University in Priština - Kosovska Mitrovica, Faculty of Sciences, Kosovska Mitrovica, Serbia \\ 2 University of Niš, Faculty of Science, Niš, Serbia \\ 3 Tigar A.D., Pirot, Serbia \\ 4 University of Belgrade, Institute of Nuclear Science Vinča, Belgrade, Serbia \\ 5 University of Novi Sad, Faculty of Technology, Novi Sad, Serbia
}

\section{https://doi.org/10.21175/rad.abstr.book.2021.15.19}

The montmorillonite as a 2:1 smectite type clay has two tetrahedral sheets of silica sandwiching the alumina octahedral sheet. The particles of this mineral are plate-shaped with the thickness of $0.96 \mathrm{~nm}$ and an average diameter about $1 \mu \mathrm{m}$. This type of clay (K10) is using to improve the characteristics of different materials in the fields of catalysis, food additives, polymers, sorbents, etc. In this study thermally activated montmorillonite (DK10) was used because the thermal treatment alters its properties. The degree of activation was determined using specific surface measurement (Sear's method). The specific surface area (SSA) of K1O was $119 \mathrm{~m}^{2} / \mathrm{g}$ but after the thermal treatmant it was $317.4 \mathrm{~m}^{2} / \mathrm{g}$. The effect of UV-irradiation on the thermal stability of a urea-formaldehide resin (UF) nanocomposites based on DTK10 was assessed. For this purpose UF nanocomposites were synthesized and irradiated using UV light at two wavelengths l (254 and $366 \mathrm{~nm}$ ). Characterization of nanocomposites with modified and not modified clay was performed using XRD, FTIR, and TG/DTA analysis. The peaks at $2 \mathrm{q}$ values of $26.74,26.6,26.54,26.6$ originate from quartz in sample DTK10, non-irradiated UF/DTK10 and UV-irradiated sample at $\mathrm{l}=254 \mathrm{~nm}$ and $\mathrm{l}=366 \mathrm{~nm}$, respectively. From the IR spectra it was assessed that intensity of the carboxyl group at $1630 \mathrm{~cm}^{-1}$ decreased with UVirradiation in modified nanocomposite. The aliphatic stretch band about $2956 \mathrm{~cm}^{-1}$ and $-\mathrm{C}-\mathrm{O}$ band at $1130 \mathrm{~cm}^{-1}$ also decreases due to the formation of hydrogen bonds with the hydroxyl groups on the DTK10 surface. Based on thermogravimetric measurements it was estimated that before and after UV-irradiation modified UF/DTK10 nanocomposites have almost identical temperature intervals in which degradation processes are occurred. The total mass loss for non-irradiated and irradiated UF/DTK10 was 60.18, 55.26 and $58.6 \%$, respectively. 


\title{
Compact universal digital X-ray diagnostic system
}

\section{Yuri Kovalenko', Sergii Miroshnichenko², Andrii Nevhasymyy3}

\author{
1 P.L. Shupyk National Medical Academy of Postgraduate Education, Kyiv, Ukraine \\ 2 National Aviation University, Kyiv, Ukraine \\ 3 Teleoptic PRC Ltd, Kyiv, Ukraine
}

\section{https://doi.org/10.21175/rad.abstr.book.2021.16.1}

Purpose of work. In medicine, X-ray remote control tables are widely used, which are universal $\mathrm{X}$-ray diagnostic systems for the performing of radiographic, fluoroscopic and tomographic studies. However, they are bulky and expensive.

In recent years, cone beam tomographs have become increasingly used in clinical practice, which can expand the diagnostic capabilities of X-ray diagnostics. However, these are specialized X-ray machine on which radiography and fluoroscopy cannot be performed.

The aim of the work was to create a compact universal X-ray diagnostic system with advanced diagnostic capabilities, on which you can perform radiography, fluoroscopy and cone beam tomography.

Materials and methods. The pilot unit included high-frequency X-ray generator with pulsefluoro mode, a universal x-ray stand with a fixed distance "source-detector" of $100 \mathrm{~cm}$ and rotation "source-detector" console with motor control, an x-ray tube with a rotating anode, a table of a patient that can be rotated 90 degrees, X-ray tube with rotating anode, dynamic digital receptor (DDR) with $43 \times 36 \mathrm{~cm}$ field of view and 3700x2400 matrix, as well as a workstation with back projection method image reconstruction software. X-ray test target was used to assess the spatial resolution of the experimental assembly. Several animals were examined.

Results. In radiography mode it was received images of examined subjects with $360 \times 432 \mathrm{~mm}$ field of view and with pixel size 117x150 micron. In fluoroscopy mode with image size 216x160 mm pixel size was $350 \times 300$ micron. Image frequency varied within 6 to 20 per second. In tomography mode the console 'source-detector' rotated during 30 seconds around the examined subject by 210 degrees, thereafter in four minutes $3 \mathrm{D}$ images of examined subjects were recovered using ultrafast CT volume recovery algorithms. Tomography zone diameter made up to $300 \mathrm{~mm}$. Voxel size didn't exceed 500 microns.

Conclusions. The pilot unit that was investigated in this work allows performing $\mathrm{X}$-ray, fluoroscopy and tomography studies and can be used as universal digital X-ray diagnostic system. Currently, it is already used in veterinary clinics. After conducting clinical trials, it may be used in medicine. 


\section{Neutron source VITA for Boron Neutron Capture Therapy and other applications}

\section{Sergey Taskaevi,2}

1 Budker Institute of Nuclear Physics, Novosibirsk, Russia

2 Novosibirsk State University, Novosibirsk, Russia

\section{https://doi.org/10.21175/rad.abstr.book.2021.16.2}

An accelerator based neutron source has been proposed and developed for boron neutron capture therapy (BNCT) and other applications at the Budker Institute of Nuclear Physics. The neutron source comprises a Vacuum Insulated Tandem Accelerator, a lithium target, and a neutron beam shaping assembly. The proton beam energy can be varied within a range of $0.6 \mathrm{MeV}-2.3 \mathrm{MeV}$ keeping a high-energy stability $0.1 \%$. The beam current can also be varied in a wide range from $0.3 \mathrm{~mA}$ to $10 \mathrm{~mA}$ with high current stability (0.4\%). The accelerator is also capable of generating a deuteron beam. The neutron source served as a prototype for a facility created for Neuboron Xiamen BNCT Center (Xiamen, Fujian, China), one of the first six BNCT clinics in the world.

A proton beam was used for in situ observation of blisters growth in metals and for study their effect on the neutron yield from the lithium layer.

In the device, neutron flux with yield up to $210^{12} \mathrm{~s}^{-1}$ is generated as a result of the ${ }^{7} \mathrm{Li}(\mathrm{p}, \mathrm{n})^{7} \mathrm{Be}$ reaction. A beam shaping assemblies with magnesium fluoride moderator or a plexiglas moderator are applied to convert this flux into a beam of epithermal neutrons with characteristics suitable for clinical testing of BNCT. A beam of monoenergetic neutrons with an energy variable from $10 \mathrm{keV}$ to $100 \mathrm{keV}$ is obtained with kinematic collimation. This beam is suitable for calibrating a dark matter detector and is planned to be used for boron imaging by prompt gamma-ray spectroscopy. Fast neutron flux is obtained as a result of the $7 \mathrm{Li}(\mathrm{d}, \mathrm{n})$ reaction. This beam is used in studies of activation of $\mathrm{B}_{4} \mathrm{C}$ ceramics and SS 316L-ITER grade steel by fast neutrons. It is planned to use the fast neutron flux for radiation tests of fibers of the laser calorimeter calibration system of the CMS electromagnetic detector developed for the High-Luminosity Large Hadron Collider in CERN.

A flux of $478 \mathrm{keV}$ photons is produced in the ${ }^{7} \mathrm{Li}\left(\mathrm{p}, \mathrm{p}\right.$ 'g) ${ }^{7} \mathrm{Li}$ reaction. This beam is used for in situ measuring the thickness of a lithium layer and for measurement of the high-LET particles dose.

It can be concluded that the device has a wide range of applications including BNCT, material science, characterization of neutron detectors, detailed study of the neutronless fusion ${ }^{11} \mathrm{~B}(\mathrm{p}, \mathrm{a}) \mathrm{aa}$ reaction, etc.

Acknowledgments: The study was supported by the grant from the Russian Science Foundation (project No. 19-72-30005). 


\title{
Development and RBS study of ultra-sensitive high-speed perovskite film X-ray detectors for radiation technologies
}

\author{
T.Yu Zelenyak, V.A. Kinev², P.P. Gladyshev \\ T.N. Vershinina1, A.C. Doroshkevich1, P.S. Dzhumaev3, \\ R.Sh. Isayev ${ }^{1,3,4}$, M. Balasoiu' ${ }^{1,5}$, A. Stanculescu 6 \\ 1 Joint Institute for Nuclear Research, Dubna, Moscow Region, Russia \\ 2 State University “Dubna”, Dubna, Moscow Region, Russia \\ 3 National Research Nuclear University, Moscow, Russia \\ 4 National Nuclear Research Center, Baku, Azerbaijan \\ 5 Horia Hulubei National Institute for R\&D in Physics and Nuclear Engineering (IFIN-HH), Bucharest \\ Romania \\ 6 National Institute for Materials Physics (NIMP), Măgurele, Romania
}

\section{https://doi.org/10.21175/rad.abstr.book.2021.16.3}

Currently, X-ray detectors have a place in medical radiography and medical diagnostics. Array semiconductor detectors are already being developed to replace conventional X-ray films [1]. Perovskite $\mathrm{CH}_{3} \mathrm{NH}_{3} \mathrm{PbI}_{3}$ has a successful combination of properties - a fast photoresponse and a large $\mathrm{X}$-ray photon absorption cross section due to the presence of heavy lead and iodine atoms in its composition. superior charge carrier lifetime, tunable bandgap and low temperature fabrication process. In particular, the possibility of creating solid-state $\mathrm{X}$-ray detectors using single-crystal perovskites $\mathrm{CH}_{3} \mathrm{NH}_{3} \mathrm{PbI}_{3}, \mathrm{CH}\left(\mathrm{NH}_{2}\right)_{2} \mathrm{PbI}_{3}$, treated with iodine $\mathrm{CH}_{3} \mathrm{NH}_{3} \mathrm{PbBr}_{3}$, was demonstrated [2].

In this work, based on perovskite $\mathrm{CH}_{3} \mathrm{NH}_{3} \mathrm{PbI}_{3}$, homogeneous functional structures of the glass/ITO/TiO $/ \mathrm{CH}_{3} \mathrm{NH}_{3} \mathrm{PbI}_{3}$ type were obtained for solid-state X-ray detectors in the form of thin films; their structure and elemental composition were studied by XRD, XRD, RBS, and EDS, respectively.

In view of the high spatial homogeneity of the films obtained, the latter can be considered as elements for an X-ray detector.

\author{
References \\ [1] J. P. Moy, Nucl. Instrum. Methods Phys. Res., 442 (2000) 26. \\ [2] S. Yakunin, et al., Nat. Photon., 9 (2015) 444.
}

Acknowledgments: The study was performed in the scope of the Poland- JINR and RO-JINR Projects within the framework of themes FLNP JINR 03-4-1128-2017/2022. 


\title{
Therapy of neuroendocrine tumors with ${ }^{90} \mathrm{Y}$ DOTA-TATE
}

\author{
Vera Artiko \\ Center for Nuclear Medicine with PET, University Clinical Center of Serbia, Faculty of Medicine University of \\ Belgrade, Belgrade, Serbia
}

\section{https://doi.org/10.21175/rad.abstr.book.2021.17.1}

Aim. The aim of this work is presentation of the preliminary results of the therapy of NETs with 90Y DOTA TATE.

Patients and methods. The therapy was applied in 15 patients with various NETs, after discovering high tumor uptake during scintigraphy with somatostatin analogues.

The therapy was performed with 2-4.5 GBq ${ }^{90} \mathrm{Y}$ DOTA TATE per patient per one cycle, in the slow infusion in the physiological liquid $(150 \mathrm{ml} / 15 \mathrm{~min})$. Between the cycles, there was a time delay of 6-8 weeks. Before the therapy, patients received the infusion of amino acids.

Results. Analysis of the "bremsstrahlung" images showed uptake of the radiopharmaceutical in the liver, but the most of the activity was observed in the regions of the "hot spots" (planar $\mathrm{T} / \mathrm{B}=1.70 \pm 0.9$, SPECT $\mathrm{T} / \mathrm{B}=2.9+/-0.8$ ) registered with previous $99 \mathrm{~m} \mathrm{Tc}$ Tektrotyd and/or ${ }^{111}$ In Octreoscan images.

After the therapy, progressive disease (PD - 2 with neuroendocrine pancreatic carcinoma with liver mets and 2 with NET of unknown origin) occurred in four patients. In the majority of patients, stable disease (SD) was registered (one with bronchial carcinoid and liver mets, one with pancreatic gastrinoma, one with NET of unknown origin and liver lung and bone mets, two with neuroendocrine pancreatic carcinomas with liver mets, one with NET of unknown origin with liver mets, one with lung neuroendocrine carcinoma with liver and bone mets, one with NET of unknown origin with bone and skin metastases), and in three partial remission (PR - coecal carcinoid with liver mets and 2 with NET of unknown origin with liver mets). Up to now, there were no major clinical side-effects of hepatic function. Transient pancytopenia occurred in two patients, and impairment of kidney function in one.

Conclusion. Treatment with ${ }^{90} \mathrm{Y}$ DOTA TATE is feasible for the management of patients with inoperable or disseminated NETs. 


\title{
Organizational and technical ways of providing the population with accessible and high-quality radiodiagnosis in conditions of scarce resources
}

\author{
Sergii Balashov', Yury Kovalenko²
}

1 X-ray Technology Center of the Association of Radiologists of Ukraine, Kyev, Ukraine

2 P.L. Shupyk National Medical Academy of Postgraduate Education, Kyev, Ukraine

\section{https://doi.org/10.21175/rad.abstr.book.2021.17.2}

The purpose of the work. Over the past 5 years, the number of $x$-ray units in the country has decreased by one thousand. The number of radiologists is reduced by almost 200 people annually.

Today, for one radiologist in the country there are 4 units of X-ray equipment. Almost half a million people in the country receive new housing annually and move away from X-ray units. However, road conditions do not allow the patient to be quickly delivered to the X-ray room, even on short distances.

Therefore, the urgent task is to find ways to increase the availability and effectiveness of radiodiagnosis.

Materials and methods. The paper summarizes the experience gained during the last three years of introducing new technological and organizational measures aimed at improving the effectiveness of radiodiagnosis, in particular, the use of light-weight digital radiodiagnostic complexes, radiodiagnostic complexes with the mode of tomosynthesis and teleradiology.

Results. The introduction into clinical practice of light-weight digital radiodiagnostic complexes on the basis of ward x-ray equipment, for which it is sufficient to have a room for storage and operation, allowed us to bring the radiodiagnosis closer to the patients at the expense of equipping them with primary care centers. In addition, such equipment has become financially available for radiologists, entrepreneurs, who also place their offices near family-owned outpatient clinics and primary care centers.

In the municipal consultative-diagnostic center, where there is no radiologist, teleradiology is implemented: diagnostic images are sent to him via the Internet. This reduces the time for family doctors to obtain X-ray results by 2-24 hours.

At the end of 2017, a digital X-ray diagnostic complex with a mode of tomosynthesis was established at the Kyiv clinical base of the Center for X-ray Technologies of the Association of Radiologists of Ukraine and the experience of its use was analyzed within two years. In two years, 321 studies were performed to clarify the information obtained by radiography. In 68 cases (21.2\%), the previous diagnosis made after radiography was withdrawn, in 99 cases (30.8\%) the diagnosis was confirmed and in 154 cases (48\%) additional data on the detected pathology were obtained. The use of tomosynthesis made it possible to greatly reduce the number of referrals of patients for computed and magnetic resonance imaging. After receiving positive results of the test use of tomosynthesis in Kyiv at the end of 2019, an X-ray diagnostic complex with the mode of tomosynthesis was purchased for each municipal consultative-diagnostic center.

Taking into account the annual reduction in the number of doctors in the country, teleradiology is gradually being introduced, i.e. remote processing of diagnostic images from several $x$-ray machines by one radiologist. This allows you to optimize workloads for staff, make use of second thoughts in difficult cases, and compensate for staff shortages.

Conclusions. Installation of light-weight digital radiodiagnostic complexes in or near primary care centers, use of equipment with the mode of tomosynthesis and teleradiology in the X-ray units allows us to bring the high-quality radiodiagnosis closer to the population and make it more effective. 


\title{
Visibility of the lateral pterygoid muscle in temporomandibular disorders by magnetic resonance imaging
}

\author{
Josip Ivacic', Aleksandar Knezevic², \\ Daniela Djurovic Koprivica3, Aleksandra Maletin3, Milica Jeremic Knezevic3
}

\author{
1 General Hospital Senta, Serbia, Senta, Serbia \\ 2 University of Novi Sad, Faculty of Medicine Novi Sad, Novi Sad, Serbia : Clinical Centre of Vojvodina, \\ Medical Rehabilitation Clinic, Novi Sad, Serbia \\ 3 University of Novi Sad, Faculty of Medicine, Novi Sad, Serbia
}

\section{https://doi.org/10.21175/rad.abstr.book.2021.17.3}

Background. Temporomandibular disorders (TMD) are a heterogeneous group of diseases involving temporomandibular joint (TMJ) and related muscles, characterized by pain, joint movement alterations and articular sounds. Lateral pterygoid muscle (LPM) is a masticatory muscle, one of the main depressors of lower jaw. It has two heads: one inserts on the articular disc and fibrous capsule of TMJ and the other on the mandibular condyle. Visualization of the LPM using magnetic resonance imaging (MRI) enables evaluation of the insertion, presence of partial or complete tear, hypertrophy, atrophy of the muscle and the presence of contracture.

Methods. Fibers of LPM upper head may enter the anterior-medial part of the disc directly and participate in kinematics of TMJ. During contraction, upper head of LPM pushes the disc forward (protraction) and limits the posterior movement of the disc (retraction). Although the importance of LPM for the function of the disc is clear, the exact role of LPM insertion in TMDs is not yet explained. MRI is a powerful tool for the evaluation of TMJ changes, able to depict in detail anatomic relations of LPM to the adjacent structures.

Results. Insertion of the LPM upper head has been evaluated in order to explain the presence of the anterior disc dislocation in TMD; however, most studies failed to prove significant correlations between this insertion and disc dislocation. 


\title{
The role of contrast-enchased computer tomography in the management of intestinal obstruction
}

\author{
Ana Lazarova \\ University Clinic for Surgery Disease St. Naum Ohridski Skopje, Skopje, North Macedonia
}

https://doi.org/10.21175/rad.abstr.book.2021.17.4

Introduction. Intestinal obstruction is a condition in which intestine is obstructed because of any mechanical or non-mechanical reasons. It accounts for $20 \%$ of all surgical admissions for acute abdomen. Computed tomography (CT) is currently the exam of choice for bowel obstruction because has higher specificity in reporting the etiology, site and grade of obstruction, and the presence of bowel ischemia, affecting the surgical decision.

Objective: To determine the sensitivity and specificity of contrast-enchased CT for diagnosing the small and large bowel obstruction.

Materials and methods. This is prospective study which includes 100 patients diagnosed with bowel obstruction in the period from January 2016 to July 2020 in the University Clinic for Surgery Disease St.Naum Ohridski Skopje. All of the patients were with clinic presentation of acute abdomen, primary conventional X- ray on abdomen was done and bowel distention with developed air-fluid levels was found. This was an indication for CT so the reason for bowel obstruction to be found as well as the level, site and grade of obstruction. 76 from 100 patients underwent operative treatment whereas 14 patients were treated conservatively and with nasogastric tube decompression. The results of surgical treatment in resolving intestinal obstruction and the finding for etiology were taken as the gold standard in evaluating the accuracy of CT in detecting the causes of intestinal obstruction.

Results. This study included a 100 patients aged from 44 to 81 years. 55 from the patients were male and 45 female .60 from the patients had small bowel obstruction and 30 patents had large bowel obstruction, whereas in 10 patients there was bowel obstruction on the small and large intestine in the same time. In 39 patients small bowel obstruction was caused by adhesive bends and in that case CT scan made prediction for that reason made on exclusion. In two cases ileus was as a result of pseudomembranous colitis, one as small bowel obstruction and one was small and large bowel obstruction. There were 3 patients with acute diverticulitis on sigmoid part of the colon. In one case it was small bowel obstruction and in two patients there were small and secondary large bowel obstruction. One case was presented with peritoneal carcinomatosis as a reason for bowel obstruction. Seven from the patients with bowel obstruction were with intestinal ischemia as a result of mesenteric thrombosis. Five of them were only with small bowel obstruction a two were with small primary and then developed large bowel obstruction.11 patients presented with bowel obstruction as a result of herniation. In two patients complications from Morbus Chron were the reason for small bowel obstruction. There were 30 patients present with large bowel obstruction. The main cause of it in 24 patients was neoinfiltrative process in the wall of the bowel. In 6 patients the reason for large bowel obstruction was sigmoid volvulus.

Conclusion. This study is a clear indication of the importance of contrasts enhanced CT in the evaluation of intestinal obstruction. High accuracy in detecting the etiology of intestinal obstruction makes it the most important diagnostic method for adequate resolution of intestinal obstruction. 


\title{
High-frequency ultrasound scanning in eyelids assessment
}

\author{
Tatiana Kiseleva', Yulia Stepanova², Natalya Guseva', Ksenya Lugovkina'
}

1 Helmholtz National Medical Research Center of Eye Diseases, Moscow, Russia

2 A.V. Vishnevsky National Medical Research Center of Surgery, Moscow, Russia

\section{https://doi.org/10.21175/rad.abstr.book.2021.17.5}

The development and search of possible non-invasive examinations of eyelids and periorbital area are currently in progress. These techniques are essential and accurate methods in differential diagnosis of neoplastic, tumor-like processes and inflammatory diseases of eyelids.

Purpose: to study the normal ultrasound image of anatomical structures of eyelids using high-frequency ultrasound scanning.

Materials and methods. We observed 48 healthy volunteers (96 eyes) aged from 17 to 46 years.

Ultrasound methods which included high-frequency grayscale B-scan, color Doppler imaging (CDI) and ultrasonic density measurement or echodensitometry (ED) of eyelids and periorbital tissues were performed on the Voluson diagnostic system (GE Healthcare) using linear transducer SP $10-16 \mathrm{MHz}$ with the high resolution zoom function. Echographic anatomy of eyelid layers, i.e. skin, orbicularis oculi muscle, tarsus, and orbital fat, was described in detail. Depth and ultrasonic density values for all layers were provided.

Results. Considering anatomical data and echographic features of different tissues of eyelids we determined the ultrasound image of eyelid structures and the mean indices of the thickness of eyelid layers in the middle line on longitudinal and transversal scans. The thickness of upper eyelid was $2.90 \pm 0.2 \mathrm{~mm}$, skin with subcutaneous tissue - $0.98 \pm 0.17 \mathrm{~mm}$, orbicularis oculi muscle $-0.76 \pm 0.1 \mathrm{~mm}$, tarsal plate with conjunctiva $-1.09 \pm 0.15 \mathrm{~mm}$. Results of lower eyelid examination were the following: thickness of eyelid - 3.30 $\pm 0.24 \mathrm{~mm}$, skin with subcutaneous tissue $-1.12 \pm 0.16 \mathrm{~mm}$, orbicularis oculi muscle - $0.98 \pm 0.14 \mathrm{~mm}$, tarsal plate with conjunctiva $-1.19 \pm 0.17 \mathrm{~mm}$. ED assessment of eyelid showed follow indices: epidermis $-99.7 \pm 22$ c.u.; papillary

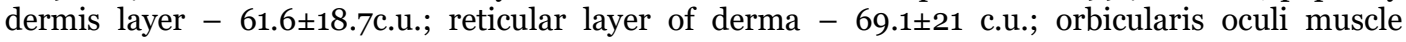
$-41.6 \pm 17$ c.u.; tarsal plate $-97.2 \pm 22$ c.u. Echographic image of eyelids was lamellar. Epidermis, reticular layer of derma and orbital fat, tarsal plate with conjunctiva were hyperechoic. Papillary layer and orbicularis oculi muscle were hypoechoic. There was no statistically significant difference between ultrasonic density indices of the upper eyelid layers and the lower ones ( $>0.05)$. CDI demonstrated vessels with arterial blood flow waveform between anterior and posterior lamella of eyelid.

Conclusions. Based on echographic detailed identification of normal anatomic features of eyelid structures, complex ultrasound examination including high frequency B-scan, ED and CDI can be used successfully in clinical practice. We suggest performing a high frequency ultrasound scanning of eyelids prior to reconstructive and plastic surgery in order to facilitate the choice of surgical tactics. 


\title{
Possibilities of sonoelastography in the evaluation of the lower third of face soft tissue changes determining the most effective method of correction
}

\author{
Daria Kiseleva, Yulia Stepanova, Naida Sultanova
}

A.V. Vishnevsky National Medical Research Center of Surgery, Moscow, Russia

https://doi.org/10.21175/rad.abstr.book.2021.17.6

Subject. This study discusses the use of shear wave elastography in evaluation of age-related atrophic processes in muscle tissue in the lower third of face in women of different age.

Objective: To explore the possibility of using sonoelastography in the study of age-related changes in elasticity of muscle tissue of the lower third of the face in women, correlating with atrophic processes.

Materials and methods. 45 women divided into three equal groups by age (20-30, 30-40 and 40-60 years old) were involved in the study. The division into groups is necessary to estimate the differences in the parameters of elasticity depending on age. Elasticity of muscle tissue in the area of buccinator muscle was assessed using shear wave elastography with linear probe. The results were statistically processed and evaluated in terms of practical application.

Results. There was a statistically significant decrease in the elasticity of muscle tissue in the lower third of the face, associated with the processes of age-related atrophy (the average value of the stiffness of the muscle in vertical position: group $1-10.58 \pm 1.78 \mathrm{kPa}$, group $2-8.09 \pm 1 \mathrm{kPa}$, group $3-5.8 \pm 1.24 \mathrm{kPa}$ ). There were almost no differences in elasticity while changing the position from vertical to horizontal in the older age group (group 3). It is associated with progressive age-related changes in the tissues and is clearly displayed by ultrasound examination (stiffness of the muscle in group 3 in vertical position - $5.8 \pm 1.24 \mathrm{kPa}$, in horizontal - $5.44 \pm 1.21 \mathrm{kPa}$ ).

Conclusion. Using sonoelastography it is possible to display atrophic processes in facial muscle tissue. It may be helpful in the future in preoperative diagnosis and choosing the optimal method of correction. Sonoelastography is a promising method for assessing age-related changes in soft tissues in plastic surgery, aesthetic medicine and cosmetology. Further studies for the application of this method are required and may lead to its implementation in routine practice in this field of medicine. 


\title{
Radiology diagnostics at the stages of extracorporal resection of a single kidney in the treatment of renal cell cancer
}

\author{
Yulia Stepanova, Vlada Raguzina, Tatiana Baitman, Aleksandr Gritskevich
}

A.V. Vishnevsky National Medical Research Center for Surgery, Moscow, Russia

\section{https://doi.org/10.21175/rad.abstr.book.2021.17.7}

Treatment of patients with cancer of a solitary kidney: an organ-sparing approach is preferable, but doesn't always comply with the oncological radicalism. The technique of extracorporeal renal resection (ECRR) followed by autologous transplantation was developed to preserve renal function in patients with obligatory indications for organ-preserving treatment.

Purpose: to evaluate the results of ECRR with orthotopic replantation of renal vessels in conditions of pharmaco-cold ischemia in renal cell carcinoma (RCC); to determine the possibilities and the role of radiology diagnostic at the stages of surgery.

Materials and methods. The study included 20 patients treated with RCC in 2013-20 (average age $59.5 \pm 8$ years). Men prevailed (70\%). Multiple primary metachronous cancer occurred in 14 (70\%) cases, multiple primary synchronous cancer - in 2 (10\%), previous nephrureterectomy was performed in connection with benign kidney diseases (primary contracted kidney, hydronephrosis) - in 2 (10\%), a congenital single kidney was in 2 (10\%) patients. Previously underwent surgery on a single kidney for a malignant neoplasm of the same etiology for which $4(20 \%)$ patients are being treated in this hospitalization. All of the patients underwent US and MSCT at the preoperative stage, in 7 cases MRI. Morphological verification of the lesions: clear cell RCC - 18 (90\%) cases, papillary RCC - 1 (5\%); B-cell lymphoma - 1 (5\%).

Results. Staging according to the TNM system: pT1a-T3vNo-2Mo-1G1-3, of which the tumor size exceeded $7 \mathrm{~cm}$ in 10 (50\%) patients, distant metastases were in 8 (40\%) cases. Reno-caval tumor thrombus was detected in 3 patients.

The average duration of cold ischemia was $102.6 \pm 42.7$ min. Bloodloss - 613 $\pm 300.1 \mathrm{ml}$. Intraoperative US was performed at the stages of surgery. In 3 cases, ECRR was performed simultaneously with thrombectomy and resection of the inferior vena cava for reno-caval tumor thrombus. In 4 cases, renal vessel replacement was performed. There were no intraoperative complications.

All patients underwent US- monitoring on the 1st, 3rd and 5th days after surgery, more often and further as needed. Laboratory control of indicators was carried out. Postoperative complications $\geq$ grade II according to Clavien-Dindo classification were observed in 9 (45\%) patients: II - 1 (5\%), IIIa - 4 (20\%), IVa - 4 (20\%). Complications of IVa degree were characterized by the development of acute renal injury, which required renal replacement therapy.

The follow-up period (US, MSCT) was 19-85 months (53.3 \pm 17.2$)$. Tumor progression occurred in $3(15 \%)$ cases. One patient died due to the progression of the tumor process 20 months after the operation.

Conclusion. Radiology diagnostic methods make it possible to control all the stages of ECRR of a single kidney under conditions of pharmaco-cold ischemia with orthotopic replantation of renal vessels. The results obtained are satisfactory, which indicates the advisability of further development of organ-saving treatment. 


\title{
Quantitative analysis of the breast tissue chemical composition based on the spectral decomposition of X-ray tomographic breast images
}

\author{
Stevan Vrbaski ${ }^{1,2}$, Renata Longo ${ }^{1,3}$, Adriano Contillo² \\ 1 University of Trieste, Trieste, Italy \\ 2 Elettra Sincrotrone Trieste, Trieste, Italy \\ 3 INFN, Trieste, Italy
}

\section{https://doi.org/10.21175/rad.abstr.book.2021.17.8}

Breast cancer accounts for the largest number of malignancies in women worldwide. The diagnostic outcome of conventional X-ray imaging relies heavily on relative attenuation differences in nearby tissues. Since the contrast difference between cancerous and glandular tissues is often not evident, the diagnosis is mainly based on morphological characteristics. Recently, advanced imaging techniques were being developed making the extraction of quantitative information a feasible task. We aim to exploit a technique of spectral decomposition to quantify the difference in chemical composition between healthy and malignant dense tissues.

Our very first study, based on the tissue-equivalent custom-made phantom, showed promising results in extracting an effective atomic number and density from multi-energy CT images. Although being very useful in mimicking the attenuation properties of breast tissues, plastic inserts fail to reproduce the valid local inhomogeneity observable in organic tissues. In this communication the first attempt to quantitatively describe breast mastectomy samples was reported. Imaging was performed at Elettra, the Italian synchrotron facility, using monochromatic beams of several energies in the breast CT energy range (22-38 keV). With help of a radiologist, the regions of interest for quantitative evaluation were selected. Three samples were processed by a spectral decomposition algorithm, resulting in composition maps in terms of a selected pair of basis materials. Using a dedicated mathematical procedure, we managed to decouple the information about the material density and its chemical composition. Finally, a calibration allowed us to retrieve the effective atomic number and density associated with each reconstructed voxel.

The range of effective atomic numbers among the plastics matched the slight differences among tissue regions within the breast mastectomies. The region-based decomposition procedure is an important intermediate step toward the application of the method to the full sample volume. The presence of structural noise, coming from the intrinsic variability of the tissue region was observed. However, its presence did not affect the overall stability of our decomposition method. The procedure allowed an accurate discrimination of the chemical composition of considered anatomical regions.

The decoupling of the information about the chemical composition allows very accurate discrimination of similar tissues composing the breast, opening the possibility of significant contributions to a breast cancer diagnosis. 


\title{
Various examination methods applied for the detection of pathological changes in the nasal mucosa and patency of the nasal passages in patients with the reduced sense of smell
}

\author{
D. Mihaylov ${ }^{\text {, V. Stoyanov' }}$, D. Thomas², M. Mitev3
}

\begin{abstract}
1 Department of Otorhinolaryngology, Medical Faculty, Trakia University, Stara Zagora, Bulgaria 2 Department of Neurology and Psychiatry, Medical Faculty, Trakia University, Stara Zagora, Bulgaria 3 Department of Medical Physics, Biophysics, Roentgenology and Radiology, Faculty of Medicine, Department of Diagnostic Imaging, University Hospital 'St. Kirkovich', Trakia University - Stara Zagora, Stara Zagora, Bulgaria
\end{abstract}

\section{https://doi.org/10.21175/rad.abstr.book.2021.17.9}

Introduction. Decreased or missing sense of smell is a current problem which is a result of various causes and diseases. The identification of these causes leading to dysfunction of the olfactory analyzer is carried out by applying various research methods (smell tests; MRI, rhinomanometry, etc.).

Material and methods. Thirty-six patients with impaired sense of smell were studied. Smell tests, MRI with Siemens Magnetom Essenza 1.5 Tesla and other methods were used.

Results. MRI in all patients revealed thickening of the mucosa in the area of the nasal conchae, and the anterior and posterior ethmoidal cells. In some patients, similar changes have been observed in the frontal and maxillary sinuses, manifested by intermediate and high signal intensity findings in the T2w sequences.

Conclusions. MRI findings are nonspecific. Although MRI cannot differentiate changes and deliver specific information, the findings obtained by this method in patients with impaired sense of smell can be used for orientating ENT specialists to diagnose the causes of peripheral damage to the olfactory analyzer.

Keywords: MRI, impaired sense of smell, smell test

\section{References}

[1] Henkin RI, Levy LM, Lin ChS. Taste and Smell Phantoms Revealed by Brain Functional MRI (fMRI). Journal of Computer Assisted Tomography. 2020; 24(1):106-123. 


\title{
Radiological examination at the stages of surgical treatment of the consequences of the soft tissue augmentation with vaseline oil for aesthetic purposes
}

\author{
Yulia Stepanova, Nora Arutiunian, Naida Sultanova, Alexey Kopyltsov
}

A.V. Vishnevsky National Medical Research Center of Surgery, Moscow, Russia

https://doi.org/10.21175/rad.abstr.book.2021.17.10

Introduction. Despite the regulations governing the provision of aesthetic non-surgical medical services, the number of patients affected by the augmentation of vaseline oil into soft tissues to correct the shape of organs and soft tissues has increased.

Objective: development of an algorithm for radiological examination of patients at the stages of surgical correction of the consequences of the augmentation with vaseline oil into soft tissues for aesthetic purposes.

Materials and methods. In a retrospective study, the results of the examination and surgical treatment of 17 women were evaluated. The patients were treated at various times with the consequences of the augmentation with vaseline oil in mammary glands, buttocks and shins. There were $11(64.7 \%)$ patients with vaseline oil introduced into one region, into two regions 5 (29.4\%), and $1(5.9 \%)$ into three regions. The migration of vaseline oil to adjacent anatomical areas was observed in 13 out of $17(76.5 \%)$ patients. All patients underwent US, MSCT, and/or MRI at the stages of surgical treatment.

Results. Multiple-stages ( from 1 to 8 ) surgical treatment was performed and in addition to excision of tissues affected by fibrosis and oleogranulomas, also reconstructive surgeries to restore the volume and contours of the organs were performed. Overall, 51 surgical interventions were performed.

MSCT and MRI studies can identify oleogranulomas and determine the volume of soft tissue damage. If it's possible to choose the method of radiology, preference should be given to MRI, it's carried out without radiation exposure and the introduction of a contrast agent and allows you to determine the full volume of soft tissue damage, as well as the presence of fibrous tissue, in the mode without fat suppression.

US is the easiest to perform, however, an effective method for diagnosing complications after soft tissues augmentation. And it's effective when searching for individual fat fragments during surgery and evaluating the intervention area around the perimeter.

In the postoperative period, US revealed limited fluid accumulations ranging in size from 12 to $36 \times 57 \mathrm{~mm}$. Only in 6 cases during 51 interventions, it was considered appropriate to evacuate the contents under US-control. Good and satisfactory results were obtained in 6 patients (MRI data and visual effect), and interventions, mainly of a reconstructive nature, are expected in 11 patients.

Conclusion. The difficulties and multi-stage surgical treatment in patients after soft tissue augmentation with vaseline oil are associated with a large volume of tissue damage with a violation of their trophic function, the migration of vaseline oil to adjacent anatomical areas, the inability to eliminate the lesion and simultaneous reconstruction. MRI makes it possible to determine the extent of the lesion at all stages of surgical treatment, US is an important pre/intraoperative navigation during surgical treatment, and also allows postoperative management of patients. 


\title{
Dose measurement in radiotherapy using various thermoluminescence dosimeters
}

\author{
Aslı Bozkurt', Şule Kaya Keleş², Gaye Özgür Çakal², Ayşe Kaşkaş²
}

\author{
1 Dr. Abdurrahman Yurtaslan Ankara Oncology Training and Research Hospital, Ankara, Turkey \\ 2 Ankara University, Institute of Nuclear Sciences, Ankara, Turkey
}

\section{https://doi.org/10.21175/rad.abstr.book.2021.18.1}

Exposing cancer tissue to ionizing radiation is a commonly used method in cancer treatment. The radiation dose to be given to the tumor is determined by using planning systems. During the verification of the dose, passive dosimeters with atomic numbers close to the tissue, such as TLD-100, are generally used in addition to the active dosimeters. In this study, the applicability of using commercial dosimeters; namely $\mathrm{BeO}$ (Thermolox 995), LiF:Mg,Ti (TLD-100), $\mathrm{Li}_{2} \mathrm{~B}_{4} \mathrm{O}_{7}$ : $\mathrm{Cu}$,In, $\mathrm{Li}_{2} \mathrm{~B}_{4} \mathrm{O}_{7}: \mathrm{Mn}$ (TLD-800), $\mathrm{MgB}_{4} \mathrm{O}_{7}: \mathrm{Dy}$, Na with effective atomic numbers close to the soft tissue and $\mathrm{CaF}_{2}$ :Dy (TLD-200), $\mathrm{Al}_{2} \mathrm{O}_{3}: \mathrm{C}$ (TLD-5O0) with effective atomic numbers close to the bone tissue for dose measurement in radiotherapy have been investigated. For this purpose, the thermoluminescence (TL) dosimeters were irradiated between 0.5 Gy and 50 Gy in a Varian Trilogy linear accelerator system using $6 \mathrm{MV}$ X-rays. The TL dosimeters were placed between the water equivalent phantoms and the phantom thickness above the TL dosimeters was adjusted to $1.6 \mathrm{~cm}$, which is the build-up point for $6 \mathrm{MV} \mathrm{X}$-rays. An irradiation field of $10 \times 10 \mathrm{~cm}^{2}$ was opened and source to skin distance was adjusted to $100 \mathrm{~cm}$. TL readouts were performed by using Harshaw 3500 TLD reader between $50^{\circ} \mathrm{C}$ and $350^{\circ} \mathrm{C}$ at a heating rate of $10^{\circ} \mathrm{C} / \mathrm{s}$. The evaluations were made using the total area of the glow curves. Then, the radiation dose response curves were obtained and the linearity of the curves was examined. As seen, $\mathrm{Li}_{2} \mathrm{~B}_{4} \mathrm{O}_{7}: \mathrm{Cu}$, In and $\mathrm{Li}_{2} \mathrm{~B}_{4} \mathrm{O}_{7}: \mathrm{Mn}$ (TLD-80o) between $0.5 \mathrm{~Gy}$ to $50 \mathrm{~Gy}$ had linear dose response curves. In the other dosimeters, linear dose responses up to 4 Gy and supralinearity behaviours above 4 Gy were observed. To conclude, the investigated lithium tetraborate dosimeters, $\mathrm{Li}_{2} \mathrm{~B}_{4} \mathrm{O}_{7}: \mathrm{Cu}$, In and $\mathrm{Li}_{2} \mathrm{~B}_{4} \mathrm{O}_{7}: \mathrm{Mn}$ (TLD-80o), can preferably be used compared to the well-known TLD-100 dosimeter for dose measurement in radiotherapy due to their linearity in a wide dose range and high TL sensitivity. 


\section{The FOOT experiment - Light nuclei fragmentation measurements}

\section{Matteo Franchini}

University of Bologna, Bologna, Italy

https://doi.org/10.21175/rad.abstr.book.2021.18.2

Effects of nuclear fragmentation in particle therapy are still an open issue. Nuclear interactions in the beam entrance channel produce fragments with high Relative Biological Efficiency (RBE), whose contribution to the unwanted damage is hard to evaluate. Accurate data on fragmentation cross sections of carbon and oxygen up to energies of about $400 \mathrm{Mev} / \mathrm{A}$, are needed to improve the RBE models and the clinical treatment plans. Similarly, in an energy range up to $700 \mathrm{MeV} / \mathrm{A}$, precise fragmentation cross section data would be of great importance in order to optimise the design of the shielding of spaceships, especially in view of long distance space travels (i.e. Mars human missions) where radio-protection issues are of utmost importance.

Currently under construction, the FOOT (FragmentatiOn Of Target) detector is designed to measure fragmentation cross sections in a beam energy range of 50-700 MeV/A with about $5 \%$ uncertainty, eventually taking advantage of an inverse-kinematic approach for proton-nucleus interaction. Target fragmentation will be measured by inverse Lorentz boost on carbon and oxygen beams fragmentation on polyethylene $\left(\mathrm{C}_{2} \mathrm{H}_{4}\right)$ and graphite $(\mathrm{C})$ targets.

The FOOT layout includes a start counter that provides the trigger signal and the start of the Time-Of-Flight (TOF) measurement when crossed by a primary particle. A drift chamber placed before the target provides the exact direction of the incoming particle, while three sets of silicon pixel and strip detectors plus a two-magnets system allow a precise tracking of nuclear fragments via a Kaman filter algorithm. A thin double layer of plastic scintillator bars provide the stop-time of the TOF system and the measurement of $\mathrm{dE} / \mathrm{dx}$, needed for fragment charge identification. The last detector element, a BGO calorimeter, provide the particle kinetic energy measurement. In dedicated runs, an emulsion spectrometer will be inserted after the target to measure the production of low $\mathrm{Z}$ fragments that wouldn't cross the whole spectrometer.

A partial setup of the FOOT detector ( including the start counter, beam-monitor, scintillator and vertex detector elements) has been assembled and tested using both protons, Carbon and Oxygen ions both at CNAO and GSI in an energy range of $[60-400] \mathrm{MeV} / \mathrm{u}$ in order to study its response in a wide range of operation. The main results obtained in these data takings will be presented. The calibration data collected allowed to reach, and often improve, the expected performance on TOF and fragment identification. Preliminary evaluation on fragments yield will be discussed. The foreseen measurement campaign for this and next year will be also presented. 


\title{
Measurement of urinary excretion and blood clearance after peptide receptor radionuclide therapy
}

\author{
Marija Jeremic', Milovan Matovic, Dragoslav Nikezic², \\ Dragana Krstic², Tatjana Miladinovic3, Suzana Pantovic4
}

\author{
1 Clinical Center Kragujevac, Department of Nuclear Medicine, Kragujevac, Serbia \\ 2 University of Kragujevac, Faculty of Science, Kragujevac, Serbia \\ 3 University of Kragujevac, Institute of Information Technologies, Department of Science, Kragujevac, Serbia \\ 4 University of Kragujevac, Faculty of Medical Sciences, Kragujevac, Serbia
}

\section{https://doi.org/10.21175/rad.abstr.book.2021.18.3}

Background. Measurement of radiopharmaceutical activity in patient's blood and urine is the starting point in all dosimetric assessments in nuclear medicine. This is the most reliable way to determinate the rate of elimination of unbound residual radioactivity in the patient's body. Peptide Receptor Radionuclide Therapy (PRRT) is a targeted molecular radionuclide therapy administered to patients with neuroendocrine tumors (NET). It is applied in several cycles and isotopes ${ }^{90} \mathrm{Y},{ }^{177} \mathrm{Lu}$ or a combination.

Materials and methods. Measurement of blood and urine samples is most reliable in liquid scintillation beta counter, due to its very high measurement efficiency, which in our case during calibration was 90.34\%. A beta counter (LSC-Liquid Scintillation Counter) Rack Beta Wallac (LKB, Finland) was used and blood and urine samples were measured in 5 patients who received $5.55 \mathrm{GBq}$ ${ }^{90}$ Y-DOTATOC and 5 patients who received $5.55 \mathrm{GBq}{ }^{177 \mathrm{Lu}-D O T A T O C .}$ Blood was sampled at the time of application of therapy, as well as $1 h, 2 h, 3 h$ and $24 h$ after completion of radiopharmaceutical administration. Urine was sampled by patients at the time of urination and recorded the time of urination and the volume of urine.

Results. In all patients, there were significant variations in measured blood and urine activities depending on tumor size and location. The highest activities in both blood and urine were recorded in the first samples. In the blood, immediately after the completion of the application of radiopharmaceuticals, values were measured in the range of $6.31 \%$ of the applied activity to $67.47 \%$, on average $29.31 \%$. While in urine, depending on the moment of urination, these values ranged from $3 \%$ of the applied activity, in a patient who urinated immediately after radiopharmaceutical administration to $29 \%$ in a patient who urinated 4 hours after completion of application, on average 23.64\%. There were no significant differences depending on the isotopes used.

Conclusion. Peptides have very fast biokinetics and in a very short time there is a transport of radiolabeled peptides from the blood to the tumor tissue, where they remain bound and contribute to the desired therapeutic effect. 


\title{
WIDMApp (Wearable Individual Dose Monitoring Apparatus): An innovative approach for individual dose monitoring in Molecular Radiotherapy
}

\author{
Carlo Mancini-Terracciano, ${ }^{1,2}$, Francesco Collamati², \\ Riccardo Faccini' ${ }^{1,2}$, Giuseppe Iaccarino3, Riccardo Mirabelli², \\ Francesca Nicolanti,2, Massimiliano Pacilio4, \\ Elena Solfaroli Camillocci ${ }^{1,2,5}$, Antonella Soriani3 ${ }^{3}$,Silvio Morganti²
}

\author{
1 Department of Physics, Sapienza University of Rome, Rome, Italy \\ 2 INFN Section of Rome, Rome, Italy \\ 3 Laboratory of Medical Physics and Expert Systems, IRCCS Regina Elena National Cancer Institute, Rome, \\ Italy \\ 4 Medical Physics Division, Azienda Ospedaliera-Universitaria Policlinico Umberto I, Rome, Italy \\ 5 Specialty School of Medical Physics, Sapienza University of Rome, Rome, Italy
}

\section{https://doi.org/10.21175/rad.abstr.book.2021.18.4}

We will present the proof-of-concept of WIDMApp (Wearable Individual Dose Monitoring Apparatus), a multi-channel detector and data processing system, aimed at allowing an accurate determination of absorbed dose per organ in Molecular Radiotherapy (MRT). The precise estimation of dose absorbed by the target and healthy organs is essential to increase therapeutic effectiveness, optimize the treatment planning and establish dose-effect relationships for tumor and normal tissues. Moreover, the European regulations (EU Directive 59/2013) considers mandatory treatment planning and verification in all patients' exposures for radiotherapeutic purposes, including MRT. Finally, WIDMApp will strongly reduce the need of nuclear medicine imaging systems reducing the need of resources of nuclear medicine departments (i.e., technology, uptime and human resources) to perform individualized biokinetics studies.

To perform the feasibility study, we used the male anthropomorphic MIRD phantom to develop a Monte Carlo simulation of a ${ }^{131} \mathrm{I}$ thyroid treatment. The simulation provides the Time dependent Counts Curves (TCC) that would have been measured by seven WIDMApp-like sensors placed and oriented to face each one of 5 emitting organs plus two thyroid lobes. The WIDMApp data analysis tool is then applied on this simulated data set to perform the deconvolution of the TCC and to reconstruct the Time-Activity Curves (TAC) of each organ. Deviations of the reconstructed TAC parameters from their actual values are studied as a function of the initial conditions used to initialize the data analysis algorithm and as a function of the fluctuation of the TCC data points.

The study demonstrates that it is possible, at least in the simple simulated scenario, to infer the organ cumulated activity by measuring the TCC recorded by few detectors placed in selected position over the patient body. The accuracy on the cumulated activity of $5 \%$ is measured even with a $20 \%$ error on the TCC data points and a variation of $50 \%$ on the initial guess on the deconvolution algorithm parameters. The possibility to perform in-vivo sampling more frequently than conventional biokinetics studies would increase the number of time-points and so the accuracy in TAC estimations. 


\title{
Effective dose as a reporting tool in radiology: Myth or reality
}

\author{
Natasa Brasik \\ University Hospital Vienna-AKH, Vienna, Austria \\ https://doi.org/10.21175/rad.abstr.book.2021.18.5 \\ The awareness for radiation protection rose among the medical community and public authorities \\ after the publishing the European Basic Safety Standards (EU-BSS) in 2014. Austria, as other EU \\ Member states implemented the European Basic Safety Standards (EU-BSS) and translated it into \\ the Austrian Medical Radiation Protection Law (Medizinischestrahlenschutzverordnung).
}

According to the Austrian Medical Radiation Protection Law, the patient dose should be part of the radiological report for each patient.

The problem arises when we think of a term "dose". It is very general term and physicists talk about Air Kerma Dose, Equivalent Dose, organ Dose etc, medical personnel and patients speak about risk.

We need trained personnel, who can translate the risk from each radiological exam for each patient using one quantity.

Is the effective dose the most convenient parameter we can use to communicate risk?

This aim of this study is to try to find the answer, which value shall we report in the future.

For reporting purposes, we have to take into consideration two types of health effects from radiation: stochastic and deterministic.

Stochastic effects are connected with the risk of cancer, and deterministic effects are related directly to the absorbed radiation dose and the severity of the effect increases as the dose increases.

Effective dose was introduced in 1977 by ICRP, as a parameter that reflects the risk of a nonuniform radiation exposure in terms of a whole-body exposure. Effective dose represents a weighted combination of organ and tissue doses for a defined set of radiosensitive organs and tissues. The sum of these weighting factors is always equal to 1.o. Calculating effective dose requires estimating doses to each of the specified organs or tissues. The concept of effective dose allows comparison between examinations of different techniques (such as CT vs fluoroscopy vs nuclear medicine cardiac examinations). Effective dose can be also used for informing judgments on justification, and establishing constraints for careers and volunteers in medical research. Where doses from the same technique are being compared, measurable quantities are preferred.

However, the medical community must realise that an effective dose estimate represents a generic estimate of risk from a given procedure to a generic model of the human body. It doesn't represent a risk to any individual patient. Effective dose should not be used for epidemiologic studies or the estimation of population risks due to the inherent uncertainties and oversimplifications involved. For epidemiologic studies or individual risk estimates, modeling of doses to specific tissues and organs in an individual, taking into account that individual's body size and shape, must be performed to estimate the various organ and tissue doses. 


\title{
Investigation of the applicability to study the FLASH effect with protons on LINAC-4 accelerators at CERN and with carbon ions on NUCLOTRON at JINR (Dubna)
}

\author{
Ivan Tsanev, Borislav Pavlov, Peicho Petkov, Leandar Litov
}

Sofia University “St. Kliment Ohridski”, Sofia, Bulgaria

\section{https://doi.org/10.21175/rad.abstr.book.2021.18.6}

The interest of the radiation oncology community in the application of FLASH radiotherapy is constantly growing. In FLASH therapy the prescribed dose is deliveries to the entire treatment volume in a very short time interval. Early preclinical data show that these extremely high doses rates provide significant sparing of healthy tissue compared to conventional radiation therapy without reducing cancer cell damage. This sparked also an interest in proton and hadron therapy community

The aim of this work is to investigate the possibilities of studying the FLASH effect with heavy charged particles on available particle accelerators. The accelerators should be able to deliver high dose for a very short time to investigate FLASH, but also should be able to deliver the same dose for longer time interval in order to reproduce the conditions during the standard therapy. The number of available accelerators in the World satisfying the above requirements is limited. Two accelerators are considered in the study - LINAC-4 at CERN and NUCLOTRON in Dubna.

To achieve the objectives of the work, numerous radio-dosimetric MC simulations were performed using GEANT4 and FLUKA. Initially, a set of simulations of a water phantom placed in the path of a wide beam of charged particles was performed. The design beam parameters of LINAC4 and NUCLOTRON are used as an input for the simulations. The results of this initial set of simulations served to estimate qualitatively and quantitatively the radiological dose in the aqueous phantom in terms of FLASH effect requirements. After an assessment of the simulation results a new set of simulations has been performed where a detailed phantom composed by a vessel with appropriate dimensions and a cuvette to place in-vitro cell culture has been simulated. The volume and position of the cuvette has been optimised in such a way that the dose in it is maximal, homogeneous, and measurable by a radio-biological method.

The results of the simulations convince us that it is possible to study the FLASH effect with protons on the LINAC-4 accelerators at CERN and carbon ions on NUCLOTRON at JINR (Dubna).

Keywords: FLASH effect, Heavy charged particles, CERN, JINR, Dubna, GEANT4, FLUKA 


\title{
Cyber Knife - A proposal of dosimetric audit with shane phantom
}

\author{
Maria Piziorska ${ }^{1,2}$, Marcin Szymański, Oskar Madetko1, \\ Wioletta Ślusarczyk-Kacprzyki,3, Wojciech Bulski3
}

\author{
1 Masovian Oncology Hospital (MSO), Wieiszew, Poland \\ 2 Central Clinical Hospital of Ministry of Internal Affairs and Administration (CSKMSWiA), Warsaw, Poland \\ 3 Maria Sklodowska-Curie National Research Institute of Oncology, Warsaw, Poland
}

\section{https://doi.org/10.21175/rad.abstr.book.2021.18.7}

Dosimetry audit is one of the major element of quality assurance in radiotherapy. IAEA (The International Atomic Energy Agency), and WHO (The World Health Organization), for a number of years, have been active in the efforts to enhance the quality and effectiveness of radiotherapy all over the world. Most of the audit methods are designed to be carried out on classical linear accelerators. At present, there is no commonly accepted and used scheme of dosimetry audit for such unconventional accelerators as the CyberKnife.

The aim of this study is a proposal for the dosimetry audit scheme for the CyberKnife system. For this purpose, an anthropomorphic phantom SHANE by CIRS, designed for comprehensive testing of radiotherapy quality was used. The Phantom has inbuilt anatomical structures in the head and neck region which enables the verification of the patient position with the use of the 6D-Skull procedure, one of the techniques used for this purpose on the CyberKnife accelerator. The phantom also has inserts for placing the ionization chamber and dosimetric film.

In order to verify the dosimetric and geometric parameters of the whole process of radiotherapy, a tomography of the SHANE phantom was performed. Five different PTVs (Planning Target Volume) of different volumes were drawn on the CT images (target volumes approximately $88 \mathrm{~cm}^{3}, 44 \mathrm{~cm}^{3}$, $\left.15 \mathrm{~cm}^{3}, 7 \mathrm{~cm}^{3}, 1.5 \mathrm{~cm}^{3}\right)$. For these target volumes, the treatment plans were prepared with the MultiPlan Ver. 4.6.1. system. The FIXED collimator system was used. In each plan number of collimators and their size were adapted to the contour of the PTV. Doses were calculated using the RayTracing algorithm. Each plan was verified with a QA (Quality Assurance) plan for slab phantom, according to the standard protocol used in the clinic. In case of the positive result of the QA test (acceptable dose difference up to $5 \%$ ), the dose was measured with TM31010 Semiflex 0,125 $\mathrm{cm}^{3}$ ionization chamber (irradiation time ranged between 27 and 68 minutes).

The percentage differences between the dose measured in the SHANE phantom and calculated with the TPS were: $4.29 \%,-1.42 \%,-0.7 \%, 1.37 \%,-1.88 \%$ (for PTV: $88 \mathrm{~cm}^{3}, 44 \mathrm{~cm}^{3}, 15 \mathrm{~cm}^{3}, 7 \mathrm{~cm}^{3}$, $1.5 \mathrm{~cm}^{3}$, respectively), were found to meet the criteria adapted in our center. Analyzing various methods of dosimetric audits for small fields to be found in the literature (percentage difference in the range $3-5 \%$ ) we are of the opinion that the proposed dosimetric audit of the CyberKnife system, using SHANE phantom, is a correct and acceptable method of the verification of the process of radiotherapy. 


\title{
The differences in dose distribution caused by uncorrected rotations
}

\section{Maria Poncyljusz ${ }^{\text {, Wojciech Bulski² }}$}

\begin{abstract}
1 The Central Clinical Hospital of the Ministry of the Interior and Administration in Warsaw, Department of Medical Physics, Warsaw, Poland

2 The Maria Skłodowska-Curie Memorial Cancer Centre and Institute of Oncology, Department of Medical Physics, Warsaw, Poland
\end{abstract}

\section{https://doi.org/10.21175/rad.abstr.book.2021.18.8}

One of the advantages of Image-guided radiotherapy (IGRT) is the opportunity to use it to correct patient set-up errors. The information about $\mathrm{x}, \mathrm{y}, \mathrm{z}$ translations as well as roll, pitch and yaw rotations is received after set-up verification. Unfortunately, most machines are equipped with couches that do not allow correcting roll and pitch rotations. This results in shifts of the CTV points. Substantial shifts occur at a distance of about $10 \mathrm{~cm}$ from the rotation axis. Fifteen patients with prostate cancer treated with VMAT technique had daily cone beam CT scans (CBCT scans) prior to treatment delivery (420 CBCTs in total). Dose distributions were calculated for maximal value of rotation. The dose coverage of planning target volume (PTV) and the dose delivered to the pelvic bones were analysed. The differences in the delivered dose depend on the sign and value of the uncorrected rotations. 


\title{
Computational breast models dedicated to contrast enhanced spectral mammography
}

\author{
Kristina Bliznakova, Yanka Baneva, Georgi Todorov
}

Medical University, Varna, Bulgaria

https://doi.org/10.21175/rad.abstr.book.2021.18.9

Purpose. Contrast-enhanced spectral mammography is a method where the contrast media is introduced in the blood circulatory system of the patient and the accumulation of the contrast agent is exploited to detect cancerous cells. Female breasts differ in size and elemental composition; thus xray technicians must be confident that the obtained image after the injecting of the tracer is of very good quality. An important factor in this procedure is the clinical apparatus, which needs both regular quality control and well defined clinical protocols. Physical phantoms for contrast-enhanced mammography are currently under constructions and thus not widely available. A preliminary step in the design of such physical phantom is the design of a computational prototype with characteristics closed to the characteristics of the real patient breast tissues. The purpose of this study is the design and evaluation of computational breast phantoms for contrast-enhanced spectral mammography.

Materials and Methods. Three different computational phantoms for contrast-enhanced spectral mammography were designed with the use of an in-house developed radiology computational tool. The first phantom has a semi cylindrical shape with a radius of $50 \mathrm{~mm}$ and height of $45 \mathrm{~mm}$, made from polymethyl methacrylate (PMMA). Six iodinated inserts with a radius of $10 \mathrm{~mm}$ and different heights are inserted inside the PMMA container. The second phantom is a PMMA parallelepiped with a base dimensions of $203 \mathrm{~mm} \times 152 \mathrm{~mm}$ and a thickness of $25 \mathrm{~mm}$. In this PMMA container, six iodinated inserts are modelled with radius of $9.5 \mathrm{~mm}$ and heights which vary from $0.5 \mathrm{~mm}$ to $20 \mathrm{~mm}$. The third phantom is heterogenous breast phantom which is replica of the first one with included heterogeneous background in the form of spheres with different diameters. The iodinated inserts were modelled from Omnipauque for all the models. Each phantom was subjected to two simulated $\mathrm{x}$-ray radiographs: one with an energy below the K-edge of the iodine $-20 \mathrm{keV}$ and one with an energy above the K-edge $-34 \mathrm{keV}$. The images were processed to a recombined iodine image.

Results. The obtained contrast-enhanced images of the three computational phantoms showed excellent suppression of the surrounding background tissue, while bringing in focus the iodinated test objects. Compared to the low-energy images of the phantoms, the simulated spectral images demonstrated improved object contrast improvement. The simulations with the inhomogeneous model revealed that the heterogeneous background has been successively depressed while improving the visibility of the iodine inserts

Conclusions. The heterogenous breast phantom might be used as a reference tool for information about the needed iodine concentration to be inserted during the procedure to obtain significant enhancement in the suspicious area. Current work includes the construction of the physical breast phantoms and their thorough evaluation.

Keywords: Breast computational phantom, contrast-enhanced spectral mammography, iodine concentration, modelling, simulation 


\title{
A rare type of mesiodens - case report: Diagnosis and treatment
}

\author{
Miona Vuletic ${ }^{1}$, Milca Veličković1, Aleksandar Acović1, \\ Vladimir Ristić ${ }^{1}$, Tamara Ristić ${ }^{2}$, Tatjana Kanjevac ${ }^{1}$
}

1 University of Kragujevac, Serbia, Faculty of Medical Sciences, Department of Dentistry, Kragujevac, Serbia
2 Department of Clinical Dentistry, Faculty of Dentistry Pancevo, Pancevo, Serbia

\section{https://doi.org/10.21175/rad.abstr.book.2021.19.1}

Supernumerary teeth are defined as an increased number of teeth, more than 20 teeth in primary or more than 32 in permanent dentition and can be found in almost any region of the dental arch. Our study presents a case of a missing central incisor due to the presence of excessive teeth. A 12year-old boy reports to the Department of Dentistry due to lack of a permanent maxillary right central incisor. Based on clinical and radiographic examination, a diagnosis of multiple mesiodens was obstructed, as the main cause of impaction of a permanent central incisor. Extraction of the residual root of the primary incisor and removal of the lobe was accessed, and further extraction of supernumerary teeth and surgical exposure of the central incisor, which allowed the lock to be placed on the central incisor in the same act during surgery. Mesiodens is the most common type of supernumerary teeth that often causes numerous abnormalities in the dental arch. Use of CBCT secure detailed examinations and evaluations of supernumerary teeth, local dental and bone structure which are very beneficial in diagnosis and treatment planning and preventing complications which may occur.

Keywords: Hyperdontia, Supernumerary tooth, CBCT 


\title{
A rare case of orbital mucocele
}

\author{
Ana Lazarova \\ University Clinic for Surgery Disease St. Naum Ohridski Skopje, Skopje, North Macedonia
}

https://doi.org/10.21175/rad.abstr.book.2021.19.2

Introduction. Orbital mucoceles are cysts which are filled with mucous fluid that cannot naturally drain from the nose. Most common arising from the ethmoid or frontal sinuses they grow and slowly cause mass effect of the adjacent orbital tissues.

Case report. This is a case of 46 years old patient who present with symptoms of exophthalmos and pain in the right eye, in a period of one year. After ophthalmology examination he was send for magnetic resonance imaging (MRI) of the orbital cavity to be done. MRI was done in standard T1 and T2 waited images and waited images after contrast administration. In the right orbital cavity next to the ethmoidal cellules, oval homogenous tumor formation with the diameter of $26 \mathrm{~mm}$ was found. This formation was with signal characteristics of solid tumor, and was localized in the extraconal space. There was no invasion of the optic nerve or other conal eye structures, but there was mass effect of the medial rectus muscle with the displacement of all conal structures lateral. Also computer tomography (CT) scan was preformed, as additional investigation but no microcalcifications were found, and the density of solid tissue was confirmed. Neurosurgery with Misonix ultrasonic knife was performed and encapsulated cystic formation was found and send to pathohistology verification with the result for mucocele.

Conclusion. This is an interesting case of atypical presentation of sinus mucocele which can mimic benign orbital cavity tumor mass and MRI diagnosis of mucocele in orbital cavity should be considered especially when communication with nearby sinus can be predicted on the images. In spite of being benign, the expansive nature of mucocele may cause paranasal sinuses bone erosion by compression and consequential bone resorption, leading to compromise of adjacent structures. Early diagnosis is essentially for adequate treatment. 


\title{
Clinical case of ovarian cancer after in-vitro fertilization: Forensic medical malpractice - clinical case and data review
}

\author{
Rossen Hadjiev', Margarita Tankova', \\ Margarita Taushanova², Lyubomir Damyanov3
}

\author{
1 Multifunctional Hospital for Active Treatment 'Losenetz', Department of Forensic Medicine, Sofia, Bulgaria \\ 2 University Hospital 'Tsaritsa Yoanna - ISUL', Department of Oncology, Sofia, Bulgaria \\ 3 Medical Center for Human Reproduction Ltd, Sofia, Bulgaria
}

\section{https://doi.org/10.21175/rad.abstr.book.2021.19.3}

Background. Ovarian cancer is a leading cause of death amongst women. The extreme hormonal stimulation plays an important role for the cancerous development if there is an existing predisposition.

Introduction. In-vitro fertilization procedures (IVF) are a part of the assisted reproductive technologies (ART). Factors that may increase the risk of development of ovarian cancer are, but are not limited to, genetic mutations, lack of pregnancy, hormone replacement therapy in menopause, oral contraceptives and inherited mutations in the BRCA-1 and BRCA-2 genes.

Case report. A 45-year-old woman with no family history of cancer, with almost diminished ovarian reserve started IVF procedures. The doses of the hormone medications that were used - Merional and Cetrotide, do not exceed the recommended values. The subsequent IVF attempt was successful and the patient had a normal pregnancy and delivery.

Three months later, during a gynecological ultrasound examination, formations were found as follows: $30.5 \mathrm{~mm}$ in the right ovary and $20 \mathrm{~mm}$ in the left ovary - unilocular cysts. Tumor markers indicated slightly higher level of ROMA - 12.36\%, Ca-125 - 37.34, HE4 - 61.8. Therapy with Dufaston was prescribed with the assumption that the ovarian cysts indicate Luteinized unruptured follicle (LUF) syndrome.

After another 3 months, during a gynecological ultrasound examination it was established that the formations persisted: in the right ovary $-49 \mathrm{~mm}$, in the left ovary $-15 \mathrm{~mm}$.

Half a year later, ultrasound diagnostics revealed a formation measuring $25 \mathrm{~cm} / 15 \mathrm{~cm}$. The tumor markers indicated as follows: Ca-125 - 38.54, HE4 - 106.3. A total hysterectomy with bilateral tumor adnexectomy was performed. During the surgery two formations were found - one originating from the right ovary with dimensions $25 \mathrm{~cm} / 25 \mathrm{~cm}$, and a solid formation $5 \mathrm{~cm} / 5 \mathrm{~cm}$ from the left ovary. Histological examination confirmed the diagnosis: malignant mixed Müllerian tumor.

Discussion. There are known cases of cystic and malignant formations in the ovaries, as well as ovarian hyper stimulation syndrome, after IVF procedures due to the usage of ovulation stimulating drugs. There is a theory that hyper stimulation with the purpose of provoking repeated ovulations lead to malignant transformation of the ovarian parenchyma.

Conclusion. Each IVF procedure needs to be tailored to the individual patient with regards to the specific health conditions. It is a necessity for a detailed medical history of inherited oncological diseases to be established, as well as for genetic testing of BRCA-1 and BRCA-2 mutations to be performed. The strict and regular disease control is important if pathological changes are confirmed. It is a mandatory condition for implementing a good clinical practice.

Keywords: Ovarian cancer, in-vitro fertilization, ovarian hyper stimulation, forensic medical malpractice 


\title{
Physical processes of the action of laser radiation on biological systems
}

\author{
Volodymyr Timaniuk', Ella Romodanova', \\ Stanislav Pogorelov' ${ }^{1}$, Tatiana Dubko² ${ }^{2}$ Mykola Kokodii ${ }^{1}$
}

\author{
1 National University of Pharmacy, Kharkiv, Ukraine \\ 2 Institute for Cryobiology and Cryomedicine of the National Academy of Sciences of Ukraine, Kharkiv, \\ Ukraine
}

\section{https://doi.org/10.21175/rad.abstr.book.2021.20.1}

Introduction. Low-intensity laser radiation is widely used in therapy. In the infrared region, the photon energy is only sufficient to increase the vibrational energy of biological molecules, resulting in an increase in temperature. Visible light induces physico-chemical processes, which are divided into primary (light) and secondary (dark). The primary processes are excitation of the electronic state of molecules, ionisation (photoelectric effect) and decomposition into ions or radicals. The GrotkusDreiner rule, the Bunsen-Rosko rule, the Stark-Einstein law and the phenomenon of intermolecular energy migration are valid for these processes. In addition, it is the optical range of radiation whose quantum energy is sufficient for the transition of oxygen molecules from the triplet state to the excited singlet state. The occurrence of singlet oxygen promotes the formation of reactive oxygen species, which induces free-radical processes.

Purpose: To carry out a comparative analysis of the contribution of various physical processes in the formation of the action of laser radiation of the optical range in the methods of therapy.

To analyse the role of changes in water properties when exposed to laser radiation in the development of biological effects.

Materials and methods. Water is known to be present in large quantities in biological tissues. There is a developed system of hydrogen bonds of water, which arise spontaneously under certain conditions. Under the action of electromagnetic fields this system of bonds changes, which leads to changes in the structure and properties of water itself. The effect of low-intensity laser radiation on the properties of water and substances dissolved in it has been investigated by spectral methods.

\section{Results.}

1. analysis of changes in water absorption spectra shows that laser irradiation affects the hydrogen bonding system of water;

2. changes in the properties of water under low-intensity laser radiation enhances the effects of tryptophan fluorescence quenching;

3. changes in the spectral properties of whey albumin aromatic amino acid residues when dissolved in pre-irradiated water can be estimated as being related to a change in the macromolecule and to the transition from the native state to the molten globule state; probe.

4. pre-irradiation of water affects the interaction of human serum albumin with a fluorescent

Conclusion. The interaction of laser radiation with biosystems is complex, multidimensional, and multifaceted, which can manifest itself because of changes in the structure and properties of water.

Keywords: Laser radiation, water, effect, optical range, biological system 


\title{
Testing femtosecond delay line for diffractive imaging and atomic, molecular and optical sciences as ELI Beamlines
}

\author{
Michaela Lastovickova
}

Headington School Oxford, Oxford, United Kingdom

\section{https://doi.org/10.21175/rad.abstr.book.2021.20.2}

The research group for application in molecular, biomedical and material science at ELI Beamlines needs to control the delay of synchronized laser beams down to femtosecond precision. The aim of this student project was to apply interferometry in femtosecond delay line stage tests for atomic, molecular, and optical sciences and for coherent diffractive imaging. For this purpose, a Michelson Interferometer was built using ELI in-house resources. Using a beam splitter, a laser beam was split into two arms and then recollected to create an interference pattern. This pattern was detected by a fast $2 \mathrm{GHz}$ Thorlabs photodiode, DETo25A/M, with a 400-1000 nm sensitivity range and rise/fall times of $150 \mathrm{ps}$, in order to calibrate a femtosecond delay line stage. The Aerotech PRO19oSL/SLE was used as the translation linear stage. This stage served as the main component of the delay line tests. Further, Attocube sensors based on the Fabry-Perot Interferometer technique were added to the set-up to improve the precision and reliability of the calibration method. The advantages, disadvantages and limits of this method as well as lessons learned will be discussed from both an educational and scientific point of view. 


\title{
Genotoxicity assessment of combined exposure to WiFi and UV radiation on human keratinocytes in vitro
}

\author{
Zsófia Szilágyi', Györgyi Kubinyi', Erika Szabó', \\ Bertalan Pintér' ${ }^{1}$ Brahim Selmaoui², György Thuróczy
}

\author{
1 National Public Health Center, Department of Radiobiology and Radiohygiene, Non-ionizing Unit, \\ Budapest, Hungary \\ 2 Unité de Toxicologie Expérimentale, L'Institut National de l'Environnement Industriel et des Risques, \\ Verneuil en Halate, France
}

\section{https://doi.org/10.21175/rad.abstr.book.2021.20.3}

In the last decades, the exposure to radiofrequency (RF) radiation emitted by wireless devices has increased in the human environment, whereupon has raised concerns about its health effects. The International Agency for Research on Cancer classifies RF radiation as "possible carcinogenic" (Group 2B), and the ultraviolet (UV) radiation as "carcinogenic" (Group 1) respectively. Here, we investigated these two radiations in combination. We tested whether consecutive exposures of UV radiation and WiFi field have any effect on human keratinocytes. The cells were exposed to 1 SED UV irradiation (as first exposure), which was followed immediately by a 24-hour of 20 min switched $\mathrm{ON} / \mathrm{OFF}$ intermittent $2450 \mathrm{MHz} \mathrm{RF}$ exposure with $4 \mathrm{~W} / \mathrm{kg}$, as consecutive exposure. Genotoxic evaluation was carried out by classical in vitro methods (Alkaline Comet Assay, Cytokinesis-block Micronucleus Assay, $\gamma \mathrm{H} 2 \mathrm{AX}$ Assay). Comet Assay assessment showed that RF radiation did not induce any additional effect to the damage caused by UV radiation. A slight but not significant increase of the genotoxic damage was assessed by Micronucleus and $\gamma \mathrm{H} 2 \mathrm{AX}$ Assay. Overall, we found no conclusive and coherent evidence for the induction of DNA damages, but in some cases we reported slight tendency of the adverse effect of WiFi irradiation. Comparative studies like our approach of consecutive exposure to UV and RF may be important in the future, concerning the upcoming $5 \mathrm{G}$ and $6 \mathrm{G}$ technologies, where the radiation will be absorbed mainly in the skin. This study was carried out as part of the SKIN-RF project, funded by ANSES. 


\title{
The effect of intermediate frequency electromagnetic field at $22 \mathrm{kHz}$ on human fibroblast cells in vitro
}

\author{
Bertalan Pintér'1, Zsófia Szilágyi', Erika Szabó', \\ Györgyi Kubinyi ${ }^{1}$, Yves Le Drean², György Thuróczy ${ }^{1}$
}

\author{
1 National Public Health Center, Department of Radiobiology and Radiohygiene, Non-ionizing Radiation \\ Unit, Budapest, Hungary \\ 2 Université de Rennes I, Institut de Recherche sur la Santé, l’Environnement et le Travail, Rennes, France
}

\section{https://doi.org/10.21175/rad.abstr.book.2021.20.4}

There are a number of household appliances that use the IF range, such as induction cookers, compact fluorescent lamps, anti-theft devices, which are commonly installed in areas accessible to people. The number of devices using inverter technology (air condition systems, refrigerator etc.) have also grown significantly in recent years producing IF electromagnetic exposure. So far only a limited number of studies have examined the possible genotoxic effect of IF, therefore comprehensive research in this topic is important. The aim of this study was to examine whether Intermediate Frequency (IF) Electromagnetic Field (EMF) has any effect on DNA of human fibroblast cells in vitro. This study is focused on the possible genotoxic effect of IF-EMF in itself on human fibroblast cells. The possible adaptive response induced by IF radiation was also investigated. The phenomena of adaptive response was first described in connection with ionizing radiation, where a relative low dose of treatment induces a kind of adaptation (as resistance) against a subsequent higher dose of treatment. During our experiments human fibroblast cells were exposed to $22 \mathrm{kHz}$, $100 \mathrm{mT}$ IF radiation (as adaptive dose) for 24 hours and 4 hours later were challenged by 2.5 Gy X-ray ionizing radiation. Evaluation of the possible DNA damage was performed by Fpg-modified alkaline comet assay. Data were analysed with Comet Assay IV software. This is an ongoing experiment; evaluation of the data is still in progress and the final results will be presented at the meeting. This study was carried out as part of the FiGe project, funded by ANSES. 


\title{
2-5G electromagnetic field chronic exposure biological effects assessment
}

\author{
Nina Rubtsova, Sergey Perov, Olga Belaya
}

FSBSI, Moscow, Russia

https://doi.org/10.21175/rad.abstr.book.2021.20.5

The problem of general public electromagnetic safety due to $5 \mathrm{G}$ communication systems implementation is important.

In order to study the possible adverse effects of electromagnetic fields (EMF) emitted by $5 \mathrm{G}$ mobile communication systems, there were carried out comparative assessment of 2-4G (3 frequency bands) and $5 \mathrm{G}$ (3 frequency bands) EMF multifrequency chronic biological effects. Experiments were carried out in 180 Wistar male rats divided into three groups: 2 exposed $\left(250 \mu \mathrm{W} / \mathrm{cm}^{2}\right.$ power density) and 1 sham exposed; 24h/day exposure, 4 months and 1 month post exposure. Neuroendocrine system (corticosterone and ACTH content in the peripheral blood serum) and rat's research activity parameters were evaluated after 1;2;3;4 months of exposure and 1-month postexposure (12 animals in each group every time).

Rats exposed to EMF corresponding to 2-4G observed corticosterone secretion significant elevation after the 2nd and 3rd months of exposure, with tendency to increase after 1st and 4th months of exposure and decrease after 1 month after. In group exposed to $5 \mathrm{G}$ EMF, significant corticosterone content increase was founded after 1st month of exposure and 1 month postexposure period, with tendency to increase after 2nd month of exposure. ACTH content in of rat' blood serum during the exposure period did not differ significantly from control values, with tendency to significant decrease after the 3rd month of 2-4G EMF exposure, while after 3 months of $5 \mathrm{G}$ EMF exposure there was ACTH content tendency to increase only, replaced by decrease after 4 months of exposure, remaining 1 month after the end of exposure.

The revealed corticosteroid secretion wave-form changes, manifested in immediate increase with followed depression of corticosteroid response to normal or subnormal values and secondary hormonal secretion increase indicate hypothalamic-pituitary-adrenal system stress reaction to EMF more expressed in case of 2-4G EMF chronic exposure.

The results showed rats oriented research activity (open field test) wave-form changes, indicating that EMF in frequency spectrum corresponding to 2-4G exposure causes sufficiently evident excitatory effect after 1 month of exposure, which, with continued exposure up to 4 months replaced by inhibitory effect, and after the end of exposure again leads to an increase in activity, which most likely indicates that the used EMF parameters lie above the threshold of the central nervous system sensitivity and approach the threshold of adverse effects. $5 \mathrm{G}$ frequency range EMF exposure caused similar, but less severity changes of rats' research activity. 


\title{
The effect of laser radiation on drug emulsions
}

\author{
Andra Dinache, Tatiana Tozar, Adriana Smarandache, \\ Ionut Andrei, Mihai Boni, Mihail Lucian Pascu, Angela Staicu
}

National Institute for Laser, Plasma and Radiation Physics, Magurele, Romania

https://doi.org/10.21175/rad.abstr.book.2021.20.6

The interaction of laser radiation with drug solutions can be resonant, leading to molecular modifications and generation of photoproducts, or unresonant, having a mechanical effect.

We employed the unresonant effect of laser radiation to design a laser-assisted device for emulsion generation. For this, the wavelength of laser radiation was chosen according to the absorbance spectra of the two immiscible solutions so the effect of laser radiation on emulsions to be of mechanical nature.

The drug emulsions were generated with a double syringe system, mixing a drug (e.g., sodium tetradecyl sulphate - STS, Vancomycin) in water solution with an oily phase. The system allows to set mixing speed and number of mixing cycles. The generated emulsion was then exposed to laser radiation [1]. The unirradiated and irradiated samples were analyzed through optical microscopy, dynamic light scattering (DLS), surface tension analysis, UV-Vis reflectance spectroscopy, FTIR and Raman spectroscopy.

Optical microscopy analysis and mean hydrodynamic diameters measured through DLS showed that droplets are smaller after exposing the emulsion to laser radiation (141.8 $\mathrm{nm}$ after irradiation, in comparison with $269.5 \mathrm{~nm}$ before exposure, in the case of STS emulsions). Zeta potential measured before and after irradiation of emulsions, as well as surface tension measured with the pendant drop technique, demonstrated that irradiated emulsions are more stable than the unirradiated ones [1]. FTIR analyses of emulsions allowed the identification of vibrational bands specific to each of the immiscible components, further providing a real-time evaluation of the emulsion's destabilization. Raman spectroscopy enabled the characterization of the modifications at the molecular level, through identification of the corresponding vibrations from the functional groups of emulsion components. The difference between the UV-Vis-NIR reflectance spectra of the unirradiated and irradiated emulsions (correlated with previous measurements) showed that the physical characteristics of the emulsions changed during exposure to laser radiation. The increase in the reflectance signal suggests that the dimensions of the droplets decreased, leading to an emulsion with better time stability [2].

\section{References}

[1] A. Dinache et al., "Laser assisted generation of micro/nanosize emulsions," Colloids and Surfaces A: Physicochemical and Engineering Aspects, vol. 577, pp. 265-273, Sep. 2019, doi: 10.1016/j.colsurfa.2019.05.083.

[2] A. Dinache et al., "Spectroscopic Characterization of Emulsions Generated with a New Laser-Assisted Device," Molecules, vol. 25, no. 7, p. 1729, Apr. 2020, doi: 10.3390/molecules25071729.

Acknowledgments: This research was funded by Romanian National Authority for Scientific Research and Innovation, CNCS/CCCDI-UEFISCDI, projects PN-III-P2-2.1-PED-2019-4771, PN-III-P2-2.1-PED-2019-1264 and Nucleu Programme, ctr. No. 16N /08.02.2019. 


\title{
Analysis of DNA damage response following proton radiation exposure in an in vitro neuronal model
}

\author{
Mihaela Temelie', Tiberiu Esanu1, \\ Liviu Craciun', Nicoleta Moisoi²,1, Diana Savu'
}

1 National Institute of Physics and Nuclear Engineering Horia Hulubei, Magurele, Romania

2 De Montfort University, Leicester, United Kingdom

\section{https://doi.org/10.21175/rad.abstr.book.2021.21.1}

Radiation effects are a main research theme in the context of cancer therapy that involves studying the rapidly diving tumour cell lines. Recently developed particle therapy started to gain terrain in the treatment of some brain cancers due to its improved control of the irradiation area. Anyhow, adverse effects cannot be neglect, since several studies from the field of space radiobiology linked neurodegeneration with exposure to particle radiation in the head area. Moreover, the non-dividing neural cells appear to show particular sensitivity to protons exposure compared to $\mathrm{X}$ irradiation linked to radiation characteristics (higher LET).

Our aim here was to analyse proton radiation effects on DNA damage response and cellular death/survival in a differentiated neuronal model.

Human neuroblastoma cell line $\mathrm{SH}_{-} \mathrm{SY}_{5} \mathrm{Y}$ was differentiated to a neuronal phenotype using a protocol based on addition of all-trans retinoic acid for 7 days and validated by immunofluorescence staining of specific markers (b-III-tubulin and tyrosine hydroxylase). Cells were irradiated using the proton beam line of a TR19 Cyclotron (IFIN-HH) with doses of 2-10 Gy. The viability was evaluated using the MTT assay and by cell counting in a hemocytometer. Immunofluorescence and Western Blot assay were used for assessing DNA damage response through visualisation of proteins specifically involved in pathways of DNA repair: 53BP1, gH2AX, RAD51, Ku70.

Results. The protocol of cellular differentiation lead to increased percentage of b-III-tubulin and $\mathrm{TH}$ positive cells, change of cellular morphology, and decrease of cellular proliferation. The dose-response curve was established for protons irradiation at several time-point following exposure $(24 \mathrm{~h}, 48 \mathrm{~h}, 72 \mathrm{~h})$. The dose of 2 Gy of proton irradiation, corresponding to a reduction of cellular viability to about $80 \%$ of control, was selected for further studies concerning DNA damage response. Number of DNA repair foci highly increased shortly after irradiation (3h), with a time-dependent decrease. However, even at longer time-points following irradiation (24 hours ) DNA repair is not complete. Expression of several proteins associated with pathways of DNA damage signalling were found to be increased following proton irradiation in neuronal differentiated cells.

Perspective. While the study represents an evaluation of DNA damage mechanism in differentiated cells, we must acknowledge the fact that our model is not composed exclusively of fully differentiated mature neurons. A future microscopy and/or flowcytometric analysis will be used in order to distinguish specific DNA repair mechanism in dependence to cellular phenotype.

Acknowledgments: The study was supported by Romanian Ministry of Research National Grant PD 46 / 2020 "BRAIN AGEING UNDER RADIATION: PINK1 role in DNA damage response following proton-induced neurodegeneration”. 


\title{
Determination of neutron flux distribution in a ${ }^{252} \mathrm{Cf}$ irradiator using GATE
}

\begin{abstract}
Menekşe Şenyiğit
Ankara University Department of Physics, Ankara, Turkey

https://doi.org/10.21175/rad.abstr.book.2021.22.1

Calculation of neutron flux at the irradiation positions of a neutron activation system is essential for material irradiations. This study aims to investigate the determination of neutron flux distribution and the absorbed dose rates in a ${ }^{252} \mathrm{Cf}$ irradiator. GATE simulation toolkit was used to estimate the neutron flux distribution and the absorbed dose rates for various moderators such as paraffin, light water, heavy water, and graphite. The simulation work was done to carry out the moderation of ${ }^{252} \mathrm{Cf}$ neutron source at various distances from the neutron source position in the specified moderator sphere. The flux and the dose rate of heavy water and paraffin at different distances from the neutron source were also obtained for thermal, epithermal, and fast neutron
\end{abstract} cases. 


\title{
Time to energy conversion method for neutron Time of Flight measurements
}

\author{
Elizabeth Musacchio Gonzalez
}

INN-LNL, Padua, Italy

\section{https://doi.org/10.21175/rad.abstr.book.2021.22.2}

One of the most interesting topics in nuclear astrophysics is related to nucleosynthesis of the elements beyond iron, which are mainly produced via neutron-capture reactions. The neutron capture cross section data are the fundamental ingredient for the calculation of the stellar reaction rates and thus the possibility of reproducing the observed abundance of the elements in the Universe. The Maxwellian-Averaged Cross Section (MACS) is the quantity needed for the calculation of the stellar reaction rate. A direct measurement of the MACS, under certain conditions, can be performed by neutron activation analysis (NAA) providing a neutron beam with the stellar spectrum, i.e. a Maxwell-Boltzmann Neutron Spectrum (MBNS). Previous to perform any MACS measurement, neutron spectrum determination is mandatory, and this is the purpose of this work. A new method to convert the temporal neutron spectrum to an energy spectrum was implemented. Monte Carlo simulations using MCNPX were performed for the implementation of the conversion method, and also for comparison with experimental data found in the literature. Preliminary results of an experimental measurement will be reported in the talk. 


\title{
Single moderator active neutron spectrometer for therapeutic beam characterization in Boron Neutron Capture Therapy facilities
}

\author{
Valeria Monti',2, Roberto Bedogni3, Marco Costa1,2, \\ Andrea Pola4, Davide Bortot4, Alessandro Lega3, Saverio Altieri5,6
}

\author{
1 University of Turin, Physics Department, Torino, Italy \\ 2 INFN, Torino, Italy \\ 3 INFN, Frascati (RO), Italy \\ 4 Politecnico di Milano, Milano, Italy \\ 5 University of Pavia, Pavia, Italy \\ 6 INFN, Pavia, Italy
}

\section{https://doi.org/10.21175/rad.abstr.book.2021.22.3}

In the framework of the ENTER_BNCT project (INFN-Italy), a directional neutron spectrometer, tailored for BNCT beams QA/QC measurements, has been designed and constructed.

This device, which arises from the optimization of previously developed single moderator spectrometers, condenses the functionality of a Bonner Spheres Spectrometer into a single moderator, embedding multiple thermal neutron detectors.

The geometry of the spectrometer has been optimized through MCNP6 simulations and consists in a cylindrical collimator followed by a cylindrical core which contains, along his axis, 6 silicon detectors made sensitive to thermal neutrons through a $6 \mathrm{LiF}$ deposited layer. The position of the detector has been studied in order to optimize the spectrometer response in the epithermal range. As the internal thermal neutron sensors are simultaneously acquired, the instrument is suited to operate as a real-time neutron spectrometer.

The simulation model of the spectrometer has been experimentally verified by exposing the prototype in the reference neutron field of $241 \mathrm{Am}$-Be of the Politecnico di Milano. The count rate in the spectrometer internal detectors, as calculated from the simulation model, coincided with the experimental ones within about $\pm 2 \%$, confirming the high degree of accuracy of the simulation model. The results are shown in this communication together with aspects related to the future use of the device in therapeutic neutron beams. 


\title{
The concept of the development and practical testing of the "Fast neutron activation analysis (FNAA)" method at the EG-5 accelerator, FLNP JINR
}

\author{
I.A. Chepurchenko', A.S. Doroshkevich", ${ }^{1,2}$ M. Kulik,3, T.YuZelenyak, \\ A.I. Madadzada',4, A.N. Lihachev', M.A. Balasoui ${ }^{5}$, E.V. Lychagin' ${ }^{1}$, P. Badica6 ${ }^{6}$, \\ M. Stef7, B. Jasinska3, T.V. Phuc',8,9, L.H. Khiem ${ }^{8,9}$, P.L. Tuan',1o \\ 1 Joint Institute for Nuclear Research, Dubna, Russia \\ 2 Donetsk Institute for Physics and Engineering named after O.O. Galkin, Kiev, Ukraine \\ 3 Institute of Physics, Maria Curie-Skłodowska University, Lublin, Poland \\ 4 National Center for Nuclear Research, Baku, Azerbaijan \\ 5 Horia Hulubei National Institute for R\&D in Physics and Nuclear Engineering (IFIN-HH), Bucharest \\ Romania \\ 6 National institute of materials physics, Măgurele, Romania \\ 7 West University of Timisoara, Timisoara, Romania \\ 8 Graduate University of Science and Technology, Vietnam Academy of Science and Technology, Ha Noi, \\ Vietnam \\ 9 Institute of Physics, Vietnam Academy of Science and Technology, Ha Noi, Vietnam \\ 10 Vietnam Atomic Energy Institute, Hanoi, Vietnam
}

\section{https://doi.org/10.21175/rad.abstr.book.2021.22.4}

At the moment, FLNP is practicing some types of nuclear-physical methods of matter elemental analysis, such as neutron-activation analysis with irradiation of samples directly in the "hot" zone of the reactor, X-ray fluorescence, activation on thermal neutrons with instant registration.

The work of the "Accelerator EG-5" group was carried out on the theoretical justification and feasibility of developing a new method for the laboratory neutron activation analysis using fast neutron with instantaneous gamma rays on the electrostatic particle accelerator.

The method of obtaining a neutron flux is not new and is well studied. The neutron flux density when using a gas target an energy of the order of $4-5 \mathrm{MeV}$ is $4^{*} 10^{7} \mathrm{~cm} 2 / \mathrm{sec}$. with. When using a solid target, a flux of $1^{*} 10^{9 \mathrm{~cm} 2} / \mathrm{sec}$ can be achieved with an energy of $10-14 \mathrm{MeV}$.

FNAA complements existing methods of elemental analysis with work with the determination of light particlesisotopes, combining all the advantages of the described methods, such as: completely indestructible sample, simple sample preparation, as well as an extremely low degree of activation with the possibility of further work with the material.

Acknowledgments: The study was performed in the scope of the Poland-JINR and the RO-JINR Projects within the framework of themes FLNP JINR: 04-4-1140-2020/2022, 04-4-1143-2021/2025 and 03-4-11282017/2022. 


\title{
Our solution for automatic capsules filling with high activities of ${ }^{131}$ I
}

\author{
Milovan Matovic', Miroslav Ravlic ${ }^{2}$, Vladimir Zdravkovic3, \\ Aleksandar Vukadinović4, Marija Jeremić ${ }^{1}$, Sanja Vraneš-Đurićc
}

\author{
1 Clinical Center Kragujevac, Center of Nuclear Medicine, Kragujevac, Serbia \\ 2 Prizma Company, Kragujevac, Serbia \\ 3 Zastava Weapons, Kragujevac, Serbia \\ 4 Institute of Nuclear Sciences, Belgrade, Serbia
}

https://doi.org/10.21175/rad.abstr.book.2021.23.1

Aim/Introduction. It is fact that production of capsules which contains high activity ${ }^{131} \mathrm{I}$ is important, but dangerous activity of our colleagues such as physicists, chemists, physico-chemists, pharmacists, technicians, etc..., who works in this kind of industry. They can be exposed to high level of radiation exposure doses during manual preparation of capsules with high activities of 131I. There are just few commercial solutions for automatic capsule filling, but those devices are very expensive or unavailable in our country. We tried to develop and produce our own automatic system for this purpose. Goal of this paper is to present and shortly describe function of our system dedicated to automatic capsules filling with high activities of 131I.

Materials and Methods. Our system consists of PC controlled device which is dedicated to precise dosage of 131I solution and filling of capsules with different amounts of radioiodine activities, which are previously defined. Whole device is located into dedicated chamber, shielded by lead bricks. We use two CCD cameras to remotely surveillance whole process. Known fact that solution volume in microliters closely correlates with activity in $\mathrm{mCi}$, which is basic principle of our system. First step in the whole process is precise filling of known volume of ${ }^{131}{ }^{1}$ solution into syringe. Next step is filling-out needed volume (i.e. activity) in the capsule and closing it with appropriate cap. In order to avoid possible contamination, because of 131I is volatile, we decided to use two capsules. One smaller capsule filled with absorber which is inside one bigger capsule which cap will cover small hole which remains after filling of first capsule. Next step is measurement of ${ }^{131}$ I activity in the filled capsule using dose calibrator and printing it value on the self-adhesive label. Final step is transport of capsule to lead container and covering it with appropriate cover. Whole process is automated, controlled by PC, which is equipped by appropriate software developed using Delphia program language.

Results. Our system enables to avoid unnecessary radiation exposure of personnel as well as to avoid errors caused by subjective or objective reasons. Mistakes are often possible when capsules filling with high activities of ${ }^{131} \mathrm{I}$ are not automated process, but a manual activity of personnel.

Conclusion. Based on our preliminary testing and routine usage of this system, we can conclude that system could be useful tool in production of capsules filled with high activities of radioiodine ${ }^{131}$ I. 


\section{Photonuclear production of ${ }^{47} \mathrm{Sc}$ isotope}

\section{Gohar Hovhannisyan', Tiruhi Bakhshiyan²}

1 Yerevan State University, Yerevan, Armenia

2 Armenian National Agrarian University, Yerevan, Armenia

\section{https://doi.org/10.21175/rad.abstr.book.2021.23.2}

${ }^{47} \mathrm{Sc}$ is one of the proposed theragnostic radionuclides which have emissions that allow low-dose pre-therapy imaging, as well as higher dose therapy $\left(\mathrm{T}_{1 / 2}=3.35\right.$ days, $\mathrm{E}_{\beta}$ avg $=162 \mathrm{keV}, \mathrm{E}_{\gamma}=159 \mathrm{keV}$, $\mathrm{I}_{\gamma}=68.4 \%$ ). Production of ${ }^{47} \mathrm{Sc}$ by neutron capture via ${ }^{47 \mathrm{Ti}}(\mathrm{n}, \mathrm{p}){ }^{47} \mathrm{Sc}$ requires high energy neutron flux

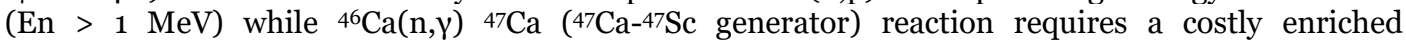
${ }^{46} \mathrm{Ca}$ target. Cyclotron production methods via ${ }^{48} \mathrm{Ti}(\mathrm{p}, 2 \mathrm{p}){ }^{47} \mathrm{Sc},{ }^{11} \mathrm{~V}(\mathrm{p}, 3 \mathrm{p} 2 \mathrm{n}){ }^{47} \mathrm{Sc}$ and ${ }^{\mathrm{nat}} \mathrm{Ca}(\mathrm{p}, 2 \mathrm{n}){ }^{47} \mathrm{Sc}$ lead to coproduction of ${ }^{46} \mathrm{Sc}(\mathrm{T} 1 / 2=83.79$ days $)$ and ${ }^{48} \mathrm{Sc}\left(\mathrm{T}_{1 / 2}=43.67\right.$ hours $)$. An alternative method for ${ }^{47} \mathrm{Sc}$ production is the use of linear electron accelerators. We discuss photonuclear production of ${ }^{47} \mathrm{Sc}$ at the bremsstrahlung end-point energies of 30 and $40 \mathrm{MeV}$. We performed irradiation of natTi targets at the linear electron accelerator of the A.I. Alikhanian National Science Laboratory (Yerevan, Armenia). The natTi were irradiated by bremsstrahlung photons for an hour. The specific activity of ${ }^{47} \mathrm{Sc}$ at the end of bombardment was measured. The yield of $47 \mathrm{Sc}$ was $446(1338) \mathrm{kBq} /(\mu \mathrm{A} \mathrm{h} \mathrm{g})$ at $\mathrm{E}_{\mathrm{gmax}}=30(40) \mathrm{MeV}$, respectively. Fraction of the main impurities ${ }^{46} \mathrm{Sc}$ and ${ }^{48} \mathrm{Sc}$ at the end of bombardment was $1.3(1.09) \%$ and $9.1(11.9) \%$ of ${ }^{47} \mathrm{Sc}$ radioactivity, respectively. It is possible to produce an impurity free ${ }^{47 \mathrm{Sc}}$ with this method by using an enriched ${ }^{48} \mathrm{Ti}$ target and keeping the beam energy lower than ${ }^{48} \mathrm{Ti}(\gamma, \mathrm{pn}){ }^{46} \mathrm{Sc}$ reaction threshold $(22.1 \mathrm{MeV})$. 


\title{
Copper, zinc and bismuth complexes with new macrocyclic chelators for radiopharmaceuticals: In vitro and in vivo stability
}

\author{
Gleb Aleshin', Bayirta Egorova1, Lyubov Zamurueva', \\ Sofia Khabirova', Anastasia Zubenko², Olga Fedorova², Stepan Kalmykov
}

1 Lomonosov Moscow State University, Moscow, Russia

2 A. N. Nesmeyanov Institute of Organoelement Compounds of Russian Academy of Sciences, Moscow, Russia

\section{https://doi.org/10.21175/rad.abstr.book.2021.23.3}

Use of the same conjugate in both diagnostics and radionuclide therapy is promising for nuclear medicine. However, it requires compounds with radiometals with emission appropriate for both

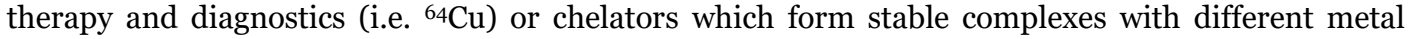
ions. In this way macrocyclic ligands are of high interest as a part of radiopharmaceutical. Copper isotopes are very intensively investigated to apply in PET, SPECT, and $\beta$-therapy. At the same time, zinc radiopharmaceuticals are very poorly studied despite the nuclear physical characteristics of some zinc isotopes suitable for diagnostics. Bismuth isotopes ${ }^{212} \mathrm{Bi}$ and ${ }^{213} \mathrm{Bi}$ are effective for targeted alpha-therapy. In this work we studied the stability (including complexation constants, stability in serum and in vivo biodistribution) of copper, zinc and bismuth complexes with three azacrown ethers containing 5 (L1 and L2) and 6 (L3) heteroatoms in macrocycle.

Labeled complexes were prepared with ${ }^{64 \mathrm{Cu}}{ }^{65 \mathrm{Zn}}$ and ${ }^{207} \mathrm{Bi}$. Stability constants for the complexes with L1, L2 and L3 were determined by potentiometric titration. Labeling yield was determined using TLC with gamma-spectrometry. For in vitro tests complexes solutions were added to fetal bovine serum and incubated at $37^{\circ} \mathrm{C}$; then at the fixed time points proteins were precipitated by ethanol and the radioactivity of supernatant was measured and compared with initial sample. For in vivo experiments normal male mice were used; complexes or blank solutions were injected intraperitoneally, and after 1 or 6 hours after injection the mice were euthanized, major organs and tissues were collected and measured by gamma-spectrometry.

High labeling yields (more than 90\%) for the studied complexes were at ligands concentration of $1 \cdot 10^{-4}-1 \cdot 10^{-3} \mathrm{M}$. Despite of higher stability constants copper complexes were unstable in serum while ZnL2 and ZnL3 didn't show transchelation of $65 \mathrm{Zn}$ by proteins. BiL2 and BiL3 were moderately stable in serum. In vivo studies showed that complexes ZnL2 and ZnL3 demonstrate high stability in organism. In vivo stability of BiL3 was demonstrated, however BiL2 was completely unstable in organism despite of the same complexation constant and in vitro behavior. Thus, L2 and L3 are promising as bifunctional chelators for radiopharmaceuticals with ${ }^{62} \mathrm{Zn}$ and ${ }^{213} \mathrm{Bi}$.

Acknowledgments: This work was funded by Russian Science Foundation, project no. 18-73-10035. 


\title{
Quality control of non-imaging intraoperative gamma probes
}

\begin{abstract}
Milena Dimcheva
Sofia Cancer Center, Department of Nuclear medicine, Sofia, Bulgaria

https://doi.org/10.21175/rad.abstr.book.2021.23.4

The Quality Control (QC) measurements for non-imaging intraoperative gamma probe are based on procedures described in the standards publication NU-3-2004 published by the US National Electrical Manufacturers Association. The standard applies for probes equipped with both scintillation detectors: thallium-doped sodium iodide $\mathrm{NaI}(\mathrm{Tl})$, thallium-doped caesium iodide CsI(Tl), and semiconductor detectors such as cadmium telluride (CdTe), cadmium zinc telluride (CdZnTe ) and mercuring iodine $\left(\mathrm{HgI}_{2}\right)$. To evaluate the quality control of non-imaging intraoperative gamma probe, we measured and discussed the following parameters: overall sensitivity (cps/kBq), spatial resolution (FWHM of PSF, mm), both: radial and lateral, energy resolution $\left(100 \times \Delta \mathrm{E} / \mathrm{E}_{\gamma}\right)$ and signal to noise ratio SNR. Radial resolution is the width of the measurement cone where the radiation is detected at a defined distance. Lateral spatial resolution is the capability to accurately localize the position of a target source and to separate two adjacent sources. Energy resolution is the capacity of the gamma detection system to discriminate between radiations of different energies. The signal to noise ratio relates to the ability of the probe to discriminate the signal arising from the target with respect to the noise represented by the background radiation within the surrounding tissue. Results are compared with those reported in literature. The following QC procedures should be performed regularly to ensure that the non-imaging intraoperative gamma probe is functioning safely and within the manufacturers' specifications.
\end{abstract}

Keywords: Non-imaging intraoperative gamma probe, quality control, semiconductor detectors 


\title{
Radiolabelled strontium hexaferrite nanoparticles for radiopharmaceutical applications
}

\author{
Sofia Khabirova1, Gleb Aleshin', Evgeny Anokhin², \\ Anna Shchukina ${ }^{3}$, Anastasia Zubenko3
}

\author{
1 Moscow State University, Faculty of Chemistry, Moscow, Russia \\ 2 Moscow State University, Faculty of Materials Science, Moscow, Russia \\ 3 A.N.Nesmeyanov Institute of Organoelement Compounds of Russian Academy of Sciences, Moscow, Russia
}

\section{https://doi.org/10.21175/rad.abstr.book.2021.23.5}

The possibilities for diagnostic and therapy of oncological diseases are based on the accumulation of radionuclides in the affected tissues. Nanoparticles can be considered as a platform for combined radiopharmaceuticals both for diagnostic and therapy. Due to their small size, nanoparticles can deliver the radionuclide closer to the tumor cell nucleus what can reduce harmful effect of ionizing radiation on healthy tissues. The radionuclide can be bound to the surface of the nanoparticles by surface modification using bifunctional chelators which binds to the nanoparticle and quickly binds the metal into a stable complex.

The properties of magnetic nanoparticles make it possible to use them both in diagnostics as a contrast for MRI and magneto-mechanical therapy. Strontium hexaferrite nanoparticles are one of the few hard magnetic materials on the basis of which biocompatible stable colloidal solutions can be obtained.

In this work, conjugates of nanoparticles of strontium hexaferrite coated with silicon dioxide (the plate-like shape of nanoparticles with an average diameter of $40 \mathrm{~nm}$ ) and azacrown ether L with six nitrogen atoms in a macrocycle were obtained by chemical surface modification.

Modified strontium hexaferrite nanoparticles were analyzed by IR and Raman spectrometry. Confirmation of the modification of the surface of nanoparticles at each stage were also studied by a series of $\zeta$-potentials measurements at different $\mathrm{pH}$ values in the range from 2 to $11(\mathrm{I}=0.01 \mathrm{M})$ and determination of the isoelectric point by dynamic light scattering. It was shown that the modification of the particles surface with amino groups leads to a shift of the isoelectric point to the region of higher $\mathrm{pH}$ values, while the modification of nanoparticles by the ligand shifts it to the region of lower $\mathrm{pH}$ values. It was demonstrated by competitive liquid extraction that the resulting conjugates form a stable complex with ${ }^{207} \mathrm{Bi}^{3+}$ at bismuth concentrations of $10^{-9} \mathrm{M}$ and particle concentrations of $10^{-3} \mathrm{~g} / \mathrm{l}$. Furthermore, the labeled nanoparticle-ligand-radionuclide structure was investigated for their stability in biologically significant media by thin layer chromatography. Thus, conjugates of strontium hexaferrite nanoparticles and azacrown ether L can be further studied as a potential component of radiopharmaceutical for theranostics.

Acknowledgments: The research was funded by RFBR and Moscow city Government, project number 21-33-70023. 


\title{
Consideration of reactor and photonuclear pathways to produce ${ }^{195 \mathrm{~m}} \mathrm{Pt}$ for theranostics applications
}

\author{
Alexander Madumarov, Nikolay Aksenov, Gospodin Bozhikov
}

Joint Institute for Nuclear Research, Dubna, Russia

https://doi.org/10.21175/rad.abstr.book.2021.23.6

Platinum-based drugs are widely used in chemotherapy for the treatment of various malignancies. It is crucial to know how these biomolecules spread in the body and assess the individual response to the treatment conducted. Diagnostic platinum radioisotopes such as ${ }^{191} \mathrm{Pt}$, ${ }_{195 \mathrm{mPt}}{ }_{197} \mathrm{Pt}$, attached to a drug would allow us to carry out molecular imaging. It was mentioned that the high yield of ${ }^{195 \mathrm{~m} P t}$ Auger electrons (33 electrons per decay) makes this radioisotope a suitable candidate for theranostics [1].

There are reactor and accelerator methods to produce ${ }^{195 \mathrm{mPt}}$, which include enriched ${ }^{194} \mathrm{Pt}$ irradiation at a nuclear reactor, and enriched ${ }^{192} \mathrm{Os}$ bombardment by $\alpha$-particles. The neutron cross section for the reaction ${ }^{194} \mathrm{Pt}(\mathrm{n}, \mathrm{g})^{195 \mathrm{~m} P t}$ is $0.09 \mathrm{~b}$ and leads to the product with a carrier and thus low specific activity. Reaction ${ }^{192} \mathrm{Os}(\mathrm{a}, \mathrm{n}){ }^{195 \mathrm{~m} P t}$ provides specific activity by two orders of magnitude higher compared to the reactor production but the yield and cross section being $3.7 \mathrm{mb}$ is much lower.

Therefore, conventional methods are currently unable to provide enough specific activity to meet demands for radionuclide therapy, so it is relevant to continue research on ${ }^{195 \mathrm{~m}} \mathrm{Pt}$ production possibilities.

In this work, two novel promising methods are addressed: neutron irradiation of iridium enriched in ${ }^{193} \mathrm{Ir}$ and photonuclear production based on natural platinum irradiation.

The first method of iridium irradiation is based on the reaction ${ }^{193} \operatorname{Ir}(n, \gamma)^{194} \operatorname{Ir}(n, \gamma)^{195 \mathrm{~m}} \operatorname{Ir} \beta^{-} \rightarrow{ }^{195 \mathrm{~m} P t}$. In order to make conclusion on the perspectives of this method it is crucial to know cross sections of all the reactions involved. So the aim of this work was to determine previously unknown parameters of the reaction ${ }^{194} \operatorname{Ir}(n, \gamma)^{195 \mathrm{~m}} \mathrm{Ir}$. Two targets of enriched iridium with and without Cd cladding respectively were irradiated for 17 days at the reactor IBR-2. Because of the enormous radiation background, one had to separate the product ${ }^{195 \mathrm{mPt}}$ from iridium bulk radiochemically. The targets were processed separately, the procedure included electrochemical dissolution followed by separation by extraction chromatography on TEVA of no-carrier-added platinum from macroamounts of iridium. Then based on the radioactivity of ${ }^{195} \mathrm{mPt}$ in both targets and using Cd difference technique to separate resonance neutrons effect from thermal neutrons one we found that production on thermal neutrons is almost negligible and estimated for the first time cross section on resonance neutrons of the reaction ${ }^{194} \operatorname{Ir}(n, \gamma)^{195 \mathrm{~m}} \mathrm{Ir}$ to be 2940.

The second method of photonuclear production involved irradiating a mixture of cisplatin and cryptomelane. The latter served as a recoil nuclei collector; therefore, a higher specific activity was achieved due to the Szilard-Chalmers effect.

\section{References}

[1] Nadar, R.A., Farbod, K., der Schilden, K.Cv. et al. Targeting of radioactive platinum-bisphosphonate anticancer drugs to bone of high metabolic activity. Sci. Rep. 10, 5889 (2020). https://doi.org/10.1038/s41598$\underline{020-62039-2}$ 


\section{The concept of developing a security plan for nuclear medicine facilities in the Republic of Serbia}

\section{Vojislav Antic', Milos Mladenovic², Mirjana Petrovic', Zivorad Savic ${ }^{1}$}

1 University Clinical Center of Serbia, Beograd, Serbia

2 Nuclear Facilities of Serbia, Beograd, Serbia

\section{https://doi.org/10.21175/rad.abstr.book.2021.23.7}

In nuclear medical diagnostics, the security risk correspond with 99mTc / 99Mo generator

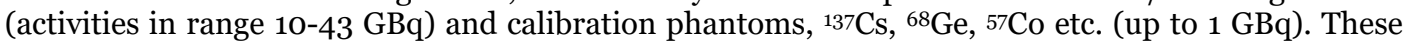
are the second and primary third category sources, by both relevant approaches to categorization (activity coefficient or application). Due to the low risk, from a security point of view, relevant issues have not been addressed in the literature by now. On the other hand, the hospital environment is favorable for potential sabotage, and such activities, if successful, would, at the very least, lead to the cancellation of the clinical program and unwanted attention and image in the media. Therefore, (and) with the dedication of authors, taking into account the definition of an incident by the International Atomic Energy Agency, a new national legislation envisages security plan writing for a nuclear medicine facility. The imperative that is imposed is to achieve defense in depth and balanced protection, with relatively small investment, to ensure adequate interaction with the police in order to respond promptly, as well as to internally raise the level of safety culture. 


\title{
Express production of medical Bi-213 using various $\mathrm{Ac} / \mathrm{Bi}$ generators
}

\author{
Aleksandr Vasilievi,2, Stanislav Ermolaev1, \\ Aino Skasyrskaya ${ }^{1}$, Elena Lapshina ${ }^{1}$, Boris Zhuikov ${ }^{1}$
}

\author{
1 Institute for Nuclear Research of the Russian Academy of Sciences, Moscow, Russia \\ 2 Lomonosov Moscow State University, Moscow, Russia
}

\section{https://doi.org/10.21175/rad.abstr.book.2021.23.8}

The development and trial of radiopharmaceuticals based on radionuclides with high linear energy transfer and short range in tissue (alpha-particles or Auger-electrons emitters) on the one hand, and highly specific delivery molecules on the other, is an essential step towards personalized medicine in the field of cancer treatment. Ac-225 $\left(\mathrm{T}_{1 / 2}=9.9\right.$ days $)$ and its decay product Bi-213 (46 min.) are proven to be promising for radiotherapy. More than 500 patients were treated with radiopharmaceuticals containing these radionuclides (up to 2018).

A crucial problem of Ac-225 and Bi-213 wide applications is their low availability. The generator method of Ac-225 production from Th-229 cannot satisfy the needs of even ongoing clinical trials. A prospective method of producing ${ }^{225} \mathrm{Ac}$ (more than $1 \mathrm{Ci}$ for a 10-day run) by irradiation of natural thorium with medium-energy protons followed by chemical isolation has been developed at the Institute for Nuclear Research of the Russian Academy of Sciences (INR RAS). Ac-225 with a small ( 0.2\%) impurity of Ac-227, which also forms under the irradiation, is appropriate as a mother radionuclide for Ac-225/Bi-213 generator.

The literature describes generator systems Ac-225/Bi-213 based on both ion exchange and extraction chromatographic resins (Actinide Resin; UTEVA Resin, Triskem Int.). The initial activity of Ac-225 has been so far limited by the capabilities of production as well as the radiation resistance of the sorbents used in the generator.

The most studied is the Ac-225/Bi-213 generator based on a cation exchange resin of the type AG MP-50 (BioRad), and clinical tests are carried out using such generators. Systems of so-called multicolumn selective inversion generators (MSIG) were developed at PNNL, USA. Significant drawbacks of the existing generators are that they are designed for a radiochemically pure parent radionuclide with initial activity below $100 \mathrm{mCi}$.

Two unique Ac-225/Bi-213 generators have been developed in INR RAS. The first one - termoxide inverse generator (TIG) - is based on a modified inorganic sorbent made from annealed zirconium and yttrium oxides [1]. The other one named "Afrabis" is a two-column generator where Ac-225 is firmly retained by Actinide Resin on the first column, and Bi-213 is accumulated and concentrated due to the separation and decay of the short-lived Fr-221 on the second column with AG MP-50 resin [2]. These generator systems demonstrate high radiation resistance, express production of Bi-213, and reliability.

\section{References}

[1] Vasiliev, A. N., Ermolaev, S. V., Lapshina, E. V., Zhuikov, B. L., \& Betenekov, N. D. (2019) 225Ac/213Bi generator based on inorganic sorbents. // Radiochimica Acta, - vol. 107, no. 12. - p. 1203-1211.

[2] Ermolaev, S.V., Skasyrskaya, A.K., Vasiliev A.N. A Radionuclide Generator of High-purity Bi-213 for Instant Labeling. // Pharmaceutics, - in press.

Acknowledgments: This work has been supported by the Russian Science Foundation under Contract № 19-73-00348. 


\title{
Production of ${ }^{99 \mathrm{~m}} \mathrm{Tc}$ via ${ }^{100} \mathrm{Mo}(\gamma, \mathrm{n}){ }^{99} \mathrm{Mo}$ reaction using ${ }^{100} \mathrm{MoO}_{3}$ target at microtron MT-25
}

\author{
Ilya Chuprakovi,2, Alexander Madumarov',

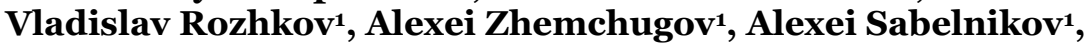 \\ Gospodin Bozhikov' ${ }^{1}$ Alexander Bodrov ${ }^{1}$, Nikolay Aksenov ${ }^{1}$
}

1 Joint Institute for Nuclear Research, Dubna, Russia

2 Institute for Nuclear Physics, Almaty, Kazakhstan

\section{https://doi.org/10.21175/rad.abstr.book.2021.23.9}

Currently the importance and scope of radionuclide diagnostics procedures increases which leads to a higher demand in medical radionuclides production. The main diagnostics radionuclide for radiopharmaceutical production is ${ }^{99 \mathrm{~m}} \mathrm{Tc}$ due to a mild monoenergetic gamma radiation (140 $\mathrm{keV}$ ) and a short half-life period $(6 \mathrm{~h})$ which allows to perform diagnostics with a lower patient radiation dose. That is why exploration of different accelerator techniques to produce aimed radionuclide

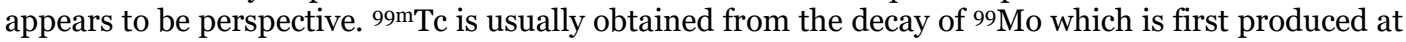
a nuclear facility and then used for the production of isotopic generator ${ }^{99} \mathrm{Mo} / 99 \mathrm{mTc}$. Photonuclear reaction ${ }^{100} \mathrm{Mo}(\gamma, n){ }^{99} \mathrm{Mo}$ as well as production of ${ }^{99} \mathrm{Mo} /{ }^{99 \mathrm{~m}} \mathrm{Tc}$ generator was earlier investigated by our co-author A. V. Sabelnikov [1]. This method provides carrier free $99 \mathrm{~m}$ Tc with activities high enough for large scale production and is preferable with respect to the chemical purity of the radionuclide of interest.

The important task of our experiment was to demonstrate potential of a position sensitive semiconductor pixel detector Timepix with a sensor of $2 \mathrm{~mm}$ CdTe for conducting SPECT imaging as a new approach for better resolved diagnostics [2]. For this goal we implemented 99mTc produced by irradiation of enriched ${ }^{100} \mathrm{Mo}$ at the electron accelerator MT-25 in FLNR JINR. The powder of ${ }^{100} \mathrm{MoO}_{3}$ was irradiated for 6 days at $10.5 \mathrm{mkA}$ current by $23 \mathrm{MeV}$ bremsstrahlung. After the irradiation we dissolved ${ }^{100} \mathrm{MoO}_{3}$ in $2 \mathrm{M} \mathrm{NaOH}$ and used this solution to make an isotope generator of ${ }^{99 \mathrm{~m}} \mathrm{Tc}$. Isotope generator was prepared using a known technique [3] that we optimised to better suit the conditions of our experiment. Based on the activity of molybdenum solution after the irradiation we calculated the yield of ${ }^{99} \mathrm{Mo}$ to be $583 \mathrm{~Bq} /(\mathrm{mkA} \cdot \mathrm{mg} \cdot \mathrm{h}) .{ }^{99 \mathrm{mT}} \mathrm{Tc}$ was later used to prepare samples to test the Timepix detector. The results will be shown at the conference.

\section{References}

[1] А.В. Сабельников, О.Д.Маслов, Л.Г. Молоканова, М.В. Густова, С.Н. Дмитриев. // Радиохимия. 2006. 48(2), 172-175.

[2] V. Kraus et al. FITPix - fast interface for Timepix pixel detectors// Journal of Instrumentation. 2011. p.1-6.

[3] Михеев Н.Б. и др. Генератор технеция-99м // Радиохимия. 1971. Т. 13. - 4. С. 631-633. 


\title{
Hair samples' retrospective analysis for the determination of drug consumption by applying enzymatic hydrolysis methods
}

\author{
Marina Krysko, Olga Strelova, Iuliia Slustovskaia
}

Saint-Petersburg State Chemical-Pharmaceutical University, Saint-Petersburg, Russia

https://doi.org/10.21175/rad.abstr.book.2021.24.1

In the last 20 years, a great deal of evidence has proved that the number of abusive people has increased. Therefore, it is necessary to develop new monitoring procedures that can determine short-term consumption of drugs, as well as a long-term one.

The aim of our research was to prove the possibility of utilizing hair for establishing long-term consumption (weeks/months) of drugs. All experiments were performed using Agouti Guinea pigs with different types of natural hair colour. The extracts obtained from the hair samples were analyzed by applying Gas Chromatography with mass-selective detection method.

All laboratory animals were kept in the experimental biological clinic (vivarium) that met the requirements of international laboratory rules (Good Laboratory Practice, GLP). Guinea pigs were divided into two separate groups and each group was fed a water solution of phenobarbital and diphenhydramine (the concentration of the solution corresponded with the maximum daily dose for humans). Then, after 6-months of consuming the aforementioned substances, the feeding of guinea pigs was stopped, and their urine was collected for a duration of two weeks. According to the results, phenobarbital was determined in the urine samples up to 11 days after the last consumption, and diphenhydramine up to 4 days after.

The guinea pigs' hair was collected 6 weeks after ending their consumption of the solution. The hair gathered $(3 \mathrm{~cm})$ was then divided into two segments - the root and the end of the strands (1.5 $\mathrm{cm}$ each). All segments were destroyed separately by applying enzymatic hydrolysis methods developed by the authors as follows: a solution of each enzyme $(0.5 \mathrm{mg} / \mathrm{ml}$; phosphatic buffer, $\mathrm{pH} 7.4)$ - trypsin, chymotrypsin and chymopsin - and a solution of papain enzyme $(0.5 \mathrm{mg} / \mathrm{ml}$; acetic buffer, $\mathrm{pH} 4.7$ ). These were blended with the hair samples ( $0.4 \mathrm{~g})$ and maintained for three hours at $37^{\circ} \mathrm{C}$ twice. The total time of hydrolysis was 6 hours. The methods of acidic and alkali hydrolysis were used at the same time as the enzymatic ones to enable an effective comparison between the two. Phenobarbital and diphenhydramine were isolated from the hydrolysates by liquidliquid extraction (at selective $\mathrm{pH}$ ).

The methods of acidic and alkali hydrolysis were ineffective for the retrospective analysis application since the amount of substances were 2-3 times less in comparison with the results of the enzymatic ones. Moreover, the RSD (\%) of acidic and alkali hydrolysis methods did not meet the necessary requirements.

The developed methods of enzymatic hydrolysis allowed to establish the fact of long-term drugs' consumption. In fact, phenobarbital was isolated more from the root segments, whereas diphenhydramine was mainly extracted from the end segments. Thus, these methods presented their effectiveness and applicability for the retrospective analysis of various natural hair colour samples to prove long-term drug usage. 


\title{
Feasibility of introducing telepharmacy elements in low-income countries
}

\section{Iryna Timanyuk, Iryna Bondarieva, Volodymyr Malyi}

\author{
National University of Pharmacy, Kharkiv, Ukraine
}

https://doi.org/10.21175/rad.abstr.book.2021.24.2

Relevance. The majority of people in low-income countries are currently unable to pay adequate attention to their health. In particular, this is due to the accessibility of the necessary specialist in the area of residence, difficulties in finding and purchasing of necessary medicines. Integration of telepharmacy services will improve accessibility and quality of health care services.

Purpose: To justify the possibility of introducing elements of telepharmacy in low-income countries, using Ukraine as an example.

Results. Currently, telepharmacy in Ukraine is practically non-existent, only some of its components, such as telephone counselling. In other countries, however, the so-called "telephone pharmacy" has long been in operation as a way of delivering pharmaceutical products and services via telecommunications. In this way, patients can receive their medicines and other pharmaceutical products without leaving a place where they can easily obtain these services. There are services that deliver medicines to remote locations as well as labelling them for use. These services are mainly provided by retail pharmacies, but can also be provided by nursing homes, hospitals and other health care facilities. Telepharmacy is also used by pharmacies not only to provide pharmaceutical services, but also for training, education, and management services.

The introduction of vending machines can be considered as an element of telepharmacy in lowincome countries. Two main types have been considered in the paper: machines that have the possibility to contact a pharmacist, machines that only dispense medicines.

When the dispensers are of the closed type (the machines are built into buildings and are protected as much as possible), prescription medicines can be sold. Their main function is to facilitate the work of pharmacists in selling medicines for chronic diseases, where the additional consultation of a pharmacist is not required. In this case, the patient can receive the same medication using his code or other identifier at any convenient time for him, without standing in queues. Such machines can hold up to 70 different types of medicines.

Other vending machines can be placed indoors or outdoors to sell over-the-counter medicines and medical products. Such vending machines can be placed in remote and inaccessible towns and villages, as well as in airports, train stations and other crowded places. This is applicable when it is not feasible to maintain a pharmacist, pay rent for premises and incur other costs.

Conclusion. The results of these studies have demonstrated the feasibility and necessity of introducing self-service facilities in low-income countries to improve access to pharmaceutical services for people living in remote areas. 


\title{
Estimation of the reproducibility for the identificational method of paracetamol-based drugs
}

\author{
Viktoria Tihonova, Anna Saushkina
}

Federal State Budgetary Educational Establishment of Higher Education "St. Petersburg State ChemicalPharmaceutical University", Saint Petersburg, Russia

\section{https://doi.org/10.21175/rad.abstr.book.2021.24.3}

53 commercial names of paracetamol drugs are circulated on the Russian pharmaceutical market. They are exposed in 10 dosage forms: granules for preparation of a suspension for oral administration, infusion solution, solution for oral administration, syrup, rectal suppositories, suspension for oral administration, tablets, film-coated tablets, soluble tablets, effervescent tablets. Variety and affordability have determined the choice of paracetamol preparations for assessing the capabilities of Raman spectroscopy in the pharmaceutical analysis [1].

Comparative evaluation of the substance's Raman spectra and the investigated paracetamol preparations showed characteristic intense bands in the terahertz range $(5-250 \mathrm{~cm}-1)$. In our opinion, their similarity is resulting from the presence of paracetamol as the main component. The other spectral regions have significant differences which can be caused by excipients and fillers.

The spectra of paracetamol solution for parenteral use and paracetamol syrup recorded by a similar method [2] showed their lack of information, since the water in their composition masks the spectral pattern due to intense fluorescence.

The reproducibility of the Raman spectra was studied at the position of 5 maxima of analytical signals $(85.8 \pm 2 ; 391.3 \pm 2 ; 858.2 \pm 2 ; 1169.3 \pm 2 ; 1655.2 \pm 2) \mathrm{cm}-1$ on four substances of paracetamol produced by different manufacturers at exposure time to laser radiation 20,60 and 120 seconds. It was found that the best reproducibility is observed in the middle spectral range for any duration of analysis, and the shape of the spectral signals did not change at the same duration of exposure to laser radiation both in each series and between series. The maximum deviation in the position of the spectrum maxima of $1.13 \%$ indicates a good reproducibility of the analytical method [3].

Our investigation has shown the capability of identifying pharmaceutical substances and paracetamol solid dosage forms by the method of Raman spectroscopy.

\section{References}

[1] Gosudarstvennyj reestr lekarstvennyh sredstv. Available at: https://grls.rosminzdrav.ru/Default.aspx (Accessed 20 January 2021).

[2] Tihonova V.V. Razrabotka metodiki identifikacija sostava mnogokomponentnyh tverdyh lekarstvennyh form metodom spektroskopii kombinacionnogo rassejanija na primere tabletok rastvorimyh "Panadol" / V.V. Tihonova, A.S. Saushkina, I.S. Shul'c // Izvestija rossijskoj voenno-medicinskoj akademii. - 2020.- №1.- C. 175-181. (In Russ).

[3] Gosudarstvennaja Farmakopeja RF: T. I-IV. 14-e izd. Federal'naja jelektronnaja medicinskaja biblioteka. Available at: http://www.femb.ru/feml (Accessed 20 January 2021). 


\title{
The development of a certified reference material for Pharmaceutical Quality Assurance based on genistein
}

\author{
Anna Zhigalina, Ol'ga Strelova, Alexsandr Grebenuk, Elizaveta Cekhanskaya
}

Federal State Budgetary Educational Establishment of Higher Education "St. Petersburg State ChemicalPharmaceutical University”, St. Petersburg, Russia

\section{https://doi.org/10.21175/rad.abstr.book.2021.24.4}

One of the priority directions in the development of modern medicines is a search for highly effective drugs with a wide spectrum of pharmacological action and low toxicity. Naturally occurring substances, genistein in particular, are of great interest. Experimental and clinical studies have demonstrated that genistein has radioprotective properties. The possibility of creating a medicine based on it as addition to radiation therapy of tumors has been shown [1]. The use of certified reference materials (CRMs) ensures metrological traceability and comparability of analysis results. However, development, registration and practical application of CRMs face problems such as the lack of a unified understanding of the term CRM in various regulatory acts of the Russian Federation, the diversity of the classification of CRMs. Translation of existing in European Pharmacopoeia (EP), United States Pharmacopoeia (USP), International Pharmacopoeia, and ISO Guide 30:2015 international terms refer to CRM into Russian gives several parallel terms. The aim of our study is development of the CRM and regulatory documentation for synthetic genistein to ensure quality assurance of medicines. Natural genistein is an isoflavone derived from plants of the legume (Fabaceae). A comparative analysis of natural genistein and synthetic genistein was carried out. In the course of the study, quality attributes of synthetic genistein were determined: characteristics, solubility, identification (color reactions, UV spectrophotometry and IR spectroscopy), melting point, specific absorbance [2]. The study of samples by gas chromatography with mass selective detection showed the peaks of the same type for the molecular ion (trimethylsilyl derivative) with $\mathrm{m} / \mathrm{z} 486.2$, the base peak 471.1 and the splinter peaks of ions 73.0, 207.0, 228.0, 281.0, 399.1. Automatic processing using a specialized library "NISTo8.L" using the AMDIS program showed that the studied mass spectra with a probability of at least $90 \%$ coincided with the spectrum of $5,7,4^{\prime}$-tri (trimethylsilyl) genistein [3]. Thus, the creation of CRMs for synthetic genistein will ensure the quality of drugs and dietary supplements containing this substance and will be more widely used in medicine.

\section{References}

[1] Grebenjuk A.N., Basharin V.A., Tarumov R.A. [i dr.]. Ocenka antioksidantnyh svojstv otechestvennogo sinteticheskogo genisteina na modeljah in vitro i in vivo // Vestnik Rossijskoj Voenno-medicinskoj akademii. 2013. № 2. S. 83-87.

[2] Gosudarstvennaja Farmakopeja RF: T. I-IV. 14-e izd. Federal'naja jelektronnaja medicinskaja biblioteka. Available at: http://www.femb.ru/feml (Accessed 21 January 2021).

[3] Strelova O.Ju., Volkova K.V., Grebenjuk A.N., Teslov L.S. Ocenka pokazatelej kachestva perspektivnoj farmacevticheskoj substancii na osnove sinteticheskogo genisteina // Butlerovskie soobshhenija. 2016. T. 48, № 12. S. 94-101. 


\title{
Diffusion in POEGMA hydrogels prepared by Radiation Induced Crosslinking Polymerization
}

\author{
Krzysztof Piechocki', Marcin Kozanecki', \\ Kaloian Koynov², Justyna Piechocka ${ }^{3}$
}

\author{
1 Department of Molecular Physics, Lodz University of Technology, Lodz, Poland \\ 2 Max Planck Institute for Polymer Research, Mainz, Germany \\ 3 Department of Environmental Chemistry, University of Lodz, Lodz, Poland
}

\section{https://doi.org/10.21175/rad.abstr.book.2021.25.1}

Stimuli responsive hydrogels based on poly(olygoether methacrylates) (POEGMAs) obtained by Radiation Induced Crosslinking Polymerization (RICP) are regarded as promising biomaterials for storage, transport and release of low-molecule substances. Due to biomedical applications, like drug delivery systems, the radiation-induced methods of synthesis offering simultaneous product sterilization are extremely desired to prepare such materials. The process of controlled release is induced by thermally generated Volume Phase Transition, that is a consequence of instable balance between polymer and water. The polymer network collapse and water with low-molecule substance is released to external environment. When considering POEGMA hydrogels as transport and release systems, diffusion processes in the hydrogel matrix are very important.

This project is focused on an influence of POEGMAs structure and radiation dose used for RICP process, and as a consequence polymer concentration for diffusion in hydrogel matrix. POEGMA hydrogels were prepared by RICP and after cleaning swollen by fluorescent dye solvent for free diffusion studies or by ibuprofen sodium salt solvent for release studies. Free diffusion was studied with Fluorescence Correlation Spectroscopy, whereas release was observed by HPLC. Obtained results show that structure of polymer (length of side groups) impacts on diffusion processes. Free diffusion and kinetic of release may be adjusted by dose of radiation used for RICP. It is also good to point out that radiation dose shifts the balance between polymer network and low molecule substance in the hydrogel matrix.

Acknowledgments: The Project is carried out under the PROM Programme - International Scholarship Exchange of PHD Candidates and Academic Staff, co-financed granted from the European Union, including the European Social Fund within the framework of the Knowledge Education Development Operational Programme, non-competitive project entitled: International Scholarship Exchange of PhD Candidates and Academic Staff, contract number POWR.03.03.00-00-PN13/18. 


\title{
Reduction of silver ions using chitosan and investigation of their reactivity
}

\section{Irina G. Antropova, Natalia V. Panferova, Eldar P. Magomedbekov}

\author{
Mendeleev University of Chemical Technology of Russia, Moscow, Russia
}

https://doi.org/10.21175/rad.abstr.book.2021.25.2

Chitosan (CS) is a chitin derivative and it is the second most common polysaccharide after cellulose. Its qualities are non-toxicity, biodegradability, antibacterial activity, ability to form complexes, etc.

Currently, there are many works describing the useful properties of CS, including as an acceptor or solution for stabilizing metal nanoparticles (NP). In this work, attention is paid to the reduction of silver ions using an aqueous solution of low molecular weight CS with a molecular weight of $11 \mathrm{kDa}$ (CS-11) and $48 \mathrm{kDa}(\mathrm{CS}-48)$ with a degree of deacetylation of $3 \%$ (Bioprogress, Russia). The process of obtaining nanosilver particles was carried out by photo- and X-ray exposure to the systems in the presence of oxygen. It is shown that the Ag-NPs size is $2040 \mathrm{~nm}$ under X-ray exposure to the $\mathrm{CS} / \mathrm{Ag}+$ system.

The work revealed the antioxidant properties of an aqueous chitosan solution due to the interception of the hydroxyl radical. In this work, the relative rate constant of the interaction of $\mathrm{CS}$ with $\mathrm{OH}$ radicals was determined by the method of competing reactions using terephthalic acid. It has been shown that the molecular weight of CS affects its reactivity in the reaction with a hydroxyl radical; the higher the molecular weight, the higher it is. When studying the CS system with silver ions in water, it was shown that the relative rate constant of the CS-11 / Ag+ reactions with the $\mathrm{OH}$ radical is 2 times higher than the rate constant of the CS-48 / $\mathrm{Ag}+$.

Acknowledgments: The work was supported by Mendeleev University of Chemical Technology of Russia. Project Number 2020-16. 


\title{
TBA-MDA test for lipid peroxidation detection induced by UV-A and UV-B irradiation of Protoporphyrin IX in SUV liposomes
}

\author{
Aleksandar Lazarević, Sanja Petrović, \\ Jelena Stanojević, Dragan Cvetković, Jelena Zvezdanović
}

University of Niš, Faculty of Technology Leskovac, Serbia

\section{https://doi.org/10.21175/rad.abstr.book.2021.25.3}

Protoporphyrin IX (PPIX) is a well-known photosensitiser with great potential for use in photodynamic therapy (PDT). However, PPIX is a highly hydrophobic and cannot be dissolved in water which represents an environment suitable for PDT applications. So liposomes (as multilamellar or unilamellar phospholipidic submicroscopic vesicles) allow the incorporation of lipophilic and hydrophilic drugs such as PPIX in their matrix with potential for the delivery of various bioactive molecules used for therapeutic applications. Because of that it can be very important to investigate how PPIX incorporated inside the liposomes lipid bilayers influences lipid peroxidation (LP) - process applied in various fields in medicine - PDT and pharmacy. The most prominent and currently used assay for detection and recording of LP is the spectrophotometric thiobarbituric acid-malondialdehyde test (TBA-MDA).

PPIX-loaded liposomes were prepared using dry PPIX-lipid film method and extrusion through filters with $100 \mathrm{~nm}$ pores to obtain SUV (small unilamellar vesicles) liposomes. Lipid peroxidation was initiated by photosensitization reaction of PPIX-SUV system with continual UV-A and UV-B irradiation. The formation of the LP product malon-aldehyde (MDA), was measured spectrophotometrically (TBA-MDA test). UV-A and UV-B irradiation treatments of PPIX-SUV mixtures (with or without the photosensitizer PPIX) was performed in cylindrical photochemical reactor with emission maximum at 350 and $300 \mathrm{~nm}$, with 10 symmetrically placed $\mathrm{Hg}$-lamps and the corresponding flux at $12.9 \mathrm{Wm}^{-2}$ and $15 \mathrm{Wm}^{-2}$, respectively.

The continual UV-A and UV-B irradiation of PPIX-SUV complex resulted in lipid peroxidation process - continuous increase in absorbance band responsible for LP product. Lipid peroxidation process obeys first-order kinetics. The calculated rate constants of LP products growth are 0.0105 $\mathrm{min}^{-1}$ and $0.0439 \mathrm{~min}^{-1}$ for UV-A and UV-B irradiation, respectively. Lipid peroxidation process was not observed in the samples without photosensitizer, or samples with photosensitizer kept in dark. The results are in accordance with the known fact that UV-B irradiation is more energetic in comparison with UV-A and due to that photosensitiser PPIX in SUV liposomes is more excited causing faster lipid peroxidation process. Further, it is shown that PPIX-SUV complex is a stable with PDT potential, and photosensitiser can express oxidative potential.

Acknowledgments: This work was supported by the Ministry of Education, Science and Technological Development of the Republic of Serbia under the Program of financing scientific research work, number 451-03-68/2020-14/200133. 


\section{Press molded $\mathrm{TIBr}$ semiconductor crystals for flat panel gamma-ray imaging detectors}

\section{Toshiyuki Onodera', Keitaro Hitomi²}

1 Tohoku Institute of Technology, Sendai, Japan

2 Tohoku University, Sendai, Japan

\section{https://doi.org/10.21175/rad.abstr.book.2021.26.1}

Thallium bromide (TlBr) is a compound semiconductor characterized with high gamma-ray absorption, wide band-gap energy, low melting point. In this study, press mold method was used for crystalizing $\mathrm{TlBr}$ from a starting powder material for the first time. $\mathrm{TlBr}$ powder with nominal purity of 99.99 \% was loaded into a metallic mold made from stainless steel (SUS316) and sandwiched between plates made from ELMAX. After pressing the materials up to $50 \mathrm{kN}$ at around $1 \mathrm{~Pa}$, the materials were heated up to around $400{ }^{\circ} \mathrm{C}$ for $20 \mathrm{~min}$. and cooled by turning off a furnace. Size of $\mathrm{TlBr}$ crystals molded in this procedure were around $20 \mathrm{~mm} \times 20 \mathrm{~mm} \times 2 \mathrm{~mm}$. Planar type $\mathrm{TlBr}$ detectors were fabricated from the TlBr crystals. Detector performance has been evaluated by testing gamma-ray energy spectral response. 


\title{
Development of a shielding design for a boron-10 zinc sulfide silver-activated $\left[{ }^{10} \mathrm{~B} / \mathrm{ZnS}(\mathrm{Ag})\right]$ detector using radiation counting and $M C N P$ modeling
}

\author{
Alexander Macris' ${ }^{1}$ Kevin McKay', \\ Cheryl Brabec², William Charlton', Sheldon Landsberger'
}

1 University of Texas at Austin, Austin, Texas, United States

2 Los Alamos National Laboratory, Los Alamos, New Mexico, United States

\section{https://doi.org/10.21175/rad.abstr.book.2021.26.2}

For radiological surveying application, neutron detectors require shielding to minimize contributions from sources outside the area of interest. When taking radiation measurements using it is often useful to have a MCNP simulation by which to benchmark the results. To best model a detector's response to incident radiation, a detailed MCNP geometry must be developed to account for the different types of interactions that occur within the detector materials. In this research, MCNP models for a ${ }^{10} \mathrm{~B} / \mathrm{ZnS}(\mathrm{Ag})$ neutron detector were developed and tested against experimental counts recorded from two PuBe (plutonium-beryllium) sources. Using data from these experiments as well as information obtained directly from the detector's manufacturer (Bridgepoint Instruments, Austin), two models for this type of detector were developed-- both of which returned values within $7 \%$ of the experimental data. 


\title{
A preliminary investigation for the use of gamma-gamma coincidence spectrometry to determine ${ }^{239} \mathrm{Pu}$
}

\author{
Conny Egozi',2, Francis Martinez², Brandon De Luna', Sheldon Landsberger'
}

1 The University of Texas at Austin, Austin, United States

2 Los Alamos National Laboratory, Los Alamos, United States

\section{https://doi.org/10.21175/rad.abstr.book.2021.26.3}

Gamma-ray spectrometry is one of the most effective ways to determine the activity of ${ }^{239} \mathrm{Pu}$. However, often high backgrounds in complex spectra with low amounts of ${ }^{239} \mathrm{Pu}$ can increase detection limits. The effectiveness of the use of gamma-gamma spectrometry in the characterization of ${ }^{239} \mathrm{Pu}$ has been initiated. The gamma-gamma system was set up with a ${ }^{60} \mathrm{Co}$ source positioned equidistant between the two detectors, which were at $180^{\circ}$ to each other using the digital PIXIE XIA system. Spectra were generated at various detector to source distances, then the net area counts from each spectrum were used at the ${ }^{60} \mathrm{Co} 1173 \mathrm{keV}$ energy peak to create graphs for both single and coincidence gammas for the 1173 and $1332 \mathrm{keV}$ energy peaks. Measurements were taken at $2 \mathrm{~cm}$ and $4 \mathrm{~cm}$ and found that the ratio $2 \mathrm{~cm}: 4 \mathrm{~cm}$ for singles was 2.03, whereas the ratio for coincidence was 3.91. This clearly shows that the distance between gamma ray spectrometers has a much bigger effort in counting statistics for the gamma-gamma coincidence detectors than a single detector. In addition, background radiation measurements were taken without any shielding around the system. And it was demonstrated that the background radiation to be virtually negligible, meaning gamma-gamma spectroscopy can still be very effective without requiring the usual lead shielding. Other measurements were taken to assure Poisson statistics were attained in the digital system. Preliminary measurements revealed very low detection limits for the measurement of ${ }^{239} \mathrm{Pu}$. 


\title{
Silicon Drift Detectors system for high precision nuclear physics spectroscopy measurements: The SIDDHARTA-2 experiment at the DAФNE Collider
}

\begin{abstract}
Marco Miliucci
LNF-INFN, Frascati, Italy

https://doi.org/10.21175/rad.abstr.book.2021.26.4

The SIDDHARTA-2 Collaboration is going to perform for the first time the precision X-ray spectroscopy measurement of the kaonic deuterium transition from the $2 \mathrm{p}$ to $1 \mathrm{~s}$ level, at the DAФNE Collider of the LNF-INFN. Besides its high scientific outcome in the nuclear physics field, the new Silicon Drift Detectors (SDD) systems specially developed by the SIDDHARTA-2 Collaboration for this project represents a significant improvement in the field of X-ray detectors, thanks to their excellent spectroscopic performance even when exposed to the high radiation environment of the particle colliders. We shall present the results of the detailed characterizations of these new SDDs together with its dedicated front end electronics in laboratory and on the DAФNE collider, together with the updated SIDDHARTA-2 activities.
\end{abstract}




\title{
Corrections of HPGe detector efficiency curve due to true coincidence summing by program EFFTRAN and Monte-Carlo simulations
}

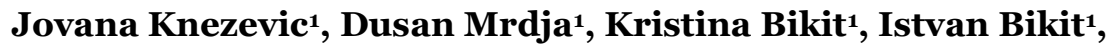 \\ Jaroslav Slivka1, Sofija Forkapic ${ }^{1}$, Predrag Kuzmanovic',2
}

\begin{abstract}
1 Department of Physics, Faculty of Sciences, University of Novi Sad, Novi Sad, Serbia
2 Academy of Professional Studies Šabac, Department of Medical and Business-Technological Studies,

Laboratory for Physics, Sabac, Serbia
\end{abstract}

\section{https://doi.org/10.21175/rad.abstr.book.2021.26.5}

Efficiency calibration of the HPGe detector is an important task in the field of gamma spectrometry, providing possibility of quantitative determination of radionuclide activity within different samples. Besides the influence of sample geometry and matrix, the effects of true coincidence summing of gamma quanta should be taken into account. In this work we calibrated large-volume HPGe detector by certified cylindrical source in contact geometry, containing the following radionuclides of known activity: Eu-152, Y-88, Ce-139, Co-60, Cd-109, Sn-113, Co-57, Cs137, and Am-241. The obtained detection efficiency curve covered energy region of $60 \mathrm{keV}-1850 \mathrm{keV}$. In the second step, EFFTRAN software was used for determination of true coincidence summing correction factors, leading to the modified efficiency curve. The relative differences between uncorrected and corrected efficiency values were found. The most prominent discrepancy was found for Eu-152: up to 40\%. These results showed that this kind of efficiency curve corrections should not be neglected in gamma spectrometry measurements. In addition, Monte-Carlo simulations of the radioactive decay of above-cited radionuclides were performed in order to obtain and compare detection efficiency curve without taking into account coincidence summing. Furthermore, by choosing the pure gamma-rays emitted from corresponding radionuclides, the true coincidence summing effects were considered, thus the simulated and experimental curve were analyzed and compared in this case as well. 


\title{
Design and characterization of a compact radiation monitor for space rockets
}

\author{
Abdulrahman Albarodi' ${ }^{1}$ M. Bilge Demirköz', Aziz Ulvi Çalışkan², \\ Uğur Kılıç', Deniz Orhun Boztemur'1, Ahmet Baran Can'1, \\ Egecan Karadöller'1, Mehmet Güntekin Kabuli², Levent Tavacioğlu²
}

\author{
1 Middle East Technical University, Ankara, Turkey \\ 2 Tubitak Yital, Istanbul, Turkey
}

\section{https://doi.org/10.21175/rad.abstr.book.2021.26.6}

A simple radiation monitor which incorporates a Geiger counter and silicon detectors was designed and tested for radiation measurements on Turkish space rockets. The device houses two large area silicon PIN detectors, each with 4 quadrants produced in Turkey by TÜBİTAK BİLGEM UEKAE YITTAL laboratories, vertically aligned inside a thin aluminum shielding, separated by 3 PCBs as degraders, to perform coincidence logic and energy discrimination. Each quadrant is amplified separately to reduce the noise on readout cards designed by METU IVMER which generate logical signals per layer, which are then coincided in a 7.8ns time window by an FPGA. The prototype also incorporates a Geiger tube sensitive to electrons and gammas to compare the counts of particles outside the box measured during the test period. The I-V and C-V characterization of the PIN diodes, as well as detailed calibration of the readout electronics were performed. The device was tested at the METU-DBL proton beamline with 15 and $30 \mathrm{MeV}$ proton beams as well as radioactive alpha and beta sources and shown to be sensitive to different particle species. The dynamic range, which has been demonstrated up to $1.3^{*} 10^{\wedge} 6 \mathrm{p} / \mathrm{cm} 2 / \mathrm{s}$ lays the foundation for a robust radiation measurement with more detector and degrader layers for a larger energy range on a satellite over the South Atlantic Anomaly as well as the van Allen belts. 


\title{
Characterization of $\mathrm{GdAlO}_{3}$ as beta radiation detector
}

\section{Daniel Nolasco Altamirano', Teodoro Rivera Montalvo', Olivia Amanda García Garduño², Alejandro Alonso Sotolongo3, Juan Zárate Medina3}

\author{
1 Instituto Politecnico Nacional CICATA-Legaria, Ciudad de México, Mexico \\ 2 Instituto Nacional de Neurología y Neurocirugía Manuel Velasco Suárez, Ciudad de México, Mexico \\ 3 Universidad Michoacana de San Nicolás de Hidalgo, Morelia, Mexico
}

\section{https://doi.org/10.21175/rad.abstr.book.2021.26.7}

One of the most widely exploited methods for radiation detection and dosimetry is those based on luminescence phenomena. Among them, the most important for practical applications of ionizing radiation monitoring is the thermoluminescence technique. For this sense, various materials have been submitted under investigation as radiation detectors. High quality inorganic halide perovskite brings an opportunity for ideal electronic radiation detection due to its large carrier mobility, lifetime, and heavy atoms. Here, we report on the $\mathrm{GdAlO}_{3}$ perovskite powders synthesized using the

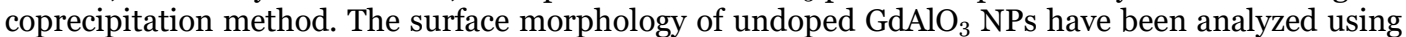
SEM images. SEM images were obtained for different calcination temperature. These images manifested that clusters are formed on the surface in large number by spherical particles but the most are formed by irregular forms. It is observed that the particles are highly agglomerated due to the high surface interaction between the nanoparticles. The X-ray diffraction (XRD) studies show that the synthesized powders can be indexed to nearly single-phase orthorhombic $\mathrm{GdAlO}_{3}$. The TL characteristics following ${ }^{90} \mathrm{Sr} /{ }^{\circ} \mathrm{Y}$ beta irradiation were studied. The TL intensity response of the synthesized samples are recorded for an irradiation at $11.1 \mathrm{~Gy}$ dose using beta radiation with heating rate $15^{\circ} \mathrm{C} / \mathrm{s}$. TL intensity is found to depend on the calcination temperature. The highest TL response was observed for powders submitted at $1500^{\circ} \mathrm{C}$. TL glow curve beta irradiated showed four peaks centered at $98,141,245$, and $306^{\circ} \mathrm{C}$ respectively. The two lower temperature peaks lose information during 24 hours, remaining the rest of the two peaks to study dosimetric properties. TL dose responses of the phosphors to beta doses from 1.1 up to $44 \mathrm{~Gy}$ showed fairly linear behavior. The kinetic parameters of the $\mathrm{GdAlO}_{3}$ samples were estimated by the free glow curve deconvolution, the curve fitting, and the peak shape methods. The results indicate that these phosphors can be considered to be promising as good candidates as a beta radiation detector. 


\title{
Angular dependence of Light-Dependent Resistors irradiated by $6 \mathrm{MV}$ photon beams
}

\author{
Juan Román-Raya1, Catalina A. Rodríguez-Cano', Isidoro Ruiz-García², \\ Alberto J. Palma², Miguel A. Carvajal², Damián Guirado1
}

1 San Cecilio Clinical University Hospital, Granada, Spain

2 University of Granada, Granada, Spain

\section{https://doi.org/10.21175/rad.abstr.book.2021.26.8}

Introduction. Light-Dependent Resistors (LDRs) are widely used as visible light detectors, due to its low cost and high sensitivity. In our previous works, it was experimentally tested that some LDR devices exhibit a promising performance as dose rate sensors, employing a digital multimeter or a clinical electrometer as reader units. In this work, a clinical electrometer was used to study the $\mathrm{LDR}$ radiation response dependence with the angle of incidence.

Materials and methods. LDR devices NSL-19M51 (Luna Optoelectronics, USA) and VT43N2 (Excelitas Technologies, USA) were characterized for $6 \mathrm{MV}$ photon beams produced by an Artiste linear accelerator (Siemens, Germany). LDRs were connected to a PC Electrometer (Sun Nuclear, USA) placed inside the treatment room. LDRs were painted with black nail polish in order to reduce the effect of environmental illumination and fixed inside a cylinder phantom. The gantry was turned from 0 to $2 \pi \mathrm{rad}$ in steps of $\pi / 6 \mathrm{rad}$. A CC13 chamber (IBA, Germany) was employed as reference chamber.

Results and conclusions. LDRs were first studied with different bias voltages between 30 and $150 \mathrm{~V}$. A higher sensitivity when high voltages were applied was observed. Therefore, a voltage of $150 \mathrm{~V}$ was chosen to monitor the angular dependence. Dose rate was calculated from both, the average current in the flat region during irradiation and the ratio between the time integral of the current when it was higher than three times the standard deviation of the base-line current and the irradiation time. To characterize the angular dependence, the devices were irradiated with a dose rate of $285 \mathrm{cGy} / \mathrm{min}$, obtaining a detector response variation up to $25 \%$ for both devices. The high sensitivity and the low price of LDRs make them a promising candidate for low cost dose measurements. It is important to carry out a complete physical characterization before studying its clinical use.

Acknowledgments: This work has been partially supported by the Consejería de Salud (PI-0505-2017) and the Consejería de Conocimiento, Investigación y Universidad (FQM387) of the Junta de Andalucía, the Spanish Ministerio de Ciencia y Competitividad (FPA2015-67694-P), the European Regional Development Fund (ERDF) and FEDER/Junta de Andalucía-Consejería de Economía y Conocimiento Project B-TIC-468-UGR18. 


\title{
Temperature compensation in dose-rate measurements based on commercial photodiodes using a modified reader unit
}

\author{
Isidoro Ruiz-García1, Pablo Escobedo, Juan Román-Raya², Stefan Illić3, \\ Damian Guirado², Alberto José Palma1, Miguel Ángel Carvajal1
}

1 University of Granada, Granada, Spain

2 San Cecilio Clinical University Hospital, Granada, Spain

3 Applied Physics Laboratory, Faculty of Electronic Engineering, University of Niš, Niš, Serbia

\section{https://doi.org/10.21175/rad.abstr.book.2021.26.9}

Introduction. The main application of photodiodes is to measure visible, ultraviolet or infrared light intensity. However, some authors have reported their use as dose rate sensor for X-ray photon beams (M. Andjelkovic et al., Radiation Measurements 75, 2015). Our research group previously developed a reader unit for MOSFET dosimeters that used the parasitic diode of DMOS transistors to apply a thermal compensation (M.A. Carvajal et al., Sensors and Actuators A 249, 2016). This compensation method measures the silicon temperature, not the room temperature, without external temperature sensors.

Due to the strong dependence of the photocurrent produced by photodiodes with temperature, base line will be strongly affected by the temperature in dose rate and absorbed dose measurements. Consequently, a thermal compensation during dose reading should be mandatory for accurate dose measurement by photodiodes as affordable skin dosimeter. In the present work, a thermal characterization of the photodiode BPW34S (Vishay Siliconix, USA) has been carried out. In addition, a new reader unit has been designed to implement this additional thermal compensation technique.

Experimental setup. A preliminary study has been done with five samples of the BPW34S photodiode. The semiconductor analyser B1500 (Agilent Technologies, Santa Clara, CA, USA) was used for devices biasing at $\mathrm{V}_{\mathrm{R}}=10 \mathrm{~V}$ and to measure the dark current. Temperature sweeps from 10 to $50{ }^{\circ} \mathrm{C}$ were conducted with a climatic chamber VCL4006 (Vötsch Industrietechnik, BalingenFrommern, Germany).

System design. In order to measure the internal temperature of the photodiode, a sink current source with a LM334 (Texas Instruments, Dallas, TX, USA) has been added to the reader unit. The working principle for thermal compensation is as follows: after dose measurement, the photodiode used as sensor is disconnected from both bias voltage source and current to voltage converter and connected between ground and sink current source. Then, the forward biased photodiode has a direct voltage drop proportional to temperature, typical of $\mathrm{p}$ - $\mathrm{n}$ junctions.

Results and conclusions. In the preliminary study, an exponential dependence was found, resulting in an average dark current at $25{ }^{\circ} \mathrm{C}$ of $(17 \pm 3)$ nA.. The designed reader unit will be tested to measure the temperature of the photodiode used as dose measurement sensor during an irradiation session. This would allow applying the temperature compensation algorithm to obtain an accurate dose rate and absorbed dose measurements minimizing the thermal uncertainty without requiring additional temperature sensors.

Acknowledgments: This work has been partially supported by the Junta de Andalucía with projects PI-0505-2017 and FQM387, the Spanish Government (FPA2015-67694-P) the European Regional Development Fund and FEDER/Junta de Andalucía Project B-TIC-468-UGR18. This work was also conducted in the framework of ELICSIR project funded by the European Union with grant No. 857558 . 


\title{
Semiconductor detectors for monitoring radiation processing
}

\author{
Roberto Bedogni ${ }^{1}$, Alessandro Lega ${ }^{1}$, \\ Alessandro Calamida ${ }^{1}$, Alessia Cemmi², Ilaria Di Sarcina²
}

\author{
1 INFN-LNF, Frascati, Italy \\ 2 ENEA Casaccia FSN-FISS-SNI, Roma, Italy
}

\section{https://doi.org/10.21175/rad.abstr.book.2021.26.10}

Radiation processing is commonly used in a variety of sectors, from sterilization to polymer processing and nanotechnology. According to the application, dose rate values from $\mathrm{Gy} / \mathrm{h}$ up to $\mathrm{MGy} / \mathrm{h}$ can be applied. Although a number of passive dosimetry techniques have been established, very few or no direct-reading dosemeters are available for the very high dose rates encountered in this field. In the framework of ENEA-INFN (Italy) collaboration, a test campaign was organised to evaluate the performance of semiconductor-based gamma detectors as active dosemeters for radiation processing. Gamma detectors based on Silicon, Silicon Carbide and GaAsP substrates were manufactured at the LEMRAP Laboratory (INFN Frascati) and tested at the ENEA Calliope ${ }^{60} \mathrm{Co}$ gamma irradiator (ENEA Casaccia) at dose rates up to few $\mathrm{kGy} / \mathrm{h}$. This communication reports the results of the tests focusing on sensitivity, radiation resistance and scalability to multi-dosemeter systems. 


\title{
$\mathrm{LaAlO}_{3}: \mathrm{Pr}^{3+}$ powder $\mathrm{TL}$ response for electron beam
}

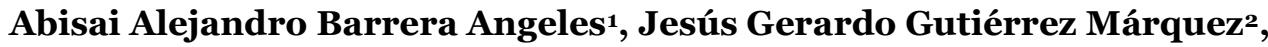 \\ Teodoro Rivera Montalvo', Daniel Nolasco Altamirano', Marco Antonio \\ Ugalde Valdés ${ }^{1}$, Alfredo Morales Hernández³, Daniel Barrera Hernández
}

\author{
1 IPN CICATA Legaría, Mexico City, Mexico \\ 2 IMSS, Mexico City, Mexico \\ 3 TNM-ITM, Morelia, Michoacan, Mexico
}

\section{https://doi.org/10.21175/rad.abstr.book.2021.26.11}

Review of the thermoluminescent (TL) characteristics of praseodymium doped lanthanum aluminate exited by higth-energy electron beams is reported. $\mathrm{LaAlO}_{3}: \mathrm{Pr}^{3+}$ powders samples were prepared using modified Pechinis's method. The formations of the obtained samples were confirmed by an X-ray diffraction patterns. TL glow curve and dosimetric characteristics of $\mathrm{LaAlO}_{3}: \mathrm{Pr}^{3+}$ powder samples were investigated under electron beam irradiation effects. All TL measurements were made using a Harshaw TL reader 3500. TL glow curves were obtained using a constant heating rate $10{ }^{\circ} \mathrm{C} / \mathrm{s}$ from $100^{\circ} \mathrm{C}$ temperature up to $400^{\circ} \mathrm{C}$ in a nitrogen atmosphere. Powders were irradiated using a linear accelerator, model Clinac IX. Dosimetry characteristics of the samples were found to be weakly dependent on the electron beam energy. The effect of electron beam dose rate was reported. Experimental results of TL dosimetric characteristics of the powder samples appear to have potential application for electron dose measurements. The technique is low cost, faster and produces well very homogeneous particles TL materials that can be used for electron checking.

Keywords: Lanthanum aluminates, electron dosimetry, thermoluminescence, synthesis 


\title{
Radiation exposure causes developmental alterations in size and shape of wings and structures associated with song production in male crickets (Acheta domesticus)
}

\author{
Tamara Fuciarelli, David Rollo
}

McMaster University, Hamilton, Canada

\section{https://doi.org/10.21175/rad.abstr.book.2021.27.1}

Environmental disasters and growth of nuclear power contribute to increasing levels of radiation pollution in the biosphere. Major impacts concern development, fertility, and survivorship in diverse species. Subtle impacts are less appreciated even though they may have profound effects on individuals and populations. Here, we examined radiation impacts on male crickets, Acheta domesticus (L.) (Orthoptera: Gryllidae), specific to wing development. Alterations in global wing morphology are detectable, but this is a flightless species. However, mating success of males is highly dependent on specialized courtship songs generated by structures associated with the opposing forewings. Such signals identify males as both conspecific and 'attractive/fit' mates. Highly specialized structures found on male forewings include the chord, harp, mirror, plectrum, and file. These work in concert to generate the species-specific male courtship song. Even slight alterations in wing morphology can alter these signals, and variation may also vary acoustic signals among populations. Here, we analyze the impacts of early-life radiation exposure (o-10 Gy) on male forewing shape and size using morphometric analysis. A canonical variate analysis (CVA) illustrated that wing shape was significantly altered in a dose-dependent basis: $10 \mathrm{~Gy}(\mathrm{P}<0.0001), 7 \mathrm{~Gy}$ $(\mathrm{P}<0.0001)$, and $2 \mathrm{~Gy}(\mathrm{P}=0.0001)$. Principal component $(\mathrm{PC})$ analysis as well as the CVA indicated that most variation in wing structure was associated with the mirror, one of the two main resonating regions on the forewing. Analysis of centroid size found significant reductions with 7 and $10 \mathrm{~Gy}$ radiation exposures. Significant radiation-induced increases in fluctuating asymmetry were also detected, with fluctuating asymmetry values increasing with dose. Due to the known specialized nature of song-producing structures and its association with both courtship songs and mating, it is likely that the alterations observed in shape and size will have larger impacts on male reproductive success. We are currently quantifying this and analyzing recordings of song structure to detect and characterize specific alterations by radiation. 


\title{
Malignant neoplasms in the offsprings of female Mayak workers
}

\author{
Svetlana Sosnina, Pavel Okatenko, Mikhail Sokolnikov
}

Southern Urals Biophysics Institute, Ozyorsk, Russia

https://doi.org/10.21175/rad.abstr.book.2021.27.2

Parental preconceptional exposure is considered as one of the potential risk factors for malignant neoplasms in offspring. Mayak Production Association (Mayak PA) is the first nuclear cycle enterprise in Russia. The cohort of Mayak PA workers includes about 25\% of female workers who is one of the few world resources to estimate such risks directly.

Aim: assessment of cancer risk among the offspring of female Mayak workers exposed to longterm occupational radiation exposure prior to conception.

Methods. A retrospective epidemiological analysis was performed in a cohort of offspring born in 1949-1990 that contained 2061 children from 1404 female Mayak workers. Mothers of 1145 children had accumulated doses of preconception external gamma-exposure to the ovaries (Study group); the remaining 916 children were regarded as internal control (Control group) as the offspring of female Mayak workers that were not exposed to preconception irradiation of gonads. The vital status and cancer incidence in the offspring were followed up to 31.12.2018. The number of personyears of follow up in the cohort made 67740 years. An analysis of the structure and crude rates of cancer incidence were carried out. The relative risk (RR) and the excess relative risk (ERR) to a dose unit of external gamma-exposure with a 95\% confidence interval were calculated using the AMFIT module of the EPICURE software.

Results. The range of maternal doses of preconception external gamma-exposure to the ovaries varied greatly: maximum dose in male offspring reached $2955 \mathrm{mGy}$ and $4076 \mathrm{mGy}$ in female offspring. A total of 92 cancer cases were registered in 1949-2018 among offspring of female Mayak workers. Average age of the observed cohort is 32 years for men and 33 years for women. Significant increase of cancers was indicated after the offspring had reached the age of 50 years. The malignancies of digestive organs and breast were registered most often in the study group. Among offspring of control group we had indicated a significant increase of brain tumors in male offspring and of the malignancies of corpus uteri among female offspring. Calculation of RR of cancers in the offspring of the study group showed no significant difference from the control group for all malignancies, for solid cancers separately, and for other most frequent cancers. RR in dose categories below $25 \mathrm{mGy}$ and 140-450 mGy was the highest but was not statistically significant. Assessment of ERR coefficients in relation to maternal accumulated absorbed dose of preconception external gamma radiation to the ovaries had revealed no statistically significant increase of cancer incidence in study group.

Conclusion. We did not find any response to preconceptional prolonged external gammaexposure to the gonads in female Mayak workers of risk of malignancies in their offspring. However, the observed cohort of the offspring has relatively young average age, which requires continued monitoring. 


\title{
Interventional radiology unit teams occupationally exposed to low-dose ionizing radiation: A cytogenetic view
}

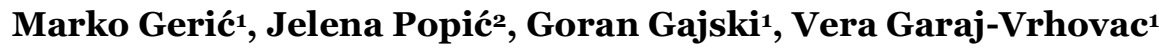 \\ 1 Institute for Medical Research and Occupational Health, Zagreb, Croatia \\ 2 University of Zagreb, School of Medicine, Clinical Hospital Merkur, Zagreb, Croatia
}

\section{https://doi.org/10.21175/rad.abstr.book.2021.27.3}

Multidisciplinary European Low Dose Initiative (MELODI) stressed out the importance of research in low-dose exposed human populations, particularly to assess cancer risks in occupational settings. Interventional radiology teams operate in proximity to low-dose ionizing radiation sources. Recent studies showed increased risks of brain malignancies that are associated with occupational radiation exposure. The advance in applied technology and procedures, as well as better dosimetry, decreased absorbed doses, however the number of interventions increased dramatically. Since the cytogenetic methods are proven to have cancer risk predictive potential, the aim of the study was to assess cytogenetic changes in interventional radiology team and to relate them with occupational effective doses.

We recruited volunteers employed at interventional teams from Zagreb hospitals ( $\mathrm{N}=\mathbf{2 4}, 6$ female and 18 male) on average $41 \pm 10$ years old, and $11 \pm 11$ years exposed to occupational radiation. To minimize impacts of possible confounders, we matched control, unexposed group for number of volunteers, sex, age, BMI, and smoking status. The readouts from volunteers' personal thermoluminiscent dosimeters showed no one reached limits set by legislation, and the average annual effective dose ranged o $-13.87 \mathrm{mSv}$. As for the biomarkers of DNA damage, we observed 1.38-fold increase in nuclear buds frequency and 1.1-fold increase in comet assay's tail length and both of them reached statistical significance $(\mathrm{p}<0.05)$. We also detected significant positive correlation of micronucleus frequency with and the length of occupational exposure.

Taken together, we managed to detect molecular and structural changes in occupationally exposed groups, with quite high inter-individual variability of the results. Further studies are needed in order to improve occupational safety of populations exposed to low-dose radiation while acknowledging ALARA principles. 


\title{
Use of gamma radiation for the characterization of biofortified tomato plants intended for space cultivation
}

\author{
Riccardo Pagliarello',2, Ilaria Di Sarcina3, Elisabetta Bennici', Giuseppe \\ Ferrara $^{3}$, Luca Nardi ${ }^{1}$, Eugenio Benvenuto', Alessia Cemmi ${ }^{1}$, Silvia Massa ${ }^{1}$
}

\begin{abstract}
1 ENEA, Italian National Agency for New Technologies, Energy and Sustainable Economic Development Biotechnology and Agro-Industry Division, Casaccia Research Center, Rome, Italy

2 University of Tuscia, DAFNE - Department of Agriculture and Forest Sciences, Viterbo, Italy, Viterbo, Italy 3 ENEA, Italian National Agency for New Technologies, Energy and Sustainable Economic Development -

Fusion and Nuclear Safety Technologies Department, FSN-FISS-SNI, Casaccia Research Center, Rome, Italy
\end{abstract}

\section{https://doi.org/10.21175/rad.abstr.book.2021.27.4}

Future long-term space missions will focus to the solar system exploration with the Moon and Mars as the leading goals. Lunar and Martian missions will be characterized by extended durations and by radiation concerns. The cultivation of higher plants will provide fresh food and bio-active molecules to the crew members, actively contributing to their psychological well-being.

Efforts to figure out how plants might perform predictably upon extraterrestrial environment and, possibly, to support life in the confined and unhealthy environment of a space outpost, are needed.

Although the effects of cosmic radiation on plant growth, development and reproduction are reported in literature, these results have been mostly obtained upon seed irradiation.

Here, two tomato (Solanum lycorpesicum cv. MicroTom) lines engineered for the constitutive bioaccumulation of anthocyanins (very valuable for their nutritional and anti-oxidant properties), were used. We firstly characterized the antioxidant properties of engineered fruits upon ex-vivo high dose radiation (i.e. $2 \mathrm{kGy}$ at Calliope ${ }^{60} \mathrm{Co}$ gamma irradiation facility, Casaccia R. C. Rome, Italy). Engineered fruits were endowed with free radicals scavenging activity and lower accumulation of reactive oxygen species with respect to wild types, as demonstrated by Electron Spin Resonance Spectroscopy (ESR). Moreover, luminescence analysis showed decreased protein folding damage compared to wild type.

Gamma radiation was also used to study the biological and metabolic dose-response effect of in vivo experiments at either o Gy, $0.5 \mathrm{~Gy}$ (dose rate: $6 \mathrm{~Gy} / \mathrm{h}$ ), 5 Gy or $30 \mathrm{~Gy}$ (dose rate: $60 \mathrm{~Gy} / \mathrm{h}$ ) on both engineered and wild type plants. These conditions were applied to tomato plants onto two developmental stages: 30 and 60 days after sowing. Biometric analysis (height, leaf area, number of leaves, number of flowers, number of fruits, fruit weight, fruit diameter, number of seeds) were performed at one-week intervals, starting from the day before irradiation. To determine the effect of radiation on anthocyanins, flavonols and chlorophyll content, fluorometric non-destructive analyses were conducted. The effects of treatments on photosynthetic maximum photochemical efficiency (Fv/Fm) and quantum yield electron transport (Y_Fm) were also evaluated, revealing that plants maintained a healthy status of the photosynthetic apparatus.

The results obtained allowed to figure out the best candidate for 'agro-space' applications within the genotypes studied giving an indication that bioengineering of plants through the manipulation of metabolic pathways can be a promising approach to generate varieties for space cultivation. 


\title{
Does altitude have an effect on pigment content of wild growing plants in Rila Mountain?
}

\author{
Tsveta Angelova1, Christo Angelov², Svetla Gateva', Gabriele Jovtchev
}

\begin{abstract}
1 Institute of Biodiversity and Ecosystem Research at the Bulgarian Academy of Sciences, Sofia, Bulgaria 2 BEO-Moussala, Institute of Nuclear Research and Nuclear Energy, Bulgarian Academy of Sciences, Sofia, Bulgaria
\end{abstract}

\section{https://doi.org/10.21175/rad.abstr.book.2021.27.5}

Rila Mountain is the highest mountain on the Balkan Peninsula and is characterized with specific microclimate. It has been revealed that with the increase of the altitude, the differences in environmental conditions change at a great extent. In mountain conditions plants have to cope with combined environmental factors such as altitude, temperature, prolonged UV irradiation, and etc.

The aim of this study is to assess if different altitudes could led to changes in pigment content in wild growing species. Five wild species, characteristic of the ecosystems in Rila Mountain: Fragaria vesca L. (Rosaceae), Myosotis sylvatica Ehrh. (Boraginaceae), Achillea millefolium L. (Asteraceae), Epilobium angustifolium L. (Onagraceae) and Dactylis glomerata L. (Poaceae) were used as plant material. Plants were collected from three different altitudes (Sofia-595 m; Rila Mountain-1500 m a.s.l. and $1782 \mathrm{~m}$ a.s.l.) in July-August in growing season of 2020. Sofia was chosen as control altitude. Pigment content was applied as endpoint. Our data showed that the levels of total chlorophylls, chl. $a$, chl. $b$ and total carotenoids for plants growing at $1500 \mathrm{~m}$ a.s.l. were lower or similar to those measured at Sofia altitude for $F$. vesca, $M$. sylvatica, A. millefolium, $E$. angustifolium and D. glomerata L. Higher levels of total chlorophylls, chl. $a$, chl. $b$ and total carotenoids in comparison with those at the lowest altitude (Sofia) were obtained for genotype $D$. glomerata growing at $1782 \mathrm{~m}$ a.s.l. There is no change in chlorophyll $a / b$ ratio detected in plants at $1500 \mathrm{~m}$ a.s.l. altitude and $1782 \mathrm{~m}$ a.s.l. in five investigated species. Genotype variability in the response between wild plant species was found in different altitudes. Based on our data it could be suggested that probably the five wild genotypes have different adaptive strategies at studied altitudes measured as pigment content. On the other hand no change in the chlorophyll a/b ratio could be an indication that altitudes have no permanent damage on the leaf photochemical system at this time of the year. It is known that chlorophyll content in plants is an indicator of their response to the habitat, weather, anthropogenic conditions. Because of the fact that in mountain conditions the effect of altitude is combined with other abiotic factors and that pigment content is very variable depending on many factors further studies are needed for better understanding of the mechanisms of interaction between factors and plant response.

Keywords: Photosynthetic pigments, altitude, wild plant species

Acknowledgments: The present study was supported by the Bulgarian Ministry of Education, Youth and Science under contract grant number DN 04/1 entitled: "Study of the combined effects of the natural radioactive background, UV radiation, climate change and cosmic rays on model groups of plant and animal organisms in mountain ecosystems". 


\title{
Ground-based preliminary tests to simulate space microgravity and radiation effects on plants in the seed to seedling transition
}

\author{
Giulio Metelli',2, Ilaria Di Sarcina', Marco Garegnani1,3, \\ Giuseppe Ferrara', Alessia Cemmi' ${ }^{1}$, Luca Nardi', Eugenio Benvenuto ${ }^{1}$
}

\author{
1 ENEA, Rome, Italy \\ 2 Tuscia University of Viterbo, Viterbo, Italy \\ 3 Politecnico of Milan, Milan, Italy
}

\section{https://doi.org/10.21175/rad.abstr.book.2021.27.6}

The space colonization Era has just begun and in the near future the first colonies will rise on the Moon with the ambition of reaching Mars. The colonization of space will depend on the ability to provide fresh and healthy foods for the crew members, with minimal re-supply from Earth. This result can be achieved through Bioregenerative Life Support Systems (BLSS) in which plants and microorganisms could be used to produce oxygen and also food, and to treat human and vegetable waste. The efficient development of plants in space is constrained by different factors (i.e., microgravity, ionizing radiation, etc.) that are recognized to deeply influence organism's growth at molecular, morpho-structural and physiological levels.

Studies on the effects of space environment on plants growth and development have already been carried out, but there is a lack of information about the cumulative effects of the different stresses on plant physiology particularly at the seedling level (i.e., ionizing radiation and microgravity together). It is well known that resistance to environmental factors of plant seeds is stronger due to structural and metabolic traits compared with organs at different life stages and, since the most harmful moment of plant development is represented by the seed to seedling transition, we have chosen microgreens to study the effect of different stress combinations.

Microgreens are fast-harvesting vegetables able to accumulate high concentration of phytochemicals such as antioxidants and vitamins, and we study them as a model food production system inside a space colony that would help astronauts face the problems of life in extreme environments.

The ongoing activities are conducted inside the "Calliope" 6oCo gamma irradiation facility (ENEA "Casaccia", Rome, Italy) with microgreens growing inside a confined controlled environment on a $2 \mathrm{D}$ clinostat to simulate microgravity and chronic and acute exposure of seeds and seedlings to gamma rays. To monitor the combined effects of both stressors, morphometric and non-destructive analysis (Fluorometric, Thermal, visible light imaging) will be conducted during the cultivation process. Preliminary results of the cumulative stress effects on microgreens plants will be reported. 


\title{
Role of glutathione S-transferase M1 (GSTM1), P1 (GSTP1), and T1 (GSTT1) gene polymorphisms in the development of the bronchial hyperreactivity in children residing at radiologically contaminated territories of Ukraine
}

\author{
Yevgenya Stepanova, Igor Kolpakov, Vitaliy Vdovenko, \\ Valentina Kondrashova, Victor Zygalo, Olena Leonovych
}

National Research Center for Radiation Medicine of the National Academy of Medical Sciences of Ukraine, Kyiv, Ukraine

\section{https://doi.org/10.21175/rad.abstr.book.2021.27.7}

Background. Vast territories of Ukraine were exposed to radioactive contamination as a result of the Chernobyl disaster. About 400 thousand children still live there. The long-term studies have shown a high incidence of obstructive lung disorders throughout the period after the Chernobyl disaster in children-residents of $\mathrm{Cs}^{137}$ contaminated territories. However, the pathophysiological mechanisms of these biomedical effects remain completely unclear. In recent years, more and more publications have appeared pointing to the link of some polymorphic variants of glutathione-S-transferase genes with the onset the bronchial hyperreactivity in children under the impact of hazardous environmental factors.

Aim. Establishing the possible role of glutathione-S-transferase M1 (GSTM1), P1 (GSTP1), and T1 (GSTT1) gene polymorphisms in the development of the bronchial hyperreactivity in children residing at radioactively contaminated territories was the research objective.

Materials and methods. School age children-residents of radioactively contaminated areas (RCA), without clinical signs of respiratory pathology were examined. Molecular genetic studies were carried out by polymerase chain reaction (PCR) and restriction fragment length polymorphism (RFLP) for further analysis. The GSTT1, GSTM1 gene deletion polymorphism was investigated using multiplex PCR. PCR and PCR-RFLP analyses were performed in the study of the GSTP1 gene $A 313 G$ polymorphism. The ventilation lung capacity was examined by the computer spirometry method according to the analysis of "the flow-volume" loop. The pharmacologic inhalation test with bronchodilator drug, affecting the $\beta_{2}$-adrenergic lung receptors was used to detect the early changes in the ventilation lung capacity - the bronchial hyperreactivity. Studies were conducted in children $(\mathrm{n}=145)$ living in settlements with a soil contamination density by $\mathrm{Cs}^{137}$ from 185 to $555 \mathrm{kBq} / \mathrm{m}^{2}$. The control group included children $(n=126)$ living in the "clean" territories. The age of children was 10-17 years old.

Results. It was found that in children of the main group the bronchial hyperreactivity were revealed more often than in the control group ( $\mathrm{p}<0.01)$. Molecular genetic studies have shown a significantly higher incidence of the GSTM1 gene deletion genotype and the GSTP1 gene $A 313 G$ polymorphism in subgroup of children-residents of Cs $^{137}$ contaminated territories having got the bronchial hyperreactivity than either in children no having the bronchial hyperreactivity or in children in the control group ( $\mathrm{p}<0.05)$. There was no statistically significant difference in the incidence of GSTT1 gene deletion polymorphism in all subgroups of children.

Conclusion. Thus, in children living in conditions of environmental contamination by $\mathrm{Cs}^{137}$ the bronchial hyperreactivity are detected more often in the presence of GSTM1 gene deletion genotype and the GSTP1 gene $A 313 G$ polymorphism. 


\title{
Effect of electron beam radiation on the mechanical and thermomechanical properties of proton exchange membranes
}

\author{
Ingars Reinholds',2, Elina Pajuste3,2, Liga Avotina3, \\ Guntars Vaivars3,4, Einars Sprugis3,4, Rossi Mikko5, Kettunen Heikki5
}

1 Baltic Scientific Instruments, Riga, Latvia

2 Faculty of Chemistry, University of Latvia, Riga, Latvia

3 Institute of Chemical Physics, University of Latvia, Riga, Latvia

4 Institute of Solid State Physics, University of Latvia, Riga, Latvia

5 Department of Physics, University of Jyväskylä, Jyväskylä, Finland

\section{https://doi.org/10.21175/rad.abstr.book.2021.27.8}

Introduction. Proton exchange membranes (PEMs) like Nafion ${ }^{\circledR}$ and other membrane materials are commonly tested for fuel cell applications. PEMs can be operated under harsh environments such as nuclear facilities that may lead to deterioration of material endurance. For example, oxidation may play an important role on the radiation induced degradation of Nafion ${ }^{\circledR}$ and release of fluorine products of radiolysis (1). Sulfonated poly-ether-ether ketone (SPEEK) containing non-fluorinated aromatic backbone is promising candidate for the applications under harsh environments (2).

The aim of this study was to evaluate the influences of electron beam irradiation on the mechanical and thermomechanical properties of Nafion ${ }^{\circledR}$ and SPEEK membranes.

Methods. Nafion ${ }^{\circledR}$ and laboratory made SPEEK membranes with a high degree of sulfonation were irradiated by accelerated electrons at a dose range of 50-500 kGy. The tensile tests, dynamic mechanical analysis (DMA) and differential scanning calorimetry (DSC) were used to characterize change of PEM material properties depending on the dose of irradiation.

Results. Preliminary analysis of the irradiated PEM samples by infra-red spectroscopy and their water solutions by ion chromatography indicated high differences in susceptibility to radiation of two PEMs - the Nafion ${ }^{\circledR}$ indicated oxidation and release of fluoride and sulfate anions with increase of the dose comparing to less pronounced changes in the structure of SPEEK membrane. The tensile tests at room temperature and DMA analysis, a notable reduction of mechanical properties (tensile strength, strain, storage modulus) was determined in the case of Nafion ${ }^{\circledR}$ due to the radiation induced oxidation and chain scission. The increase of the radiation dose affected improvements of the DMA characteristics of SPEEK. The study of thermal transitions including glass transition of SPEEK also confirmed that radiation mainly possessed little effect on the thermomechanical behavior as compared to that of negative effect on Nafion ${ }^{\circledR}$. From the DSC data it was determined that Nafion ${ }^{\circledR}$ exhibited an endothermic peak of the first heating assigned to molecular rearrangements inside the polar clusters of the polymer. A gradual decrease from 167 to $139^{\circ} \mathrm{C}$ was determined with increase of the radiation dose from 50 to $500 \mathrm{kGy}$ associated with radiation induced chain scission. There was not determined notable effect of the radiation dose on the first heating transformations of the SPEEK with exception of determined shift of endothermic peak from 102 to $110^{\circ} \mathrm{C}$ with increase of dose associated with the cluster changes due to the moisture content. As shift of the SPEEK glass transition temperature from 209 to $215{ }^{\circ} \mathrm{C}$ was indicated at $500 \mathrm{kGy}$ during the comparison of secondary heating cycle. That may be due to the induced transformation of macromolecular arrangements.

Conclusion. This study provides evidence of radiation induced degradation of Nafion ${ }^{\circledR}$ structure at irradiation doses exceeding $100 \mathrm{kGy}$. For comparison, SPEEK membrane presented rather high stability and improvement of mechanical properties at irradiation doses up to $250 \mathrm{kGy}$.

\section{References}

[1] Fox, E. B., Greenway, S. D., \& Clark, E., Fusion Science and Technology, 2010, 57(2), 103-111.

[2] Khomein, P., Ketelaars, W., Lap, T., \& Liu, G. Renewable and Sustainable Energy Reviews, 2020, 110471.

Acknowledgments: This research was supported by ERDF Project No.1.1.1.1/19/137 "Graphene-based electrochemical pumping system for radioactive hydrogen isotope separation". 


\title{
Investigation of neutron-irradiated beryllium pebble oxidation in dry and humid air at elevated temperatures for LOVA/LOCA estimation and corresponding safety protocol development
}

\author{
Rudolfs Janis Zabolockis, Elina Pajuste, Liga Avotina, Gunta Kizane
}

University of Latvia Institute of Chemical Physics, Riga, Latvia

\section{https://doi.org/10.21175/rad.abstr.book.2021.27.9}

Beryllium is a key nuclear fusion reactor material intended as a neutron multiplicator as well as a plasma-facing material. Beryllium has multiple advantages; however, it can pose risks associated with the release of fusion fuels and toxic beryllium compound dust formation under extreme conditions such as loss of vacuum (LOVA) and loss of coolant (LOCA) accidents. Thermal analysis methods can be used to evaluate the processes beryllium could undergo in LOVA/LOCA conditions.

Neutron irradiated beryllium pebbles (diameter $\sim 1 \mathrm{~mm}$, mass $\sim 1 \mathrm{mg}$ ), as well as non-irradiated pebbles of the same size and grade, were selected for investigation. Both types of pebbles were thermally treated in SEIKO EXSTAR 6300 thermogravimetric/differential thermal analyzer in dry (relative humidity $<5 \%$ ) and humid $(>95 \%)$ airflow conditions $(12 \mathrm{~L} / \mathrm{h})$ up to $1548 \mathrm{~K}, 10 \mathrm{~K} / \mathrm{min}$. The surface microstructure analysis was performed with Hitachi S-4800 scanning electron microscope with EDS system Bruker XFlash Quad $5040123 \mathrm{eV}$.

Results distinctly show the profound effect of relative humidity and neutron irradiation-induced defects on the rate of oxidation, oxidation starting temperature, phase change, and melting temperature of beryllium pebbles. The thermal treatment results in a substantial mass and volume increase of the pebbles and the creation of an extremely finely structured surface. Elevated humidity and neutron irradiation achieve a significant combined effect to produce the largest mass and volume increase.

Results obtained herein this study are to be implemented in nuclear fusion device safety solution development as well as LOVA/LOCA impact assessment on fusion fuel release. 


\title{
Electronic, structural and magnetic transitions in X-ray irradiated solid materials
}

\author{
Beata Ziaja-Motyka',2
}

\author{
1 DESY, Hamburg, Germany \\ 2 IFJ PAN, Kraków, Poland
}

https://doi.org/10.21175/rad.abstr.book.2021.27.10

$\mathrm{X}$-ray induced structural and magnetic transitions in solids are in focus of this talk. Depending on the dose absorbed, an irradiation with a femtosecond X-ray pulse can trigger an ultrafast electronic or structural transition in solid materials. In magnetic materials, an X-ray triggered ultrafast demagnetization can also occur. In this talk, selected study cases for these transitions are presented. Dedicated theoretical modeling reveals complex multistage evolution of the irradiated systems, confirmed by experimental measurements performed at FERMI and at other XFEL facilities. Challenges remaining for the modeling and quest for further improvements of the necessary diagnostics tools are discussed. 


\title{
Uncertainties in radiation exposure estimates of the German uranium miners' cohort
}

\section{Veronika Deffner', Sabine Hoffmann², Peter Scholz-Kreisel'1, Nora Fenske1}

\author{
1 Federal Office for Radiation Protection, München (Neuherberg), Germany \\ 2 Ludwig-Maximilians-Universität, München, Germany
}

\section{https://doi.org/10.21175/rad.abstr.book.2021.27.11}

Studies of uranium miners provide a substantial epidemiologic basis for the health risk estimation due to radon exposure. The German uranium miners cohort is one of the largest cohort studies of uranium miners who have been occupationally exposed to radon; the cohort includes 58,974 males accumulating over 2 million person years at risk (follow-up period 1946-2013). Exposure to radon progeny was retrospectively assessed for all cohort members based on an extensive job-exposure matrix (JEM) and individual detailed job histories. Although the individual exposure estimates are well-grounded, they may involve potential uncertainties. Current research addresses the possible impact of exposure uncertainties on health risk estimates within the German uranium miners cohort.

We will focus on the following aspects: 1 . Identification of sources of potential uncertainties in the two-stage exposure assessment procedure; 2. Overview on our strategy to account for exposure uncertainties in risk estimation.

Exposure assessment in the German uranium miners' cohort can be roughly divided into two steps. First, the JEM was derived by experts' estimations as well as by contemporary area measurements of radon progeny and contains the annual mean exposure to radon progeny for each occupational subgroup of the cohort (depending on mining facility, work place and job). In the second step, the individual exposure to radon progeny of a uranium miner was estimated by linkage of the exposure value of the assigned occupational subgroup with the individual job history.

The two-stage exposure assessment may be subject to potential uncertainties at each stage. Generalization error may arise from the generalization of exposure measurements in the first step and assignment error may occur from the assignment of the values from the JEM to individual miners. In both stages, further uncertainties may exist, including parameter uncertainties, transfer errors, documentation errors, experts' evaluation errors and procedural measurement errors, which are referred to as estimation errors.

A Bayesian hierarchical approach is currently developed to account for exposure uncertainties in health risk estimation. In addition to the quantification of uncertainties, the measurement error model plays a central role for this approach and is discussed here. The measurement error model describes the estimation procedure for individual exposure including the corresponding uncertainties. We will present the measurement error model for the German uranium miners cohort, which was developed based on the exposure assessment procedure of the cohort.

These findings represent groundwork towards the estimation of lung cancer risk depending on radon exposure taking measurement error into account. 


\title{
Terrestrial background radiation study in Bathalegoda, North-Western province of Sri Lanka
}

\author{
Thiwanka Weerakkody ${ }^{1}$, Champa Dissanayake', \\ A.G. Chandrapala ${ }^{2}$, Maheshika Kalpage ${ }^{1}$, \\ Thilaka Attanayaka ${ }^{1}$, Indrani Dissanayake ${ }^{1}$, Kasun Binduhewa ${ }^{1}$
}

1 Sri Lanka Atomic Energy Board, Colombo, Sri Lanka

2 Natural Resource Management center, Department of Agriculture, Peradeniya, Sri Lanka

\section{https://doi.org/10.21175/rad.abstr.book.2021.27.12}

Terrestrial radiation originates from natural radio-nuclides present in soils, rocks, air, and water. For all the living beings, the major source of radiation exposure is natural radioactivity in their living environment. Higher levels of radioactivity levels are harmful to human health.

Bathalegoda area lies between longitudes 80.10190 East and latitudes 7.59950 North. The area located in the North western province, Kurunegala, Sri Lanka. Superficial soil samples from twentyone locations from Bathalegoda area were analysed by gamma spectrometry. Top $2 \mathrm{~cm}$ of soil was scraped off from a $1 \mathrm{~m}^{2}$ area and $1 \mathrm{~kg}$ of soil samples were taken and the samples were immediately brought to the laboratory for analysis. Samples were homogenized by hand mixing and allowed them to air dry and then oven dried for 24 hours. $2 \mathrm{~mm}$ mesh was used to separate the gravel from soil. A sub sample from the weighed fine fraction were filled into a specific container and kept for 21 days to achieve secular equilibrium .The activity concentrations of radionuclides were measured using a Hyper Pure Germanium (HPGe) detector installed at the Gamma spectroscopy laboratory (17025:2017 accredited laboratory from 2006), Life Sciences Division of the Sri Lanka Atomic Energy Board, with the relative efficiency of $30 \%$ and the resolution of $2.20 \mathrm{KeV}$ at the gamma energy of $1332.5 \mathrm{KeV}$ of ${ }^{6 \circ} \mathrm{Co}$. Each sample was counted for 72,000 seconds. The ISOCS/LabSOCS software calibration method was used for efficiency calibration. Soil 6 was used as a reference material for detector calibration. The spectra were analysed by using the Genie 2000 software.

The median activity concentrations (range) of ${ }^{232} \mathrm{Th},{ }^{40} \mathrm{~K},{ }^{226} \mathrm{Ra} \&{ }^{210} \mathrm{~Pb}$ were $46.9 \mathrm{~Bq} / \mathrm{kg}$ (30.6-67.5), $1028.6 \mathrm{~Bq} / \mathrm{kg}(882.9-1213.0), 16.5 \mathrm{~Bq} / \mathrm{kg}(11.8-20.8)$, and $27.9 \mathrm{~Bq} / \mathrm{kg}(19.8-44.3)$ respectively. Activity concentration of ${ }^{232} \mathrm{Th}$ was higher than the world average of $30 \mathrm{~Bq} / \mathrm{kg}$ and the activity concentration of ${ }^{40} \mathrm{~K}$ was higher than the world average of $400 \mathrm{~Bq} / \mathrm{kg}$. All the soil samples from Bathalegoda area showed the radium equivalent activity below $37 \mathrm{OBq} / \mathrm{kg}(\mathrm{NEA})$ and it was ranged from $130.8-192.5 \mathrm{~Bq} / \mathrm{kg}$. Criteria formula was less than 1 in all the soil samples. Median Activity Utilization Index (AUI) in the area was below the recommended limit indicating that the dose levels in the soil samples were safe for the environment. The median external hazardous index $\left(\mathrm{H}_{\mathrm{ex}}\right)$ in Bathalegoda area was below the recommended limit of 1 and the annual effective dose was within the safe limit of $1 \mathrm{mSv} / \mathrm{y}$. Soil from this area is safe for human health. 


\title{
Optimization of neutron activation analysis of rare-earth elements
}

\author{
Cassidy Reis, Sheldon Landsberger
}

University of Texas at Austin, Austin, United States

\section{https://doi.org/10.21175/rad.abstr.book.2021.28.1}

During neutron activation analysis (NAA), if the material being analyzed is partially composed of a lanthanide content optimization of rare-earth analyses is crucial. To enhance these procedures a database was created to help assess the likelihood that a certain lanthanide isotope and gamma ray is indeed the best one to be determined by NAA. First, the most common isotopes through the (n, $\gamma)$ reaction of each element was determined, along with the strongest and most abundant gamma rays to be used, and a chart was formulated with their relevant properties. A weighting factor was constructed based on seven characteristics of the properties of the lanthanides: thermal cross section, resonance integral, natural abundance of the element itself, half-life of the activation product, the branching ratio, the Igamma(relative) value, and relative detector efficiency of the photon spanning from $80-1596 \mathrm{keV}$. The seven individual characteristics were then added together and divided by the total possible value to get their total weighting factor. In addition, the application of gammagamma coincidence and Compton suppression was also evaluated to determine which radionuclides may be better evaluated. 


\title{
Radiometric characterization for the decommissioning of the Scanditronix MC17 medical cyclotron of the Italian National Cancer Institute of Milano
}

\author{
Davide Bortot ${ }^{1}$, Andrea Pola', Stefano Pasquato', Davide Mazzucconi ${ }^{1}$, \\ Anna Brusa ${ }^{2}$, Carlo Chiesa ${ }^{2}$, Fabio Zanellati ${ }^{2}$, Leonardo Baldassarre ${ }^{3}$
}

1 Politecnico di Milano, Milano, Italy

2 Istituto Nazionale dei Tumori di Milano, Milano, Italy

3 LB Servizi per le Aziende, Roma, Italy

https://doi.org/10.21175/rad.abstr.book.2021.28.2

Aim and background. This work aims at presenting the first radiometric characterization of the non-self-shielded Scanditronix MC17 medical positive ions cyclotron of the National Cancer Institute of Milano, conceived for producing ${ }^{11} \mathrm{C}$ and ${ }^{18} \mathrm{~F}$ for PET. This study allowed to carry out one of the very first decommissioning of a medical cyclotron in Italy.

Constituent metals of the cyclotron, the walls of the vault and all the auxiliary structures inside (pumps, water and gas pipes) have been exposed for 20 years to intense and mixed radiation fields mainly given by fast neutrons (energy up to about $10 \mathrm{MeV}$ ) produced by the $17 \mathrm{MeV}{ }^{18} \mathrm{O}(\mathrm{p}, \mathrm{n}){ }^{18} \mathrm{~F}$ nuclear reaction. Metals inside the acceleration chamber were exposed also to direct and scattered proton beam. The consequent activation, not crucial during normal machine operation, became a fundamental issue to face for a safe management of the accelerator decommissioning.

For this reason, after the cyclotron definitive shutdown, a qualitative and quantitative characterization of the radioactivity concentration of its main components was performed to define and optimize decommissioning.

Materials and methods. Several representative samples of different compositions (bolts, screws, slabs, connectors) were removed from the external surface and from acceleration chamber. The experimental characterization involved high resolution gamma spectrometry by means of the ISOCS detector (In-situ Object Counting System, Mirion Technologies). This was followed by Monte Carlo simulations performed with the FLUKA code, in order to compare experimental and simulated results.

Results. This study highlighted the presence of long-lived isotopes, in particular ${ }^{60} \mathrm{Co},{ }^{54 \mathrm{Mn}}$ and ${ }^{6} \mathrm{Zn}$, whose activity concentration was always above the reference clearance levels, equal to $0.1 \mathrm{Bg} \mathrm{g}^{-1}$ (IAEA RS-G-1.7, 2004). These values resulted to be strongly dependent on the material composition and on the position with respect to the cyclotron target, leading to very different time scenarios. Components with low amount of Nickel showed the predominance of $54 \mathrm{Mn}$, produced by the ${ }^{54} \mathrm{Fe}(\mathrm{n}, \mathrm{p})$ reaction, whose relatively short half-life of $0.86 \mathrm{y}$ allows to bring the activity concentration below the clearance levels in few years. On the contrary, on materials with non-negligible Nickel content, the major contribution is due to the ${ }^{60} \mathrm{Ni}(\mathrm{n}, \mathrm{p}){ }^{60} \mathrm{Co}$ reaction $\left({ }^{60} \mathrm{Co}\right.$ half-life $\left.5.28 \mathrm{y}\right)$, which keeps the activity concentration above the clearance levels for tens of years. Very different activity concentration levels were measured, even for similar components, especially for samples removed from the acceleration chamber. This is due to the strong dependence of the activation on the primary beam trajectory and on the spatial distribution of beam losses, which produce a large amount of $65 \mathrm{Zn}$ from the ${ }^{65} \mathrm{Cu}(\mathrm{p}, \mathrm{n})$ reaction. 


\title{
Experimental characterization of a wall-less TEPC for nanodosimetric measurements
}

\author{
Davide Mazzucconi ${ }^{1,2}$, Davide Bortot ${ }^{1}$, \\ Andrea Pola', Stefano Agosteo', Valeria Conte ${ }^{2}$
}

\author{
1 Politecnico di Milano, Milano, Italy \\ 2 INFN - Laboratori Nazionali di Legnaro, Legnaro, Italy
}

\section{https://doi.org/10.21175/rad.abstr.book.2021.28.3}

The deep understanding of the interaction between ionizing radiation and soft tissue is of primary importance for radiation therapy and in particular, hadron therapy. These cancer treatments exploit beams of intense energy to cause severe damage in the DNA of malignant cells and inactivate them. It is recognized that the observable radiobiological effects of ionizing radiations are strongly correlated to the clustering of damages in micrometer- and nanometer-sized subcellular structures, hence to the particle track structure. The characteristic properties of track structure are directly measurable nowadays with bulky experimental apparata, which cannot be easily operated in a clinical environment. With this in mind, an avalanche-confinement Tissue Equivalent Proportional Counter (TEPC) was constructed to simulate biological target sites of size down to $25 \mathrm{~nm}$. This range of operation offers the means to perform a direct comparison between microdosimetric spectra and track-nanodosimetric distributions.

A study on experimental gains of the filling gas of the detector has been conducted aiming at verifying the agreement with theoretical gains evaluated through different models. The TEPC was irradiated with an isotopic ${ }^{244} \mathrm{Cm}$ alpha source at different impact parameters (i.e. distances between its center and the trajectory of the primary particle) showing that, once calibrated, it is capable of assessing a nanodosimetric cluster size distribution for some tenths of nanometers in simulated size. Several Monte Carlo simulations have been performed, showing a good agreement with experimental results. Moreover, the behavior of nanodosimetric distributions as certain parameters and conditions vary was studied.

Finally, cluster size distributions produced by ${ }^{244} \mathrm{Cm}$ alpha particles were simultaneously measured with the Startrack nanodosimeter (INFN-Laboratori Nazionali di Legnaro, Italy) and with the avalanche confinement TEPC. The comparison of pairwise measurements shows an excellent agreement when the signals from the TEPC are processed in order to include the reduced detection efficiency and the dead time of the Startrack detector. This result suggests a minor influence of the gas-avalanche stochastics on the measured distributions and encourages the use of proportional counters for the experimental characterization of therapeutic hadron beams at the nanometer level. Further measurements with different light ions are ongoing to confirm this finding. A direct and systematic comparison between nanometric-domain microdosimetric distributions and tracknanodosimetric measurements will allow investigating the feasibility of using the nanomicrodosimetric spectrum to derive track-structure quantities at the nanometer level (for instance the mean cluster size M1 and the cumulative distributions F1, F2 and F3) which have been observed to correlate strongly with the radiation damage. 


\title{
Influence of beam spoiler and air gap on dose distribution in build-up region for X6 MV static field
}

\author{
Edyta Dąbrowska-Szewczyki, Pawel Kukolowicz'1, \\ Anna Zawadzka', Piotr Kowalczyk², Rafal Podgorski², \\ Michal Wojasiński², Tomasz Ciach $^{2}$, Tomasz Zawistowski3
}

\author{
1 Maria Sklodowska-Curie National Research Institute of Oncology, Warsaw, Poland \\ 2 Warsaw University of Technology, Warsaw, Poland \\ 3 Creotech Instruments S.A., Warsaw, Poland
}

https://doi.org/10.21175/rad.abstr.book.2021.28.4

Purpose/Objective. Based on CBCT images we noticed that for postmastectomy patients there are several different problems with the reproducibility of bolus positioning (E. Dąbrowska, et al., Investigation of reproducibility of bolus position based on $\mathrm{kV} \mathrm{CBCT}$ imaging, Radiotherapy \& Oncology, Journal of the European Society for Radiotherapy and Oncology, Volume 123, Supplement 1, 2017), especially air gap occurrence. This study aimed to determine the influence of air gaps between bolus and phantom on dose distribution in the build-up region for X6 MV. The novelty of the work is using a different density of boluses fabricated with the $3 \mathrm{D}$ printer.

Materials and Methods. Depth dose measurements were performed for X6 MV $10 \times 10 \mathrm{~cm}^{2}$ open field, generated with Varian CL 2300 C/D. The measurements were carried out with the Markus plane-parallel ionization chamber (PTW) (0.055 $\mathrm{cm}^{3}$ volume), connected to Unidos (PTW) electrometer. As a $1 \mathrm{~cm}$ thick beam spoilers we used $3 \mathrm{D}$-printed thermoplastic polyurethane (TPU) cuboids filled with $5 \%, 10 \%, 15 \%$ and $20 \%$ honeycomb structure (thickness of $0.5 \mathrm{~g} / \mathrm{cm}^{2}, 0.17 \mathrm{~g} / \mathrm{cm}^{2}$, $0.22 \mathrm{~g} / \mathrm{cm}^{2}$ ). To generate air gap we used homemade styrofoam frames. Measurements were performed at a physical depth of $1,5,10$, and $15 \mathrm{~mm}$ for all beam spoilers without air gap and with 10 , 20, 30, and $40 \mathrm{~mm}$ air gaps, at constant $90 \mathrm{~cm}$ SSD.

Results. The results of measurements will be presented on graphs. The beam spoilers increased the dose in the build-up region. The bigger is spoiler filling the larger is surface dose. The same dependence was present irrespective of air gap size. Regardless of spoiler percentage filling with honeycomb structure, the larger air gap size the smaller dose was on $1 \mathrm{~mm}$ depth.

Conclusions. The influence of beam spoiler filling and air gap on dose distribution in the buildup region for X6 MV was investigated. Beam spoiler increased the dose in the build-up region. The larger thickness of the beam spoiler (larger filling) the larger influence on the build-up dose. The occurrence of air gaps lowers the surface dose. However, the influence of the air gap in the range of $10-40 \mathrm{~mm}$ is small. For bolus with the density of $0.22 \mathrm{~g} / \mathrm{cm}^{2}$ (filling $15 \%$ and $20 \%$ ) regardless of air gap size the dose on $1 \mathrm{~mm}$ depth was always larger than $95 \%$ of the maximum dose. 


\title{
Modeling Geant4 based GATE simulation for total gamma efficiency of phoswich type detector system
}

\author{
Nuray Yavuzkanat
}

Bitlis Eren University, Bitlis, Turkey

https://doi.org/10.21175/rad.abstr.book.2021.28.5

In nuclear physics, phosphor-sandwich (phoswich) (PW) type detector systems can measure more than one type of radiation at the same time. In addition to the use of these detectors in lowactivity environmental radiation measurements, these detectors, which are made with three or two layers, are also used in medical imaging, nuclear power plants, and environmental safety after nuclear accidents. Being able to measure more than one type of radiation at the same time, this type of detector system is also used to separate gamma radiations in different energy ranges as in large detector systems such as PARIS. It is necessary to obtain the total gamma efficiency curve for the calibration of the wide range of use of the PW type detector systems. It can be determined in four different methods, such as experimental, empirical, analytical, and Monte Carlo (M.C) simulation. In this study, the efficiency values and the fitting parameters were found out by modeling the phoswich detector system, including cylindrical $\mathrm{NaI}(\mathrm{Tl})$ and $\mathrm{CaF}_{2}(\mathrm{Eu})$ scintillations, in the Geant 4 based GATE simulation program. The total gamma efficiency of the Phoswich detector system has been obtained for point and disc-shaped radioactive sources that emit photons isotropically at different energies from $50 \mathrm{keV}$ to $3000 \mathrm{keV}$. In addition, these results were compared with the results in the literature and it was observed that the results were compatible with each other. 


\title{
The new matrix method for deriving counting rate equations describing coincidence summing of gamma and X-rays for germanium spectrometers
}

\begin{abstract}
Dragana Jordanov, Laslo Nadjdjerdj
Vinča Institute of Nuclear Sciences, National Institute of Republic of Serbia, University of Belgrade, Belgrade, Serbia

https://doi.org/10.21175/rad.abstr.book.2021.28.6

The method for deriving counting rate equations in coincidence summing of gamma and X-rays that we have developed has made it much easier to obtain the results as well as the measurement process itself. This method allows us to determine the activity of a radioactive source directly without calibration of the detector and also to simultaneously to determine the efficiency of detection. We have successfully applied the method to the radionuclide with a simpler decay scheme such as ${ }^{139} \mathrm{Ce}$, ${ }^{57} \mathrm{Co},{ }^{133} \mathrm{Ba}$. The application of this method to the ${ }^{152} \mathrm{Eu}$ as a radionuclide with a much more complex

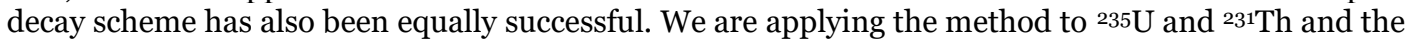
preliminary results show that it is possible to apply this method to these radionuclides as well.
\end{abstract}




\title{
Measurements of natural background radiation in the underground laboratories of the BSUIN and EUL projects
}

\author{
Katarzyna Szkliniarz, Kinga Polaczek-Grelik, Agata Walencik-Łata, Jan Kisiel
}

University of Silesia in Katowice, August Chełkowski Institute of Physics, Katowice, Poland

\section{https://doi.org/10.21175/rad.abstr.book.2021.28.7}

Natural radioactivity surrounds us everywhere. However, there are places where there is or maybe less of it. These are underground facilities, laboratories, research \& educational mines and test-sites bring. Such sites are used, among other things, for scientific research but can also provide unique environments for various businesses, production of thermal energy, or facilitating food production. To increase access to underground laboratories (ULs) operating in the Baltic Sea region and facilitate their service offer, the BSUIN (Baltic Sea Underground Innovation Network) project was created, involving 6 underground laboratories operating in the Baltic States. This project was completed in 2020 but is still being continued as the EUL (Empowering Underground Laboratories Network Usage) project. One of the activities of both projects is the characterization of the natural background radiation of the ULs participating in them. Natural radioactivity tests were carried out in selected underground locations and included: (a) in-situ gamma-ray measurements using gamma spectrometry and semiconductor high purity germanium detector (HPGe), (b) measurements of radon concentration in the air with the use of $\mathrm{RAD} 7$ detector, (c) as well as laboratory measurements of rock and water samples taken from the tested underground locations with the use of alpha / beta and gamma spectrometry techniques.

During the presentation, the results of measurements of natural background radiation in several ULs will be discussed. 


\title{
Comparison of coincidence summing correction factors calculated by EFFTRAN and GESPECOR software
}

\author{
Mirjana Đurašević, Aleksandar Kandić, Igor Čeliković, \\ Zorica Obradović, Tamara Milanović, Ivana Vukanac
}

Vinča Institute of Nuclear Sciences, National Institute of Republic of Serbia, University of Belgrade, Belgrade, Serbia

\section{https://doi.org/10.21175/rad.abstr.book.2021.28.8}

Gamma ray spectrometry with high purity germanium (HPGe) detector is one of the most widely used methods for environmental samples measurements. It is basically a non-destructive method and usually there is no need for the extensive sample preparation. True coincidence summing effect has a significant effect in gamma ray spectrometry analysis, especially in low source-to-detector geometry. Many authors have paid attention to this problem mainly by analyzing and determining coincidence summing correction factors for artificial radionuclides. However, the effect of true coincidence summing can significantly affect the result obtained by gamma ray spectrometry of environmental samples. Therefore, special attention should be paid to the determination of correction factors for coincidence summing of radionuclides from natural series (uranium, thorium and actinium series).

Coincidence summing correction factors can be determined in several manners. Application of appropriate software packages is one of the most common. Software packages EFFTRAN and GESPECOR are among the most used ones. These software are based on Monte Carlo simulation. EFFTRAN is efficiency transfer code with semiempirical approach, whereas GESPECOR is dedicated code specifically tailored to solve most of the problems concerning gamma spectrometric measurements. The values of the correction factors depend on the detector geometry as well as the relevant data related to analyzed sample. The geometry of the detector implies the parameters of the detector itself (crystal dimensions, thickness of the dead layer, the thickness of the detector window). On the other hand, the relevant data related to analyzed sample includes dimensions of the container in which the sample is packed, sample density, chemical composition.

The aim of this work is to compare coincidence summing factors obtained by using EFFTRAN and GESPEOR software in the case of p-type semiconductor HPGe detector with $30 \%$ relative efficiency. All coincidence summing correction factors were calculated for soil sample in the cylindrical geometry and for radionuclides which are usually analyzed for environmental samples:

- for the uranium series: ${ }^{214} \mathrm{~Pb}$ for energies $295.22 \mathrm{keV}$ and $351.93 \mathrm{keV}$; ${ }^{214} \mathrm{Bi}$ for energies $609.32 \mathrm{keV}, 1120.29 \mathrm{keV}$ and $1764.54 \mathrm{keV;}{ }^{234 \mathrm{mPa}}$ for energies $766.36 \mathrm{keV}$ and $1001.03 \mathrm{keV}$.

- for the thorium series: ${ }^{212} \mathrm{~Pb}$ for energies $238.63 \mathrm{keV}$ and $300.09 \mathrm{keV}$; ${ }^{208} \mathrm{Tl}$ for energy $583.19 \mathrm{keV} ;{ }^{212} \mathrm{Bi}$ for energy $727.33 \mathrm{keV} ;{ }^{228} \mathrm{Ac}$ for energies $911.2 \mathrm{keV}$ and $968.97 \mathrm{keV}$.

- for the actinium series: ${ }^{235 \mathrm{U}}$ for energies $143.76 \mathrm{keV}, 163.33 \mathrm{keV}, 185.71 \mathrm{keV}$ and $205.31 \mathrm{keV}$.

The obtained values of the correction factors for coincidence summing showed a good agreement between these two software packages. Mutual deviations between correction factors calculated by using EFFTRAN and GESPECOR software were within $\pm 3 \%$. 


\title{
Characterization and applications of electron and X-ray beams of the REX facility
}

\author{
Monia Vadrucci' ${ }^{1}$ Fabio Borgognoni ${ }^{1}$, Alessia Cemmi², \\ Ilaria Di Sarcina², Beatrice D'orsi3 ${ }^{3}$, Luigi Picardi'
}

\begin{abstract}
1 APAM Laboratory, Application of Radiations Technical Unit, ENEA, Frascati (RM), Italy
2 SNI Laboratory, Technologies, Plants and Materials for Nuclear Fission Unit, ENEA, Rome (RM), Italy

3 Sapienza/INRS, Rome, Italy
\end{abstract}

\section{https://doi.org/10.21175/rad.abstr.book.2021.28.9}

The REX source is a versatile irradiation machine employed for experimental campaigns in different research fields thanks to its customizable radiation beam.

The system is based on a linear electron accelerator and a removable head containing an electron to X-ray converter with its collimator.

The machine and the irradiation chamber were manufactured and commissioned at ENEA Frascati Research Center.

Some of the REX applications we performed are related to irradiation of biological systems, development of in-beam monitoring devices, test of beam monitors, material radiation damage studies and cultural heritage. Experiments are carried out for specimen treatment with as by electrons as by X-rays.

Alanine-ESR and other secondary dosimetric systems have been applied to control the intensity of both electron and X-ray beams and a preliminary calibration with the absolute Fricke solution has been performed at the ENEA Calliope gamma irradiation facility.

The characterization of the two radiation qualities produced by REX source in the current operating configuration is presented in this work. 


\title{
Evaluation of the precision of radioactive deposition measurements
}

\author{
Dorottya Jakab, Zsuzsa Endrődi, \\ Tamás Pázmándi, László Tósaki, Péter Zagyvai
}

\author{
Centre for Energy Research, Budapest, Hungary
}

\section{https://doi.org/10.21175/rad.abstr.book.2021.28.10}

Sampling and measurement of radioactive deposition (fallout) plays an important role in environmental radiation monitoring. It provides information on the existing environmental radioactive contamination levels and detects any changes in the activity concentrations of radionuclides deposited on the ground, and consequently enables the assessment and execution of actions for the protection of public. By its joint monitoring with airborne concentrations by active sampling and prevailing meteorological conditions, the movement of radionuclides through the environmental compartments, following a discharge to the atmosphere, can also be investigated.

To ensure the adequate fulfillment of the above objectives, the precision of the radioactive deposition measurements has been examined in a series of experiments over several years, the obtained results will be summarized in this work. We experimentally quantified the uncertainty arising from the deposition sampling process and evaluated its relation to the uncertainty associated to the analytical measurement, the gamma spectrometric analysis. Key sources of the uncertainty of the measurement process were identified which should be the target of reduction in order to enhance the overall performance of the method.

As part of the environmental radiological monitoring system operated in the KFKI Campus in Budapest, the sampling of deposition is carried out at 4 compound measurement stations using identical stainless steel collectors with a surface area of $0.2 \mathrm{~m}^{2}$. Routine sampling frequency varies from weekly to monthly periods at the different stations. In addition to the existing samplers for combined sampling of the total deposition, some of which are located at the same measurement station for parallel collection, we also installed a collector capable of separate sampling of wet and dry deposition on the site. These measurements facilitated the examination of the deposition processes, furthermore, the validation of dry and wet deposition models and model parameters (dry deposition velocity and scavenging coefficient). 


\title{
Monte Carlo simulation methods for gamma-ray metrology
}

\author{
Ciprian Cosar'1, Aurelian Luca², Catalin Matei3, Maria Sahagia4
}

\author{
1 University of Bucharest, Faculty of Physics, Horia Hulubei National Institute for R\&D in Physics and \\ Nuclear Engineering (IFIN-HH), Bucharest, Romania \\ 2 Horia Hulubei National Institute for R\&D in Physics and Nuclear Engineering (IFIN-HH), Bucharest, \\ Magurele, Romania \\ 3 IFIN-HH / Extreme Light Infrastructure Nuclear Physics, (ELI-NP), Bucharest Magurele, Romania \\ 4 Faculty of Physics, University of Bucharest, Magurele, Ilfov, Romania; Horia Hulubei National Institute for \\ R\&D in Physics and Nuclear Engineering (IFIN-HH), Bucharest Magurele, Romania
}

\section{https://doi.org/10.21175/rad.abstr.book.2021.28.11}

Gamma-ray metrology at energies above the $3 \mathrm{MeV}$ threshold is novel and difficult task to undertake, mainly because there are few available radioisotopes emitting gamma-rays from $3 \mathrm{MeV}$ up to $5 \mathrm{MeV}$ and no known radioisotopes available for energies higher than $5 \mathrm{MeV}$. We are presenting our results using 3 different sources in secular equilibrium and one used as a standard source.

Another important characteristic for gamma-ray metrology is the detector material, which needs to be carefully chosen, as high efficiency and high-resolution are needed in order to perform accurate measurements of these radiations.

The Monte Carlo simulation methods are well known and established by now and are being used to solve various problems, to compare theoretical and experimental results and to support a wide range of activity standardization techniques such as those used in gamma-ray metrology.

Here, we propose new Monte Carlo simulation methods and guides to support and improve gamma-ray metrology at interval energies from $60 \mathrm{keV}$ up to the $3 \mathrm{MeV}$, based on comparisons with published efficiency and resolution data for High Purity Germanium Detectors (HPGe) (M. C. Lépy et. al., Appl. Radiat. Isot. 154 (2019) 108850). This type of semiconductor detector is well known for gamma-ray spectrometry and was selected because it has a high resolution for the energy ranges of the gamma-rays emitted by selected radionuclides.

For a better representation, the code MCNP was chosen, since it has been used for a long time and is well understood by now, and it is validated for various topics in nuclear physics and engineering.

For the present work, sources of $152 \mathrm{Eu}, 226 \mathrm{Ra}$ and daughters in secular equilibrium, 232Th (Thorium Ore) and daughters in secular equilibrium, and $(238 \mathrm{U}+235 \mathrm{U}+234 \mathrm{U}$ ) (Natural (not enriched) Uranium Ore) and daughters in secular equilibrium, as well as the corresponding gammaray spectra recorded with a predefined HPGe detector characteristics (http://www.lnhb.fr/icrm gs wg/icrm gs wg benchmarks/) were generated/simulated with the help of SuperSYNTH interface software for MCNP and of the code MCNP Version 6.2. The detection efficiency and the coincidence summing corrections corresponding to the gamma-rays from all spectra generated were determined using the FitzPeaks software (for $186.21 \mathrm{keV}$ (226Ra) no coincidence summing corrections are necessary).

The outcome of the methods employed here can be adapted further ahead to characterize all types of detectors in a wide energy range of the gamma-rays. The final goal is to be able to characterize detectors resolution and efficiency for gamma-rays with energies of $10 \mathrm{MeV}$ to $20 \mathrm{MeV}$. 


\title{
Broadband and band-selective measurements of radiofrequency EM field with drone system around $5 \mathrm{G}$ base station
}

\author{
Peter Necz ${ }^{1}$, Balázs Gyulai², József Krausz², György Thuroczy \\ 1 National Public Health Center, Budapest, Hungary \\ 2 National Media and Infocommunications Authority, Budapest, Hungary
}

\section{https://doi.org/10.21175/rad.abstr.book.2021.28.12}

Introduction. The aim of present study was to develop the method of measuring the $\mathrm{RF}$ electromagnetic field and exposure patterns of fixed outdoor RF sources by a drone system. Previous study was taken in 2018 about the feasibility of this method. The easily available drone systems today may support using them in the measurements of several environmental exposure patterns. Our trial was to use the drone system to perform RF exposure measurements in the vicinity of $5 \mathrm{G}$ base station. Another aim was to check how to use a lightweight personal exposimeter and a spectrum analyzer in the study.

Methods. In September 2020 environmental RF exposure around a base station was investigated during a series of flights by RF field meters attached to a drone. It was strong enough to carry the following two devices:

1. Narda SRM-3006 spectrum analyzer (Narda-STS, New York, USA) with a Narda 3502/01 Three-axis Antenna.

\section{ExpoM-RF Personal RF Exposure Meter (Fields at Work GmbH, Zurich, Switzerland).}

The drone was to fly from $3 \mathrm{~m}$ to $36 \mathrm{~m}$ above ground level in three distinct columns around a rooftop mounted base station. At the investigated base station a $5 \mathrm{G}$ base station was deployed at 3.6 GHz band (FR1) beyond other $2 \mathrm{G}-4 \mathrm{G}$ base stations. A test mobile device nearby was forced to communicate with the base station continuously. We choose vertical columns for each of the flights. The distance of the columns from the base station was $80 \mathrm{~m}, 60 \mathrm{~m}$ and $40 \mathrm{~m}$ respectively. The height of the first measurement point was $3 \mathrm{~m}$ above ground level, and after then the drone stopped at every $3 \mathrm{~m}$ up to $36 \mathrm{~m}$.

Results. Because the drone communicates with the remote control and the driver by Wi-Fi technology, we measured relatively high electric field strength values at ISM $2.4 \mathrm{GHz}$ band. That's why it is recommended to exclude from the evaluation the recordings of Wi-Fi band.

The results shows that during a flight, raising from the ground level the electric field strength in Mobile $3.5 \mathrm{GHz}$ band was increasing then at the top levels it started to decrease. This may be caused by the main beam of the base station, which is downwards from the base station (at the roof of the building) in the direction of ground. With the rising the drone approaches the main beam, and after moving away from it. Comparing flights with each other we can see that the electric field strength at this band is increasing and the position of the peak depends on the distance presumably due to the radiation pattern of the beam. 


\title{
Optimization of thyroid measurements with Monte Carlo simulation in in-vivo monitoring
}

\author{
Anna Pantya, Tamás Pázmándi, Laura Horváth, Péter Zagyvai
}

Centre for Energy Research, Budapest, Hungary

https://doi.org/10.21175/rad.abstr.book.2021.28.13

Ionizing radiations can cause external or internal exposure to the human body. The internal exposure is typically determined in two steps. In the first step the actual activity present in the body is determined by direct or indirect monitoring methods. By direct measurements, the activity in the whole or part of the human body can be determined (in-vivo). In the second step the intake value and the associated committed dose can be estimated on the basis of measured data and considering necessary assumptions on exposure conditions (time and route of intake, chemical form etc.). In case of the in-vivo measurement the low activity of the human body, the non-standard geometry and the limited measurement times considerably increase the measurement uncertainty. Thyroid measurements are also influenced by various parameters such as the age of the patient, the shape of thyroid, position of the thyroid inside the human body, the detector distance from the body surface, the distribution of the activity within the organ. Physical phantoms can be used for efficiency calibration of the measurement system.

After efficiency calibration of the thyroid counter with physical phantoms, efficiency calibration was performed by numerical methods to optimize the measurements. In order to take into account uncertainties introduced by these factors in the estimation of dose attributed to different radioactive isotopes of iodine, numerical simulations based on Monte Carlo photon transport techniques were performed, detector response and corresponding detection efficiencies were calculated. During our studies uncertainties due to differences in the distance of the thyroid from neck surface $(0.6-2.2 \mathrm{~cm})$, different size and shape of thyroid (e.g. variant thyroid shapes of children, teenagers and adults) and the detector distance from the body surface $(4-12 \mathrm{~cm})$ were analysed. All these factors affect the accuracy of dose estimation as well. The computed uncertainties due to various parameters should be taken into account while estimating the activity of iodine isotopes in the thyroid. 


\title{
Verification method to increase uniformity of foodstuff irradiation
}

\author{
Ulyana Bliznyuk ${ }^{1}$, Polina Borchegovskaya ${ }^{1}$, \\ Felix Studenikin1,2, Alexander Chernyaevi,2, Vadim Khankin²
}

1 M.V. Lomonosov Moscow State University, Physics Department, Moscow, Russia

2 Skobeltsyn Institute of Nuclear Physics of Lomonosov Moscow State University, Moscow, Russia

\section{https://doi.org/10.21175/rad.abstr.book.2021.28.14}

Increasing the shelf life of food products while maintaining their quality is a task of current interest directly related to the most important aspects of life and health of people around the world. Therefore, it is necessary to constantly develop and improve existing food processing methods. Irradiation of products with beams of accelerated electrons is one of the most effective treatment methods. To ensure the appropriate quality of irradiated products, it is important to carefully monitor the absorbed radiation dose throughout the entire volume of products, since an uneven distribution of the absorbed dose can lead to undesirable changes in the physical, chemical, and organoleptic characteristics of the product.

The purpose of the research carried out at Moscow State University (MSU) in collaboration with the D.V. Skobeltsyn Scientific Research Institute of Nuclear Physics, is to study the effect of ionizing radiation on biological objects and develop methods for enhancing the efficiency of radiation processing.

It is proposed to use aluminum modifier plates of various thicknesses installed between the irradiated object and the accelerated electron beam to increase the degree of irradiation homogeneity.

A phantom of PETG plastic was made for experimental verification of the method, consisting of $502.5 \mathrm{~mm}$ thick plates allowing for the placement of dosimetric films in $0.5 \mathrm{~mm}$ deep grooves.

A series of irradiation treatment sessions were carried out on the phantom with and without the addition of $1 \mathrm{~mm}, 2 \mathrm{~mm}$, and $3 \mathrm{~mm}$ thick aluminum modifier plates. An industrial continuous electron accelerator UELR-10-15-S-1 was chosen as a source of ionizing radiation, operating on a mode with maximum effective processing energy of $9.5 \mathrm{MeV}$ and a surface dose of $35 \mathrm{kGy}$.

The experimentally obtained values of the absorbed dose depending on the depth in layers of cubic phantoms made of PETG plastic (after beam processing) demonstrated that the addition of a $2 \mathrm{~mm}$ thick aluminum plate leads to an increase in the degree of irradiation homogeneity (the ratio of the dose on the phantom's surface to the maximum absorbed dose in the phantom's volume) from 0.864 to 0.963 .

This work clearly shows that it is possible to use aluminum modifier plates to increase the homogeneity of the absorbed dose distribution over the depth of the irradiated object during processing with accelerated electrons.

Acknowledgments: This research has been supported by the Interdisciplinary Scientific and Educational School of Moscow University "Photonic and Quantum technologies. Digital medicine". 


\title{
Monitoring studies to assess the condition of drinking water supply sources in potentially radon-hazardous areas by specific gross alpha activity
}

\author{
Lidiya Karl,2, Tatiana Lashchenova3,1, Albert Marenny²
}

\author{
1 RUDN University, Moscow, Russia \\ 2 State Research and Technical Center of Radiation-Chemical Safety and Hygiene FMBA, Moscow, Russia \\ 3 A.I. Burnasyan Federal Medical Biophysical Center FMBA, Moscow, Russia
}

\section{https://doi.org/10.21175/rad.abstr.book.2021.28.15}

The greatest contribution to the formation of internal radiation dose due to the consumption of drinking water is made by natural radionuclides ${ }^{222} \mathrm{Rn},{ }^{226} \mathrm{Ra},{ }^{238} \mathrm{U}$. The content of radionuclides in natural waters varies in a wide range. The WHO recommends taking measures to reduce the radioactivity of drinking water. Monitoring of drinking water supply in potentially radon hazardous areas is an important and urgent problem.

Assessment of drinking water quality by radiation safety parameters is carried out by specific gross alpha and beta activity according to the requirements of the main regulatory documents. The currently used methods for measuring $\sum \alpha$ in water require a long-term sample preparation, while allowing no more than 2-4 samples to be analyzed simultaneously.

In this work, the methodology of monitoring studies in potentially radon-hazardous areas is developed, the advantage of which is to obtain reliable information on the assessment of $\sum \alpha$ of sources of drinking water supply of the population simultaneously a large number of samples, which significantly reduces the economic costs of conducting research.

The purpose of this work is the monitoring of drinking water supply sources of the population in potentially radon-hazardous areas according to the parameter of specific $\sum \alpha$.

The method is based on the measurement of specific $\sum \alpha$ by dipping tracked nitrocellulose detectors of LR-115-2 type into water samples, with the addition of stabilizing solutions. The detectors are incubated in the water samples for the time necessary for the detector to form a statistically significant number of tracks. The detectors are chemically etched after exposure. The number of tracks on the detectors is determined by electrospark breakdown. Based on the number of tracks, the specific $\sum \alpha$ of the samples is calculated.

Studies in this work were aimed at determining the chemical resistance of the film material LR-115-2 in distilled water, in different concentrations of acids $\mathrm{HCl}, \mathrm{HNO}_{3}, \mathrm{C}_{6} \mathrm{H}_{8} \mathrm{O}_{7}$, for their possible use as stabilizing additives for water samples. The registration characteristics of the track detector were studied. It was found that the film material LR-115-2 is chemically stable when held in distilled water, $0.2 \mathrm{M} \mathrm{C}_{6} \mathrm{H}_{8} \mathrm{O}_{7}, 0.1 \mathrm{M} \mathrm{HCl}$ for a long time. Studies have shown the fundamental possibility of using film detectors of LR-115-2 type to measure the specific $\sum \alpha$ of natural waters in the submerged version of the measurements. At exposure time of 10 days and 30 days the minimal detectable $\sum \alpha$ of water sample is 0.013 and $0.0043 \mathrm{~Bq} / \mathrm{ml}$ respectively. Using a track method with a submersible detector for measuring alpha activity of natural waters requires a technical design of the measurement process that provides the ability to measure a small volume of liquid (0.1-10 ml), obtained by methods of concentrating radionuclides from a large volume sample.

Acknowledgments: The reported study was funded by RFBR, project number 20-38-90291. 


\title{
Optimizing the size of cylindrical sucrose solid state/EPR dosimeters for high energy ionizing radiation
}

\author{
Yordanka Karakirova, Velislava Yordanova \\ Institute of Catalysis, Bulgarian Academy of Sciences, Sofia, Bulgaria
}

\section{https://doi.org/10.21175/rad.abstr.book.2021.28.16}

Electron paramagnetic resonance (EPR) spectroscopy has proved to be a very effective method of investigation of free radicals created in materials during irradiation with high energy radiation. In the years sucrose has been studied for a long time as a dosimeter in radiation accident and in high-dose dosimetry by using different analytical techniques. The sucrose is a tissues equivalent material and it is involved in the DNA structure. That is why it is important to study its radiation properties. In the last years sucrose was considered as one of the most promising radiation sensitive materials (RSM) for EPR dosimetry as an alternative material of alanine. It is know that there is a saturation of dose response of irradiated sucrose Solid State/Electron Paramagnetic Resonance (SS/EPR) dosimeters at doses higher than $10 \mathrm{kGy}$. One of the possibilities to dissolve this problem is adding of internal standard $\mathrm{Mn}^{2+}$ magnetically diluted in $\mathrm{MgO}$ to the RSM. However no many laboratories have such kind of standard and it is not easy to be prepared. Therefore the aim of the present work is to achieve a linear dose response of the cylindrical dosimeters at possible the widest interval of doses, only through change their diameter and height and without to use an internal standard. In view of this it has been researched cylindrical sucrose dosimeters with different sizes (diameters 2, 3 and $4 \mathrm{~mm}$ and heights 5,10 and $14 \mathrm{~mm}$ ). From the obtained results have been found that with decreased of the diameter of the dosimeters from 4 to $2 \mathrm{~mm}$ the intensity of the EPR signal became linearly depended from the absorbed dose radiation in the dose range 1-50 kGy. In the dosimeters with diameters 3 and $4 \mathrm{~mm}$ a weak saturation of the dose response curves at higher doses have been observed. This confirms our previous claim that the saturation is due to simultaneously impact of the deeper penetration of the samples in the electric field in the EPR cavity and of the irradiation with high dose gamma rays. The increased of the height of the dosimeters from 5 to $14 \mathrm{~mm}$ aims to increase the sensitivity and to some extent to compensate the loss of sensitivity due to the smaller diameter. Charcoal samples with the same shape for comparison have been prepared. 


\section{The internal dose rate in quartz grains: Implications for luminescence dating}

\section{Agnieszka Szymak, Piotr Moska, Konrad Tudyka, Grzegorz Poręba, Grzegorz Adamiec}

Institute of Physics - Centre for Science and Education, Silesian University of Technology, Gliwice, Poland

\section{https://doi.org/10.21175/rad.abstr.book.2021.28.17}

This work presents how the internal alpha and beta dose rates in quartz grains obtained from sandy sediments impacts luminescence dating. In order to determine the internal dose rates, the innovative $\mu$ Dose systems were used.

Our findings clearly indicate and confirm that etched quartz grains contain radioactive isotopes, hence the assumption that the only source of radioactivity is radioisotopes from outside is an erroneous approximation.

Presented results show that the internal radioactivity has a relevant influence on determining the age of the tested samples. Ignoring the internal alpha and beta components during the total dose rate assessing can overestimate the luminescence age. The internal dose rate to total dose rate ratio in our measurements often exceeds $10 \%$. This means that the correction for internal dose rates should always be applied, otherwise the final dose rates will be underestimated, and the final results overestimated. 


\title{
The effect of water gases on radon measurements in electrostatic cell radon monitor
}

\section{Robert Csaba Begy ${ }^{1,2}$, Codrin Fabian Savin²,1, David-Karoly Sule ${ }^{2,1}$}

\author{
1 Interdisciplinary Research Institute on Bio-Nano-Sciences, University of Babes-Bolyai, Cluj-Napoca, \\ Romania \\ 2 Faculty of Environmental Science and Engineering, University of Babes-Bolyai, Cluj-Napoca, Romania
}

\section{https://doi.org/10.21175/rad.abstr.book.2021.28.18}

Radon is a radioactive, naturally occurring, chemically inert gas that is present in water as a result of natural processes, such as decay of its parent nuclide ${ }^{226} \mathrm{Ra}$ and dissolution from the surrounding geological environment. Beside radon, other naturally-occurring gases can be present in water, such as carbon dioxide $\left(\mathrm{CO}_{2}\right)$ and hydrogen sulfide $\left(\mathrm{H}_{2} \mathrm{~S}\right)$. When measuring radon activities in water using $\mathrm{RAD} 7$, these gases are collected by the built-in air pump and entering the internal cell of the device. In this study, thirty spring water samples were subjected to radon measurements using two methods, Liquid Scintillation Counting (LSC) and $\mathrm{RAD}_{7}$ device. After performing the measurements, the resulted values showed a lack of similarity, RAD7 activities being significantly lower, and therefore suggesting an inverse correlation with the gas content of the samples. The aim of the present study is to investigate the effect of two gases, hydrogen sulfide and carbon dioxide, on the RAD7 radon measurements in water. In this regard, $\mathrm{CO}_{2}$ concentrations between $0.649 \mathrm{~g} / \mathrm{l}$ and $5.19 \mathrm{~g} / \mathrm{l}$ were generated in water samples containing ${ }^{222} \mathrm{Rn}$ activities ranging from $23.4 \pm 0.9 \mathrm{~Bq} / \mathrm{l}$ to $6.7 \pm 0.9 \mathrm{~Bq} / \mathrm{l}$, after which the samples were measured with the RAD7 device and the results compared to the initial values of the samples. The same procedure was followed for hydrogen sulfide concentrations of $0.125 \mathrm{mg} / \mathrm{l}$ to $3 \mathrm{mg} / \mathrm{l}$. In both cases, a major impact on the radon measured activity was observed, which decreased with $24.5 \%$ for a concentration of $1.29 \mathrm{~g} / \mathrm{l} \mathrm{CO}_{2}$ in water, and up to $57 \%$ for $5.19 \mathrm{~g} / \mathrm{l}$ $\mathrm{CO}_{2}$. For hydrogen sulfide, the radon measured values decreased with $42.5 \%$ for concentrations as low as $0.5 \mathrm{mg} / \mathrm{l}$, culminating in a reduction of $86.9 \%$ for $3 \mathrm{mg} / \mathrm{l} \mathrm{H}_{2} \mathrm{~S}$. The explanation for the obtained results can be found in Po-218 neutralization processes which are reducing the electrostatic efficiency of the silicon detector system. To eliminate the interferences of these gases on RAD7 radon measurements in water, a sample preparation method was developed for the precipitation and deposition of $\mathrm{CO}_{2}$ using $\mathrm{Ca}(\mathrm{OH})_{2}$, respectively for $\mathrm{H}_{2} \mathrm{~S}$ using $\mathrm{Pb}\left(\mathrm{NO}_{3}\right)_{2}$. This procedure, if applied prior to the RAD7 measurements, leads to the removal of the effect of carbon dioxide and hydrogen sulfide on $\mathrm{RAD} 7$ radon measured activities. 


\title{
Random coincidence and angular correlation corrections in the sum-peak method using Monte Carlo simulations - A new approach
}

\author{
Tomas Nemeš ${ }^{1}$, Dušan Mrđa² ${ }^{\text {, Ištvan Bikit }}{ }^{2}$
}

\author{
1 Faculty of Technical Sciences, Novi Sad, Serbia \\ 2 Faculty of Sciences, Novi Sad, Serbia

\section{https://doi.org/10.21175/rad.abstr.book.2021.28.19}

The sum-peak method is a technique for measuring the absolute activity of gamma cascade emitting sources with a single gamma spectrometer. The effects of angular correlations and random coincidences, if not taken into account, can significantly reduce the accuracy of the method. However, we show that Monte Carlo simulations can reproduce the spectral data including pile-up events without using Poisson distribution with a sufficient quality to perform the required corrections. The new method for forming the count rate equations and performing Monte Carlo simulations leads to a generalized formula for the sumpeak method, including random coincidences of any order. 


\section{Radiation monitoring system}

\section{Katarina Karadzic}

School of Electrical Engineering, Belgrade, Serbia

https://doi.org/10.21175/rad.abstr.book.2021.28.20

Radiation sources can be natural and man-made. Undetected radiation in real-time can cause many problems which are related to the general health conditions of the whole environment. That is one of the reasons why real-time radiation monitoring has an essential role in the detection of dangerously high levels of radiation. This paper provides a proposal of a radiation monitoring system including sensor, gateway and IoT Cloud design. Also, the radiation measurement results from real-life scenarios will be shown.

Keywords: Radiation monitoring, beta, gamma, X-ray, IoT Cloud, radiation measurement 


\title{
Determination of radon emanation for phosphogypsum produced in Serbia
}

\section{Predrag Kuzmanović1,2, Igor Čeliković3, Sofija Forkapićc1, Nataša Todorovićc1, Leposava Filipović Petrovićc ${ }^{2}$, Dušan Mrđaa ${ }^{1}$, Jovana Knežević1}

\author{
1 University of Novi Sad, Faculty of Sciences, Department of Physics, Novi Sad, Serbia \\ 2 Academy of Professional Studies Šabac, Department of Medical and Business-Technological Studies, Šabac, \\ Serbia \\ 3 University of Belgrade, Vinča Institute of Nuclear Sciences, Laboratory for Nuclear and Plasma Physics, \\ Beograd, Serbia
}

\section{https://doi.org/10.21175/rad.abstr.book.2021.28.21}

Phosphogypsum is a waste that is generated in the production of fertilizers and is very similar to the natural gypsum, thus its use in the construction industry is possible. It usually contains an increased concentration of ${ }^{226} \mathrm{Ra}$ in its composition. The paper presents the results of measuring the radon emanation coefficients for phosphogypsum, natural gypsum and a mixture of natural gypsum and phosphogypsum (50\%), that could be potentially used for the production of gypsum boards that are widely applied in construction. Using the method of gamma spectrometry the concentration of ${ }^{226} \mathrm{Ra}$ in the samples was determined, while the concentration of emanated radon from the samples was measured in the specially constructed chambers of different volumes (1.5 l, 2.5 l, 4.25 l, and $30 \mathrm{l}$ ), using active radon monitors RAD7 (Durridge Company) and RTM1688-2 (Sarad GmbH). Radon emanation measurements were performed for about 100 cycles of $1 \mathrm{~h}$, after which the emanation curve was fit and the equilibrium concentration of radon activity after one month was theoretically estimated. The effect of chamber volumes on the obtained values of the radon emanation coefficient was investigated. On the basis of the obtained emanation coefficients, it is concluded that these values are strongly dependent on the volume of the measuring chamber and that they increase with expanding the chamber volume. Therefore, it is recommended to use larger volumes of measuring chambers when determining the emanation coefficient for phosphogypsum or natural gypsum. In this way, the real conditions in rooms that are potentially constructed of such materials are better represented, while at the same time the existing effect is neglected. 


\title{
Thyroid scintigraphy using Technetium-99m
}

\section{Florina-Gianina Elfarra',2, Radu Mutihac', Ileana Radulescu3}

\author{
1 University of Bucharest, Faculty of Physics - Doctoral School of Physics - Department of Biophysics and \\ Medical Physics, Bucharest, Romania \\ 2 St. John's Emergency Clinical Hospital, Bucharest, Romania \\ 3 National Institute for Physics and Nuclear Engineering Horia Hulubei IFIN-HH, Magurele-Bucharest, \\ Romania
}

\section{https://doi.org/10.21175/rad.abstr.book.2021.29.1}

The choice of radiopharmaceutical for visualization of the thyroid gland is conditioned by: the degree of its concentration in the thyroid; the need for low irradiation of both the gland and the whole body; the emission of sufficiently high gamma radiation for increased detection efficiency; suspected pathology; procureability and cost, as well as the doctor's preference. Currently, nuclear medicine uses Tc-99m. Tc-99m pertechnetate has the following physical and radiobiological benefits: - emits $140 \mathrm{keV}$ gamma radiation, allowing to obtain good quality images; - produces low irradiation of the thyroid (0.3 rad / mCi) and of the whole body (0.011-0.018 $\mathrm{rad} / \mathrm{mCi}$ ); - even allows the repetition of the scintigraphy after a short period of time, due to T1/2 less than $6 \mathrm{~h}$; - makes it possible to visualize the thyroid after about 20 minutes, without undergoing the process of organification in the gland (iodine enters the cell and participates in cell metabolism producing thyroid hormones, while 99m Tc only sticks to the cell surface); - the dose used 1-10 mCi for Tc-99m.

Thyroid nodules are a very common problem in adults [1]. Their prevalence increases with age and for this reason the thyroid scintigraphy is a technique that allows the evaluation of the functional characteristics [2]. In this study, a clinical trial with two different thyroid disorders were preferred, one patient with toxic thyroid nodule in the right lobe, the second patient was with left thyroid nodule agenesis. The radiopharmaceutical chosen for performing scintigraphy was pertechnetate Tc99m, and was administered intravenously. After waiting for 20 minutes, the patient was placed on the table of the scintigraphy device with his head bent on his back, so that the area of the thyroid lodge is under the detector as central as possible. To assess the position and size of the thyroid on the image from the previous incidence, mark the upper edge of the sternum, the collarbones, the upper edge of the thyroid cartilage and the lateral edges of the neck. When using the scintillation chamber, it is preferable that each image obtained should contain 200,000 pulses.

In conclusion, it was found that total lobe suppression, in patients with the toxic thyroid nodule, would certainly happen if the toxic nodule had accumulated much more radioactivity than the normal lobe. Also, covering the toxic nodule with lead plate may allow the observation of radioactivity in the suppressed lobe.

\section{References}

[1] Tunbridge WM, Evered DC, Hall R. The spectrum of thyroid disease in a community: The Whickham survey. Clin Endocrinol. 1977; 7: 481-493

[2] Moreno-Reyes R, Kyrilli A, Lytrivi M, Bourmorck C, Chami C, Corvilain B, Is there still a role for thyroid scintigraphy in the workup of a thyroid nodule in the era of fine needle aspiration cytology and molecular testing? F100oResearch 2016, 5: 763-770 


\title{
Cancer in the countries of the SEE (Balkans) region and the prospective of the Particle Therapy Center - SEEIIST
}

\author{
Mimoza Ristova \\ Faculty of Natural Sciences and Maths, University Ss Cyril and Methodius, Skopje, North Macedonia
}

https://doi.org/10.21175/rad.abstr.book.2021.29.2

Purpose. A recent initiative was launched for establishing the South-East European International Institute for Sustainable Technologies (SEEIIST) which will provide a cutting-edge hadron radiotherapy treatment and research institute for treating cancer patients with Hadron Therapy (HT). To justify the initiative for building the SEEIIST facility, a study was conducted to estimate the number of cancer patients from the SEE region that would be eligible for HT.

Methods and materials. Two different methods for projecting the future annual cancer incidence have been applied: (a) using the Globocan model of the IARC@WHO which employs country's demographic factors, and (b) averaging the crude incidence data of three SEE countries with available national cancer registries, using a linear regression model of combined incidence per 100,000 , and applying it to the entire SEE region. Cancer epidemiology data were collected and studied by using the countries' cancer datasheets from the World Health Organization (WHO.) The top 10 cancers were presented for the SEE region. Studies of other countries were used to develop a primordial model for estimating the number of SEE patients that could be treated most successfully with HT upon SEEIIST commissioning in 2030.

Results. A model was developed to estimate the number of eligible patients for HT from SEE. It is estimated that 2900-3200 patients per year would be eligible for HT in the new SEEIIST facility in 2030.

Conclusion. After commissioning, SEEIIST will initially treat approximately 400 patients/year, progressing towards 1000. Creation of SEEIIST dedicated patient selection criteria will be both necessary and highly challenging. 


\title{
Study of the ion source complex radioactive contamination in the SPES facility
}

\author{
Lisa Centofante1,2, Antonietta Donzella',3, Aldo Zenoni1,3, \\ Matteo Ferrari4, Michele Ballan², Stefano Corradetti², \\ Fabio D'Agostini'2, Giordano Lilli5,2, Mattia Manzolaro², Alberto Monetti², \\ Luca Morselli, ${ }^{6,2}$, Daniele Scarpa ${ }^{2}$, Alberto Andrighetto
}

\author{
1 Università degli Studi di Brescia, Brescia, Italy \\ 2 Istituto Nazionale di Fisica Nucleare - Laboratori Nazionali di Legnaro, Legnaro (PD), Italy \\ 3 Istituto Nazionale di Fisica Nucleare - Sezione di Pavia, Pavia, Italy \\ 4 CERN, Geneva, Switzerland \\ 5 Università degli studi di Padova, Padova, Italy \\ 6 Università degli Studi di Ferrara, Ferrara, Italy
}

\section{https://doi.org/10.21175/rad.abstr.book.2021.30.1}

The ISOL (Isotope Separation On Line) technique is today established as one of the primary techniques for the production of high intensity and high quality radioactive beams. The main reaction channels used for the production of radioactive isotopes (spallation, fragmentation and fission) produce, for a given amount of the desired isotope, many orders of magnitude of different radioactive isotopes. Due to residual activity generated by the interactions of the primary beam, by activation due to the intense neutron fields and by deposition of the produced radioactive ions inside the magnetic separators and beam line elements, an ISOL facility in operation becomes an intense radioactive source. As a consequence, the biological hazard imposes severe radiological safety challenges to the design, operation, ordinary and extraordinary maintenance of such facilities. In this respect, one of the most challenging component is the ion source complex, where part of the fragments produced in the target is ionized and subsequently extracted. In particular, the ion extraction electrode sub-component has the crucial function of extracting radioactive ions from the ion source volume by means of an intense electric field providing also the first acceleration to the extracted beam. The radioactive contamination of this sub-component is studied, by means of the FLUKA Monte Carlo code, in the case of the SPES (Selective Production of Exotic Species) facility, in advanced construction phase at INFN-LNL (Istituto Nazionale di Fisica Nucleare, Laboratori Nazionali di Legnaro), Padua, Italy. The developed model includes: the isotope production by the interactions of a $40 \mathrm{MeV}$ energy proton beam on a ${ }^{238} \mathrm{UC}_{\mathrm{x}}$ target; the selection of the produced radioactive isotopes able to stick on the ion extraction electrode tip during one year of operation of the facility; the time evolution of the implanted isotopes during the operation period as well as appropriate cooling periods before maintenance interventions; the evaluation of the ambient dose equivalent rate generated by the contamination of the ion extraction electrode. Basing on these results, the possibility of manual interventions for maintenance and emergency versus the use of a remote handling system is discussed with reference to the ALARA principle. An automatic system designed and realized at LNL for the ion extraction electrode tip replacement is shortly described. 


\title{
A competition between two- and three-body dissociation channels in photo-double-ionization of tetrahydro-2H-pyran and 3,4-dihydro-2 $\mathrm{H}$-pyran molecules
}

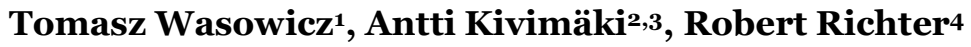

\author{
1 Division of Complex Systems Spectroscopy, Institute of Physics and Applied Computer Science, Gdansk \\ University of Technology, Gdansk, Poland \\ 2 Nano and Molecular Systems Research Unit, University of Oulu, Oulu, Finland \\ 3 MAX IV Laboratory, Lund University, Lund, Sweden \\ 4 Elettra - Sincrotrone Trieste, Area Science Park Basovizza, Trieste, Italy
}

\section{https://doi.org/10.21175/rad.abstr.book.2021.30.2}

Various molecules possess structures consisting of the heterocyclic rings containing oxygen atoms. These substances often play a significant role in many different fields of chemistry, medicine, and biology. Due to a specific atomic composition and bond arrangement, many of these molecules retain unique electronic properties, which may be probed by diverse spectroscopic techniques, including those utilizing synchrotron radiation. In particular, the photoelectron-photoion coincidence (PEPICO) technique provides detailed information about the presence of specific bonds, the reactivity of these molecules, as well as their electronic structure and decomposition mechanisms.

In the present communication, we present results on the dissociative photo-double-ionization of the six-membered heterocyclic molecules containing oxygen, namely 3,4-dihydro- $2 H$-pyran (DHP, $\mathrm{C}_{5} \mathrm{H}_{8} \mathrm{O}$ ) and tetrahydro-2 $\mathrm{H}$-pyran (THP, $\mathrm{C}_{5} \mathrm{H}_{10} \mathrm{O}$ ). Both molecules exhibit a wide spectrum of targets and broad biological, chemical, and engineering activities. For example, the reaction of alcohols and DHP gives 2-tetrahydropyranyl ethers usually used as protecting groups in organic synthesis. A tetrahydropyran ring system is the core of pyranose sugars, such as glucose as well as anthracyclines, a class of antitumor agents that induce, among other things, indirect damage to DNA via reactions involving reactive oxygen species. The experiments were carried out at the Gas Phase Photoemission beamline at the Elettra-Sincrotrone radiation facility exploiting the VUV excitation and the ion time-of-flight spectrometry combined with the PEPICO technique. The dissociative processes where only one electron is released are quite well known. However, double ionization is a more complex mechanism producing the doubly charged parent ion after two correlated electrons' emission. These doubly charged ions are usually very reactive, short-living objects that easily dissociate into the ionic fragments via many-body fragmentation pathways. The present experimental study aims at elucidating the mechanisms of these dissociation channels in DHP and THP. In particular, we show how the character of the bond arrangement in DHP and THP influences the generation of two- and three-body charge separation reactions occurring at the lowest energies. $\mathrm{C}_{5} \mathrm{H}_{8} \mathrm{O}$ has a non-aromatic ring with five carbon atoms and one oxygen atom. It contains one double bond. By removing the double bond by two more hydrogen atoms, we get a saturated six-membered ring of tetrahydro- $2 \mathrm{H}$-pyran. The analysis reveals that this small change in the bond rearrangement makes available a distinct stabilization of the DHP ring compared to the THP structure, featuring the activation of a two-body fragmentation pathway with competition to a three-body breakup.

Acknowledgments: We are grateful to the Elettra-Sincrotrone Trieste for providing beamtime no. 20190430. The research leading to this result has been supported by the project CALIPSOplus under Grant Agreement 730872 from the EU Framework Programme for Research and Innovation HORIZON 2020. The assistance of the staff of the Sincrotrone Trieste is also gratefully acknowledged. 


\title{
Ionization and fragmentation of the six-membered heterocycles containing oxygen - Comparative studies of electron and photon impact on the 3,4-dihydro-2 $\mathrm{H}$-pyran molecules
}

\author{
Michal Jurkowski, Tomasz J. Wasowicz
}

\begin{abstract}
Division of Complex Systems Spectroscopy, Institute of Physics and Applied Computer Science, Gdansk University of Technology, Gdańsk, Poland
\end{abstract}

\section{https://doi.org/10.21175/rad.abstr.book.2021.30.3}

The development of civilization has a significant impact on the lifestyle and environment in which we live. Increased life pace, too many obligations, stress, processed foods, and environmental pollution enhance cancer occurrence [1]. Therefore, there is a growing interest in various cancer treatments. One of the oncological cure methods is radiotherapy, which damages the tumour cells using ionizing radiation. This radiation's interaction with biological material produces structural and chemical modifications in the DNA helix. The impact of the primary photons and the secondary particles (electrons, free radicals, ions) produced along the photon tracks cause DNA damage by bond ruptures. They all generate many alterations to the carcinogenic cells that may lose the ability to replicate and consequently further die.

Therefore, it is essential to explore the processes leading to the fragmentation of DNA components and their analogues to characterize the underlying mechanisms. In this perspective, we present the fragmentation of the gas-phase 3,4-dihydro-2 $\mathrm{H}$-pyran $\left(\mathrm{C}_{5} \mathrm{H}_{8} \mathrm{O}\right)$ heterocyclic ether whose ring is the base of compounds found in living organisms and also is used in the synthesis of anticancer drugs [2,3]. Electron impact ionization and ionic fragmentation of $\mathrm{C}_{5} \mathrm{H}_{8} \mathrm{O}$ molecules were measured in the energy range from ionization threshold up to $70 \mathrm{eV}$ using electron mass spectrometer. The cation mass spectra, ionization, and ionic fragmentation efficiencies were determined over this energy range. Well-resolved mass peaks were detected in the mass range 10-90 amu and assigned to corresponding ionic molecular fragments. Appearance energies of selected ionic fragments were also determined. We will compare these results to the mass spectra recorded in the VUV photon-induced ionization and fragmentation of 3,4-dihydro-2H-pyran. Possible ionic fragmentation processes will also be discussed.

\section{References}

[1] G. Danaei, S. Vander Hoorn, A. D. Lopez, C.J. L. Murray, M. Ezzati "Causes of cancer in the world: comparative risk assessment of nine behavioral and environmental risk factors'. Lancet 366, 1784-93 (2005).

[2] Man Jung Han et al., "Syntheses and Biological Activity of Poly(methyl 3,4-dihydro-2H-pyran-2carboxylate-alt-maleic anhydride) Derivative' Journal of Bioactive and Compatible Polymers 5, 80-88 (1990).

[3] Jing $\mathrm{Li}$ et al., "Synthesis of biscoumarin and dihydropyran derivatives with promising antitumor and antibacterial activities' Bioorganic \& Medicinal Chemistry Letters 25, 5520-5523 (2015). 


\title{
Radiation defects in GaP, GaAsP, InGaN LEDs
}

\author{
Roman Vernydub', Olena Kyrylenko', Oksana Konoreva², \\ Oleksandr Radkevych3, Dmytro Stratilat ${ }^{2}$, Volodymyr Tartachnyk²
}

\author{
1 National Pedagogical Dragomanov University, Kyiv, Ukraine \\ 2 Institute for nuclear research of the NAS of Ukraine, Kyiv, Ukraine \\ 3 SE 'SRI of Microdevices' STC 'Institute for Single Crystals' of the NAS of Ukraine, Kyiv, Ukraine
}

\section{https://doi.org/10.21175/rad.abstr.book.2021.30.4}

The GaP, GaAsP, and InGaN LEDs can be successfully used as model objects for studying the effect of accelerated particles on charge transfer processes in objects with the $p$-n junction, radiative recombination, and radiation resistance.

The GaP output LEDs doped with $\mathrm{N}$ (green) and $\mathrm{Zn}, \mathrm{O}$ (red), GaAsi-x $\mathrm{P}_{\mathrm{x}}$ - orange $(\mathrm{x}=0.45)$ and yellow ( $\mathrm{x}=0.85$ ), InGaN (blue) and irradiated with electrons with $\mathrm{E}=2 \mathrm{MeV}, \mathrm{F}=10^{15} \div 10^{16} \mathrm{~cm}^{-2}$ have been studied.

On the current-voltage characteristics (CVC) at low temperatures (90-77 K) in GaP and GaAsP LEDs, arise of negative differential resistance (NDR) of the S-type [1], in the case of GaP is N-type has been revealed.

The arise of S-similarity in GaP diodes can be caused by the structural features of the C- band; the formation of the N-region is associated with tunneling transitions of carriers of the band-defect-band type. The detected area of NDR in the GaAsP solid solution is due to the presence of the GaP component.

Electron irradiation leads to the increase in the DR of the LEDs under study and to a decrease in the reverse currents.

A breakdown section arises on the CVC of GaP diodes at significant reverse voltages (U $=15 \div 20$ V); as the sample temperature rises, it shifts to the region of lower voltages, indicating the predominance of the tunneling breakdown mechanism.

The value of the coefficient of the imperfection of GaP LEDs within the currents of $10^{-7} \div 10^{-4} \mathrm{~A}$ is in the range of $n=1.6 \div 1.7$ which indicates the predominance of the tunnel-recombination component of the full current.

By controlling the voltage change depending on the annealing temperature ( $\left.\mathrm{T}_{\text {anneal }}\right)$ at a constant current through the $\mathrm{GaP}$ sample, the $\mathrm{U}\left(\mathrm{T}_{\text {anneal }}\right)$ curve can be divided into stages $20 \div 100^{\circ} \mathrm{C}$ and $160 \div$ $300{ }^{\circ} \mathrm{C}$. The emergence of the first stage can be interpreted as a process of decay of unstable defect formations localized near large-scale structural disturbances, such as dislocations or dislocation networks. Annealing in the range of $150 \div 350^{\circ} \mathrm{C}$, where the diffuse fluxes $\mathrm{V}_{\mathrm{P}}$ and $\mathrm{V}_{\mathrm{Ga}}$ coincide, is the main one in terms of the scale of restoration of the CVC of the irradiated samples.

The temperature dependence of the bandgap of the GaAsP solid solution is well described by the Varshni equation for $\mathrm{A}^{\mathrm{II}} \mathrm{B}^{\mathrm{V}}$ semiconductor compounds has been shown.

The irradiation of GaAsP LEDs leads to the expansion of the spectral lines due to the action of fields of structural defects of radiation origin and a decrease in the glow intensity has been revealed. Compared to GaP diodes GaAsP LEDs are radiation-resistant their average radiation damage coefficient of the lifetime is close to $1.2 \cdot 10^{-10} \mathrm{~cm}^{2} \mathrm{~s}^{-1}$ and for $\mathrm{GaP}$ is $8 \cdot 10^{-8} \mathrm{~cm}^{2} \mathrm{~s}^{-1}$.

Irradiation of InGaN LEDs with quantum wells causes a monotonic decreasing in the radiation intensity as a result of interbarrier tunneling of current carriers.

\section{References}

[1] G. Gaydar et al. Superlat and Microstr. 104 (2017) 316. 


\title{
The application of voxel and ORNL phantoms to the internal and external dosimetry
}

\author{
Dragana Krstic1, Dragoslav Nikezic1,2,

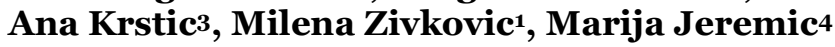

\author{
1 University of Kragujevac, Faculty of Science, Kragujevac, Serbia \\ 2 The State University, Novi Pazar, Serbia \\ 3 KVARK doo, Kragujevac, Serbia \\ 4 Clinical Center Kragujevac, Kragujevac, Serbia
}

\section{https://doi.org/10.21175/rad.abstr.book.2021.30.5}

The present study is based on application of mathematical and adult voxel models to the internal and external dosimetry. The ORNL is mathematical phantom developed at the Oak Ridge National Laboratory which consists of three main sections: an elliptical cylinder is a trunk; a circular cylinder and an elliptical cylinder capped by half an ellipsoid are neck and head and two truncated circular cones representing the legs. All organs of ORNL phantom were represented by simplified mathematical equations and inequalities. External shapes of organs were represented with planes, spheres, cylinders, cones, ellipsoids, elliptical cylinders, tori and combinations of these geometrical bodies.

ICRP Publication 110 describes the development of the adult voxel phantoms of the Reference Computational Male (RCP-AM) and Reference Computational Female (RCP-AF).Voxel models are constructed from computed tomographic (CT) or magnetic resonance imaging (MRI) data of real human body. From these data, voxel phantoms were segmented and then adjusted to the reference values.

Both types of phantoms were used in internal and external dosimetry.

The well-known MNCP6 Monte Carlo Neutron Particle code was performed for particle transport from the sources to the individual target organs of phantoms.

The great differences were found between ORNL-type and voxel models, due to the larger distance between ORNL phantom organs than in the real case, and due to simplified organ shapes.

\section{References}

[1] MCNP6.2 Monte Carlo N-Particle Transport Code System; Version 6.2. Report LA-UR-17-29981. Los Alamos, 2018.

[2] Eckerman K F, Cristy M, Ryman J C. Oak Ridge National Laboratory. TN 37831, USA; 1996.

[3] ICRP 110: Adult Reference Computational Phantoms. Realistic reference phantoms: An ICRP/ICRU joint effort. Ann. ICRP 39 3-5; Elsevier. 2009. 


\title{
Modelling the radioluminescence of alpha particles in the air by the Monte Carlo method
}

\author{
Ioana Lalau1,2, Mihail-Razvan Ioan'1
}

1 Horia Hulubei National Institute for R\&D in Physics and Nuclear Engineering, Magurele, Romania

2 University of Bucharest, Faculty of Physics, Magurele, Romania

\section{https://doi.org/10.21175/rad.abstr.book.2021.30.6}

In the last period, the alpha-induced luminescence of air was proposed to be utilized for remote detection of alpha emitting from radioactive waste sources or for rapid radon detection.

The aim of this study is to investigate the possibility to discriminate between the radioluminescence signal and Cherenkov photons due to the simultaneous presence of alpha and beta sources in the same container.

Alpha particles induce radioluminescence when absorbed in air. The photons are emitted in the near ultraviolet region by nitrogen molecules excited by secondary electrons. The accurate knowledge of the radioluminescence yield is of the utmost importance for novel radiation detection applications utilizing this effect.

On the other hand, the energetic electrons from the beta spectra can produce Cherenkov radiation in air in the same wavelengths as the photons emitted by deexcitation of nitrogen molecules.

When alpha and beta emitting radionuclides are simultaneously present in the sample, the detector must be able to discriminate between these contributions.

We determine the number of photons produced per unit area (photon flux) by deexcitation of nitrogen molecules and also the number of photons in the same wavelength range produced by beta particles when passing through air. Monte Carlo method is used to simulate the experimental setup and to calculate the sensitivity of the detector as a function of the activities of alpha and beta sources, of the energies of the emitted radiations, of the distances between detector and sources, and also of the sensitivities of the detector in different spectral ranges. 


\title{
Computed tomography in population living close to the nuclear production complex: Cohort profile
}

\author{
Mikhail Osipov1,2, Evgeniy Fomin², Mikhail Sokolnikov'
}

1 Southern Urals Biophysics Institute, Ozyorsk, Russia

2 Clinical Hospital \# 71, Ozyorsk, Russia

https://doi.org/10.21175/rad.abstr.book.2021.31.1

Purpose. The profile describes the data collection procedure and the sources of information for the Ozyorsk Computed Tomography Cohort study (OCTC study) designed to assess the low-dose effects of exposure to ionizing radiation due to computed tomography (CT) in population living within $10 \mathrm{~km}$ from the "Mayak" Production Association - the $1^{\text {st }}$ nuclear weapon production complex in the former USSR.

Materials and methods. The main data source for the OCTC study is the "CT Register" database which currently contains 26,626 records of Ozyorsk district residents exposed to diagnostic CT. The data have been retrospectively collected from five clinical hospitals located in Chelyabinsk region of the Russian Federation. The cohort data were linked to the local Cancer registry of Ozyorsk to obtain information on cancer incidence, and to the Mayak Worker Cohort data to obtain information on occupational exposure to protracted gamma- and alpha- ionizing radiation. The vital status and the cause of death have been derived from Ozyorsk clinical hospital no.71 and from the local death causes registry. The medical and dosimetric data as well as the information on main cancer risk factors have been detailed in the profile.

Results. 16,624 patients of both sexes at the age from o to 99 exposed to diagnostic radiation due to CT examinations during the period from 1993 to 2019 have been included in the cohort. The maximum number of CT examinations per patient reached up to 29 while $67.9 \%$ of persons were single-time examined. The individual radiation dose burden to the end of follow-up reached up to $150 \mathrm{mSv} .347$ patients (2.1\%) had been exposed to additional internal radiation due to PET/CT. 25\% of the cohort were the MPA staff occupationally exposed to ionizing radiation.

To the end of follow-up, 2,842 cancer cases had been accumulated in the cohort with $31.6 \%$ of cases diagnosed after the date of the $1^{\text {st }} \mathrm{CT}$ with $2.8 \%$ of second cancer cases. Vital status has been established for $60.1 \%$ of patients, and $30.3 \%$ of the study cohort had died to the end of follow-up with registered cause of death.

Discussion. The OCTC study cohort has several weak points related to possible loss information on individuals examined outside Chelyabinsk region, and those who had migrated from Ozyorsk as well. The vital status has not yet been established for about $40 \%$ of individuals. $14.2 \%$ of the cases had died during the $1^{\text {st }}$ year of follow-up. The radiation dose absorbed in the organs due to CT has not been yet calculated as of the end of the follow-up.

Conclusions. The OCTC cohort offers information on the main medical, dosimetric, demographic and occupational factors for the population of Ozyorsk district. The data can be used for an epidemiological study of late effects of exposure to low doses of diagnostic radiation. 


\title{
Evaluation of various atmospheric radon monitors
}

\author{
Ileana Radulescu, Mihail-Razvan Ioan, Aurelian Luca
}

National Institute for Physics and Nuclear Engineering Horia Hulubei IFIN-HH, Magurele-Bucharest, Romania

\section{https://doi.org/10.21175/rad.abstr.book.2021.31.2}

This work presents the results obtained in intercomparison measurements performed with various radon monitors types. The results presented are important from the point of view of choosing the best suitable monitor when monitoring the radon concentration in the outdoor environment, in such way to obtain the most reliable data. This work is in tight connection with the activities involved within traceRadon project that is aiming to develop the use of a radon transfer standard monitor to increase the metrological capacity of radon monitoring networks. Properties such as environmental parameters, activity levels, stability, accuracy, achieved uncertainty and portability for the field application of the commercially available radon monitors were considered to construct a matrix that will also be used to identify the required parameters for a transfer standard for the traceable calibration of atmospheric radon monitors according to IEC 61577. The purpose of this paper is to summarize the current state of conceptualization of such a transfer standard in environmental radon monitoring.

In the intercomparison test three laboratories have participated with four devices portable atmospheric radon measurement systems: 1346 model PYLON AB-5, manufactured by Pylon Electronics Inc. Canada - Lab. SALMROM (IFIN-HH); 1367 - Pylon System - Lab. LGABG - ICSI Ramnicu Valcea; 1292 - Pylon System and a System AlphaGuard - Lab. DMDR (IFIN-HH). The results showed the variation of radon activity concentration as function of temperature and relative humidity. The recorded ranges of the radon activity concentration for the measurements performed with AlphaGuard and Pylon monitors did not show a clear dependency on temperature and relative humidity. In the case of Radon Scout monitor, the temperature variation had an influence on the data measured. Another important aspect regarding the results of the measurements is the fact that the AlphaGuard data from one hour to another are less scattered than for the Radon Scout at concentrations above $300 \mathrm{~Bq} / \mathrm{m}^{3}$ the hourly difference being higher than $20 \%$. The data shows that after $40 \mathrm{~h}$ the radon activity concentration in the room with ventilation off, reached a steady-state, with an average value of $410 \mathrm{~Bq} / \mathrm{m}^{3}$.

Another important aspect when testing the radon monitors is related to the measurement uncertainties. For radon activity concentrations above $200 \mathrm{~Bq} / \mathrm{m}^{3}$ for a $10 \mathrm{~min}$. measurement the uncertainty (for $\mathrm{k}=1$ ) is about $20 \%$ for the AlphaGuard monitor. The corresponding measurement uncertainty of Pylon monitor is in the order of $17 \%$ (with $\mathrm{k}=1$ ) for a 10 min measurement, while for Radon Scout monitor this is also $20 \%$, but for a $1 \mathrm{~h}$ measurement $(\mathrm{k}=1)$. In all cases, the total uncertainty is limited by the sensitivity uncertainty and the statistical uncertainty component due to the background counts recorded by the monitor.

Acknowledgments: The results presented in this work are developed in the framework of the 19ENVo1 traceRadon project. This project has received funding from the EMPIR program co-financed by the Participating States and from the European Union's Horizon 2020 research and innovation program. 


\title{
Microdosimetric investigation of the radiation quality of low-medium energy electrons using Geant4-DNA
}

\author{
Ioanna Kyriakou', Ioanna Tremi², \\ Alexandros Georgakilas ${ }^{2}$, Dimitrios Emfietzoglou'
}

\begin{abstract}
1 Medical Physics Laboratory, Department of Medicine, University of Ioannina, Ioannina, Greece
2 DNA Damage Laboratory, Physics Department, School of Applied Mathematical and Physical Sciences,

National Technical University of Athens, Athens, Greece
\end{abstract}

\section{https://doi.org/10.21175/rad.abstr.book.2021.31.3}

The increasing clinical use of low-energy photon and electron sources (below few tens of keV) has raised concerns on the adequacy of the existing approximation of an energy-independent radiobiological effectiveness. In this work, the variation of the quality factor $(Q)$ and relative biological effectiveness (RBE) of electrons over the low-medium energy range $(0.1 \mathrm{keV}-1 \mathrm{MeV})$ is examined using several microdosimetry-based Monte Carlo methodologies with input data obtained from Geant4-DNA track-structure simulations. The sensitivity of the results to the different methodologies, Geant4-DNA physics models, and target sizes is examined. Calculations of $Q$ and RBE are based on the ICRU Report 40 recommendations, the Kellerer-Hahn approximation, the site version of the theory of dual radiation action (TDRA), the microdosimetric kinetic model (MKM) of cell survival, and the calculated yield of DNA double strand breaks (DSB). The stochastic energy deposition spectra needed as input in the above approaches have been calculated for nanometer spherical volumes using the different electron physics models of Geant4-DNA. Results are normalized at $100 \mathrm{keV}$ electrons which is here considered the reference radiation. It is shown that in the energy range $\sim 50 \mathrm{keV}-1 \mathrm{MeV}$, the calculated $Q$ and RBE are approximately unity (to within 1-2\%) irrespective of the methodology, Geant4-DNA physics model, and target size. At lower energies, $Q$ and RBE become energy-dependent reaching a maximum value of $\sim 1.5-2.5$ between $\sim 200-700 \mathrm{eV}$. The detailed variation of $Q$ and RBE at low energies depends mostly upon the adopted methodology and target size, and less so upon the Geant4-DNA physics model. Overall, the DSB yield predicts the highest RBE values (with RBEmax $\approx 2.5$ ) whereas the MKM the lowest RBE values (with RBEmax $\approx 1.5$ ). The ICRU Report 40, Kellerer-Hahn, and TDRA methods are in excellent agreement (to within $1-2 \%$ ) over the whole energy range predicting a $Q \max \approx 2$. In conclusion, the approximation $Q=\mathrm{RBE}=1$ was found to be valid only above $\sim 50 \mathrm{keV}$ whereas at lower energies both $Q$ and RBE become strongly energy-dependent. It is envisioned that the present work will contribute towards establishing robust methodologies to determine theoretically the energy-dependence of radiation quality of individual electrons which may then be used in subsequent calculations involving practical electron and photon radiation sources.

Acknowledgments: This research is co-financed by Greece and the European Union (European Social Fund - ESF) through the Operational Programme "Human Resources Development, Education and Lifelong Learning 2014-2020" in the context of the project "Microdosimetry using the GEANT4-DNA Monte Carlo code for $\mathrm{RBE}$ calculations of non-conventional radiation in radiotherapy" (MIS 5047632). 


\title{
DNA repair capacity of Saccharomyces cerevisiae after single and combined treatment with zeocin and different concentrations of Clinopodium vulgare L. extract
}

\author{
Teodora Todorova1, Iviyana Ivanova1,2, Mariya Yovkova1, \\ Maria Dimitrova ${ }^{1}$, Petya Parvanova ${ }^{1}$, Martin Dimitrov ${ }^{1,2}$, Stephka Chankova ${ }^{1}$
}

\author{
1 Institute of Biodiversity and Ecosystem Research, Bulgarian Academy of Sciences, Sofia, Bulgaria \\ 2 Faculty of Biology, Sofia University “St. Kliment Ohridski”, Sofia, Bulgaria
}

\section{https://doi.org/10.21175/rad.abstr.book.2021.31.4}

The aim of the present work was to evaluate whether the DNA protective potential of Clinopodium vulgare L. extract is related to changes in the repair capacity of Saccharomyces cerevisiae. Single and combined treatment with 10,100 and $1000 \mu \mathrm{g} / \mathrm{ml}$ plant extract and zeocin was performed on two yeast strains - haploid and diploid one. The role of incubation conditions and repair capacity were studied based on double-strand breaks induction in DNA.

The results revealed that both strains did not differ in their DNA susceptibility measured as primary induced DSB levels. Differences were obtained in the repair capacity and the role of the incubation conditions was established.

New evidence is provided that the diploid strain D7ts1 possesses better-expressed repair capacity than the haploid strain 551. The combined treatment with Clinopodium vulgare L. extract at concentrations' range $10-1000 \mu \mathrm{g} / \mathrm{ml}$ and Zeocin protects the yeast DNA from double-strand breaks. When incubation is performed on ice the repair processes are impeded but not fully blocked. Such a study confirms that the difference in the repair capacity of the cells could be one of the possible mechanisms, participating in the resistance formed against different DNA damaging factors. 


\title{
Radiation dose optimization in interventional cardiology using artificial intelligence
}

\author{
Teodoro Rivera ${ }^{1}$, Eduardo Uruchurtu ${ }^{2}$, \\ Rocio Toledo ${ }^{1}$, Ricardo Garcia ${ }^{1}$, Isidro Gomez ${ }^{1}$
}

1 IPN, Mexico, Mexico

2 Hospital Juarez de Mexico, Mexico, Mexico

\section{https://doi.org/10.21175/rad.abstr.book.2021.31.5}

Interventional cardiology (IC) procedures, during which live images are acquired, involve exposure to x-rays. The use of fluoroscopy in IC can cause high radiation doses to patients and operators because of the prolonged duration of x-ray exposure. Artificial Intelligence (AI) is a family of operations using advanced mathematical algorithms that have allowed for the enhancement of cognitive output. AI tools have different goals as well as prediction of the system, classification and regression of the problems, and enhancement of clinical decisions. In the present work, the artificial intelligence tools in the interventional cardiology lab are being implemented. The application of AI in our interventional cardiology (IC) room can be divided into 3 main branches, virtual and physical, imaging decision, and abnormalities detection. Notwithstanding, the virtual and physical branch is best represented for robotic interventional procedures, in our case, we can generate some very powerful data for protocol optimization and quality control, by being able to actually integrate the information about the radiation dose, contrast injection, and image quality. Results can be predicted outcomes for future interventional procedures. Imaging decision, clinical decision support systems with computing data are under development and include self-learning systems using ML and DL in order to improve IC procedures, besides help us find dose variations in IC procedures attributed to the acquisition imaging mode (CINE) and cardiologist's experience. Abnormalities detection - more recent advances have given rise to superior algorithms that learn through direct navigation of data to "recognize" some human error findings. AI has been utilized in the practice to analyzing of ESAK measurements for patients undergoing diagnostic and therapeutic interventional procedures, considering variables such as the anatomy of the patient (height, weight, gender, BMI, age), the complexity of the procedure, radiological parameters as well as cardiologist's expertise. The implementation of AI tools in IC suggested reduction variability and improve the quality, safety, and efficiency of care around radiation dose management. Besides, our findings were used to assist IC staff to be able to visualize and navigate procedures more safely and efficiently with significantly less radiation dose exposure. 


\title{
Calculating safe handling radiation dosage limits from neutron activation analysis
}

\author{
Amaya Sinha, Jose Parga, Sheldon Landsberger \\ University of Texas, Austin, United States
}

https://doi.org/10.21175/rad.abstr.book.2021.31.6

This project's primary purpose is to use computational modeling in Python to determine limits on radiation exposure when performing neutron activation analysis (NAA) on samples. In typical laboratories, radioactive sources are usually composed of one to two isotopes to only a few. These sources are well understood and characterized, which leads to safe handling procedures and dose limits. However, in NAA laboratories, samples tend to be made up of many isotopes, which usually cannot be anticipated readily. NAA samples' complexity leads to little or no guidance and exposure limits on how to handle the samples safely. This work aims to develop a deterministic method of calculating doses from these samples. Creating a deterministic method to model radiation transport from NAA samples is important as stochastic methods such as Monte Carlo N-Particle (MCNP) are computationally expensive to run and require variance reduction techniques. In this work, we utilized standard reference materials (SRM) from the National Institute of Standards and Technology (NIST) to model the most commonly irradiated samples at an NAA laboratory. The percentage by mass and neutron cross-section values for common isotopes found within the SRM samples were determined. Next, each isotope activity level in the SRM samples was calculated in Becquerels (Bq) for a given irradiation time and neutron flux. The radiation dose can then be determined as a function of time after irradiation. The next step in the experiment will be to model the human hand to calculate safe handling dose limits. To do this, we will model a cross-section of the human hand, including tissue and bones composed of the International Commission on Radiological Protection (ICRP) and the International Commission on Radiation Units and Measurements (ICRU) standards. The results will then be compared to MCNP Monte Carlo simulations to validate the deterministic method developed in this work. 


\title{
Redox-active oxide nanoparticles improving short- and long-term survival of $\mathrm{X}$-ray irradiated rats
}

\author{
Ganna Grygorova, Vladimir Klochkov, Svetlana Yefimova
}

Institute for Scintillation Materials NAS of Ukraine, Kharkiv, Ukraine

https://doi.org/10.21175/rad.abstr.book.2021.31.7

Nanomedicine utilizes the remarkable properties of nanomaterials for the diagnosis, treatment, and prevention of disease. Many nanomaterials have robust antioxidative properties, potentially functioning as strong scavengers of reactive oxygen species (ROS). Conversely, some nanomaterials to promote the generation of reactive oxygen species, which may precipitate the onset of oxidative stress, a state that is thought to contribute to the development of a variety of adverse conditions. In our previous papers, we reported on biological activity of rare-earth orthovanadate nanoparticles and cerium oxide (pro-/antioxidant properties, effects on bioenergy processes in cell mitochondria, selective accumulation in cell nuclei, etc.) that makes such redox nanomaterials very prospective for biomedical applications and requires deep understanding the mechanisms of their redox activity [1-4].

In this work, we report on the effectiveness of $\mathrm{CeO}_{2-\mathrm{x}}$ and $\mathrm{Gd}_{0.9} \mathrm{Eu}_{0.1} \mathrm{VO}_{4}$ nanoparticles in radioprotection in model animals during radiation exposure. Nanoparticles were injected per os by two different schemes - once and during 15 days. For the 15 -days scheme of $\mathrm{Gd}_{0.9} \mathrm{Eu}_{0.1} \mathrm{VO}_{4}$ nanoparticles injection during 30 days of observation the survival of animals irradiated with a dose of 6.0 Gy was $100 \%$; for ceria it was $90 \%$ for $\mathrm{CeO}_{2-x}$ nanoparticles. In addition to the analysis of the 30-day survivability, different signs of acute radiation sickness were monitored, such as a ruffled fur (a common indicator for the state of the body), bloating, diarrhea, swelling of mouth and eyes. It was revealed that 7.0 Gy radiation dose is lethal. For an irradiation by 7.0 Gy the signs of acute radiation sickness did not differ from those of the controls.

These results can serve as a motivation to consider inorganic nanoparticles as the basis for new drugs possessing by radioprotective effect. In general, the radioprotective effect of considered nanoparticles corresponds to, and even possibly exceeds the level of protection provided by conventionally used organic radioprotectors.

\section{References}

[1] P.O. Maksimchuk, K.O. Hubenko, G.V. Grygorova, V.K. Klochkov [et al.], Impact of Eu $3^{+}$Ions on Prooxidant Activity of $\mathrm{ReVO}_{4}$ : Eu3 ${ }^{+}$Nanocrystals // J. Phys. Chem. C. - 2021. - V.125 (2). - P. 1564-1569.

[2] G.V. Grygorova, V.K. Klochkov, Ye. Mamotyuk, Yu.V. Malyukin, Cerium Dioxide $\mathrm{CeO}_{2-x}$ and Orthovanadate $\left(\mathrm{Gd}_{0.9} \mathrm{Eu}_{0.1} \mathrm{VO}_{4}\right)$ Nanoparticles for Protection of Living Body from X-Ray Induced Damage // in: Nanomaterials for Security, Springer. - 2016. - p. 289-296.

[3] V.K. Klochkov, A.V. Grigorova, O.O. Sedyh, Yu.V. Malyukin, The Influence of Agglomeration of Nanoparticles on Their Superoxide Dismutase-Mimetic Activity // Colloids Surf. A: Physicochem. Eng. Aspects. - 2012. - V.409. - P. 176-182.

[4] K. Hubenko, S. Yefimova, T. Tkacheva, P.Maksimchuk [et al.], Reactive oxygen species generation in aqueous solutions containing $\mathrm{GdVO}_{4}: \mathrm{Eu}^{3+}$ nanoparticles and their complexes with methylene blue //Nanoscale Res. Lett. - 2018. - V.13. - p. 100.

Acknowledgments: This research was supported by National Research Foundation of Ukraine, Grant №2020.02/0052 for Leading and Young Scientists Research Support. 


\title{
Support for a European Metrology Network on reliable radiation protection: Gaps in radiation protection metrology
}

\author{
Behnam Khanbabaee', Annette Röttger'1, Rolf Behrens', Stefan Röttger'1, \\ Sebastian Feige ${ }^{2}$, Oliver Hupe ${ }^{1}$, Hayo Zutz'1, Paula Toroi ${ }^{3}$, Paul Leonard4, \\ Liset de la Fuente Rosales' , Pete Burgess5, Vincent Gressier6, \\ Jose Luis Gutierrez Villanueva7, Rodolfo Cruz Suárez ${ }^{8}$, Dirk Arnold ${ }^{1}$
}

\author{
1 Physikalisch-Technische Bundesanstalt (PTB), Braunschweig, Germany \\ 2 Federal Office for Radiation Protection, Berlin, Germany \\ 3 Radiation and Nuclear Safety Authority (STUK), Helsinki, Finland \\ 4 Corporate Risk Associates Limited (CRA), London, United Kingdom \\ 5 Radiation Metrology Ltd, Oxford, United Kingdom \\ 6 Institut de Radioprotection et de Sûreté Nucléaire (IRSN), Fontenay-aux-Roses, France \\ 7 Radonova Laboratories AB (radonova), UPPSALA, Sweden \\ 8 International Atomic Energy Agency (IAEA), Vienna, Austria
}

\section{https://doi.org/10.21175/rad.abstr.book.2021.31.8}

The European regulation on ionizing radiation is mainly laid down in the COUNCIL DIRECTIVE 2013/59/EURATOM. This Directive implements the basic safety standards for the protection of the health of workers and the general public against the dangers arising from exposure to ionizing radiation.

Reliable and quality-assured metrology is the basis for fulfilling the legal tasks defined in the EURATOM regulations. To avoid difficulties in practice, the implementation of regulations always needs to consider the metrological implications. To overcome these, the consortium of the EMPIR project 19NETo3 supportBSS[1] is currently planning to develop a joint and sustainable European metrology network underpinning radiation protection regulation. This is urgently needed as a single point of contact to overcome all metrological gaps and to add further needs to radiation protection metrology. The final aim of the network is a harmonized, sustainable, coordinated and specialized infrastructure to meet the demands expressed in the COUNCIL DIRECTIVE 2013/59/EURATOM and the EURATOM Treaty.

Such a metrology network under the umbrella of EURAMET is currently in the founding phase. One important task in the preparation of this network is to identify and to analyse the gaps in the metrological services as well as the research needs related to the services and the stakeholders.

The metrological gaps identified up to now exist in 8 areas of interest:

1. Activity standards,

2. Reference fields where the complexity arises due to the new radiation practices and new technological developments, for instance pulsed fields in medical, industrial and technical applications,

3. Are new operational quantities in radiation protection needed?

4. Measuring devices for radiation protection in medical or industry applications of ionizing radiation, i.e. especially suitable for pulsed radiation,

5. Measuring devices for environmental monitoring, i.e. capable of nuclide specific dose determination,

6. Type testing: harmonization of national requirements with international standards (IEC and ISO) and radiation protection legislation

7. Harmonized handling, transmission, storage and availability of measurement data,

8. Education and training needs.

This list summarizes the results of the scientific stakeholder workshop and is meant to open a continuous and constructive dialogue on the identified stakeholder needs and gaps in metrology.

Acknowledgments: This project 19NETo3 supportBSS has received funding from the EMPIR programme co-financed by the Participating States and from the European Union's Horizon 2020 research and innovation programme. 19NETo3 supportBSS denotes the EMPIR project reference. 


\title{
A survey of the current status in the management of radioactive wastes from linear accelerators in Korea
}

\author{
Na Hye Kwon', Dong Oh Shin², Jin Sung Kim', \\ Dong Wook Kim¹, Sang Hyoun Choi3
}

\author{
1 Department of Radiation Oncology, Yonsei Cancer Center, Yonsei University College of Medicine, Seoul, \\ South Korea \\ 2 Department of Radiation Oncology, Kyung Hee University Hospital, Seoul, South Korea \\ 3 Department of Radiation Oncology, Institute of Radiological and Medical Sciences, Seoul, South Korea
}

\section{https://doi.org/10.21175/rad.abstr.book.2021.31.9}

When X-ray energy above $8 \mathrm{MV}$ is used, photoneutrons are generated by the photonuclear reaction, which activates the components of linear accelerator (LINAC). Measurement and analysis is important procedure while disposing of the LINAC or replacing the components. But in Korea, radioactive material management standards are not clear, making it difficult to safely manage of radioactive materials.

Herein, we investigated the management status of radioactive wastes in domestic medical LINACs. A survey was conducted on 53 radiation oncology departments to understand the status of radioactive materials management from medical LINACs. For some questions, duplicate responses were allowed.

48 institutions responded among the 53 institutions. Of the responded 48 institutions, 23 responded that they had experience in disposing of LINACs and components, 16 of them disposing of the entire of LINAC, and 18 of them responding that they had partially disposed of the parts of the LINAC. 12 organizations responded that they used a survey meter to evaluate radio-activation. The other 10 organizations did not perform the measurement, and one organization responded that the measurement was performed using the HPGe detector. There were 18 organizations that responded that the information on the management and treatment of radioactive waste in the LINAC was in the radiation safety management regulations, and 8 of them responded that they had procedures and standards. Most of the institutions which have an experience of disposing of LINAC found that they don't have a specific procedure or guideline. Also, since the disposal criteria did not exist, the radioactive material from LINAC was either stored within the institution or collected by the manufacturer.

Results suggest that most domestic institutions did not have the regulations for disposing radioactive waste from LINAC or the management procedures and standards were unclear. For the safe management of medical LINACs, it is necessary to establish safety management regulations. In Korea, the standards for disposal, such as radiation or dose limits, are required. A system for clearance when disposing at a medical institution should be prepared and further study is to lay the foundation for radioactive waste management from medical LINACs. 


\title{
Case study with CARC software for verifying compliance with atmospheric release criteria of nuclear installations
}

\author{
Csilla Rudas, Tamás Pázmándi
}

Centre for Energy Research, Budapest, Hungary

https://doi.org/10.21175/rad.abstr.book.2021.31.10

A new software titled Calculating Atmospheric Release Criteria (CARC) has been developed in recent years for the verification of compliance with atmospheric release criteria of nuclear installations. The method implemented in the software was developed in response to the articles about preventing the release of radioactive material from nuclear facilities published in the Council Directive 2014/87/Euratom. The main objective of the developed software was to produce a simple and quick tool to use in deterministic safety assessments of nuclear facilities. The software is capable of taking into account site specific parameters, a large number of meteorological scenarios and calculates various percentiles of the resulting dose quantities.

In this work, a case study was conducted with a hypothetical atmospheric release from a nuclear power plant to show the practical application of the method and the software. The source term applies to Design Extension Conditions and was taken from literature and for the meteorological conditions, real measurement data from a weather station collected over a period of 5 years was used. The $95^{\text {th }}$ percentile of the effective dose containing exposure pathways of cloudshine, groundshine and inhalation was evaluated. Results were compared with the corresponding safety criteria for Design Extension Conditions. 


\title{
Modelling the sensitivity of semiconductor detectors for in-situ monitoring of strontium-90 contamination at nuclear decommissioning sites
}

\author{
Graeme Turkington, Kelum A. A. Gamage, James Graham
}

University of Glasgow, Glasgow, United Kingdom

https://doi.org/10.21175/rad.abstr.book.2021.31.11

In-situ characterisation of strontium-90 contamination in groundwater at nuclear decommissioning sites would represent a novel and cost-saving technology for the nuclear industry. This research considers the application of semiconductor detectors for in-situ detection where the device is placed in groundwater boreholes for monitoring. Monte Carlo simulations were used to model this scenario and the potential performance of such detectors and their sensitivity to strontium-90 and yttrium-90. The limit of detection for a cadmium telluride sensor upon exposure to strontium-90 decay in groundwater was calculated as $323 \mathrm{~Bq} / \mathrm{L}$ after a 1 hour measurement and $66 \mathrm{~Bq} / \mathrm{L}$ after a 24 hour measurement. This performance is less sensitive than widely adopted methods of liquid scintillation, but results can be achieved in a much smaller time frame - days as opposed to weeks. 


\title{
TLD-100 post-irradiation fading characteristics according to IEC 62387:2020 standard
}

\author{
Jelena Stanković Petrović1, Marko Krajinovićé,2, \\ Nikola Kržanović1, Miloš Živanović1, Andrea Kojićc,3, Predrag Božović1
}

\author{
1 Vinca Institute of Nuclear Sciences - National Institute of the Republic of Serbia, University of Belgrade, \\ Belgrade, Serbia \\ 2 School of Electrical Engineering, University of Belgrade, Belgrade, Serbia \\ 3 Faculty of Physics, University of Belgrade, Belgrade, Serbia
}

\section{https://doi.org/10.21175/rad.abstr.book.2021.31.12}

The results of the post-irradiation fading of whole body dosemeters based on two TLD-100 (Thermo Scientific ${ }^{\mathrm{TM}}$ Harshaw $^{\mathrm{TM}}$, USA) detectors are presented. The dosemeters are regularly used by accredited individual monitoring service (IMS) at Vinca Institute of Nuclear Science (VINS), Belgrade, Serbia. The testing of post-irradiation fading was carried out according to International Electrotechnical Commission (IEC) 62387:2020 standard. The irradiations are done in S-Cs ${ }^{137}$ field at secondary standard dosimetry laboratory (SSDL) at VINS, according to International Organization for Standardization document ISO 4037-3:2019. The chosen reference personal dose equivalent value was $3 \mathrm{mSv}$. The research was carried out in the period from October 2020 to February 2021, and it lasted 128 days. The frequency of irradiations was approximately 7 days, while 3 periods between irradiations were longer than 10 days, due to COVID-19 pandemic. The irradiations were performed at different dates; thus, all dose readouts were done on the same day to prevent influence of reader's instabilities. The irradiated dosemeters were stored at the same room where the average temperature was nearly $20^{\circ} \mathrm{C}$. There were 14 groups with 6 dosemeters for irradiation and 2 dosemeters for natural background radiation level correction. The reader was Harshaw TLD ${ }^{\mathrm{TM}}$ Model 6600 Plus Automated Reader (Thermo Fisher Scientific, USA). The whole glow curve was used for dose estimation (all of 200 channels). The time as influence quantity was considered to be of type F, thus the range of relative response was limiting factor in the analysis. The results showed that the maximum measurement time $t_{\max }$ is 72 days for deep dose $\left(H_{\mathrm{p}}(10)\right)$ detector, and 85 days for shallow dose $\left(H_{\mathrm{p}}(0.07)\right)$ detector. Thus, the standard's requirement is satisfied, as it is required minimum of 30 days. The relative response range for all of the 14 groups was from 0.82 to 1.14 and from 0.83 to 1.17, for deep and shallow dose, respectively. The research has limitation as the irradiations were organized aligned to COVID-19 working schedule. One of the consequences of this timetable is lack of 7 days' time point, thus the values from 16 days' time point was used as referential. 


\title{
An Optically Stimulated Luminescence study of costal sand as a potential fortuitous dosimeter
}

\section{Monica Vidotto', Emanuela Bortolin², Nadica Maltar-Strmečki1}

\author{
1 Ruđer Bošković Institute, Zagreb, Croatia \\ 2 Istituto Superiore di Sanità, Roma, Italy
}

\section{https://doi.org/10.21175/rad.abstr.book.2021.31.13}

In the case of a mass-casualty radiological or nuclear $(\mathrm{R} / \mathrm{N})$ event, the grouping of individuals into those requiring medical care and those who were not affected during the accident is urgent. Often, the people involved do not own a professional dosimeter and therefore the dose assessment relies on fortuitous dosimeters [1].

This study focuses on the possibility of using costal sand as a fortuitous dosimeter in a $\mathrm{R} / \mathrm{N}$ emergency. A significant number of papers regarding the optically stimulated luminescence (OSL) response of dating of sediments [2] has been published, but none on the application proposed in this work. Advantages of using a portable OSL reader are the rapidity of the measurement, the undemanding sample preparation and the user friendliness of the instrument.

The signals obtained from irradiated (1 Gy) and unirradited sand were acquired right after irradiation and compared for three different light stimulations (infrared, blue light and both combined). Moreover, the signal of the irradiated sample was recorded $24 \mathrm{~h}$ after irradiation to study the signal stability. Results indicated that it is possible to differentiate between unirradiated sand and the one irradiated with $1 \mathrm{~Gy}$. Moreover, the signal appears to be stable $24 \mathrm{~h}$ after the irradiation (changes withing $1 \mathrm{SD}$ ). Therefore, the obtained results, along with the advantages of the instrument, suggest that this method could be convenient for a fast categorization of civilians in a $\mathrm{R} / \mathrm{N}$ accident.

\section{References}

[1] Bailiff IK, Sholom S, McKeever SWS. Retrospective and emergency dosimetry in response to radiological incidents and nuclear mass-casualty events: a review. Radiation Measurements 2016; 94(83-139). doi: 10.1016/j.radmeas.2016.09.004

[2] Madsen AT, Murray AS. Optically stimulated luminescence dating of young sediments: A review. Geomorphology. 2009;109(1-2):3-16. doi:10.1016/j.geomorph.2008.08.020

Acknowledgments: This study was supported by NATO Science for Peace and Security Programme (BioPhyMeTRE project (grant No. G5684)). 


\title{
S-heteryl modified cysteamine protects total body irradiation-induced hematopoietic system injury in rats
}

\author{
Nataliya Uzlenkova', Maryna Kornet², Nataliya Skorobogatova1, Olexandr

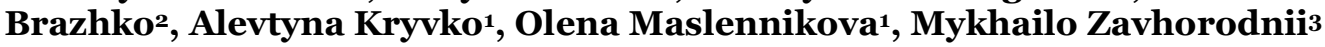

1 SO "Grigoriev Institute for Medical Radiology and Oncology of the National Academy of Medical Sciences of Ukraine”, Kharkiv, Ukraine

2 Zaporizhzhia National University, Zaporizhzhia, Ukraine

3 Khortytsya National Academy, Zaporizhzhia, Ukraine

\section{https://doi.org/10.21175/rad.abstr.book.2021.31.14}

Radiation exposure poses a significant threat to human health, especially the hematopoietic system. Hematopoietic injury is one of the major manifestations of acute radiation syndrome following total body irradiation (TBI). The doses greater than 1 Gy induce bone marrow (BM) cell injury and BM suppression is also the main symptom in the clinic under radiotherapy. Protection and/or mitigation of hematopoietic system from radiation injury is an important goal in the development of medical countermeasure agents. In this study the effectiveness of S-heteryl modified cysteamine (S-HMC) as a novel agent in enhancing hematopoietic reconstitution in rats following a lethal dose of irradiation was investigated.

Methods. The white female rats, $160-180 \mathrm{~g}$, were used. All animal studies were conducted following the international principles of the "European Convention for the protection of vertebrate animals used for experimental and other scientific purposes" (Strasbourg, 1998) and the Law of Ukraine "On protection against the rude treatment" (Kyiv, 2006). The rats were total body irradiated (TBI) on CLINAC (6 MeV) at a dose of $7.0 \mathrm{~Gy}$ with a dose rate of $0.76 \mathrm{~Gy} / \mathrm{min}$. S-HMC was administrated intraperitoneal (i.p.) at a dose of $150 \mathrm{mg} / \mathrm{kg}$ in $30 \mathrm{~min}$ before irradiation. A haematology analyzer RT-760o (Rayto, China) was used for the peripheral blood cell count. The clonogenic function of mesenchymal stromal cells (MSCs) in BM was assayed by counting colonyforming unit ( $\mathrm{CFU})$ in vitro. Hematoxylin and eosin staining was used for BM analysis. The myelokaryocytes apoptosis was determinate with acridine orange (AO). The BM cellularity was counted by standard calculation in Goryaev's chamber.

Results. Compared with the TBI group, administration of S-HMC mitigated radiation-induced lethality. S-HMC improved TBI-induced skewed the ratios of early and differentiated elements of granulocytic, lymphoid and erythroid lineages in BM and accelerated hematopoietic self-renewal and regeneration. Recovery of granulocyto-, lympho- and erythropoiesis was in shorter terms and it prevented the BM pool depletion. It was increased the total myelokaryocytes number by 2.2 times on day 3 and normalization of this parameter on day 7 after irradiation. Under in vitro culture conditions survival of a greater number (2.7 times) of the clonogenic BM MSCs was determined. S-HMC administration led to enhanced recovery of peripheral blood cell counts and mitigated TBI-induced myelosuppression due to myelokaryocytes apoptosis decreasing. Thus, efforts to reduce apoptosis of myelokaryocytes not only helped to attenuate TBI-induced BM depletion, but also relieve TBI-induced BM injury in the hematopoietic system.

Conclusion. This study suggests that S-HMC could be used as a potentially effective agent to protect the hematopoietic system against TBI-induced bone marrow suppression. 


\title{
Ten years of the Fukushima nuclear accident: Global impact of a lesson learnt
}

\author{
Jozef Sabol \\ Police Academy of the Czech Republic in Prague, Prague, Czech Republic
}

https://doi.org/10.21175/rad.abstr.book.2021.31.15

On 11 March 2011, Japan was struck by a massive earthquake, which initiated a tsunami caused significant devastation and led to a severe accident at the Fukushima Daiichi Nuclear Power Plant. Post-accident analyses have verified that the radiation and radioactivity released from the accident has not produced such devastating direct impact on human health as previous reports and evaluations suggested. However, the accident severely affected health and well-being of more than 150 ooo people living in surrounding areas including some early deaths as a result of evacuations from the afflicted area. While there have not been any death cases due to the nuclear accident alone, there were a number of deaths and other health problems mainly due to the lack of health care or medicines, stress-related complications, and other causes not related to radiation exposure.

The paper is trying to assess the consequences of the Fukushima accident realistically and to interpret the resulting exposure and radioactive contamination in relation and comparing them to the natural background as to both radiation level and natural radioactive concentration in the environment as well as exposures normally received by professionals handling radiation sources in accordance with international radiation protection standards. Unfortunately, this accident has been extensively exaggerated and misused by various anti-nuclear organizations opposing the peaceful use of nuclear energy. 


\title{
Features of individual psychological adaptation and periods of staged hematological syndrome in two patients with severe acute radiation sickness and moderate acute radiation sickness and acute leukemia who suffered from the Chernobyl accident (22 years and 30 years of observation)
}

\author{
Nely Metlyaeva, Andrey Bushmanov, Irina Galstyan, \\ Michail Konchalovsky, Anahit Davtyan, Olga Shcherbatykh
}

\author{
A.I. Burnasyan Federal Medical Biophysical Center, Moscow, Russia
}

https://doi.org/10.21175/rad.abstr.book.2021.32.1

Objective. The aim of the work is to assess the psychophysiological adaptation of two patients with severe degree ARS and ARS of moderate severity and acute leukemia affected in the Chernobyl accident according to the characteristics of their individual mental adaptation and stages of the hematological syndrome (22 and 30 years of observation).

Materials and Methods. Two patients were examined, former employees of the Chernobyl emergency shift. One of them acted as an engineer (Yu.A.P., 1961), the second - the deputy head of the turbine shop (D.R.I, 1950). They are 04/26/1986 were subjected to acute external relatively uniform gamma-beta irradiation due to the Chernobyl accident. Yu.A.P. he received Acute Radiation Sickness of the III degree of severity, multiple radiation burns of the I - II degree (40\%) and the III - IV degree (15\%) of the body surface. D.R.I. - ARS II severity and oropharyngeal syndrome I degree. The radiation dose, according to the cytogenetic study, was 4.3 Gy (Yu.A.P.) and 3.4 Gy (D.R.I.). Education in both is higher. Disabled persons of group II were with $100 \%$ disability. Married, in families of 1 and 2 children, children were born before the Chernobyl accident, were at the age of 2 and 7-10 years. Yu.A.P. in January 2007 he was diagnosed with chronic myeloid leukemia PH-positive in the acceleration phase. At D.R.I. - in June 2016 AML, transformation of myelodysplastic syndrome (MDS). Despite ongoing therapy, the death of Yu.A.P. came in November 2008, in D.R.I. in March 2017. Psychophysiological examination was conducted in 1999, 2001, 2016, i.e. 13,15 and 30 years after the Chernobyl accident. Psychophysiological research was carried out using the method of MMPI, Cattell, Raven test, sensorimotor reactions of the automated programmethodical complex "Expert", designed to study the personality characteristics of a person, cognitive and intellectual personality traits 22 and 30 years after the radiation accident in dynamics.

Results. A clinical and psychophysiological assessment of the personality and the actual mental state made it possible to determine the following two types of impairment of psychophysiological adaptation: the type of asthenic depression and the demonstrative type of impairment of psychophysiological adaptation.

Conclusion. Psychophysiological assessment of personality and actual mental state determined individual personality traits in two patients in the form of asthenic depression with depressivehypochondriac tendencies in one and a demonstrative type of violation of psychophysiological adaptation with pronounced hypochondria and a tendency to anxiety-depressive type of behavior in the other who had ARS, local radiation injuries of severe and extremely severe and acute leukemia after 22 and 30 years after the Chernobyl NPP accident. 


\title{
The effect of proton and gamma irradiation on the behavior, learning ability and pregnancy in rats
}

\author{
Viktoria Panfilova, Olga Kolganova, Olga Chibisova
}

\begin{abstract}
A. Tsyb Medical Radiological Research Center - branch of the National Medical Research Radiological Center of the Ministry of Health of the Russian Federation, Obninsk, Russia
\end{abstract}

\section{https://doi.org/10.21175/rad.abstr.book.2021.32.2}

Revealing the possible influence of brain irradiation on the Prometheus proton device and the Novak electron accelerator (gamma radiation) on the behavior and learning ability of female Wistar rats, as well as on the course and outcome of their pregnancy.

In the experiment, 42 female and 21 male Wistar rats were used. At the first stage of the experiment, females were irradiated once in the brain area at a dose of 3 Gy on the Prometheus proton complex (proton energy $165 \mathrm{MeV}$ ) and the Novak electron accelerator (dose rate $0.2 \mathrm{~Gy} / \mathrm{min}$ ). Control females were subjected to sham irradiation under conditions similar to those of the experimental animals.

To assess the effect of proton and gamma radiation on the learning ability of laboratory animals 1 , 3 , and 14 days after irradiation, their cognitive (memory) brain functions were assessed according to the ability to develop and reproduce the conditioned active avoidance reflex (ACAI). To assess the motor and orientation-exploratory activity, the rats were tested in the "Open Field". In the course of the next stage of the experiment, the analysis of the effects induced by radiation was carried out in terms of embryonic toxicity: the level of pre- and post-implantation mortality of embryos was determined, as well as a set of criteria characterizing the course of pregnancy and childbirth in females.

Irradiation at the Prometheus proton device and at the Novak electron accelerator at a dose of 3 Gy does not cause statistically significant differences from control animals both in learning speed and in indicators characterizing the cognitive functions of the brain, short-term and long-term memory. According to all indicators of the behavior of rats in the "Open Field", rats from the experimental groups did not differ from the control animals. The only thing that can be noted is the change in the number of bowel movements (an indicator of emotionality). This difference turned out to be statistically insignificant; however, an upward trend was observed in the irradiated rats. Embryogenesis in females of the experimental groups proceeds without statistically significant deviations from the norm and from the indicators of the control group. The total number of viable fetuses does not differ from similar control indicators. Also, pregnancy and childbirth in females of the experimental groups proceeds normally, not differing from the course of pregnancy in females of the control group.

Irradiation of the brain of female rats on the Prometheus proton device and on the Novak electron accelerator at a dose of 3 Gy does not significantly differ from control animals in terms of learning rate, indicators of short-term and long-term memory, as well as in terms of behavior of rats under moderate stress condition situations. It was also possible to establish that local irradiation of the brain at a dose of $3 \mathrm{~Gy}$ does not have a statistically significant effect on the course and outcome of pregnancy in irradiated females. 


\title{
Inhibition of cell recovery from densely ionizing radiation by copper sulfate
}

\author{
Svetlana V. Belkina, Marianna D. Pronkevich
}

\begin{abstract}
A. Tsyb Medical Radiological Research Center - branch of the National Medical Research Radiological Center of the Ministry of Health of the Russian Federation, Obninsk, Russia
\end{abstract}

\section{https://doi.org/10.21175/rad.abstr.book.2021.32.3}

The effectiveness of radiotherapy for oncological diseases can be increased by inhibiting cell regeneration after exposure to ionizing radiation. Densely ionizing radiation has the greatest damaging power since high linear energy transfer of this radiation leads to extensive cell damage and inhibition of their recovery. To enhance such recovery chemical inhibitors can be used. This study aimed to estimate the mechanism of cell recovery after exposure to densely ionizing radiation and copper sulfate.

Cell recovery after consequence action of alpha radiation (4.1 MeV) and copper sulfate $(0.02 \mathrm{mg} / \mathrm{ml}$ ) was investigated by the cells clonogenic activity test. Diploid Saccharomyces cerevisiae yeast cells wild type XS80o in the stationary growth phase were used. To study the kinetics of cell recovery, the exposed cells were kept in a non-nutrient medium at $30^{\circ} \mathrm{C}$ at various time intervals up to 60 hours.

Data on cells ability to recover after densely ionizing radiation either in a non-nutrient medium or in a medium containing $\mathrm{CuSO}_{4}$ were used to estimate the share of unrepaired radiation lesions. The limiting value of this parameter, i.e. an irreversible component is equal to 0.56 under the action of only densely ionizing radiation, and 0.72 after the combined action of radiation and copper sulfate. This means that in the presence of this chemical agent, the share of radiation lesions from which the cells could not recover (72\%), increased compared to the condition without copper sulfate (56\%). It also has been estimated that the share of repaired radiation lesions exponentially decreases with recovery time and does not depend on the presence of a chemical radiosensitizer. The rate of cell recovery $(\beta)$ was $0.04 \mathrm{~min}^{-1}$. That means that the increase in the radiosensitivity of the cell is due to a slight increase in the proportion of irreversibly damaged cells, while the process of post-radiation recovery remained unchanged ( $\beta=$ const).

The mechanism of cell recovery inhibition by copper sulfate after exposure to densely ionizing radiation is not associated with inhibition of the recovery process itself but occurs due to an increase in the number of unrepairable lesions. 


\title{
Participation of molecule excitation in the biological effects of ionizing radiation
}

\section{Polina Kuptsova, Galina Zhurakovskaya, Olga Pereklad, Marianna Pronkevich}

\author{
A. Tsyb Medical Radiological Research Center - branch of the National Medical Research Radiological \\ Center, the Ministry of Health of the Russian Federation, Obninsk, Russia
}

\section{https://doi.org/10.21175/rad.abstr.book.2021.32.4}

Introduction. Under the action of ionizing radiation, atoms or molecules are ionized or excited. While charged particles move in media at a speed exceeding the phase velocity of light, the Vavilov - Cherenkov effect occurs. Thus, the effect of high-energy ionizing radiation can actually be represented as a combined effect of ionization and excitations. This phenomenon is used in luminescent Cherenkov imaging, as well as for visualizing the surface organs of patients treated with beta-minus radiopharmaceuticals. The biological effect of the Vavilov-Cherenkov radiation is practically not studied. The purpose of this study was to determine the participation of the excitation of molecules in the biological action of ionizing radiation.

Materials and methods. The excitation of molecules was modeled using a short-wave UV light $(254 \mathrm{~nm})$. Diploid yeast cells of Saccharomyces cerevisiae were exposed to various types of radiation: UVC (254 nm), $\alpha$-particles $(4.1 \mathrm{MeV})$ and $\gamma$ - ray $(1.33 \mathrm{MeV})$. Chemical substances: cystamine, cysteamine and cysteine, known for their radioprotective properties against ionizing radiation. Test: clonogenic assay.

Results. A search was made for a chemical that modifies the effect of both ionizing radiation and UV light equally well. Such a chemical substance turned out to be cystamine, which has protective properties to both types of radiation. Quantitative assessment of the cystamine Dose-Modifying Factor is as follows: $\alpha$ - particles $=1.0, \gamma$ - ray $=1.8$ and UV light $=1.9$. Dose-Modifying Factor under the action of $\gamma$-ray and UV light are statistically identical. The reason for the sensibility of cystamine to UV light is explained by the fact that the most sensibility to short-wave UV light are disulfide bridges ( $\mathrm{S}-\mathrm{S}-$ ), which are broken when a photon of light is absorbed. This disulfide bridge is contained in the cystamine molecule. So, the cystamine molecules in the solution can compete for photons of light with the amino acids that make up the cells, contributing to their protection. If we adhere to the hypothesis that the effect of ionizing radiation is a combination of the effects of ionization and excitation of molecules, and UV light only from their excitation, then we can assume that the protective effect of cystamine is aimed more at protecting cells from the inhibitory effect of excitations caused by UV component of ionizing radiation.

Conclusions. The presented opportunity to equally affect the sensitivity of cells to damage caused by both UV light and gamma rays, confirmed our assumption about a more significant participation of the excitation of atoms and molecules in the biological action of ionizing radiation. The first results are obtained, requiring extension. 


\title{
Enhancement of $\gamma$-irradiation genotoxicity in human lung carcinoma A 549 cells in the presence of gold nanoparticles
}

\author{
Anna Rzianina, Gennady Mytsin, Karine Voskanyan, Victor Gaevskiy
}

Joint Institute for Nuclear Research, Dubna, Russia

https://doi.org/10.21175/rad.abstr.book.2021.32.5

Introduction. One of the main problems of radiotherapy is the lack of selectivity in the absorption of radiation energy by tumor cells due to the similar energy-absorbing properties of tumor and healthy tissues. An increase in the absorbed dose can be achieved by administering or delivering substances to tumor cells with a significantly larger radiation absorption cross-section than in normal cells. These are chemical elements with a high atomic number $\mathrm{Z}$, more than 52 (I, Gd, Au, Pt, etc.). Due to the greater probability of interaction of such elements with radiation, it is possible to achieve a local increase in the absorbed dose in the area of accumulation of a substance with a high atomic number. The resulting secondary short-range radiation localizes the energy release near these elements and affects only nearby biological structures.

The aim of the research is to study the genotoxic effect of $\gamma$-irradiation on tumor cells of human lung carcinoma A 549 in the presence of gold nanoparticles.

Materials and methods. Human lung carcinoma cells A549 were cultured under standard conditions. Gold nanoparticles (Au/PEG600o/W 200/20 nm) were added to the culture medium 24 hours before irradiation at a concentration of $0.1 ; 2.5 ; 5 ; 10 ; 15 ; 30 \mu \mathrm{g} / \mathrm{ml}$. Before irradiation, the medium with nanoparticles was removed, the cells were washed twice of PBS and a fresh medium was added. The cells were exposed to ${ }^{60} \mathrm{Co} \gamma$-radiation at a dose of $2 \mathrm{~Gy}$. The genotoxic effect of gold nanoparticles (GNP) was studied by a cytokinesis-blocking micronucleus test (CBMN).

Results. The number of micronucleus in A549 cells cultured in the presence of GNP in the concentration of $0.1-30 \mu \mathrm{g} / \mathrm{ml}$ is at the control level. There is an increase in the number of micronucleus under $\gamma$-irradiation of cells treated with GNP at a dose of 2 Gy compared to untreated cells. The GNP concentration of $15 \mu \mathrm{g} / \mathrm{ml}$ is optimal for increasing the efficiency of $\gamma$-irradiation at a dose of 2 Gy for cells A549. The proliferation index (CBPI) was calculated for cells exposed to $\gamma$-radiation at a dose of 2 Gy for two groups of cells, untreated and treated with GNP. The proliferation index within each group is approximately the same. However, in cells irradiated in the presence of GNP, the proliferation index is lower than in untreated cells. At a concentration of 0.1, $2.5,5,10 \mu \mathrm{g} / \mathrm{ml}$, the cytostatic effect of GNP does not significantly differ from the control samples (irradiation of 2 Gy without GNP). The tendency to increase cytostatic activity is observed at concentrations of 15 and $30 \mu \mathrm{g} / \mathrm{ml}$ in combination with $\gamma$ irradiation.

Conclusions. GNP in combination with $\gamma$-irradiation enhances the genotoxic effect on the tumor cells A549. The frequency of micronucleus increases and the proliferative index decreases in cells irradiated together with GNP. 


\title{
Research on the combined effects of radiation and chemotherapy on tumor cells
}

\author{
Anzhelika Melnikova, Luidmila Komarova
}

Obninsk Institute for Nuclear Power Engineering, Obninsk, Russia

https://doi.org/10.21175/rad.abstr.book.2021.32.6

Carbon-12 ion therapy is the most effective and accurate method of radiotherapy for deep-seated and radioresistant tumors. A significant advantage of this therapy is the penetration of the beam of accelerated ${ }^{12} \mathrm{C}$ ions to the required depth with maximum damage to the tumor cells. At the end of the run, the carbon ions experience a sharp deceleration, transferring most of the energy to the absorbed substance (Bragg peak). Thus, there is a selective effect on the deep-located tumor.

It is known that the combined effect of ionizing radiation and medicinal antitumor drugs increases the radiosensitivity of tumor cells. As a result, it is relevant to research the combined effect of ionizing radiation and antitumor agents. Due to the wide spectrum of action against many types of tumors, we chose doxorubicin as a chemotherapy drug. The cytotoxic effect of doxorubicin is to suppress the synthesis of DNA and RNA mainly in the S-phase due to the formation of free radicals. In the case of some tumors, it is possible to develop resistance to the drug. The solution to this problem can be a combination of different methods of therapy, which is used to increase the radiosensitivity of tumor cells in the treatment of resistant forms of malignant neoplasms of various localizations.

The purpose of our scientific research is to research the nature of the combined effect of ionizing radiation of accelerated ${ }^{12} \mathrm{C}$ ions with the antitumor agent doxorubicin on malignant cells of various origins.

The object of the study was three tumor cell cultures: MCF-7 (human breast duct adenocarcinoma), Hela (cervical cancer), and HUH-7 (hepatocarcinoma).

The procedure of irradiation of cell cultures was carried out on the U-70 carbon ion accelerator (Institute of high energy physics, Protvino). The work identified three groups of research: Group 1-irradiation of tumor cells with carbon-12 ions in the Bragg peak at doses of 1, 2, 4 Gy; group 2-incubation of cell cultures with doxorubicin at a concentration of $0.004 \mathrm{mg} / \mathrm{ml}$; group 3-combined action of doxorubicin and ionizing radiation.

The integral indicator of the loss of clonogenic activity and death by cells in our study was chosen - the doubling time of cell cultures.

It is shown that the value of the doubling time in the combined action of agents on Hela and MCF-7 tumor cells is higher than the theoretically expected index of additive addition of effects, which indicates an increased radiosensitivity of tumor cells to ionizing radiation after the action of an antitumor drug. This effect may be due to the short generation time combined with high cell death. 


\title{
Cytogenetic follow-up study for over 4 years of three individuals accidentally exposed to ${ }^{60} \mathrm{Co}$ in Bulgaria
}

\author{
Albena Staynova, Dimka Georgieva, Ljubomira Popova, Rositsa Hristova
}

National Center of Radiobiology and Radiation Protection, Sofia, Bulgaria

https://doi.org/10.21175/rad.abstr.book.2021.32.7

On 14 June 2011, a serious radiation accident occurred in an industrial irradiation facility, containing ${ }^{60} \mathrm{Co}$ sources with very high activity. The accident resulted in the exposure of five persons to acute high-dose and whole-body external irradiation. The purpose of this study was to examine the frequency of dicentrics and rings (dic $+r)$ over time after a real case of accidental exposure.

Materials and Methods. Conventional chromosomal aberration analysis was performed by scoring dic $+\mathrm{r}$ in peripheral blood lymphocytes from three of the victims. The course of unstable chromosomal frequency (dic+r) was analysed at four time-points after the first examination. Only metaphases with complete number of 46 chromosomes were analyzed.

Results. The results show that unstable chromosomal aberrations declined during the research period. One year after exposure, the dic $+r$ frequencies decreased to $41-65 \%$ of their initial levels and still showed dose dependence. For the person with the highest dose -5.6 Gy $(4.94-6.25)$ the frequency of aberrations fell faster as a function of time and four years post-irradiation it was reduced with 95\%. For the subjects who had been exposed to lower doses $-3.37 \mathrm{~Gy}(2.85-3.89)$ and 2.47 Gy (2.04 - 2.9), the elimination course was slower. Although we observed gradual disappearance in the frequency of unstable chromosomal aberrations, at the end of the studied period it was several times higher than the control level, found in Bulgarian population.

Conclusions. The follow-up study extending over four years revealed a reduction in the frequencies of dic $+r$ in lymphocytes from three victims, accidentally exposed to ${ }^{\circ} \mathrm{Co}$. The kinetic attenuating frequency of unstable chromosomal aberrations with the time seems to depend on the dose received by the individual. 


\title{
Effect of curcumin on proteasome activity in irradiated or non-irradiated lymphocytes
}

\author{
Nevena Aneva, Nora Kostova, Ilonka Ivanova, \\ Ljubomira Popova-Hadjiiska, Rositsa Hristova
}

National Center of Radiobiology and Radiation Protection, Sofia, Bulgaria

\section{https://doi.org/10.21175/rad.abstr.book.2021.32.8}

Proteasome is a multicatalytic enzyme complex that is responsible for decomposition of damaged or improperly folded proteins and is crucial for cell cycle, cell survival and other important cellular processes. Curcumin is known to have strong antioxidant, anti-inflammatory and anti-tumor effects and can be used as radioprotector. There is evidence that curcumin can inhibit NF- $\kappa \mathrm{B}$ transcriptional pathway and affect, in this way, inflammatory pathways through proteasome-dependent proteolytic degradation.

The aim of this study is to analyze proteasome activity with fluorescent substrate $\mathrm{N}$-succinyl-Leu-Leu-Val-Tyr-7-amino-4-ethylcoumarin (Suc-LLVY-AMC) in peripheral blood mononuclear cells after incubation with different concentrations of curcumin $(0 ; 0.5 ; 10 ; 20$ and $100 \mu \mathrm{g} / \mathrm{ml}$ ) and $\gamma$ - irradiation of cells with different doses (o; 0.05; 0.5; 1 and $2 \mathrm{~Gy}$ ) in three donors.

We found significant differences in proteasome activity between the three donors. In vitro irradiation with doses ranging from 0.05 Gy to $1 \mathrm{~Gy} \gamma$-rays in curcumin-untreated cells of the first donor show inhibition of proteasome activity in varying degrees. There is also concentration dependent increase in the proteasome activity in the cells that are non-irradiated or irradiated with low doses (0.05 and 0.5 Gy). The effect of ionizing radiation on proteasome activity in curcuminuntreated cells from the second donor shows that at doses of $0.5 \mathrm{~Gy}$ and $1 \mathrm{~Gy}$ the activity of the proteasome is slightly increased, while at doses of $0.05 \mathrm{~Gy}$ and $2 \mathrm{~Gy}$ there is suppression. Treatment of the cells with $100 \mu \mathrm{g} / \mathrm{ml}$ curcumin shows decrease of proteasome activity regardless of the dose. In the peripheral blood mononuclear cells of the third donor, we didn't observe significant changes in proteasome activity. We can conclude that radiation and/or curcumin induced changes in proteasome activity can be very different in individuals. 


\title{
Evaluating change in genomic frequency of translocations in 20 patients exposed to low-dose $\mathrm{X}$-ray irradiation during routine vertebroplasty
}

\author{
Ilonka Ivanova', Hristo Hristov², Hristo Tsonev², Rositsa Hristova1
}

1 National Center of Radiobiology and Radiation Protection, Sofia, Bulgaria

2 University Hospital St. Ivan Rilski, Department of Neurosurgery, Sofia, Bulgaria

https://doi.org/10.21175/rad.abstr.book.2021.32.9

Aim. Fluoroscopic X-ray imaging is progressively used in routine noninvasive medical procedures such as vertebroplasty. There is still no consensus among researchers on the long-term effects of low-dose irradiation ( $<100 \mathrm{mGy})$ considering the factor of a possible increase in stable chromosomal aberrations in the human cell which are recognized as potentially carcinogenic. The aim of this study is to evaluate the change in genomic frequency of translocations (GF) in the genetic material in peripheral blood mononuclear cells - lymphocytes of patients exposed to low-dose X-ray irradiation during vertebroplasty.

Methods. Peripheral blood samples were acquired immediately before and after the medical intervention from 20 patients of whom 15 women and 5 men within the range of 60-91 years old. The average cumulative dose received was $25.49 \pm 20.26 \mathrm{mGy}$. Blood samples were cultured following dicentrics assay protocol and three-colored FISH was applied for 2 microscopic slides for each patient. The fluorescent analysis was carried out on 32378 cells in total and GF was calculated according to Loucas et al. equation for whole-genome equivalency. Statistical analysis for correlation was performed.

Results. The calculated average genomic frequency of translocations in peripheral blood mononuclear cells analyzed from 20 patients undergoing vertebroplasty medical procedure was $0.008 \pm 0.010$ before and $0.018 \pm 0.020$ after the intervention. In $65 \%$ of all cases (13 patients), there was an average increase in GF by nearly twofold. In 3 cases there was no change and in 4 cases there was a decrease in GF. No significant correlation was found between age, genomic frequency of translocations, and cumulative dose ( $\mathrm{p}>0.050)$.

Conclusion. After analyzing blood samples from 20 patients undergoing vertebroplasty we found an average increase in the genomic frequency of translocations by nearly twofold in $65 \%$ of all cases (13 of all the patients). This preliminary research shows a tendency for an increase in stable chromosomal aberrations in peripheral blood mononuclear cells after low-dose X-ray irradiation during a routine medical procedure. Because of the small group of subjects and the limited amount of data more patients undergoing noninvasive fluoroscopically guided interventions need to be included in this research. 


\title{
Does curcumin protect cellular DNA against $\gamma$-ray induced damage?
}

\author{
Nora Kostova, Dimka Georgieva, Albena Staynova, \\ Ljubomira Popova, Ilonka Ivanova, Rositsa Hristova
}

National Centre of Radiobiology and Radiation Protection, Sofia, Bulgaria

https://doi.org/10.21175/rad.abstr.book.2021.32.10

Background. The cells are highly sensitive to ionizing radiation (IR). Radiation exposure causes double strand breaks (DSBs) in DNA. DSBs can remain non-repaired or misrepaired by cell repair system, that results in either loss or rearrangement of genetic information, cell death or malignant transformation. On the other hand, IR is widely used in medical diagnostics, cancer therapy and also has additional industrial applications. The scientific interest is focused on radioprotectors that could limit the deleterious effect of IR. Curcumin is a natural compound of turmeric Curcuma longa. It could be a potential radioprotective agent because of its high antioxidant activity and ability to neutralize the free active oxygen radicals resulting from various mutagenic factors, particularly IR.

Aim. The aim of present study is to evaluate curcumin effect on cellular DNA after in vitro exposure to $\gamma$-rays. In response of DNA damage, histone protein $\gamma$-H2AX becomes phosphorylated and attracts other reparative proteins forming 'foci' at the sites of DSBs. Our interest is directed on $\gamma$-H2AX immunolocalization for assessment of DSBs. We are also focused on stable chromosomal aberrations such as translocations that appear as a consequence of misrepaired DSBs. They are related with increased cancer risk and could be found in dividing cells many years after irradiation. Thus, curcumin effect on both DSBs and chromosomal translocations frequency has been investigated.

Methods. Human peripheral blood samples were pre-treated with different concentrations of curcumin $(0.5 \mu \mathrm{g} / \mathrm{ml} ; 10 \mu \mathrm{g} / \mathrm{ml} ; 20 \mu \mathrm{g} / \mathrm{ml}$ and $100 \mu \mathrm{g} / \mathrm{ml})$. Thereafter they were exposed to ${ }^{60} \mathrm{Co} \gamma$-rays using various irradiation doses (0.05 Gy; 0.5 Gy; 1 Gy and $2 \mathrm{~Gy}$ ). DSBs were analyzed in isolated lymphocytes through sensitive $\gamma$-H2AX/53BP1 foci assay. Curcumin effect on stable translocations after irradiation was studied by tri-colored FISH. About 30000 cells totally for both methods were examined manually using fluorescent microscopy.

Results: Curcumin pre-treatment leads to significant lower $\gamma-\mathrm{H} 2 \mathrm{AX} / 53 \mathrm{BP} 1$ foci appearance compared to untreated lymphocytes, after exposure to $\gamma$-rays regardless of dose used. Decreased number of radiation-induced $\gamma$-H2AX foci respectively DSBs has been found at all curcumin concentrations. Curcumin pre-treatment also reduced translocations frequency in irradiated cells. The reduction of total translocations frequency at $1 \mathrm{~Gy}$ irradiation and $10 \mu \mathrm{g} / \mathrm{ml}$ curcumin is $42 \%$. We found that at $2 \mathrm{~Gy}$ irradiation, the most protective concentration is $5 \mu \mathrm{g} / \mathrm{ml}$ curcumin. In this case, the number of translocations dropped almost twofold compared to curcumin non-treated cells.

Conclusion. The present in vitro study shows that curcumin reduces both $\gamma$-H2AX/53BP1 foci and translocations occurrence in peripheral blood lymphocytes, after exposure to $\gamma$-rays. Our results support the understanding about radioprotective effect of curcumin on cellular DNA. More experiments are needed to make a general conclusion. 


\title{
Cytogenetic effects of protons at low doses
}

\section{Ekaterina Koryakina, Marina Troshina, Raisa Baykuzina, Vladimir Potetnya, Alexey Solovev, Sergey Koryakin}

\begin{abstract}
A. Tsyb Medical Radiological Research Centre - branch of the National Medical Research Radiological Centre of the Ministry of Health of the Russian Federation, Obninsk, Russia
\end{abstract}

\section{https://doi.org/10.21175/rad.abstr.book.2021.32.11}

Proton therapy is a highly effective method of radiation therapy. The proton Bragg peak allows conformal irradiation of tumors including the deep-seated ones. At the same time, the radiation dose to the surrounding tissues is much lower.

The biological effect of low-LET radiation may be non-linear dose-dependent in the range of 0.05-1 Gy, and shows the phenomena of hypersensitivity and induced radioresistance (HRS and IRR). It was demonstrated by cell survival and induction of chromosome aberrations for X-rays and gamma radiation, but contradictory results were obtained for protons with different LET. It is important to study the HRS/IRR effects of protons because, with multi-field proton irradiation, healthy tissues are irradiated at doses below 1 Gy per fraction. Direct extrapolation of the biological effect from higher doses can lead to errors in predicting damage to normal tissues.

The research aim was to investigate the cytogenetic effects of scanning beam protons in mammalian cells at doses up to $1 \mathrm{~Gy}$.

Chinese hamster ovary cells (CHO-K1) and fibrosarcoma cells (B14-150) in the stationary growth stage were used. Scanning proton beam (ø 4-7 $\mathrm{mm}$ ) was produced using the "Prometheus" proton accelerator ("Protom" Ltd.). The proton energy was $83 \mathrm{MeV}$ (LET $\sim 2 \mathrm{keV} / \mu \mathrm{m}$ ) under the experimental conditions. Cell monolayers were irradiated at the Bragg curve plateau. After irradiation, cells were removed from the growth surface with a trypsin solution, were reseeded in fresh medium, and were incubated during 24 hours at $37^{\circ} \mathrm{C}$ in an atmosphere of 5 per cent $\mathrm{CO}_{2}$. Slides of first mitosis metaphases were prepared using standard procedures. All types of structural chromosomal aberrations visible by Giemsa staining were scored.

The dose-response curves for the total frequency of chromosome aberrations in CHO-K1 and B14-150 cells under the scanning proton beam irradiation were similar. They show HRS regions with sharp effect increase at $0.1-0.15 \mathrm{~Gy}$, IRR plateaus at dose ranges from 0.1 to $0.3 \mathrm{~Gy}$ for B14-150 cells and from 0.1 to 0.5 Gy for CHO-K1 cells, and finally the curves turn into linear-quadratic ones. Analogous data patterns were obtained for the frequency of dicentrics and deletions (acentric fragments).

Thus, we established the irregular shape of the dose curves with the HRS and IRR regions for protons by the induction of chromosome aberrations both in normal (CHO-K1) and tumor (B14-150) cells. The effect is similar to those observed for different cell types exposed to low-LET radiations at low doses. 


\title{
Cytogenetic effects in mammalian cells irradiated with carbon SOBP beam
}

\author{
Marina Troshina1, Ekaterina Koryakina1, Vladimir Potetnya1, \\ Aleksey Solovevi, Vladimir Pikalov', Sergey Koryakin'
}

\author{
1 A. Tsyb Medical Radiological Research Center - branch of the National Medical Research Radiological \\ Center of the Ministry of Health of the Russian Federation, Obninsk, Russia \\ 2 Institute for High Energy Physics named by A.A. Logunov of National Research Centre "Kurchatov \\ Institute", Protvino, Russia
}

\section{https://doi.org/10.21175/rad.abstr.book.2021.32.12}

Carbon ion therapy is a promising treatment for patients with malignant neoplasms. The first center of ion radiotherapy in Russia is currently being developed and created based on the U-70 accelerator at the Institute of High Energy Physics (Protvino). Since 2014, physical, dosimetric, and radiobiological experiments have been carried out at a carbon ions beam extracted from U-70. The study aim was to determine the relative biological efficiency (RBE) of carbon ions at different positions of the spread-out Bragg peak (SOBP) of the beam where the LET is close to that of the therapeutic beams.

Chinese hamster B14-150 fibrosarcoma cells in the stationary phase growth of culture were used. Cell monolayers were irradiated at the proximal regions, central and distal edge of a $30 \mathrm{~mm}$ SOBP. Doses were 0.5, 1, 1.5 and $2 \mathrm{~Gy}$. The dose-average $\mathrm{LET}_{\mathrm{d}}$ for each irradiation position were calculated using Geant4. RBE was determined by the induction of chromosome aberrations (CA). Metaphase chromosomes were stained with Giemsa solution. The frequency and type of chromosome aberrations were analyzed by examining at least 200 cells for each radiation dose.

Dose-response curves for the induction of chromosome aberrations by carbons were linear for all exposure positions. Terminal deletions and exchange type aberrations (dicentrics, centric rings) make the major contribution to the CA frequency, $60-65 \%$ and $20-25 \%$, respectively. The RBEs determined at $1 \mathrm{CA}$ per cell were 1.4 for the proximal edge $(37-41 \mathrm{keV} / \mu \mathrm{m}), 1.8$ for the SOBP center $(53-55 \mathrm{keV} / \mu \mathrm{m})$ and 2.7 for the distal edge $(91-107 \mathrm{keV} / \mu \mathrm{m})$. The RBEs for the effect corresponding to 2 Gy of ${ }^{60} \mathrm{Co}$ gamma radiation were $2.4,3.1$, and $4.3 \mathrm{~Gy}$. Thus, even low doses of ions have a significant damaging effect. 


\title{
DNA double-strand break repair efficiency in cancer cells exposed to laser-driven ultrashort electron beams
}

\author{
Andreyan Osipov', Nelly Babayan², Natalia Vorobyeva3, \\ Bagrat Grigoryan4, Anna Chigasova-Grekhova5, Margarita Pustovalova6, \\ Sofya Rodneva ${ }^{3}$, Yuriy Fedotov3, Gohar Tsakanova ${ }^{2}$, Rouben Aroutiounian7
}

\author{
1 N.N. Semenov Federal Research Center for Chemical Physics Russian Academy of Sciences, Moscow, Russia \\ 2 Institute of Molecular Biology NAS RA, Yerevan, Armenia \\ 3 State Research Center - Burnasyan Federal Medical Biophysical Center of Federal Medical Biological \\ Agency, Moscow, Russia \\ 4 CANDLE Synchrotron Research Institute, Yerevan, Armenia \\ 5 Emanuel Institute for Biochemical Physics Russian Academy of Sciences, Moscow, Russia \\ 6 Moscow Institute of Physics and Technology, Dolgoprudny, Russia \\ 7 Yerevan State University, Yerevan, Armenia
}

\section{https://doi.org/10.21175/rad.abstr.book.2021.32.13}

Laser-driven accelerators allow to generate ultrashort (from femto- to picoseconds) high peak dose-rate (up to tens of GGy/s) accelerated particle beams. However, the radiobiological effects of ultrashort pulsed irradiation are still poorly studied. The aim of this work was to compare the formation and elimination of $\gamma \mathrm{H} 2 \mathrm{AX}$ and $53 \mathrm{BP} 1$ foci (well known markers for DNA double-strand breaks (DSBs)) in Hela cells exposed to ultrashort pulsed electron beams generated by Advanced Research Electron Accelerator Laboratory (AREAL) accelerator (electron energy $3.6 \mathrm{MeV}$, pulse duration $450 \mathrm{fs}$, pulse repetition rates 2 or $20 \mathrm{~Hz}$ ) and quasi-continuous radiation generated by Varian accelerator (electron energy $4 \mathrm{MeV}$ ) at doses of 250-1000 $\mathrm{mGy}$. Additionally, a study on the dose-response relationships of changes in the number of residual $\gamma \mathrm{H} 2 \mathrm{AX}$ foci in HeLa and A549 cells $24 \mathrm{~h}$ after irradiation at doses of 500-10,000 mGy were performed. We found no statistically significant differences in $\gamma \mathrm{H} 2 \mathrm{AX}$ and $53 \mathrm{BP} 1$ foci yields at $1 \mathrm{~h}$ after exposure to $2 \mathrm{~Hz}$ ultrashort pulse vs. quasi-continuous radiations. In contrast, $20 \mathrm{~Hz}$ ultrashort pulse irradiation resulted in 1.27-fold higher foci yields as compared to the quasi-continuous one. After $24 \mathrm{~h}$ of pulse irradiation at doses of 500-10,000 mGy the number of residual $\gamma \mathrm{H} 2 \mathrm{AX}$ foci in Hela and A549 cells was 1.7-2.9 times higher compared to that of quasi-continuous irradiation. Overall, the obtained results suggest the slower repair rate for DSBs induced by ultrashort pulse irradiation in comparison to DSBs induced by quasi-continuous irradiation.

Acknowledgments: This research was funded by the Russian Science Foundation, grant no. 19-14-00151. 


\title{
Blood and buccal micronuclei as biomarkers in monitoring of children exposed to diagnostic radiation in clinical settings
}

\author{
Goran Gajski' ${ }^{1}$, Mirta Milić ${ }^{1}$, Marko Gerić ${ }^{1}$, Marijana Nodilo² \\ Željka Kneževićén ${ }^{2}$ Natko Beck ${ }^{3}$, Vera Garaj-Vrhovac ${ }^{1}$, Saveta Miljanićé ${ }^{2}$ \\ Mariá Ranogajec-Komor ${ }^{2}$, Đurđica Milkovićc 4
}

\author{
1 Institute for Medical Research and Occupational Health, Zagreb, Croatia \\ 2 Ruđer Bošković Institute, Zagreb, Croatia \\ 3 University Hospital "Sveti Duh", Zagreb, Croatia \\ 4 Srebrnjak Children's Hospital, Zagreb, Croatia
}

\section{https://doi.org/10.21175/rad.abstr.book.2021.32.14}

It has been postulated that micronuclei frequency is related to the increasing presence of carcinogens. The lymphocyte micronucleus cytome (L-MN Cyt) assay has become one of the bestvalidated methods for measuring chromosome damage; however, the buccal micronucleus cytome (B-MN Cyt) assay has been gaining more attention in recent years, especially as it is minimally invasive, hence more appropriate for children biomonitoring. As children might be more sensitive to radiation, there is a need for constant biomonitoring of young populations receiving X-ray diagnostic examinations. Therefore, we aimed to evaluate the effects of diagnostic chest and sinus X-ray exposure on lymphocytes and buccal cells using both MN Cyt assays. Additionally, doses were measured using thermoluminescence and radiophotoluminescent dosimetry systems and were in satisfactory agreement. Using L-MN Cyt assay, it was shown that the mean number of micronuclei, nucleoplasmic bridges and nuclear buds were significantly higher after the diagnostic procedure. Furthermore, the B-MN Cyt assay was done in order to evaluate DNA damaging, replicative, cytostatic, and cell death effects. Micronuclei as well as other biomarkers of DNA damage (nuclear buds and so-called "broken eggs") and genomic instability (normal basal cells, normal differentiated cells, binucleated cells, cells with condensed chromatin, pyknotic cells, cells with karyorrhectic chromatin and karyolitic cells) were analysed. The only significant increase was noted in cells with condensed chromatin, indicating more cells undergoing early stages of apoptosis. It should be pointed out that interindividual differences existed for each monitored child in both assays. Based on our results, MN Cyt assay could be very useful in acute events where children are exposed to genotoxic agents from physical sources. Besides, B-MN Cyt assay could be used for monitoring genetic damage in children who are often exposed to diagnostic procedures, as it is a minimally invasive method of sample collection. 


\title{
Protective role of isoflurane after combined exposure with 1 or 2 Gy of ionizing radiation assessed with alkaline comet assay in vivo
}

\author{
Mirta Milićé, Nada Oršolićé, \\ Nikola Borojevićc3, Anica Horvat Kneževićc ${ }^{2}$, Vesna Benkoviće
}

\author{
1 Institute for Medical Research and Occupational Health, Zagreb, Croatia \\ 2 Faculty of Science, University of Zagreb, Zagreb, Croatia \\ 3 Warrington and Halton Teaching Hospitals NHS Foundation Trust, Warrington, United Kingdom
}

\section{https://doi.org/10.21175/rad.abstr.book.2021.32.15}

Cancer yearly increase is connected also with radiotherapy increase, and younger patients such as children are also not spared from the yearly cancer and radiotherapy increase. To ease their pain and to make them comfortable during the radiotherapy, and also the lower the number of the dose treatment, by precise localization of the treatment, sometimes it is necessary to use the volatile anaesthetics as part of general anaesthesia. In past, those anaesthetics were considered safe, but there are new findings demonstrating that this is not always true. We decided to use the most used VA and to examine in vivo on animal model whether there is synergistic effect in combined exposure to isoflurane and to exposure to 1 or 2 Gy of ionizing radiation, usually used in fractioned radiotherapy. Using healthy Swiss male albino mice 60 days old, cca. 25 grams of body weight, we exposed them for 2 hours to $1.7 \%$ of inhaled isoflurane mixture with oxygen and air and afterwards some of them were also exposed to 1 or 2 Gy of ionizing radiation and were sacrificed immediately after exposure, 2, 6 and 24 hours from the exposure. We had 5 animals per group and radiation source was cobalt, with the dose rate of $1.88 \mathrm{~Gy} /$ minute on the Theratron Phoenix teletherapy unit, Atomic Energy Ltd., in at the Clinical Hospital "Sveti Duh", Zagreb, Croatia. Study was approved by Ethical Committee of the Faculty of Science (University of Zagreb, Croatia) and was designed in accordance with the relevant Croatian guidelines: The Animal Protection Act (NN 102/17.) and the Ordinance on the protection of animals used for scientific purposes (NN 55/13; 39/17). We measured primary DNA damage level using alkaline comet assay, well established method for in vivo genotoxicity assessment according to OECD guidelines on 200 comets per each time point and treatment. DNA damage was assessed using fluorescence microscope Olympus BX 40 connected with CCD camera with Comet Assay IV software and with parameters of tail length, tail intensity and tail moment. We took blood samples and liver samples for the alkaline comet assay. The results demonstrated that both single exposure to isoflurane and ionising radiation caused significantly higher DNA damage levels when compared to the control samples, with isoflurane and 1 Gy ionizing radiation having similar levels, while 2 Gy exposure caused the highest DNA damage levels. Surprisingly, combined exposure of isoflurane and ionizing radiation caused lower levels of DNA damage when compare to only radiated samples. These findings implicate that isoflurane can cause protective effect and cause lower level of DNA damage and should be used in radiotherapy. The mechanism by which isoflurane caused this effect is probably adaptive response, by activating DNA repair mechanisms and the levels of scavengers of reactive free oxygen radicals, covering on that way both types of which IR exposure can cause DNA damage effects (indirect and direct IR effect on DNA). 


\title{
Combined exposure to halothane and 1 or $2 \mathrm{~Gy}$ ionizing radiation causes synergistic effect in DNA damage in both blood and liver of Swiss albino mice
}

\author{
Vesna Benkovic ${ }^{1}$, Nikola Borojeviće ${ }^{2}$, Nada Oršolić ${ }^{1}$ \\ Gordana Brozović3 3,4 , Anica Horvat Knežević ${ }^{1}$, Mirta Milić
}

\author{
1 Faculty of Science, University of Zagreb, Zagreb, Croatia \\ 2 Warrington and Halton Teaching Hospitals NHS Foundation Trust, Warrington, United Kingdom \\ 3 Department of Anaesthesiology, Reanimatology and ICU, University Hospital for Tumours, Sestre \\ Milosrdnice University Hospital Centre, Zagreb, Croatia \\ 4 Faculty of Dental Medicine and Health, Osijek, Croatia \\ 5 Institute for Medical Research and Occupational Health, Mutagenesis Unit, Zagreb, Croatia
}

\section{https://doi.org/10.21175/rad.abstr.book.2021.32.16}

Patient immobilization by general volatile anaesthesia (VA) during medical radiology treatment is sometimes necessary and thus far it has had quite an annual increase. With ionizing radiation exposure there will be for sure some level of DNA damage since IR is a well-known genotoxic and cytotoxic agent, although the doses used are maintained at the minimum, with good localization in order to spare as much as possible healthy tissue and organs that do not need to be exposed. Recently, there is a growing number of studies demonstrating that volatile anaesthetics can cause also DNA damage effect in patients, and in occupationally exposed personnel. Since there are no studies on IR and VA combined effects, we decided to use animal model and check whether there are elevated levels of DNA damage after combined exposure by mimicking the real conditions of exposure during the radio-treatment using animal model of Swiss albino mice. Healthy mice were anaesthetized by inhaling $2.4 \%$ halothane mixture with oxygen and air for 2 hours and then were exposed to either 1 or 2 Gy of ionizing radiation ( ${ }^{60} \mathrm{Co}$ source, Theratron Phoenix teletherapy unit, Atomic Energy Ltd., in at the Clinical Hospital "Sveti Duh", 1.88Gy/min doze rate). With 5 animals per group, there were groups exposed only to halothane, only to radiation of 1 or 2 Gy and to combined exposure of halothane and 1 Gy or 2 Gy radiation, and there were groups examined immediately after exposure, 2, 6 and 24 hours after the exposure. Blood was taken from the tail immediately after the exposure, and liver after sacrifice of the animal. Study was approved by Ethical Committee of the Faculty of Science (University of Zagreb, Croatia) and was designed in accordance with the relevant Croatian guidelines: The Animal Protection Act (NN 102/17.) and the Ordinance on the protection of animals used for scientific purposes (NN 55/13; 39/17). Single cell samples were prepared for the alkaline comet assay, and DNA damage was assessed with the Comet Assay IV software. The results performed in duplicate slide, in total od 200 comets per each point, demonstrated that both halothane and IR demonstrated elevated levels of DNA damage, and that combined treatment caused synergistic effect that increased with the dose of radiation and was not repaired not even after 24 hours from the exposure. These data are confirming the concern about safety of combined VA and IR exposure, and calling for further investigation on the safety and proper use of the type of the anaesthetic necessary used during radiotherapy. 


\title{
Functional changes in brain microvascular endothelial cells upon low-energy accelerated proton-irradiation
}

\author{
Roberta Stoica', Mihai Radu', Beatrice Mihaela Radu²
}

\author{
1 Department of Life and Environmental Physics, 'Horia Hulubei' National Institute for Physics and Nuclear \\ Engineering, Magurele, Romania \\ 2 Department of Anatomy, Animal Physiology and Biophysics, Faculty of Biology, University of Bucharest, \\ Bucharest, Romania
}

\section{https://doi.org/10.21175/rad.abstr.book.2021.32.17}

Nowadays, it has become a common practice to use ionizing radiation during justified diagnostic and therapeutic medical procedures. The ionizing radiation exposure can induce endothelial activation, dysfunction, reactive oxygen species formation, blood brain barrier permeability elevation and tight junction morphology changes $[1,2]$. The neuro-oncology specialists intend to develop a controlled method to disrupt the BBB in strategic places in the brain or tumor bed before the administration of chemotherapeutics in order to increase their distribution and efficacy. Thus, a complex characterization of the brain microvasculature after irradiation is imposed.

The main objective of our study is to understand how accelerated proton beams affect the brain microvascular endothelial cells functionality. We obtained preliminary results after low energy accelerated proton irradiation $(<10 \mathrm{MeV})$ regarding the cytotoxic, genotoxic and functional effects upon an in vitro model of murine cerebral microvasculature (bEnd.3 cell line, ATCC). One beam line of a TR19 cyclotron was adapted to irradiate cell cultures in controlled conditions. We exposed the cell cultures to various doses in the range $0-10 \mathrm{~Gy}$, the dose rate being of $1 \mathrm{~Gy} / \mathrm{min}$. After irradiation, the cellular capacity of proliferation and surviving in stress-induced conditions were tested using clonogenic assay method. Also, to assess the ionizing radiation-induced DNA damage, we measured the expression of micronuclei and g- $\mathrm{H} 2 \mathrm{AX}$ foci. It is questionable the cellular functionality after irradiation and thus calcium ions dynamics was observed at different time intervals post-irradiation. As results, we observed a significant modification of the measured parameters with increasing the dose and the LET (linear energy transfer) values.

Our study presents preliminary results for genotoxicity and cytotoxicity after proton irradiation which will contribute to our on-going study regarding the post-irradiation functional changes in endothelial cells. Considering that bEnd. 3 is a popular cell line used for BBB model, our data are valuable in understanding the barrier behavior in oxidative stress conditions.

Keywords: Blood-brain barrier, proton irradiation, calcium, bEnd.3 cells

\section{References}

[1] Baselet Bjorn et al., Pathological effects of ionizing radiation: endothelial activation and dysfunction, Cellular and Molecular Life Sciences (2019), 76(4): 699-728.

[2] Appelboom Geoff et al., Stereotactic modulation of blood-brain barrier permeability to enhance drug delivery, Neuro-Oncology (2016), 18(12): 1601-1609. 


\title{
Comparison of methods for assessing the effect of chronic radiation exposure on the veining of leaves of Phragmites australis (Cav.) Trin. ex Steud
}

\author{
Dmytro Ganzha', Dmytro Ganzha²
}

1 Ivano-Frankivsk Department of the Ukrainian Geographical Society, Ivano-Frankivsk, Ukraine

2 Institute of Hydrobiology National Academy of Sciences of Ukraine, Kiyv, Ukraine

\section{https://doi.org/10.21175/rad.abstr.book.2021.32.18}

The aim of the study: to compare methods for assessing the effect of chronic internal radiation exposure from incorporated ${ }^{90 S r}$ and ${ }^{137} \mathrm{Cs}$ on the veining of common reed leaves "Phragmites australis (Cav.) Trin. ex Steud”.

During 2012-2013 and 2017-2019, changes in the veining of common reed leaves in ecosystems of six reservoirs with different levels of radionuclide contamination were studied in the Chornobyl Exclusion Zone. In the selected 28 samples of common reed leaves by beta spectrometry measured the concentration of ${ }^{9} \mathrm{Sr}$ and ${ }^{137} \mathrm{Cs}$, with a total uncertainty of measurements (u), not more than $18 \%$. According to the measurement results, the dose of internal irradiation relative to the weight of raw plants was calculated. In freshly selected reed leaves, the veining was photographed in transmitted light: by the photographic method - with the help of a scanner and a digital camera, by the method of microscopy at 30 times magnification. 1100 leaf samples were examined by scanning and photography, and 412 by microscopy. Statistical analysis of measurement results by the Monte Carlo method.

The median value of the internal irradiation of reed leaves at the observation sites was: for ${ }^{90} \mathrm{Sr}-0.016$ for ${ }^{137} \mathrm{Cs}-0.093$, at $\mathrm{u}=94$ and $77 \%$, respectively.

Correlation analysis by the Spearman method of sampling the dose load for ${ }^{\circ} \mathrm{Sr}$ on the one hand and samples of the number of veins per centimeter obtained by photomethod and microscopy showed a close relationship with coefficients of 0.9 and 0.7 , respectively. The same parameter with the width of the veins by photomethod and microscopy showed the inverse of the mean strength and a close relationship with the coefficients -0.6 and -0.8, respectively; with fractal dimension of veining by the method of microscopy - the average strength of the ligament with a factor of o.6. No correlation with ${ }^{90} \mathrm{Sr}$ or ${ }^{137} \mathrm{Cs}$ was found with the fractal dimension of veining by the photomethod.

Correlation analysis of pairs of samples obtained by photography and microscopy showed the parameters of the number of veins per centimeter and the width of the veins of close and medium strength relationship with coefficients of 0.5 and 0.7, respectively. Samples of both named veining parameters also have the same medians and types of statistical distribution. There is no connection between the values of the fractal dimension of the veins obtained by both methods. The total uncertainty in the measurement of veining is mainly influenced by: hardware inaccuracies of the means of survey, inhomogeneity of veining and damage to the leaf blade. In the photomethod, the total uncertainty for these parameters averages $18 \%$, in microscopy - up to $7 \%$. Thus, the data obtained by microscopy are more reliable, because when photographing and scanning the leaves of reeds, the result is significantly affected by factors not related to veining, such as dechromation and necrosis of the leaves. 


\title{
Hypoxia and antioxidant signaling in human colon carcinoma HCT116 cells exposed in vitro to $64-\mathrm{Cu}$
}

\author{
Gina Manda', Dana Niculae², Ionela-Victoria Neagoe', Radu Serban², \\ Dragos-Andrei Niculaes, Ramona Dusman², Elena Milanesi', Maria Dobre
}

\author{
1 Victor Babes National Institute of Pathology, Bucharest, Romania \\ 2 Horia Hulubei National Institute for R\&D in Physics and Nuclear Engineering, Magurele Bucharest, \\ Romania \\ 3 Carol Davila University of Medicine and Pharmacy, Bucharest, Romania
}

\section{https://doi.org/10.21175/rad.abstr.book.2021.32.19}

Background. $64-\mathrm{Cu}$ has been lately demonstrated to be a valuable theranostic agent in solid tumors, combining imagistic and therapeutical properties. Nevertheless, there is an urgent need to describe the molecular background underlining the action mechanisms of $64-\mathrm{Cu}$ for further identifying candidate molecular targets aimed at improving the therapeutical outcome as well as the toxicological profile of $64-\mathrm{Cu}$.

Aim. To identify in vitro using a transcriptomic approach the response of normal and tumor cells to $64-\mathrm{Cu}$, with a particular focus on hypoxia signaling and redox responses.

Method. Human colon carcinoma HCT116 cells and human BJ fibroblasts (ATCC) were treated with $20 \mathrm{MBq} 64-\mathrm{CuCl}_{2}$. Adherent cells were harvested in TRIzol at $24 \mathrm{~h}$ for gene expression studies performed by qRT-PCR using an array of 84 stress genes (Stress and toxicity pathway finder, Qiagen) and GAPDH / ACTB as housekeeping genes (RefFinder analysis). Only genes with a fold of change (treated versus non-treated) $>1.7$ were considered for interpretation.

Results. Treatment of cells with $20 \mathrm{MBq} 64-\mathrm{Cu}$ drastically reduced the number of viable cells in culture, both tumor and normal cells, HCT116 tumor cells being slightly more sensitive to the treatment than normal fibroblasts. The genotoxic stress inflicted by $64-\mathrm{Cu}$ in vitro was highlighted by the up-regulation of $C D K N 1 A$ and $D D B 2$. Both adherent viable HCT116 and BJ cells had at $24 \mathrm{~h}$ a gene expression profile characterized by marked hypoxia signaling evidenced by up-regulation of the HMOX1, SERPINE1, SLC2A1 and VEGFA genes. The hypoxia-related genes ADM, EPO and MMP9 were found distinctively over-expressed only in tumor HCT116 cells. A molecular fingerprint of antioxidant response was detected in HCT116 tumor cells (up-regulated GSR, HMOX1 and PRDX1), with the HMOX1 gene at the crossroad of hypoxia and redox signaling. Meanwhile, FTH1 and NQO1 were distinctively over-expressed in BJ fibroblasts. This antioxidant signature of the transcription factor NRF2 points out that $64-\mathrm{Cu}$ induces an oxidative stress against which both tumor and normal cells are reacting with common and distinctive molecular tools.

Conclusion. In vitro exposure of human normal and tumor cells to 64-Cu triggers was shown to trigger genotoxic stress accompanied by hypoxia and oxidative stress in HCT116 colon carcinoma cells and normal BJ fibroblast, with distinctive patterns of gene expression in normal and tumor cells. Pharmacologic modulation of these signaling pathways may enhance the therapeutic effect of $64-\mathrm{Cu}$ in tumors or could protect normal cells against the deleterious effect radiotoxicity of $64-\mathrm{Cu}$.

Acknowledgments: Work was supported by UEFISCDI through the grant PCCDI_64/2018, project 3, and by the Ministry of Education and Research through the NUCLEU grant PN 19.29.02.02/2019. 


\title{
Genetic markers associated with the development of stochastic effects
}

\author{
Evgeniya Blinova',2, Vladislav Nikiforov',2, \\ Mariya Yanishevskau', Alexandr Akleyevi,2
}

\author{
1 Urals Research Center for Radiation Medicine of FMBA of Russia, Chelyabinsk, Russia \\ 2 Chelyabinsk State University, Chelyabinsk, Russia
}

\section{https://doi.org/10.21175/rad.abstr.book.2021.32.20}

Main system of cell protection includes DNA reparation, cell cycle control and apoptosis, antioxidant and immune systems. It is known that human population is genetically heterogeneous that is why the efficiency of work of the systems that maintain the genome integrity may differ greatly from person to person. It means that the response to radiation exposure and individual radiosensitivity may depend not only on sex, age and health status, but also on genetic peculiarities of a particular human being.

This study involved investigation of the connection between SNP genes that regulate reparation, apoptosis, cell cycle, antioxidant and immune systems (28 polymorphic regions) and risk of malignant neoplasms development. Expression of mRNA of ATM, TP53, MDM2, CDKN1A, BAX, $B C L-2, X P C, O G G 1, S T A T_{3}, G A T A 3, M A P K 8, N F-k B 1$ and PADI4 genes was also studied in chronically exposed individuals.

Evaluation SNP studies were performed in 632 chronically exposed members of the Techa River Cohort for whom the dose to RBM had been reconstructed using TRDS 2016. The association of SNP with the risk of malignant neoplasm development was investigated in 248 patients (median exposure dose to RBM was $0.87 \mathrm{~Gy}$, dose range 0.002-4.6 Gy) with various types of solid cancers in the past history: breast cancer (49 people); skin cancer (46 people); GIT cancer (45 people); uterus cancer and cancer of ovaries (32 people); lung cancer (26 people). Comparison group consisted of 384 people without cancer (median exposure dose to RBM was $0.86 \mathrm{~Gy}$, dose range 0.001-4.2 Gy). Gene transcription activity was studied in 163 people (median exposure dose to RBM was 0.72, dose range 0.08-3.51 Gy). Comparison group consisted of 146 people residing in the same settlements with cumulative dose to RBM less than $7 \mathrm{cGy}$.

The preformed study revealed the connection between polymorphic region rs1952133 of the OGG1 gene, rs2279744 of the MDM gene, rs2279115 of the BCL2 gene, rs361525 of the TNF $\alpha$ gene and rs1050450 of the GPX1 gene and increased risk of cancer development; and association between polymorphic region rs13312840 of the NBS1 gene and rs1801270 of the CDKN1A gene with decreased risk of cancer development.

The most pronounced changes of the transcriptome in exposed persons were noted in genes that regulate apoptosis of $B C L-2, B A X, N F-k B 1$ and PADI4.

The genes that we have studied play the key role in the system of cell protection from radiation exposure. Radiation-induced changes in the transcription activity as well as the presence of tumorassociated allelic variants of genes may impede effective work of protective mechanisms of a cell, which in its turn results in the development of late effects of radiation exposure. 


\title{
Direct and bystander effects of human chondrosarcoma cell line irradiated with protons
}

\author{
Mihaela Tudor',2, Roxana Popescu', \\ Mihaela Temelie', François Chevalier3,4, Diana Savu'
}

\author{
1 Horia Hulubei National Institute for R\&D in Physics and Nuclear Engineering (IFIN-HH), Magurele, \\ Romania \\ 2 University of Bucharest, Faculty of Biology, Bucharest, Romania \\ 3 UMR6252 CIMAP, CEA - CNRS - ENSICAEN - Université de Caen Normandie, Caen, France \\ 4 LARIA, iRCM, François Jacob Institute of biology, DRF-CEA, Caen, France
}

\section{https://doi.org/10.21175/rad.abstr.book.2021.32.21}

Ionizing radiation induces complex cellular effects in targeted cells as well as neighbouring "bystander" cells which have received signals produced by irradiated cells. Characterising both direct and non-targeted bystander effects might improve the overall outcome of radiation exposure which could have implications in radiotherapy and radioprotection.

In this study, we focused on analysing direct and bystander cellular effects of proton irradiation of chondrosarcoma cells. Chondrosarcoma is resistant towards conventional chemo-/radiotherapy and can be un-operable. Charged particle radiotherapy is now a good alternative for chondrosarcoma. The advantages of this method include higher specificity (given by its specific Bragg pick) and increased biological efficacy (due to the high LET).

Two different chondrosarcoma cell lines (SW1353 and L835) were irradiated with protons at doses in the range 0.1-2 Gy. To study the bystander phenomena we used a medium transfer protocol. The bystander supernatant containing signals emitted by proton irradiated chondrosarcoma cells, was transferred to non-irradiated bystander normal chondrocyte (T/C-28a2) and endothelial (EA.hy) cells. Cell survival and DNA damage were assessed in directly irradiated chondrosarcoma cells and in bystander chondrocytes and endothelial cells.

A dose dependent direct effect was observed in chondrosarcoma cells while the bystander cells showed a less pronounced effect then directly irradiated cells. Our results showed that protonirradiated chondrosarcoma cells have the ability to release stress factors inducing bystander responses in the non-irradiated surrounding chondrocyte or endothelial cells.

Acknowledgments: We acknowledge the Romanian Ministry of Research and Innovation: PN 19060203/2019, 543 PED/2019, 18PCCDI/2018. 


\title{
75 years after the bombings of Hiroshima and Nagasaki
}

\begin{abstract}
Masaki Tan
NAKADA Geriatric Health and Welfare Facilities, Miyagi, Japan

https://doi.org/10.21175/rad.abstr.book.2021.32.22

75 years have passed since the atomic bombings of Hiroshima and Nagasaki. The use of a weapon was contemplated to assure decrease of the cost of American lives. Two types of bombs were developed. Little Boy: gun-type weapon that used Uranium-235 and Fat Man: implosion-type that used Plutonium. On July 26, 1945, Allied leaders issued the Potsdam Declaration, as an ultimatum stating that without surrender, the Allies would attack Japan, resulting in the complete destruction of the Japanese armed forces and the utter devastation of the Japanese homeland. The Declaration was rejected. On August 6, 1945, The Little Boy was dropped on Hiroshima and on August 9, 1945, Fat Man was dropped over Nagasaki. The Pacific War ended on August 15, 1945. The bombing caused Japan surrender, thereby preventing casualties that an invasion of Japan would have involved. Opposing belief is that the bombings are fundamentally immoral, and counted as war crimes, and state terrorism. Concerning the ethical and legal justification for them, they have been the subject of
\end{abstract} debate. 


\title{
Acute and chronic health effects of extracellular vesicles after irradiation
}

\author{
Dávid Kis, Eszter Persa, Tünde Szatmári, Rita Hargitai, \\ Ilona Barbara Csordás, Géza Sáfrány, Katalin Lumniczky
}

National Public Health Center, Department of Radiobiology and Radiohygiene, Unit of Radiation Medicine, Budapest, Hungary

\section{https://doi.org/10.21175/rad.abstr.book.2021.32.23}

Introduction. It is becoming increasingly clear that radiation-induced bystander effects (RIBE), as a consequence of intercellular signaling, contribute significantly to the biological response to radiation exposure. The role of extracellular vesicles (EVs) in RIBE was extensively studied in the last decade, although most of these studies were done in vitro. Here we report an in vivo bystander model using $\mathrm{C}_{57} \mathrm{BL} / 6$ suitable to investigate the role of bone marrow-derived EVs in mediating acute and chronic systemic radiation-induced bystander effects. In this study we focused on the capacity of EVs to induce changes in the bone marrow stem cells and progenitors after the in vivo transfer from irradiated to naive mice.

Methods. Male $\mathrm{C}_{57} \mathrm{Bl} / 6$ mice were exposed using 0; 0.1; 0.25 and 2 Gy X-ray doses. Mice were sacrificed 4 or 24 hours or 3 month after the irradiation, bone marrow and spleen as well as the EVs from bone marrow were isolated. The EVs were injected into the tail vein of non-irradiated mice (bystanders) and the bystander bone marrow and spleen were isolated 4 or 24 hours or 3 month after injection. The effects of EVs on the bone marrow cells of these bystander mice were compared to radiation effects in the directly irradiated animals. Apoptosis, senescence and stem / progenitor cell frequencies were measured by multicolor flow cytometry.

Results. The role of EVs in transmitting radiation effects was confirmed in the bystander mice.

EVs 4 or 24 hours after irradiation. As an acute effect we detected increased rate of apoptosis in the stem cell and progenitor populations 4 hours after injection of EVs isolated from exposed mice. Migratory stem cells were detected in the spleen 24 hours after injection of EVs. The composition of hematopoietic stem cells has changed significantly due to the decrease in multipotent progenitor population 24 hours after injection of EVs as well as moderately 3 months after. The identified chronic effects were the increased senescence frequencies and the decrease in cell number of lymphoid- and myeloid progenitors 3 months after injection of EVs.

EVs 3 months after irradiation. Interestingly, these EVs did not affect bystander animals. Neither did they induce apoptosis, migration, cell number or phenotypical changes.

Conclusion. We show here that the injection of EVs from irradiated mice after 4 or 24 hours is able to mediate acute and chronic changes in the bone marrow of non-irradiated mice, however EVs isolated 3 months after irradiation are lost this ability. This study sheds light on possible mechanisms of radiation-induced bystander damage in the bone marrow.

Acknowledgments: The presentation was supported by: NTP-NFTÖ-20-B-0295. The work described in this abstract has been supported by the European Commission, within the CONCERT project. This project has received funding from the Euratom research and training programme 2014-2018 under grant agreement No 662287. 


\title{
Approaches for the estimation of the cytogenetic effects in lymphocytes of cancer patients due to combined radiation and chemotherapy
}

\author{
Nataliya Maznyk, Tetiana Sypko, Viktor Starenkiy, \\ Inna Gukova, Nataliia Bohatyrenko, Serhii Artiukh, \\ Liudmyla Cherkasko, Irina Krugova, Larysa Zabobonina
} Grigoriev Institute for Medical Radiology and Oncology of National Academy of Medical Science of Ukraine,
Kharkiv, Ukraine

\section{https://doi.org/10.21175/rad.abstr.book.2021.32.24}

The investigations of cytogenetic effects in lymphocytes of cancer patients induced by radiation therapy lead to the better understanding the mechanisms of cytogenetic damage formation in patients' normal tissues. Fractionated partial body irradiation is complicated radiation scenario and additional mutagen influence when chemotherapy is a part of the treatment required auxiliary approaches for study design.

The aim was to develop and test in vivo and ex vivo study design for assessment the effect of previous chemotherapy on the character of radiation- and chemical-induced cytogenetic effects during radiation therapy.

Cytogenetic analysis was performed in lymphocytes of 10 patients with lung cancer and with head and neck cancer who undergoing chemotherapy followed by radiation treatment. Blood sampling was done during megavolt radiotherapy course on linear accelerator: before radiotherapy and at the end of radiation therapy course. Dose per fraction was 1.8 - 2 Gy for both tumor localizations. The part of blood samples taken before treatment was irradiated at the doses $1 \mathrm{~Gy}$ and $2 \mathrm{~Gy}$. Both conventional and G2-assay were applied for ex vivo studies and conventional chromosome aberrations test was used for in vivo studies.

The increase of radiation-induced chromosome aberrations from the beginning to the end of radiotherapy course in patients with lung cancer and less pronounced enhancement for the patients with head and neck tumors was demonstrated.

Against the background of the absence of a clearly noticeable effect of chemotherapeutic treatment on the frequency of radiation-nonspecific chromatid type damages during radiotherapy course a dependence of these parameters on the tumor localizations was observed.

The informativeness of ex vivo irradiation in study design varied depending on the dose and cytogenetic tests applied. The peculiarities of conventional and G2-assays in the evaluation of cytogenetic effects in cancer patients will be discussed.

The data obtained are necessary for correct assessment of the impact of therapeutic irradiation on the chromosomal level. 


\title{
Optimization of Sr-90 precipitation in nitric acid using experiment design and speciation modeling for radioactive waste characterization
}

\author{
Emilie Baudat', Céline Gautier', Isabelle Billard², Pascal Fichet \\ 1 Des - Service d'Etudes Analytiques et de Réactivité des Surfaces (SEARS), CEA, Université Paris-Saclay, \\ Gif-sur-Yvette, France \\ 2 Univ. Grenoble Alpes, Univ. Savoie Mont Blanc, CNRS, Grenoble INP, LEPMI, Grenoble, France
}

\section{https://doi.org/10.21175/rad.abstr.book.2021.33.1}

Numerous radiochemical procedures have been developed and published to measure Sr-90 in environmental and food samples but not many for nuclear waste. The standard method is a series of precipitation steps with a separation of Sr and Ca using fuming nitric acid [1]. It is fast and does not require complex material but implies the handling of fuming nitric acid, highly harmful. Since the 1990s, the extraction chromatrographic resin developed by Eichrom [2], called Sr-resin ${ }^{\circledR}$, has been widely applied for the separation of Sr-90. Despite the number of advantages, the implementation of separation column can be time-consuming and pre-treatments are necessary. Therefore, difficulties are still encountered to characterize Sr-90 in some matrices, particularly nuclear wastes. For this purpose, an in-depth understanding of them is necessary.

A large number of publications mentioned $\mathrm{Sr}$ and $\mathrm{Ca}$ separation using precipitation with nitric acid, but the mechanism and the experimental conditions applied are not clearly outlined. Discrepancies are observed between publications as some articles recommend fuming nitric acid [3] when others demonstrate that concentrated acid is sufficient for precipitation [4].

The presentation will describe the Design Of Experiment (DOE) theory used to prove the efficiency of nitric acid at $69 \%$ instead of fuming nitric acid. DOE enabled to identify the influent factors just by performing a limited number of experiments and optimize them by response surface methodology. It will be compared to a second strategy of optimization using speciation. The difficulties to implement speciation calculations with high ionic strengths will be highlighted. The use of speciation modeling helped to determine the behavior of the analytes and the involved interfering species during the reactions. These two approaches provided optimal experimental conditions to separate efficiently $\mathrm{Sr}$ from $\mathrm{Ca}$ in various radioactive wastes.

\section{References}

[1] Sunderman, D. N. \& Meinke, W. W. Evaluation of Radiochemical Separation Procedures. Anal. Chem. 29, 1578-1589 (1957).

[2] Horwitz, E. P., Dietz, M. L. \& Chiarizia, R. The application of novel extraction chromatographic materials to the characterization of radioactive waste solutions. J. Radioanal. Nucl. Chem. 161, 575-583 (1992).

[3] Lehto, J. \& Hou, X. Chemistry and Analysis of Radionuclides: Laboratory Techniques and Methodology. (John Wiley \& Sons, 2011).

[4] Bojanowski, R. \& Knapinska-Skiba, D. Determination of low-level ${ }^{90} \mathrm{Sr}$ in environmental materials: A novel approach to the classical method. J. Radioanal. Nucl. Chem. 138, 207-218 (1990). 


\title{
Dynamic test of alkaline HLW processing with hydroxycalix[6]arenes based solvent
}

\author{
Igor Smirnov',2, Maria Karavan',2
}

1 Ozersk Technological Institute, Ozersk, Chelyabinsk obl., Russia

2 Khlopun Radium Institute, St. Petersburg, Russia

\section{https://doi.org/10.21175/rad.abstr.book.2021.33.2}

The amount of accumulated alkaline HLW is significantly less than that of acidic ones, but their processing is a very difficult technological task due to the extremely high content of salts of nonradioactive elements. To date, "Mayak" Production Association stores more than $18000 \mathrm{~m}^{3}$ of alkaline HLW, generated during the implementation of the Atomic Project of the USSR. This waste contains up to $6 \mathrm{~mol} / \mathrm{L}$ of sodium and aluminum salts and more than $1 \mathrm{Ci} / \mathrm{L}$ of long-lived radionuclides: principally ${ }^{137} \mathrm{Cs},{ }^{90} \mathrm{Sr}$ and transuranium elements. Recovery of $99.8 \%$ of radionuclides from alkaline HLW will make it possible to transfer them to the category of low-level waste and significantly simplify their final disposal.

Hydroxycalix[n]arenes with high chemical and radiation resistance are promising extractants for the long-lived radionuclides recovery from alkaline media. We studied the regularities of ${ }^{137 \mathrm{Cs},}{ }^{90} \mathrm{Sr}$ and ${ }^{241} \mathrm{Am}$ extraction from alkaline media with p-alkylcalix[n]arenes $(\mathrm{n}=4,6,8)$. All investigated calix[4]arenes are very poorly soluble in hydrocarbon diluents. Calix[8]arenes with isononyl substituents at the lower rim are highly soluble in organic diluents, but cesium is poorly extracted. The optimal combination of high extraction ability towards cesium and good solubility in a paraffin diluent demonstrated p-isononylcalix[6]arene. The effect of the aqueous and organic phases composition on the efficiency of ${ }^{137} \mathrm{Cs}$ extraction was studied. It has been shown that the main factor affecting the efficiency of radionuclides extraction is the $\mathrm{pH}$ of the aqueous phase: the maximum is observed at $\mathrm{pH} 12 \div 14$.

During static tests on real alkaline HLW of the "Mayak" PA, high purification factors were achieved: 15000 for ${ }^{137} \mathrm{Cs}$ and beta-emitting and 100 for alpha-emitting radionuclides, which allows us to transfer this waste to the low-level category, which permits its cementation and surface burial.

The principal technological flowsheet of alkaline HLW extraction processing has been developed, including only extraction and striping blocks, without extract washing and recycle solvent regeneration.

Countercurrent flowsheet test using 16-stage of settling mixers extractors (10 extraction and 6 stripping stages) with pulsating phase mixing and simulated alkaline HLW was performed. During 105 hours of test, the setup worked stably; there were no formation of precipitates, poorly separated emulsions or mutual phase entrainment. HLW purification factor for ${ }^{137 \mathrm{Cs}}$ and beta-emitting radionuclides of about 500 and a 10 -fold concentration of ${ }^{137} \mathrm{Cs}$ in a strip product were achieved.

The test results confirm the principal possibility of using the solvent based on pisononylcalix[6]arene for the processing of "Mayak" PA alkaline HLW.

Acknowledgments: The study was supported by the Russian Science Foundation (project No. 20-63-46006). 


\title{
The role of ligand conformational mobility in the process of $A n(I I I) / L n(I I I)$ extraction
}

\author{
Nikolai Andreadi, Artem Mitrofanov, Petr Matveev, Nataliya Borisova
}

Lomonosov Moscow State University, Department of Chemistry, Moscow, Russia

https://doi.org/10.21175/rad.abstr.book.2021.33.3

A major problem of nuclear power chemical engineering is the management of high-level wastes (HLW), which are formed during the reprocessing of spent nuclear fuel. It was proposed to extract long-lived radionuclides from HLW with their subsequent fractionation and transmutation in special reactors with high neutron fluxes. This strategy will reduce the economic and environmental pressure at the stage of HLW disposal, but the question of choosing the chemical and technological procedures for the implementation of this approach remains open. Over the past 40 years, a large number of extractants have been studied. In particular, many investigations were devoted to establishing "structure-extraction properties" relationship though there is still no single theory explaining complexation efficiency or selectivity covering significant series of ligands.

This study presents a new approach for in silico modeling of the Am(III)/Eu(III) extraction efficiency. We developed a hybrid method for studying the conformational behavior of small organic molecules in solution. We also did not include any calculations for metal complexes, as they can be a major source of calculation errors. Moreover, we used relatively simple computational methods and minimized human influence on the result. Finally, we performed extraction experiments with 12 phosphine oxides and 3 monoamides as extractants. The obtained theoretical results showed agreement with the experimental ones.

The study revealed the predominance of preorganization and solvation effects over electrostatic ones as the driving force behind the efficiency of ligand extraction, so we suggested to use the sum of preorganization and solvation energies as a value to assess the efficiency of complexation in the target solvent. Thus, the conformational mobility of the ligand, which depends on the nature of the ligand and its substituents, plays a key role in the efficiency of extraction. We can also conclude that the absence of any correlation between the calculated energies and selectivity is evidence that the origin of selectivity is in a direct interaction between the metal cation and the extractant. The proposed method of modelling provides a simple approach to testing of the extraction efficiency (not selectivity) of new extractants and requires only a 2D structure of an organic molecule and can be useful at the pre-synthetic stage of new ligands, which leads to minimization of both computational and experimental costs.

Acknowledgments: This work was supported by RFBR (grant 20-33-90151). 


\title{
Yttrium-90 separation in carbonate media by solvent extraction
}

\author{
Igor Smirnov',2,3, Ahmed Hamdy Aly Harb3,4, \\ Igor Balantsev², Maria Karavan',2,3
}

\author{
1 Ozersk Technological Institute, Ozersk, Russia \\ 2 Khlopin Radium Institute, St. Petersburg, Russia \\ 3 St. Petersburg State University, St. Petersburg, Russia \\ 4 Atomic Energy Authority, Hot Laboratories Center, Nuclear Chemistry Department, Inshas, Cairo, Egypt
}

\section{https://doi.org/10.21175/rad.abstr.book.2021.33.4}

Yttrium-90 $\left(\mathrm{T}_{1 / 2}=64\right.$ hours, $\left.\mathrm{E}_{\beta \max }=2.28 \mathrm{MeV}\right)$ is of great interest for the analysis of strontium-90 $\left(\mathrm{T}_{1 / 2}=28.8\right.$ years $)$ in natural samples, since it is its daughter decay product. It is also a therapeutic radioisotope due to its pure $\beta^{-}$-particle emission, so the problem of yttrium extraction is more urgent than ever.

Usually, one can recover yttrium-90 from nitric acid media with crown ethers and D2EHPA (di-2-ethyl-hexyl-phosphoric acid) as extractants. However, the pair yttrium-90 - strontium-90 can be separated in carbonate media as well. In our work we examined our method of ${ }^{\circ 0} \mathrm{Y} / 9^{\circ} \mathrm{Sr}$ separation in alkaline carbonate media. We used 8-HQ (8-hydroxyquinoline), PAN (pyridylazonaphthol), PAR (pyridylazoresorcinol) and chromotropic acid as potential yttrium extractants, but only 8-HQ demonstrated sufficient extraction ability towards to yttrium under these conditions.

Preliminary experiments on yttrium oxides dissolution kinetics showed thatin $1 \mathrm{M} \mathrm{Na}_{2} \mathrm{CO}_{3}, \mathrm{~K}_{2} \mathrm{CO}_{3}$ and $\left(\mathrm{NH}_{4}\right)_{2} \mathrm{CO}_{3}$ solutions the solubility was $1.7,0.9,2.8 \mathrm{~g} / \mathrm{L}$ with dissolution rate of $0.21,0.26$, $0.24 \mathrm{~g} \cdot \mathrm{m}^{2} / \mathrm{s}$, respectively, in $2 \mathrm{M} \mathrm{Na}_{2} \mathrm{CO}_{3}, \mathrm{~K}_{2} \mathrm{CO}_{3}$ and $\left(\mathrm{NH}_{4}\right)_{2} \mathrm{CO}_{3}$ solutions the solubility these values were $4.9,1.4,8.2 \mathrm{~g} / \mathrm{L}$ and dissolution rate of $0.43,0.46,0.62 \mathrm{~g} \cdot \mathrm{m}^{2} / \mathrm{s}$, respectively.

Based on the high yttrium oxide solubility, we studied yttrium extraction from $\mathrm{Na}_{2} \mathrm{CO}_{3}, \mathrm{~K}_{2} \mathrm{CO}_{3}$ and $\left(\mathrm{NH}_{4}\right)_{2} \mathrm{CO}_{3}$ with 8-HQ. Butyl acetate and 2-nitrotoluene were used as diluents. $\mathrm{K}_{2} \mathrm{CO}_{3}$ possess highest distribution ratio (D), followed by $\mathrm{Na}_{2} \mathrm{CO}_{3}$, but a third phase formation was observed for $\left(\mathrm{NH}_{4}\right)_{2} \mathrm{CO}_{3}$ solution. The system "0.01 M 8-HQ in organic diluent - $0.001 \mathrm{M}$ yttrium in $0.5 \mathrm{M} \mathrm{K}_{2} \mathrm{CO}_{3}$ aqueous solution" was chosen for extraction experiments. The distribution ratio logarithm was 2.60 and 2.03 for 2-nitrotoluene and butyl acetate, respectively. The main trend of yttrium extraction dependence on $\mathrm{pH}$ shows the increase at $\mathrm{pH} \sim 10\left(8-\mathrm{HQ} \mathrm{pK}_{\mathrm{a}}\right.$ is 9.9) with maximum at $\mathrm{pH}=13$ in 2-nitrotoluene and $\mathrm{pH}=13.5$ in butyl acetate. When the organic phase was completely saturated with yttrium a correlation between yttrium and 8-HQ reached 4.1 in 2-nitrotolueneand 4.2 in butyl acetate.

Experimental results allow to consider extraction systems, based on 8-HQ, as perspective solution for yttrium recovery from alkaline carbonate media.

Acknowledgments: The study was supported by the Russian Science Foundation (project No. 20-63-46006). 


\title{
Fast method for studying the extraction of the main HLW components with crown ethers in new fluorine-containing diluents
}

\author{
Vladislav Timoshenko', Alexandr Brechalov', \\ Yuri Ermolenko', Igor Smirnovi,2
}

1 Saint Petersburg State University, Saint Petersburg, Russia

2 Khlopin Radium Institute, Saint Petersburg, Russia

\section{https://doi.org/10.21175/rad.abstr.book.2021.33.5}

The study of the interphase distribution of a large number of target and impurity components of highly radioactive wastes is a necessary and very laborious stage in the development of new extraction systems. We have developed an express method for determining the distribution ratios of metals using inductively coupled plasma mass spectrometry (ICP-MS). A feature of the proposed method is the joint extraction of trace amounts of all studied metals, followed by direct ICP-MS analysis of equilibrium aqueous and organic phases.

We used the developed express method when studying a new extraction system designed for the processes of cesium-137 and strontium-90 separation from high-level waste (HLW) after spent nuclear fuel processing. The growing interest in such processes is due to the implementation of the "Balanced NFC" concept in Russia. This concept implies the isolation, solidification, and separate handling of cesium-strontium fraction management. There is also a growing commercial demand for the strontium-90 radioisotope.

The studied extraction systems consisted of known crown ethers (CE): 4,4'(5')-di-tert-butyldibenzo-18-crown-6 (DtBuDB18C6) and 4,4'(5')-di-tert-butyl-dicyclohexyl-18-crown-6 (DtBuDCH18C6) in new fluorinated diluents: bis-tetrafluoropropyl carbonate (BK-1= 2,2,3,3tetrafluoropropan-1-ol carbonate) and bis-tetrafluoropropyl formal $(\mathrm{FN}-1=1,1,2,2,8,8,9,9$ octafluoro-4,6-dioxanonane). Extraction was carried out from aqueous solutions containing 0.1 - 5.0 $\mathrm{M} \mathrm{HNO}_{3}$ and $5-70 \mathrm{mg} / \mathrm{L}$ of each of the 28 metals present in most HLW. After special preparation of equilibrium aqueous and organic phases, the concentrations of metals were determined by ICP-MS analyzer (Agilent Technologies 7900).

The studies have shown that among the stable components of HLW, the lead cation demonstrates the highest coextraction with radionuclides. None of the studied extraction mixtures extracted Na, $\mathrm{Cr}, \mathrm{Ni}, \mathrm{Co}, \mathrm{Fe}, \mathrm{Mn}, \mathrm{Eu}, \mathrm{Y}, \mathrm{Zr}, \mathrm{Mo}$, Pd from nitric acid media. In the series of studied crown ethers, the minimum selectivity is observed for DtBuDCH18C6 ( $\mathrm{Pb}, \mathrm{Ba}, \mathrm{Ag}, \mathrm{K}$ are coextracted with strontium). Benzo-substituted DtBuDB18C6 possesses higher selectivity: only rubidium is noticeably coextracted with cesium. Crown ethers extraction systems, based on formal FN-1 exhibit higher selectivity. We observed that most of the studied metals have maximum extraction at 0.5 to $2 \mathrm{M}$ $\mathrm{HNO}_{3}$. Only lead is well extracted even from very dilute nitric acid, i.e. its reextraction with water is impossible and requires chelating agent solutions.

The studies performed have confirmed the possibility of simultaneous determination of distribution ratios of a large number of metals using ICP-MS according to our express method.

Acknowledgments: The study was supported by the Russian Science Foundation (project No. 20-13-00143). 


\title{
Development of a DFT functional for quantum radiochemistry
}

\author{
Artem Mitrofanov, Andreadi Nikolay, \\ Vadim Korolev, Petr Matveev, Stepan Kalmykov
}

Lomonosov Moscow State University, Moscow, Russia

https://doi.org/10.21175/rad.abstr.book.2021.33.6

Computational and, in particular, chemistry is an especially convenient tool in radiochemistry due to the possibility to reduce the number of required experiments and, thereby, reduce the dose absorbed by personnel. On the other hand, the usage of quantum chemical methods is limited in radiochemistry due to relativistic effects, the multiconfigurational character of the wavefunction of heavy elements or just limited computational resources. Density functional theory (DFT) seems to be an example of an efficient balance between accuracy and computational time. The problem of DFT approaches is their semiempirical and interpolation character, defined by data used for functional parameters fitting. Here we would like to propose an investigation aimed at developing new functional, designed especially for f-elements computations.

The design process is based on three main stages: collecting a reference database, developing a fitting algorithm and finally fitting the target functional. During the investigation, we collected a database with geometries and electron structures of actinide compounds calculated by a robust ab initio method. The database contains 104 compounds including single atoms from Ac to $\mathrm{Cf}$ and the most probable ionic forms. Each compound is presented in a single directory. The compound directory contains information about the structure in the struct.xyz file; energy (in $\mathrm{kcal} / \mathrm{mol}$ ), charge and multiplicity in the ecm.dat text file, and molecular orbitals in the Molden format.

The developed fitting approach is based on a Bayesian optimization with stochastic subsampling. The algorithm allows considering the "history" of fitting steps, reduces the computation time for each step, and avoids overfitting. While general testing the effectiveness of the approach, we showed an example of training specialized DFT functionals that are superior to the popular ones.

As a result, we used the database and the developed method to "retrain" popular PBEo functional and increase its accuracy nearly twice relatively to the original one. The obtained accuracy (in terms of gas-phase geometry and thermodynamics of gas-phase reactions) appeared to be comparable with the experimental one.

Acknowledgments: The research was carried out using the equipment of the shared research facilities of HPC computing resources at Lomonosov Moscow State University. The research was supported by the Russian Science Foundation (Grant 19-73-20115). 


\title{
Purification of uranium(VI) from impurities of fission product surrogates by solvent extraction in the CARBEX process
}

\author{
Alexander Boyarintsev, Sergey Stepanov, Sergey Frankiv, Nikita Chervyakov
}

Mendeleev University of Chemical Technology, Moscow, Russia

https://doi.org/10.21175/rad.abstr.book.2021.33.7

Deep purification of uranium from impurities of all fission products (FPs) is an important task in the spent nuclear fuel (SNF) reprocessing technology. In recent decades, there has been an increase in worldwide interest in developing alternative non-acidic hydro-chemical approaches for the SNF processing. In most of these approaches, the transition from nitric acid media to carbonate or carbonate-alkaline media is contemplated. One of the options for SNF reprocessing in carbonate media is CARBEX (CARbonate extraction) process in which it is proposed to use of solvent extraction technique for separation and purification of uranium/plutonium from FPs impurities. The solvent extraction stage in the CARBEX process with usage of methyltrioctylammonium (MTOA) carbonate as an extractant, involves two successive cycles: (i) extraction/partial purification of $\mathrm{U} / \mathrm{Pu}$ from impurities and (ii) deep purification of $\mathrm{U} / \mathrm{Pu}$ from impurities.

Carrying out modeling of multistage solvent extraction process on a laboratory cascade in various options and in extraction/washing/stripping mode, allowed to reach values of uranium purification factors from impurities of some FPs surrogates (Mo, Cs, Sr, Sn, Ba, Al, Cr, Cu, Sb, Re, Te, Mn, Zr, REEs) and also from sodium at value level $10^{5}-10^{6}$. The obtained results are close to those of the industrial PUREX technology.

On the basis of the carried out studies, a scheme of solvent extraction reprocessing of multicomponent carbonate solutions containing of FPs surrogates was developed and optimized. This scheme showed a high efficiency of the solvent extraction technique for fractionating of the fission products in the CARBEX process.

Acknowledgments: The research was supported by Mendeleev University of Chemical Technology. Project № T-2020-009. 


\section{Oxidative dissolution of $\mathrm{U}_{3} \mathrm{O} 8$ in carbonate-alkaline solutions}

\section{Alexander Boyarintsev, Sergey Stepanov, Sergey Frankiv, Nikita Chervyakov}

Mendeleev University of Chemical Technology, Moscow, Russia

\section{https://doi.org/10.21175/rad.abstr.book.2021.33.8}

Oxidative dissolution is the key stage of uranium oxide spent nuclear fuel (SNF) reprocessing technology in carbonate media. The ability to quickly and completely dissolve the main component of the SNF matrix - $\mathrm{UO}_{2}$ in sodium or ammonium carbonate solutions in the presence of hydrogen peroxide at room temperature made it possible to substantiate carbonate media as an alternative to nitric acid media in the SNF reprocessing technology. As a preliminary stage, it is proposed to carry out voloxidation (volumetric oxidation) before SNF oxidative dissolution stage. One of the tasks of voloxidation is the conversion of $\mathrm{UO}_{2}$ into $\mathrm{U}_{3} \mathrm{O}_{8}$, as well as the removal of volatile and gaseous fission products. The voloxidation temperature, depending on the task, can vary from 480 to $1200^{\circ} \mathrm{C}$.

The kinetic study of the oxidative dissolution of $\mathrm{U}_{3} \mathrm{O}_{8}$ samples (obtained in the various temperature at the range $480-1200^{\circ} \mathrm{C}$ ) in solutions $\mathrm{Na}_{2} \mathrm{CO}_{3} / \mathrm{NaHCO}_{3}-\mathrm{H}_{2} \mathrm{O}_{2}$ made it possible to establish a correlation between the treatment temperature of $\mathrm{U}_{3} \mathrm{O}_{8}$ (the specific surface area of the oxide powder sample) and the process rate.

The study of the dynamics of the oxidation/reduction potential (ORP) change and the determination of a correlation between the ORP value and the dissolution rate of $\mathrm{U}_{3} \mathrm{O}_{8}$ for various carbonate systems and conditions made it possible to select the most effective modes of the process at which the maximum rate and completeness of the $\mathrm{U}_{3} \mathrm{O}_{8}$ dissolution are achieved.

The use of sodium percarbonate $\left(\mathrm{Na}_{2} \mathrm{CO}_{3} \cdot 1.5 \mathrm{H}_{2} \mathrm{O}_{2}\right)$ as an alternative carbonate and oxidizing reagent for dissolution of $\mathrm{U}_{3} \mathrm{O}_{8}$ can be justified and does not reduce the efficiency of the process. At the same time, in the presence of sodium peroxide $\left(\mathrm{Na}_{2} \mathrm{O}_{2}\right)$, the oxidative dissolution rate of $\mathrm{U}_{3} \mathrm{O}_{8}$ is significantly reduced due to secondary (indirect) chemical processes.

The general kinetic and chemical regularities of $\mathrm{U}_{3} \mathrm{O}_{8}$ oxidative dissolution process in carbonate, bicarbonate and carbonate-alkali media established in the research can be of scientific and practical importance in the field of spent nuclear fuel reprocessing and high-level radioactive waste management.

Acknowledgments: The work was carried out with the financial support of the Russian Science Foundation. Grant number 20-63-46006. 


\title{
Production of Cu-64 on an automated irradiation and processing system - Process validation
}

\author{
Radu A. Leonte, Diana S. Cocioaba, Ramona D. Dusman, \\ Bogdan G. Burghelea, Simona I. Baruta, \\ Roxana M. Cornoiu, Liviu S. Craciun, Dana Niculae
}

Horia Hulubei National Institute for Physics and Nuclear Engineering, Magurele, Romania

https://doi.org/10.21175/rad.abstr.book.2021.33.9

An automated process for the production and purification of copper radioisotopes produced by irradiating solid targets in a variable energy (14-19 MeV) cyclotron has been validated, using a commercially available automated solid target system comprising modules for electrodeposition, pneumatic transfer, irradiation, dissolving and purification. The initial process-script was modified as result of the local set-up and particularities.

Copper, as a natural bioelement in the human body, incorporated into a variety of proteins, is involved in key biological processes based on its redox properties. Its trafficking, accumulation and clearance are tightly controlled in normal health but often disturbed in disease states. These mechanisms can be exploited for both imaging and therapy, based on several radioisotopes of copper which are of certain medical interest. Some of them emit positrons: $\mathrm{Cu}-60$ (93\%), half-life $23.6 \mathrm{~min}$; Cu-61 (93\%) half-life $3.3 \mathrm{~h}$; Cu-62 (98\%) but only 9.76 min half-life; Cu-64 (19\%) half-life $12.8 \mathrm{~h}$. $\mathrm{Cu}-64, \mathrm{Cu}-66$ and $\mathrm{Cu}-67$ have therapeutic emissions. They can be obtained by the protons beam of a variable, medium energy cyclotron $(14-19 \mathrm{MeV})$ by ${ }^{60} \mathrm{Ni}(\mathrm{p}, \mathrm{n}),{ }^{61} \mathrm{Ni}(\mathrm{p}, \mathrm{n}),{ }^{6} \mathrm{Cu}(\mathrm{p}, 2 \mathrm{n})^{62} \mathrm{Zn}(\mathrm{EC}){ }^{62} \mathrm{Cu}$,

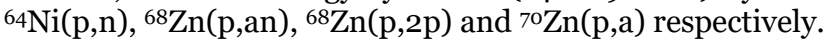

Copper-64 has several unique attributes: a complex decay scheme, with electron capture, betaemission and positron emission branches; the positron emission has a low energy allowing high resolution images and there are no abundant gamma emissions that impair the imaging properties; the combination of positron, beta- and of high LET Auger electrons emissions imparts a high local radiation dose at the cellular level making it suitable for targeted radionuclide therapy, with high cytotoxic potency if the radionuclide is located within or close to cell nuclei.

A reliable process for production of ${ }^{64} \mathrm{Cu}$ by nuclear reaction ${ }^{64} \mathrm{Ni}(\mathrm{p}, \mathrm{n})$, with low metallic impurities and of high specific activity, for biomedical application, is described. The process involves the plating of enriched ${ }^{64} \mathrm{Ni}(99.5 \%)$ on the target, pneumatic transfer to the irradiation station, mounted on a slope-down proton beam, irradiation at $14 \mathrm{MeV}$ (degraded with an $\mathrm{Al}$ foil), at $70 \mu \mathrm{A}$, and uses automated module based on ion exchange chromatography to purify the resulted copper-64 chloride. The amount of enriched material on target was 40-50 mg, resulting in a total radioactivity greater than $15 \mathrm{GBq}$, with specific activity up to $6 \mathrm{GBq} / \mu \mathrm{moL}$. The copper isotopes were separated from the nickel via ion exchange chromatography using dedicated single-use disposable kit and the radioisotopic purity was assessed by gamma spectroscopy. The process is to be translated for production of $\mathrm{Cu}-61$, by ${ }^{61} \mathrm{Ni}(\mathrm{p}, \mathrm{n})^{61} \mathrm{Cu}$ nuclear reaction at $14.7 \mathrm{MeV}$ proton beam, and is intended to be further adapted for the production of the other copper radioisotopes of medical interest.

Acknowledgments: This work was supported by a grant of the Romanian Ministry of Research and Innovation UEFISCDI project number 68PCCDI/2018. 


\title{
Production of $\mathrm{Zr}-89$ radioisotope using a variable energy cyclotron (TR-19)
}

\section{Ramona-Daniela Dusman',2, Diana Cocioaba', Alina Raicu', Mirela Mihon', Livia Chilug', Radu Leonte', Liviu Craciun¹, Dana Niculae}

\author{
1 Horia Hulubei National Institute for Physics and Nuclear Engineering, Radiopharmaceutical Research \\ Centre, Magurele, Romania \\ 2 University Politehnica of Bucharest, Faculty of Applied Chemistry and Materials Science, Bucharest, \\ Romania
}

\section{https://doi.org/10.21175/rad.abstr.book.2021.33.10}

Introduction. The increasing number of nuclear medicine procedures employing radiolabelled targeting agents demand the use of radioisotopes with longer half-lives (usually within hours and days).

Such radioisotope is ${ }^{89} \mathrm{Zr}$, which is an emergent radioisotope with potential use in the field of PET imaging. Its decay properties $\left(t_{1 / 2}=78.41 \mathrm{~h}, \mathrm{EC}=76.6 \%, \beta^{+}=22.3 \%, E_{\max }\left(\beta^{+}\right)=897 \mathrm{keV}, E_{\text {ave. }}\left(\beta^{+}\right)=\right.$ $\left.396.9 \mathrm{keV}, R_{\text {ave. }}\left(\beta^{+}\right)=1.18 \mathrm{~mm}, E_{\gamma}=908.97 \mathrm{keV}, I_{\gamma}=100 \%\right)$ make it suitable for the design of novel radiotracers, such as nanoparticules and monoclonal antibodies (mAb). Therefore, many ${ }^{89} \mathrm{Zr}-$ radiolabelled PET agents are currently under development, in different stages, aiming to obtain better targeting agents for various types of cancer.

This work presents our experience in the field of production of $89 \mathrm{Zr}$ at TR-19 cyclotron and further radiochemical processing to obtain pharmaceutical grade ${ }^{89} \mathrm{Zr}$-oxalate.

Methods. ${ }^{89} \mathrm{Zr}$ was obtained by irradiation of natY foils (99.9\%, Alpha Aesar) at TR-19 cyclotron by ${ }^{n a t} Y(p, n)^{89} \mathrm{Zr}$ nuclear reaction with following parameters: $\mathrm{I}=25 \mu \mathrm{A} ; \mathrm{t}=4 \mathrm{~h}$. The protons were extracted at 14.2 MeV and degraded for optimal cross-section to $12.7 \mathrm{MeV}$ when reaching the target, by using a pure aluminium degrader. After irradiation, the target was dissolved using $2 \mathrm{M} \mathrm{HCl}$ followed by light heating $\left(60^{\circ} \mathrm{C}\right)$ until the solution became clear. The obtained ${ }^{89} \mathrm{ZrCl}_{4}$ solution was passed on a ZR1-R10-S cartridge (Triskem) were zirconium ions were trapped. The recovery of zirconium was carried out with $0.5 \mathrm{M}$ oxalic acid. Quality control of the final product was carried out. Irradiation yield, radiolabelling yield, $\mathrm{pH}$, radiochemical purity and radionuclide purity were assessed.

Results. Using the semi-automated solid target system, $1.28 \mathrm{GBq}{ }^{89} \mathrm{ZrCl}_{4}$ were obtained, while with the fully-automated solid target system, 2.7 GBq. The yield after irradiation and processing the target using fully-automated solid target system was $71.8 \%$. The radiochemical purity, assessed by radio-HPLC, was higher than $99 \%$ after purification.

Conclusions. The characteristics of the $\left[{ }^{89} \mathrm{Zr}\right] \mathrm{ZrCl}_{4}$ solution meet the requirements for the biomolecules radiolabelling (antibodies) and comply to the translation to radiopharmaceutical production line, aiming to develop new generation of PET agents for preclinical testing and clinical trials. 


\title{
Prospects and challenges of chemistry of superheavy element research at FLNR
}

\author{
Nikolay Aksenov \\ Flerov Laboratory of Nuclear Reactions, Joint Institute for Nuclear Research, Dubna, Russia
}

\section{https://doi.org/10.21175/rad.abstr.book.2021.33.11}

Relatively high stability of new superheavy elements with atomic numbers $113-118$, as well as new long-lived isotopes of elements 104-112, recently discovered at Flerov Laboratory of Nuclear Reactions, present unique possibilities to study chemical properties of transactinide elements which completes $7^{\text {th }}$ period of the D.I. Mendeleev's Periodic System. The main focus is to compare the transactinide elements properties with the properties of their light homologues in the groups of the Periodic Table and thus test the influence of relativistic effects on the law of periodicity of chemical properties in the field of superheavy elements.

Results of recently performed experiments at FLNR on the chemical identification of ${ }^{288} \mathrm{Mc}$, based on liquid-phase separations of its decay product ${ }^{268} \mathrm{Db}$, and the results of pioneering gas-phase experiments on the chemistry of elements $\mathrm{Cn}, \mathrm{Nh}$ and $\mathrm{Fl}$ are summarized in my talk. Detailed studies will be continued at the new accelerator complex SuperHeavy Element Factory at FLNR at significantly higher statistical level. The basis for future study of the chemical properties of SHE, including new elements 115-118, will be new gas-filled separator for radiochemical research. Research in this field is interdisciplinary and the potential of new research facilities will only be realized if they are accompanied by theoretical research and model experiments with homologues, the developments of new accelerator targets and beams, new setups and radiochemical laboratories. 


\section{Radiochemical separation of heavy actinides produced in multi-nucleon transfer reactions}

\section{Aleksandr Bodrov,2, Gospodin Bozhikov, Nikolay Aksenov, Yurii Albin¹}

1 Joint Institute for Nuclear Research, Flerov Laboratory of Nuclear Reactions, Dubna, Russia

2 Moscow State University, Moscow, Russia

\section{https://doi.org/10.21175/rad.abstr.book.2021.33.12}

Multinucleon transfer reactions are one of several accelerating methods to produce neutron-rich isotopes heavier einsteinium [1]. Predictable half-lives of new isotopes of actinides after Es are low and we need to improve classical technique based on cation-exchange chromatography with ammonium $\alpha$-hydroxyisobutyrate ( $\alpha$-HIB) solution as an eluent [2] for separation of actinide (III) series. However, the activity of producing isotopes is different and positions of elements such as Md, No, Lr are bad-known on chromatogram. New technique is required to be highly sensitive and effective for actinide separation and to allow making quick separation and predicting positions of $\mathrm{Md}$, No and Lr.

Having used equation of mass transfer equation and numerical methods, we improved this technique and determined distribution coefficients, heights of theoretical plates and influence of equal concentration of $\alpha$-HIB on distribution coefficients. To choose likelihood chromatogram we used minimum of chi-square criterium.

\section{References}

[1] Myeong-Hwan Mun, Kyujin Kwak, G. G. Adamian, and N. V. Antonenko//Phys.Rev.C.99., $2019,054627$.

[2] M.Schädel, W.Brüchle, B.Haefner //Nuclear Inst. and Methods in Physics Research, A, V.264, pp. 308-318. 


\title{
The synthesis of encapsulated radiolabeled compounds to be used for diagnosis of Parkinson's disease
}

\author{
Emre Uygur'1, Kadriye Büşra Karatay², Emine Derviş², \\ Cansu Kayaş², Fazilet Zümrüt Biber Müftüler²
}

\author{
1 Biomedical Device Technologies, Manisa Celal Bayar University, Manisa, Turkey \\ 2 Institute of Nuclear Science, Ege University, İzmir, Turkey
}

\section{https://doi.org/10.21175/rad.abstr.book.2021.33.13}

Parkinson's disease is a common neurodegenerative disease that affects about 6 million people worldwide. Last decades, the identification and purification of natural compounds obtained from medical plants with radiolabeled by radioisotopes such as Tc-99m, I-125, and I-131 have been of great importance in the diagnosis of the disease. One of these medicinal plants is Centella Asiatica (Gotu Kola). This plant includes saponins. Saponins have anti-inflammatory, antimicrobial, antioxidant, analgesic and neuron protective effects on the body. Madecassoside (MA) is also a saponin obtained from Centella Asiatica. It has a pharmacological effect on the central nervous system as neuron protective properties, especially on the dopaminergic cells in the brain Substantia Nigra Pars Compacta (SNc) and Stratium regions. Blood-Brain Barrier (BBB) plays an important role in the drug transport mechanism in the brain. Particularly in drug delivery mechanisms using nanoparticles (NPs) increasing the likelihood of the active substance reaching the brain. In the literature, biodegradable polyester NPs such as poly-lactic-co-glycolic acid (PLGA) have been widely used in the last thirty years because of their controlled biodegradability, excellent biocompatibility, high safety, to be one of the few biodegradable synthetic polymers and biomechanical applications. The current study was carried out in order to determine the in vitro potential of encapsulated radiolabeled compounds of plant origin which can be used in the diagnosis of Parkinson's disease. In the experimental steps, MA and Levodopa were conjugated. Levodopa (L-DOPA) compound is already used in the diagnosis and treatment of the disease as a commercial drug. Conjugation yield and purity analyses were performed by Thin-layer chromatography (TLC), High-performance liquid chromatography (HPLC), Fourier-transform infrared spectroscopy (FTIR), Proton nuclear magnetic resonance ( $\left.{ }^{1} \mathrm{H}-\mathrm{NMR}\right)$, and Liquid chromatography-mass spectrometry (LC-MS) analyses. MA and MA-L-DOPA compounds were encapsulated with PLGA. The analyses of encapsulated compounds (MA-PLGA and MA-L-DOPA-PLGA) were performed by Dynamic light scattering $(D L S)$ and Scanning Electron Microscopy (SEM) analyses. MA, MA-L-DOPA, MA-PLGA, and MA-L-DOPA-PLGA compounds were radiolabeled with Tc-99m. Radiolabeling yields, stability and lipophilicity studies of compounds were performed by using Thin-layer Radio chromatography (TLRC), High-performance liquid Radio chromatography (HPLRC), methods. Experimental results show that MA (\% 94.04 $\pm 1.60, \mathrm{n}=3$ ), MA-L-DOPA (\%99.35 $\pm 0.37, \mathrm{n}=3)$, MA-PLGA (\%99.20 \pm 0.34 , $\mathrm{n}=3$ ) and MA-L-DOPA-PLGA (\%99.71 $\pm 0.17, \mathrm{n}=3$ ) compounds can be radiolabeled with Tc-99m. In in vitro studies, cytotoxicity and cell incorporation of PLGA encapsulated MA and MA-L-DOPA molecules and Tc-99m radiolabeled encapsulated compounds were performed on human neuroblastoma ( $\left.\mathrm{SH}_{-} \mathrm{SY}_{5} \mathrm{Y}\right)$ and rat pheochromocytoma (PC12) cell lines. Increased concentrations of MA, MA-L-DOPA, MA-PLGA, and MA-L-DOPA-PLGA compounds have been determined to increase their cytotoxicity on the $\mathrm{SH}_{-} \mathrm{SY}_{5} \mathrm{Y}$ cell line. The increase binding value of the conjugation product (99mTc-MA-L-DOPA) and encapsulated compounds (99mTc-MA-PLGA and 99mTc-MA-L-DOPA-PLGA) on both cell lines (SH-SY5Y and PC-12) are promising as the 99mTc-MA-L-DOPA and encapsulated compounds which is a plant-derived agent for the diagnosis of the disease. As a result, it was concluded that the potential use of $99 \mathrm{mTc}-\mathrm{MA}$ and ${ }^{99 \mathrm{~m} T c-M A-L-D O P A}$ and encapsulated radiolabeled compounds in the diagnosis of Parkinson's disease must be supported by more extensive in vitro and in vivo experimental animal model studies. 


\title{
Effects of ionizing radiation and weather-climatic factors on radial growth of Scots pine in the East Ural Radioactive Trace
}

\author{
Vladimir Kukarskih, Nadezhda Devi, Makar Modorov, \\ Nadezhda Shimalina, Ludmila Mikhailovskaya, Vera Pozolotina
}

Institute of Plant and Animal Ecology, Ural Branch of the Russian Academy of Sciences, Ekaterinburg, Russia

https://doi.org/10.21175/rad.abstr.book.2021.34.1

Introduction. The East Ural Radioactive Trace (EURT) is a result of the explosion at the "Mayak" Production Association in September 1957 (the Kyshtym accident). The majority of the Scots pine stands near the epicenter of the accident died in 1958-1959. Currently, we have found several small pine-birch stands with pine trees that are over 70 years old. When estimating radiation effects on tree-ring formations, one should consider the influence of the weather-climatic conditions, as the climate synchronizes the dynamics of tree growth. The aim of this study is to evaluate the response of the radial growth of pines that survived the Kyshtym accident and have been growing in sites with different levels of soil contaminations by ${ }^{90} \mathrm{Sr}$; moreover we compared the effect of high doses of radiation with the influence of extreme climatic events.

Materials and methods. We used the model proposed by F. Tikhomirov and G. Romanov (1993) for assessing the dose loads received by the EURT pines in the initial period after the accident in which the density of soil contamination by ${ }^{90} \mathrm{Sr}$ was used as a predictor. The Scots pine cores were analyzed employing dendrochronological methods to detect the effects of climate and ionizing radiation on the annual growth of trees. Tree-ring chronologies were studied for two control and three impact sites with different levels of radioactive contamination.

Results. Hydrothermal conditions of the growth period have the main influence on the value of the Scots pine radial growth both in zone EURT and in adjacent background areas. Acute radiation exposure in the first years after the Kyshtym accident significantly reduced radial growth in trees in two contaminated sites, where initial contamination levels of ${ }^{90} \mathrm{Sr}$ measured $3.7-26.6 \mathrm{MBq} \mathrm{m}^{-2}$; synchrony of the chronologies from the contaminated and control sites were disturbed. The reaction of the trees' radial growth to acute radiation was delayed by two years after accident; it was clearly manifested in 1959-1965. Effect of radiation was comparable to the effect of droughts, which are the main extreme climatic event in this region. Background radial growth values were reached within 7-8 years after accident and in the subsequent period, all chronologies have been highly synchronous. The severity and duration of radiation effects on radial growth of trees was considerably dependent on the dose received in the "acute period". The morphological structure of stands had a significant impact on the response of pine growth to acute radiation exposure in EURT. The young trees were sheltered by the forest canopy from radioactive aerosols and were able to survive the accident even with the high density of radioactive contamination.

Conclusion. The results of analysis shown that the radial growth of Scots pine trees in the EURT zone has been limited by both climate and ionizing radiation. The effect of acute irradiation on the radial growth of trees was comparable to the effects of droughts or exceeded their.

Acknowledgments: This study was performed within the frameworks of state contract with the Institute of Plant and Animal Ecology, Ural Branch of RAS and the Russian Foundation for Basic Research (project № 19-05-00469). 


\title{
Parameters of ${ }^{137} \mathrm{Cs}$ specific activity changes in the fish from the Kiev water reservoir
}

\author{
Olena Volkova', Vladimir Belyaev', Serge Pryshlyak, Vladimir Skyba²
}

\author{
1 Institute of Hydrobiology of the NAS of Ukraine, Kyiv, Ukraine \\ 2 BilaTserkva National Agrarian University, BilaTserkva, Ukraine
}

\section{https://doi.org/10.21175/rad.abstr.book.2021.34.2}

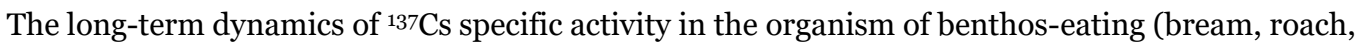
silver bream, tench) and carnivorous (pike, pike-perch) fish of the Kiev water reservoir for 1986-2020 was analyzed.

Before the accident at the Chernobyl nuclear power plant on April 26, 1986, the ${ }^{137} \mathrm{Cs}$ activity in the fish of the Dnieper water reservoirs was 1-3 Bq/ $\mathrm{kg}$, and 40-50 days after the accident it made, on average, $1590 \mathrm{~Bq} / \mathrm{kg}$ in benthos and $160 \mathrm{~Bq} / \mathrm{kg}$ in the predatory fish. The accumulation levels of ${ }^{137 \mathrm{Cs}}$ in benthos-eating fish decreased to $10 \mathrm{~Bq} / \mathrm{kg}$ during the period June 1986-2021. The specific activity

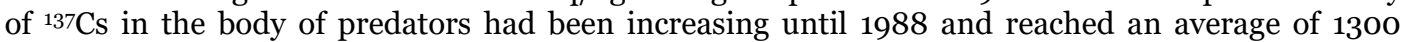
$\mathrm{Bq} / \mathrm{kg}$, though it decreased to $20 \mathrm{~Bq} / \mathrm{kg}$ during the period from 1988 to 2021.

Exponential relationship can describe the decrease in the content of the radionuclide in fish. In this case, the rate of decrease in the specific activity is inversely proportional to the period during which the specific activity decreases by half. Calculations showed that the rate of decrease in the

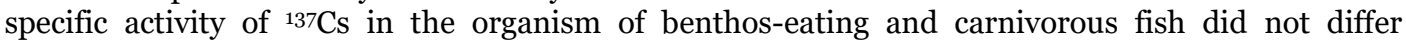
significantly and amounted to $0.13 \pm 0.01$ year $^{-1}$ for the entire post-accident period. However, three periods with different parameters of changes in the ${ }^{137} \mathrm{Cs}$ content in fish can be distinguished in the research. The highest rate of ${ }^{137} \mathrm{Cs}$ decrease was observed in non-predatory fish during 1986-1991 - it amounted $0.37 \pm 0.12$ year $^{-1}$, in 1991-2001 this value decreased by 2.5 times. In 2001-2020 the rate of ${ }^{137} \mathrm{Cs}$ decrease in non-predatory fish $\left(0.043 \pm 0.008\right.$ year $\left.^{-1}\right)$ was 9 times less than during the first 5 years after the accident. In predatory fish species, the highest rate of ${ }^{137} \mathrm{Cs}$ decrease was observed in the period 1988-1993 and made $0.20 \pm 0.04$ year $^{-1}$, in 1993-2002 the rate of ${ }^{137 C s}$ decline decreased by $25 \%$. In the period from 2002 to 2020 , the rate of ${ }^{137}$ Cs specific activity decrease in predatory fish species was $0.082 \pm 0.008$ year $^{-1}$.

Analysis of the long-term dynamics of ${ }^{137} \mathrm{Cs}$ specific activity in fish from the Kiev water reservoir suggests the rate of the radionuclide content decrease in the populations slows down in the course of time.

The results obtained are essential in predictive assessment of the ichthyofauna radioactive contamination. 


\section{Influence of rainfall on the temporal variability of atmospheric beryllium-7 ( $\left.{ }^{7} \mathrm{Be}\right)$ activity concentration}

\section{Dmitrii Kremenchutskii}

Marine Hydrophysical Institute, Russian Academy of Sciences, Sevastopol, Russia

https://doi.org/10.21175/rad.abstr.book.2021.34.3

Beryllium-7 (7Be) is a radionuclide of cosmogenic origin. It is formed in the atmosphere as the result of the spallation of nitrogen and oxygen nuclei by cosmic ray flux. The scientific interest in this radionuclide is due to the possibility of its use as a tracer of physical processes occurring in the atmosphere on time scales from synoptic to seasonal. In particular, it finds application in studies of vertical exchange in the troposphere, in the exchange between the stratosphere and the troposphere, in estimating the resident time of atmospheric aerosols in the troposphere and stratosphere, etc. Atmospheric precipitations are one of the main factors controlling the temporal variability of $7 \mathrm{Be}$ on synoptic time scales. This study aims to obtain quantitative estimates of the influence of rainfall on the temporal variability of atmospheric $7 \mathrm{Be}$ activity concentration.

The data for this study were obtained in the city of Sevastopol, Russia during the period 2011-2016. Aerosol samples were taken daily using a high-performance air filtration unit $\left(525 \mathrm{~m}^{3} \mathrm{~h}^{-1}\right)$ on the Petryanov FPP-15-1.5 fiber filter. The radionuclide activity on aerosols was determined using a low-background gamma-spectrometer with a $\mathrm{NaI}(\mathrm{Tl})$ scintillation detector. The data on meteorological parameters were obtained by an automatic meteorological station located near the aerosols sampling site.

During the study period, the $7 \mathrm{Be}$ activity concentration in individual samples varied from 0.2 to $10.2 \mathrm{mBq} \mathrm{m}^{-3}$ with a mean value of $3.5 \pm 1.7 \mathrm{mBq} \mathrm{m}^{-3}$. Increased values of the concentration of 7Be were typical for the spring-summer period, decreased for the winter. When analyzing the effect of rainfall, only those precipitation cases were considered, the duration of which did not exceed one day. It was found that precipitation leads to a decrease in the daily values of $7 \mathrm{Be}$ by an average of $60 \%$. On average, the radionuclide activity concentration on atmospheric aerosols after precipitation was restored to its before-precipitation levels in two days. The daily activity concentration values increase on average to $60 \%$ of their before-precipitation levels on the first day and to $84 \%$.on the second one.

Acknowledgments: The time-series data were obtained within the framework of the state assignment of the Ministry of Science and Higher Education of the Russian Federation project No. 0555-2021-0004; the data analysis was carried out within the framework of the Russian Science Foundation project No. 20-77-00024. 


\title{
Influence of meteorological parameters on the temporal variability of bismuth-214 $\left({ }^{214} \mathrm{Bi}\right)$ on atmospheric aerosols
}

\author{
Gennady Batrakov, Dmitrii Kremenchutskii, Yuliya Kurinnaya
}

Marine Hydrophysical Institute, Russian Academy of Sciences, Sevastopol, Russia

https://doi.org/10.21175/rad.abstr.book.2021.34.4

Bismuth-214 $\left.{ }^{214} \mathrm{Bi}\right)$ is a daughter product of the decay of radon-222 $\left.{ }^{222} \mathrm{Rn}\right)$. It is one of the main radionuclides that form the natural gamma background of the surface atmosphere. This study aims to obtain quantitative estimates of the influence of meteorological parameters on the temporal variability of atmospheric ${ }^{214} \mathrm{Bi}$ activity concentration.

The data for this study were obtained in the city of Sevastopol, Russia. Aerosol samples were taken daily using a high-performance air filtration unit $\left(525 \mathrm{~m}^{3} \mathrm{~h}^{-1}\right)$ on the Petryanov FPP-15-1.5 fiber filter. The radionuclide activity on aerosols was determined using a low-background gammaspectrometer with a $\mathrm{NaI}(\mathrm{Tl})$ scintillation detector. The reported activity concentration values correspond to the period of 9-11 AM local time zone $($ UTC +3$)$. The data on meteorological parameters (air temperature, atmospheric pressure, relative humidity, total precipitation) were obtained by an automatic meteorological station located near the aerosols sampling site. The data on the zonal component of wind speed, the meridional component of wind speed, solar radiation flux to the Earth's surface, cloudiness, the height of the cloud bottom, $0-7 \mathrm{~cm}$ soil layer temperature and moisture content, atmospheric boundary layer height, upward heat flux, downward UV radiation flux to the surface were taken from ERA5 reanalysis.

For the period 2007-2020, the ${ }^{214} \mathrm{Bi}$ activity concentration varied in the range from 0.1 to 11.4 $\mathrm{Bq} \mathrm{m}^{-3}$ and averaged $2.7 \pm 1.5 \mathrm{~Bq} \mathrm{~m}^{-3}$. The coefficient of variation is $54 \%$ and indicates significant variability in ${ }^{214} \mathrm{Bi}$ activity concentration over the study period. Relatively increased values of ${ }^{214} \mathrm{Bi}$ were typical for the period July-October (3.1-3.5 $\mathrm{Bq} \mathrm{m} \mathrm{m}^{-3}$ ), relatively low values for the period December-April (2.1-2.2 $\left.\mathrm{Bq} \mathrm{m}^{-3}\right)$.

The relationship between changes in the ${ }^{214} \mathrm{Bi}$ activity concentration on atmospheric aerosols and the number of parameters listed above was investigated. From the results of the correlation analysis, it follows that soil moisture, which determines the input of ${ }^{222} \mathrm{Rn}$ into the atmosphere, is the dominant factor of temporal variability of ${ }^{214} \mathrm{Bi}$ at mesoscale, synoptical, monthly, seasonal and yearly time scales. On a seasonal time scale, a significant role is also played origin of air masses (continental or marine) arriving in the region under study, which is reflected by the presence of a strong statistically significant correlation with the meridional component of wind speed. The intensity of vertical exchange in the troposphere plays an important role on the synoptic time scale. This is indicated by the presence of statistically significant correlations with air temperature, solar radiation flux and the height of the atmospheric boundary layer. Air humidity, which determines the rate of coagulation and removal of atmospheric aerosol from the atmosphere, is essential on the mesoscale time scale.

Acknowledgments: The study was carried out within the framework of the state assignments of the Ministry of Education and Science of Russia No. 0827-2020-0004 with the financial support of the Russian Foundation for Basic Research and the city of Sevastopol in the framework of the scientific project No. 20-45-920007. 


\title{
Fine effects governing Cs sorption onto aluminosilicates
}

\author{
Anna Semenkova', Irina Tonyan', \\ Victoria Krupskaya1,2, Anna Romanchuk', Stepan Kalmykov
}

\author{
1 Lomonosov Moscow State University, Moscow, Russia \\ 2 Institute of Geology of Ore Deposits, Petrography, Mineralogy and Geochemistry, Russian Academy of \\ Science, Moscow, Russia
}

\section{https://doi.org/10.21175/rad.abstr.book.2021.34.5}

Nowadays, the isolation of radioactive waste (RW) from the biosphere is one of the main problems of nuclear industry. Following the requirements of radiation safety geological isolation of RW requires a multi-barrier safety system, and natural clays are considered to be one of the most promising components. Bentonite clays, whose properties are provided by the presence of smectite group minerals, are considered to be the most promising material. A lot of works are devoted to radionuclides interaction with bentonite clays and their components.

In this work, Cs interaction with various aluminosilicates (smectite, kaolinite, illite, and zeolite) was compared. During this study we performed experiments with natural bentonite clays of different deposits: previously non-widely studied deposits from Russia (Khakassia, Dash-Salakhinskiy, Zyryanskiy) and Kazakhstan (Dinosaur) as well as widely studied samples MX-8o (USA), KunipiaF (Japan), FEBEX (Spain). The cesium sorption was studied in a wide range of concentrations $\left(10^{-14-10^{-2}} \mathrm{M}\right)$ and $\mathrm{pH}(2-10)$.

During the study significant differences in cesium behavior on the various samples were observed. Differences in sorption were especially evident at trace concentrations of cesium. Decrease in cesium sorption in the row illite-montmorillonite zeolite-kaolinite in the whole range of studied concentrations was observed. Despite differences in structure, similar sorption dependencies were obtained for samples MX-80 and zeolite. For some bentonites (FEBEX, Zyryanskiy, Dinosaur, DashSalakhinskiy, and Khakassia deposits), 2 plateaus on sorption isotherms can be observed, which indicates the existence of different sorption sites. MX-80 and KunipiaF have a similar mineral composition, but only one sorption site can be found, and the sorption values are significantly lower. Significant differences were also observed for cesium sorption on samples Khakassia and Dinosaur that have similar mineral composition. Thus, the significant difference in Cs sorption on bentonite samples with similar composition should be noted. This may be due to the influence of the fine structure of the sample. The correlation between sorption of trace cesium concentrations and the clay layer charge distribution was revealed.

The data obtained allowed us to carry out modeling that adequately describes the sorption of cesium on a large set of aluminosilicates in various conditions.

Acknowledgments: This work was supported by the Russian Science Foundation (project 20-73-00135). 


\title{
Features of application and durability of metallic silver on bentonite and silica aerogel for obtaining radioiodine getters
}

\author{
Ekaterina Tyupina, Artem Pryadko, \\ Daria Marchenko, Polina Parshina, Pavel Kozlov
}

D. Mendeleev University of Chemical Technology of Russia, Moscow, Russia

\author{
https://doi.org/10.21175/rad.abstr.book.2021.34.6
}

Radioactive iodine isotope ${ }^{129}$, which poses a significant threat due to its long half-life and organotropism, is immobilized into radioactive waste (RW) in potentially mobile anionic $\mathrm{I}^{-}$and $\mathrm{IO}_{3}{ }^{-}$ forms. Engineering barriers that prevent migration of radionuclides from RW repositories are supposed to be constructed from compacted bentonite clays, that have high water-proof and cation exchange properties, but do not uptake anions. Addition of sorbent based on silver compounds that have high iodine uptake properties to the engineering barrier will protect the environment from radioactive iodine and retain performance characteristics of bentonites. The first step in obtaining of silver-containing sorbent is deposition of metallic silver onto the surface of a material. Silver can subsequently transferred to another chemical form, e.g. oxidized to $\operatorname{Ag}_{2} \mathrm{O}$, which captures anionic and organic iodine species effectively.

The aim of this work is to compare behavior of reaction of silver application and its durability on bentonite and silica aerogel in order to choose the framework material for silver-containing sorbent.

Both bentonite and silica aerogel have high specific surface areas, are cheap, available and chemically inactive and that makes them advantageous for modification. Our technique for silver application is based on reduction of silver ammine complex by formaldehyde evolving upon HMTA thermal hydrolysis and allows to apply silver uniformly and quantitatively onto materials with high surface area. For determination of durability of silver the silver-containing material was treated with nitric acid solutions of various concentrations in various condition and the percent of dissolved silver was determined.

Deposition of silver onto silica aerogel was found to demand less time than deposition of silver onto bentonite because silica aerogel hydrates during 2 hours while bentonite swells during 24 hours. The silver reduction reaction itself completes within 18 hours in both cases. The dissolution experiments conducted in the most severe conditions showed high durability of applied silver: $6 \mathrm{M}$ nitric acid can remove $87 \%$ of silver applied on silica aerogel in 3 hours and $89 \%$ of silver applied on bentonite in 48 hours. Structural features of materials cause different time necessary for both application and removal of silver: silica aerogel has porous structure which absorbs water easily while layered structure of bentonite swells more slowly.

Thus, the developed technique allows to apply silver on both bentonite and silica aerogel effectively and ensures high durability of silver. That allows to transfer applied silver in form of oxide and to obtain sorbents for localization of iodine-containing anionic RW in nuclear waste repositories. 


\title{
Comparison of sampling and measurement methods for atmospheric ${ }^{14} \mathrm{C}$ activity
}

\author{
Damir Borković, Ines Krajcar Bronić, Andreja Sironić, Jadranka Barešić
}

Ruđer Bošković Institute, Zagreb, Croatia

https://doi.org/10.21175/rad.abstr.book.2021.34.7

Radiocarbon $\left.{ }^{14} \mathrm{C}\right)$ is both the cosmogenic and anthropogenic isotope. Anthropogenic ${ }^{14} \mathrm{C}$ is produced (1) in atmospheric nuclear bomb tests, and (2) in various nuclear facilities. The bombproduced ${ }^{14} \mathrm{C}$ has been globally distributed across the planet and can be considered as a new-natural level (clean-air sites). Furthermore, combustion of fossil fuels that do not contain ${ }^{14} \mathrm{C}$ causes increase of atmospheric $\mathrm{CO}_{2}$ concentration and consequently depletion of local ${ }^{14} \mathrm{C}$ levels. Naturally produced $\mathrm{CO}_{2}$ and that formed by fossil fuel combustion are characterized by different content of the stable isotope ${ }^{13} \mathrm{C}\left(\mathrm{d}^{13} \mathrm{C}\right.$ values). Therefore, the carbon isotopes ${ }^{13} \mathrm{C}$ and $\left.{ }^{14} \mathrm{C}\right)$ of the atmospheric $\mathrm{CO}_{2}$ can indicate sources of $\mathrm{CO}_{2}$ at each location, if it is far from nuclear facilities.

${ }^{14} \mathrm{C}$ activity in the atmospheric $\mathrm{CO}_{2}$ in Zagreb, Croatia, has been monitored since 1985 . Recently we started monitoring at several other locations with the aim of determining influence of fossil fuel combustion on atmospheric ${ }^{14} \mathrm{C}$ activity and $\mathrm{d}^{13} \mathrm{C}$ values at clean-air sites and urban sites supposing that the urban sites are affected by fossil fuel uses.

A static sampling method has been used for monitoring ${ }^{14} \mathrm{C}$ activity - concentrated $\mathrm{NaOH}$ is exposed in a tray for 3-4 weeks. The method is simple and requires neither electric power nor any control, so it can be used at remote sites. However, the method introduces large isotope fractionation in ${ }^{13} \mathrm{C}$ due to high alkalinity of $\mathrm{NaOH}$. Thus, it cannot be applied for monitoring ${ }^{13} \mathrm{C}$. For the new monitoring program we would like to have a method that can simultaneously deliver both $\delta^{13} \mathrm{C}$ and ${ }^{14} \mathrm{C}$ activity data.

A dynamic method of continuous $\mathrm{CO}_{2}$ collection on $\mathrm{NaOH}(3 \mathrm{M})$ with a constant air-flow requires source of electricity for pumping and heating, and occasional control of sampler performances. Control of air flow velocity and temperature enables determination of the absorbed $\mathrm{CO}_{2}$ amount. Both static and dynamic sampling methods give an average ${ }^{14} \mathrm{C}$ activity over the sampling period. The amount of collected sample allows benzene synthesis for liquid scintillation spectrometry (LSC-B) that requires about $4 \mathrm{~g}$ of carbon. We also developed an instant sampling of atmospheric air in 10-L bags. However, the amount of $\mathrm{CO}_{2}$ is enough for measurement by accelerator mass spectrometry (AMS) that requires about $2 \mathrm{mg}$ of carbon.

Comparison of LSC-B and AMS measurement methods resulted in good agreement of ${ }^{14} \mathrm{C}$ activities for samples collected by the static method. The ${ }^{14} \mathrm{C}$ activity of the instant sample revealed comparable results with the samples collected by the static method indicating that the two sampling methods could be combined. $\mathrm{d}^{13} \mathrm{C}$ values of samples collected by the dynamic method showed no fractionation in ${ }^{13} \mathrm{C}$ and comparable ${ }^{14} \mathrm{C}$ activities with the static method. Instant samples can provide simultaneously ${ }^{14} \mathrm{C}$ activity and $\mathrm{d}^{13} \mathrm{C}$ values. All ${ }^{14} \mathrm{C}$ activity and $\mathrm{d}^{13} \mathrm{C}$ data will be used to differentiate carbon of global origin and carbon from local fossil fuel combustion. 


\title{
Development of the decommissioning concept of the specialized enterprise for radioactive waste management UE "Ekores" in the Republic of Belarus
}

\author{
Natallia Kuzmina, Mikhail Zhemzhurov
}

The Joint Institute for Power and Nuclear Research - Sosny of NAS of Belarus, Minsk, Belarus

https://doi.org/10.21175/rad.abstr.book.2021.34.8

The specialized enterprise for radioactive waste management UE "Ekores" (hereinafter "Ekores") is located $2 \mathrm{~km}$ away from Minsk city and was founded in 1963 to store radioactive waste (hereinafter - RW) resulted from the activities of the research reactor of the former Nuclear Energy Institute of the Academy of Sciences of the BSSR.

The existing capacities of "Ekores" will ensure storage of RW generated in organizations of the Republic up to about 2030. However, further storage of RW in the old mothballed and being decommissioned near-surface storage facilities "Ekores" of the "Radon" type may cause a deterioration of the radioecological situation in the region.

In 2019 in order to determine the objective level of radiation and environmental safety, as well as obtain initial data for the development of a RW extraction project Stock company "Logistics Center of NFC" (Russian Federation) together with Stock company "PDC UGR", Limited liability company AP "KVARK", FSUE "RADON" (Russian Federation) carried out works on a comprehensive engineering and radiation research (hereinafter - CERR) of mothballed and being decommissioned "Ekores" storage facilities.

The state scientific institution "JIPNR - Sosny" of NAS of Belarus carried out scientific and methodological support of the mentioned activities on CERR.

The recommended option for the decommissioning of "Ekores" is the option of its deferred dismantling, which assumes that there are deviations from the design solutions and the requirements of regulatory legal acts, while the RW storage is in a satisfactory condition and will ensure the radiation safety of the population and the environment in short term.

This paper proposes a conceptual technological scheme for organizing decommissioning of mothballed "Ekores" storage facilities, provides conceptual design solutions for the extraction of the RW from the storage cells, gives recommendations for sorting, conditioning and packaging of the recovered RW, identifies measures to ensure radiation safety of the personnel involved in the work. A set of recommended measures for strengthening, replacing and repairing engineering structures and equipping "Ekores" storage facilities, as well as improving the radiation monitoring system, ensuring radiation safety of the population and the environment in the medium term, is presented.

After carrying out a set of measures for the extraction, identification and inventory of solid RW and spent sources of ionizing radiation, there is a potential possibility to use the structures of the decommissioned storage facilities again as a RW storage facility for the orderly storage of RW and spent sources of ionizing radiation in containers until they are sent for placement at the RW final disposal facility.

The proposed conceptual solutions will serve as the basis for the development of the concept for the decommissioning of "Ekores". 


\title{
Accumulation of ${ }^{90} \mathrm{Sr}$ by Pinus sylvestris and Betula pendula in the zone of radioactive contamination (East Ural Radioactive Trace, Russia)
}

\author{
Ludmila Mikhailovskaya ${ }^{1}$, Vera Pozolotina ${ }^{1}$, \\ Makar Modorov', Vladimir Kukarskih', Valentina Guseva ${ }^{1}$, \\ Zinaida Mikhailovskaya ${ }^{1}$, Nadezhda Shimalina ${ }^{1}$, Oleg Tarasov ${ }^{2}$
}

\author{
1 Institute of Plant and Animal Ecology, Ural Branch of the Russian Academy of Sciences, Ekaterinburg, \\ Russia \\ 2 Federal State Unitary Enterprise, Ozersk, Russia
}

\section{https://doi.org/10.21175/rad.abstr.book.2021.34.9}

Introduction. The studies were carried out in 2010-2020 on the territory of the East Ural radioactive trace (EURT), formed in 1957 as a result of an accident at the "Mayak Production Association". Currently ${ }^{90} \mathrm{Sr}$ is main pollutant. Forest ecosystems are mainly represented by secondary birch and mixed forests. Purpose of the study: to study the patterns of ${ }^{90} \mathrm{Sr}$ accumulation by woody plants (Pinus sylvestris and Betula pendula), which have been growing in the gradient of radioactive contamination for more than 60 years.

Materials and methods. We have selected test sites in automorphic territories located at a distance of $5-30 \mathrm{~km}$ from the epicenter of the accident. The soil contamination density decreased with distance from 70 to $0.4 \mathrm{MBq} / \mathrm{m}^{2}$. Control plots $\left(0.002 \mathrm{MBq} / \mathrm{m}^{2}\right)$ were selected outside the EURT area. Few Scots pine stands were found in the head part of the EURT only in areas where the soil contamination density does not exceed $10 \mathrm{MBq} / \mathrm{m}^{2}$. We took samples of soils and aboveground organs of trees: leaves (needles), small branches 1-5 years old, large branches $5+$ years old, trunk (wood, bark). The ${ }^{90} \mathrm{Sr}$ content in soil and plant samples was determined by the radiochemical method. The beta activity of the preparations was measured on a UMF-200o radiometer (Russia) with a lower detection limit of $0.2 \mathrm{~Bq}$.

Results. Concentration of ${ }^{90} \mathrm{Sr}$ in air-dry matter and plant ash increased with increasing soil contamination density in accordance with the power function. Evaluation of ${ }^{90} \mathrm{Sr}$ accumulation in various organs of pine and birch growing on the territory of EURT showed that the radionuclide concentrations decrease in the following order: bark> needles (leaves) $>$ small branches $>$ large branches $>$ wood. With an increase in the soil contamination density by ${ }^{\circ} \mathrm{Sr}$ (from background values to $70 \mathrm{MBq} / \mathrm{m}^{2}$ ), the aggregated transfer factors for aboveground organs of $P$. sylvestris decreased from $1 \cdot 10^{-2}$ to $1 \cdot 10^{-4}$, and for $B$. pendula, from $5 \cdot 10^{-2}$ to $5 \cdot 10^{-5}$. Similar results were obtained by us earlier for herbaceous plants, mosses and lichens.

Conclusion. White birch accumulates ${ }^{90} \mathrm{Sr}$ in larger quantities than Scots pine. The concentrations of ${ }^{90} \mathrm{Sr}$ in air-dry matter and plant ash were increased with increasing soil contamination density in accordance with the exponential dependence.

Acknowledgments: This study was performed within the frameworks of state contract with the Institute of Plant and Animal Ecology, Ural Branch, RAS (№ AAAA-A19-119032090023-0) and the Russian Foundation for Basic Research (project № 19-05-00469). 


\title{
Soil-to-plant transfer factors in arid regions using stable isotopes of caesium and strontium in local vegetables
}

\author{
Nemeer Padiyath, Prasoon Raj, Diana Francis \\ Emirates Nuclear Technology Center (ENTC), Khalifa University, Abu Dhabi, United Arab Emirates
}

\section{https://doi.org/10.21175/rad.abstr.book.2021.34.10}

With the recent catastrophes the world had experienced through severe radiation exposures from Chernobyl and Fukushima to name a few, it became a major concern to understand the effects of fallout radionuclides on us and our ecosystem. Several countries all over the world have collected transfer factor (TF) data which are key pre-requisites to develop a radioecological database for their region, and produce environmental impact assessment models. With the absence of relevant published data and the recent installation of the Barakah NPP in the UAE, there is a necessity for such a database. Since there is no standard protocol for studying soil-to-plant TF values in UAE, an extensive pilot root uptake experiment was conducted to study the transfer of anthropogenic nuclides in local vegetables from the soil. In addition to inhalation and external radiation, one of the major radionuclide pathways to humans is via ingestion of plant and animal matter. This TF depends on several parameters such as local climate, type of nuclides, and soil and plant characteristics. Factors such as soil types, vegetative seasons, local biodiversity, agricultural and pastoral practices, and local dietary habits were considered. For the soil, Torripsamments, a red sandy desert soil with high mineral content was used with bio-compost due to its high availability for local farmers and low salinity as compared to the 9 other great soil groups in UAE. Tomatoes, cucumbers, and eggplants were grown outdoors. These plants were decided as they are some of the most locally produced vegetable crops in UAE. The control group was watered daily with deionized water and the experimental group with spiking solutions of stable isotopes, Sr-88 and Cs-133, respectively, for a period of 20 weeks. The stable isotopes simulate the long-lived fallout radionuclides, $\mathrm{Sr}-90$ and Cs137, which are assumed to exhibit the same transfer kinetics. Fertilizers were added throughout the study as per local agricultural practices. The ratio of isotopic concentrations in the vegetables/leaves/stem/roots to the soil will give us the required TF values. Larger scale farm experiments with radionuclides will be undertaken in the future using this established protocol. 


\title{
$\mathrm{RH}$ analysis of natural radionuclides in animal feed and animal feed additives
}

\author{
Mihajlo Vićentijević1, Dubravka Vuković1, Vujadin Vuković1, \\ Marija Pavlović, Jasna Kureljušić, Dragan Živanov², Branislava Slavata²
}

1 Scientific Institute of Veterinary Medicine of Serbia, Belgrade, Serbia

2 Faculty of Veterinary Medicine, University of Belgrade, Belgrade, Serbia

\section{https://doi.org/10.21175/rad.abstr.book.2021.34.11}

The main route of introduction of radioactive substances into the body of animals occurs through animal feed. Over $70 \%$ of natural and artificial radionuclides enter the body through ingestion. Radioactive substances thus introduced are actively involved in the metabolism of animals and lead to a range of health disorders, and are deposited in critical organs and tissues. In this way, indirect contamination of products and raw materials of animal origin occurs and through them, endangers human health. In today's global pollution environment, the radiation protection of animal feeds is imperative in safeguarding animal health. This paper presents the results of measurements and methods for the early detection and control of the presence of natural radionuclides $\left({ }^{238} \mathrm{U}\right.$ and $\left.{ }^{\circ} \mathrm{K}\right)$ in animal feed, through the implementation of continuous RH monitoring and measures under regular conditions. Radiation-hygiene monitoring under regular conditions should be performed in accordance with the Rulebook on the limits of radionuclide content in drinking water, foodstuffs, animal feed, medicines (Official Gazette of RS 36/18). In the period from 2016-2020, a total of 263 samples of animal feed with its additives were tested. All samples were in accordance with the rulebook. Out of this number, only 40 samples were with higher activity levels of ${ }^{238} \mathrm{U}$ but still within the limits allowed by the rulebook. 


\title{
Radiological survey of spring water from post-volcanic areas in Romania
}

\section{Robert Csaba Begy',2, Codrin Fabian Savin',1, David-Karoly Sule²,1}

\author{
1 Interdisciplinary Research Institute on Bio-Nano-Sciences, University of Babes-Bolyai, Cluj-Napoca, \\ Romania \\ 2 Faculty of Environmental Science and Engineering, University of Babes-Bolyai, Cluj-Napoca, Romania
}

\section{https://doi.org/10.21175/rad.abstr.book.2021.34.12}

The Eastern Carpathians incorporates the Neogene and Quaternary volcanic chain, which hosts important resources of mineral spring waters. Due to the post-volcanic gas emanations in the area, these resources mainly consist of $\mathrm{CO}_{2}$-rich natural springs. The interactions with the aquifer geological environment can lead to elevated radioisotopes concentrations in water, explained by the naturally higher ${ }^{238} \mathrm{U}$ and ${ }^{232} \mathrm{Th}$ content in volcanic rocks. The aim of the present work is to perform a radiological water-quality assessment on 64 spring water samples collected from the Eastern Carpathians Neogene volcanic range area in Romania. The spring water samples were collected from four counties in Romania, namely Covasna, Harghita, Bistrița-Năsăud and Maramureș. These areas are situated in the vicinity of the Neogene Călimani-Gurghiu-Harghita and Oaş-Gutâi-Țibleș mountain ranges, and are abundant in $\mathrm{CO}_{2}$-rich mineral spring waters, frequently consumed by the locals. The radiological analysis consisted in the measurement of gross alpha and gross beta activities, alongside with ${ }^{222} \mathrm{Rn},{ }^{226} \mathrm{Ra}$, and ${ }^{210} \mathrm{Po}$ concentrations. Gross alpha activities were measured through alpha counting, resulted values ranged between $0.018 \pm 0.2-7.3 \pm 0.4 \mathrm{~Bq} / \mathrm{l}$. Gross beta activities were in the $0.032 \pm 0.003-5.8 \pm 0.17 \mathrm{~Bq} / \mathrm{l}$ interval, and were determined by subtracting alpha activities from the total activity, which was measured by liquid scintillation counting. ${ }^{222} \mathrm{Rn}$ and ${ }^{226} \mathrm{Ra}$ were measured by liquid scintillation counting, and the activity values ranged between $0.6 \pm 0.02$ and $81 \pm 3.2 \mathrm{~Bq} / \mathrm{l}$ for ${ }^{222} \mathrm{Rn}, 3 \pm 0.1$ and $1154 \pm 57.7 \mathrm{mBq} / \mathrm{l}$ for ${ }^{226} \mathrm{Ra}$ respectively. Alpha spectrometry was used to determine ${ }^{210}$ Po activities, which were in the $1.5 \pm 0.1-64.2 \pm 7 \mathrm{mBq} / \mathrm{l}$ interval. The results are highlighting that 14 of 64 samples are exceeding the WHO threshold value of $0.5 \mathrm{~Bq} / \mathrm{l}$ for gross-alpha activity in drinking water, and 12 water samples the WHO limit of $1 \mathrm{~Bq} / 1 \mathrm{for}$ gross-beta activities. 


\title{
Investigation of the effect of anthropogenic land use on the Pănăzii Lake (Romania) catchment area using Cs-137 and $\mathrm{Pb}-210$ radionuclides
}

\author{
Robert Csaba Begy ${ }^{1,2}$, Codrin Fabian Savin²,1, Szabolcs Kelemen²,1, Daniel \\ Veres $^{1,3}$, Octavian Liviu Muntean², Cristian Valeriu Malos², Tibor Kovacs ${ }^{4}$ \\ 1 Interdisciplinary Research Institute on Bio-Nano-Sciences, University of Babes-Bolyai, Cluj-Napoca, \\ Romania \\ 2 Faculty of Environmental Science and Engineering, University of Babes-Bolyai, Cluj-Napoca, Romania \\ 3 Romanian Academy, Institute of Speology “Emil Racoviță”, Cluj-Napoca, Romania \\ 4 Institute of Radiochemistry and Radioecology, University of Pannonia, Veszprém, Hungary
}

\section{https://doi.org/10.21175/rad.abstr.book.2021.34.13}

The problem of soil degradation has accentuated over recent decades. Aspects related to soil erosion and its relation to changes in land use as well as anthropogenic influence constitute a topic of great interest. The current study is focused on a soil erosion assessment in relation to land use activities in the Pănăzii Lake catchment area. Fallout radionuclides were used to provide information on soil erosion as well as redistribution rates and patterns. Variations in the sedimentation rate of the lake were also investigated as these reflect periods in which massive erosion events took place in the lake catchment area. The novelty of this study is the construction of a timescale with regard to the soil erosion events to better understand the relationship between soil erosion and land use activities. In this study, 10 soil profiles and one sediment core from the lake were taken. Soil parameters ( $\mathrm{pH}$, organic matter, inorganic matter and soil particle size) were determined for each sample. The activities of ${ }^{210} \mathrm{~Pb},{ }^{137} \mathrm{Cs}$ and ${ }^{226} \mathrm{Ra}$ were measured by gamma spectroscopy. For low ${ }^{210} \mathrm{~Pb}$ activities, measurements via ${ }^{210} \mathrm{Po}$ using an alpha spectrometer were performed. Soil erosion rates were determined by the ${ }^{137} \mathrm{Cs}$ method and the sedimentation rate calculated by the Constant Rate of Supply (CRS) model. A soil erosion rate of $13.5 \mathrm{t} \cdot \mathrm{ha}^{-1} \cdot \mathrm{yr}^{-1}$ was obtained. Three distinct periods could be observed in the evolution of the sedimentation rate. For the first period, between 1880 and 1958, the average deposition rate was 9.2 tons/year, followed by a high deposition period (1960-1991) of 29.6 tons/year and a third period, consisting of the last 30 years, during which the sedimentation rate was 15.7 tons/year. These sedimentation rates fluctuated depending on the main land use activity, which can also be seen in the soil erosion rates that had almost doubled by the time agricultural activities were performed in the area. 


\title{
Baseline soil-to-plant transfer of NORM radionuclides in Abu Dhabi date-palms
}

\author{
Prasoon Raj, Nemeer Padiyath, Yacine Addad, Diana Francis \\ Emirates Nuclear Technology Center (ENTC), Khalifa University, Abu Dhabi, United Arab Emirates
}

https://doi.org/10.21175/rad.abstr.book.2021.34.14

Field experiments form the primary source of radioecological concentration ratios (CR) used in the environmental decision support systems of nuclear entities. With four nuclear power units erected, of which one is already operational, and another is in the phase of clod tests, the UAE has made considerable progress towards a large-scale, safe, and sustainable national nuclear energy program. Thus, it becomes critical to assess the environmental impacts of a planned or accidental release of radionuclides in the UAE, from reactor-related facilities or NORM-employing industries (such as oil and gas). However, given its unique geography and dry arid climate, comparable data for the UAE are rather rare to find. Also, the food and agriculture ecosphere of the country has been changing rapidly over the last decade. To fulfill this gap in the arid region databases of the international radioecological monitoring bodies, we have designed UAE-specific pilot experiments for the soil-to-plant transfer of radionuclides in local produces, fruits, and vegetables.

In this paper, we present the measurement of the baseline soil-to-plant CR value for the main commercial crop of the UAE: date-palms (Phoenix dactylifera L.). With about 40 million trees of over 100 gene-varieties, UAE's dates constitute about $12 \%$ of the world's total production. Few recent studies have recorded NORM $\left({ }^{238} \mathrm{U},{ }^{232} \mathrm{Th}\right.$ and ${ }^{40} \mathrm{~K}$ chains) concentrations separately in the soil as well as in the date-fruit samples, but their ratios, i.e. the CR data, are scarce or unavailable. For this purpose, we have sampled 14 different palm trees and the surrounding soils, from the Um al Naar Sas al Nakhl region of the Abu Dhabi city, following the standard IAEA environmental sampling and monitoring guidelines. This activity is a running pilot project started in the summer of 2020. With mature fruits (Khalal or later stage), the pedicels, leaflets, leaf-stems, and barks of the trees have also been picked. Considering the lack of detailed knowledge for such tasks in the UAE, many aspects of work are careful alterations of similar measurements with fruits like apples and strawberries in Europe and elsewhere. The soil samples are being prepared for NORM measurements through the standard sieving and drying procedures. As a first-of-its-kind work, we present here the development of a consistent and reproducible protocol for the baseline date-palm soil-to-plant CR measurements in Abu Dhabi, along with a preliminary set of output CR data from our experiments. We also outline the long-term program to create a database of CR values for the UAE agricultural crops. Such a database can also be applied for other arid and semiarid regions around the world. 


\title{
Transfer from radioactively contaminated soil and accumulation of ${ }^{137} \mathrm{Cs}$ in agricultural crops with edible belowground part
}

\author{
Olga Komissarova', Tatiana Paramonova ${ }^{1}$, \\ Natalia Kuzmenkova ${ }^{2}$, Leonid Turykin ${ }^{3}$
}

\begin{abstract}
1 Soil Science Faculty of Lomonosov Moscow State University, Moscow, Russia
2 Chemistry Faculty of Lomonosov Moscow State University, Moscow, Russia

3 Geography Faculty of Lomonosov Moscow State University, Moscow, Russia
\end{abstract}

\section{https://doi.org/10.21175/rad.abstr.book.2021.34.15}

Crops with edible belowground part are of particular concern under growing on radioactively contaminated agricultural lands due to the possibility of direct passive transport of ${ }^{137} \mathrm{Cs}$ into the consumable tissues. Meanwhile, similar plants are currently found in the structure of crop rotation in the post-Chernobyl territories of the chernozem zone of Russia.

To assess bioaccumulation of ${ }^{137} \mathrm{Cs}$ in edible belowground parts of agricultural crops, a study was carried out in 2016-2019, $\approx 30$ years after the Chernobyl accident, in the area of the "Plavsky radioactive hotspot" (Tula region). Agrosystems under study were located in the central (potatoes, carrot and beetroot) and peripheral (onion) sections of the contaminated area with the initial level of ${ }^{137} \mathrm{Cs}$ accumulation $185-555 \mathrm{kBq} / \mathrm{m}^{2}$ and the current level of $80-170 \mathrm{kBq} / \mathrm{m}^{2}$, which is still $2-4.5$ times higher than the permissible value of the radionuclide content in soil.

An important radioecological feature of the study area is the deep rehabilitative plowing of soils

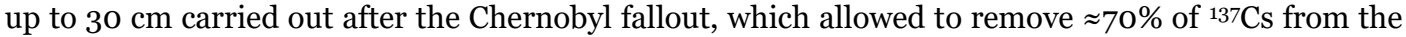
main $10-\mathrm{cm}$ rooting zone of cereals and $\approx 35-40 \%$ from the $20-\mathrm{cm}$ arable horizon of other crops of field rotation. However, potatoes, onions and root vegetables are cultivated with plowing up to 30; as a result, these crops interact with all ${ }^{137} \mathrm{Cs}$ accumulated in soils, the specific activity of which in the arable layer ranges from 170 (onions) to $390-420 \mathrm{~Bq} / \mathrm{kg}$ (potatoes, carrot and beetroot).

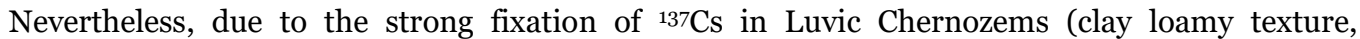
$\mathrm{pH}$ 6.5-7.2, humus 6-7\%) and the adaptive capacity of the crops under study, the transfer of the radionuclide into the plant biomass is characterized by a low intensity. Transfer factors (TFs) of ${ }^{137} \mathrm{Cs}$ from soil to total biomass are estimated as 0.32 for potatoes, 0.31 for carrot, 0.26 for beetroot, and 0.18 for onions. The minimum TF for onion appears to be primarily related to its biological

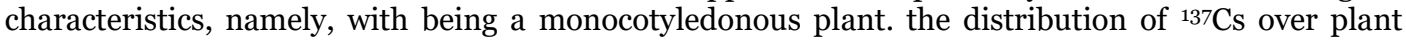
tissues has a sharply different character for onions and other listed crops: the radionuclide's specific activity in onion roots is 2.5 times higher than in shoots (bulb and leaves), while ${ }^{137} \mathrm{Cs}$ concentrations in the aerial parts of potatoes, carrot and beetroot are $\approx 3.5,6$ and 6.5 times higher than in the roots, correspondently. The values of TFs in consumed potato tubers, taproots of carrot and beetroot, as well as onion bulbs (0.012, 0.011, 0.011, and 0.003, correspondently) are less than the respective TFs in the total biomass of these crops.

In this regard, the accumulation of ${ }^{137} \mathrm{Cs}$ in the yield of the studied crops does not exceed $5 \mathrm{~Bq} / \mathrm{kg}$, which fully complies with the sanitary safety standards and decreases the radioecological risk of their cultivation on radioactively contaminated lands.

Acknowledgments: The reported study was funded by RFBR, project number 20-35-90119. 


\title{
Dose planning system for the proton beam therapy complex
}

\author{
Aleksandr Khalikovi, Natalya Mamedova ${ }^{2}$, Fedor Pak ${ }^{2}$ \\ 1 Petersburg Nuclear Physics Institute named by B.P.Konstantinov of NRC “Kurchatov Institute”, Saint- \\ Petersburg, Russia \\ 2 Petersburg Nuclear Physics Institute named by B.P.Konstantinov of NRC "Kurchatov Institute", Gatchina, \\ Russia
}

\section{https://doi.org/10.21175/rad.abstr.book.2021.35.1}

Proton beam therapy (PBT) is developed at Petersburg Nuclear Physics Institute named by B.P.Konstantinov of NRC "Kurchatov Institute" in close cooperation with Russian Scientific Center of Radiology and Surgical Technologies n.a. acad. A.M. Granov. Currently, the complex modernization process is in final stages of upgrading the equipment and software to be used for irradiation of intracranial targets with $1 \mathrm{GeV}$ proton beam using the "fly-through" method. This treatment method differs from commonly accepted proton therapy method - so called method of "Bragg peak". The "fly-through" method is based on a rotation technique allowing one to rotate the patient using a special medical installation and a fixed beam extraction system.

Dose fields formed by two-axis rotation of the irradiated object in relation to a fixed $1 \mathrm{GeV}$ proton beam are calculated using an algorithm developed for this purpose and are measured experimentally using a dose detection system with high spatial resolution and weak energy dependence of detectors. Dose fields acquired at such irradiation characteristically exhibit a sharp peak of absorbed dose in the center of rotation. Depending on the programme of irradiation, it is possible to acquire fields of different forms and sizes corresponding to the size of pituitary adenomas.

As the method of treatment using $1 \mathrm{GeV}$ proton beam is unique and therefore does not fall within the norms of existing requirements, the existing commercial software cannot be applied to this method.

Planning system is a designated software product that performs simulation and calculation of dose distribution in the zone of interest, makes choice of the optimal irradiation plan for performance of PBT upon malignant and benign neoplasms using $1 \mathrm{GeV}$ beam energy.

A planning system allows one to assess the quality of irradiation programme by calculating the dose-volume histogram (DVH). Minimal set of parameters needed for the planning quality assessment includes the inconsistency of the dose in the planned target and DVH. The system estimates the possibilities of local tumor control and complications in healthy tissues. Using this data, medical physicist can choose the most optimal programme for the planned target to be fully covered by a set isodose with calculated dose distribution so that the adjacent tissues and structures are exposed to a minimal possible dose.

The planning system being developed is designed for application in PBT centers possessing installations with fixed proton beam extraction system and using rotation technology that allows one to rotate a patient on a patient's table. The system will be used by radiologists and medical physicists performing irradiation planning with the aim of gaining optimal combination of a dose field and the target and minimizing the dose to critical head structures. 


\title{
Proton beam therapy complex at $1000 \mathrm{MeV}$ proton beam
}

\author{
Natalya Kuzora', Aleksandr Khalikov², Natalya Mamedova1, Djan Karlin' \\ 1 Petersburg Nuclear Physics Institute named by B.P.Konstantinov of NRC "Kurchatov Institute”, Gatchina, \\ Russia \\ 2 Petersburg Nuclear Physics Institute named by B.P.Konstantinov of NRC "Kurchatov Institute", Saint- \\ Petersburg, Russia
}

\section{https://doi.org/10.21175/rad.abstr.book.2021.35.2}

Synchrocyclotron SC 1000 with the proton energy of $1000 \mathrm{MeV}$ based in Leningrad Nuclear Physics Institute was put into operation in 1970. As it turned out, it could be used not only in order to conduct research in field of fundamental physics, but also for medical purposes.

Synchrocyclotron SC 1000 medical proton beam with fixed energy of $1000 \mathrm{MeV}$ was put into operation in January 1973 and since 1975 it has been used for medical purposes.

A unique "fly-through" method based on irradiation of specific brain sections with protons with fixed energy of $1000 \mathrm{MeV}$ was developed and used for treatment of patients. Low scattering of protons penetrating through an irradiated object combined with the rotation technique of irradiation allows one to provide a very high ratio of the dose in the irradiated zone to the dose at the object's surface. It allows one to irradiate tumors in immediate proximity to the life-critical organs.

At the installation for stereotaxic proton therapy, the beam is centered precisely at the target zone. The main element of the installation, the patient's table, provides oscillation in the horizontal plane within the range of $\pm 40^{\circ}$. The anteriour part of the table represents a head-fixation device performing oscillation about the $\mathrm{X}$-axis within $\pm 36^{\circ}$. The crossover of both oscillation axes, vertical and horizontal, as well as the irradiation beam axis perpendicular to them both, is the center of rotation (isocenter).

Since 1975 until 2013, 1394 patients were treated with the course of proton therapy at the PNPI synchrocyclotron. 477 cases were the patients with pituitary adenomas, 502 cases - the patients with arteriovenous malformations, 350 patients experienced treatment for the purposes of pain management. In 151 cases the treatment was repeated.

1394 patients are almost $26 \%$ of all the patients who experienced proton therapy in Russia and $2 \%$ of the patients treated using this therapy in the world.

The treatment was performed without damaging the skull and brain tissues. None of the patients had any life-critical complications or complications causing disability. All this proves the efficiency of the method and validity of its application.

The results of clinical application of $1000 \mathrm{MeV}$ proton beam in treatment of intracranial abnormal and normal structures allow us to promote further development of this field. Stereotaxic therapy with a narrow high-energy proton beam is a method alternative to surgical treatment. 


\section{Results of the radiology monitoring of breasts in women with gynecological malignant tumors}

\section{Viktor Myakynkov}

Taurt Medical Regional Oncology Hospital, Kherson, Ukraine

https://doi.org/10.21175/rad.abstr.book.2021.35.3

Purpose. There are contradictory data of breast cancer (BC) appearance frequency in women with gynecological malignant tumors (GMT). The purpose of this research is evaluation of BC appearance frequency in women with GMT depending on the time passed after GMT detection, age when GMT were diagnosed, GMT type, definition of examination tactic.

Methods and materials. Breast examination results of 2412 women with GMT were analysed (ovarian cancer - 442 patients, cervical carcinoma - 998 patients, endometrial carcinoma - 972 patients. During 5 years following the operation 868 women were being observed (36\%), 6-10 years 699 (29\%), 11-15 years - 531 (22\%), over 15 years - 314 (13\%). Mammography, ductography, pneumocystography and sonography were used.

Results. Breast cancer was revealed in 27 (1.1\%) women; with ovarian cancer - $10(2.2 \%)$, cervical carcinoma - 7(0.7\%), endometrial carcinoma - 10 (1.0\%). During 5 years following the operation 7 cases $(0.8 \%), 6-10$ years $-7(1.0 \%)$, $11-15$ years $-7(1.3 \%)$, over 15 years $-6(1.9 \%)$. The frequency of disease for $\mathrm{BC}$ at the moment of revealing the GMT before the age of $34-0.7 \%$, $35-44-1.3 \%, 45-54-0.8 \%, 55-64-1.6 \%, 65$ and older $-0.8 \%$.

Conclusion. Frequency of breast cancer appearance in women with gynecological malignant tumors prevails the morbidity of female population at our region by 19 times and has 2 peaks. The highest rate of revealing of breast cancer was observed when gynecological malignant tumors were detected on the age $55-64$ years. The highest risk of breast cancer appearance belongs to patients with ovarian cancer (2.2\%).

Taking into account high percent of the breast cancer, diagnosed in women with gynecological malignant tumors, it is necessary to examine the patients carefully before operation.

For breast monitoring the following tactic of using radiology methods of diagnostic was recommended annual mammography (if necessary sonography and MRI). 


\title{
Monte Carlo simulations of biological samples irradiation using a new setup at the 3 MV Tandetron ${ }^{\mathrm{TM}}$ of IFIN-HH
}

\author{
Călin Mircea Rusu1, Mihai Straticiuc², Mihaela Bacalum¹, Mihai Radu1
}

\author{
1 Department of Life and Environmental Physics, "Horia Hulubei” National Institute of Physics and Nuclear \\ Engineering, Măgurele, Romania \\ 2 Department of Applied Nuclear Physics, "Horia Hulubei” National Institute of Physics and Nuclear \\ Engineering, Măgurele, Romania
}

\section{https://doi.org/10.21175/rad.abstr.book.2021.35.4}

The capability of a more precise estimation of the delivered dose constitutes an advantage of hadrontherapy over photon-based radiotherapy techniques. In order to maximize the benefits of hadron therapy, the effectiveness of the irradiation process has to be assessed through radiobiology experiments. This can be pursued by evaluating connected factors such as the dose, Linear Energy Transfer (LET) and endpoint effects at the tissue level. In cell irradiation experiments, an optimized local control of the beam allows for a precise dose delivery, as the position of the Bragg peak can be adjusted in regard to the positioning of the sample. A radiobiology experimental setup was implemented at the 3 MV Tandetron ${ }^{\mathrm{TM}}$ accelerator facility of the National Institute for Physics and Nuclear Engineering (IFIN-HH). Geant4 simulations were performed for the calculation of the LET, Bragg peak profile and lateral energy distribution, as well as for the beam evaluation at the interface between the target volume and exterior medium. We developed a particle tracking strategy, to calculate the dose and LET of a proton beam along the propagation axis. For this purpose, we provided our custom geometry description for the irradiation setup, along with volume properties and specific materials configurations. We simulated a $3 \mathrm{MeV}$ disc-shaped general particle source from which the beam was launched through a $6.7 \mu \mathrm{m}$ thin Au foil, in accordance with the experimental conditions. Simulated quantities were recorded in replica of the scoring volume along the propagation axis. In each replica, we recorded the dose, LET and particle fluence. We also evaluated the kinetic energy of each particle before entering the scoring volume in order to evaluate the spatial sampling of the beam as well as its energy distribution. In real experiments, the Bragg peak was drawn from measurements of the dose with a Markus chamber placed behind a $6 \mu \mathrm{m}$ incrementing layer of Mylar, with thickness varying from o to $60 \mu \mathrm{m}$. Following the simulations, the recorded data superimposed well over the experimentally recorded values $(r=0.997)$. The positioning of the Bragg peak was reported in both experimental and simulation conditions to be appearing at a depth of $36 \mu \mathrm{m}$ from the air-Mylar interface. An average energy of $1.95 \pm 0.1 \mathrm{MeV}$ was recorded by simulation at the air-Mylar interface, and evaluated from the kinetic energy distribution of each individual particle. The scoring of the LET values in each replica of a $1 \mu \mathrm{m}$ water scoring volume shows the maximum positioned at $\sim 60 \mu \mathrm{m}$, ensuring that the first $10 \mu \mathrm{m}$ of the volume, where the $2 \mathrm{D}$ cell culture usually resides, will receive $\sim 17.94 \mathrm{keV} / \mu \mathrm{m}$. The experimental records of the Bragg peak were confirmed by the Geant 4 simulations. Provided these favorable predictions, we were able to further implement our simulations using a higher flux of protons or alpha particles. 


\title{
Diagnosis of non-traumatic hemorrhages in the brain parenchyma
}

\author{
Živorad Savić1, Katarina Savić ${ }^{\text {, }}$ \\ Sofija Savić3, Mirjana Petrović4, Vojislav Antić5
}

\author{
1 University Clinical Center of Serbia, Department of Emergency Radiology, Belgrade, Serbia \\ 2 General Hospital Pančevo, Service for Radiological Diagnostics, Pančevo, Serbia \\ 3 Volunteer Specialization in Radiology, Faculty of Medicine, University of Belgrade, Belgrade, Serbia \\ 4 University Clinical Center of Serbia, Belgrade, Serbia \\ 5 University Clinical Center of Serbia, Center for Nuclear Medicine, Belgrade, Serbia
}

\section{https://doi.org/10.21175/rad.abstr.book.2021.35.5}

The most urgent indications for diagnosis are actually non-traumatic hemorrhages, because timely identification is important information for further treatment of the patient. Depending on the etiology of the cause, we have several diagnostic methods for diagnosing them:

1. Lumbar puncture

2. DSA - gold standard (Moniz and Lima 1927 and modification according to Seldinger 1953 through the femoral vein).

3. CT angiography (thanks to Sir Godfrey Hounsfield, Nobel Prize for it in 1979.). With the advent of spiral CT scanners, new techniques such as Maximum Intensity Projection-MIP; Volume Rendering-VR; MRA - Bloch and Purcell 1952 and Sir Peter Menfield gives the first finding on a man in 1976.

In principle, we have three types of hemorrhage, SAH, ICH and IVH. The most common causes of nontraumatic hemorrhage in the brain parenchyma are:

\section{Rupture of aneurysms}

2. Vascular malformations (Basic types of AVM, dural - dAVM, cavernous - CM, venous malformations and capillary telangiectasia).

The AVM is actually a network of vascular channels between the feeding arteries and the draining veins. In aneurysms, about $10 \%$ are present, and $85 \%$ are in the cerebellar hemispheres, and about $50 \%$ show signs of hemorrhage, which gives a cumulative risk of $2-4 \%$ annually.

dAVF when the feeding arteries flow directly into the venous canals without intervening vascular networks. They make 10-15\%. of all VMS. Acquired lesions are created either through existing micro shunts or by angiogenesis, we most often meet them at the age of 40-60, and the risk of hemorrhage is about $2 \%$ for each year.

Cavernous malformations CM- They cause recurrent bleeding, they are natively presented as wellseparated structures from the surrounding tissue. After radiation, the possibility of hemorrhage is $0.25-0.7 \%$ annually. Cavernomas are angiographically occult.

Venous malformations are thin-walled and the risk of hemorrhage is about $0.34 \%$ annually.

Capillary telangiectasias are found in autopsies of $16-20 \%$ of all vascular malformations.

\section{Hypertension}

They are most common at the age of 45-70 years and make up about 50\% of intracranial non-traumatic hemorrhages, where about $75 \%$ are localized in the basal ganglia putamen / extrnal capsules $60-65 \%$.

\section{Embolic infarction with reperfusion}

It is presented as a hemorrhagic transformation of the initial ischemic lesion, which occurs 24 hours after the onset of ischemia. At autopsies it is in 50-70\% of all arterial infarcts.

\section{Anticoagulant therapy and coagulopathies}

One of the causes of hemorrhage is anticoagulant trapia (heparin, warfarin, aspirin).

Coagulopathy is present in hepatocellular disease, vitamin $\mathrm{K}$ deficiency as well as DIC - disseminated intravascular coagulopathy.

\section{Bleeding in the tumor itself}

The pathology is unclear, it occurs in high-grade tumors due to neovascularization, rapid tumor growth and necrosis, plasminogen activation or invasion of vascular structures. The frequency is $1-15 \%$.

Other causes of non-traumatic bleeding in the brain parenchyma are:

7. Cortical vein thrombosis

8. Amyloid angiopathies

9. Pituitary apoplexy 


\title{
Development of functionalized resins for the purification of innovative radiopharmaceuticals
}

\author{
Ella Moreau', Eugen Andreiadis', Vincent Blet ${ }^{2}$
}

1 CEA, DES, ISEC, DMRC, Univ. Montpellier, Marcoule, France

2 CEA, DES, DDSD, DFDE, Univ. Montpellier, Marcoule, France

\section{https://doi.org/10.21175/rad.abstr.book.2021.36.1}

Vector-driven internal radiotherapy is a rapidly growing field that completes the conventional techniques for cancer treatment such as surgery, chemotherapy or external radiotherapy. The appropriate radionuclide is coupled with a specific vector (monoclonal antibody, peptide...) in order to deliver the radioactivity specifically to the tumor cells. Contrary to the other methods, radioimmunotherapy allows the treatment of diffuse or metastatic tumors, limiting damage on the healthy tissues. Recent developments in isotope production now provide access to novel radionuclides and open new opportunities for theranostics. However, the production of novel radiopharmaceuticals is often limited by a short half-life (few days), high specific activities (> GBq/g) and high levels of isotopic purity required (> 99.9\%). Therefore, reliable and efficient separation and purification processes are needed. Solid phase extraction processes are an attractive solution for the purification of radionuclides, as they allow using simple, compact equipment, with obvious advantages for radioprotection and handling. Moreover, this type of processes produces less waste and effluents compared to solvent-type extraction and are largely operated using remote control.

An isotope of ruthenium has been selected for the development of a new radiopharmaceutical, in agreement with several criteria established by physicians, radiochemists, and nuclear engineers. Ruthenium can be produced from an irradiated metallic target. After dissolution of the irradiated material in nitric acid, ruthenium can be extracted and purified by solid phase extraction using a series of organic resins, either commercially available or specifically developed for this application. Results concerning the performance of the various resins in surrogate solutions will be presented and discussed. The present studies pave the way to the development of novel ruthenium separation processes and contribute to a better understanding of the factors influencing the extraction properties of ruthenium isotopes. 


\title{
Antioxidant behavior of $\mathrm{GdYVO}_{4}: \mathrm{Eu}^{3+}$ nanoparticles under $\mathrm{X}$-ray and UV irradiation: Proposed mechanism
}

\author{
Svitlana Yefimova, Pavel Maksimchuk, Kateryna Hubenko, Alexander Sorokin
}

Institute for Scintillation Materials NAS of Ukraine, Kharkiv, Ukraine

\section{https://doi.org/10.21175/rad.abstr.book.2021.36.2}

Recently, redox nanomaterials (NMs) have revealed growing interest in biomedical area as a novel class of theranostic agents with antioxidant action, which can eliminate the negative effects of oxidative stress decreasing the level of Reactive Oxygen Species (ROS) in living organism. As NMs with inherent antioxidant properties and catalase-like behavior (CAT-mimetics), $\mathrm{CeO}_{2}, \mathrm{Mn}_{3} \mathrm{O}_{4}$, $\mathrm{Co}_{3} \mathrm{O}_{4}, \mathrm{Fe}_{3} \mathrm{O}_{4}$ nanoparticles (NPs) have been reported, whereas for $\mathrm{Mn}_{3} \mathrm{O}_{4}$ and $\mathrm{V}_{2} \mathrm{O}_{5}$ NPs glutathione peroxidase activity (GPX -mimetics) has been mentioned. Superoxide dismutase like behavior (SOD-mimetics) has been described for $\mathrm{Mn}_{3} \mathrm{O}_{4}, \mathrm{Co}_{3} \mathrm{O}_{4}$, carbon nanotubes, and functionalized fullerenes. Since the overproduction of ROS, such as superoxide $\left(\mathrm{O}_{2}^{--}\right)$, hydroxyl radical $(\mathrm{OH})$, hydrogen peroxide $\left(\mathrm{H}_{2} \mathrm{O}_{2}\right)$, etc., in cells is associated with the development of various diseases including cancer and age-related ones, finding new therapeutics enable to control ROS level is an important challenge.

In present study, we report an antioxidant action of $\mathrm{GdYVO}_{4}: \mathrm{Eu}^{3+} \mathrm{NPs}(\mathrm{d}=2 \mathrm{~nm})$ at both X-ray and UV-irradiation in water solutions, model system of cell membranes and in cells suspensions. The ability of $\mathrm{GdYVO}_{4}: \mathrm{Eu}^{3+} \mathrm{NPs}$ to decrease the ROS concentration in test media has been revealed by spectroscopic methods using various ROS sensors. The mechanism of ROS scavenging for $\mathrm{GdYVO}_{4}: \mathrm{Eu}^{3+} \mathrm{NPs}$ is proposed and discussed. Antioxidant action of $\mathrm{GdYVO}_{4}: \mathrm{Eu}^{3+} \mathrm{NPs}^{3}$ is ascribed to the presence of ions with changeable valence states $\left(\mathrm{V}^{3+} / \mathrm{V}^{4+} / \mathrm{V}^{+}\right)$in the crystal lattice of the NPs and reversible switching between $V^{3+} \leftrightarrow V^{4^{+}}$and $V^{4+} \leftrightarrow V^{5^{+}}$vanadium oxidation states that provides GdYVO NPs enzyme-like activity.

Antioxidant action of $\mathrm{GdYVO}_{4}: \mathrm{Eu}^{3+} \mathrm{NPs}$ make them prospective in biomedical applications to decrease the ROS level in living organism and prevent oxidative stress development. $\mathrm{GdYVO}_{4}: \mathrm{Eu}^{3+}$ NPs are also revealed to be effective ROS scavengers in conditions of X-ray or UV irradiation that make them unique therapeutics with radioprotective effects.

Acknowledgments: This research was supported by National Research Foundation of Ukraine, Grant No. 2020.02/0052 for Leading and Young Scientists Research Support. 


\title{
Creation of complexes of "scintillation nanoparticles $\mathrm{LaF}_{3}: \mathrm{Tb}^{3+}-$ Rose Bengal" for X-ray activated PDT
}

\author{
Iryna Bespalova, Pavel Maksimchuk, \\ Kateryna Hubenko, Alexander Sorokin, Svetlana Yefimova
}

Institute for Scintillation Materials National Academy of Sciences of Ukraine, Kharkiv, Ukraine

https://doi.org/10.21175/rad.abstr.book.2021.36.3

The application of various drug delivery systems, which have been developed recent decades, offers many potential advantages in cancer treatment strategies including Photodynamic therapy (PDT) [1]. PDT is a method for cancer, infections, and some other diseases treatment, which combines the use of a specific agent - photosensitizer (PS), usually organic molecules, and action of light with appropriate wavelength to excite photosensitizer, which then transfers the absorbed energy to molecular oxygen generating cytotoxic reactive oxygen species (ROS), especially highly toxic singlet oxygen $\left({ }^{1} \mathrm{O}_{2}\right)$ [2]. The main advantage of nanoparticles (NPs) application in PDT is associated with scintillation NPs (SNPs) and their composites with conventional PSs that make possible the treatment of deep tumors. In such SNPs-based systems, which are excited by X-ray, excitation energy is transferred from the excited SNPs to organic PS molecule via the Förster resonance energy transfer (FRET) mechanism with subsequent ROS generation by PS molecules [3, 4].

Here, we report on the creation of an effective complexes on the base of scintillation nanoparticles $\mathrm{LaF}_{3}: \mathrm{Tb}^{3+}$ and organic photosensitizer Rose Bengal (RB), which could be potentially applied in X-ray induced PDT for deep-seated tumour.

The resulting complexes on the base of scintillation $\mathrm{LaF}_{3}: \mathrm{Tb}^{3+} \mathrm{NPs}$ (average diameter sized between 10 and $15 \mathrm{~nm}$ ) and organic photosensitizer Rose Bengal have been created in water solutions using physical dyes adsorption on the surface of SNPs, which reveal $100 \%$ FRET from $\mathrm{LaF}_{3}: \mathrm{Tb}^{3+}$ SNPs to organic PS RB under UV-light or X-ray irradiation. It has been shown that upon UV-light or X-ray irradiation, created $\mathrm{LaF}_{3}: \mathrm{Tb}^{3+}-\mathrm{RB}$ (40:1) complexes reveal the efficient singlet oxygen ${ }^{1} \mathrm{O}_{2}$ generation. Moreover, $\mathrm{LaF}_{3}: \mathrm{Tb}^{3+} \mathrm{SNPs}$ reveal efficient hydroxyl radical $\mathrm{OH}$ generation that increases the total toxicity of the created $\mathrm{LaF}_{3}: \mathrm{Tb}^{3+}-\mathrm{RB}$ complexes and could serve the problem of treatment of hypoxic areas in tumor cell population during X-ray induced PDT.

\section{References}

[1] Y. Zheng et al. Nanoparticle-based drug delivery systems for controllable photodynamic cancer therapy. Eur J Pharm Sci. 144 (2020):105213-16.

[2] N.L. Oleinick et al. The role of apoptosis in response to photodynamic therapy: what, where, why, and how. Photochem Photobiol Sci. 1 (2002):1-22.

[3] M. Q. Mesquita et al. An insight on the role of photosensitizer nanocarriers for Photodynamic Therapy. An Acad Bras Cienc. 90 (2018):1101-1130.

[4] P. Figueiredo et al. The emerging role of multifunctional theranostic materials in cancer nanomedicine, In Handbook of Nanomaterials for Cancer Theranostics; Elsevier Science Publishing Company: Oxford, UK, 2018, pp. 1-31.

Acknowledgments: This research was supported by National Research Foundation of Ukraine, Grant № 2020.02/0052 for Leading and Young Scientists Research Support. 


\title{
Optical system for quick and precise assessment of mechanical isocenter of accelerators
}

\author{
Mikołaj Tarchalski', Paweł Kukołowicz²
}

1 NaviRation, Warsaw, Poland

2 Maria Sklodowska-Curie National Research Institute of Oncology (MSCNRIO), Warsaw, Poland

\section{https://doi.org/10.21175/rad.abstr.book.2021.36.4}

Purpose. Mechanical isocenter of linear accelerators have to be verified regularly. There are several different techniques for determination of the isocenter of accelerators. The optical system for measurements of the accelerator isocenter has been designed. The aim of this work is to present the system optical system called NaviRation designed for making all mechanical tests of linear accelerator.

Materials and methods. The system consists of two optical cameras mounted on a horizontal arm at a distance of $1 \mathrm{~m}$ from each other and from the marker set. The cameras are positioned at distance of about 2 meters from the isocenter of the accelerator. The marker set is placed sequentially on the arm of accelerator, on the top of the treatment table and on the collimator. During the rotation of the each part of the accelerator the position of the marker set captured by the cameras are transferred to the computer. With the specialized software the actual position of rotation axis (isocenter) is calculated. Finally, the actual position of the axes of all three rotational parts of the accelerators is numerically and graphically presented.

Results. The precision of our system obtained with the coordinate-measuring machine reviled that the imprecision is smaller than $0.1 \mathrm{~mm}$. We have performed all feasibility tests using two accelerators installed in the National Cancer Center in Warsaw with two Varian accelerators Clinac $2300 \mathrm{CD}$ and Edge. Comparison of our measurements with the measurements carried out by the Varian service with the specialized micrometer system showed the conformity of both measurements. Our measurements were 10 times shorter than the measurements performer with the system used by Varian service.

Conclusion. The results of the performed measurements fully confirmed the suitability of our system for the rapid performance of mechanical tests of the accelerator. 


\title{
Comparison of the results of calculations using GEANT4 and SRNA
}

\author{
Grigorii V. Merzlikin', Sergey V. Akulinichev',2, Ivan A. Yakovlev',2
}

1 Institute for Nuclear Research of RAS, Troitsk, Russia

2 Hospital of RAS, Troitsk, Russia

\section{https://doi.org/10.21175/rad.abstr.book.2021.36.5}

The aim of this work was to study the dependence of possible differences in the results of Monte Carlo calculations on the choice of a specific software package based on this method. The specific task of the study was to compare the results of calculations using the software packages GEANT4 and SRNA [1]. The beam profiles at different depths, the depth dose distributions of protons, and the isodose curves for the proton therapy unit based on the linear proton accelerator of the INR RAS were calculated. This facility is now of additional interest, because of the prospects of its use for FLASH therapy [2]. The main parameters of the nucleon-nucleon interactions were the same in both cases, so the possible differences in the calculation results can be attributed to the properties of the programs themselves. As a model installation, as well as a sensitive detector, a water phantom and a scattering plate made of PMMAs with a thickness of 0.1 to $2 \mathrm{~cm}$ were used for various model experiments. The initial proton energy was $209 \mathrm{MeV}$. Preliminary results show that in SRNA, compared to GEANT4, there is a dip of $5-10 \%$ in the depth dose distributions in the region of the plateau before the Bragg peak and approximately the same increase in the width of the beam profiles at greater depths. This may indicate a stronger contribution of proton scattering in SRNA compared to GEANT4.

\section{References}

[1] R.D. Ilić, D. Lalić, S.J. Stanković// SRNA - Monte Carlo codes for proton transport simulation in combined and voxelized geometries//Nuclear Technology \& Radiation Protection, 17(1-2)(2002)27-36.

[2] S.V. Akulinichev, V.N. Vasiliev, Yu.K. Gavrilov et al. // Possibilities of Proton FLASH Therapy on the Accelerator at the Russian Academy of Sciences' Institute for Nuclear Research//Bull. Russ. Acad. Sci. Phys. 84, 1325-1329 (2020). 


\title{
Multimodal approach in treatment of skin cancer
}

\author{
Julya Zuenkova' ${ }^{1}$ Lev Izurov ${ }^{2}$
}

1 RUDN University, Moscow, Russia

2 Sverdlovsk Oncology Regional Dispensary, Ekaterinburg, Russia

\section{https://doi.org/10.21175/rad.abstr.book.2021.36.6}

It has been established that the patient's pathways may differ from their formal routing. The multimodal interdisciplinary approach to skin cancer treatment mitigates the risks of medical care delay, helps to develop patients' adherence, use personalization treatment approach and safe human resources.

Purpose of the study was to describe the state of skin cancer care at the oncology dispensary, reveal risks of medical care in time provision, formulate requirements for the optimal organization of oncological care and propose its improvement taking into account quality, accessibility, and patient adherence.

Materials and methods. Patient routing was assessed using observation, description and measurement methods. Based on the medical site statistics, the main risks of compliance with the requirements for routing and the timing were identified. Using the methods of system analysis, a model of the optimal organization of skin cancer medical care in the region was built.

Results. Due to the high incidence of skin cancer, the improvement of the oncodermatological service in the region and the optimization of patient routing can be considered as a priority task. The main risks of the existing organization of the patients flow are the risk of exceeding the period of diagnostics, the risk of exceeding the treatment start, the risk of low patient adherence and the poor follow-up, inability to provide a personalized medical care which corresponds to patient's individual needs. Excessive patient's logistic during the entire cycle of diagnosis and treatment, excessive costs of highly specialized human resources and their remoteness from the main patient flow are considered as the basis for organizing a multimodal skin cancer oncology care.

Discussion. The developed model is based on a value-oriented approach, risk-oriented approach and contributes to the achievement of a synergistic effect at the regional level through a comprehensive solution to the problems of introducing innovations in the industry.

Conclusion. The presented multimodal design of the skin cancer care is aimed to achieve a synergistic effect due to the maximum concentration of competencies and resources. Allocation resources in accordance with the patients needs should contribute to improving the healthcare quality and accessibility, and develop patient adherence. 


\title{
Determination of tissue volume in $100 \%$ isodose for application with brachytherapy cylinder
}

\author{
Zoran Stefanovski, Petar Chakalaroski, Violeta Klisarovska
}

University Clinic of Radiotherapy and Oncology, Skopje, North Macedonia

https://doi.org/10.21175/rad.abstr.book.2021.36.7

Introduction. Among the minimum requirements for reporting gynecological brachytherapy treatment is the reporting of the size of the volume occupied by $100 \%$ isodose line $\left(\mathrm{V}_{100}\right)$. This requirement also applies when a cylinder applicator is used to irradiate, most commonly, endometrial cancer. This volume is easily found from DVH plot. However, the total reported volume also includes the part of the volume occupied by the cylinder applicator. But we should report only tissue volume receiving at least $100 \%$ dose.

This work describes the procedure for obtaining this volume and makes statistics to see what the deviations of the reported volume between different patients are. The most often clinically used cylinders were cylinders with diameters of 3.5 and $3 \mathrm{~cm}$. The reference points at which the dose was normalized were at distances of 5 or $10 \mathrm{~mm}$ from the top of the applicator for the dose of $7 \mathrm{~Gy}$.

Materials and Methods. Treatment planning is carried out on the Varian BrachyVision treatment planning system. To determine only the volume of tissue covered by $100 \%$ isodose, it is necessary to set one side of the calculation grid to pass through the intersection of $100 \%$ isodose and the surface of the cylinder. When this is done, we can measure the distance from the top of the cylinder to the bottom edge of the grid. In this way we can calculate the volume of the cylinder that is enclosed by the $100 \%$ isodose curve. The difference in volume covered by $100 \%$ isodose surface and the volume of the calculated part of the cylinder gives the actual volume $V_{100, t}$ of tissue irradiated with at least $100 \%$ of the prescribed dose.

Results and Discussion. For a cylinder with a diameter of $3.5 \mathrm{~cm}$ and a normalization point $5 \mathrm{~mm}$ from the top, the mean value of $\mathrm{V}_{100, t}$ was $7.17 \mathrm{~cm}^{3}$ and the standard deviation was $2.84 \mathrm{~cm}^{3}$. For a cylinder with a diameter of $3 \mathrm{~cm}$ and a normalization point of $5 \mathrm{~mm}$, the mean was $11.19 \mathrm{~cm}^{3}$ and the standard deviation was $6.18 \mathrm{~cm}^{3}$. For a cylinder with a diameter of $3.5 \mathrm{~cm}$ and a normalization point of $1 \mathrm{~cm}$ from the top, the mean value of $\mathrm{V}_{100, t}$ was $26.53 \mathrm{~cm}^{3}$ and the standard deviation was $5.46 \mathrm{~cm}^{3}$. For a cylinder with a diameter of $3 \mathrm{~cm}$ and a normalization point of $10 \mathrm{~mm}$, the mean value was $26.76 \mathrm{~cm}^{3}$ and the standard deviation $4.38 \mathrm{~cm}^{3}$. Comparison of confidence intervals suggests that in both cases the volume covered by $100 \%$ isodose originated in the same population $(\mathrm{p}=0.061)$. The volume of $100 \%$ isodose calculated for the whole grid, $\mathrm{V}_{100}$, in average is $(3.65+/-1.26)$ times the $\mathrm{V}_{100, t}$ for a $3.5 \mathrm{~cm}$ cylinder and $(3.34+/-1.45)$ for a $3 \mathrm{~cm}$ cylinder. 


\title{
Comparison of dose distribution verification methods for high-precision radiotherapy
}

\author{
Kinga Polaczek-Grelik, Aneta Kawa-Iwanicka
}

NU-Med Cancer Diagnosis and Treatment Centre Katowice, Katowice, Poland

https://doi.org/10.21175/rad.abstr.book.2021.36.8

Introduction. The verification of the absolute dose distribution planned in external beam treatment planning system (TPS) could be realized either indirectly - as a calculation of monitor units / irradiation time or a measurement of a fluence map, or directly - through the measurement of dose distribution during the treatment plan realization by a medical accelerator. Only the second one enables to review at the same time the physical ability of a particular accelerator to implement often a very complex treatment plan and the exactness of linac model implemented in TPS. The final evaluation of the dosimetric verification of a treatment plan is influenced by the applied measurement method itself and by its spatial resolution. The difference between dose-to-water and dose-to-medium calculations, and tissues inhomogeneities (tumor location) on the results of dosimetric plan verification are studied to set a path towards better accuracy of photon radiotherapy.

Materials and methods. Stereotactic body radiation therapy (SBT) plans were taken into analysis. These plans incorporate small field issue and steep dose gradients, making the dose distribution verification more demanding. Lung, bone and pancreas tumours cases were under consideration to study the tissue inhomogeneities problem.

RT plans were prepared in Monaco v.5.11 treatment planning system and realized on Elekta Synergy linac with Agility multileaf collimator ( $5 \mathrm{~mm}$ leaf width).

The following dosimetric methods were compared:

- 2D measurement of dose distribution in the plane of ionization chambers of MatriXXEvolution ${ }^{\circledR}$ device placed in MultiCube ${ }^{\mathrm{TM}}$ phantom,

- $\quad 3 \mathrm{D}$ dose reconstruction in Compass ${ }^{\circledR}$ dosimetric system on the base of measurements using MatriXXEvolution ${ }^{\circledR}$ (1024 ion chambers array) and Dolphin ${ }^{\circledR}$ (transmission 1513 ion chambers) devices.

The following criteria of global gamma index: $\mathrm{DTA}=3 \mathrm{~mm} / \Delta \mathrm{D}=3 \%, \mathrm{DTA}=2 \mathrm{~mm} / \Delta \mathrm{D}=3 \%$, $\mathrm{DTA}=2 \mathrm{~mm} / \Delta \mathrm{D}=2 \%$ and $\mathrm{DTA}=1 \mathrm{~mm} / \Delta \mathrm{D}=5 \%$ were used to investigate the influence of spatial resolution of applied methods as well as the precision of absolute dose reconstruction.

Results and conclusions: Gamma criterion of $3 \mathrm{~mm} / 3 \%$ not allows to investigate the differences between planned and delivered dose distribution in SBRT modality - tighter criteria should be used. However, the value of DTA criterion which could be fulfilled in a particular plan verification is closely correlated with the resolution of the measurement device, i.e. the distance between detectors and volume-averaging phenomenon.

It is possible to fulfill the tolerance of 95\% passing points using SBRT gamma index criterion even using measuring device with limited resolution, provided that the dose is reconstructed with an independent algorytm by the dosimetric system having its own linac model (independent from the TPS). 3D map of gamma index on patient CT is the best choice for plan verification - visualization of the regions where the result of dose deposition is uncertain helps to conduct RT in a safer way. 


\title{
Prostate cancer FLASH therapy treatments with electrons of high energy: A feasibility study
}

\author{
Gaia Franciosini1,2, Patrizia De Maria²,3, Micol De Simoni1,4, Marta Fischetti4,5, \\ Michela Marafini4,6, Massimiliano Pacilio7, Damiana Rubeca5, Alessio Sarti4,5, \\ Angelo Schiavi4,5, Adalberto Sciubba5,8, Vincenzo Tombolini9, Marco Toppi5,8, \\ Giacomo Traini4, Antonio Trigilio ${ }^{1,4}$, Vincenzo Patera5,4
}

\author{
1 Sapienza University of Rome, Physics, Rome, Italy \\ 2 INFN Istituto Nazionale di Fisica Nucleare, Sezione di Roma I, Rome, Italy \\ 3 Sapienza University of Rome, Post-graduate School in Medical Physics Department of Medico-Surgical \\ Sciences and Biotechnologies, Rome, Italy \\ 4 INFN Istituto Nazionale Fisica Nucleare, Sezione di Roma I, Rome, Italy \\ 5 Sapienza University of Rome, Scienze di Base e Applicate per l'ingegneria, Rome, Italy \\ 6 Museo Storico della Fisica e Centro Studi e Ricerche, Rome, Italy \\ 7 Azienda Ospedaliera-Universitaria Policlinico Umberto I, Unità di Fisica Sanitaria, Rome, Italy \\ 8 INFN Istituto Nazionale Fisica Nucleare, Sezione dei Laboratori di Frascati, Rome, Italy \\ 9 Sapienza University of Rome, Scienze Radiologiche- Oncologiche e Anatomo Patologiche, Rome, Italy
}

\section{https://doi.org/10.21175/rad.abstr.book.2021.36.9}

Over the past decades, technological advances have improved the efficacy of radiation therapy (RT) cancer treatment. Nevertheless, the RT potential is still limited by normal tissue complications: the absorbed dose in fact peaks few $\mathrm{mm}$ after entering the patient body while, afterwards, it exponentially decreases irradiating the healthy tissues placed along the beam direction before or after the target volume. On the other side the specific interactions of charged particles with matter (resulting in the so-called Bragg Peak distribution) can help in sparing the healthy tissues, while keeping the same target volume coverage. This characteristic has been already exploited in Particle Therapy (PT) which uses protons and carbon ion beams. Very High Energy Electron (VHEE) beams have thus been explored as well as other charged particles, as they have enough energy to reach deep seated tumours. However, an advanced and effective technology with dedicated facilities (acceleration lines, treatment rooms, gantry, etc.) is needed to treat with proton, carbon or electron beams, resulting in a high cost per treatment.

In this context, FLASH radiotherapy represents a promising perspective, thanks to the achievable significant reduction of the healthy tissue damage: several pre-clinical studies recently claimed that the toxicity in healthy tissues related to tumour treatments can be significantly reduced (from $80 \%$ down to $60 \%$ ), while keeping the same efficacy in cancer killing, if the dose rate is radically increased $(\sim 10 \mathrm{~Gy} / \mathrm{s}$, or even more) with respect to conventional treatments ( 0.01 Gy/s). Several laboratories are performing first tests of FLASH irradiation. A possible limitation in delivering protons at FLASH rates is currently represented by the treatment delivery approach performed, so far, using the pencil beam scanning approach: to fully cover the planned treatment volume several seconds are needed and this reduces the dose-rate which may be far too low to induce the FLASH effect. For what concern photon beams instead, the implementation is currently limited by technical aspects related to the delivery of FLASH intensities.

In this contribution an approach to cure deep-seated tumors exploiting the FLASH effect, using VHEE beams with energies above $50 \mathrm{MeV}$ will be shown. In particular the contribution will focus on how recent developments in electron beam therapy could reshape the landscape of prostate treatments. An example of VHEE treatment optimised combining an accurate Monte Carlo simulation with a simple modelling of the FLASH effect will be compared with conventional RT. The results demonstrate that FLASH therapy with VHEE beams of $70-130 \mathrm{MeV}$ could represent a valid alternative to standard RT allowing a better sparing of the healthy tissues surrounding the tumour, in the framework of an affordable technological development. 


\title{
Boron compound as a sensitizer to proton radiation for cancer cells in vitro
}

\author{
Luiza Garaeva1,2,3, Vladimir Burdakovi,2, Eva Kuus',3,4, \\ Andrey Volnitskiy,2, Alina Garina1,3, Hau Nhan Tran3, Georgy Andreev4, \\ Andrey Lubinskiy4, Konstantin Shabalin',2, Victor Ezhov ${ }^{1}$, \\ Dmitry Lebedevi,2, Nicolay Verlovi,2, Andrey Konevega ${ }^{1,2,3}$, Tatiana Shtam ${ }^{1,2}$
}

\author{
1 Petersburg Nuclear Physics Institute named by B.P. Konstantinov of National Research Center "Kurchatov \\ Institute", Gatchina, Russia \\ 2 National Research Center "Kurchatov Institute", Moscow, Russia \\ 3 Peter the Great St. Petersburg Polytechnic University, St. Petersburg, Russia \\ 4 Proton therapy center MIBS, St. Petersburg, Russia
}

\section{https://doi.org/10.21175/rad.abstr.book.2021.36.10}

Radiotherapeutic methods play a significant role in cancer treatment. Proton therapy (PT) is being administered since many years to the patients with different types of cancer, including brain tumors. Due to the remarkable physical properties of proton beam, allowing for the delivery of the radiation to specific and very precise brain volume with almost no exit dose, protons are particularly appropriate for these tumors. Proton boron capture therapy (PBCT) for suggested few decades ago for cancer treatment based on specific nuclear reaction of proton captured with boron-11 atoms in the Bragg peak region. The effect of boron is based on the reaction of nuclear fusion. Emission of Linear Energy Transfer (LET), $\alpha$-particles, related by proton-boron fusion reaction in the Bragg peak region causes irreparable clustered DNA damages expected increase in effectiveness in killing of the tumor cell. Nowadays there are few publications showing effectiveness of proton beam irradiation boron-11-containing (sodium borocaptate, $\left.\left(\left(\mathrm{B}_{12} \mathrm{H}_{11} \mathrm{SH}\right) \mathrm{Na}_{2}\right), \mathrm{BSH}\right)$ cancer cells [1,2]. Single number of studies on this issue and the contradictoriness of the obtained results raises question on the effectiveness of BSH sensitization in proton irradiation, as well as the physical nature of the described effect.

In present study, we have examined the possibility to enhance biological effects of proton irradiation in boron-containing cultured glioma cells.

At first, the cytotoxicity of BSH for two glioma cell lines (A172, Gl-Tr) and normal epithelial cells (DF-2) was determined. The cytotoxic effects of $\mathrm{BSH}$ at $0-1000 \mathrm{ppm}$ of ${ }^{11} \mathrm{~B}$ concentration have been studied by the AlamarBlue $®$ Assay after 7 and 18 hours of incubation with BSH. Sodium borocaptate appears to be non-toxic for the normal morphology DF-2 epithelial cells. Insignificant toxicity of BSH was determined for glioma cells at the concentration of boron more than $160 \mathrm{ppm}$. There was no effect of BSH on cell cycle distribution in both glioblastoma cell lines after 7 and 18 hours of incubation in the presence of sodium borocaptate.

Next, the glioma cells were irradiated using clinical proton beam setup in the presence of BSH. Glioma cells were preincubated with BSH (boron concentration $80 \mathrm{ppm}$ ) for 7 and 18 hours and then irradiated in 6-well plates in a medium containing boron with a clinical proton beam at Bragg peak in a dose range of o-6 Gray. Control cells were cultured and irradiated under the same conditions, but without the addition of BSH to the culture medium. To test whether the physical nuclear reaction results in an enhancement of the cell death by high-energy proton beam irradiation the glioma cell lines were also irradiated with graded doses o-6 Gray using lift-up type ${ }^{60} \mathrm{Co}$-based $\gamma$-ray setup. The radiation sensitivity was determined by the AlamarBlue $\AA$ Assay and cologenic assays using crystal violet for staining. As expected, no radio sensitizing effect of borocaptate was observed for both cell lines under gamma irradiation. Some ability of BSH to activate the cancer cell death with protons at the Bragg peak irradiation was shown for one glioma cell line (Gl-Tr). At the same time, for the second cell line (A172), the observed effect of increased cell death upon irradiation of cells with protons in the presence of borocaptate was even less significant. In any case, the sensitizing effect of borocaptate detected for glioma cells upon irradiation with a proton beam was significantly weaker than the described effect for prostate cancer cells [2].

Based on the results obtained in this study and on previously published data, it is obvious that the prospects of boron proton capture therapy still require further study and confirmation.

\section{References}

[1] D.-K. Yoon et al., Appl. Phys. Lett. 105, 223507 (2014).

[2] G.A.P. Cirrone et al., Sci. Rep. 8, 1141 (2018).

[3] A. Mazzone et al, Eur. Phys. Plus. 134:361 (2019)

Acknowledgments: The work was carried out with the support of NRC "Kurchatov Institute" (order no. 1363). 


\title{
Case report of a renal cell carcinoma skin metastasis treated locally with contact brachytherapy
}

\author{
Petar Chakalaroski, Violeta Klisarovska, Zoran Stefanovski
}

University Clinic for Radiotherapy and Oncology - Skopje, Skopje, North Macedonia

https://doi.org/10.21175/rad.abstr.book.2021.36.11

Introduction. Skin malignancies have been a radiotherapy target for a long period of time. Usually radiotherapy treats non-melanoma skin cancers, particularly as an adjuvant treatment or when primary surgical treatment fails to confine the malignant spread. Brachytherapy due to its characteristics is an ideal radiotherapy treatment of choice. However, sometimes also metastatic tumors arise in superficial and skin tissues.

Such was the case of 68 years old male patient, who came to our clinic - It was a case of a metastatic recidivant tumor. Tumor was presented in patient's right supraorbital arch with dimensions of $4 \times 3 \times 3 \mathrm{~cm}$ and it was vulnerable to touch. Patient had pain originating from his right supraorbital arch. Histopathology showed to be a secondary (metastatic) tumor of a previously surgically treated kidney cancer (Clear Cell renal adenocarcinoma).

Objective. Due to patient's comorbidities there were no options for surgical or systemic oncological treatment, thus it was decided to reduce the pain by treating the tumor with brachytherapy in palliative setting.

Methods. Patient received a total of 5 fractions (once a week) over the course of 28 days of HDR-brachytherapy by using a surface contact applicator (BA 40mm) with a prescribed dose of $7 \mathrm{~Gy}$ on a depth of $7 \mathrm{~mm}$ and an estimate surface dose of $11.6 \mathrm{~Gy}$ per fraction. Patient received total brachytherapy dose of $35 \mathrm{~Gy}$ (an estimated surface dose of $58 \mathrm{~Gy})$ and estimated EQD2 dose $(\alpha / \beta=5)$ of $60 \mathrm{~Gy}$.

Results/Clinical outcome. On the first check-up patient reported that the pain subsided to a significant level of decrease (almost non-existent) while locally there was an evident tumor shrinkage with a small residual mass present (measuring several millimeters across) situated at the bottom of the operative lacunar defect. Sadly, patient did not show on the next appointments.

Conclusion. We may conclude that even a considered radioresistant tumors with low rate $\alpha / \beta$ ratios like in this presenting case, can be successfully treated with HDR-brachytherapy (if it is site applicable). 


\title{
Hydrogeochemical background of ${ }^{222} \mathrm{Rn}$ in groundwaters from fore-sudetic monocline area in Poland
}

\author{
Agata Kowalska, Piotr Maciejewski \\ Wrocław University of Science and Technology, Faculty of Geoengineering, Mining and Geology, Wrocław, \\ Poland
}

\section{https://doi.org/10.21175/rad.abstr.book.2021.37.1}

This work presents results of radon activity concentration in groundwaters from fore-sudetic monocline area in Poland. Authors collected 52 samples of groundwaters from polish part of fore-sudetic monocline, located in western Poland. This area, as a geological unit, is completely covered by Tertiary and Quaternary sediments. Water samples were taken mostly from private wells, therefore it could confirm that all these waters are shallow circulationg waters and their reservoir rocks are younger than the monocline itself.

In this research ${ }^{222} \mathrm{Rn}$ activity concentrafion were measured, as well as ${ }^{226} \mathrm{Ra}$ activity concentration and basic water parameters were checked (ORP, $\mathrm{pH}, \mathrm{T}$, conductivity). To determine activities concentrations of both isotopes authors used LSC method.

Range of ${ }^{222} \mathrm{Rn}$ and ${ }^{226} \mathrm{Ra}$ activities concentrations in these groundwaters were respectively from 0.18 to $19.78 \mathrm{~Bq} / \mathrm{dm}^{3}$ and from 0.08 to $0.75 \mathrm{~Bq} / \mathrm{dm}^{3}$ (in the case of ${ }^{226} \mathrm{Ra}$ only three results were above the limit of detection which is $0.05 \mathrm{~Bq} / \mathrm{dm}^{3}$ ). According to Przylibski's classiffication, most of these waters are poor-radon waters (24 samples), 19 of them are free-radon waters, and 9 samples represents low-radon water type. The range of hydrogeochemical background of radon in groundwaters from fore-sudetic monocline is from o to $11.0 \mathrm{~Bq} / \mathrm{dm}^{3}$. 


\title{
Assessment of geogenic radon potential based on granulometric analysis of soil in Vojvodina region
}

\author{
Robert Lakatoš1, Sofija Forkapićčn, Selena Samardžićć, \\ Kristina Bikit-Schroeder², Aleksandra Mihailovićć, Jordana Ninkov3
}

\author{
1 University of Novi Sad, Faculty of Technical Sciences, Novi Sad, Serbia \\ 2 University of Novi Sad, Faculty of Sciences, Department of Physics, Novi Sad, Serbia \\ 3 Institute of Field and Vegetable Crops, Novi Sad, Novi Sad, Serbia
}

\section{https://doi.org/10.21175/rad.abstr.book.2021.37.2}

A method based on soil permeability estimates and radon in soil concentration measurements was used to assess radon potential for the first time in our region. The methodology for soil permeability evaluation proposed in the literature is based on determining the weight percentage of fine fraction (particles smaller than $65 \mu \mathrm{m}$ ) in soil samples and classifying of soil permeability index for the radon according to it. Soils with the weight percentage of the fine fraction below $15 \%$ were classified as high permeable, in the range $15-65 \%$ as medium permeable, and in the case of the fine fraction above $65 \%$ as low permeable. A soil grain size distribution analysis was performed by laser diffraction dry method using a particle size analyzer Mastersizer 2000, Malvern Instruments. Radon concentrations in soil gas were measured in situ by an active RAD7 radon monitor with stainless soil gas probe inserted down to a depth of $80 \mathrm{~cm}$ using grab protocol which considers sniff mode of pumping and four five-minute cycles of counting. Combining the obtained results for soil permeability indexes and soil radon concentrations, the radon potential in the Vojvodina region was estimated at 14 locations, indicating that the radon risk is in the low and medium class. Parent material for all analyzed types of soil is loess - a typical example of soil with a high amount of the fine fraction which corresponds to low permeability, but in great dependence on soil humidity. Two additional locations in the Southern part of Serbia were included also in this study with a different geological substrate. 


\title{
Comparison of indoor radon measurement methods
}

\author{
Ivana Vukanac, Igor Čeliković, \\ Gordana Pantelić, Miloš Živanović, Jelena Krneta Nikolić
}

"Vinca" Institute of Nuclear Sciences, National Institute of the Republic of Serbia, University of Belgrade, Belgrade, Serbia

\section{https://doi.org/10.21175/rad.abstr.book.2021.37.3}

Radon is the radioactive gas originating from the decay chain, mainly uranium and thorium series. The main source of population exposure to ionizing radiation (more than $80 \%$ ) is the natural radioactivity. Radon and its short lived progeny contribute with more than $50 \%$ to the radiation dose received by the general population from all sources, and the World Health Organization has recognized radon as the second most important cause of lung cancer.

The European Council has laid down a basic safety standards (BSS, Council Directive 2013/59/Euratom) for the protection against the dangers arising from exposure to ionizing radiation, obliging member states, among other, to investigate the exposure of members of the public and workers to indoor radon, to develop a radon action plan and to inform the public about radon levels they are exposed to. As a consequence, this has led to increased number of indoor radon measurements in recent years.

There are numerous methods for indoor radon measurement which can be performed either by direct measurement of radon or indirectly by measuring radon progeny, with or without radon presence. The choice of method used depends on the purpose of the measurements, available instrumentation and time.

In order to assess the reliability of indoor radon measurement methods available in "Vinca" Institute of Nuclear Sciences, comparative measurement were conducted. Indoor radon concentration was measured in four working rooms in Vinca Institute, two offices and two laboratory premises, in the period of October-November 2020. In all locations continuous radon measurements were performed with tree different active measurement devices: RTM1688-2 and Radon Scout from SARAD GmbH, and RadonEye from RadonFTLAB, while integrated radon measurements were conducted with charcoal canisters. In addition, Equilibrium Equivalent Radon Concentration EERC was measured with radon progeny monitor RPM2200 (SARAD GmbH). Measurement by all active devices was performed simultaneously and lasted around 4 days with 1 hour sampling time, while measuring time for charcoal canisters was 2 days. In one office and one laboratory measurements were repeated and six sets of results were obtained.

Very good agreement between results obtained by active devices was observed, with correlation ranging from $0.72-0.98$. A certain discrepancy was observed between averaged radon concentration from active devices and results from charcoal canisters only in one office. 


\title{
High indoor radon concentration in residential houses
}

\author{
Dusica Spasic, Ljiljana Gulan
}

University of Pristina, Faculty of Sciences and Mathematics, Kosovska Mitrovica, Serbia

https://doi.org/10.21175/rad.abstr.book.2021.37.4

People are mainly exposed to radon in indoor environments. Exposure to radon and its decay products is the second leading cause of lung cancer, and there is a strong synergistic effect with smoking. The most significant source of indoor radon is usually the soil around building; radon penetrates into houses directly from the soil through fractures and cracks. In some cases the source of higher radon could be household water from drilled wells or exhalation from building materials. In the European legislation on radiation protection (Directive 2013/59/EURATOM), EU Member States are obliged to identify areas/buildings (dwellings and workplaces) in which the mean annual radon concentration exceeds the relevant national reference level and to provide information locally and nationally about radon exposure and corresponding health risks. Currently, the situation with COVID-19 pandemic, and the people's tendency to stay more indoors in low air exchange rate conditions, enhanced risk to radon exposure.

In this paper we presented the results of continuous indoor radon measurements performed in a residential house near the town Kosovska Mitrovica (mining area of Trepča). The house was built in the 1970's with the walls from stones taken from the nearby hills and without concrete slab. Radon detector Airthings Corentium Home was used for radon measurements in living room and bedroom during spring 2020 (from March to June). Diurnal radon concentrations varied from $368-1290 \mathrm{~Bq} / \mathrm{m} 3$ in living room and from $796-2330 \mathrm{~Bq} / \mathrm{m} 3$ in bedroom during measuring period. These variations are presumably influenced by meteorological conditions, lifestyle and occupant's habits. The values of long-term average radon for living room and bedroom were $709 \mathrm{~Bq} / \mathrm{m} 3$ and $1377 \mathrm{~Bq} / \mathrm{m} 3$, respectively. These values are higher than these proposed by the WHO $(100 \mathrm{~Bq} / \mathrm{m} 3)$, and by the Council of the European Union of $300 \mathrm{~Bq} / \mathrm{m} 3$ (Directive 2013/59/EURATOM).

In the near future, an implementation of effective remedial solutions should be provided for this house. Also, more new houses should be included in radon monitoring in the mining area, because according to these and some previously published results, a high radon area is suspected. 


\title{
Characterization of radioactivity in thermal springs and the implementation of the European Directive 2013/59/Euratom
}

\author{
Ana Sofia Silva, Maria de Lurdes Dinis
}

\begin{abstract}
CERENA-Polo FEUP - Centre for Natural Resources and the Environment, FEUP - Faculty of Engineering, University of Porto, Porto, Portugal
\end{abstract}

\section{https://doi.org/10.21175/rad.abstr.book.2021.37.5}

The therapeutic properties of thermal mineral waters have been known since ancient times and they are quite famous as spa resorts. However, in many cases, these waters contain a certain degree of natural radioactivity attributable to elements from uranium and thorium natural decay series. Tectonics (and particularly active structures, for thermal waters, in a geological sense) are closely related to the occurrence of thermal springs. Radioactivity in thermal water is due mostly to dissolved radon and radon daughters. Spatial variations in radon concentration are generally related to changes in geology, soil type, and structural controls. High radon concentrations in groundwater and soil are observed above structural planes like fault, fracture, fold, and lineaments.

In the northern region of Portugal, the occurrence and circulation of waters are controlled by deep faults generated in metamorphic and/or magmatic environments while in the central and southern Portugal, the mineral and spring waters are mostly influenced by rock dissolution processes.

In Portugal there are 33 different types of bottled water, 18 of which are classified as natural mineral water and the remaining as spring water. The majority of these waters are sulphurous, with low mineralization, with some bicarbonate and gasocarbonic.

The purpose of this study was to characterize the levels of radioactivity in thermal springs located in the Centre region of Portugal, within the structural unit of the Hesperic Massif. A total of 54 of water samples were collected from springs/boreholes located at 16 Portuguese spas. Radon concentrations were measured by liquid scintillation counting techniques LSC) using an ultra-low-level spectrometer (Quantulus 1220), at the Natural Radioactivity Laboratory of the Department of Earth Sciences, University of Coimbra, Portugal. Radon levels in thermal water presented a wide range of variation, from values below the detection limit up to a maximum of approximately $7000 \mathrm{~Bq} / \mathrm{L}$.

Gamma activity levels were also assessed with a GAMMA SCOUT® (GS3) equipment for gamma dose rates $(\mu \mathrm{Sv} / \mathrm{h})$, with hourly readings during 45 days. The results ranged from 0.083 to $1.484 \mu \mathrm{Sv} / \mathrm{h}$. According to the European Directive 2013/59/EURATOM the reference level applying to indoor external exposure to gamma radiation emitted by building materials, in addition to outdoor external exposure, shall be $1 \mathrm{mSv}$ per year. In average, the value of $1 \mathrm{mSv} / \mathrm{y}$ was exceeded in all monitored locations.

The results are to be compared with those of an ongoing follow-up study.

Acknowledgments: This work was financially supported by: Base Funding - UIDB/04028/2020 of the Research Center for Natural Resources and Environment - CERENA - funded by national funds through the FCT/MCTES (PIDDAC). 


\title{
Using experts' consensus to evaluate the Readiness Factors of Lean Implementation in Healthcare
}

\author{
Eni Bushi', Klejda Hudhra1, Andreas Kakouris², Delina Xhafaj3
}

\author{
1 University of Medicine Tirana, Faculty of Medicine, Department of Pharmacy, Tirana, Albania \\ 2 University of the Aegean, Department of Business Administration, Chios, Greece \\ 3 Albanian University, Faculty of Medical Sciences, Department of Pharmacy, Tirana, Albania
}

\section{https://doi.org/10.21175/rad.abstr.book.2021.38.1}

Rationale, aims, and objectives. The challenge for increased quality of healthcare services, coupled with the pressure to achieve more with limited resources, has oriented healthcare organizations towards new and more efficient management strategies of providing care, such as Lean. However, the study of readiness factors for implementing Lean in this sector is still poor and remains one of the reasons for Lean failure. This research aims to identify and validate the readiness factors that influence the successful and sustainable implementation of Lean in healthcare organizations.

Methodology. A systematic review of the literature was utilized to generate a synthesis of readiness factors that help healthcare organizations to implement Lean. The multidisciplinary search engine Google Scholar is used to identify and collect articles published during the period from 2005 to 2020. Only articles from peer-reviewed journals of credible publishing houses were selected. After carefully analyzing the content of 22 selected articles, 11 readiness factors were identified, as the main contributors to the success of Lean implantation in healthcare. This work is further extended by using the Delphi survey to validate the identified factors from the perspective of 10 healthcare experts. The internal consistency of survey questions was assessed using Cronbach's alpha. The consensus level was measured through Kendall's Coefficient of Concordance W.

Results. The systematic review of the literature, revealed 11 readiness factors as important contributors to the success of Lean implantation in healthcare. After 3 rounds of Delphi, a moderate consensus (Kendall's $\mathrm{W}=0.519 ; \mathrm{P}<0.001$ ) were achieved among experts' panel over 9 readiness factors, from which 7 factors were extracted from the literature (including support and commitment of leadership, organizational culture, and effective communication), while 2 factors were derived from experts' suggestions indicating the presence of strategic approaches in sustaining Lean improvement over time.

Conclusion. Investigation of organizational readiness to implement Lean will help healthcare organizations to be better prepared in their initiation of the Lean journey, and will also enable the sustainability of improvements.

Limitations. Firstly, a further understanding is needed whether readiness factors should be set before adopting Lean tools, or tools and readiness factors should be implemented synchronously. Secondly, it would be interesting to discover if an organization needs to be more focused on some or all the factors. 


\title{
$\mathrm{Re}^{3+}$-doping and $\mathrm{pH}$ effect on the redox activity of nanoceria $\left(\mathrm{CeO}_{2-\mathrm{x}}\right)$
}

\section{Vladyslav Seminko, Pavel Maksimchuk, Ganna Grygorova, Yuri Malyukin}

\author{
Institute for Scintillation Materials NAS of Ukraine, Kharkiv, Ukraine
}

https://doi.org/10.21175/rad.abstr.book.2021.38.2

Ceria $\left(\mathrm{CeO}_{2-\mathrm{x}}\right)$ nanoparticles are widely studied by both physicists and biologists due to their surprising redox activity, which was shown recently in the number of papers providing a basement for the new interdisciplinary field of nanoenzymology. However, detailed mechanism of ceria antioxidant action is absent up to now due to large number of possible intermediate steps of $\mathrm{CeO}_{2-\mathrm{x}}$ interaction with reactive oxygen species (ROS) of different kinds and absence of consensus between different authors. Here we have used a new approach to the investigation of catalase-mimetic activity (ability to decompose hydrogen peroxide) and dynamics of ceria- $\mathrm{H}_{2} \mathrm{O}_{2}$ interaction based on the combined luminescence and absorption spectroscopy techniques.

We have determined that the redox activity of nanoceria and $\mathrm{Re}^{3^{+}}$-doped ceria colloidal nanoparticles depends strongly on the $\mathrm{pH}$, size of nanoparticle, type and concentration of doped ions, and presence of laser irradiation. While $\mathrm{Re}^{3+}$ doping improves the efficiency of scavenging of hydroxyl radicals $\left({ }^{\circ} \mathrm{OH}\right.$ ), it leads to sufficient slowing-down of $\mathrm{H}_{2} \mathrm{O}_{2}$ decomposition. This effect was explained by the main role of $\mathrm{Ce}^{3+} / \mathrm{Ce}^{4+}$ ratio in ${ }^{\circ} \mathrm{OH}$ scavenging, and $\mathrm{Ce}^{3+}-\mathrm{V}_{0}-\mathrm{Ce}^{3+}$ complexes in the processes of hydrogen peroxide decomposition. The role of $\mathrm{Ce}^{3+}-\mathrm{V}_{0^{-}}-\mathrm{Ce}^{3+}$ complexes is similar to the role of active sites of enzymes. The continuous laser irradiation with specific wavelengths leads to formation of additional oxygen vacancies, and so, of additional $\mathrm{Ce}^{3+}-\mathrm{V}_{0}-\mathrm{Ce}^{3+}$ complexes providing better antioxidant activity of pre-irradiated ceria nanoparticles. $\mathrm{pH}$ decrease from 7.5 to 4.5 changes the type of redox activity of nanoceria from antioxidant ( ${ }^{\circ} \mathrm{OH}$ scavenging) to prooxidant ( $\mathrm{OH}$ generation).

Overall, our results have shown that the dynamics of nanoceria-oxidant interaction is determined by the interplay of the content of $\mathrm{Ce}^{3+}-\mathrm{V}_{0}-\mathrm{Ce}^{3+}$ complexes in nanoceria, oxidant concentration in colloidal solution and temperature. Doping by different ions and/or size variation open the way to modify the antioxidant properties of nanoceria, and to obtain nanoparticles with improved catalaselike activity in both water solutions and biological environment.

Acknowledgments: This research was supported by National Research Foundation of Ukraine, Grant № 2020.02/0052 for Leading and Young Scientists Research Support and National Academy of Sciences of Ukraine (Grant for Young Scientists "Development of redox-active nanomaterials with controlled pro-/antioxidant activity based on oxide nanocrystals", 0120U100725). 


\title{
Preliminary criticality safety assessment for a nuclear fuel production facility
}

\section{Ibrahim Alrammah}

King Abdulaziz City for Science and Technology (KACST), Riyadh, Saudi Arabia

\author{
https://doi.org/10.21175/rad.abstr.book.2021.38.3
}

The purpose of performing a criticality safety assessment is to ensure protection from criticality accident consequences, initially by preventing the accident. The application of criticality safety techniques across fuel cycle operations at a varied range of facilities is a crucial safety obligation. These operations involve several diverse processes and technologies. Based on the process, the fissile materials could be in the form of solid, liquid or vapor phases. Fuel cycle operations also involve the transport of fuel and waste.

There are various stages of the nuclear fuel cycle where it is required to consider applying criticality safety assessment in plant operations during the plant lifetime. Criticality safety must be confirmed in all stages and processes including fissile fuel, specifically: enrichment, fabrication, handing, transport and storage, spent fuel reprocessing, waste processing and disposal. Sub-criticality is attained by controlled operation within the operating license. Key parameters comprise: degree of moderation, control of reflectors, geometry and fissile mass. Controls required for production operations include material cross-over for grinding, machining, cutting, handling and storage of fissile fuel.

Fuel processing facilities usually have large quantities of fissile material. Criticality safety assessment is typically founded on a deterministic method in which a set of conservative assumptions and procedures for fissile material facilities is applied.

This paper uses methods of Buckling Conversion and Surface Density to estimate the critical size of the system and to define limiting conditions for fissile arrays of units.

The buckling conversion technique is founded on results of the diffusion equation which provide a relationship between the spatial neutron flux distribution in a neutron multiplying medium and a parameter named "geometric buckling". Buckling conversion method is useful to execute inclusive parametric assessments on criticality safety parameters for uncomplex fissile systems. This method is also applicable to convert the neutron leakage characteristics among various simple geometries -such as: a sphere, cylinder, or slab-, provided that critical data is available for a certain system.

Surface density technique can be utilized to determine the subcritical center-to-center spacing for regular and irregular shapes of fissile materials that are staged or stored in finite array arrangements where the array dimension in one direction is limited. This method is effectively applicable to various process configurations in which each unit is substantially subcritical.

The present paper highlights the potential hazards related to criticality and associated with a nuclear fuel production facility. It presents the determination of subcritical limits as part of nuclear criticality safety which is fundamental for ensuring that processes involving fissile material remain safe 


\title{
Achieved results in radiation protection education of the Center for Permanent Education INN "Vinča"
}

\section{Mirjana Đurašević ${ }^{1}$, Zorica Obradović1, Katarina Rajković ${ }^{2}$}

\author{
1 Vinca Institute of Nuclear Sciences, National Institute of Republic of Serbia, University of Belgrade, \\ Belgrade, Serbia \\ 2 Academy of Professional Studies Sumadija-Department in Kragujevac, Kragujevac, Serbia
}

\section{https://doi.org/10.21175/rad.abstr.book.2021.38.4}

The Center for permanent education (CPE) of the "Vinča" Institute of nuclear sciences is a unique educational institution in Serbia. The main objective of the CPE is an additional and complementary education of various profiles of specialists who use radioactive isotopes and sources of ionizing radiation in their everyday work in accordance with the Law on Radiation and Nuclear Safety and Security of Serbia.

Application of ionizing radiation in various fields of activity (industry, medicine, scientific research) carries the appropriate risks to the health and safety of those who work with ionizing radiation sources. It is therefore crucial that the handling of radioactive sources and ionizing radiation generators, as well as the handling of nuclear waste, be in line with certain safety standards aimed at protecting those exposed to ionizing radiation. Center for Permanent Education is authorized by the Directorate for Radiation and Nuclear Safety and Security of Serbia to provide additional training and education for occupationally exposed persons and persons responsible for implementing measures radiation protection. Accordingly, the activities of the Center are focused on the organization of different types of courses, depending on the category of a particular radiation activity. Permanent education program defines: additional and appropriate training before beginning work with radiation sources, training of persons responsible for implementing radiation protection measures, and periodic renewal of knowledge provided for all occupationally exposed persons at least once in five years. The special type of courses refers to courses for work with open sources of radiation, where longer and more detailed training is required.

The aim of this work is to present the achieved results related to the number of held courses and the number of participants between 2011 and 2020, as well as years with the most and the least held courses and years with the largest and the smallest number of participants. These numbers have been changed in accordance with the requirements of legal regulations related to radiation protection. The Center for permanent education has worked since 1956, but information about all courses and candidates have been kept within database program only since 2011. During that period, 1503 different courses were held related to radiation protection. The most courses (272) were held in 2018 and the least (43) in 2011. Regarding the number of candidates, 5805 candidates completed the courses in the period between 2011 and to 2020. The largest number of candidates (1036) were in 2018, and the smallest (185) in 2013. The CPE aims to improve the quality of its services and strives to continuously follow and introduce modern and new methods of education for the next period. 


\title{
Controlled redox-activity of small orthovanadate nanocrystals
}

\author{
Kateryna Hubenko, Pavel Maksimchuk, Svetlana Yefimova
}

Institute for Scintillation Materials of NAS of Ukraine, Kharkiv, Ukraine

https://doi.org/10.21175/rad.abstr.book.2021.38.5

Nanocrystals, which promote generation of reactive oxygen species (ROS) and stimulate the onset of oxidative stress responsible for the development of a variety of adverse conditions, are of great prospect for various nanomedicine applications. ROS generation in semiconducting nanocrystals is a complex process, the efficiency of which is governed by both nanocrystals characteristics (size, morphology, etc.) and external conditions, such as type of irradiation. On the other hand, in nanocrystals characterized by high amount of structural defects, for instance, oxygen vacancies $\left(\mathrm{V}_{\mathrm{O}}\right)$ in oxide nanocrystals, such defects could serve as traps for charge carriers and are responsible for ROS production in nanocrystals even without UV irradiation (in the dark). In our previous works, we have shown that $\mathrm{GdYVO}_{4}: \mathrm{Eu}^{3+}$ nanocrystals exhibit photo-catalytic activity (ROS production) at both UV-light irradiation [1, 2] and in the dark condition [3].

In this work, we report controlled redox-activity of small $(\mathrm{d}=2 \mathrm{~nm}) \mathrm{GdYVO}_{4}: \mathrm{Eu}^{3+}$ nanocrystals in aqueous solutions and biological environment. It has been revealed that depending on pre-treatment conditions (exposure to UV light or storage in the darkness), the same nanocrystals exhibit pro- or antioxidant properties.

Pro-/antioxidant activity in aqueous solutions was evaluated by UV-vis spectroscopy by the ascorbic acid (AA) oxidation test, and hydroxyl radical $(\cdot \mathrm{OH})$ generation analysis. Lipid oxidation under the effect of nanocrystals have been also analyzed.

Multi-functional $\mathrm{GdYVO}_{4}: \mathrm{Eu}^{3+}$ nanocrystals with controlled redox-activity are assumed to be a new theranostic agent in radiotherapy.

\section{References}

[1] S.L. Yefimova, P.O. Maksimchuk, V.V. Seminko, N.S. Kavok, V.K. Klochkov, K.A. Hubenko, A.V. Sorokin, I.Yu. Kurilchenko, Yu.V. Malyukin, Janus-Faced Redox Activity of $\mathrm{LnVO}_{4}: \mathrm{Eu}^{3^{+}}(\mathrm{Ln}=\mathrm{Gd}$, Y, and La) Nanoparticles, J Phys Chem C, no. 123, pp. 15323-15329, 2019.

[2] K. Hubenko, S. Yefimova, T. Tkacheva, P. Maksimchuk, I. Borovoy, V. Klochkov, N. Kavok, O. Opolonin, Yu. Malyukin, Reactive oxygen species generation in aqueous solutions containing $\mathrm{GdVO}_{4}: \mathrm{Eu}^{3+}$ nanoparticles and their complexes with methylene blue, Nanosc Res Lett, no. 13, pp. 100-109, 2018.

[3] P. O. Maksimchuk, S. L. Yefimova, K. O. Hubenko, V. V. Omelaeva, N. S. Kavok, V. K. Klochkov, A. V. Sorokin, Y. V. Malyukin. Dark Reactive Oxygen Species Generation in $\operatorname{ReVO}_{4}: \mathrm{Eu}^{3+}(\mathrm{Re}=\mathrm{Gd}, \mathrm{Y})$ Nanoparticles in Aqueous Solutions, Phys Chem C, no. 124, pp. 3843-3850, 2020.

Acknowledgments: This research was supported by National Research Foundation of Ukraine, Grant № 2020.02/0052 for Leading and Young Scientists Research Support. 


\title{
Pro-oxidant action of preliminarily UV-activated orthovanadate nanocrystals
}

\author{
Pavlo Maksymchuk, Kateryna Hubenko, \\ Vladyslav Seminko, Svetlana Yefimova
}

Institute for Scintillation Materials NAS of Ukraine, Kharkiv, Ukraine

\section{https://doi.org/10.21175/rad.abstr.book.2021.38.6}

The well-known ability of dielectric nanocrystals to generate reactive oxygen species (ROS) under $\mathrm{X}$-ray excitation enhances sufficiently the efficiency of cancer radiotherapy making such nanoparticles a promising material for biological and medical applications. However, the ionizing radiation used during radiotherapy has a great disadvantage of being dangerous not for cancer cells only, but for whole organism as well. For solving this problem, we propose orthovanadate nanocrystals with pro-oxidant activity without any external stimuli after preliminary irradiation of nanoparticles with UV-light [1].

Pro-oxidant action of preliminarily UV-activated orthovanadate nanocrystals is shown to be associated with superoxide and hydroxyl radical generation and depended on the size of nanocrystals and, consequently, on the concentration of surface defects [2]. Oxide nanocrystals contain high amount of structural defects (such as oxygen vacancies) which could serve as traps for charge carriers and are responsible for ROS production in nanocrystals even long time after irradiation. For small 2 $\mathrm{nm}$ preliminarily $\mathrm{UV}$-activated $\mathrm{GdYVO}_{4}: \mathrm{Eu}^{3+}$ nanocrystals, strong superoxide and hydroxyl radical production has been revealed, whereas for larger preliminarily UV-activated $\mathrm{GdVO}_{4}: \mathrm{Eu}^{3+}$ nanocrystals, low level of superoxide and negligible one of hydroxyl radicals production have been detected during the storage of the samples in the darkness.

Orthovanadate nanocrystals with «dark» redox activity after preliminarily UV-activation could be prospective for the application in biomedical area as multifunctional therapeutic agents for cancer treatment.

\section{References}

[1] S.L. Yefimova, P.O. Maksimchuk, K.A. Hubenko et al, Colloids and Surfaces A, 2019, 577, 630-636.

[2] Pavel O. Maksimchuk, Svetlana L. Yefimova, Kateryna O. Hubenko et al, Phys. Chem. C, 2020, 124, 6, 3843-3850.

Acknowledgments: This research was supported by National Research Foundation of Ukraine, Grant № 2020.02/0052 for Leading and Young Scientists Research Support. 


\title{
Spices as acid-base indicators in volumetric analysis
}

\author{
Aida Šap̌anin, Emina Ramić, Selma Korać, Mirsada Salihović
}

\author{
University of Sarajevo, Faculty of Pharmacy, Sarajevo, Bosnia and Herzegovina
}

\section{https://doi.org/10.21175/rad.abstr.book.2021.38.7}

Commonly used indicators for acid-base titrations are synthetic, and this work was focused to identify the natural indicators. The highly colored pigments obtained from selected spices (sumac, curry, chilli, saffron, cumin and red pepper) are found to exhibit color changes with variation of $\mathrm{pH}$. These spices extracts (methanolic and aqueous) were found to perform well in titrating strong acidstrong base rather than in weak acid-strong base. The sharp contrast between their colours in acid and base made these spices extracts suitable for use as acid-base indicators. In addition, spectrophotometric determination of the pKa values of selected spices extracts were performed. As these spices extracts have very simple, cost-effective, environment friendly extraction procedure and excellent performance with sharp colour change in end points of the titrations, it would be possible to replace the standard indicators with these natural indicators. Obtained results shows that the analytical potential of the plant extracts is very promising as seen in its application in acid-base volumetric analysis

Keywords: Spice, acid-base indicator, pKa, volumetric analysis 


\title{
Environmentally appropriate technologies for the final disposal of radioactive waste of the Belarusian NPP
}

\author{
Mikhail Zhemzhurov, Natallia Kuzmina
}

The Joint Institute for Power and Nuclear Research-Sosny of NAS of Belarus, Minsk, Belarus

https://doi.org/10.21175/rad.abstr.book.2021.38.8

The Strategy for radioactive waste management of the Belarusian NPP envisages construction of the first stage of the radioactive waste disposal facility (RWDF) by 2030.

According to the IAEA methodological approach, drafting of the conceptual design of the RWDF constitutes the initial phase of its designing. Within the framework of the pre-investment stage of the mentioned RWDF construction according to the agreement between the Ministry of Energy and the scientific institution "JIPNR - Sosny", the task to develop a conceptual design of disposal facility for very low-level, low-level and short-lived intermediate-level radioactive waste (RW) of the Belarusian NPP, generated in the process of its operation and decommissioning, based on the reference technologies and existing projects, was accomplished.

The St. Petersburg subsidiary of "Special scientific and production association "Eleron" VNIPIET took part in the development of the project.

Currently, a conceptual design for the construction of the RWDF determines the first stage of construction separately.

Within the conceptual design of the RWDF, standard solutions were developed without reference to a particular location of the facility, but taking into account the engineering-geological conditions in the areas of competitive and optimal RWDF sites. The means of conditioning the RW removed from the NPP were substantiated to ensure the compliance of the final RW packaging forms with the general acceptance criteria for disposal, and technological solutions for management of the waste at the point of disposal were suggested.

Methods and structures of the RW disposal constructions, as well as basic technical solutions for the auxiliary buildings, facilities and systems of engineering support of the specified RWDF, ensuring safe and economically feasible disposal of RW generated from the two nuclear power units during 60 years of operation and at the NPP decommissioning, were justified based on the regulatory requirements and the alternative reference technologies and the existing projects.

The suggested conceptual RWDF design included the following:

- $\quad$ evaluation of its radiation safety during operation and long-term safety after closure (during the post-operational period);

- a list of possible accidents at RWDF and necessary emergency measures was identified;

- limits for RWDF safe operation on radionuclides emissions and discharges were justified;

- $\quad$ suggestions for the organization of the radiation control and monitoring system for the RW disposal system during the RWDF operation and the post-operational period were presented.

It is clear that the suggested RWDF concept ensures safe RW disposal in the period of potential danger of the RW, taking into account possible external impacts of natural and man-made origin.

The aggregate technical and economic parameters of the suggested RWDF project were evaluated both in general and for the first stage of construction. 


\title{
ELV series accelerators and their applications in radiation technological processes
}

\author{
Evgeny Domarov, Nikoly Kuksanov, Denis Vorobiev, Michail Golkovskii, Yurii \\ Golubenko, Sergy Fadeev, Alexy Korchagin, Rustam Salimov, Alexy Semenov
}

BINP, Novosibirsk, Russia

https://doi.org/10.21175/rad.abstr.book.2021.38.9

BINP SB RAS produces industrial electron ELV accelerators for radiation technologies. The main innovative applications are cross-linking of the insulation of wires and cables, production of heat shrinkable tubes and films, production of foamed polyethylene, and radiation modification of rubber blanks for tires.

Model range of ELV accelerators covers the range of electron energies from $0.3 \mathrm{MeV}$ to $2.5 \mathrm{MeV}$, beam power up to $100 \mathrm{~kW}$, and beam current to $100 \mathrm{~mA}$. All models have similar concept but differ in overall dimensions, length of accelerator tube, and the number of high-voltage rectifying sections. This makes it easy to adapt accelerators to the requirements of technology. The system of automated control of accelerators and communication with technological lines is constantly developing.

ELV accelerator with an energy of $0.3-0.5 \mathrm{MeV}$ and beam current up to $130 \mathrm{~mA}$ was developed and tested. The accelerator is compact in size and installed in local steel shielding. Electron beam is extracted through a two-window extraction system with one titanium foil $180 \mathrm{~mm}$ wide.

Usually, extraction device with double-side irradiation is used. However, big size cables required high-energy accelerators. To reduce electron energy and improve the absorbed dose azimuthal inhomogeneity, four-side and ring irradiation systems were developed in BINP.

In addition, an accelerator was developed and tested with new extraction device of a focused electron beam into the atmosphere. At present, various experiments are run using the installation with device for the extraction of concentrated electron beam into the atmosphere for the production of nanopowders, surfacing of powder materials for metals, etc.

New accelerators of the ELV type are also being developed. In particular, the accelerator with an energy of $3 \mathrm{MeV}$ and a power of $100 \mathrm{~kW}$ is currently being designed. 


\section{ILC for ${ }^{90} \mathrm{Sr}$ determination in feedstuffs}

\section{Paweł Czerski ${ }^{1}$, Izabela Chmielewska² ${ }^{2}$ Jarosław Rachubik}

1 National Veterinary Research Institute - State Research Institute, Puławy, Poland

2 Główny Instytut Górnictwa, Katowice, Poland

\section{https://doi.org/10.21175/rad.abstr.book.2021.38.10}

Currently, the development of new research opportunities and the acquisition of new knowledge force laboratories to test and improve their competences. Meeting these expectations, laboratories regularly take part in various comparative tests, such as PT tests. These tests are probably the most important control elements for laboratories. Unfortunately, it is not always possible to perform PT tests within the scope of research on radioactivity. This is mainly due to the lack of matrices available for comparisons. Therefore, the authors made an attempt to conduct ILC research. Both laboratories have been successfully developed their work on measuring radioactivity in food for many years. This time, the ILC studies comprised the comparison of ${ }^{90} \mathrm{Sr}$ contents in feedstuff samples. The organizer prepared two feed samples (barley grain, powdered whey). All the samples were spiked with a known quantity of ${ }^{\circ} \mathrm{Sr}$ isotope. The liquid scintillation spectrometry method was used to determine the activity of ${ }^{90} \mathrm{Sr}$ in the prepared items. The methodology of the ISO/IEC 43-1: 1997 guide were used to evaluate the results of the interlaboratory comparative tests. On the basis of the ILC comparisons, it was found that the results of the comparisons were satisfactory. Obtaining reliable results allows for better monitoring and control of the presence of ${ }^{90} \mathrm{Sr}$ in the environment. 


\title{
Irradiation resistance of elastomeric composites based on NR/CSM blend and waste rubber powder
}

\author{
Gordana Markovic, Milena Marinović Cincović ${ }^{2}$, Vojislav Jovanović3, \\ Suzana Samaržija Jovanović3, Vesna Teofilović44, Marija Marković5, \\ Jelena Pavličević4, Jaroslava Budinski Simendić4
}

1 Tigar A.D., Pirot, Serbia

2 University of Belgrade, Vinča Institute of Nuclear Sciences, Vinča, Belgrade, Serbia

3 University in Priština - Kosovska Mitrovica, Faculty of Sciences, Kosovska Mitrovica, Serbia

4 University of Novi Sad, Faculty of Technology, Novi Sad, Serbia

5 University of Niš, Faculty of Electronic Engineering, Niš, Serbia

\section{https://doi.org/10.21175/rad.abstr.book.2021.38.11}

Effect of waste rubber particles (WTR) size on elastomers based on natural rubber and chlorosulfonated polyethylene rubber $(\mathrm{NR} / \mathrm{CSM}=50: 50)$ was studied. Two types of WTR with different particle size $(150-250 \mu \mathrm{m}$ and $450-600 \mu \mathrm{m})$ were used. The content of carbon black was constant but the content of WTR was varied from o to $40 \mathrm{phr}$. The compounds were prepared by tworoll mill. The crosslinked materials were obtained in hydraulic press. The irradiation of prepared composite materials was carried out using 6oCo gamma source at ambient temperature with the dose rate $10 \mathrm{kGyh}^{-1}$ and different total absorbed dose (100, 200 and $\left.400 \mathrm{kGy}\right)$. The morphology of the samples was examined by scanning electron microscopy. It was assessed that WTR with smaller size got uniform dispersion. Mechanicals properties were assessed for irradiated and not-irradiated samples. It was estimated that the waste rubber particles with smaller size affected better enhancement of the mechanical properties. However, tensile strength and elongation at break decreased with increasing of WTR content. 
TITLE: Book of Abstracts

EDITOR: Prof. Dr. Goran S. Ristić

PROOF-READING: Saša Trenčić, MA

TECHNICAL EDITING: Saša Trenčić, MA

COVER DESIGN: Vladan Nikolić, PhD

YEAR OF PUBLISHING: 2021

PUBLISHER: RAD Centre, Niš, Serbia

FOR THE PUBLISHER: Prof. Dr. Goran S. Ristić

CD BURNING AND COPYING: RAD Centre, Niš, Serbia

PRINT RUN: Electronic edition - 50 CDs (CD-R)

ISBN: 978-86-901150-2-O

www.rad-conference.org

CIP - Каталогизација у публикацији - Народна библиотека Србије, Београд

$539.16(048)(0.034 .2)$

$57+61(048)(0.034 .2)$

INTERNATIONAL Conference on Radiation in Various Fields of Research (9;2021; Herceg Novi)

Book of abstracts [Elektronski izvor] / Ninth International Conference on Radiation in Various Fields of Research, R9, [RAD 2021], June 14 18, 2021, Herceg Novi, Montenegro ; [editor Goran S. Ristić]. - Niš :RAD Centre, 2021 (Niš : RAD Centre). - 1 elektronski optički disk (CD-ROM) ; $12 \mathrm{~cm}$

Sistemski zahtevi: Nisu navedeni. - Nasl. sa naslovne strane dokumenta. - Tiraž 50.

ISBN 978-86-901150-2-O

а) Јонизујуће зрачење - Дозиметрија - Апстракти b) Биомедицина Апстракти 


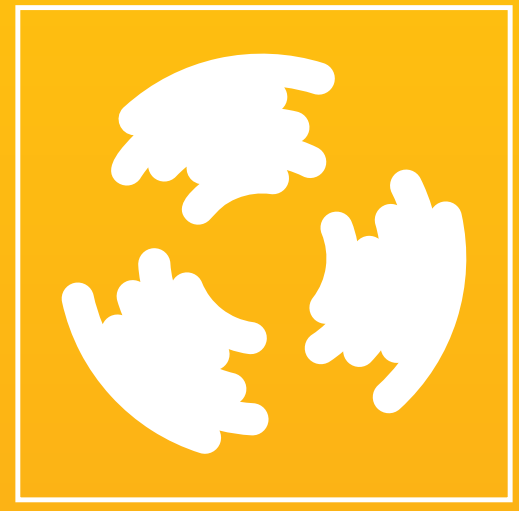

rad-conference.org 